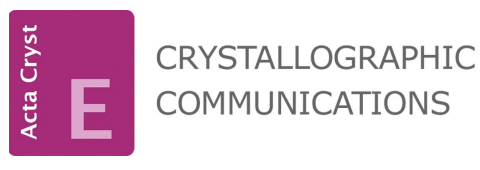

ISSN 2056-9890

Received 7 January 2020

Accepted 7 February 2020

Edited by W. T. A. Harrison, University of Aberdeen, Scotland

Keywords: crystal structure; OLED; optoelectronic; phosphorescence.

CCDC references: $1982629 ; 1982628$; $1982627 ; 1982626$

Supporting information: this article has supporting information at journals.iucr.org/e
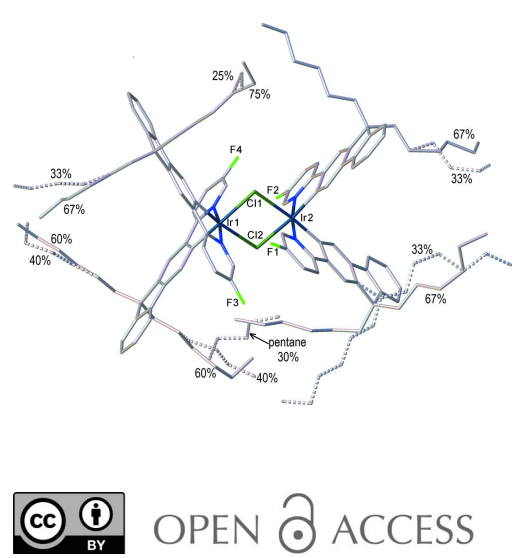

\section{Phosphorescent mono- and diiridium(III) complexes cyclometalated by fluorenyl- or phenyl-pyridino ligands with bulky substituents, as prospective OLED dopants}

\author{
Ahmed $\mathrm{M}^{\prime}$ hamedi $^{\mathrm{a}, \mathrm{b}}$ and Andrei S. Batsanov ${ }^{\mathrm{c} *}$

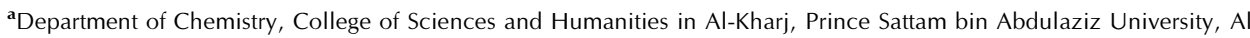 \\ Kharj 11942, Saudi Arabia, 'Laboratory of Structure Determination, Development, and Application of Molecular Material, \\ Department of Chemistry, University of Abdelhamid Ibn Badis BP 227, Mostaganem 27000, Algeria, and ${ }^{\mathbf{c}}$ Department of \\ Chemistry, University of Durham, South Road, Durham DH1 3LE, United Kingdom. *Correspondence e-mail: \\ a.s.batsanov@durham.ac.uk
}

The crystal structures of tris[9,9-dihexyl-2-(5-methoxypyridin-2-yl- $\kappa N)-9 H$ fluoren-3-yl- $\left.\kappa C^{3}\right]$ iridium pentane monosolvate, $\left[\operatorname{Ir}\left(\mathrm{C}_{31} \mathrm{H}_{38} \mathrm{NO}\right)_{3}\right] \cdot \mathrm{C}_{5} \mathrm{H}_{12}$, (I), di- $\mu_{2}$-chlorido-bis\{bis[2-(5-fluoropyridin-2-yl)-9,9-dihexyl-9H-fluoren-3-yl]iridium\} pentane 0.3-solvate, $\left[\operatorname{Ir}_{2}\left(\mathrm{C}_{30} \mathrm{H}_{35} \mathrm{FN}\right)_{4} \mathrm{Cl}_{2}\right] \cdot 0.3 \mathrm{C}_{5} \mathrm{H}_{12}$, (II), di- $\mu_{2}$-cyanatobis\{bis[9,9-dihexyl-2-(5-methoxypyridin-2-yl)-9H-fluoren-1-yl]iridium\} pentane monosolvate, $\left[\mathrm{Ir}_{2}\left(\mathrm{C}_{31} \mathrm{H}_{38} \mathrm{NO}\right)_{4}(\mathrm{NCO})_{2}(\mathrm{NCO})_{2}\right] \cdot \mathrm{C}_{5} \mathrm{H}_{12}$, (III), and $\left\{\mu-N, N^{\prime}-\right.$ bis[3,5-bis(trifluoromethyl)phenyl]oxamidato\}bis(bis\{2-[4-(2,4,6-trimethylphenyl)pyridin-2-yl]phenyl- $\kappa^{2} C^{1}, N^{\prime}$ iridium)-chlorobenzene-pentane (1/2.3/0.4), $\left[\operatorname{Ir}_{2}\left(\mathrm{C}_{20^{-}}\right.\right.$ $\left.\left.\mathrm{H}_{19} \mathrm{~N}\right)_{4}\left(\mathrm{C}_{18} \mathrm{H}_{6} \mathrm{~F}_{12} \mathrm{~N}_{2} \mathrm{O}_{2}\right)\right] \cdot 2.3 \mathrm{C}_{6} \mathrm{H}_{5} \mathrm{Cl} \cdot 0.4 \mathrm{C}_{5} \mathrm{H}_{12}$, (IV), synthesized in the quest for organic light-emitting devices, were determined. The bis- $\mu_{2}$-chloro and bis- $\mu_{2}{ }^{-}$ cyanato complexes have $\Delta \Delta$ and $\Lambda \Lambda$ configurations of the distorted octahedral Ir centres in racemic crystals, whereas the oxamido complex has a centrosymmetric (meso) structure with the $\Delta \Lambda$ configuration. The bridging oxamido moiety has a nearly planar anti geometry. All structures show substantial disorder of both host molecules and solvents of crystallization.

\section{Chemical context}

Over the two decades since the pioneering work of Baldo et al. (1998), cyclometalated Ir ${ }^{\mathrm{III}}$ complexes have been developed as emitters (phosphorescent dopants) for organic light-emitting diodes (OLEDs) or light-emitting electrochemical cells (LECs) (Li et al., 2018; Adeloye, 2019). These complexes are structurally and synthetically versatile. A large contribution of the Ir orbitals to the excited state results in efficient spin-orbit coupling, allowing in principle the harvesting of all the electrogenerated excitons and a $100 \%$ internal quantum efficiency of electro-phosphorescence. Because the luminescent properties are metal-ligand based, colour fine-tuning can be achieved by choosing the ligands (of which phenylpyridine, ppy, is by far the most important) and substituents to systematically vary the electronic and steric properties, or by incorporation of ancillary ligands. Originally the research focused on monoiridium species, because the di-iridium complexes then known (mostly bis- $\mu$-chloro bridged) proved generally poor emitters because of their electron-withdrawing bridges; for example $\left[\operatorname{Ir}(\mathrm{ppy})_{2} \mathrm{Cl}\right]_{2}$ has a quantum yield 80 times lower than fac$\operatorname{Ir}(\text { ppy })_{3}$ (King et al., 1985). 


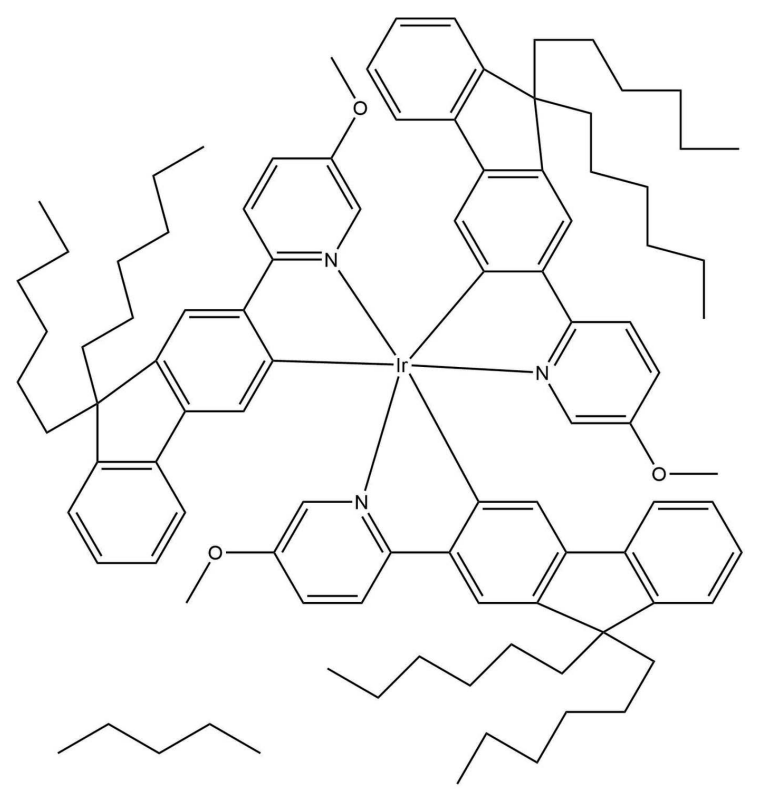

(I)

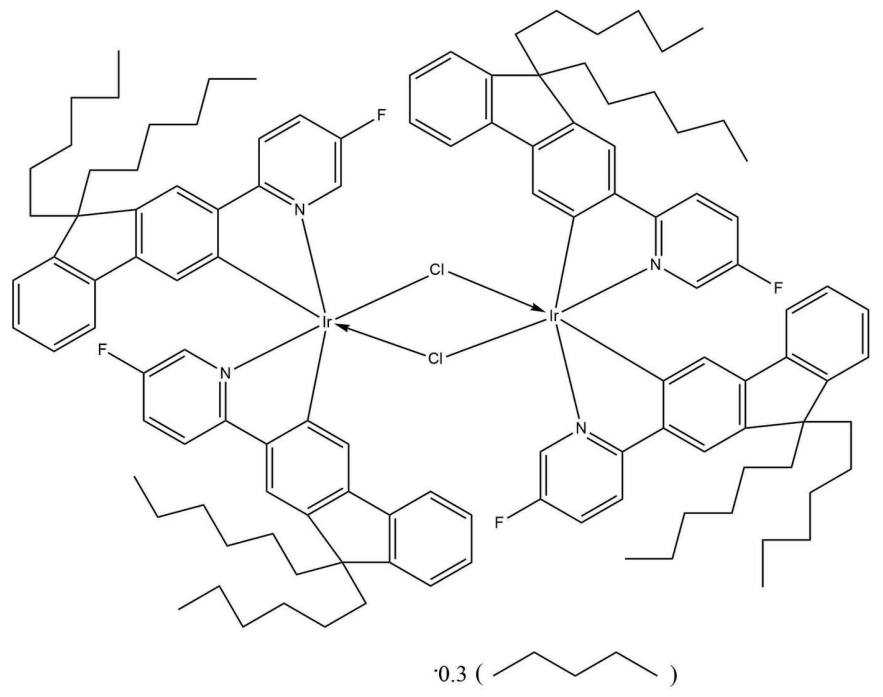

(II)

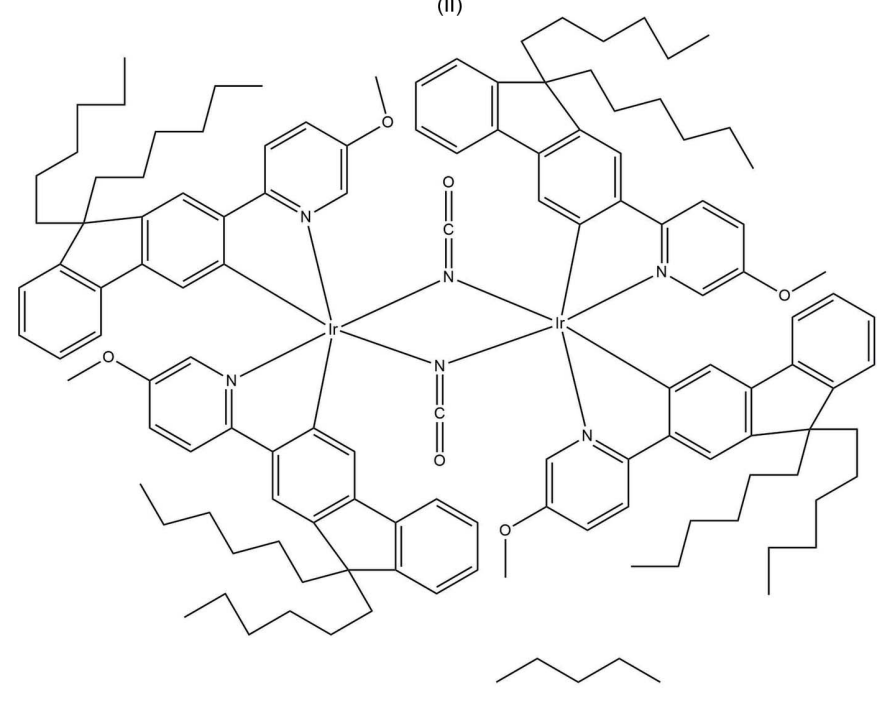

(III)

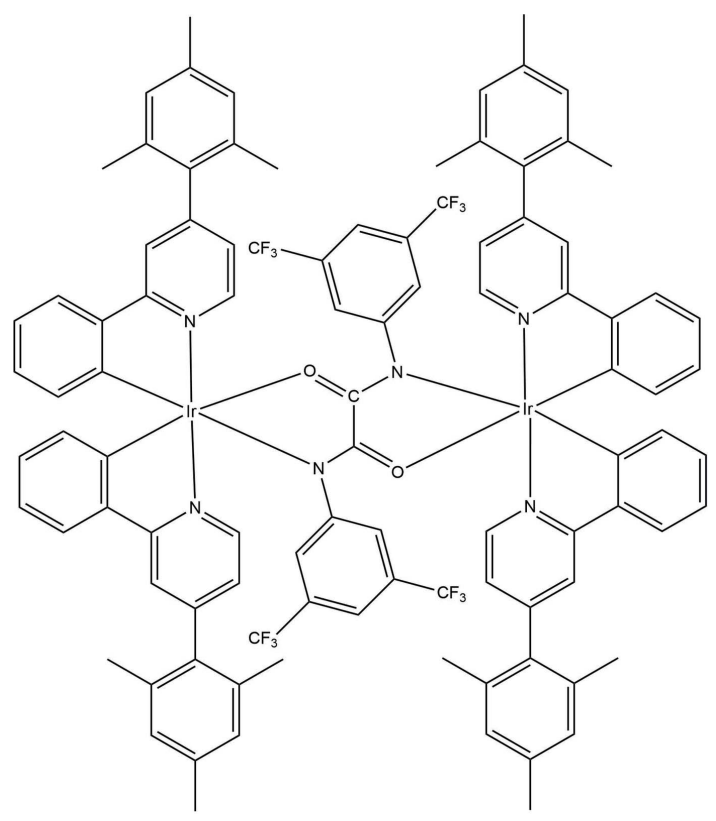<smiles>CCCCCCC</smiles>

The mono-iridium complex (I), has shown external quantum efficiency (EQE) in devices of $4.8 \%$ and current efficiency of $14 \mathrm{~cd} \mathrm{~A}^{-1}$, compared to $0.9 \%$ and $3 \mathrm{~cd} \mathrm{~A}^{-1}$ for complex (II), which is nevertheless exceptionally good for its class (M'hamedi et al., 2012). Better results $(2.6 \%$ and $8 \mathrm{~cd} \mathrm{~A}^{-1}$ ) were obtained with complex (III), although NCO ligands are also electron-withdrawing. Later some bridges were found to be propitious for high-yield phosphorescence, particularly diarylhydrazido (Congrave et al., 2017), oxamido and diaryloxamido ligands (Graf et al., 2013; M'hamedi et al., 2017). Here we report the X-ray crystal structures of complexes (I)-(IV), in relation to their optoelectronic properties.

It is noteworthy that the optoelectronic properties of such complexes depend strongly on the position of the substituents in the pyridine ring. Whereas both electron-donating (OMe) or electron-withdrawing (F) substituents are known to lower the device efficiency of mono-iridium complexes - probably by perturbing the electron and hole mobilities and hence reducing the exiton formation (Al-Attar et al., 2011) - a substituent para to the $\mathrm{N}$ atom lowers the device efficiencies drastically (M'hamedi et al., 2012).

\section{Structural commentary}

The mononuclear complex (I) (Fig. 1) crystallized as a pentane monosolvate, like its isomer $(\mathrm{I} a)$, which had the methoxy substituent in the 4- rather than 5-position of the pyridine ring (M'hamedi et al., 2012), and shows a rather similar molecular geometry. Both structures show extensive disorder; however, while in $(\mathrm{I} a)$ it is confined to the $n$-hexyl chains and the 


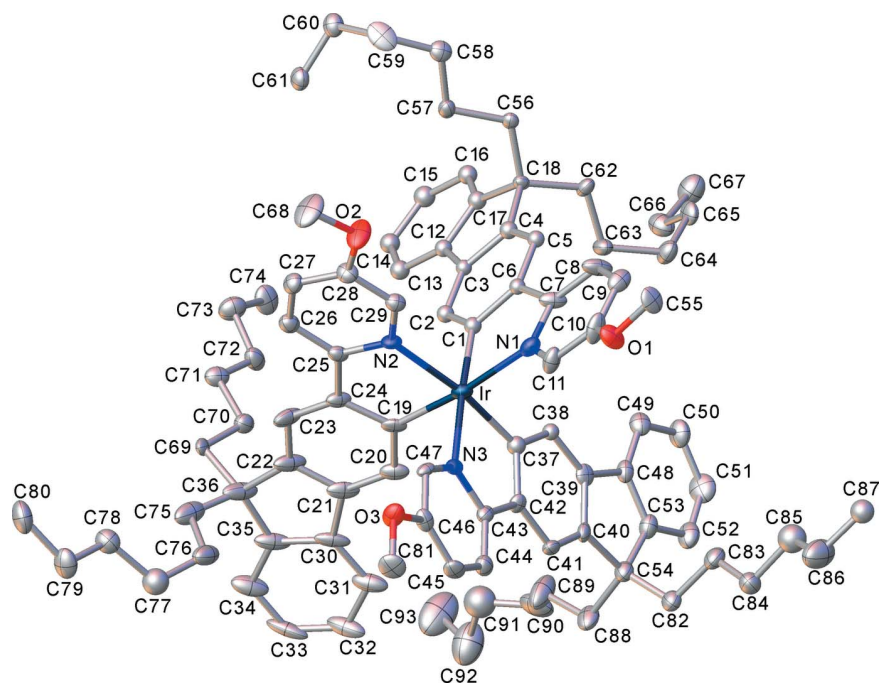

Figure 1

The molecular structure of (I), showing $50 \%$ probability displacement ellipsoids for non-H atoms. The disorder and all $\mathrm{H}$ atoms are omitted for clarity.

solvent, in (I) the disorder (between two half-occupied positions) involves one ligand entirely, most of another one (except the pyridine ring) and both $n$-hexyl chains of the third ligand, as well as the pentane molecule (Fig. 2). The Ir coordination in (I) is distorted fac-octahedral, with each $\mathrm{Ir}-\mathrm{N}$ bond in a trans orientation to an $\mathrm{Ir}-\mathrm{C}$ bond, confirming the earlier assessment from NMR spectra (M'hamedi et al., 2012). The mean distances $\mathrm{Ir}-\mathrm{N}=2.13$ (1) and $\mathrm{Ir}-\mathrm{C}=2.02$ (1) $\AA$ are slightly longer than those in ( $\mathrm{I} a)$ (2.119 and $2.006 \AA$, respectively) and similar to those in the unsubstituted $\operatorname{Ir}(\text { ppy })_{3}$, both in its trigonal (Breu et al., 2005) and tetragonal polymorphs (Berger et al., 2010; Takayasu et al., 2013; Wang et al., 2013).



Figure 2

Disorder in the structure of (I); $\mathrm{H}$ atoms are omitted. All disordered fragments have $50 \%$ occupancies

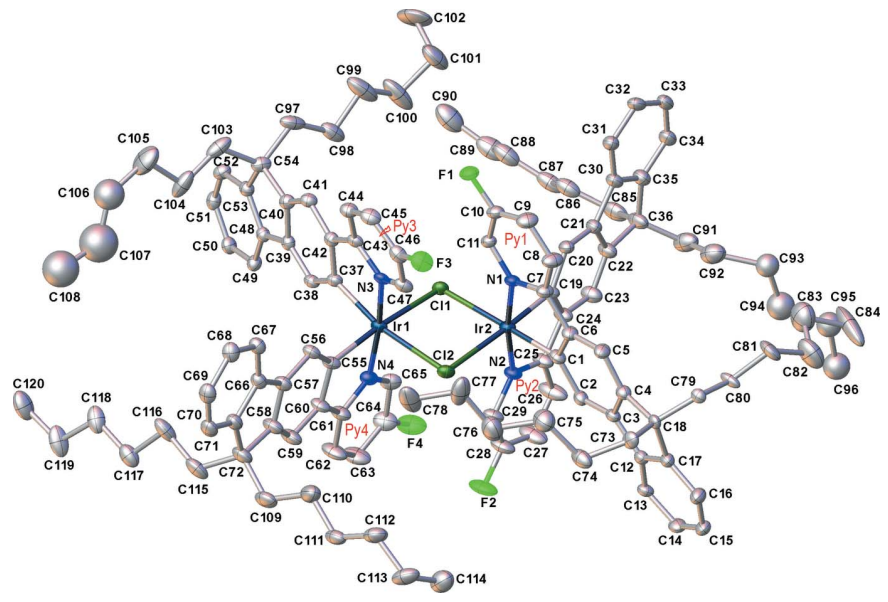

Figure 3

The molecular structure of (II), showing $30 \%$ probability displacement ellipsoids and the notation of pyridyl rings. The disorder and all $\mathrm{H}$ atoms are omitted.

Complex (II) crystallized from chlorobenzene/pentane as a non-stoichiometric pentane solvate, in a triclinic structure with one independent molecule in a general position. In contrast, its analogues with non-substituted (II $a$ ) and 4-fluorinated (II b) pyridyl rings (M'hamedi et al., 2012), which crystallized from the same solvents as the chlorobenzene solvates, as well a $\mathrm{CH}_{2} \mathrm{Cl}_{2}$ /water solvate of II $a$ ( $\left.\mathrm{II} a^{\prime}\right)$ (Bettington et al., 2006), all form isomorphous orthorhombic crystals (space group Fddd) with the crystallographic molecular symmetry 222. Notably, the analogue of (II) with 4-methoxy-substituted pyridine rings (IIc) also crystallized in a triclinic structure, with the lattice parameters and host molecules' packing broadly similar to those of (II), albeit with more solvent of crystallization (two chlorobenzene and three pentane molecules per formula unit) and correspondingly expanded unit-cell volume (M'hamedi et al., 2012). The molecular geometry of (II) (Fig. 3), as that of (IIc), only approximately conforms to $C_{2}$ symmetry: the $\mathrm{Ir}_{2} \mathrm{Cl}_{2}$ system is not planar, as in (II $a$ ) and (II $b$ ), but folded by $7.7(1)^{\circ}$ (II) or $8.26(3)^{\circ}$ (IIc) along the $\mathrm{Cl} \cdots \mathrm{Cl}$ vector. In (II), the iridium atoms have distorted octahedral coordination with the $\mathrm{N}$ atoms trans to one another and both $\mathrm{C}$ atoms trans to bridging chloro ligands. The mean bond lengths $\mathrm{Ir}-\mathrm{Cl}=$ 2.507 (2), $\mathrm{Ir}-\mathrm{N}=2.036$ (5) and $\mathrm{Ir}-\mathrm{C}=1.998(6) \AA$ are not substantially different from those in (II $a$ ) [2.498 (1), 2.038 (5) and 1.993 (6) §, respectively] or (II $b)$ [2.498 (2), 2.016 (7) and 1.977 (8) $\AA$, respectively], although the $\operatorname{Ir} 1 \cdots \operatorname{Ir} 2$ distance in (II) $[3.740$ (1) $\AA]$ is appreciably longer than in (II $a$ ) or (II $b$ ) (3.675 or $3.674 \AA$, respectively). Both Ir centres in the molecule of (II) have the same configuration: either $\Delta \Delta$ or $\Lambda \Lambda$, the crystal being racemic. The $n$-hexyl chains of the ligands show complicated disorder (Fig. 4), being superimposed upon a pentane molecule of crystallization with a partial (ca 30\%) occupancy.

Near-parallel alignment of pyridyl rings coordinated to different Ir atoms [interplanar angles $\mathrm{Py} 1 / \mathrm{Py}=13.4(2)^{\circ}$, Py2/Py4 = 6.3 (3) ${ }^{\circ}$, see Fig. 3], may seem propitious to intramolecular $\pi-\pi$ stacking, which is important for optoelectronic properties in these phases. However, with the parallel slip 


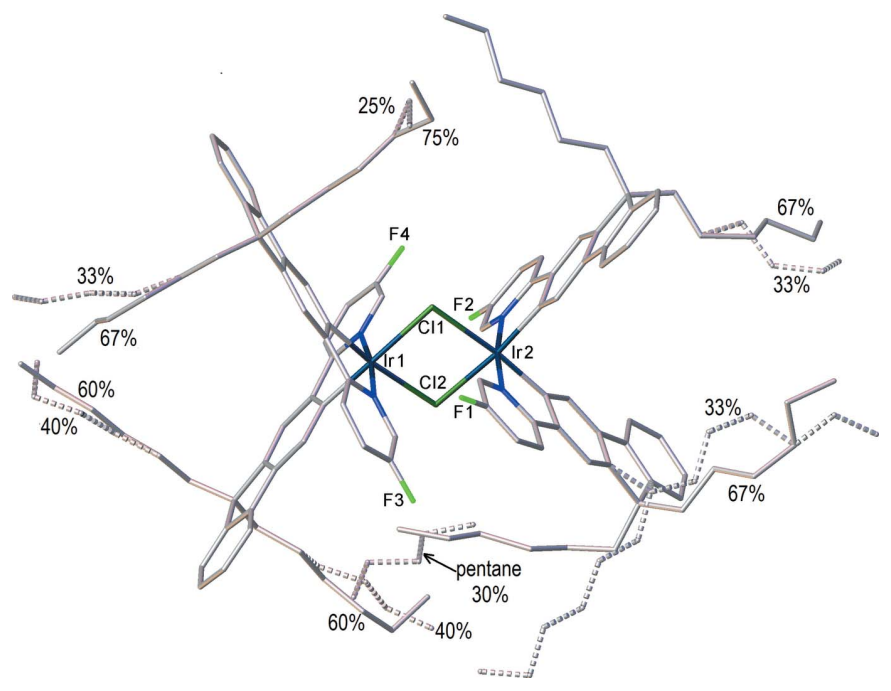

Figure 4

Disorder in the structure of (II), showing occupancies. $\mathrm{H}$ atoms are omitted.

distance of $2.73 \AA$ between rings Py 1 and Py3, and of $3.62 \AA$ between Py 2 and Py4, the $\pi$-systems of the former pair overlap only on the fringes and the latter not at all. The closest contacts are $\mathrm{C} 10 \cdots \mathrm{C} 46=3.35$ (1) and $\mathrm{C} 11 \cdots \mathrm{C} 47=3.10$ (1) $\AA$ in the Py1/Py3 pair and $\mathrm{C} 28 \cdots \mathrm{C} 64=3.51(1)$ and $\mathrm{C} 29 \cdots \mathrm{C} 65=$ 3.21 (1) § for Py2/Py4. Other dichloro-bridged complexes show a similar pattern: in ( $\mathrm{II} a$ ) and $(\mathrm{II} b)$, the $\mathrm{Py} / \mathrm{Py}$ angles are 9.4 (3) and $6.3(4)^{\circ}$, with parallel slips of 2.94 and $2.88 \AA$, respectively. In (IIc), the two Py/Py pairs are even more unequal than in (II): one showing a fringe overlap [Py/Py angle $=5.1(1)^{\circ}$, slip $=3.0 \AA$, shortest $\mathrm{C} \cdots \mathrm{C}$ contacts of $3.36(1)$ and $3.42(1) \AA]$ and the other a more substantial overlap $\left[\mathrm{Py} / \mathrm{Py}\right.$ angle $=7.6(1)^{\circ}$, slip of only $\left.2.03 \AA\right]$ and the mean interplanar separation being $3.21 \AA$.

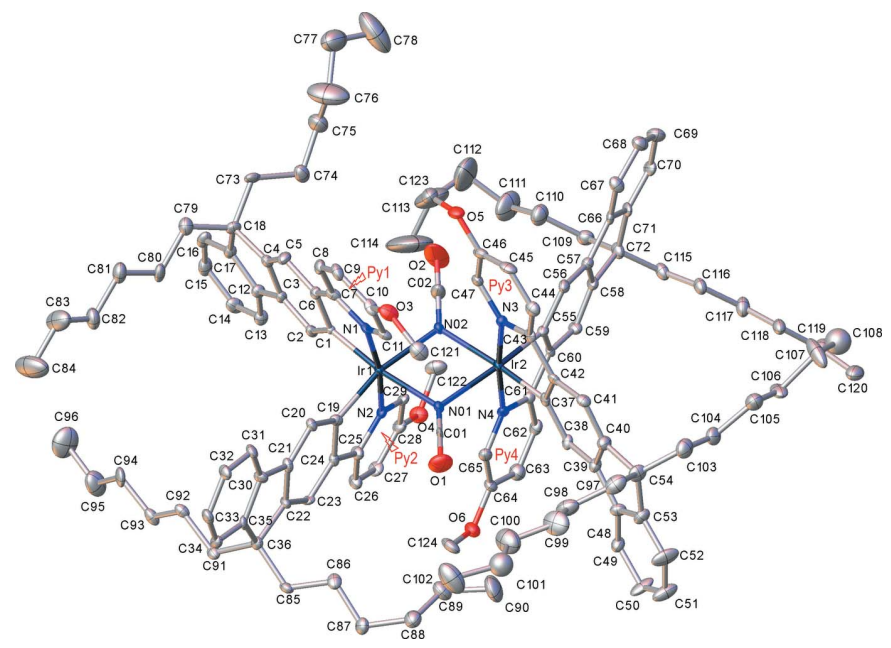

Figure 5

The molecular structure of (III), showing $30 \%$ probability displacement ellipsoids for non-H atoms. The disorder and all $\mathrm{H}$ atoms are omitted for clarity.



Figure 6

Disorder in the structure of (III), showing occupancies. $\mathrm{H}$ atoms are omitted.

Complex (III) (Fig. 5) crystallized as a pentane monosolvate in a centrosymmetric monoclinic structure with two molecules per asymmetric unit. Like (II), (III) crystallized as a racemate; the molecule has no crystallographic symmetry but an approximate local twofold symmetry relating the iridium centres, which have the same configurations. Thus, molecules with $\Delta \Delta$ or $\Lambda \Lambda$ configurations are equally present, whereas the previously reported analogues of (III) with non-substituted (III $a$ ) or 4-fluorinated (III $b$ ) pyridyl rings (M'hamedi et al., 2012) gave non-solvated chiral crystals (space group $P 2_{1}$ ), which were isomorphous and contained one independent molecule each. The precision of structure (III) is limited, due to massive disorder, generally weak diffraction intensities and, possibly, incommensurate modulation along the $\mathbf{a}^{*}$ direction, as indicated by 'streaky' reflection peaks. The intramolecular Ir *..Ir distances in (III), 3.410 (1) and 3.432 (1) $\AA$, are similar to those in (III $a)$ [3.402 (1) $\AA]$ and III $b[3.425$ (1) $\AA$ ] and $c a$ $0.3 \AA$ shorter than in chloro-bridged complexes. Similarly to (II) and especially to (II c), in each independent molecule one pair of pyridyl rings shows substantial $\pi-\pi$ stacking [interplanar Py/Py angles of 11.4 (3) and 10.7 (4) , slips of $1.90 \AA$, mean interplanar separations of $3.25 \AA]$, the other only a fringe overlap $\left[\mathrm{Py} / \mathrm{Py}\right.$ angles $=13.9(3)$ and $19.5(4)^{\circ}$, slips of 2.88 and $2.83 \AA$. In this structure, the $n$-hexyl chains also show extensive disorder (Fig. 6), which could be only imperfectly resolved. These chains surround well-defined voids containing disordered solvent, which was masked. The electron density maps, and the shape and size of the voids suggest the solvent to be pentane rather than chlorobenzene.

In fact, for such diiridium complexes with monoatomic bridges, both metal centres have to adopt the same chirality: the meso diastereomer with opposite $(\Delta \Lambda)$ configurations of the two iridium centres would have sterically impossible short intramolecular contacts between the cyclometalating ligands. With longer bridges, however, both the $\Delta \Delta / \Lambda \Lambda$ and meso isomers are sterically possible - and in some cases have been isolated and structurally characterized (Congrave et al., 2017). Thus, the molecule of (IV) (Fig. 7) is crystallographically centrosymmetric, i.e. meso. As in complexes (II) and (III), the Ir atoms in (IV) have a distorted octahedral coordination with the pyridine $\mathrm{N}$ atoms in trans positions to each other. The 


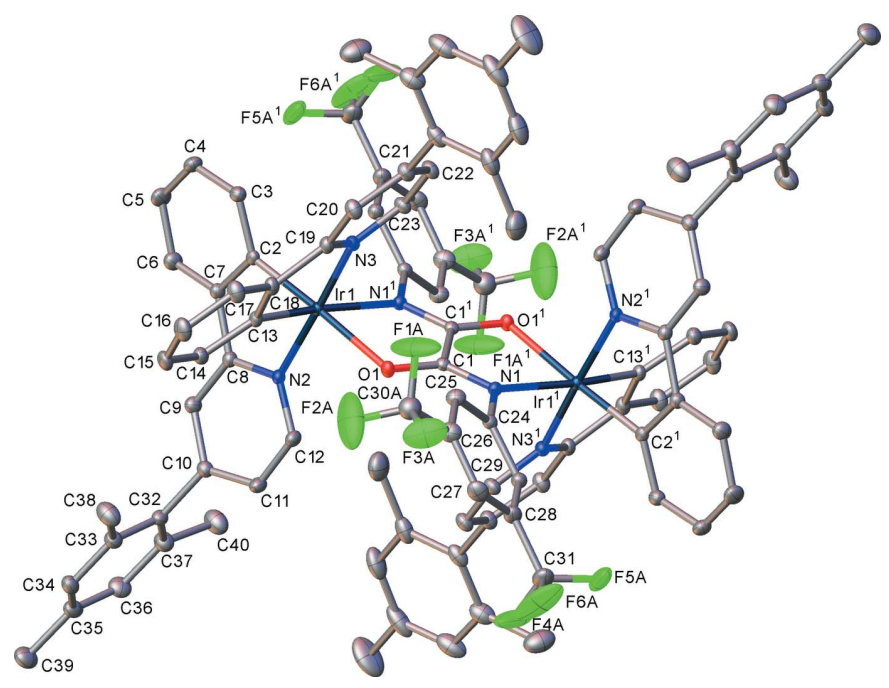

Figure 7

The molecular structure of (IV), showing $50 \%$ probability displacement ellipsoids for non-H atoms. Symmetry code $\left({ }^{1}\right): 1-x, 1-y, 1-z$.

bridging oxamido ligand adopts the anti geometry, its central $\mathrm{C}_{2} \mathrm{~N}_{2} \mathrm{O}_{2}$ moiety is almost planar, the 3,5-trifluoromethylated arene ring is inclined to its plane by $47.3(1)^{\circ}$ and the Ir atom deviates from it by 0.334 (4) $\AA$, which amounts to an $11.2(1)^{\circ}$ fold of the chelate ring along the $\mathrm{O} 1 \cdots \mathrm{N} 1^{1}$ vector [symmetry code: (i) $1-x, 1-y, 1-z]$. In both independent cyclometalating ligands, the mesityl substituent is almost perpendicular to the pyridine ring [dihedral angles 85.9 (1) and $\left.81.5(1)^{\circ}\right]$. The $\operatorname{Ir} 1-\mathrm{N} 1^{\mathrm{i}}$ bond of $2.182(2) \AA$ is substantially longer than in the analogue with a 4-tert-butylphenyl substituent at this $\mathrm{N}$ atom [2.147 (3) $\AA$, see above]. The $\mathrm{CF}_{3}$ groups and the solvent of crystallization are disordered (Fig. 8).

Recently, we prepared and characterized two close analogues of (IV), which proved efficient as dopants in phosphorescent organic light-emitting devices (M'hamedi et al., 2017), viz. tetrakis[2-phenyl-4-(mesityl)pyridine- $\left.C^{2}, N^{\prime}\right]$-bis $(\mu$-oxamidato)diiridium (IV $a$ ) with an unsubstituted oxamidato bridge, and its derivative with an $N, N^{\prime}$-bis(4-tert-butylphenyl)substituted bridge (IVb). As in (IV), both have an anticonfiguration of the bridge: (IV $b$ ) certainly, (IVa) most probably, although in the latter the bridge is disordered, as in ( $\mu_{2}$-oxamidato)tetrakis(5-methyl-2-(pyridin-2-yl)phenyl)diiridium (V), reported by Graf et al. (2013). It is noteworthy that both (IVb) and (V), like (IV), are meso $(\Delta \Lambda)$ diastereomers where the Ir centres are related by a crystallographic inversion centre (IVb) or mirror plane $(\mathrm{V})$, whereas molecule (IVa) lies on a crystallographic twofold axis in the centrosymmetric space group $P b c m$, i.e. the crystal is a racemate of $\Delta \Delta$ and $\Lambda \Lambda$ diastereomers.

\section{Supramolecular features}

The packing of (I)-(III) is defined by the flexible and extensively disordered $n$-hexyl substituents, with the remaining gaps filled by pentane solvent molecules. There are few inter-
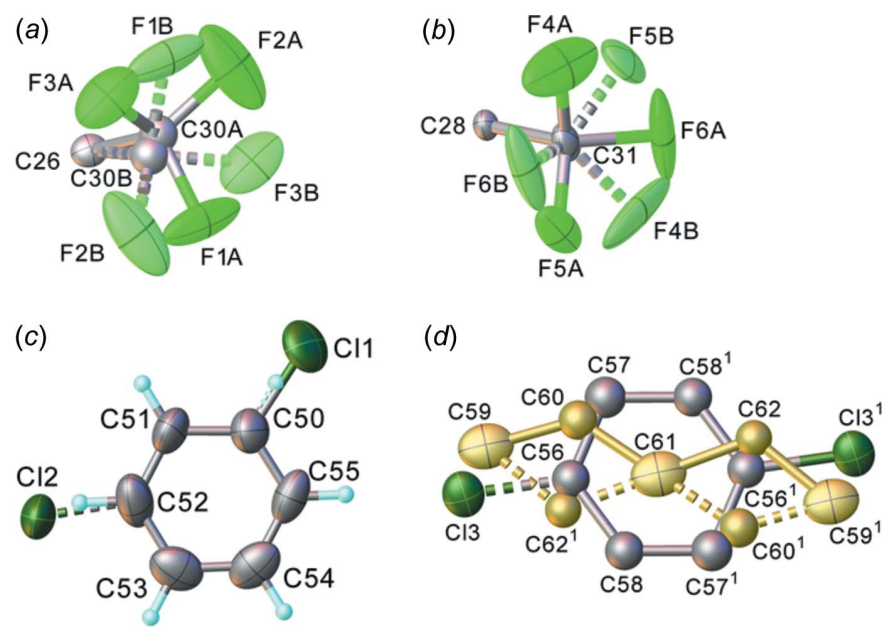

Figure 8

The disorder of the $\mathrm{CF}_{3}$ groups $(a),(b)$, chlorobenzene of crystallization $(c)$ and chlorobenzene/pentane site-sharing $(d)$ [symmetry code $\left(^{1}\right):-x$, $-y,-z]$ in the structure of (IV).

molecular contacts between aromatic moieties, and those of the edge-to-edge kind; no $\pi-\pi$ stacking or intercalation exists. This promises better solubility and compatibility with the host polymer and, indeed, can explain the relatively high efficiency of these complexes in optoelectronic devices (M'hamedi et al., 2012). The structure of (IV) is also devoid of any $\pi-\pi$ stacking; the contacts between all aromatic systems, whether ppy moieties, mesityl substituents or chlorobenzene molecules of crystallization, are of edge-to-face $(' \pi-\sigma$ ') type.

\section{Database survey}

The Cambridge Crystallographic Database (CSD, version 5.40, including updates up to August 2019; Groom et al., 2016) was searched for bis-chloro-bridged diiridium complexes with cyclometalating fluorenyl-pyridino ligands. No structures were found besides ACISUB (II $a^{\prime}$ ), NIFKEU (II $a$ ), NIFKAQ (II $b$ ) and NIFJUJ (IIc), discussed above. A search for similar biscyanato-bridged complexes returned structures NIFJOD (III $a$ ) and NIFJIX (III $b$ ), also discussed above. No $\operatorname{Ir}\left(\mu_{2}-X\right) \operatorname{Ir}$ analogues with other $X$ ligands were found. A search for antioxamido-bridged diiridium complexes with two chelating phenylpyridyl moieties (with any substituents) at each Ir atom, returned three structures discussed above, viz. SAWDIG (IVa), SAWDEC (IV $b$ ) and GIRMIF (V). The only monoiridium complexes cyclometalated by three fluorenyl-pyridino derivatives, are NIFJET (I $a$, see above) and tris(2-(pyridin-2yl)spiro[fluorene-9, $9^{\prime}$-xanthen]-3-yl)-iridium, SAWRIU (Ren et al., 2017).

Of the four structures of $f a c-\operatorname{Ir}(\mathrm{ppy})_{3}$ present in the CSD, $\mathrm{KAVJOH}$ is the trigonal polymorph (Breu et al., 2005), KAVJOH01 (Berger et al., 2010) and KAVJOH03 (Takayasu et al., 2013) refer to the same tetragonal form (space group $\bar{P} 42{ }_{1} c$ ) at 100 and $193 \mathrm{~K}$, respectively, whereas KAVJOH02 (Wang et al., 2013) is probably the latter structure solved in a wrong space group $(\bar{P} 4)$. 


\section{Synthesis and crystallization}

Complexes (I), (II) and (III) were synthesized as described elsewhere (M'hamedi et al., 2012), viz. (I) and (II) by the reaction of the corresponding fluorenylpyridine with $\operatorname{Ir}(\mathrm{acac})_{3}$ or $\mathrm{IrCl}_{3}$, respectively, (III) by reaction of the methoxysubstituted analogue of (II) (i.e. di-chloro-bridged complex) with $\mathrm{Bu}_{4} \mathrm{~N}^{+} \mathrm{NCO}^{-}$. All three complexes were recrystallized from the mixed solvents chlorobenzene/pentane.

Complex (IV) was prepared following the previous procedure for the synthesis of diiridium(III) complexes with bridging oxamidato ligands (M'hamedi et al., 2017): a mixture of sodium methoxide $(20.8 \mathrm{mg}, 3.0$ eq.) in methanol $(2 \mathrm{ml})$, and $N, N^{\prime}$-bis(3,5-bis(trifluoromethylphenyl)oxamide $(98.6 \mathrm{mg}$, 1.5 eq.) in methanol $(6 \mathrm{ml})$ and the corresponding dichlorobridged dimer (Rota Martir et al., 2016) (200 mg, $0.128 \mathrm{mmol}$ ) in THF (15 ml) gave a product that was purified by column chromatography over silica gel, eluting with dichloromethane (saturated with $\mathrm{K}_{2} \mathrm{CO}_{3}$ and $2 \% \mathrm{Et}_{3} \mathrm{~N}$ ) to give (IV) as a yelloworange coloured solid (141 mg, 58\% yield). Crystals were grown from a chlorobenzene/pentane solution. MS (MALDITOF) $m / z$ 1984.2 ([M+ H, $\left.\left.{ }^{191} \mathrm{Ir},{ }^{193} \mathrm{Ir}\right], 100 \%\right)$. Calculated for $\mathrm{C}_{98} \mathrm{H}_{78} \mathrm{~F}_{12} \mathrm{Ir}_{2} \mathrm{~N}_{6} \mathrm{O}_{2} m / z$ 1984.53.

\section{Refinement}

Crystal data, data collection and structure refinement details are summarized in Table 1.

In structure (I), the entire cyclometalating ligand $\mathrm{N} 3{ }^{\wedge} \mathrm{C} 37$, ligand $\mathrm{N} 1{ }^{\wedge} \mathrm{C} 1$ except the pyridyl ring and the methyl atom C67, and $n$-hexyl substituents (except atoms C75 and C76) in the ligand $\mathrm{N} 2{ }^{\wedge} \mathrm{C} 19$ are disordered equally between two positions (one of them labelled $B$ ). In the pentane solvent molecule, all atoms except $\mathrm{C}(1 S)$ are also disordered between two positions (alternative carbon positions are primed).

In structure (II), atom C18 of the fluorene ring and both $n$ hexyl chains attached to it (except the $\mathrm{C} 82$ atom), chains C93-C94-C95-C96 and C105-C106-C107-C108 (with their respective $\mathrm{H}$ atoms) are disordered over two sets of sites with occupancies $2 / 3$ and $1 / 3$. The butyl chain at $\mathrm{C} 110$ is disordered between positions $\mathrm{C} 111-\mathrm{C} 112-\mathrm{C} 113-\mathrm{C} 114$ and $\mathrm{C} 211-\mathrm{C} 212-\mathrm{C} 213-\mathrm{C} 214$, the butyl chain at $\mathrm{C} 116$ is disordered between positions $\mathrm{C} 117-\mathrm{C} 118-\mathrm{C} 119-\mathrm{C} 120$ and C217-C218-C219-C220 (with respective $\mathrm{H}$ atoms); both in a 0.6:0.4 ratio, respectively. The ethyl group at $\mathrm{C} 100$ is disordered between positions $\mathrm{C} 101-\mathrm{C} 102$ and $\mathrm{C} 201-\mathrm{C} 202$ in a 0.75:0.25 ratio. All minor positions are labelled $B$. The pentane molecule of crystallization, $\mathrm{C} 1 S-\mathrm{C} 2 S-\mathrm{C} 3 S-\mathrm{C} 4 S-\mathrm{C} 5 S$ (with respective $\mathrm{H}$ atoms) shares the site with disordered $n$ hexyl groups and was refined with a 0.3 occupancy.

In structure (III), the ethyl group at $\mathrm{C} 100$ is disordered between positions $\mathrm{C} 101-\mathrm{C} 102$ and $\mathrm{C} 125-\mathrm{C} 126$ and the butyl group at $\mathrm{C} 104$ between positions $\mathrm{C} 105-\mathrm{C} 106-\mathrm{C} 107-\mathrm{C} 108$ and $\mathrm{C} 10 A-\mathrm{C} 10 B-\mathrm{C} 10 C-\mathrm{C} 10 D$, with occupancies $2 / 3$ and $1 /$ 3 , respectively, in both cases. A methylene group is disordered between positions $\mathrm{C} 80^{\prime}$ and $\mathrm{C} 80 \mathrm{~B}$ with occupancies 0.6 and 0.4 and a dimethylene fragment is disordered between positions C204-C205 and $\mathrm{C} 20 B-\mathrm{C} 20 \mathrm{C}$ with occupancies 0.7 and 0.3 , respectively. The propyl chain at $\mathrm{C} 211$ is disordered between positions $\mathrm{C} 212-\mathrm{C} 213-\mathrm{C} 214$ and $\mathrm{C} 21 B-\mathrm{C} 21 C-\mathrm{C} 21 D$, and the butyl chain at $\mathrm{C} 216$ is disordered between positions $\mathrm{C} 217-\mathrm{C} 218-\mathrm{C} 219-\mathrm{C} 220$ and $\mathrm{C} 21 E-\mathrm{C} 21 F-\mathrm{C} 21 G-\mathrm{C} 21 H$ with equal (0.5) occupancies in both cases. The structure contains enclosed solvent-accessible voids of $374 \AA^{3}$ (four per unit cell), occupied by disordered solvent, which could not be refined at atomic resolution and was masked using the OLEX2 SMTBX solvent-masking procedure based on Rees et al. (2005). The diffuse electron density in the voids being too low for chlorobenzene, the integral of 70 e per void can be interpreted as two pentane molecules (42 e each).

In structures (I)-(III), all $\mathrm{H}$ atoms were permitted to ride in geometrically idealized positions with $\mathrm{C}-\mathrm{H}=0.95,0.99$ and $0.98 \AA$ for aromatic, methylene and methyl $\mathrm{C}$ atoms, respectively.

In structure (IV), aromatic $\mathrm{H}$ atoms were permitted to ride in geometrically idealized positions with $\mathrm{C}-\mathrm{H}=0.95 \AA$. Methyl groups were ascribed idealized geometry $(\mathrm{C}-\mathrm{H}=$ $0.98 \AA$ A) and were permitted to rotate around the $\mathrm{C}-\mathrm{C}$ bonds (with a common refined $U_{\text {iso }}$ for all $\mathrm{H}$ atoms of each group), except the $\mathrm{C}_{48} \mathrm{H}_{3}$ group, which was treated as ideally disordered between two opposite orientations. For the latter and aromatic $\mathrm{H}$ atoms, $U_{\text {iso }}(\mathrm{H})=1.2 U_{\text {eq }}(\mathrm{C})$. The $\mathrm{C}_{31 \mathrm{~F}_{3}}$ group is disordered (by rotation about the $\mathrm{C}-\mathrm{C}$ bond) between orientations $A$ and $B$ with occupancies of 0.774 (5) and 0.226 (5), respectively, while the $\mathrm{C}_{30} \mathrm{~F}_{3}$ group is disordered by a similar rotation and tilt of the $\mathrm{C}-\mathrm{C}$ bond, between orientations $A$ and $B$ with occupancies of 0.586 (15) and 0.414 (15), respectively. The chlorobenzene molecule in a general position has the chlorine atom disordered equally between positions $\mathrm{Cl} 1$ and $\mathrm{Cl} 2$. The void around the inversion centre $(0,0$, 0 ) with the solvent-accessible volume of $204 \AA^{3}$ is shared by $c a$ 0.15 of a chlorobenzene and 0.20 of a pentane molecule, refined at atomic resolution; the occupancies are in agreement with the integral electron density of $33.4 \mathrm{e}$ per void, as estimated by OLEX2 SMTBX. The benzene ring has crystallographic inversion symmetry: the $\mathrm{Cl}$ atom is disordered between two positions related by this inversion. The central pentane atom C61 lies at the inversion centre, the adjacent atom is disordered equally between two positions with the terminal atom ordered. The pentane $\mathrm{H}$ atoms were not

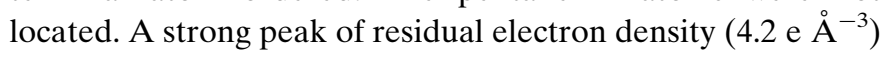
near the Ir1 atom can be interpreted as an alternative position, Ir 1 , of this atom, due to a whole-molecule disordered by a $12^{\circ}$ rotation around its (crystallographic) inversion centre. Refinement of the occupancies of Ir1 and Ir2 (assuming equal ADPs) converged at 0.9817 (6) and 0.0183 (6), respectively, with a $\operatorname{Ir} 1 \cdots \operatorname{Ir} 2$ distance of $0.62 \AA$.

\section{Acknowledgements}

The authors acknowledge helpful discussions with Professor Martin R. Bryce (Durham University, UK). 
Table 1

Experimental details.

(I) (II) $\quad$ (III)

Crystal data

Chemical formula

$M_{\mathrm{r}}$

Crystal system, space group

Temperature (K)

$a, b, c(\AA)$

$\alpha, \beta, \gamma\left({ }^{\circ}\right)$

$V\left(\AA^{3}\right)$

Z

Radiation type

$\mu\left(\mathrm{mm}^{-1}\right)$

Crystal size (mm)

Data collection

Diffractometer

Absorption correction

$T_{\min }, T_{\max }$

No. of measured,

independent and observed

$[I>2 \sigma(I)]$ reflections

$R_{\text {int }}$

$(\sin \theta / \lambda)_{\max }\left(\AA^{-1}\right)$

Refinement

$R\left[F^{2}>2 \sigma\left(F^{2}\right)\right], w R\left(F^{2}\right), S$

No. of reflections

No. of parameters

No. of restraints

$\mathrm{H}$-atom treatment

$\Delta \rho_{\max }, \Delta \rho_{\min }\left(\mathrm{e} \AA^{-3}\right)$

$\begin{array}{ll}{\left[\operatorname{Ir}\left(\mathrm{C}_{31} \mathrm{H}_{38} \mathrm{NO}\right)_{3}\right] \cdot \mathrm{C}_{5} \mathrm{H}_{12}} & {\left[\mathrm{Ir}_{2}\left(\mathrm{C}_{30} \mathrm{H}_{35} \mathrm{FN}\right)_{4} \mathrm{Cl}_{2}\right] \cdot 0.3 \mathrm{C}_{5} \mathrm{H}} \\ & \\ 1586.21 & 2191.30 \\ \text { Triclinic, } P \overline{1} & \text { Triclinic, } P \overline{1} \\ 120 & 120 \\ 16.4880(5), 17.0003(5), & 12.2744(7), 17.6132(10), \\ 17.0288(5) & 25.3966(15) \\ 91.9252(12), 97.8137(12), & 105.119(2), 93.787(2), \\ 117.3489(11) & 90.779(2) \\ 4175.2(3) & 5286.2(5) \\ 2 & 2 \\ M o K \alpha & \operatorname{Mo~K\alpha } \\ 1.65 & 2.62 \\ 0.11 \times 0.07 \times 0.04 & 0.14 \times 0.1 \times 0.04\end{array}$

Bruker SMART CCD 6000

Multi-scan ( $S A D A B S$;

Krause et al., 2015)

$0.803,0.850$

46917, 19128, 13949

0.071

0.650

$0.042,0.076,0.91$
19128
1358
1114
$\begin{gathered}\text { H-atom parameters } \\ \text { constrained }\end{gathered}$

$1.73,-0.86$

$1.39,-0.76$
Bruker SMART CCD 6000

Integration ( $S A D A B S$;

Krause et al., 2015)

$0.735,0.927$

48264, 18623, 12096

0.065

0.595

$0.041,0.103,0.95$
18623
1342
2905
$\begin{gathered}\text { H-atom parameters } \\ \quad \text { constrained }\end{gathered}$

$\left[\mathrm{Ir}_{2}\left(\mathrm{C}_{31} \mathrm{H}_{38} \mathrm{NO}\right)_{4}(\mathrm{NCO})_{2}\right]--$ $\mathrm{C}_{5} \mathrm{H}_{12}$

2303.07

Monoclinic, $P 2_{1} / c$

120

38.976 (6), 21.615 (3),

$28.759(4)$

90, 108.920 (3), 90

$22920(6)$

8

Mo $K \alpha$

2.38

$0.2 \times 0.1 \times 0.01$

Bruker SMART CCD 6000

Multi-scan (SADABS;

Krause et al., 2015)

$0.809,1.000$

95765, 40676, 19348

0.143

0.601

$0.077,0.185,0.97$
40676
2479
2600
$\begin{gathered}\text { H-atom parameters } \\ \quad \text { constrained }\end{gathered}$

(IV)

\author{
$\left[\mathrm{Ir}_{2}\left(\mathrm{C}_{20} \mathrm{H}_{19} \mathrm{~N}\right)_{4}\left(\mathrm{C}_{18} \mathrm{H}_{6} \mathrm{~F}_{12}-\right.\right.$ \\ $\left.\left.\mathrm{N}_{2} \mathrm{O}_{2}\right)\right] \cdot 2.3 \mathrm{C}_{6} \mathrm{H}_{5} \mathrm{Cl} \cdot-$ \\ $0.4 \mathrm{C}_{5} \mathrm{H}_{12}$ \\ 2271.78 \\ Triclinic, $P \overline{1}$ \\ 120 \\ 11.8734 (5), 14.2267 (6), \\ $16.6076(7)$ \\ $110.386(2), 106.524$ (2), \\ $96.303(2)$ \\ $2452.00(18)$ \\ 1 \\ Mo $K \alpha$ \\ 2.85 \\ $0.12 \times 0.08 \times 0.04$
}

Bruker D8 Venture

Integration ( $S A D A B S$;

Krause et al., 2015)

$0.770,0.913$

54411, 14405, 12696

0.032

0.705

$0.028,0.073,1.06$

14405

702

593

$\mathrm{H}$ atoms treated by a mixture of independent and constrained refinement $0.96,-1.01$

Computer programs: SMART (Bruker, 2001), SAINT (Bruker, 2001, 2008, 2016, 2017), APEX3 (Bruker, 2016), SHELXS (Sheldrick, 2008), SHELXT2018 (Sheldrick, 2015a), SHELXL2018 (Sheldrick, 2015b) and OLEX2 (Dolomanov et al., 2009).

\section{Funding information}

Funding for this research was provided by: Deanship of Scientific Research at Prince Sattam Bin Abdulaziz University (grant No. 11187/01/2019 to Ahmed M'hamedi).

\section{References}

Adeloye, A. O. (2019). Materials, 12, 2734.

Al-Attar, H. A., Griffiths, G. C., Moore, T. N., Tavasli, M., Fox, M. A., Bryce, M. R. \& Monkman, A. P. (2011). Adv. Funct. Mater. 21, 2376-2382.

Baldo, M. A., O'Brien, D. F., You, Y., Shoustikov, A., Sibley, S., Thompson, M. E. \& Forrest, S. R. (1998). Nature, 395, 151-154.

Berger, R. J. F., Stammler, H.-G., Neumann, B. \& Mitzel, N. W. (2010). Eur. J. Inorg. Chem. pp. 1613-1617.

Bettington, S., Tavasli, M., Bryce, M. R., Batsanov, A. S., Thompson, A. L., Al Attar, H. A., Dias, F. B. \& Monkman, A. P. (2006). $J$. Mater. Chem. 16, 1046-1052.

Breu, J., Stössel, P., Schrader, S., Starukhin, A., Finkenzeller, W. J. \& Yersin, H. (2005). Chem. Mater. 17, 1745-1752.

Bruker (2001). SMART and SAINT. Bruker AXS Inc., Madison, Wisconsin, USA.

Bruker (2001). SAINT. Bruker AXS Inc., Madison, Wisconsin, USA. Bruker (2016). SAINT. Bruker AXS Inc., Madison, Wisconsin, USA.
Bruker (2017). APEX3. Bruker AXS Inc., Madison, Wisconsin, USA.

Congrave, D. G., Hsu, Y., Batsanov, A. S., Beeby, A. \& Bryce, M. R. (2017). Organometallics, 36, 981-993.

Dolomanov, O. V., Bourhis, L. J., Gildea, R. J., Howard, J. A. K. \& Puschmann, H. (2009). J. Appl. Cryst. 42, 339-341.

Graf, M., Czerwieniec, R. \& Sünkel, K. (2013). Z. Anorg. Allg. Chem. 639, 1090-1094.

Groom, C. R., Bruno, I. J., Lightfoot, M. P. \& Ward, S. C. (2016). Acta Cryst. B72, 171-179.

King, K. A., Spellane, P. J. \& Watts, R. J. (1985). J. Am. Chem. Soc. 107, 1431-1432.

Krause, L., Herbst-Irmer, R., Sheldrick, G. M. \& Stalke, D. (2015). J. Appl. Cryst. 48, 3-10.

Li, G., Congrave, D. G., Zhu, D., Su, Z. \& Bryce, M. R. (2018). Polyhedron, 140, 146-157.

M'hamedi, A., Batsanov, A. S., Fox, M. A., Bryce, M. R., Abdullah, K., Al-Attar, H. A. \& Monkman, A. P. (2012). J. Mater. Chem. 22, 13529-13540.

M'hamedi, A., Fox, M. A., Batsanov, A. S., Al-Attar, H. A., Monkman, A. P. \& Bryce, M. R. (2017). J. Mater. Chem. C, 5, 6777-6789.

Rees, B., Jenner, L. \& Yusupov, M. (2005). Acta Cryst. D61, 1299 1301. 
Ren, B.-Y., Guo, R. D., Zhong, D.-K., Ou, Ch.-J., Xiong, G., Zhao, X.-H., Sun, Y.-G., Jurow, M., Kang, J., Zhao, Y., Li, Sh.-B., You, L.-X., Wang, L.-W., Liu, Y. \& Huang, W. (2017). Inorg. Chem. 56, 8397-8407.

Rota Martir, D., Momblona, C., Pertegás, A., Cordes, D. B., Slawin, A. M. Z., Bolink, H. J. \& Zysman-Colman, E. (2016). Appl. Mater. Interfaces, 8, 33907-33915.

Sheldrick, G. M. (2008). Acta Cryst. A64, 112-122.
Sheldrick, G. M. (2015a). Acta Cryst. A71, 3-8.

Sheldrick, G. M. (2015b). Acta Cryst. C71, 3-8.

Takayasu, S., Suzuki, T. \& Shinozaki, K. (2013). J. Phys. Chem. B, 117, 9449-9456.

Wang, P., Wang, F.-F., Chen, Y., Niu, Q., Lu, L., Wang, H.-M., Gao, X.-C., Wei, B., Wu, H.-W., Cai, X. \& Zou, D. (2013). J. Mater. Chem. $C, \mathbf{1}, 4821-4825$. 


\section{supporting information}

Acta Cryst. (2020). E76, 392-399 [https://doi.org/10.1107/S2056989020001784]

Phosphorescent mono- and diiridium(III) complexes cyclometalated by fluorenyl- or phenyl-pyridino ligands with bulky substituents, as prospective OLED dopants

\section{Ahmed M'hamedi and Andrei S. Batsanov}

Computing details

Data collection: SMART (Bruker, 2001) for (I), (II), (III); APEX3 (Bruker, 2016) for (IV). Cell refinement: SAINT (Bruker, 2008) for (I); SMART (Bruker, 2001) for (II); SAINT (Bruker, 2016) for (III); SAINT (Bruker, 2017) for (IV). Data reduction: SAINT (Bruker, 2008) for (I); SAINT (Bruker, 2001) for (II); SAINT (Bruker, 2016) for (III); SAINT (Bruker, 2017) for (IV). Program(s) used to solve structure: SHELXS (Sheldrick, 2008) for (I), (II); SHELXT2018 (Sheldrick, 2015a) for (III); SHELXS2013 (Sheldrick, 2008) for (IV). For all structures, program(s) used to refine structure: SHELXL2018 (Sheldrick, 2015b); molecular graphics: OLEX2 (Dolomanov et al., 2009); software used to prepare material for publication: OLEX2 (Dolomanov et al., 2009).

$\backslash$ Tris[9,9-dihexyl-2-(5-methoxypyridin-2-yl- $\kappa N)-9 H$-fluoren-3- $\left.\backslash y \mathrm{l}-\kappa C^{3}\right]$ iridium pentane monosolvate (I)

\section{Crystal data}

$\left[\operatorname{Ir}\left(\mathrm{C}_{31} \mathrm{H}_{38} \mathrm{NO}\right)_{3}\right] \cdot \mathrm{C}_{5} \mathrm{H}_{12}$

$M_{r}=1586.21$

Triclinic, $P \overline{1}$

$a=16.4880(5) \AA$

$b=17.0003(5) \AA$

$c=17.0288(5) \AA$

$\alpha=91.9252(12)^{\circ}$

$\beta=97.8137(12)^{\circ}$

$\gamma=117.3489(11)^{\circ}$

$V=4175.2(3) \AA^{3}$

\section{Data collection}

Bruker SMART CCD 6000 diffractometer

Radiation source: sealed X-ray tube Parallel,graphite monochromator Detector resolution: 5.6 pixels $\mathrm{mm}^{-1}$ $\omega$ scans Absorption correction: multi-scan

(SADABS; Krause et al., 2015) $T_{\min }=0.803, T_{\max }=0.850$
$Z=2$

$F(000)=1672$

$D_{\mathrm{x}}=1.262 \mathrm{Mg} \mathrm{m}^{-3}$

Mo $K \alpha$ radiation, $\lambda=0.71073 \AA$

Cell parameters from 9920 reflections

$\theta=2.4-26.4^{\circ}$

$\mu=1.65 \mathrm{~mm}^{-1}$

$T=120 \mathrm{~K}$

Block, yellow

$0.11 \times 0.07 \times 0.04 \mathrm{~mm}$

46917 measured reflections 19128 independent reflections 13949 reflections with $I>2 \sigma(I)$

$R_{\text {int }}=0.071$

$\theta_{\max }=27.5^{\circ}, \theta_{\min }=1.2^{\circ}$

$h=-21 \rightarrow 21$

$k=-22 \rightarrow 22$

$l=-22 \rightarrow 22$ 


\section{Refinement}

Refinement on $F^{2}$

Least-squares matrix: full

$R\left[F^{2}>2 \sigma\left(F^{2}\right)\right]=0.042$

$w R\left(F^{2}\right)=0.076$

$S=0.91$

19128 reflections

1358 parameters

1114 restraints

Special details

Experimental. The data collection nominally covered full sphere of reciprocal space, by a combination of 4 runs of narrow-frame $\omega$-scans (scan width $0.3^{\circ} \omega, 60$ s exposure, $2 \times 600+552+50$ scans), every run at a different $\varphi$ and/or $2 \theta$ angle. Crystal to detector distance $4.84 \mathrm{~cm}$.

Geometry. All esds (except the esd in the dihedral angle between two 1.s. planes) are estimated using the full covariance matrix. The cell esds are taken into account individually in the estimation of esds in distances, angles and torsion angles; correlations between esds in cell parameters are only used when they are defined by crystal symmetry. An approximate (isotropic) treatment of cell esds is used for estimating esds involving l.s. planes.

Refinement. extensive disorder

Fractional atomic coordinates and isotropic or equivalent isotropic displacement parameters $\left(\AA^{2}\right)$

\begin{tabular}{llllll}
\hline & $x$ & $y$ & $z$ & $U_{\text {iso }}{ }^{*} / U_{\text {eq }}$ & Occ. $(<1)$ \\
\hline Ir & $0.31386(2)$ & $0.66708(2)$ & $0.41146(2)$ & $0.01857(4)$ & \\
O2 & $0.13331(19)$ & $0.60982(18)$ & $0.66372(15)$ & $0.0417(7)$ & \\
N1 & $0.36370(19)$ & $0.7927(2)$ & $0.47826(16)$ & $0.0199(7)$ & \\
N2 & $0.21147(18)$ & $0.59240(19)$ & $0.48141(16)$ & $0.0171(6)$ & $0.0247(8)$ \\
C7 & $0.3206(2)$ & $0.8412(3)$ & $0.4527(2)$ & $0.0332(10)$ \\
C8 & $0.3470(2)$ & $0.9236(3)$ & $0.4933(2)$ & $0.040^{*}$ \\
H8 & 0.315976 & 0.956674 & 0.475663 & $0.0279(9)$ & \\
C9 & $0.4172(2)$ & $0.9587(3)$ & $0.5587(2)$ & $0.033^{*}$ \\
H9 & 0.435109 & 1.015300 & 0.586099 & $0.0314(9)$ & \\
C10 & $0.4604(2)$ & $0.9088(3)$ & $0.5834(2)$ & $0.0274(9)$ & \\
C11 & $0.4323(2)$ & $0.8266(2)$ & $0.5417(2)$ & $0.0215(8)$ & \\
H11 & 0.462678 & 0.792825 & 0.558575 & $0.0264(8)$ \\
C19 & $0.2515(2)$ & $0.5464(2)$ & $0.3469(2)$ & $0.032^{*}$ \\
C20 & $0.2749(2)$ & $0.5202(3)$ & $0.2775(2)$ & $0.0308(9)$ \\
H20 & 0.325118 & 0.562731 & 0.255432 & $0.0381(10)$ \\
C21 & $0.2255(3)$ & $0.4334(3)$ & $0.2407(2)$ & $0.0361(10)$ \\
C22 & $0.1493(3)$ & $0.3698(3)$ & $0.2695(2)$ & $0.043^{*}$ \\
C23 & $0.1240(2)$ & $0.3930(3)$ & $0.3370(2)$ & $0.0237(8)$ \\
H23 & 0.072209 & 0.350170 & 0.357125 & $0.0193(7)$ \\
C24 & $0.1752(2)$ & $0.4793(2)$ & $0.3762(2)$ & $0.0241(8)$ \\
C25 & $0.1543(2)$ & $0.5059(2)$ & $0.4501(2)$ & $0.029^{*}$ \\
C26 & $0.0853(2)$ & $0.4518(2)$ & $0.4915(2)$ & $0.0238(8)$ \\
H26 & 0.044333 & 0.392169 & 0.469197 & $0.029^{*}$ \\
C27 & $0.0751(2)$ & $0.4820(2)$ & $0.5630(2)$ & \\
H27 & 0.028585 & 0.443697 & 0.591056 & \\
C28 & $0.1344(3)$ & $0.5694(2)$ & $0.5937(2)$ & \\
& & & & \\
\end{tabular}

Hydrogen site location: mixed

$\mathrm{H}$-atom parameters constrained

$w=1 /\left[\sigma^{2}\left(F_{\mathrm{o}}^{2}\right)+(0.026 P)^{2}\right]$

where $P=\left(F_{\mathrm{o}}^{2}+2 F_{\mathrm{c}}^{2}\right) / 3$
$(\Delta / \sigma)_{\max }=0.004$

$\Delta \rho_{\min }=-0.85$ e $\AA^{-3}$ 


\begin{tabular}{|c|c|c|c|c|c|}
\hline $\mathrm{C} 29$ & $0.2009(2)$ & $0.6224(2)$ & $0.55021(19)$ & $0.0203(8)$ & \\
\hline $\mathrm{H} 29$ & 0.240932 & 0.682756 & 0.570954 & $0.024 *$ & \\
\hline $\mathrm{C} 30$ & $0.2415(3)$ & $0.3895(3)$ & $0.1730(2)$ & $0.0333(9)$ & \\
\hline $\mathrm{C} 31$ & $0.3118(3)$ & $0.4221(3)$ & $0.1271(2)$ & $0.0375(10)$ & \\
\hline H31 & 0.357650 & 0.482873 & 0.135077 & $0.045^{*}$ & \\
\hline $\mathrm{C} 32$ & $0.3126(3)$ & $0.3625(3)$ & $0.0698(2)$ & $0.0446(12)$ & \\
\hline H32 & 0.359715 & 0.383465 & 0.037724 & $0.054^{*}$ & \\
\hline $\mathrm{C} 33$ & $0.2469(3)$ & $0.2742(3)$ & $0.0580(2)$ & $0.0477(12)$ & \\
\hline H33 & 0.250002 & 0.234826 & 0.018991 & $0.057^{*}$ & \\
\hline $\mathrm{C} 34$ & $0.1762(3)$ & $0.2424(3)$ & $0.1028(2)$ & $0.0430(11)$ & \\
\hline H34 & 0.130227 & 0.181637 & 0.093934 & $0.052^{*}$ & \\
\hline $\mathrm{C} 35$ & $0.1742(3)$ & $0.3005(3)$ & $0.1605(2)$ & $0.0377(10)$ & \\
\hline $\mathrm{C} 36$ & $0.1066(3)$ & $0.2798(3)$ & $0.2189(3)$ & $0.0441(11)$ & \\
\hline C67 & $0.0931(3)$ & $1.0219(3)$ & $-0.0702(3)$ & $0.0469(12)$ & \\
\hline H67A & 0.059846 & 0.981690 & -0.119432 & $0.070^{*}$ & 0.5 \\
\hline H67B & 0.144946 & 1.076060 & -0.082482 & $0.070^{*}$ & 0.5 \\
\hline $\mathrm{H} 67 \mathrm{C}$ & 0.050716 & 1.037630 & -0.046762 & $0.070^{*}$ & 0.5 \\
\hline H67D & 0.045886 & 0.985100 & -0.116102 & $0.070^{*}$ & 0.5 \\
\hline H67E & 0.139036 & 1.076500 & -0.087852 & $0.070^{*}$ & 0.5 \\
\hline H67F & 0.063606 & 1.037290 & -0.030382 & $0.070^{*}$ & 0.5 \\
\hline C68 & $0.0762(3)$ & $0.5530(3)$ & $0.7158(2)$ & $0.0499(13)$ & \\
\hline H68A & 0.010813 & 0.529120 & 0.691859 & $0.075^{*}$ & \\
\hline H68B & 0.088056 & 0.587280 & 0.767255 & $0.075^{*}$ & \\
\hline H68C & 0.090669 & 0.503698 & 0.723869 & $0.075^{*}$ & \\
\hline C75 & $0.1016(3)$ & $0.2037(3)$ & $0.2673(3)$ & $0.0473(12)$ & \\
\hline $\mathrm{H} 75 \mathrm{~A}$ & 0.076496 & 0.148649 & 0.230131 & $0.057^{*}$ & \\
\hline H75B & 0.056836 & 0.193699 & 0.303961 & $0.057^{*}$ & \\
\hline C76 & 0.1918 & $0.2166(3)$ & $0.3164(3)$ & $0.0440(12)$ & \\
\hline H76A & 0.237510 & 0.227415 & 0.280549 & $0.053^{*}$ & \\
\hline H76B & 0.216390 & 0.270365 & 0.355089 & $0.053^{*}$ & \\
\hline $\mathrm{O} 1$ & $0.5212(7)$ & $0.9382(8)$ & $0.6548(6)$ & $0.030(2)$ & 0.5 \\
\hline $\mathrm{C} 1$ & $0.2209(6)$ & $0.7022(5)$ & $0.3565(5)$ & $0.0169(11)^{*}$ & 0.5 \\
\hline $\mathrm{C} 2$ & $0.1481(5)$ & $0.6589(6)$ & $0.2929(5)$ & $0.0171(11)^{*}$ & 0.5 \\
\hline $\mathrm{H} 2$ & 0.132963 & 0.600682 & 0.271090 & $0.020^{*}$ & 0.5 \\
\hline $\mathrm{C} 3$ & $0.0975(5)$ & $0.7001(5)$ & $0.2611(4)$ & $0.0143(18)^{*}$ & 0.5 \\
\hline $\mathrm{C} 4$ & $0.1153(5)$ & $0.7841(5)$ & 0.2928 (4) & $0.0179(16)^{*}$ & 0.5 \\
\hline $\mathrm{C} 5$ & $0.1847(5)$ & $0.8282(5)$ & 0.3568 (4) & $0.0170(16)^{*}$ & 0.5 \\
\hline H5 & 0.197133 & 0.885457 & 0.379083 & $0.020^{*}$ & 0.5 \\
\hline C6 & $0.2368(5)$ & $0.7866(5)$ & 0.3899 (4) & $0.0146(19)^{*}$ & 0.5 \\
\hline $\mathrm{C} 12$ & $0.0208(5)$ & $0.6692(5)$ & 0.1928 & $0.0177(19)^{*}$ & 0.5 \\
\hline $\mathrm{C} 13$ & $-0.0233(6)$ & $0.5908(6)$ & $0.1417(5)$ & $0.0215(12)^{*}$ & 0.5 \\
\hline H13 & -0.005164 & 0.545589 & 0.148270 & $0.026^{*}$ & 0.5 \\
\hline $\mathrm{C} 14$ & $-0.0937(6)$ & $0.5791(6)$ & $0.0814(5)$ & $0.0220(12)^{*}$ & 0.5 \\
\hline H14 & -0.126014 & 0.524478 & 0.047708 & $0.026^{*}$ & 0.5 \\
\hline $\mathrm{C} 15$ & $-0.1180(6)$ & $0.6469(5)$ & $0.0693(5)$ & $0.025(2)^{*}$ & 0.5 \\
\hline H15 & -0.170229 & 0.635552 & 0.030328 & $0.030^{*}$ & 0.5 \\
\hline $\mathrm{C} 16$ & $-0.0749(5)$ & $0.7244(5)$ & 0.1199 (4) & $0.0204(17)^{*}$ & 0.5 \\
\hline H16 & -0.092159 & 0.770173 & 0.112675 & $0.025^{*}$ & 0.5 \\
\hline
\end{tabular}




\begin{tabular}{|c|c|c|c|c|c|}
\hline $\mathrm{C} 17$ & $-0.0054(5)$ & $0.7359(5)$ & $0.1821(4)$ & $0.0196(16)^{*}$ & 0.5 \\
\hline $\mathrm{C} 18$ & $0.0503(5)$ & $0.8155(5)$ & $0.2457(5)$ & $0.0155(16)$ & 0.5 \\
\hline $\mathrm{C} 55$ & $0.5425(8)$ & $1.0169(9)$ & $0.7041(7)$ & $0.029(3)$ & 0.5 \\
\hline $\mathrm{H} 55 \mathrm{~A}$ & 0.569555 & 1.068714 & 0.674356 & $0.043^{*}$ & 0.5 \\
\hline H55B & 0.586821 & 1.023926 & 0.751723 & $0.043^{*}$ & 0.5 \\
\hline $\mathrm{H} 55 \mathrm{C}$ & 0.485599 & 1.012279 & 0.719937 & $0.043^{*}$ & 0.5 \\
\hline C56 & $-0.0105(6)$ & $0.8302(6)$ & $0.2995(5)$ & $0.0164(18)$ & 0.5 \\
\hline H56A & -0.046493 & 0.855852 & 0.268975 & $0.020^{*}$ & 0.5 \\
\hline H56B & 0.030757 & 0.874492 & 0.345076 & $0.020^{*}$ & 0.5 \\
\hline $\mathrm{C} 57$ & $-0.0772(5)$ & $0.7467(5)$ & $0.3321(5)$ & $0.0207(16)$ & 0.5 \\
\hline H57A & -0.125993 & 0.706548 & 0.287690 & $0.025^{*}$ & 0.5 \\
\hline H57B & -0.043343 & 0.714948 & 0.354620 & $0.025^{*}$ & 0.5 \\
\hline C58 & $-0.1226(5)$ & $0.7682(5)$ & 0.3964 (4) & $0.0243(16)$ & 0.5 \\
\hline H58A & -0.072599 & 0.813072 & 0.437373 & $0.029^{*}$ & 0.5 \\
\hline H58B & -0.159139 & 0.796432 & 0.371733 & $0.029^{*}$ & 0.5 \\
\hline $\mathrm{C} 59$ & $-0.1837(8)$ & $0.6938(7)$ & $0.4382(6)$ & $0.033(2)$ & 0.5 \\
\hline H59A & -0.148403 & 0.664144 & 0.462273 & $0.039^{*}$ & 0.5 \\
\hline H59B & -0.202253 & 0.718134 & 0.482033 & $0.039^{*}$ & 0.5 \\
\hline $\mathrm{C} 60$ & $-0.2756(6)$ & $0.6198(5)$ & 0.3798 (4) & $0.0272(17)$ & 0.5 \\
\hline $\mathrm{H} 60 \mathrm{~A}$ & -0.294383 & 0.650373 & 0.338183 & $0.033^{*}$ & 0.5 \\
\hline H60B & -0.326133 & 0.594073 & 0.411543 & $0.033^{*}$ & 0.5 \\
\hline C61 & $-0.2663(5)$ & $0.5447(5)$ & 0.3399 (4) & $0.0271(17)$ & 0.5 \\
\hline H61A & -0.253114 & 0.510742 & 0.380440 & $0.041^{*}$ & 0.5 \\
\hline H61B & -0.215474 & 0.569153 & 0.309228 & $0.041^{*}$ & 0.5 \\
\hline $\mathrm{H} 61 \mathrm{C}$ & -0.324225 & 0.505468 & 0.303913 & $0.041 *$ & 0.5 \\
\hline C62 & $0.1071(5)$ & $0.9027(6)$ & $0.2087(5)$ & $0.0211(17)$ & 0.5 \\
\hline H62A & 0.144851 & 0.950717 & 0.252725 & $0.025^{*}$ & 0.5 \\
\hline H62B & 0.063441 & 0.919887 & 0.177735 & $0.025^{*}$ & 0.5 \\
\hline C63 & $0.1717(6)$ & $0.8980(6)$ & $0.1551(6)$ & $0.026(2)$ & 0.5 \\
\hline H63A & 0.210984 & 0.874685 & 0.184062 & $0.031 *$ & 0.5 \\
\hline H63B & 0.133554 & 0.854905 & 0.107832 & $0.031^{*}$ & 0.5 \\
\hline C64 & $0.2333(5)$ & $0.9862(5)$ & $0.1264(5)$ & $0.0309(18)$ & 0.5 \\
\hline H64A & 0.269725 & 1.029477 & 0.174036 & $0.037^{*}$ & 0.5 \\
\hline H64B & 0.277655 & 0.977667 & 0.097966 & $0.037^{*}$ & 0.5 \\
\hline $\mathrm{C} 65$ & $0.1846(6)$ & $1.0276(6)$ & $0.0722(5)$ & $0.031(2)$ & 0.5 \\
\hline H65A & 0.231777 & 1.086562 & 0.061309 & $0.037^{*}$ & 0.5 \\
\hline H65B & 0.141057 & 1.037312 & 0.100839 & $0.037^{*}$ & 0.5 \\
\hline C66 & $0.1328(13)$ & $0.9721(13)$ & $-0.0070(7)$ & $0.037(3)$ & 0.5 \\
\hline H66A & 0.173953 & 0.954937 & -0.032029 & $0.044^{*}$ & 0.5 \\
\hline H66B & 0.079943 & 0.916637 & 0.003621 & $0.044^{*}$ & 0.5 \\
\hline C69 & $-0.0006(6)$ & $0.2390(6)$ & $0.1885(6)$ & $0.0221(16)$ & 0.5 \\
\hline H69A & -0.033745 & 0.235853 & 0.233445 & $0.026^{*}$ & 0.5 \\
\hline H69B & -0.025685 & 0.178243 & 0.160815 & $0.026^{*}$ & 0.5 \\
\hline $\mathrm{C} 70$ & $-0.0110(8)$ & $0.3013(7)$ & $0.1311(7)$ & $0.026(2)$ & 0.5 \\
\hline $\mathrm{H} 70 \mathrm{~A}$ & 0.019842 & 0.362440 & 0.159028 & $0.031^{*}$ & 0.5 \\
\hline $\mathrm{H} 70 \mathrm{~B}$ & 0.021482 & 0.301800 & 0.086078 & $0.031^{*}$ & 0.5 \\
\hline C71 & $-0.1113(5)$ & $0.2761(6)$ & $0.0978(5)$ & $0.0272(18)$ & 0.5 \\
\hline $\mathrm{H} 71 \mathrm{~A}$ & -0.144306 & 0.212378 & 0.076447 & $0.033^{*}$ & 0.5 \\
\hline
\end{tabular}




\begin{tabular}{|c|c|c|c|c|c|}
\hline H71B & -0.141306 & 0.283668 & 0.141947 & $0.033^{*}$ & 0.5 \\
\hline $\mathrm{C} 72$ & $-0.1224(9)$ & $0.3302(7)$ & $0.0331(9)$ & $0.030(3)$ & 0.5 \\
\hline $\mathrm{H} 72 \mathrm{~A}$ & -0.084753 & 0.394031 & 0.053396 & $0.036^{*}$ & 0.5 \\
\hline $\mathrm{H} 72 \mathrm{~B}$ & -0.096263 & 0.318871 & -0.012414 & $0.036^{*}$ & 0.5 \\
\hline $\mathrm{C} 73$ & $-0.2200(5)$ & $0.3136(6)$ & $0.0028(5)$ & $0.036(2)$ & 0.5 \\
\hline $\mathrm{H} 73 \mathrm{~A}$ & -0.260510 & 0.248566 & -0.008842 & $0.044^{*}$ & 0.5 \\
\hline $\mathrm{H} 73 \mathrm{~B}$ & -0.242470 & 0.335676 & 0.045218 & $0.044^{*}$ & 0.5 \\
\hline $\mathrm{C} 74$ & $-0.2285(11)$ & $0.3576(9)$ & $-0.0708(8)$ & $0.038(3)$ & 0.5 \\
\hline $\mathrm{H} 74 \mathrm{~A}$ & -0.185485 & 0.421548 & -0.060777 & $0.057^{*}$ & 0.5 \\
\hline H74B & -0.292076 & 0.348574 & -0.084530 & $0.057^{*}$ & 0.5 \\
\hline $\mathrm{H} 74 \mathrm{C}$ & -0.213449 & 0.331304 & -0.115111 & $0.057^{*}$ & 0.5 \\
\hline O1B & $0.5424(7)$ & $0.9298(8)$ & $0.6356(6)$ & $0.025(2)$ & 0.5 \\
\hline C1B & $0.2381(6)$ & $0.7177(5)$ & $0.3512(5)$ & $0.0169(11)^{*}$ & 0.5 \\
\hline $\mathrm{C} 2 \mathrm{~B}$ & $0.1680(5)$ & $0.6738(6)$ & $0.2846(5)$ & $0.0171(11)^{*}$ & 0.5 \\
\hline $\mathrm{H} 2 \mathrm{~B}$ & 0.152520 & 0.614587 & 0.265781 & $0.020^{*}$ & 0.5 \\
\hline $\mathrm{C} 3 \mathrm{~B}$ & $0.1205(5)$ & $0.7151(5)$ & $0.2456(5)$ & $0.020(2)^{*}$ & 0.5 \\
\hline C4B & $0.1434(5)$ & $0.8031(5)$ & $0.2701(4)$ & $0.0184(16)^{*}$ & 0.5 \\
\hline C5B & $0.2109(5)$ & $0.8491(5)$ & $0.3350(4)$ & $0.0177(16)^{*}$ & 0.5 \\
\hline H5B & 0.226039 & 0.908583 & 0.352689 & $0.021 *$ & 0.5 \\
\hline C6B & $0.2578(5)$ & $0.8070(5)$ & $0.3752(4)$ & $0.0160(19)^{*}$ & 0.5 \\
\hline C12B & $0.0443(5)$ & $0.6823(5)$ & $0.1773(4)$ & $0.0175(19)^{*}$ & 0.5 \\
\hline C13B & $-0.0040(6)$ & $0.5995(6)$ & $0.1330(5)$ & $0.0215(12)^{*}$ & 0.5 \\
\hline H13B & 0.010432 & 0.552751 & 0.145305 & $0.026^{*}$ & 0.5 \\
\hline C14B & $-0.0738(6)$ & $0.5861(6)$ & $0.0706(5)$ & $0.0220(12)^{*}$ & 0.5 \\
\hline H14B & -0.107655 & 0.529471 & 0.040006 & $0.026^{*}$ & 0.5 \\
\hline C15B & $-0.0950(6)$ & $0.6540(5)$ & $0.0521(5)$ & $0.025(2)^{*}$ & 0.5 \\
\hline H15B & -0.136999 & 0.645873 & 0.004675 & $0.030^{*}$ & 0.5 \\
\hline C16B & $-0.0460(5)$ & $0.7378(5)$ & 0.0956 (4) & $0.0230(18)^{*}$ & 0.5 \\
\hline H16B & -0.060562 & 0.784464 & 0.083121 & $0.028^{*}$ & 0.5 \\
\hline C17B & $0.0236(5)$ & $0.7511(5)$ & 0.1579 (4) & $0.0205(17)^{*}$ & 0.5 \\
\hline C18B & $0.0841(6)$ & $0.8351(6)$ & $0.2160(5)$ & $0.0178(16)$ & 0.5 \\
\hline C55B & $0.5691(9)$ & $1.0092(10)$ & $0.6860(8)$ & $0.033(3)$ & 0.5 \\
\hline H55D & 0.578707 & 1.058122 & 0.653395 & $0.050^{*}$ & 0.5 \\
\hline H55E & 0.626794 & 1.023909 & 0.722320 & $0.050^{*}$ & 0.5 \\
\hline $\mathrm{H} 55 \mathrm{~F}$ & 0.520208 & 1.000070 & 0.717056 & $0.050^{*}$ & 0.5 \\
\hline C56B & $0.0236(5)$ & $0.8604(5)$ & $0.2619(5)$ & $0.0220(16)$ & 0.5 \\
\hline $\mathrm{H} 56 \mathrm{C}$ & -0.007242 & 0.886308 & 0.225153 & $0.026^{*}$ & 0.5 \\
\hline H56D & 0.064988 & 0.907698 & 0.305463 & $0.026^{*}$ & 0.5 \\
\hline C57B & $-0.0505(7)$ & $0.7856(7)$ & $0.2983(6)$ & $0.027(2)$ & 0.5 \\
\hline $\mathrm{H} 57 \mathrm{C}$ & -0.019962 & 0.762480 & 0.338476 & $0.033^{*}$ & 0.5 \\
\hline H57D & -0.089972 & 0.736280 & 0.255766 & $0.033^{*}$ & 0.5 \\
\hline C58B & $-0.1111(5)$ & $0.8154(6)$ & $0.3369(5)$ & $0.0355(19)$ & 0.5 \\
\hline $\mathrm{H} 58 \mathrm{C}$ & -0.076800 & 0.847879 & 0.389922 & $0.043^{*}$ & 0.5 \\
\hline H58D & -0.122650 & 0.857489 & 0.304392 & $0.043^{*}$ & 0.5 \\
\hline C59B & $-0.2033(6)$ & $0.7399(6)$ & $0.3489(6)$ & $0.047(2)$ & 0.5 \\
\hline $\mathrm{H} 59 \mathrm{C}$ & -0.237676 & 0.708910 & 0.295467 & $0.057^{*}$ & 0.5 \\
\hline H59D & -0.238896 & 0.766730 & 0.370417 & $0.057^{*}$ & 0.5 \\
\hline C60B & $-0.2043(8)$ & $0.6693(8)$ & $0.4012(6)$ & $0.041(2)$ & 0.5 \\
\hline
\end{tabular}




\begin{tabular}{|c|c|c|c|c|c|}
\hline $\mathrm{H} 60 \mathrm{C}$ & -0.160963 & 0.648931 & 0.385238 & $0.049^{*}$ & 0.5 \\
\hline H60D & -0.267022 & 0.617431 & 0.391178 & $0.049^{*}$ & 0.5 \\
\hline C61B & $-0.1804(9)$ & $0.6953(9)$ & $0.4853(6)$ & $0.073(4)$ & 0.5 \\
\hline H61D & -0.117047 & 0.744537 & 0.497883 & $0.110^{*}$ & 0.5 \\
\hline H61E & -0.184227 & 0.644638 & 0.513323 & $0.110^{*}$ & 0.5 \\
\hline H61F & -0.223434 & 0.714699 & 0.502326 & $0.110^{*}$ & 0.5 \\
\hline C62B & $0.1432(6)$ & $0.9140(6)$ & $0.1734(5)$ & $0.0202(18)$ & 0.5 \\
\hline $\mathrm{H} 62 \mathrm{C}$ & 0.102303 & 0.935198 & 0.144108 & $0.024^{*}$ & 0.5 \\
\hline H62D & 0.188153 & 0.962828 & 0.214268 & $0.024 *$ & 0.5 \\
\hline C63B & $0.1968(6)$ & $0.8964(5)$ & $0.1156(5)$ & $0.0254(18)$ & 0.5 \\
\hline $\mathrm{H} 63 \mathrm{C}$ & 0.234716 & 0.871229 & 0.143561 & $0.030^{*}$ & 0.5 \\
\hline H63D & 0.151956 & 0.851099 & 0.071991 & $0.030^{*}$ & 0.5 \\
\hline C64B & $0.2598(5)$ & $0.9790(5)$ & $0.0780(5)$ & $0.0308(18)$ & 0.5 \\
\hline H64C & 0.289624 & 0.961161 & 0.039218 & $0.037^{*}$ & 0.5 \\
\hline H64D & 0.309514 & 1.021191 & 0.120848 & $0.037^{*}$ & 0.5 \\
\hline C65B & $0.2108(6)$ & $1.0274(6)$ & $0.0359(5)$ & $0.0289(19)$ & 0.5 \\
\hline $\mathrm{H} 65 \mathrm{C}$ & 0.257721 & 1.082126 & 0.017999 & $0.035^{*}$ & 0.5 \\
\hline H65D & 0.181690 & 1.045795 & 0.074859 & $0.035^{*}$ & 0.5 \\
\hline C66B & $0.1374(11)$ & $0.9732(12)$ & $-0.0360(6)$ & 0.033 & 0.5 \\
\hline $\mathrm{H} 66 \mathrm{C}$ & 0.166512 & 0.956997 & -0.076448 & $0.040^{*}$ & 0.5 \\
\hline H66D & 0.090461 & 0.917377 & -0.019475 & $0.040^{*}$ & 0.5 \\
\hline C69B & $0.0147(7)$ & $0.2678(6)$ & $0.1625(6)$ & $0.0221(16)$ & 0.5 \\
\hline $\mathrm{H} 69 \mathrm{C}$ & -0.034115 & 0.248401 & 0.196286 & $0.026^{*}$ & 0.5 \\
\hline H69D & -0.003135 & 0.216261 & 0.122856 & $0.026^{*}$ & 0.5 \\
\hline $\mathrm{C} 70 \mathrm{~B}$ & $0.0043(8)$ & $0.3373(7)$ & $0.1153(7)$ & $0.025(2)$ & 0.5 \\
\hline $\mathrm{H} 70 \mathrm{C}$ & 0.002530 & 0.382285 & 0.152440 & $0.030^{*}$ & 0.5 \\
\hline H70D & 0.058910 & 0.367905 & 0.088820 & $0.030^{*}$ & 0.5 \\
\hline C71B & $-0.0838(6)$ & $0.2970(6)$ & $0.0528(6)$ & $0.034(2)$ & 0.5 \\
\hline $\mathrm{H} 71 \mathrm{C}$ & -0.135504 & 0.253261 & 0.076851 & $0.041^{*}$ & 0.5 \\
\hline H71D & -0.074584 & 0.264181 & 0.008681 & $0.041^{*}$ & 0.5 \\
\hline $\mathrm{C} 72 \mathrm{~B}$ & $-0.1113(9)$ & $0.3634(7)$ & $0.0184(8)$ & $0.029(3)$ & 0.5 \\
\hline $\mathrm{H} 72 \mathrm{C}$ & -0.129388 & 0.390013 & 0.060897 & $0.035^{*}$ & 0.5 \\
\hline $\mathrm{H} 72 \mathrm{D}$ & -0.056838 & 0.411873 & 0.001107 & $0.035^{*}$ & 0.5 \\
\hline C73B & $-0.1905(6)$ & $0.3241(6)$ & $-0.0518(6)$ & $0.040(2)$ & 0.5 \\
\hline $\mathrm{H} 73 \mathrm{C}$ & -0.173227 & 0.295604 & -0.093581 & $0.048^{*}$ & 0.5 \\
\hline H73D & -0.245497 & 0.276924 & -0.033991 & $0.048^{*}$ & 0.5 \\
\hline C74B & $-0.2172(11)$ & $0.3911(10)$ & $-0.0890(10)$ & $0.051(4)$ & 0.5 \\
\hline H74D & -0.163553 & 0.437699 & -0.107678 & $0.077^{*}$ & 0.5 \\
\hline $\mathrm{H} 74 \mathrm{E}$ & -0.237310 & 0.418021 & -0.049181 & $0.077^{*}$ & 0.5 \\
\hline $\mathrm{H} 74 \mathrm{~F}$ & -0.267920 & 0.360532 & -0.134245 & $0.077^{*}$ & 0.5 \\
\hline C77B & $0.1690(6)$ & $0.1365(6)$ & $0.3585(6)$ & $0.0401(15)^{*}$ & 0.5 \\
\hline $\mathrm{H} 77 \mathrm{~A}$ & 0.123784 & 0.129850 & 0.393728 & $0.048^{*}$ & 0.5 \\
\hline H77B & 0.142844 & 0.082120 & 0.320328 & $0.048^{*}$ & 0.5 \\
\hline C78B & $0.2593(5)$ & $0.1520(5)$ & $0.4077(5)$ & $0.0308(18)$ & 0.5 \\
\hline $\mathrm{H} 78 \mathrm{~A}$ & 0.300010 & 0.143052 & 0.374782 & $0.037^{*}$ & 0.5 \\
\hline $\mathrm{H} 78 \mathrm{~B}$ & 0.291960 & 0.214492 & 0.432702 & $0.037^{*}$ & 0.5 \\
\hline C79B & $0.2398(5)$ & $0.0900(5)$ & $0.4717(4)$ & $0.0275(17)$ & 0.5 \\
\hline $\mathrm{H} 79 \mathrm{~A}$ & 0.211373 & 0.027844 & 0.446620 & $0.033^{*}$ & 0.5 \\
\hline
\end{tabular}




\begin{tabular}{|c|c|c|c|c|c|}
\hline H79B & 0.194793 & 0.095774 & 0.501250 & $0.033^{*}$ & 0.5 \\
\hline $\mathrm{C} 80 \mathrm{~B}$ & $0.3265(5)$ & $0.1095(5)$ & $0.5296(4)$ & $0.0283(17)$ & 0.5 \\
\hline $\mathrm{H} 80 \mathrm{~A}$ & 0.310832 & 0.067892 & 0.570280 & $0.042 *$ & 0.5 \\
\hline H80B & 0.354182 & 0.170702 & 0.555290 & $0.042 *$ & 0.5 \\
\hline $\mathrm{H} 80 \mathrm{C}$ & 0.370772 & 0.102762 & 0.500630 & $0.042 *$ & 0.5 \\
\hline O3B & $0.4563(4)$ & $0.5453(5)$ & $0.6547(3)$ & $0.0266(14)$ & 0.5 \\
\hline N3B & $0.4196(6)$ & $0.6481(7)$ & $0.4829(4)$ & $0.0153(17)$ & 0.5 \\
\hline C37B & $0.4226(4)$ & $0.7280(5)$ & $0.3517(4)$ & $0.0203(12)^{*}$ & 0.5 \\
\hline C38B & $0.4236(5)$ & $0.7644(5)$ & $0.2778(4)$ & $0.0161(11)^{*}$ & 0.5 \\
\hline H38B & 0.368830 & 0.764924 & 0.252550 & $0.019^{*}$ & 0.5 \\
\hline C39B & $0.5013(6)$ & $0.7967(5)$ & $0.2408(4)$ & $0.0154(16)$ & 0.5 \\
\hline $\mathrm{C} 40 \mathrm{~B}$ & $0.5830(5)$ & $0.7975(5)$ & $0.2773(4)$ & $0.0216(15)$ & 0.5 \\
\hline C41B & $0.5878(4)$ & $0.7664(5)$ & $0.3501(4)$ & $0.0213(15)$ & 0.5 \\
\hline H41B & 0.643577 & 0.767955 & 0.375566 & $0.026^{*}$ & 0.5 \\
\hline $\mathrm{C} 42 \mathrm{~B}$ & $0.5086(4)$ & $0.7313(5)$ & $0.3860(4)$ & $0.0178(15)$ & 0.5 \\
\hline C43B & $0.5044(4)$ & $0.6867(5)$ & 0.4584 (4) & $0.0186(15)$ & 0.5 \\
\hline C44B & $0.5790(5)$ & $0.6809(5)$ & 0.5045 (4) & $0.0242(16)$ & 0.5 \\
\hline H44B & 0.638532 & 0.710291 & 0.489759 & $0.029 *$ & 0.5 \\
\hline C45B & $0.5672(5)$ & $0.6343(5)$ & 0.5697 (4) & $0.0242(16)$ & 0.5 \\
\hline H45B & 0.617500 & 0.629227 & 0.599087 & $0.029^{*}$ & 0.5 \\
\hline $\mathrm{C} 46 \mathrm{~B}$ & $0.4804(5)$ & $0.5942(5)$ & $0.5919(5)$ & $0.0195(17)$ & 0.5 \\
\hline C47B & $0.4092(9)$ & 0.6039 (11) & $0.5468(6)$ & $0.015(2)$ & 0.5 \\
\hline H47B & 0.350230 & 0.578082 & 0.563000 & $0.018^{*}$ & 0.5 \\
\hline C48B & $0.5136(5)$ & $0.8269(5)$ & $0.1618(4)$ & $0.0202(15)$ & 0.5 \\
\hline C49B & $0.4543(5)$ & $0.8379(5)$ & $0.1032(5)$ & $0.0270(18)$ & 0.5 \\
\hline H49B & 0.395309 & 0.828774 & 0.112526 & $0.032 *$ & 0.5 \\
\hline $\mathrm{C} 50 \mathrm{~B}$ & $0.4822(6)$ & $0.8619(5)$ & $0.0297(5)$ & $0.0315(18)$ & 0.5 \\
\hline H50B & 0.440859 & 0.867579 & -0.011559 & $0.038^{*}$ & 0.5 \\
\hline C51B & $0.5689(6)$ & $0.8771(6)$ & $0.0171(5)$ & $0.029(2)$ & 0.5 \\
\hline H51B & 0.586879 & 0.893595 & -0.033006 & $0.034^{*}$ & 0.5 \\
\hline C52B & $0.6305(5)$ & $0.8698(5)$ & $0.0765(4)$ & $0.0278(16)$ & 0.5 \\
\hline H52B & 0.690364 & 0.881281 & 0.067503 & $0.033^{*}$ & 0.5 \\
\hline C53B & $0.6020(6)$ & $0.8443(5)$ & $0.1496(5)$ & $0.0208(17)$ & 0.5 \\
\hline C54B & $0.6573(5)$ & $0.8318(6)$ & $0.2229(4)$ & $0.0251(17)$ & 0.5 \\
\hline C81B & $0.5305(5)$ & $0.5406(5)$ & $0.7067(5)$ & $0.0302(18)$ & 0.5 \\
\hline $\mathrm{H} 81 \mathrm{~A}$ & 0.561443 & 0.516516 & 0.675463 & $0.045^{*}$ & 0.5 \\
\hline H81B & 0.505113 & 0.501641 & 0.747820 & $0.045^{*}$ & 0.5 \\
\hline $\mathrm{H} 81 \mathrm{C}$ & 0.575410 & 0.600399 & 0.732060 & $0.045^{*}$ & 0.5 \\
\hline C82B & $0.7418(5)$ & $0.9207(6)$ & $0.2596(5)$ & 0.0337 (19) & 0.5 \\
\hline $\mathrm{H} 82 \mathrm{~A}$ & 0.789131 & 0.935908 & 0.224666 & $0.040^{*}$ & 0.5 \\
\hline H82B & 0.768811 & 0.911908 & 0.312006 & $0.040^{*}$ & 0.5 \\
\hline C83B & $0.7246(6)$ & $1.0012(6)$ & $0.2705(7)$ & $0.060(3)$ & 0.5 \\
\hline $\mathrm{H} 83 \mathrm{~A}$ & 0.719013 & 1.024677 & 0.218771 & $0.072 *$ & 0.5 \\
\hline H83B & 0.665563 & 0.982337 & 0.290781 & $0.072 *$ & 0.5 \\
\hline C84B & $0.8105(8)$ & $1.0763(6)$ & $0.3214(6)$ & $0.0469(17)^{*}$ & 0.5 \\
\hline $\mathrm{H} 84 \mathrm{~A}$ & 0.826644 & 1.050521 & 0.368550 & $0.056^{*}$ & 0.5 \\
\hline H84B & 0.861453 & 1.093154 & 0.290279 & $0.056^{*}$ & 0.5 \\
\hline C85B & $0.8124(6)$ & $1.1531(6)$ & $0.3490(6)$ & $0.062(2)$ & 0.5 \\
\hline
\end{tabular}




\begin{tabular}{|c|c|c|c|c|c|}
\hline $\mathrm{H} 85 \mathrm{~A}$ & 0.759439 & 1.137729 & 0.377472 & $0.075^{*}$ & 0.5 \\
\hline H85B & 0.803096 & 1.183469 & 0.302657 & $0.075^{*}$ & 0.5 \\
\hline $\mathrm{C} 86 \mathrm{~B}$ & $0.9014(6)$ & $1.2189(7)$ & $0.4050(6)$ & $0.065(4)$ & 0.5 \\
\hline H86A & 0.952000 & 1.206470 & 0.393914 & $0.077^{*}$ & 0.5 \\
\hline H86B & 0.917007 & 1.279617 & 0.390707 & $0.077^{*}$ & 0.5 \\
\hline C87B & $0.9015(7)$ & $1.2201(7)$ & $0.4931(6)$ & $0.051(3)^{*}$ & 0.5 \\
\hline H87A & 0.962267 & 1.264918 & 0.521503 & $0.077^{*}$ & 0.5 \\
\hline H87B & 0.853601 & 1.234770 & 0.506076 & $0.077^{*}$ & 0.5 \\
\hline $\mathrm{H} 87 \mathrm{C}$ & 0.888757 & 1.161284 & 0.509298 & $0.077^{*}$ & 0.5 \\
\hline C88B & $0.6922(4)$ & $0.7651(4)$ & $0.2036(4)$ & $0.0244(15)$ & 0.5 \\
\hline $\mathrm{H} 88 \mathrm{~A}$ & 0.732655 & 0.788386 & 0.162970 & $0.029^{*}$ & 0.5 \\
\hline H88B & 0.731412 & 0.763530 & 0.252368 & $0.029^{*}$ & 0.5 \\
\hline C89B & $0.6200(4)$ & $0.6700(4)$ & $0.1734(4)$ & $0.0250(16)$ & 0.5 \\
\hline H89A & 0.584336 & 0.642911 & 0.216253 & $0.030^{*}$ & 0.5 \\
\hline H89B & 0.576196 & 0.670753 & 0.127859 & $0.030^{*}$ & 0.5 \\
\hline C90B & $0.6619(5)$ & $0.6128(4)$ & $0.1472(4)$ & $0.0266(16)$ & 0.5 \\
\hline H90A & 0.704821 & 0.611408 & 0.193171 & $0.032 *$ & 0.5 \\
\hline H90B & 0.698829 & 0.640972 & 0.105372 & $0.032 *$ & 0.5 \\
\hline C91B & $0.5911(6)$ & $0.5174(5)$ & $0.1149(5)$ & $0.0317(19)$ & 0.5 \\
\hline H91A & 0.546491 & 0.519429 & 0.070865 & $0.038^{*}$ & 0.5 \\
\hline H91B & 0.555992 & 0.489037 & 0.157768 & $0.038^{*}$ & 0.5 \\
\hline C92B & $0.6295(6)$ & $0.4570(6)$ & $0.0840(7)$ & $0.036(3)^{*}$ & 0.5 \\
\hline $\mathrm{H} 92 \mathrm{~A}$ & 0.640372 & 0.421610 & 0.125230 & $0.043^{*}$ & 0.5 \\
\hline H92B & 0.686523 & 0.490678 & 0.061281 & $0.043^{*}$ & 0.5 \\
\hline C93B & $0.5381(6)$ & $0.3960(7)$ & $0.0148(6)$ & $0.051(3)^{*}$ & 0.5 \\
\hline H93A & 0.548316 & 0.351049 & -0.013533 & $0.076^{*}$ & 0.5 \\
\hline H93B & 0.483131 & 0.366264 & 0.040056 & $0.076^{*}$ & 0.5 \\
\hline $\mathrm{H} 93 \mathrm{C}$ & 0.528639 & 0.434370 & -0.023004 & $0.076^{*}$ & 0.5 \\
\hline $\mathrm{O} 3$ & $0.4887(4)$ & $0.5455(5)$ & $0.6272(4)$ & $0.0284(15)$ & 0.5 \\
\hline N3 & $0.4178(6)$ & $0.6324(7)$ & 0.4594 (4) & $0.0153(17)$ & 0.5 \\
\hline C37 & $0.3989(5)$ & $0.7153(5)$ & $0.3322(4)$ & $0.0203(12)^{*}$ & 0.5 \\
\hline $\mathrm{C} 38$ & $0.3904(5)$ & $0.7632(5)$ & $0.2700(4)$ & $0.0161(11)^{*}$ & 0.5 \\
\hline H38 & 0.341517 & 0.778631 & 0.263000 & $0.019^{*}$ & 0.5 \\
\hline C39 & $0.4543(6)$ & $0.7897(5)$ & $0.2188(4)$ & $0.0209(17)$ & 0.5 \\
\hline $\mathrm{C} 40$ & $0.5260(5)$ & $0.7670(5)$ & $0.2275(4)$ & $0.0204(16)$ & 0.5 \\
\hline $\mathrm{C} 41$ & $0.5384(4)$ & $0.7209(5)$ & $0.2894(4)$ & $0.0208(15)$ & 0.5 \\
\hline H41 & 0.587333 & 0.705502 & 0.295040 & $0.025^{*}$ & 0.5 \\
\hline $\mathrm{C} 42$ & $0.4747(5)$ & $0.6960(5)$ & $0.3431(4)$ & $0.0173(14)$ & 0.5 \\
\hline $\mathrm{C} 43$ & $0.4854(5)$ & $0.6533(5)$ & $0.4129(4)$ & $0.0181(14)$ & 0.5 \\
\hline $\mathrm{C} 44$ & $0.5582(5)$ & $0.6344(4)$ & $0.4404(4)$ & $0.0239(16)$ & 0.5 \\
\hline H44 & 0.605667 & 0.647729 & 0.409205 & $0.029^{*}$ & 0.5 \\
\hline $\mathrm{C} 45$ & $0.5635(4)$ & $0.5983(5)$ & $0.5103(4)$ & $0.0241(16)$ & 0.5 \\
\hline H45 & 0.613042 & 0.585522 & 0.527217 & $0.029^{*}$ & 0.5 \\
\hline $\mathrm{C} 46$ & $0.4947(5)$ & $0.5799(5)$ & $0.5561(5)$ & 0.0218 (17) & 0.5 \\
\hline $\mathrm{C} 47$ & $0.4214(8)$ & $0.5972(11)$ & $0.5268(6)$ & $0.015(2)$ & 0.5 \\
\hline H47 & 0.372680 & 0.583088 & 0.556641 & $0.018^{*}$ & 0.5 \\
\hline $\mathrm{C} 48$ & $0.4614(6)$ & $0.8414(5)$ & $0.1507(5)$ & $0.0233(16)$ & 0.5 \\
\hline C49 & $0.4061(5)$ & $0.8780(5)$ & $0.1178(4)$ & $0.0266(16)$ & 0.5 \\
\hline
\end{tabular}




\begin{tabular}{|c|c|c|c|c|c|}
\hline $\mathrm{H} 49$ & 0.353932 & 0.871422 & 0.140212 & $0.032 *$ & 0.5 \\
\hline $\mathrm{C} 50$ & $0.4281(5)$ & $0.9235(5)$ & $0.0512(4)$ & $0.0342(19)$ & 0.5 \\
\hline H50 & 0.391705 & 0.949851 & 0.028882 & $0.041^{*}$ & 0.5 \\
\hline $\mathrm{C} 51$ & $0.5024(6)$ & $0.9311(6)$ & $0.0174(5)$ & $0.039(2)$ & 0.5 \\
\hline H51 & 0.514559 & 0.960452 & -0.029407 & $0.047^{*}$ & 0.5 \\
\hline C52 & $0.5591(7)$ & $0.8965(6)$ & $0.0502(5)$ & $0.033(2)$ & 0.5 \\
\hline H52 & 0.611670 & 0.904167 & 0.027881 & $0.039^{*}$ & 0.5 \\
\hline $\mathrm{C} 53$ & $0.5369(5)$ & $0.8498(5)$ & 0.1175 (4) & $0.0264(16)$ & 0.5 \\
\hline C54 & $0.5846(6)$ & $0.8017(5)$ & $0.1604(5)$ & $0.0263(19)$ & 0.5 \\
\hline C77 & $0.1921(7)$ & $0.1391(6)$ & $0.3640(6)$ & $0.0401(15)^{*}$ & 0.5 \\
\hline $\mathrm{H} 77 \mathrm{C}$ & 0.253618 & 0.162721 & 0.398219 & $0.048^{*}$ & 0.5 \\
\hline H77D & 0.187368 & 0.092651 & 0.324339 & $0.048^{*}$ & 0.5 \\
\hline $\mathrm{C} 78$ & $0.1176(5)$ & $0.0914(5)$ & $0.4170(4)$ & 0.0337 (19) & 0.5 \\
\hline $\mathrm{H} 78 \mathrm{C}$ & 0.115246 & 0.138561 & 0.450828 & $0.040^{*}$ & 0.5 \\
\hline $\mathrm{H} 78 \mathrm{D}$ & 0.055446 & 0.057902 & 0.383688 & $0.040^{*}$ & 0.5 \\
\hline $\mathrm{C} 79$ & $0.1253(6)$ & $0.0283(5)$ & $0.4738(5)$ & $0.038(2)$ & 0.5 \\
\hline $\mathrm{H} 79 \mathrm{C}$ & 0.126868 & -0.021226 & 0.443002 & $0.045^{*}$ & 0.5 \\
\hline H79D & 0.184598 & 0.059944 & 0.511462 & $0.045^{*}$ & 0.5 \\
\hline $\mathrm{C} 80$ & $0.0469(6)$ & $-0.0102(5)$ & $0.5218(5)$ & $0.044(2)$ & 0.5 \\
\hline H80D & 0.056426 & -0.050667 & 0.557161 & $0.067^{*}$ & 0.5 \\
\hline $\mathrm{H} 80 \mathrm{E}$ & -0.012194 & -0.043207 & 0.485261 & $0.067^{*}$ & 0.5 \\
\hline $\mathrm{H} 80 \mathrm{~F}$ & 0.045656 & 0.038103 & 0.553821 & $0.067^{*}$ & 0.5 \\
\hline C81 & $0.5628(5)$ & $0.5273(6)$ & $0.6592(5)$ & $0.0313(19)$ & 0.5 \\
\hline H81D & 0.571389 & 0.490655 & 0.618836 & $0.047^{*}$ & 0.5 \\
\hline H81E & 0.547522 & 0.495383 & 0.706319 & $0.047^{*}$ & 0.5 \\
\hline $\mathrm{H} 81 \mathrm{~F}$ & 0.620121 & 0.583582 & 0.674322 & $0.047^{*}$ & 0.5 \\
\hline C82 & $0.6861(6)$ & $0.8635(5)$ & $0.1949(5)$ & $0.0267(18)$ & 0.5 \\
\hline $\mathrm{H} 82 \mathrm{C}$ & 0.721792 & 0.880255 & 0.150456 & $0.032 *$ & 0.5 \\
\hline H82D & 0.710102 & 0.830445 & 0.229276 & $0.032 *$ & 0.5 \\
\hline $\mathrm{C} 83$ & $0.7042(6)$ & $0.9493(6)$ & $0.2439(5)$ & $0.0306(19)$ & 0.5 \\
\hline $\mathrm{H} 83 \mathrm{C}$ & 0.655252 & 0.933809 & 0.277212 & $0.037^{*}$ & 0.5 \\
\hline H83D & 0.697772 & 0.990339 & 0.206622 & $0.037^{*}$ & 0.5 \\
\hline C84 & $0.7963(5)$ & $0.9979(5)$ & $0.2971(5)$ & $0.0322(18)$ & 0.5 \\
\hline $\mathrm{H} 84 \mathrm{C}$ & 0.845698 & 1.009560 & 0.265094 & $0.039^{*}$ & 0.5 \\
\hline H84D & 0.800868 & 0.959530 & 0.338174 & $0.039^{*}$ & 0.5 \\
\hline $\mathrm{C} 85$ & $0.8107(8)$ & $1.0832(7)$ & $0.3467(6)$ & $0.0469(17)^{*}$ & 0.5 \\
\hline $\mathrm{H} 85 \mathrm{C}$ & 0.817687 & 1.128705 & 0.309655 & $0.056^{*}$ & 0.5 \\
\hline H85D & 0.753272 & 1.068576 & 0.368507 & $0.056^{*}$ & 0.5 \\
\hline $\mathrm{C} 86$ & $0.8878(6)$ & $1.1235(7)$ & $0.4122(6)$ & $0.062(2)$ & 0.5 \\
\hline $\mathrm{H} 86 \mathrm{C}$ & 0.947447 & 1.133222 & 0.397195 & $0.075^{*}$ & 0.5 \\
\hline H86D & 0.873237 & 1.080411 & 0.452375 & $0.075^{*}$ & 0.5 \\
\hline $\mathrm{C} 87$ & $0.8989(7)$ & $1.2109(6)$ & $0.4505(6)$ & $0.033(2)^{*}$ & 0.5 \\
\hline H87D & 0.948489 & 1.232209 & 0.497038 & $0.050^{*}$ & 0.5 \\
\hline H87E & 0.841098 & 1.202179 & 0.467408 & $0.050^{*}$ & 0.5 \\
\hline $\mathrm{H} 87 \mathrm{~F}$ & 0.915488 & 1.255119 & 0.412038 & $0.050^{*}$ & 0.5 \\
\hline $\mathrm{C} 88$ & $0.5749(5)$ & $0.7239(5)$ & $0.1035(5)$ & 0.0364 (19) & 0.5 \\
\hline $\mathrm{H} 88 \mathrm{C}$ & 0.597295 & 0.687537 & 0.134619 & $0.044^{*}$ & 0.5 \\
\hline H88D & 0.615643 & 0.749069 & 0.063407 & $0.044^{*}$ & 0.5 \\
\hline
\end{tabular}




\begin{tabular}{|c|c|c|c|c|c|}
\hline $\mathrm{C} 89$ & $0.4748(6)$ & $0.6618(6)$ & $0.0588(5)$ & 0.059 & 0.5 \\
\hline $\mathrm{H} 89 \mathrm{C}$ & 0.435865 & 0.686623 & 0.077068 & $0.071^{*}$ & 0.5 \\
\hline H89D & 0.455698 & 0.604103 & 0.081208 & $0.071^{*}$ & 0.5 \\
\hline $\mathrm{C} 90$ & $0.4392(7)$ & $0.6358(6)$ & $-0.0397(5)$ & $0.053(3)$ & 0.5 \\
\hline $\mathrm{H} 90 \mathrm{C}$ & 0.421884 & 0.680460 & -0.061158 & $0.064 *$ & 0.5 \\
\hline H90D & 0.491393 & 0.639404 & -0.064770 & $0.064^{*}$ & 0.5 \\
\hline C91 & $0.3645(8)$ & $0.5518(8)$ & $-0.0610(7)$ & $0.059(4)^{*}$ & 0.5 \\
\hline $\mathrm{H} 91 \mathrm{C}$ & 0.306475 & 0.556601 & -0.065533 & $0.071^{*}$ & 0.5 \\
\hline H91D & 0.364286 & 0.513718 & -0.018242 & $0.071^{*}$ & 0.5 \\
\hline C92 & $0.3659(8)$ & $0.5083(8)$ & $-0.1374(6)$ & $0.075(4)$ & 0.5 \\
\hline $\mathrm{H} 92 \mathrm{C}$ & 0.382291 & 0.460083 & -0.126286 & $0.090^{*}$ & 0.5 \\
\hline H92D & 0.414164 & 0.552478 & -0.164243 & $0.090^{*}$ & 0.5 \\
\hline C93 & $0.2739(7)$ & $0.4699(8)$ & $-0.1920(6)$ & $0.088(4)$ & 0.5 \\
\hline H93D & 0.277605 & 0.441860 & -0.241621 & $0.132^{*}$ & 0.5 \\
\hline H93E & 0.226155 & 0.425312 & -0.165960 & $0.132^{*}$ & 0.5 \\
\hline $\mathrm{H} 93 \mathrm{~F}$ & 0.258014 & 0.517666 & -0.203900 & $0.132^{*}$ & 0.5 \\
\hline C1S & $0.5936(4)$ & $0.7728(5)$ & 0.7767 (4) & $0.109(3)$ & \\
\hline H1SA & 0.614669 & 0.740856 & 0.742590 & $0.164^{*}$ & 0.5 \\
\hline H1SB & 0.627082 & 0.836747 & 0.772014 & $0.164^{*}$ & 0.5 \\
\hline H1SC & 0.605520 & 0.762116 & 0.832268 & $0.164^{*}$ & 0.5 \\
\hline H1SD & 0.648018 & 0.821263 & 0.810209 & $0.164^{*}$ & 0.5 \\
\hline H1SE & 0.585660 & 0.715442 & 0.793472 & $0.164^{*}$ & 0.5 \\
\hline H1SF & 0.602180 & 0.775334 & 0.720862 & $0.164^{*}$ & 0.5 \\
\hline $\mathrm{C} 2 \mathrm{~S}$ & $0.4895(6)$ & $0.7393(8)$ & $0.7505(7)$ & $0.082(3)^{*}$ & 0.5 \\
\hline $\mathrm{H} 2 \mathrm{SA}$ & 0.455162 & 0.674596 & 0.754765 & $0.098^{*}$ & 0.5 \\
\hline $\mathrm{H} 2 \mathrm{SB}$ & 0.476792 & 0.749459 & 0.694323 & $0.098^{*}$ & 0.5 \\
\hline $\mathrm{C} 3 \mathrm{~S}$ & $0.4571(8)$ & $0.7912(8)$ & $0.8059(7)$ & $0.085(3)^{*}$ & 0.5 \\
\hline H3SA & 0.481237 & 0.790797 & 0.862303 & $0.102^{*}$ & 0.5 \\
\hline H3SB & 0.483162 & 0.853996 & 0.794146 & $0.102^{*}$ & 0.5 \\
\hline $\mathrm{C} 4 \mathrm{~S}$ & $0.3505(8)$ & $0.7492(8)$ & $0.7937(9)$ & $0.091(3)^{*}$ & 0.5 \\
\hline H4SA & 0.323615 & 0.710164 & 0.835075 & $0.109^{*}$ & 0.5 \\
\hline H4SB & 0.320263 & 0.716914 & 0.739857 & $0.109^{*}$ & 0.5 \\
\hline $\mathrm{C} 5 \mathrm{~S}$ & $0.3494(10)$ & $0.8403(8)$ & $0.8045(9)$ & $0.113(5)^{*}$ & 0.5 \\
\hline H5SA & 0.285182 & 0.829826 & 0.798957 & $0.170^{*}$ & 0.5 \\
\hline H5SB & 0.382827 & 0.870760 & 0.857631 & $0.170^{*}$ & 0.5 \\
\hline $\mathrm{H} 5 \mathrm{SC}$ & 0.379525 & 0.877409 & 0.763835 & $0.170^{*}$ & 0.5 \\
\hline $\mathrm{C} 2 \mathrm{~S}^{\prime}$ & $0.5081(6)$ & $0.7825(8)$ & $0.7854(8)$ & $0.082(3)^{*}$ & 0.5 \\
\hline $\mathrm{H} 2 \mathrm{SC}$ & 0.508701 & 0.784907 & 0.843616 & $0.098^{*}$ & 0.5 \\
\hline $\mathrm{H} 2 \mathrm{SD}$ & 0.524942 & 0.843787 & 0.772231 & $0.098^{*}$ & 0.5 \\
\hline $\mathrm{C} 3 \mathrm{~S}^{\prime}$ & $0.4089(7)$ & $0.7323(8)$ & $0.7516(7)$ & $0.085(3)^{*}$ & 0.5 \\
\hline $\mathrm{H} 3 \mathrm{SC}$ & 0.383172 & 0.674290 & 0.773778 & $0.102^{*}$ & 0.5 \\
\hline H3SD & 0.403005 & 0.719763 & 0.693299 & $0.102^{*}$ & 0.5 \\
\hline $\mathrm{C} 4 \mathrm{~S}^{\prime}$ & $0.3490(9)$ & $0.7767(9)$ & $0.7658(7)$ & $0.091(3)^{*}$ & 0.5 \\
\hline $\mathrm{H} 4 \mathrm{SC}$ & 0.296460 & 0.752759 & 0.720705 & $0.109^{*}$ & 0.5 \\
\hline H4SD & 0.386565 & 0.840719 & 0.760183 & $0.109^{*}$ & 0.5 \\
\hline $\mathrm{C}^{2} \mathrm{~S}^{\prime}$ & $0.3070(11)$ & $0.7726(12)$ & $0.8421(8)$ & $0.131(6)^{*}$ & 0.5 \\
\hline H5SD & 0.271446 & 0.805677 & 0.837885 & $0.196^{*}$ & 0.5 \\
\hline H5SE & 0.265961 & 0.710282 & 0.848592 & $0.196^{*}$ & 0.5 \\
\hline
\end{tabular}


Atomic displacement parameters $\left(\AA^{2}\right)$

\begin{tabular}{|c|c|c|c|c|c|c|}
\hline & $U^{11}$ & $U^{22}$ & $U^{33}$ & $U^{12}$ & $U^{13}$ & $U^{23}$ \\
\hline Ir & $0.01876(7)$ & $0.02774(8)$ & $0.01489(7)$ & $0.01543(6)$ & $0.00330(5)$ & $0.00433(5)$ \\
\hline $\mathrm{O} 2$ & $0.0472(18)$ & $0.0385(17)$ & $0.0263(15)$ & $0.0056(15)$ & $0.0214(13)$ & $-0.0042(13)$ \\
\hline $\mathrm{N} 1$ & $0.0156(15)$ & $0.0277(18)$ & $0.0157(15)$ & $0.0092(14)$ & $0.0029(12)$ & $0.0063(13)$ \\
\hline $\mathrm{N} 2$ & $0.0195(15)$ & $0.0244(16)$ & $0.0123(14)$ & $0.0139(14)$ & $0.0049(12)$ & $0.0003(12)$ \\
\hline $\mathrm{C} 7$ & $0.0248(19)$ & $0.039(2)$ & $0.0167(18)$ & $0.0224(19)$ & $-0.0030(15)$ & $-0.0062(16)$ \\
\hline $\mathrm{C} 8$ & $0.028(2)$ & $0.045(3)$ & $0.033(2)$ & $0.026(2)$ & $-0.0075(17)$ & $-0.0136(19)$ \\
\hline C9 & $0.023(2)$ & $0.032(2)$ & $0.0201(19)$ & $0.0065(18)$ & $0.0027(15)$ & $-0.0057(16)$ \\
\hline $\mathrm{C} 10$ & $0.020(2)$ & $0.030(2)$ & $0.024(2)$ & $-0.0027(18)$ & $-0.0071(15)$ & $0.0046(16)$ \\
\hline C11 & $0.0175(19)$ & $0.026(2)$ & $0.028(2)$ & $0.0024(17)$ & $-0.0027(15)$ & $0.0098(16)$ \\
\hline C19 & $0.0201(18)$ & $0.035(2)$ & $0.0184(18)$ & $0.0224(18)$ & $-0.0021(14)$ & $-0.0003(16)$ \\
\hline $\mathrm{C} 20$ & $0.028(2)$ & $0.046(2)$ & $0.0166(18)$ & $0.0281(19)$ & $-0.0011(15)$ & $0.0010(17)$ \\
\hline $\mathrm{C} 21$ & $0.025(2)$ & $0.055(3)$ & $0.0185(19)$ & $0.029(2)$ & $-0.0100(15)$ & $-0.0135(18)$ \\
\hline $\mathrm{C} 22$ & $0.020(2)$ & $0.053(3)$ & $0.037(2)$ & $0.020(2)$ & $-0.0084(17)$ & $-0.026(2)$ \\
\hline $\mathrm{C} 23$ & $0.0130(18)$ & $0.045(3)$ & $0.041(2)$ & $0.0080(19)$ & $0.0016(17)$ & $-0.020(2)$ \\
\hline $\mathrm{C} 24$ & $0.0152(17)$ & $0.034(2)$ & $0.0220(19)$ & $0.0139(17)$ & $-0.0037(14)$ & $-0.0100(16)$ \\
\hline $\mathrm{C} 25$ & $0.0172(17)$ & $0.0212(19)$ & $0.0207(18)$ & $0.0117(16)$ & $-0.0020(14)$ & $-0.0039(14)$ \\
\hline $\mathrm{C} 26$ & $0.0211(19)$ & $0.023(2)$ & $0.028(2)$ & $0.0114(17)$ & $0.0007(15)$ & $-0.0008(16)$ \\
\hline $\mathrm{C} 27$ & $0.024(2)$ & $0.024(2)$ & $0.0231(19)$ & $0.0107(17)$ & $0.0070(16)$ & $0.0076(16)$ \\
\hline $\mathrm{C} 28$ & $0.029(2)$ & $0.027(2)$ & $0.0169(18)$ & $0.0143(18)$ & $0.0054(15)$ & $0.0028(15)$ \\
\hline $\mathrm{C} 29$ & $0.0207(18)$ & $0.022(2)$ & $0.0182(18)$ & $0.0115(16)$ & $-0.0001(14)$ & $-0.0012(15)$ \\
\hline $\mathrm{C} 30$ & $0.038(2)$ & $0.062(3)$ & $0.0181(19)$ & $0.043(2)$ & $-0.0084(16)$ & $-0.0099(18)$ \\
\hline $\mathrm{C} 31$ & $0.057(3)$ & $0.062(3)$ & $0.019(2)$ & $0.050(2)$ & $0.0031(18)$ & $0.0022(19)$ \\
\hline $\mathrm{C} 32$ & $0.084(3)$ & $0.069(3)$ & $0.020(2)$ & $0.066(3)$ & $0.016(2)$ & $0.013(2)$ \\
\hline $\mathrm{C} 33$ & $0.088(4)$ & $0.074(3)$ & $0.021(2)$ & $0.074(3)$ & $0.003(2)$ & $-0.001(2)$ \\
\hline C34 & $0.054(3)$ & $0.064(3)$ & $0.025(2)$ & $0.046(3)$ & $-0.0112(19)$ & $-0.017(2)$ \\
\hline C35 & $0.036(2)$ & $0.064(3)$ & $0.024(2)$ & $0.038(2)$ & $-0.0114(17)$ & $-0.0190(19)$ \\
\hline C36 & $0.023(2)$ & $0.058(3)$ & $0.047(3)$ & $0.022(2)$ & $-0.0090(18)$ & $-0.035(2)$ \\
\hline C67 & $0.038(3)$ & $0.051(3)$ & $0.045(3)$ & $0.015(2)$ & $0.008(2)$ & $0.012(2)$ \\
\hline C68 & $0.051(3)$ & $0.060(3)$ & $0.025(2)$ & 0.009 (3) & $0.023(2)$ & $0.002(2)$ \\
\hline $\mathrm{C} 75$ & $0.022(2)$ & $0.050(3)$ & $0.053(3)$ & $0.007(2)$ & $0.0010(19)$ & $-0.032(2)$ \\
\hline C76 & $0.025(2)$ & $0.035(3)$ & $0.066(3)$ & $0.011(2)$ & $0.008(2)$ & -0.019 (2) \\
\hline $\mathrm{O} 1$ & $0.032(5)$ & $0.027(4)$ & $0.028(5)$ & $0.016(4)$ & $-0.009(3)$ & -0.011 \\
\hline $\mathrm{C} 18$ & $0.013(4)$ & $0.015(4)$ & $0.019(4)$ & $0.008(3)$ & $0.000(3)$ & $-0.001(3)$ \\
\hline C55 & $0.029(6)$ & $0.022(5)$ & $0.023(6)$ & $0.007(5)$ & $-0.012(4)$ & $-0.011(4)$ \\
\hline C56 & $0.015(5)$ & $0.016(5)$ & $0.020(4)$ & $0.009(4)$ & $0.002(3)$ & $0.004(4)$ \\
\hline $\mathrm{C} 57$ & $0.015(4)$ & $0.017(4)$ & $0.028(4)$ & $0.006(3)$ & $0.002(3)$ & $0.004(3)$ \\
\hline C58 & $0.026(4)$ & $0.026(4)$ & $0.019(4)$ & $0.012(3)$ & $-0.001(3)$ & $-0.004(3)$ \\
\hline C59 & $0.055(6)$ & $0.040(5)$ & $0.009(5)$ & $0.030(4)$ & $-0.002(5)$ & $-0.002(4)$ \\
\hline C60 & $0.030(4)$ & $0.025(4)$ & $0.024(4)$ & $0.011(4)$ & $0.005(3)$ & $0.004(3)$ \\
\hline C61 & $0.028(4)$ & $0.022(4)$ & $0.031(4)$ & $0.010(4)$ & $0.006(3)$ & $0.008(3)$ \\
\hline C62 & $0.015(4)$ & $0.024(5)$ & $0.026(4)$ & $0.009(4)$ & 0.007 (3) & 0.009 (4) \\
\hline C63 & $0.027(5)$ & $0.026(5)$ & $0.036(6)$ & $0.017(4)$ & $0.016(4)$ & $0.009(4)$ \\
\hline C64 & 0.025 & $0.032(5)$ & $0.037(5)$ & $0.011(4)$ & $0.011(4)$ & $0.014(4)$ \\
\hline
\end{tabular}




\begin{tabular}{|c|c|c|c|c|c|c|}
\hline C65 & $0.037(5)$ & $0.023(5)$ & $0.029(5)$ & $0.010(4)$ & $0.012(4)$ & $0.014(4)$ \\
\hline C66 & $0.045(7)$ & $0.037(6)$ & $0.035(6)$ & $0.025(6)$ & $0.012(6)$ & $0.005(6)$ \\
\hline C69 & $0.015(3)$ & $0.007(5)$ & $0.036(5)$ & $0.000(3)$ & $0.002(3)$ & $-0.006(3)$ \\
\hline $\mathrm{C} 70$ & $0.020(5)$ & $0.023(7)$ & $0.031(6)$ & $0.008(5)$ & $0.000(4)$ & -0.002 \\
\hline C71 & $0.024(4)$ & $0.031(5)$ & $0.025(4)$ & $0.013(4)$ & -0.001 & -0.007 (4) \\
\hline $\mathrm{C} 72$ & $0.023(5)$ & $0.020(7)$ & $0.041(6)$ & $0.009(5)$ & -0.004 (4) & -0.003 \\
\hline $\mathrm{C} 73$ & $0.027(4)$ & $0.048(6)$ & $0.034(5)$ & $0.019(4)$ & $0.000(4)$ & $0.000(4)$ \\
\hline C74 & $0.033(6)$ & $0.032(8)$ & $0.033(6)$ & $0.008(6)$ & $-0.015(5)$ & $-0.005(5)$ \\
\hline O1B & $0.026(4)$ & $0.024(4)$ & $0.021(4)$ & $0.011(3)$ & -0.009 (3) & -0.008 \\
\hline C18B & $0.017(4)$ & $0.018(5)$ & $0.020(4)$ & $0.010(4)$ & -0.001 & 0.003 \\
\hline C55B & $0.033(7)$ & $0.026(6)$ & $0.033(7)$ & $0.009(5)$ & $-0.003(5)$ & -0.008 \\
\hline C56B & $0.028(4)$ & $0.029(5)$ & $0.015(4)$ & $0.019(4)$ & -0.001 & -0.001 \\
\hline C57B & $0.025(6)$ & $0.027(6)$ & $0.033(5)$ & $0.015(5)$ & $0.006(4)$ & $0.002(5)$ \\
\hline C58B & $0.030(4)$ & $0.045(5)$ & $0.041(5)$ & $0.025(4)$ & 0.007 (4) & $-0.002(4)$ \\
\hline C59B & $0.055(5)$ & $0.055(6)$ & $0.060(6)$ & $0.041(5)$ & $0.038(5)$ & $0.027(5)$ \\
\hline $\mathrm{C} 60 \mathrm{~B}$ & $0.046(6)$ & $0.049(7)$ & $0.038(6)$ & $0.032(5)$ & $0.008(5)$ & $0.010(5)$ \\
\hline C61B & $0.112(10)$ & $0.115(11)$ & $0.037(6)$ & $0.094(9)$ & $0.003(7)$ & $0.006(7)$ \\
\hline C62B & $0.021(5)$ & $0.020(4)$ & $0.022(5)$ & $0.012(4)$ & -0.001 & $0.002(4)$ \\
\hline C63B & $0.026(4)$ & $0.026(4)$ & $0.031(5)$ & $0.017(4)$ & $0.005(3)$ & 0.008 \\
\hline C64B & $0.023(4)$ & $0.028(4)$ & $0.037(5)$ & 0.008 (4) & $0.006(3)$ & $0.009(4)$ \\
\hline C65B & $0.036(5)$ & $0.021(5)$ & $0.031(5)$ & $0.014(4)$ & $0.010(4)$ & 0.009 (4) \\
\hline C66B & $0.032(5)$ & $0.025(6)$ & $0.032(7)$ & $0.005(4)$ & $0.009(5)$ & $-0.004(6)$ \\
\hline C69B & $0.015(3)$ & $0.007(5)$ & $0.036(5)$ & $0.000(3)$ & $0.002(3)$ & $-0.006(3)$ \\
\hline C70B & $0.025(5)$ & $0.026(7)$ & $0.028(5)$ & $0.018(5)$ & -0.001 & $-0.006(4)$ \\
\hline C71B & $0.027(5)$ & 0.023 & $0.045(6)$ & $0.011(4)$ & $-0.012(4)$ & $-0.006(4)$ \\
\hline C72B & $0.026(5)$ & 0.020 & $0.037(6)$ & $0.012(5)$ & -0.008 & -0.005 \\
\hline C73B & $0.034(5)$ & $0.036(5)$ & $0.045(6)$ & $0.017(5)$ & $-0.012(4)$ & $-0.007(4)$ \\
\hline C74B & $0.046(8)$ & $0.068(12)$ & $0.050(9)$ & $0.042(9)$ & $-0.008(6)$ & $-0.001(6)$ \\
\hline C78B & $0.034(5)$ & $0.026(4)$ & 0.038 & 0.019 & 0.007 (4) & $0.012(3)$ \\
\hline C79B & $0.027(4)$ & $0.023(4)$ & $0.031(4)$ & $0.011(4)$ & $0.004(3)$ & $0.006(3)$ \\
\hline C80B & $0.032(4)$ & $0.024(4)$ & $0.027(4)$ & $0.013(4)$ & $0.003(3)$ & 0.003 \\
\hline O3B & $0.026(4)$ & $0.037(4)$ & $0.021(3)$ & $0.018(3)$ & $0.002(2)$ & 0.009 (3) \\
\hline N3B & $0.0166(16)$ & $0.016(4)$ & 0.014 & 0.009 & $0.001(2)$ & -0.002 \\
\hline C39B & $0.016(4)$ & 0.009 (4) & $0.017(4)$ & $0.003(4)$ & $0.002(3)$ & -0.004 \\
\hline $\mathrm{C} 40 \mathrm{~B}$ & $0.015(3)$ & $0.023(4)$ & $0.021(3)$ & $0.005(3)$ & $0.004(3)$ & 0.000 \\
\hline C41B & $0.013(3)$ & $0.028(4)$ & $0.022(3)$ & $0.010(3)$ & $-0.002(3)$ & $0.000(3)$ \\
\hline C42B & 0.011 & 0.023 & 0.015 & 0.006 & -0.001 & -0.002 \\
\hline C43B & 0.014 & $0.027(4)$ & 0.019 & $0.012(3)$ & 0.004 & $0.001(3)$ \\
\hline C44B & $0.016(3)$ & $0.037(5)$ & $0.021(4)$ & $0.014(3)$ & $0.003(3)$ & 0.004 \\
\hline $\mathrm{C} 45 \mathrm{~B}$ & $0.021(4)$ & $0.026(4)$ & $0.024(4)$ & $0.012(4)$ & -0.006 & 0.000 \\
\hline C46B & $0.023(4)$ & 0.019 (4) & 0.015 & 0.010 & $-0.002(3)$ & -0.003 \\
\hline C47B & $0.017(4)$ & $0.016(5)$ & $0.012(5)$ & 0.009 (4) & $0.000(3)$ & -0.003 \\
\hline C48B & $0.020(4)$ & $0.011(4)$ & $0.022(4)$ & $0.000(3)$ & $0.005(3)$ & 0.000 \\
\hline C49B & $0.024(4)$ & $0.016(4)$ & $0.033(4)$ & $0.002(4)$ & $0.004(4)$ & $0.010(4)$ \\
\hline $\mathrm{C} 50 \mathrm{~B}$ & $0.034(5)$ & $0.027(5)$ & $0.031(4)$ & $0.013(4)$ & $0.003(4)$ & $0.012(4)$ \\
\hline C51B & $0.035(4)$ & $0.020(5)$ & $0.025(5)$ & $0.007(4)$ & $0.006(4)$ & $0.003(4)$ \\
\hline C52B & $0.028(4)$ & $0.021(4)$ & $0.030(4)$ & $0.006(4)$ & $0.011(3)$ & 0.008 \\
\hline C53B & $0.021(4)$ & $0.013(4)$ & $0.022(3)$ & $0.002(4)$ & $0.004(3)$ & $0.001(3)$ \\
\hline
\end{tabular}




\begin{tabular}{|c|c|c|c|c|c|c|}
\hline C54B & $0.025(4)$ & $0.032(5)$ & 0.019 (4) & $0.012(4)$ & 0.007 (3) & $0.006(3)$ \\
\hline C81B & $0.028(4)$ & $0.034(5)$ & $0.026(4)$ & $0.014(4)$ & -0.004 & $0.006(4)$ \\
\hline C82B & $0.014(4)$ & $0.040(5)$ & $0.035(5)$ & $0.003(4)$ & $0.008(3)$ & -0.007 \\
\hline C83B & $0.026(5)$ & $0.035(6)$ & $0.096(9)$ & $-0.002(5)$ & $0.009(5)$ & $-0.031(6)$ \\
\hline C85B & $0.038(4)$ & $0.052(5)$ & $0.080(5)$ & $0.010(4)$ & $0.006(3)$ & -0.021 \\
\hline C86B & $0.030(5)$ & $0.062(7)$ & $0.063(7)$ & $-0.012(5)$ & $0.022(5)$ & $-0.039(6)$ \\
\hline C88B & 0.017 (4) & 0.035 & $0.020(4)$ & $0.011(3)$ & $0.004(3)$ & 0.005 \\
\hline C89B & 0.017 (4) & 0.030 & $0.022(4)$ & 0.007 (3) & $0.000(3)$ & 0.003 \\
\hline C90B & $0.026(4)$ & $0.032(4)$ & $0.024(4)$ & 0.014 (3) & 0.009 (3) & 0.007 (3) \\
\hline C91B & $0.035(5)$ & $0.039(5)$ & $0.027(5)$ & $0.022(4)$ & $0.005(4)$ & $0.008(4)$ \\
\hline $\mathrm{O} 3$ & $0.024(4)$ & $0.032(4)$ & $0.030(4)$ & 0.015 & $0.001(3)$ & $0.013(3)$ \\
\hline N3 & $0.0166(16)$ & $0.016(4)$ & $0.014(4)$ & 0.009 (2) & $0.001(2)$ & -0.002 \\
\hline C39 & $0.021(4)$ & $0.017(4)$ & $0.016(4)$ & $0.004(4)$ & -0.004 & $-0.006(3)$ \\
\hline $\mathrm{C} 40$ & $0.017(4)$ & $0.012(4)$ & $0.026(4)$ & $0.000(3)$ & 0.007 (3) & $0.001(3)$ \\
\hline $\mathrm{C} 41$ & $0.014(3)$ & $0.020(4)$ & $0.029(4)$ & $0.008(3)$ & $0.005(3)$ & $0.001(3)$ \\
\hline $\mathrm{C} 42$ & $0.012(4)$ & $0.018(4)$ & $0.017(4)$ & $0.004(3)$ & -0.001 & $0.000(3)$ \\
\hline $\mathrm{C} 43$ & $0.017(4)$ & $0.016(4)$ & 0.019 (4) & $0.006(3)$ & $0.004(3)$ & -0.001 \\
\hline $\mathrm{C} 44$ & $0.020(4)$ & $0.019(4)$ & $0.030(4)$ & 0.007 (3) & $0.002(3)$ & $0.002(3)$ \\
\hline $\mathrm{C} 45$ & $0.016(4)$ & 0.025 & $0.032(4)$ & $0.013(3)$ & -0.005 & $0.001(3)$ \\
\hline $\mathrm{C} 46$ & $0.024(4)$ & $0.017(4)$ & $0.019(4)$ & $0.008(4)$ & -0.007 & -0.001 \\
\hline C47 & $0.012(4)$ & $0.017(5)$ & $0.013(5)$ & 0.005 & $0.002(3)$ & -0.007 (4) \\
\hline $\mathrm{C} 48$ & $0.026(4)$ & $0.017(4)$ & $0.021(4)$ & $0.005(3)$ & $0.006(4)$ & $0.002(3)$ \\
\hline C49 & $0.033(4)$ & $0.024(4)$ & $0.020(4)$ & $0.011(4)$ & $0.008(3)$ & -0.001 \\
\hline $\mathrm{C} 50$ & $0.042(5)$ & $0.029(5)$ & $0.029(4)$ & $0.013(4)$ & 0.009 (4) & $0.013(3)$ \\
\hline C51 & $0.052(5)$ & $0.044(6)$ & $0.022(4)$ & $0.021(5)$ & 0.013 & $0.011(4)$ \\
\hline C52 & $0.038(5)$ & $0.028(6)$ & $0.027(5)$ & 0.009 (4) & $0.013(4)$ & $0.006(4)$ \\
\hline C53 & $0.026(4)$ & 0.023 & $0.024(4)$ & $0.006(4)$ & $0.005(3)$ & 0.003 \\
\hline C54 & 0.027 (4) & $0.024(5)$ & 0.028 & 0.010 & 0.011 & 0.003 \\
\hline C78 & $0.037(5)$ & $0.033(5)$ & $0.032(4)$ & 0.017 (4) & 0.004 (4) & $0.003(3)$ \\
\hline C79 & $0.040(5)$ & $0.032(5)$ & $0.037(5)$ & 0.015 & $-0.003(4)$ & $0.012(4)$ \\
\hline $\mathrm{C} 80$ & $0.054(6)$ & $0.024(5)$ & $0.049(6)$ & $0.013(4)$ & 0.007 (4) & $0.011(4)$ \\
\hline $\mathrm{C} 81$ & $0.029(4)$ & $0.041(5)$ & $0.028(4)$ & $0.023(4)$ & -0.009 (4) & 0.005 \\
\hline C82 & $0.026(4)$ & $0.024(5)$ & $0.031(5)$ & $0.011(4)$ & $0.011(3)$ & $0.005(3)$ \\
\hline C83 & $0.024(4)$ & $0.029(5)$ & $0.037(5)$ & $0.008(4)$ & $0.012(4)$ & $0.003(4)$ \\
\hline C84 & $0.029(4)$ & $0.027(5)$ & $0.042(5)$ & $0.014(4)$ & 0.007 (4) & $0.004(4)$ \\
\hline C86 & $0.038(4)$ & $0.052(5)$ & $0.080(5)$ & $0.010(4)$ & $0.006(3)$ & -0.021 \\
\hline C88 & 0.037 (4) & $0.031(4)$ & $0.036(4)$ & 0.008 & $0.022(4)$ & $0.000(3)$ \\
\hline C89 & $0.045(5)$ & $0.047(6)$ & $0.055(5)$ & $-0.007(5)$ & $0.022(4)$ & $-0.018(5)$ \\
\hline $\mathrm{C} 90$ & $0.066(7)$ & $0.057(7)$ & $0.064(6)$ & $0.049(6)$ & $0.016(5)$ & $0.013(5)$ \\
\hline C92 & $0.072(8)$ & $0.070(9)$ & $0.053(7)$ & $0.008(7)$ & $0.011(6)$ & $0.006(7)$ \\
\hline C93 & $0.073(7)$ & $0.100(10)$ & $0.052(7)$ & $0.010(7)$ & $0.012(5)$ & $-0.024(7)$ \\
\hline $\mathrm{C} 1 \mathrm{~S}$ & $0.091(5)$ & $0.133(6)$ & $0.151(7)$ & 0.078 & $0.064(5)$ & $0.041(5)$ \\
\hline
\end{tabular}

Geometric parameters $\left(\AA,{ }^{\circ}\right)$

\begin{tabular}{llll}
\hline $\mathrm{Ir}-\mathrm{N} 1$ & $2.121(3)$ & $\mathrm{C} 70 \mathrm{~B}-\mathrm{C} 71 \mathrm{~B}$ & $1.526(13)$ \\
$\mathrm{Ir}-\mathrm{N} 2$ & $2.135(3)$ & $\mathrm{C} 71 \mathrm{~B}-\mathrm{H} 71 \mathrm{C}$ & 0.9900 \\
$\mathrm{Ir}-\mathrm{C} 19$ & $2.020(4)$ & $\mathrm{C} 71 \mathrm{~B}-\mathrm{H} 71 \mathrm{D}$ & 0.9901
\end{tabular}




\begin{tabular}{|c|c|c|c|}
\hline $\mathrm{Ir}-\mathrm{C} 1$ & $2.017(7)$ & $\mathrm{C} 71 \mathrm{~B}-\mathrm{C} 72 \mathrm{~B}$ & $1.505(11)$ \\
\hline $\mathrm{Ir}-\mathrm{C} 1 \mathrm{~B}$ & $2.013(7)$ & $\mathrm{C} 72 \mathrm{~B}-\mathrm{H} 72 \mathrm{C}$ & 0.9895 \\
\hline $\mathrm{Ir}-\mathrm{N} 3 \mathrm{~B}$ & $2.142(7)$ & $\mathrm{C} 72 \mathrm{~B}-\mathrm{H} 72 \mathrm{D}$ & 0.9902 \\
\hline $\mathrm{Ir}-\mathrm{C} 37 \mathrm{~B}$ & $2.045(6)$ & $\mathrm{C} 72 \mathrm{~B}-\mathrm{C} 73 \mathrm{~B}$ & $1.516(15)$ \\
\hline $\mathrm{Ir}-\mathrm{N} 3$ & $2.128(7)$ & $\mathrm{C} 73 \mathrm{~B}-\mathrm{H} 73 \mathrm{C}$ & 0.9908 \\
\hline $\mathrm{Ir}-\mathrm{C} 37$ & $1.998(6)$ & $\mathrm{C} 73 \mathrm{~B}-\mathrm{H} 73 \mathrm{D}$ & 0.9899 \\
\hline $\mathrm{O} 2-\mathrm{C} 28$ & $1.362(4)$ & $\mathrm{C} 73 \mathrm{~B}-\mathrm{C} 74 \mathrm{~B}$ & $1.525(13)$ \\
\hline $\mathrm{O} 2-\mathrm{C} 68$ & $1.434(4)$ & $\mathrm{C} 74 \mathrm{~B}-\mathrm{H} 74 \mathrm{D}$ & 0.9800 \\
\hline $\mathrm{N} 1-\mathrm{C} 7$ & $1.362(4)$ & C74B-H74E & 0.9800 \\
\hline $\mathrm{N} 1-\mathrm{C} 11$ & $1.343(4)$ & $\mathrm{C} 74 \mathrm{~B}-\mathrm{H} 74 \mathrm{~F}$ & 0.9800 \\
\hline $\mathrm{N} 2-\mathrm{C} 25$ & $1.369(4)$ & $\mathrm{C} 77 \mathrm{~B}-\mathrm{H} 77 \mathrm{~A}$ & 0.9888 \\
\hline $\mathrm{N} 2-\mathrm{C} 29$ & $1.327(4)$ & $\mathrm{C} 77 \mathrm{~B}-\mathrm{H} 77 \mathrm{~B}$ & 0.9899 \\
\hline $\mathrm{C} 7-\mathrm{C} 8$ & $1.386(5)$ & $\mathrm{C} 77 \mathrm{~B}-\mathrm{C} 78 \mathrm{~B}$ & $1.508(8)$ \\
\hline $\mathrm{C} 7-\mathrm{C} 6$ & $1.505(7)$ & $\mathrm{C} 78 \mathrm{~B}-\mathrm{H} 78 \mathrm{~A}$ & 0.9898 \\
\hline $\mathrm{C} 7-\mathrm{C} 6 \mathrm{~B}$ & $1.474(7)$ & $\mathrm{C} 78 \mathrm{~B}-\mathrm{H} 78 \mathrm{~B}$ & 0.9901 \\
\hline $\mathrm{C} 8-\mathrm{H} 8$ & 0.9499 & $\mathrm{C} 78 \mathrm{~B}-\mathrm{C} 79 \mathrm{~B}$ & $1.504(10)$ \\
\hline $\mathrm{C} 8-\mathrm{C} 9$ & $1.380(5)$ & C79B-H79A & 0.9898 \\
\hline $\mathrm{C} 9-\mathrm{H} 9$ & 0.9502 & $\mathrm{C} 79 \mathrm{~B}-\mathrm{H} 79 \mathrm{~B}$ & 0.9899 \\
\hline $\mathrm{C} 9-\mathrm{C} 10$ & $1.379(5)$ & $\mathrm{C} 79 \mathrm{~B}-\mathrm{C} 80 \mathrm{~B}$ & $1.511(10)$ \\
\hline $\mathrm{C} 10-\mathrm{C} 11$ & $1.386(5)$ & $\mathrm{C} 80 \mathrm{~B}-\mathrm{H} 80 \mathrm{~A}$ & 0.9805 \\
\hline $\mathrm{C} 10-\mathrm{O} 1$ & 1.378 & $\mathrm{C} 80 \mathrm{~B}-\mathrm{H} 80 \mathrm{~B}$ & 0.9802 \\
\hline $\mathrm{C} 10-\mathrm{O} 1 \mathrm{~B}$ & $1.397(11)$ & $\mathrm{C} 80 \mathrm{~B}-\mathrm{H} 80 \mathrm{C}$ & 0.9802 \\
\hline $\mathrm{C} 11-\mathrm{H} 11$ & 0.9495 & $\mathrm{O} 3 \mathrm{~B}-\mathrm{C} 46 \mathrm{~B}$ & $1.366(9)$ \\
\hline $\mathrm{C} 19-\mathrm{C} 20$ & $1.411(5)$ & $\mathrm{O} 3 \mathrm{~B}-\mathrm{C} 81 \mathrm{~B}$ & $1.445(9)$ \\
\hline $\mathrm{C} 19-\mathrm{C} 24$ & $1.421(5)$ & $\mathrm{N} 3 \mathrm{~B}-\mathrm{C} 43 \mathrm{~B}$ & $1.376(9)$ \\
\hline $\mathrm{C} 20-\mathrm{H} 20$ & 0.9496 & $\mathrm{~N} 3 \mathrm{~B}-\mathrm{C} 47 \mathrm{~B}$ & $1.330(9)$ \\
\hline $\mathrm{C} 20-\mathrm{C} 21$ & $1.389(5)$ & $\mathrm{C} 37 \mathrm{~B}-\mathrm{C} 38 \mathrm{~B}$ & $1.420(8)$ \\
\hline $\mathrm{C} 21-\mathrm{C} 22$ & $1.394(6)$ & $\mathrm{C} 37 \mathrm{~B}-\mathrm{C} 42 \mathrm{~B}$ & $1.434(8)$ \\
\hline $\mathrm{C} 21-\mathrm{C} 30$ & $1.470(5)$ & $\mathrm{C} 38 \mathrm{~B}-\mathrm{H} 38 \mathrm{~B}$ & 0.9497 \\
\hline $\mathrm{C} 22-\mathrm{C} 23$ & $1.379(5)$ & $\mathrm{C} 38 \mathrm{~B}-\mathrm{C} 39 \mathrm{~B}$ & $1.392(9)$ \\
\hline $\mathrm{C} 22-\mathrm{C} 36$ & $1.529(5)$ & $\mathrm{C} 39 \mathrm{~B}-\mathrm{C} 40 \mathrm{~B}$ & $1.399(10)$ \\
\hline $\mathrm{C} 23-\mathrm{H} 23$ & 0.9499 & $\mathrm{C} 39 \mathrm{~B}-\mathrm{C} 48 \mathrm{~B}$ & $1.462(9)$ \\
\hline $\mathrm{C} 23-\mathrm{C} 24$ & $1.398(5)$ & $\mathrm{C} 40 \mathrm{~B}-\mathrm{C} 41 \mathrm{~B}$ & $1.371(9)$ \\
\hline $\mathrm{C} 24-\mathrm{C} 25$ & $1.460(5)$ & $\mathrm{C} 40 \mathrm{~B}-\mathrm{C} 54 \mathrm{~B}$ & $1.544(9)$ \\
\hline $\mathrm{C} 25-\mathrm{C} 26$ & $1.392(5)$ & $\mathrm{C} 41 \mathrm{~B}-\mathrm{H} 41 \mathrm{~B}$ & 0.9504 \\
\hline $\mathrm{C} 26-\mathrm{H} 26$ & 0.9502 & $\mathrm{C} 41 \mathrm{~B}-\mathrm{C} 42 \mathrm{~B}$ & $1.399(8)$ \\
\hline $\mathrm{C} 26-\mathrm{C} 27$ & $1.367(5)$ & $\mathrm{C} 42 \mathrm{~B}-\mathrm{C} 43 \mathrm{~B}$ & $1.463(9)$ \\
\hline $\mathrm{C} 27-\mathrm{H} 27$ & 0.9497 & $\mathrm{C} 43 \mathrm{~B}-\mathrm{C} 44 \mathrm{~B}$ & $1.411(8)$ \\
\hline $\mathrm{C} 27-\mathrm{C} 28$ & $1.382(5)$ & $\mathrm{C} 44 \mathrm{~B}-\mathrm{H} 44 \mathrm{~B}$ & 0.9499 \\
\hline C28-C29 & $1.385(5)$ & $\mathrm{C} 44 \mathrm{~B}-\mathrm{C} 45 \mathrm{~B}$ & $1.368(9)$ \\
\hline C29-H29 & 0.9506 & $\mathrm{C} 45 \mathrm{~B}-\mathrm{H} 45 \mathrm{~B}$ & 0.9499 \\
\hline $\mathrm{C} 30-\mathrm{C} 31$ & $1.395(5)$ & $\mathrm{C} 45 \mathrm{~B}-\mathrm{C} 46 \mathrm{~B}$ & $1.387(10)$ \\
\hline $\mathrm{C} 30-\mathrm{C} 35$ & $1.391(6)$ & $\mathrm{C} 46 \mathrm{~B}-\mathrm{C} 47 \mathrm{~B}$ & $1.390(10)$ \\
\hline C31-H31 & 0.9502 & $\mathrm{C} 47 \mathrm{~B}-\mathrm{H} 47 \mathrm{~B}$ & 0.9503 \\
\hline $\mathrm{C} 31-\mathrm{C} 32$ & $1.388(5)$ & $\mathrm{C} 48 \mathrm{~B}-\mathrm{C} 49 \mathrm{~B}$ & $1.380(10)$ \\
\hline C32-H32 & 0.9500 & $\mathrm{C} 48 \mathrm{~B}-\mathrm{C} 53 \mathrm{~B}$ & $1.395(10)$ \\
\hline $\mathrm{C} 32-\mathrm{C} 33$ & $1.377(6)$ & $\mathrm{C} 49 \mathrm{~B}-\mathrm{H} 49 \mathrm{~B}$ & 0.9497 \\
\hline C33-H33 & 0.9497 & $\mathrm{C} 49 \mathrm{~B}-\mathrm{C} 50 \mathrm{~B}$ & $1.399(10)$ \\
\hline
\end{tabular}




\begin{tabular}{|c|c|c|c|}
\hline $\mathrm{C} 33-\mathrm{C} 34$ & $1.386(6)$ & $\mathrm{C} 50 \mathrm{~B}-\mathrm{H} 50 \mathrm{~B}$ & 0.9499 \\
\hline C $34-\mathrm{H} 34$ & 0.9501 & $\mathrm{C} 50 \mathrm{~B}-\mathrm{C} 51 \mathrm{~B}$ & $1.382(11)$ \\
\hline $\mathrm{C} 34-\mathrm{C} 35$ & $1.385(5)$ & $\mathrm{C} 51 \mathrm{~B}-\mathrm{H} 51 \mathrm{~B}$ & 0.9497 \\
\hline $\mathrm{C} 35-\mathrm{C} 36$ & $1.524(6)$ & $\mathrm{C} 51 \mathrm{~B}-\mathrm{C} 52 \mathrm{~B}$ & $1.383(11)$ \\
\hline $\mathrm{C} 36-\mathrm{C} 75$ & $1.534(6)$ & $\mathrm{C} 52 \mathrm{~B}-\mathrm{H} 52 \mathrm{~B}$ & 0.9500 \\
\hline $\mathrm{C} 36-\mathrm{C} 69$ & $1.573(10)$ & $\mathrm{C} 52 \mathrm{~B}-\mathrm{C} 53 \mathrm{~B}$ & $1.402(9)$ \\
\hline C36-C69B & $1.603(11)$ & $\mathrm{C} 53 \mathrm{~B}-\mathrm{C} 54 \mathrm{~B}$ & $1.524(11)$ \\
\hline C67-H67A & 0.9805 & $\mathrm{C} 54 \mathrm{~B}-\mathrm{C} 82 \mathrm{~B}$ & $1.542(11)$ \\
\hline C67-H67B & 0.9804 & $\mathrm{C} 54 \mathrm{~B}-\mathrm{C} 88 \mathrm{~B}$ & $1.534(9)$ \\
\hline C67-H67C & 0.9797 & $\mathrm{C} 81 \mathrm{~B}-\mathrm{H} 81 \mathrm{~A}$ & 0.9800 \\
\hline C67-H67D & 0.9803 & $\mathrm{C} 81 \mathrm{~B}-\mathrm{H} 81 \mathrm{~B}$ & 0.9800 \\
\hline C67-H67E & 0.9801 & $\mathrm{C} 81 \mathrm{~B}-\mathrm{H} 81 \mathrm{C}$ & 0.9800 \\
\hline C67-H67F & 0.9798 & $\mathrm{C} 82 \mathrm{~B}-\mathrm{H} 82 \mathrm{~A}$ & 0.9899 \\
\hline C67-C66 & $1.639(16)$ & $\mathrm{C} 82 \mathrm{~B}-\mathrm{H} 82 \mathrm{~B}$ & 0.9900 \\
\hline C67-C66B & $1.425(17)$ & $\mathrm{C} 82 \mathrm{~B}-\mathrm{C} 83 \mathrm{~B}$ & $1.531(13)$ \\
\hline C68-H68A & 0.9800 & $\mathrm{C} 83 \mathrm{~B}-\mathrm{H} 83 \mathrm{~A}$ & 0.9897 \\
\hline C68-H68B & 0.9800 & $\mathrm{C} 83 \mathrm{~B}-\mathrm{H} 83 \mathrm{~B}$ & 0.9898 \\
\hline C68-H68C & 0.9800 & $\mathrm{C} 83 \mathrm{~B}-\mathrm{C} 84 \mathrm{~B}$ & $1.522(11)$ \\
\hline $\mathrm{C} 75-\mathrm{H} 75 \mathrm{~A}$ & 0.9900 & $\mathrm{C} 84 \mathrm{~B}-\mathrm{H} 84 \mathrm{~A}$ & 0.9900 \\
\hline C75-H75B & 0.9902 & $\mathrm{C} 84 \mathrm{~B}-\mathrm{H} 84 \mathrm{~B}$ & 0.9900 \\
\hline $\mathrm{C} 75-\mathrm{C} 76$ & $1.519(5)$ & $\mathrm{C} 84 \mathrm{~B}-\mathrm{C} 85 \mathrm{~B}$ & $1.357(11)$ \\
\hline C76-H76A & 0.9899 & $\mathrm{C} 85 \mathrm{~B}-\mathrm{H} 85 \mathrm{~A}$ & 0.9900 \\
\hline $\mathrm{C} 76-\mathrm{H} 76 \mathrm{~B}$ & 0.9899 & $\mathrm{C} 85 \mathrm{~B}-\mathrm{H} 85 \mathrm{~B}$ & 0.9900 \\
\hline C76-C77B & $1.476(7)$ & $\mathrm{C} 85 \mathrm{~B}-\mathrm{C} 86 \mathrm{~B}$ & $1.535(12)$ \\
\hline $\mathrm{C} 76-\mathrm{C} 77$ & $1.572(7)$ & $\mathrm{C} 86 \mathrm{~B}-\mathrm{H} 86 \mathrm{~A}$ & 0.9900 \\
\hline $\mathrm{O} 1-\mathrm{C} 55$ & $1.426(12)$ & $\mathrm{C} 86 \mathrm{~B}-\mathrm{H} 86 \mathrm{~B}$ & 0.9900 \\
\hline $\mathrm{C} 1-\mathrm{C} 2$ & $1.392(9)$ & $\mathrm{C} 86 \mathrm{~B}-\mathrm{C} 87 \mathrm{~B}$ & $1.499(13)$ \\
\hline $\mathrm{C} 1-\mathrm{C} 6$ & $1.418(9)$ & $\mathrm{C} 87 \mathrm{~B}-\mathrm{H} 87 \mathrm{~A}$ & 0.9800 \\
\hline $\mathrm{C} 2-\mathrm{H} 2$ & 0.9500 & $\mathrm{C} 87 \mathrm{~B}-\mathrm{H} 87 \mathrm{~B}$ & 0.9800 \\
\hline $\mathrm{C} 2-\mathrm{C} 3$ & $1.385(9)$ & $\mathrm{C} 87 \mathrm{~B}-\mathrm{H} 87 \mathrm{C}$ & 0.9800 \\
\hline $\mathrm{C} 3-\mathrm{C} 4$ & $1.392(8)$ & $\mathrm{C} 88 \mathrm{~B}-\mathrm{H} 88 \mathrm{~A}$ & 0.9900 \\
\hline $\mathrm{C} 3-\mathrm{C} 12$ & $1.474(8)$ & $\mathrm{C} 88 \mathrm{~B}-\mathrm{H} 88 \mathrm{~B}$ & 0.9900 \\
\hline $\mathrm{C} 4-\mathrm{C} 5$ & $1.370(8)$ & $\mathrm{C} 88 \mathrm{~B}-\mathrm{C} 89 \mathrm{~B}$ & $1.519(8)$ \\
\hline $\mathrm{C} 4-\mathrm{C} 18$ & $1.550(8)$ & $\mathrm{C} 89 \mathrm{~B}-\mathrm{H} 89 \mathrm{~A}$ & 0.9900 \\
\hline $\mathrm{C} 5-\mathrm{H} 5$ & 0.9500 & $\mathrm{C} 89 \mathrm{~B}-\mathrm{H} 89 \mathrm{~B}$ & 0.9900 \\
\hline $\mathrm{C} 5-\mathrm{C} 6$ & $1.418(8)$ & $\mathrm{C} 89 \mathrm{~B}-\mathrm{C} 90 \mathrm{~B}$ & $1.515(8)$ \\
\hline $\mathrm{C} 12-\mathrm{C} 13$ & $1.386(9)$ & $\mathrm{C} 90 \mathrm{~B}-\mathrm{H} 90 \mathrm{~A}$ & 0.9900 \\
\hline $\mathrm{C} 12-\mathrm{C} 17$ & $1.396(8)$ & $\mathrm{C} 90 \mathrm{~B}-\mathrm{H} 90 \mathrm{~B}$ & 0.9900 \\
\hline $\mathrm{C} 13-\mathrm{H} 13$ & 0.9500 & $\mathrm{C} 90 \mathrm{~B}-\mathrm{C} 91 \mathrm{~B}$ & $1.524(9)$ \\
\hline $\mathrm{C} 13-\mathrm{C} 14$ & $1.375(9)$ & C91B-H91A & 0.9900 \\
\hline C14-H14 & 0.9500 & C91B-H91B & 0.9900 \\
\hline $\mathrm{C} 14-\mathrm{C} 15$ & $1.395(9)$ & $\mathrm{C} 91 \mathrm{~B}-\mathrm{C} 92 \mathrm{~B}$ & $1.544(10)$ \\
\hline $\mathrm{C} 15-\mathrm{H} 15$ & 0.9502 & $\mathrm{C} 92 \mathrm{~B}-\mathrm{H} 92 \mathrm{~A}$ & 0.9900 \\
\hline $\mathrm{C} 15-\mathrm{C} 16$ & $1.372(9)$ & $\mathrm{C} 92 \mathrm{~B}-\mathrm{H} 92 \mathrm{~B}$ & 0.9900 \\
\hline $\mathrm{C} 16-\mathrm{H} 16$ & 0.9498 & $\mathrm{C} 92 \mathrm{~B}-\mathrm{C} 93 \mathrm{~B}$ & $1.654(10)$ \\
\hline $\mathrm{C} 16-\mathrm{C} 17$ & $1.387(8)$ & $\mathrm{C} 93 \mathrm{~B}-\mathrm{H} 93 \mathrm{~A}$ & 0.9800 \\
\hline $\mathrm{C} 17-\mathrm{C} 18$ & $1.529(9)$ & C93B-H93B & 0.9800 \\
\hline $\mathrm{C} 18-\mathrm{C} 56$ & $1.539(11)$ & C93B-H93C & 0.9800 \\
\hline
\end{tabular}




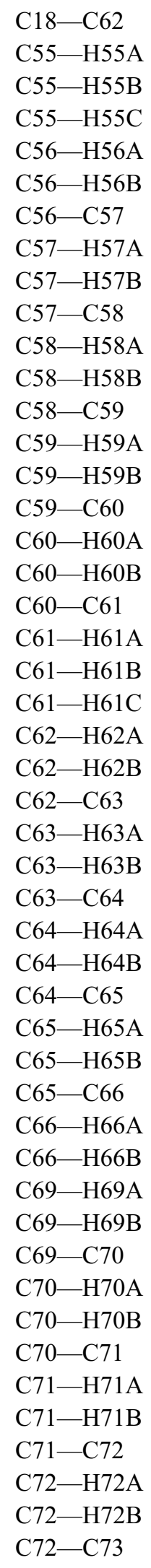

$1.555(10)$
0.9800
0.9800
0.9800
0.9903
0.9899
$1.522(10)$
0.9898
0.9907
$1.531(10)$
0.9898
0.9896
$1.486(13)$
0.9897
0.9899
$1.619(12)$
0.9901
0.9903
$1.505(10)$
0.9800
0.9800
0.9800
0.9898
0.9906
$1.522(10)$
0.9901
0.9900
$1.517(11)$
0.9905
0.9900
$1.535(11)$
0.9900
0.9899
$1.516(15)$
0.9895
0.9901
0.9895
0.9900
$1.516(11)$
0.9898
0.9912
$1.522(13)$
0.9904
0.9895
$1.504(13)$
0.9903
0.9897
$1.511(14)$

$\mathrm{O} 3-\mathrm{C} 46$

$\mathrm{O} 3-\mathrm{C} 81$

N3- 443

N3-C47

C $37-\mathrm{C} 38$

$\mathrm{C} 37-\mathrm{C} 42$

C38-H38

C $38-\mathrm{C} 39$

$\mathrm{C} 39-\mathrm{C} 40$

$\mathrm{C} 39-\mathrm{C} 48$

$\mathrm{C} 40-\mathrm{C} 41$

$\mathrm{C} 40-\mathrm{C} 54$

C41-H41

$\mathrm{C} 41-\mathrm{C} 42$

$\mathrm{C} 42-\mathrm{C} 43$

$\mathrm{C} 43-\mathrm{C} 44$

C44-H44

C44-C45

$\mathrm{C} 45-\mathrm{H} 45$

$\mathrm{C} 45-\mathrm{C} 46$

$\mathrm{C} 46-\mathrm{C} 47$

C47-H47

$\mathrm{C} 48-\mathrm{C} 49$

$\mathrm{C} 48-\mathrm{C} 53$

$\mathrm{C} 49-\mathrm{H} 49$

$\mathrm{C} 49-\mathrm{C} 50$

C $50-\mathrm{H} 50$

C $50-\mathrm{C} 51$

C $51-\mathrm{H} 51$

C51-C52

C52-H52

C $52-\mathrm{C} 53$

C53-C54

C54-C 82

C $54-\mathrm{C} 88$

C77-H77C

C77-H77D

C77-C78

C78- H78C

C78-H78D

C78-C79

C79-H79C

C79-H79D

C79-C 80

C $80-H 80 D$

C $80-\mathrm{H} 80 \mathrm{E}$

C $80-\mathrm{H} 80 \mathrm{~F}$

C81-H81D
$1.359(9)$
$1.437(8)$
$1.375(9)$
$1.320(9)$
$1.388(8)$
$1.422(9)$
0.9503
$1.383(9)$
$1.394(10)$
$1.463(9)$
$1.383(9)$
$1.550(10)$
0.9505
$1.413(8)$
$1.447(9)$
$1.405(8)$
0.9502
$1.369(9)$
0.9495
$1.386(10)$
$1.403(10)$
0.9500
$1.393(10)$
$1.386(10)$
0.9500
$1.389(9)$
0.9497
$1.378(10)$
0.9501
$1.385(12)$
0.9501
$1.410(10)$
$1.514(11)$
$1.525(12)$
$1.547(9)$
0.9897
0.9900
$1.554(8)$
0.9899
0.9904
$1.504(10)$
0.9899
0.9901
$1.522(11)$
0.9799
0.9802
0.9802
0.9800 


\begin{tabular}{|c|c|c|c|}
\hline $\mathrm{C} 73-\mathrm{H} 73 \mathrm{~A}$ & 0.9898 & C81-H81E & 0.9800 \\
\hline C73-H73B & 0.9907 & $\mathrm{C} 81-\mathrm{H} 81 \mathrm{~F}$ & 0.9800 \\
\hline $\mathrm{C} 73-\mathrm{C} 74$ & $1.503(13)$ & $\mathrm{C} 82-\mathrm{H} 82 \mathrm{C}$ & 0.9902 \\
\hline $\mathrm{C} 74-\mathrm{H} 74 \mathrm{~A}$ & 0.9800 & C82-H82D & 0.9899 \\
\hline C74-H74B & 0.9800 & $\mathrm{C} 82-\mathrm{C} 83$ & $1.538(12)$ \\
\hline $\mathrm{C} 74-\mathrm{H} 74 \mathrm{C}$ & 0.9800 & $\mathrm{C} 83-\mathrm{H} 83 \mathrm{C}$ & 0.9905 \\
\hline $\mathrm{O} 1 \mathrm{~B}-\mathrm{C} 55 \mathrm{~B}$ & $1.422(13)$ & C83-H83D & 0.9900 \\
\hline $\mathrm{C} 1 \mathrm{~B}-\mathrm{C} 2 \mathrm{~B}$ & $1.405(9)$ & $\mathrm{C} 83-\mathrm{C} 84$ & $1.494(11)$ \\
\hline $\mathrm{C} 1 \mathrm{~B}-\mathrm{C} 6 \mathrm{~B}$ & $1.431(9)$ & $\mathrm{C} 84-\mathrm{H} 84 \mathrm{C}$ & 0.9905 \\
\hline $\mathrm{C} 2 \mathrm{~B}-\mathrm{H} 2 \mathrm{~B}$ & 0.9500 & $\mathrm{C} 84-\mathrm{H} 84 \mathrm{D}$ & 0.9902 \\
\hline $\mathrm{C} 2 \mathrm{~B}-\mathrm{C} 3 \mathrm{~B}$ & $1.394(9)$ & $\mathrm{C} 84-\mathrm{C} 85$ & $1.556(10)$ \\
\hline $\mathrm{C} 3 \mathrm{~B}-\mathrm{C} 4 \mathrm{~B}$ & $1.396(9)$ & $\mathrm{C} 85-\mathrm{H} 85 \mathrm{C}$ & 0.9900 \\
\hline $\mathrm{C} 3 \mathrm{~B}-\mathrm{C} 12 \mathrm{~B}$ & $1.468(9)$ & $\mathrm{C} 85-\mathrm{H} 85 \mathrm{D}$ & 0.9900 \\
\hline $\mathrm{C} 4 \mathrm{~B}-\mathrm{C} 5 \mathrm{~B}$ & $1.372(8)$ & $\mathrm{C} 85-\mathrm{C} 86$ & $1.447(11)$ \\
\hline $\mathrm{C} 4 \mathrm{~B}-\mathrm{C} 18 \mathrm{~B}$ & $1.543(9)$ & $\mathrm{C} 86-\mathrm{H} 86 \mathrm{C}$ & 0.9898 \\
\hline $\mathrm{C} 5 \mathrm{~B}-\mathrm{H} 5 \mathrm{~B}$ & 0.9497 & $\mathrm{C} 86-\mathrm{H} 86 \mathrm{D}$ & 0.9897 \\
\hline $\mathrm{C} 5 \mathrm{~B}-\mathrm{C} 6 \mathrm{~B}$ & $1.405(8)$ & $\mathrm{C} 86-\mathrm{C} 87$ & $1.524(13)$ \\
\hline $\mathrm{C} 12 \mathrm{~B}-\mathrm{C} 13 \mathrm{~B}$ & $1.385(9)$ & C87-H87D & 0.9796 \\
\hline $\mathrm{C} 12 \mathrm{~B}-\mathrm{C} 17 \mathrm{~B}$ & $1.399(8)$ & C87-H87E & 0.9798 \\
\hline $\mathrm{C} 13 \mathrm{~B}-\mathrm{H} 13 \mathrm{~B}$ & 0.9500 & $\mathrm{C} 87-\mathrm{H} 87 \mathrm{~F}$ & 0.9806 \\
\hline $\mathrm{C} 13 \mathrm{~B}-\mathrm{C} 14 \mathrm{~B}$ & $1.384(9)$ & $\mathrm{C} 88-\mathrm{H} 88 \mathrm{C}$ & 0.9900 \\
\hline $\mathrm{C} 14 \mathrm{~B}-\mathrm{H} 14 \mathrm{~B}$ & 0.9500 & $\mathrm{C} 88-\mathrm{H} 88 \mathrm{D}$ & 0.9900 \\
\hline $\mathrm{C} 14 \mathrm{~B}-\mathrm{C} 15 \mathrm{~B}$ & $1.387(9)$ & $\mathrm{C} 88-\mathrm{C} 89$ & $1.556(9)$ \\
\hline $\mathrm{C} 15 \mathrm{~B}-\mathrm{H} 15 \mathrm{~B}$ & 0.9497 & C89- $\mathrm{H} 89 \mathrm{C}$ & 0.9900 \\
\hline $\mathrm{C} 15 \mathrm{~B}-\mathrm{C} 16 \mathrm{~B}$ & $1.392(9)$ & C89-H89D & 0.9900 \\
\hline $\mathrm{C} 16 \mathrm{~B}-\mathrm{H} 16 \mathrm{~B}$ & 0.9508 & $\mathrm{C} 89-\mathrm{C} 90$ & $1.673(10)$ \\
\hline $\mathrm{C} 16 \mathrm{~B}-\mathrm{C} 17 \mathrm{~B}$ & $1.382(8)$ & $\mathrm{C} 90-\mathrm{H} 90 \mathrm{C}$ & 0.9900 \\
\hline $\mathrm{C} 17 \mathrm{~B}-\mathrm{C} 18 \mathrm{~B}$ & $1.529(9)$ & C90-H90D & 0.9900 \\
\hline $\mathrm{C} 18 \mathrm{~B}-\mathrm{C} 56 \mathrm{~B}$ & $1.541(10)$ & $\mathrm{C} 90-\mathrm{C} 91$ & $1.384(10)$ \\
\hline $\mathrm{C} 18 \mathrm{~B}-\mathrm{C} 62 \mathrm{~B}$ & $1.526(12)$ & C91-H91C & 0.9900 \\
\hline $\mathrm{C} 55 \mathrm{~B}-\mathrm{H} 55 \mathrm{D}$ & 0.9800 & C91-H91D & 0.9900 \\
\hline $\mathrm{C} 55 \mathrm{~B}-\mathrm{H} 55 \mathrm{E}$ & 0.9800 & C91-C92 & $1.482(11)$ \\
\hline $\mathrm{C} 55 \mathrm{~B}-\mathrm{H} 55 \mathrm{~F}$ & 0.9800 & $\mathrm{C} 92-\mathrm{H} 92 \mathrm{C}$ & 0.9900 \\
\hline $\mathrm{C} 56 \mathrm{~B}-\mathrm{H} 56 \mathrm{C}$ & 0.9904 & C92-H92D & 0.9900 \\
\hline $\mathrm{C} 56 \mathrm{~B}-\mathrm{H} 56 \mathrm{D}$ & 0.9900 & $\mathrm{C} 92-\mathrm{C} 93$ & $1.502(11)$ \\
\hline $\mathrm{C} 56 \mathrm{~B}-\mathrm{C} 57 \mathrm{~B}$ & $1.526(11)$ & C93-H93D & 0.9800 \\
\hline $\mathrm{C} 57 \mathrm{~B}-\mathrm{H} 57 \mathrm{C}$ & 0.9904 & C93-H93E & 0.9800 \\
\hline $\mathrm{C} 57 \mathrm{~B}-\mathrm{H} 57 \mathrm{D}$ & 0.9900 & C93-H93F & 0.9800 \\
\hline $\mathrm{C} 57 \mathrm{~B}-\mathrm{C} 58 \mathrm{~B}$ & $1.520(11)$ & $\mathrm{C} 1 \mathrm{~S}-\mathrm{H} 1 \mathrm{SA}$ & 0.9800 \\
\hline $\mathrm{C} 58 \mathrm{~B}-\mathrm{H} 58 \mathrm{C}$ & 0.9901 & $\mathrm{C} 1 \mathrm{~S}-\mathrm{H} 1 \mathrm{SB}$ & 0.9800 \\
\hline $\mathrm{C} 58 \mathrm{~B}-\mathrm{H} 58 \mathrm{D}$ & 0.9895 & $\mathrm{C} 1 \mathrm{~S}-\mathrm{H} 1 \mathrm{SC}$ & 0.9800 \\
\hline $\mathrm{C} 58 \mathrm{~B}-\mathrm{C} 59 \mathrm{~B}$ & $1.519(11)$ & $\mathrm{C} 1 \mathrm{~S}-\mathrm{H} 1 \mathrm{SD}$ & 0.9800 \\
\hline $\mathrm{C} 59 \mathrm{~B}-\mathrm{H} 59 \mathrm{C}$ & 0.9897 & $\mathrm{C} 1 \mathrm{~S}-\mathrm{H} 1 \mathrm{SE}$ & 0.9800 \\
\hline $\mathrm{C} 59 \mathrm{~B}-\mathrm{H} 59 \mathrm{D}$ & 0.9899 & $\mathrm{C} 1 \mathrm{~S}-\mathrm{H} 1 \mathrm{SF}$ & 0.9800 \\
\hline $\mathrm{C} 59 \mathrm{~B}-\mathrm{C} 60 \mathrm{~B}$ & $1.514(13)$ & $\mathrm{C} 1 \mathrm{~S}-\mathrm{C} 2 \mathrm{~S}$ & $1.531(6)$ \\
\hline $\mathrm{C} 60 \mathrm{~B}-\mathrm{H} 60 \mathrm{C}$ & 0.9902 & $\mathrm{C} 1 \mathrm{~S}-\mathrm{C} 2 \mathrm{~S}^{\prime}$ & $1.518(8)$ \\
\hline C60B-H60D & 0.9898 & $\mathrm{C} 2 \mathrm{~S}-\mathrm{H} 2 \mathrm{SA}$ & 0.9900 \\
\hline $\mathrm{C} 60 \mathrm{~B}-\mathrm{C} 61 \mathrm{~B}$ & $1.430(13)$ & $\mathrm{C} 2 \mathrm{~S}-\mathrm{H} 2 \mathrm{SB}$ & 0.9900 \\
\hline
\end{tabular}




\begin{tabular}{|c|c|c|c|}
\hline C61B-H61D & 0.9800 & $\mathrm{C} 2 \mathrm{~S}-\mathrm{C} 3 \mathrm{~S}$ & $1.570(9)$ \\
\hline C61B-H61E & 0.9800 & $\mathrm{C} 3 \mathrm{~S}-\mathrm{H} 3 \mathrm{SA}$ & 0.9900 \\
\hline C61B-H61F & 0.9800 & $\mathrm{C} 3 \mathrm{~S}-\mathrm{H} 3 \mathrm{SB}$ & 0.9900 \\
\hline $\mathrm{C} 62 \mathrm{~B}-\mathrm{H} 62 \mathrm{C}$ & 0.9892 & $\mathrm{C} 3 \mathrm{~S}-\mathrm{C} 4 \mathrm{~S}$ & $1.543(9)$ \\
\hline $\mathrm{C} 62 \mathrm{~B}-\mathrm{H} 62 \mathrm{D}$ & 0.9892 & $\mathrm{C} 4 \mathrm{~S}-\mathrm{H} 4 \mathrm{SA}$ & 0.9900 \\
\hline $\mathrm{C} 62 \mathrm{~B}-\mathrm{C} 63 \mathrm{~B}$ & $1.516(11)$ & $\mathrm{C} 4 \mathrm{~S}-\mathrm{H} 4 \mathrm{SB}$ & 0.9900 \\
\hline $\mathrm{C} 63 \mathrm{~B}-\mathrm{H} 63 \mathrm{C}$ & 0.9901 & $\mathrm{C} 4 \mathrm{~S}-\mathrm{C} 5 \mathrm{~S}$ & $1.561(7)$ \\
\hline C63B-H63D & 0.9902 & C5S-H5SA & 0.9800 \\
\hline $\mathrm{C} 63 \mathrm{~B}-\mathrm{C} 64 \mathrm{~B}$ & $1.537(11)$ & $\mathrm{C} 5 \mathrm{~S}-\mathrm{H} 5 \mathrm{SB}$ & 0.9800 \\
\hline $\mathrm{C} 64 \mathrm{~B}-\mathrm{H} 64 \mathrm{C}$ & 0.9908 & $\mathrm{C} 5 \mathrm{~S}-\mathrm{H} 5 \mathrm{SC}$ & 0.9800 \\
\hline C64B-H64D & 0.9899 & $\mathrm{C} 2 \mathrm{~S}^{\prime}-\mathrm{H} 2 \mathrm{SC}$ & 0.9900 \\
\hline $\mathrm{C} 64 \mathrm{~B}-\mathrm{C} 65 \mathrm{~B}$ & $1.528(11)$ & $\mathrm{C} 2 \mathrm{~S}^{\prime}-\mathrm{H} 2 \mathrm{SD}$ & 0.9900 \\
\hline $\mathrm{C} 65 \mathrm{~B}-\mathrm{H} 65 \mathrm{C}$ & 0.9893 & $\mathrm{C} 2 \mathrm{~S}^{\prime}-\mathrm{C} 3 \mathrm{~S}^{\prime}$ & $1.470(9)$ \\
\hline $\mathrm{C} 65 \mathrm{~B}-\mathrm{H} 65 \mathrm{D}$ & 0.9902 & $\mathrm{C} 3 \mathrm{~S}^{\prime}-\mathrm{H} 3 \mathrm{SC}$ & 0.9900 \\
\hline $\mathrm{C} 65 \mathrm{~B}-\mathrm{C} 66 \mathrm{~B}$ & $1.520(15)$ & $\mathrm{C} 3 \mathrm{~S}^{\prime}-\mathrm{H} 3 \mathrm{SD}$ & 0.9900 \\
\hline $\mathrm{C} 66 \mathrm{~B}-\mathrm{H} 66 \mathrm{C}$ & 0.9900 & $\mathrm{C} 3 \mathrm{~S}^{\prime}-\mathrm{C} 4 \mathrm{~S}^{\prime}$ & $1.530(7)$ \\
\hline $\mathrm{C} 66 \mathrm{~B}-\mathrm{H} 66 \mathrm{D}$ & 0.9900 & $\mathrm{C} 4 \mathrm{~S}^{\prime}-\mathrm{H} 4 \mathrm{SC}$ & 0.9900 \\
\hline $\mathrm{C} 69 \mathrm{~B}-\mathrm{H} 69 \mathrm{C}$ & 0.9910 & $\mathrm{C} 4 \mathrm{~S}^{\prime}-\mathrm{H} 4 \mathrm{SD}$ & 0.9900 \\
\hline $\mathrm{C} 69 \mathrm{~B}-\mathrm{H} 69 \mathrm{D}$ & 0.9899 & $\mathrm{C} 4 \mathrm{~S}^{\prime}-\mathrm{C} 5 \mathrm{~S}^{\prime}$ & $1.543(7)$ \\
\hline $\mathrm{C} 69 \mathrm{~B}-\mathrm{C} 70 \mathrm{~B}$ & $1.510(11)$ & C5S'-H5SD & 0.9800 \\
\hline $\mathrm{C} 70 \mathrm{~B}-\mathrm{H} 70 \mathrm{C}$ & 0.9901 & $\mathrm{C} 5 \mathrm{~S}^{\prime}-\mathrm{H} 5 \mathrm{SE}$ & 0.9800 \\
\hline $\mathrm{C} 70 \mathrm{~B}-\mathrm{H} 70 \mathrm{D}$ & 0.9900 & $\mathrm{C} 5 \mathrm{~S}^{\prime}-\mathrm{H} 5 \mathrm{SF}$ & 0.9800 \\
\hline $\mathrm{N} 1-\mathrm{Ir}-\mathrm{N} 2$ & $96.55(10)$ & $\mathrm{C} 70 \mathrm{~B}-\mathrm{C} 69 \mathrm{~B}-\mathrm{H} 69 \mathrm{D}$ & 105.4 \\
\hline $\mathrm{N} 1-\mathrm{Ir}-\mathrm{N} 3 \mathrm{~B}$ & $89.3(3)$ & $\mathrm{C} 69 \mathrm{~B}-\mathrm{C} 70 \mathrm{~B}-\mathrm{H} 70 \mathrm{C}$ & 108.8 \\
\hline $\mathrm{N} 1-\mathrm{Ir}-\mathrm{N} 3$ & $99.9(3)$ & $\mathrm{C} 69 \mathrm{~B}-\mathrm{C} 70 \mathrm{~B}-\mathrm{H} 70 \mathrm{D}$ & 109.4 \\
\hline $\mathrm{N} 2-\mathrm{Ir}-\mathrm{N} 3 \mathrm{~B}$ & $93.2(2)$ & $\mathrm{C} 69 \mathrm{~B}-\mathrm{C} 70 \mathrm{~B}-\mathrm{C} 71 \mathrm{~B}$ & $112.2(8)$ \\
\hline $\mathrm{C} 19-\mathrm{Ir}-\mathrm{N} 1$ & $173.31(12)$ & $\mathrm{H} 70 \mathrm{C}-\mathrm{C} 70 \mathrm{~B}-\mathrm{H} 70 \mathrm{D}$ & 107.9 \\
\hline $\mathrm{C} 19-\mathrm{Ir}-\mathrm{N} 2$ & $79.44(13)$ & $\mathrm{C} 71 \mathrm{~B}-\mathrm{C} 70 \mathrm{~B}-\mathrm{H} 70 \mathrm{C}$ & 108.9 \\
\hline $\mathrm{C} 19-\mathrm{Ir}-\mathrm{N} 3 \mathrm{~B}$ & $96.3(3)$ & $\mathrm{C} 71 \mathrm{~B}-\mathrm{C} 70 \mathrm{~B}-\mathrm{H} 70 \mathrm{D}$ & 109.5 \\
\hline $\mathrm{C} 19-\mathrm{Ir}-\mathrm{C} 37 \mathrm{~B}$ & $98.0(3)$ & $\mathrm{C} 70 \mathrm{~B}-\mathrm{C} 71 \mathrm{~B}-\mathrm{H} 71 \mathrm{C}$ & 108.8 \\
\hline $\mathrm{C} 19-\mathrm{Ir}-\mathrm{N} 3$ & $86.1(3)$ & $\mathrm{C} 70 \mathrm{~B}-\mathrm{C} 71 \mathrm{~B}-\mathrm{H} 71 \mathrm{D}$ & 108.2 \\
\hline $\mathrm{C} 1-\mathrm{Ir}-\mathrm{N} 1$ & $81.7(2)$ & $\mathrm{H} 71 \mathrm{C}-\mathrm{C} 71 \mathrm{~B}-\mathrm{H} 71 \mathrm{D}$ & 107.5 \\
\hline $\mathrm{C} 1-\mathrm{Ir}-\mathrm{N} 2$ & $86.6(3)$ & $\mathrm{C} 72 \mathrm{~B}-\mathrm{C} 71 \mathrm{~B}-\mathrm{C} 70 \mathrm{~B}$ & $114.9(8)$ \\
\hline $\mathrm{C} 1-\mathrm{Ir}-\mathrm{C} 19$ & $92.6(2)$ & $\mathrm{C} 72 \mathrm{~B}-\mathrm{C} 71 \mathrm{~B}-\mathrm{H} 71 \mathrm{C}$ & 108.8 \\
\hline $\mathrm{C} 1-\mathrm{Ir}-\mathrm{N} 3$ & $174.5(3)$ & $\mathrm{C} 72 \mathrm{~B}-\mathrm{C} 71 \mathrm{~B}-\mathrm{H} 71 \mathrm{D}$ & 108.4 \\
\hline $\mathrm{C} 1 \mathrm{~B}-\mathrm{Ir}-\mathrm{N} 1$ & $77.9(2)$ & $\mathrm{C} 71 \mathrm{~B}-\mathrm{C} 72 \mathrm{~B}-\mathrm{H} 72 \mathrm{C}$ & 108.5 \\
\hline $\mathrm{C} 1 \mathrm{~B}-\mathrm{Ir}-\mathrm{N} 2$ & $95.1(3)$ & $\mathrm{C} 71 \mathrm{~B}-\mathrm{C} 72 \mathrm{~B}-\mathrm{H} 72 \mathrm{D}$ & 108.9 \\
\hline $\mathrm{C} 1 \mathrm{~B}-\mathrm{Ir}-\mathrm{C} 19$ & $97.0(2)$ & $\mathrm{C} 71 \mathrm{~B}-\mathrm{C} 72 \mathrm{~B}-\mathrm{C} 73 \mathrm{~B}$ & $114.1(9)$ \\
\hline $\mathrm{C} 1 \mathrm{~B}-\mathrm{Ir}-\mathrm{N} 3 \mathrm{~B}$ & $165.4(4)$ & $\mathrm{H} 72 \mathrm{C}-\mathrm{C} 72 \mathrm{~B}-\mathrm{H} 72 \mathrm{D}$ & 107.6 \\
\hline $\mathrm{C} 1 \mathrm{~B}-\mathrm{Ir}-\mathrm{C} 37 \mathrm{~B}$ & $93.8(3)$ & $\mathrm{C} 73 \mathrm{~B}-\mathrm{C} 72 \mathrm{~B}-\mathrm{H} 72 \mathrm{C}$ & 108.8 \\
\hline $\mathrm{C} 37 \mathrm{~B}-\mathrm{Ir}-\mathrm{N} 1$ & $86.7(2)$ & $\mathrm{C} 73 \mathrm{~B}-\mathrm{C} 72 \mathrm{~B}-\mathrm{H} 72 \mathrm{D}$ & 108.7 \\
\hline $\mathrm{C} 37 \mathrm{~B}-\mathrm{Ir}-\mathrm{N} 2$ & $171.0(2)$ & $\mathrm{C} 72 \mathrm{~B}-\mathrm{C} 73 \mathrm{~B}-\mathrm{H} 73 \mathrm{C}$ & 108.8 \\
\hline $\mathrm{C} 37 \mathrm{~B}-\mathrm{Ir}-\mathrm{N} 3 \mathrm{~B}$ & $78.4(3)$ & $\mathrm{C} 72 \mathrm{~B}-\mathrm{C} 73 \mathrm{~B}-\mathrm{H} 73 \mathrm{D}$ & 108.7 \\
\hline $\mathrm{N} 3-\mathrm{Ir}-\mathrm{N} 2$ & $98.4(2)$ & $\mathrm{C} 72 \mathrm{~B}-\mathrm{C} 73 \mathrm{~B}-\mathrm{C} 74 \mathrm{~B}$ & $114.8(10)$ \\
\hline $\mathrm{C} 37-\mathrm{Ir}-\mathrm{N} 1$ & $94.3(2)$ & $\mathrm{H} 73 \mathrm{C}-\mathrm{C} 73 \mathrm{~B}-\mathrm{H} 73 \mathrm{D}$ & 107.6 \\
\hline $\mathrm{C} 37-\mathrm{Ir}-\mathrm{N} 2$ & $169.1(2)$ & $\mathrm{C} 74 \mathrm{~B}-\mathrm{C} 73 \mathrm{~B}-\mathrm{H} 73 \mathrm{C}$ & 108.2 \\
\hline $\mathrm{C} 37-\mathrm{Ir}-\mathrm{C} 19$ & $89.6(3)$ & $\mathrm{C} 74 \mathrm{~B}-\mathrm{C} 73 \mathrm{~B}-\mathrm{H} 73 \mathrm{D}$ & 108.6 \\
\hline
\end{tabular}




\begin{tabular}{|c|c|}
\hline $\mathrm{C} 37-\mathrm{Ir}-\mathrm{C} 1$ & $93.6(3)$ \\
\hline $\mathrm{C} 37-\mathrm{Ir}-\mathrm{N} 3$ & $81.0(3)$ \\
\hline $\mathrm{C} 28-\mathrm{O} 2-\mathrm{C} 68$ & $116.5(3)$ \\
\hline C7-N1-Ir & $115.1(2)$ \\
\hline $\mathrm{C} 11-\mathrm{N} 1-\mathrm{Ir}$ & $125.8(2)$ \\
\hline $\mathrm{C} 11-\mathrm{N} 1-\mathrm{C} 7$ & $119.0(3)$ \\
\hline $\mathrm{C} 25-\mathrm{N} 2-\mathrm{Ir}$ & $114.2(2)$ \\
\hline $\mathrm{C} 29-\mathrm{N} 2-\mathrm{Ir}$ & $126.0(2)$ \\
\hline $\mathrm{C} 29-\mathrm{N} 2-\mathrm{C} 25$ & $119.8(3)$ \\
\hline $\mathrm{N} 1-\mathrm{C} 7-\mathrm{C} 8$ & $119.5(3)$ \\
\hline $\mathrm{N} 1-\mathrm{C} 7-\mathrm{C} 6$ & $110.9(4)$ \\
\hline $\mathrm{N} 1-\mathrm{C} 7-\mathrm{C} 6 \mathrm{~B}$ & $115.8(4)$ \\
\hline $\mathrm{C} 8-\mathrm{C} 7-\mathrm{C} 6$ & $128.6(4)$ \\
\hline $\mathrm{C} 8-\mathrm{C} 7-\mathrm{C} 6 \mathrm{~B}$ & $124.1(4)$ \\
\hline $\mathrm{C} 7-\mathrm{C} 8-\mathrm{H} 8$ & 119.1 \\
\hline $\mathrm{C} 9-\mathrm{C} 8-\mathrm{C} 7$ & $121.7(4)$ \\
\hline $\mathrm{C} 9-\mathrm{C} 8-\mathrm{H} 8$ & 119.3 \\
\hline $\mathrm{C} 8-\mathrm{C} 9-\mathrm{H} 9$ & 121.2 \\
\hline $\mathrm{C} 10-\mathrm{C} 9-\mathrm{C} 8$ & $118.0(4)$ \\
\hline $\mathrm{C} 10-\mathrm{C} 9-\mathrm{H} 9$ & 120.9 \\
\hline $\mathrm{C} 9-\mathrm{C} 10-\mathrm{C} 11$ & $119.0(3)$ \\
\hline $\mathrm{C} 9-\mathrm{C} 10-\mathrm{O} 1 \mathrm{~B}$ & $132.5(6)$ \\
\hline $\mathrm{C} 11-\mathrm{C} 10-\mathrm{O} 1 \mathrm{~B}$ & $107.4(5)$ \\
\hline $\mathrm{O} 1-\mathrm{C} 10-\mathrm{C} 9$ & $117.7(5)$ \\
\hline $\mathrm{O} 1-\mathrm{C} 10-\mathrm{C} 11$ & $122.8(6)$ \\
\hline $\mathrm{N} 1-\mathrm{C} 11-\mathrm{C} 10$ & $122.8(3)$ \\
\hline $\mathrm{N} 1-\mathrm{C} 11-\mathrm{H} 11$ & 118.5 \\
\hline $\mathrm{C} 10-\mathrm{C} 11-\mathrm{H} 11$ & 118.7 \\
\hline $\mathrm{C} 20-\mathrm{C} 19-\mathrm{Ir}$ & 128.2 \\
\hline $\mathrm{C} 20-\mathrm{C} 19-\mathrm{C} 24$ & $116.3(3)$ \\
\hline $\mathrm{C} 24-\mathrm{C} 19-\mathrm{Ir}$ & $115.5(2)$ \\
\hline $\mathrm{C} 19-\mathrm{C} 20-\mathrm{H} 20$ & 119.5 \\
\hline $\mathrm{C} 21-\mathrm{C} 20-\mathrm{C} 19$ & $121.1(4)$ \\
\hline $\mathrm{C} 21-\mathrm{C} 20-\mathrm{H} 20$ & 119.4 \\
\hline $\mathrm{C} 20-\mathrm{C} 21-\mathrm{C} 22$ & $121.2(3)$ \\
\hline $\mathrm{C} 20-\mathrm{C} 21-\mathrm{C} 30$ & $130.7(4)$ \\
\hline $\mathrm{C} 22-\mathrm{C} 21-\mathrm{C} 30$ & $108.0(4)$ \\
\hline $\mathrm{C} 21-\mathrm{C} 22-\mathrm{C} 36$ & $111.4(3)$ \\
\hline $\mathrm{C} 23-\mathrm{C} 22-\mathrm{C} 21$ & $119.3(4)$ \\
\hline $\mathrm{C} 23-\mathrm{C} 22-\mathrm{C} 36$ & $129.2(4)$ \\
\hline $\mathrm{C} 22-\mathrm{C} 23-\mathrm{H} 23$ & 120.0 \\
\hline $\mathrm{C} 22-\mathrm{C} 23-\mathrm{C} 24$ & $119.9(4)$ \\
\hline $\mathrm{C} 24-\mathrm{C} 23-\mathrm{H} 23$ & 120.1 \\
\hline $\mathrm{C} 19-\mathrm{C} 24-\mathrm{C} 25$ & $115.9(3)$ \\
\hline $\mathrm{C} 23-\mathrm{C} 24-\mathrm{C} 19$ & $122.1(3)$ \\
\hline $\mathrm{C} 23-\mathrm{C} 24-\mathrm{C} 25$ & $122.0(3)$ \\
\hline $\mathrm{N} 2-\mathrm{C} 25-\mathrm{C} 24$ & $114.9(3)$ \\
\hline $\mathrm{N} 2-\mathrm{C} 25-\mathrm{C} 26$ & $118.5(3)$ \\
\hline
\end{tabular}

$\begin{array}{ll}\text { C73B-C74B-H74D } & 109.5 \\ \text { C73B-C74B-H74E } & 109.5 \\ \text { C73B-C74B-H74F } & 109.5 \\ \text { H74D-C74B-H74E } & 109.5 \\ \text { H74D-C74B-H74F } & 109.5 \\ \text { H74E-C74B-H74F } & 109.5 \\ \text { C76-C77B-H77A } & 110.6 \\ \text { C76-C77B-H77B } & 111.2 \\ \text { C76-C77B-C78B } & 105.3(6) \\ \text { H77A-C77B-H77B } & 108.9 \\ \text { C78B-C77B-H77A } & 109.9 \\ \text { C78B-C77B-H77B } & 110.9 \\ \text { C77B-C78B-H78A } & 112.0 \\ \text { C77B-C78B-H78B } & 108.2 \\ \text { H78A-C78B-H78B } & 108.1 \\ \text { C79B-C78B-C77B } & 109.4(6) \\ \text { C79B-C78B-H78A } & 109.6 \\ \text { C79B-C78B-H78B } & 109.4 \\ \text { C78B-C79B-H79A } & 109.3 \\ \text { C78B-C79B-H79B } & 109.0 \\ \text { C78B-C79B-C80B } & 112.3(6) \\ \text { H79A-C79B-H79B } & 107.9 \\ \text { C80B-C79B-H79A } & 109.1 \\ \text { C80B-C79B-H79B } & 109.1 \\ \text { C79B-C80B-H80A } & 109.7 \\ \text { C79B-C80B-H80B } & 109.4 \\ \text { C79B-C80B-H80C } & 109.3 \\ \text { H80A-C80B-H80B } & 109.5 \\ \text { H80A-C80B-H80C } & 109.5 \\ \text { H80B-C80B-H80C } & 109.5 \\ \text { C46B-O3B-C81B } & 116.4(6) \\ \text { C43B-N3B-Ir } & 114.8(5) \\ \text { C47B-N3B-Ir } & 125.6(7) \\ \text { C47B-N3B-C43B } & 119.5(8) \\ \text { C38B-C37B-Ir } & 128.5(5) \\ \text { C38B-C37B-C42B } & 114.6(5) \\ \text { C42B-C37B-Ir } & 116.8(4) \\ \text { C37B-C38B-H38B } & 118.5 \\ \text { C39B-C38B-C37B } & 121.9(6) \\ \text { C39B-C38B-H38B } & 119.6 \\ \text { C38B-C39B-C40B } & 120.6(6) \\ \text { C38B-C39B-C48B } & 130.6(8) \\ \text { C40B-C39B-C48B } & 108.7(6) \\ \text { C39B-C40B-C54B } & 110.9(6) \\ \text { C41B-C40B-C39B } & 120.5(6) \\ \text { C41B-C40B-C54B } & 128.6(6) \\ \text { C40B-C41B-H41B } & 120.7 \\ \text { C40B-C41B-C42B } & 118.8(6) \\ & \\ \text { C } & \end{array}$




\begin{tabular}{|c|c|}
\hline $\mathrm{C} 26-\mathrm{C} 25-\mathrm{C} 24$ & $126.6(3)$ \\
\hline $\mathrm{C} 25-\mathrm{C} 26-\mathrm{H} 26$ & 118.7 \\
\hline $\mathrm{C} 27-\mathrm{C} 26-\mathrm{C} 25$ & $121.9(3)$ \\
\hline $\mathrm{C} 27-\mathrm{C} 26-\mathrm{H} 26$ & 119.4 \\
\hline $\mathrm{C} 26-\mathrm{C} 27-\mathrm{H} 27$ & 120.7 \\
\hline $\mathrm{C} 26-\mathrm{C} 27-\mathrm{C} 28$ & $118.4(3)$ \\
\hline $\mathrm{C} 28-\mathrm{C} 27-\mathrm{H} 27$ & 120.9 \\
\hline $\mathrm{O} 2-\mathrm{C} 28-\mathrm{C} 27$ & $125.8(3)$ \\
\hline $\mathrm{O} 2-\mathrm{C} 28-\mathrm{C} 29$ & $115.8(3)$ \\
\hline $\mathrm{C} 27-\mathrm{C} 28-\mathrm{C} 29$ & $118.4(3)$ \\
\hline $\mathrm{N} 2-\mathrm{C} 29-\mathrm{C} 28$ & $122.9(3)$ \\
\hline $\mathrm{N} 2-\mathrm{C} 29-\mathrm{H} 29$ & 118.7 \\
\hline $\mathrm{C} 28-\mathrm{C} 29-\mathrm{H} 29$ & 118.3 \\
\hline $\mathrm{C} 31-\mathrm{C} 30-\mathrm{C} 21$ & $130.6(4)$ \\
\hline $\mathrm{C} 35-\mathrm{C} 30-\mathrm{C} 21$ & $108.7(4)$ \\
\hline $\mathrm{C} 35-\mathrm{C} 30-\mathrm{C} 31$ & $120.7(4)$ \\
\hline $\mathrm{C} 30-\mathrm{C} 31-\mathrm{H} 31$ & 121.1 \\
\hline $\mathrm{C} 32-\mathrm{C} 31-\mathrm{C} 30$ & $117.7(4)$ \\
\hline $\mathrm{C} 32-\mathrm{C} 31-\mathrm{H} 31$ & 121.3 \\
\hline $\mathrm{C} 31-\mathrm{C} 32-\mathrm{H} 32$ & 119.0 \\
\hline $\mathrm{C} 33-\mathrm{C} 32-\mathrm{C} 31$ & $121.8(4)$ \\
\hline $\mathrm{C} 33-\mathrm{C} 32-\mathrm{H} 32$ & 119.2 \\
\hline $\mathrm{C} 32-\mathrm{C} 33-\mathrm{H} 33$ & 119.8 \\
\hline $\mathrm{C} 32-\mathrm{C} 33-\mathrm{C} 34$ & $120.3(4)$ \\
\hline C $34-\mathrm{C} 33-\mathrm{H} 33$ & 119.8 \\
\hline $\mathrm{C} 33-\mathrm{C} 34-\mathrm{H} 34$ & 120.3 \\
\hline $\mathrm{C} 35-\mathrm{C} 34-\mathrm{C} 33$ & $118.8(4)$ \\
\hline $\mathrm{C} 35-\mathrm{C} 34-\mathrm{H} 34$ & 120.9 \\
\hline $\mathrm{C} 30-\mathrm{C} 35-\mathrm{C} 36$ & $111.3(3)$ \\
\hline $\mathrm{C} 34-\mathrm{C} 35-\mathrm{C} 30$ & $120.7(4)$ \\
\hline $\mathrm{C} 34-\mathrm{C} 35-\mathrm{C} 36$ & $128.0(4)$ \\
\hline $\mathrm{C} 22-\mathrm{C} 36-\mathrm{C} 75$ & $113.3(4)$ \\
\hline $\mathrm{C} 22-\mathrm{C} 36-\mathrm{C} 69$ & $114.1(5)$ \\
\hline $\mathrm{C} 22-\mathrm{C} 36-\mathrm{C} 69 \mathrm{~B}$ & $107.8(5)$ \\
\hline $\mathrm{C} 35-\mathrm{C} 36-\mathrm{C} 22$ & $100.5(3)$ \\
\hline $\mathrm{C} 35-\mathrm{C} 36-\mathrm{C} 75$ & $112.2(3)$ \\
\hline $\mathrm{C} 35-\mathrm{C} 36-\mathrm{C} 69$ & $121.0(5)$ \\
\hline $\mathrm{C} 35-\mathrm{C} 36-\mathrm{C} 69 \mathrm{~B}$ & $102.1(4)$ \\
\hline $\mathrm{C} 75-\mathrm{C} 36-\mathrm{C} 69$ & $96.5(4)$ \\
\hline $\mathrm{C} 75-\mathrm{C} 36-\mathrm{C} 69 \mathrm{~B}$ & $118.9(4)$ \\
\hline H67A-C67-H67B & 109.4 \\
\hline $\mathrm{H} 67 \mathrm{~A}-\mathrm{C} 67-\mathrm{H} 67 \mathrm{C}$ & 109.5 \\
\hline $\mathrm{H} 67 \mathrm{~B}-\mathrm{C} 67-\mathrm{H} 67 \mathrm{C}$ & 109.5 \\
\hline H67D-C67-H67E & 109.4 \\
\hline $\mathrm{H} 67 \mathrm{D}-\mathrm{C} 67-\mathrm{H} 67 \mathrm{~F}$ & 109.5 \\
\hline $\mathrm{H} 67 \mathrm{E}-\mathrm{C} 67-\mathrm{H} 67 \mathrm{~F}$ & 109.5 \\
\hline C66-C67-H67A & 109.4 \\
\hline C66-C67-H67B & 109.4 \\
\hline
\end{tabular}

$\begin{array}{ll}\text { C42B-C41B-H41B } & 120.5 \\ \text { C37B-C42B-C43B } & 114.0(5) \\ \text { C41B-C42B-C37B } & 123.6(6) \\ \text { C41B-C42B-C43B } & 122.1(6) \\ \text { N3B-C43B-C42B } & 115.9(6) \\ \text { N3B-C43B-C44B } & 118.3(6) \\ \text { C44B-C43B-C42B } & 125.8(6) \\ \text { C43B-C44B-H44B } & 118.9 \\ \text { C45B-C44B-C43B } & 121.5(6) \\ \text { C45B-C44B-H44B } & 119.6 \\ \text { C44B-C45B-H45B } & 120.6 \\ \text { C44B-C45B-C46B } & 118.9(7) \\ \text { C46B-C45B-H45B } & 120.5 \\ \text { O3B-C46B-C45B } & 126.7(7) \\ \text { O3B-C46B-C47B } & 115.2(7) \\ \text { C45B-C46B-C47B } & 118.1(8) \\ \text { N3B-C47B-C46B } & 123.5(10) \\ \text { N3B-C47B-H47B } & 118.3 \\ \text { C46B-C47B-H47B } & 118.2 \\ \text { C49B-C48B-C39B } & 131.2(8) \\ \text { C49B-C48B-C53B } & 120.3(7) \\ \text { C53B-C48B-C39B } & 108.5(7) \\ \text { C48B-C49B-H49B } & 120.2 \\ \text { C48B-C49B-C50B } & 119.1(8) \\ \text { C50B-C49B-H49B } & 120.7 \\ \text { C49B-C50B-H50B } & 119.5 \\ \text { C51B-C50B-C49B } & 120.4(8) \\ \text { C51B-C50B-H50B } & 120.0 \\ \text { C50B-C51B-H51B } & 119.4 \\ \text { C50B-C51B-C52B } & 121.1(8) \\ \text { C52B-C51B-H51B } & 119.5 \\ \text { C51B-C52B-H52B } & 120.5 \\ \text { C51B-C52B-C53B } & 118.5(7) \\ \text { C53B-C52B-H52B } & 121.0 \\ \text { C48B-C53B-C52B } & 120.5(8) \\ \text { C48B-C53B-C54B } & 111.8(6) \\ \text { C52B-C53B-C54B } & 127.8(7) \\ \text { C53B-C54B-C40B } & 100.1(6) \\ \text { C53B-C54B-C82B } & 111.5(7) \\ \text { C53B-C54B-C88B } & 111.9(6) \\ \text { C82B-C54B-C40B } & 112.0(6) \\ \text { C88B-C54B-C40B } & 113.1(6) \\ \text { C88B-C54B-C82B } & 108.2(6) \\ \text { O3B-C81B-H81A } & 109.5 \\ \text { O3B-C81B-H81B } & 109.5 \\ \text { O3B-C81B-H81C } & 109.5 \\ \text { H81A-C81B-H81B } & 109.5 \\ \text { H81A-C81B-H81C } & 109.5 \\ & \\ & \end{array}$




\begin{tabular}{|c|c|}
\hline $\mathrm{C} 66-\mathrm{C} 67-\mathrm{H} 67 \mathrm{C}$ & 109.6 \\
\hline $\mathrm{C} 66 \mathrm{~B}-\mathrm{C} 67-\mathrm{H} 67 \mathrm{D}$ & 109.3 \\
\hline $\mathrm{C} 66 \mathrm{~B}-\mathrm{C} 67-\mathrm{H} 67 \mathrm{E}$ & 109.7 \\
\hline $\mathrm{C} 66 \mathrm{~B}-\mathrm{C} 67-\mathrm{H} 67 \mathrm{~F}$ & 109.4 \\
\hline $\mathrm{O} 2-\mathrm{C} 68-\mathrm{H} 68 \mathrm{~A}$ & 109.5 \\
\hline $\mathrm{O} 2-\mathrm{C} 68-\mathrm{H} 68 \mathrm{~B}$ & 109.5 \\
\hline $\mathrm{O} 2-\mathrm{C} 68-\mathrm{H} 68 \mathrm{C}$ & 109.5 \\
\hline $\mathrm{H} 68 \mathrm{~A}-\mathrm{C} 68-\mathrm{H} 68 \mathrm{~B}$ & 109.5 \\
\hline $\mathrm{H} 68 \mathrm{~A}-\mathrm{C} 68-\mathrm{H} 68 \mathrm{C}$ & 109.5 \\
\hline $\mathrm{H} 68 \mathrm{~B}-\mathrm{C} 68-\mathrm{H} 68 \mathrm{C}$ & 109.5 \\
\hline $\mathrm{C} 36-\mathrm{C} 75-\mathrm{H} 75 \mathrm{~A}$ & 108.4 \\
\hline $\mathrm{C} 36-\mathrm{C} 75-\mathrm{H} 75 \mathrm{~B}$ & 108.1 \\
\hline $\mathrm{H} 75 \mathrm{~A}-\mathrm{C} 75-\mathrm{H} 75 \mathrm{~B}$ & 107.4 \\
\hline $\mathrm{C} 76-\mathrm{C} 75-\mathrm{C} 36$ & $116.7(3)$ \\
\hline $\mathrm{C} 76-\mathrm{C} 75-\mathrm{H} 75 \mathrm{~A}$ & 108.0 \\
\hline $\mathrm{C} 76-\mathrm{C} 75-\mathrm{H} 75 \mathrm{~B}$ & 108.0 \\
\hline $\mathrm{C} 75-\mathrm{C} 76-\mathrm{H} 76 \mathrm{~A}$ & 108.9 \\
\hline $\mathrm{C} 75-\mathrm{C} 76-\mathrm{H} 76 \mathrm{~B}$ & 108.6 \\
\hline $\mathrm{C} 75-\mathrm{C} 76-\mathrm{C} 77$ & $119.1(5)$ \\
\hline $\mathrm{H} 76 \mathrm{~A}-\mathrm{C} 76-\mathrm{H} 76 \mathrm{~B}$ & 107.7 \\
\hline $\mathrm{C} 77 \mathrm{~B}-\mathrm{C} 76-\mathrm{C} 75$ & $106.6(5)$ \\
\hline $\mathrm{C} 77 \mathrm{~B}-\mathrm{C} 76-\mathrm{H} 76 \mathrm{~A}$ & 114.4 \\
\hline $\mathrm{C} 77 \mathrm{~B}-\mathrm{C} 76-\mathrm{H} 76 \mathrm{~B}$ & 110.5 \\
\hline $\mathrm{C} 77-\mathrm{C} 76-\mathrm{H} 76 \mathrm{~A}$ & 104.6 \\
\hline $\mathrm{C} 77-\mathrm{C} 76-\mathrm{H} 76 \mathrm{~B}$ & 107.4 \\
\hline $\mathrm{C} 10-\mathrm{O} 1-\mathrm{C} 55$ & $124.1(9)$ \\
\hline $\mathrm{C} 2-\mathrm{C} 1-\mathrm{Ir}$ & $131.1(6)$ \\
\hline $\mathrm{C} 2-\mathrm{C} 1-\mathrm{C} 6$ & $117.4(7)$ \\
\hline $\mathrm{C} 6-\mathrm{C} 1-\mathrm{Ir}$ & $111.4(5)$ \\
\hline $\mathrm{C} 1-\mathrm{C} 2-\mathrm{H} 2$ & 119.8 \\
\hline $\mathrm{C} 3-\mathrm{C} 2-\mathrm{C} 1$ & $120.5(8)$ \\
\hline $\mathrm{C} 3-\mathrm{C} 2-\mathrm{H} 2$ & 119.8 \\
\hline $\mathrm{C} 2-\mathrm{C} 3-\mathrm{C} 4$ & $121.6(7)$ \\
\hline $\mathrm{C} 2-\mathrm{C} 3-\mathrm{C} 12$ & $130.0(7)$ \\
\hline $\mathrm{C} 4-\mathrm{C} 3-\mathrm{C} 12$ & $108.4(6)$ \\
\hline $\mathrm{C} 3-\mathrm{C} 4-\mathrm{C} 18$ & $111.3(6)$ \\
\hline $\mathrm{C} 5-\mathrm{C} 4-\mathrm{C} 3$ & $120.0(6)$ \\
\hline $\mathrm{C} 5-\mathrm{C} 4-\mathrm{C} 18$ & $128.7(6)$ \\
\hline $\mathrm{C} 4-\mathrm{C} 5-\mathrm{H} 5$ & 120.5 \\
\hline $\mathrm{C} 4-\mathrm{C} 5-\mathrm{C} 6$ & $118.9(6)$ \\
\hline $\mathrm{C} 6-\mathrm{C} 5-\mathrm{H} 5$ & 120.6 \\
\hline $\mathrm{C} 1-\mathrm{C} 6-\mathrm{C} 7$ & $120.0(6)$ \\
\hline $\mathrm{C} 1-\mathrm{C} 6-\mathrm{C} 5$ & $121.5(6)$ \\
\hline $\mathrm{C} 5-\mathrm{C} 6-\mathrm{C} 7$ & $117.7(6)$ \\
\hline $\mathrm{C} 13-\mathrm{C} 12-\mathrm{C} 3$ & $131.4(7)$ \\
\hline $\mathrm{C} 13-\mathrm{C} 12-\mathrm{C} 17$ & $119.9(7)$ \\
\hline $\mathrm{C} 17-\mathrm{C} 12-\mathrm{C} 3$ & $108.6(6)$ \\
\hline & \\
\hline
\end{tabular}

$\begin{array}{ll}\text { H81B-C81B-H81C } & 109.5 \\ \text { C54B-C82B-H82A } & 108.0 \\ \text { C54B-C82B-H82B } & 108.7 \\ \text { H82A-C82B-H82B } & 107.3 \\ \text { C83B-C82B-C54B } & 116.7(8) \\ \text { C83B-C82B-H82A } & 107.4 \\ \text { C83B-C82B-H82B } & 108.5 \\ \text { C82B-C83B-H83A } & 110.2 \\ \text { C82B-C83B-H83B } & 109.1 \\ \text { H83A-C83B-H83B } & 108.1 \\ \text { C84B-C83B-C82B } & 108.3(9) \\ \text { C84B-C83B-H83A } & 104.7 \\ \text { C84B-C83B-H83B } & 116.3 \\ \text { C83B-C84B-H84A } & 106.8 \\ \text { C83B-C84B-H84B } & 106.8 \\ \text { H84A-C84B-H84B } & 106.7 \\ \text { C85B-C84B-C83B } & 122.1(10) \\ \text { C85B-C84B-H84A } & 106.8 \\ \text { C85B-C84B-H84B } & 106.8 \\ \text { C84B-C85B-H85A } & 108.4 \\ \text { C84B-C85B-H85B } & 108.4 \\ \text { C84B-C85B-C86B } & 115.7(9) \\ \text { H85A-C85B-H85B } & 107.4 \\ \text { C86B-C85B-H85A } & 108.4 \\ \text { C86B-C85B-H85B } & 108.4 \\ \text { C85B-C86B-H86A } & 107.8 \\ \text { C85B-C86B-H86B } & 107.8 \\ \text { H86A-C86B-H86B } & 107.1 \\ \text { C87B-C86B-C85B } & 118.2(8) \\ \text { C87B-C86B-H86A } & 107.8 \\ \text { C87B-C86B-H86B } & 107.8 \\ \text { C86B-C87B-H87A } & 109.5 \\ \text { C86B-C87B-H87B } & 109.5 \\ \text { C86B-C87B-H87C } & 109.5 \\ \text { H87A-C87B-H87B } & 109.5 \\ \text { H87A-C87B-H87C } & 109.5 \\ \text { H87B-C87B-H87C } & 109.5 \\ \text { C54B-C88B-H88A } & 107.9 \\ \text { C54B-C88B-H88B } & 107.9 \\ \text { H88A-C88B-H88B } & 107.2 \\ \text { C89B-C88B-C54B } & 117.5(6) \\ \text { C89B-C88B-H88A } & 107.9 \\ \text { C89B-C88B-H88B } & 107.9 \\ \text { C88B-C89B-H89A } & 109.0 \\ \text { C88B-C89B-H89B } & 109.0 \\ \text { H89A-C89B-H89B } & 107.8 \\ \text { C90B-C89B-C88B } & 112.9(5) \\ \text { C90B-C89B-H89A } & 109.0 \\ & \\ & \end{array}$




\begin{tabular}{|c|c|}
\hline $\mathrm{C} 14-\mathrm{C} 13-\mathrm{C} 12$ & $119.5(8)$ \\
\hline $\mathrm{C} 14-\mathrm{C} 13-\mathrm{H} 13$ & 120.3 \\
\hline $\mathrm{C} 13-\mathrm{C} 14-\mathrm{H} 14$ & 119.7 \\
\hline $\mathrm{C} 13-\mathrm{C} 14-\mathrm{C} 15$ & $120.5(9)$ \\
\hline $\mathrm{C} 15-\mathrm{C} 14-\mathrm{H} 14$ & 119.7 \\
\hline $\mathrm{C} 14-\mathrm{C} 15-\mathrm{H} 15$ & 119.1 \\
\hline $\mathrm{C} 16-\mathrm{C} 15-\mathrm{C} 14$ & $120.3(8)$ \\
\hline $\mathrm{C} 16-\mathrm{C} 15-\mathrm{H} 15$ & 120.0 \\
\hline $\mathrm{C} 15-\mathrm{C} 16-\mathrm{H} 16$ & 120.7 \\
\hline $\mathrm{C} 15-\mathrm{C} 16-\mathrm{C} 17$ & $119.5(7)$ \\
\hline $\mathrm{C} 17-\mathrm{C} 16-\mathrm{H} 16$ & 119.8 \\
\hline $\mathrm{C} 12-\mathrm{C} 17-\mathrm{C} 18$ & $111.6(6)$ \\
\hline $\mathrm{C} 16-\mathrm{C} 17-\mathrm{C} 12$ & $120.2(6)$ \\
\hline $\mathrm{C} 16-\mathrm{C} 17-\mathrm{C} 18$ & $128.2(6)$ \\
\hline $\mathrm{C} 4-\mathrm{C} 18-\mathrm{C} 62$ & $110.8(6)$ \\
\hline $\mathrm{C} 17-\mathrm{C} 18-\mathrm{C} 4$ & $100.1(5)$ \\
\hline $\mathrm{C} 17-\mathrm{C} 18-\mathrm{C} 56$ & $112.8(6)$ \\
\hline $\mathrm{C} 17-\mathrm{C} 18-\mathrm{C} 62$ & $112.1(7)$ \\
\hline $\mathrm{C} 56-\mathrm{C} 18-\mathrm{C} 4$ & $111.0(6)$ \\
\hline $\mathrm{C} 56-\mathrm{C} 18-\mathrm{C} 62$ & $109.8(6)$ \\
\hline $\mathrm{O} 1-\mathrm{C} 55-\mathrm{H} 55 \mathrm{~A}$ & 109.5 \\
\hline $\mathrm{O} 1-\mathrm{C} 55-\mathrm{H} 55 \mathrm{~B}$ & 109.5 \\
\hline $\mathrm{O} 1-\mathrm{C} 55-\mathrm{H} 55 \mathrm{C}$ & 109.5 \\
\hline $\mathrm{H} 55 \mathrm{~A}-\mathrm{C} 55-\mathrm{H} 55 \mathrm{~B}$ & 109.5 \\
\hline $\mathrm{H} 55 \mathrm{~A}-\mathrm{C} 55-\mathrm{H} 55 \mathrm{C}$ & 109.5 \\
\hline $\mathrm{H} 55 \mathrm{~B}-\mathrm{C} 55-\mathrm{H} 55 \mathrm{C}$ & 109.5 \\
\hline $\mathrm{C} 18-\mathrm{C} 56-\mathrm{H} 56 \mathrm{~A}$ & 108.8 \\
\hline $\mathrm{C} 18-\mathrm{C} 56-\mathrm{H} 56 \mathrm{~B}$ & 108.3 \\
\hline $\mathrm{H} 56 \mathrm{~A}-\mathrm{C} 56-\mathrm{H} 56 \mathrm{~B}$ & 107.6 \\
\hline $\mathrm{C} 57-\mathrm{C} 56-\mathrm{C} 18$ & $114.7(7)$ \\
\hline $\mathrm{C} 57-\mathrm{C} 56-\mathrm{H} 56 \mathrm{~A}$ & 108.9 \\
\hline $\mathrm{C} 57-\mathrm{C} 56-\mathrm{H} 56 \mathrm{~B}$ & 108.3 \\
\hline C56-C57-H57A & 108.9 \\
\hline C56-C57-H57B & 109.4 \\
\hline $\mathrm{C} 56-\mathrm{C} 57-\mathrm{C} 58$ & $112.0(6)$ \\
\hline $\mathrm{H} 57 \mathrm{~A}-\mathrm{C} 57-\mathrm{H} 57 \mathrm{~B}$ & 107.9 \\
\hline C58-C57-H57A & 109.1 \\
\hline C58-C57-H57B & 109.4 \\
\hline $\mathrm{C} 57-\mathrm{C} 58-\mathrm{H} 58 \mathrm{~A}$ & 107.7 \\
\hline $\mathrm{C} 57-\mathrm{C} 58-\mathrm{H} 58 \mathrm{~B}$ & 108.0 \\
\hline $\mathrm{H} 58 \mathrm{~A}-\mathrm{C} 58-\mathrm{H} 58 \mathrm{~B}$ & 107.2 \\
\hline $\mathrm{C} 59-\mathrm{C} 58-\mathrm{C} 57$ & $118.0(7)$ \\
\hline $\mathrm{C} 59-\mathrm{C} 58-\mathrm{H} 58 \mathrm{~A}$ & 107.4 \\
\hline $\mathrm{C} 59-\mathrm{C} 58-\mathrm{H} 58 \mathrm{~B}$ & 108.1 \\
\hline C58-C59-H59A & 108.9 \\
\hline С58-C59-H59B & 109.2 \\
\hline $\mathrm{C} 58-\mathrm{C} 59-\mathrm{C} 60$ & $113.1(7)$ \\
\hline$H$ & \\
\hline
\end{tabular}

\begin{tabular}{|c|c|}
\hline $\mathrm{C} 90 \mathrm{~B}-\mathrm{C} 89 \mathrm{~B}-\mathrm{H} 89 \mathrm{~B}$ & 109.0 \\
\hline $\mathrm{C} 89 \mathrm{~B}-\mathrm{C} 90 \mathrm{~B}-\mathrm{H} 90 \mathrm{~A}$ & 108.7 \\
\hline $\mathrm{C} 89 \mathrm{~B}-\mathrm{C} 90 \mathrm{~B}-\mathrm{H} 90 \mathrm{~B}$ & 108.7 \\
\hline $\mathrm{C} 89 \mathrm{~B}-\mathrm{C} 90 \mathrm{~B}-\mathrm{C} 91 \mathrm{~B}$ & $114.2(6)$ \\
\hline $\mathrm{H} 90 \mathrm{~A}-\mathrm{C} 90 \mathrm{~B}-\mathrm{H} 90 \mathrm{~B}$ & 107.6 \\
\hline $\mathrm{C} 91 \mathrm{~B}-\mathrm{C} 90 \mathrm{~B}-\mathrm{H} 90 \mathrm{~A}$ & 108.7 \\
\hline $\mathrm{C} 91 \mathrm{~B}-\mathrm{C} 90 \mathrm{~B}-\mathrm{H} 90 \mathrm{~B}$ & 108.7 \\
\hline $\mathrm{C} 90 \mathrm{~B}-\mathrm{C} 91 \mathrm{~B}-\mathrm{H} 91 \mathrm{~A}$ & 108.2 \\
\hline $\mathrm{C} 90 \mathrm{~B}-\mathrm{C} 91 \mathrm{~B}-\mathrm{H} 91 \mathrm{~B}$ & 108.2 \\
\hline $\mathrm{C} 90 \mathrm{~B}-\mathrm{C} 91 \mathrm{~B}-\mathrm{C} 92 \mathrm{~B}$ & $116.5(7)$ \\
\hline $\mathrm{H} 91 \mathrm{~A}-\mathrm{C} 91 \mathrm{~B}-\mathrm{H} 91 \mathrm{~B}$ & 107.3 \\
\hline $\mathrm{C} 92 \mathrm{~B}-\mathrm{C} 91 \mathrm{~B}-\mathrm{H} 91 \mathrm{~A}$ & 108.2 \\
\hline $\mathrm{C} 92 \mathrm{~B}-\mathrm{C} 91 \mathrm{~B}-\mathrm{H} 91 \mathrm{~B}$ & 108.2 \\
\hline $\mathrm{C} 91 \mathrm{~B}-\mathrm{C} 92 \mathrm{~B}-\mathrm{H} 92 \mathrm{~A}$ & 112.6 \\
\hline C91B - C92B $-\mathrm{H} 92 \mathrm{~B}$ & 112.6 \\
\hline $\mathrm{C} 91 \mathrm{~B}-\mathrm{C} 92 \mathrm{~B}-\mathrm{C} 93 \mathrm{~B}$ & $95.7(7)$ \\
\hline $\mathrm{H} 92 \mathrm{~A}-\mathrm{C} 92 \mathrm{~B}-\mathrm{H} 92 \mathrm{~B}$ & 110.1 \\
\hline $\mathrm{C} 93 \mathrm{~B}-\mathrm{C} 92 \mathrm{~B}-\mathrm{H} 92 \mathrm{~A}$ & 112.6 \\
\hline C93B - C92B $-\mathrm{H} 92 \mathrm{~B}$ & 112.6 \\
\hline $\mathrm{C} 92 \mathrm{~B}-\mathrm{C} 93 \mathrm{~B}-\mathrm{H} 93 \mathrm{~A}$ & 109.5 \\
\hline $\mathrm{C} 92 \mathrm{~B}-\mathrm{C} 93 \mathrm{~B}-\mathrm{H} 93 \mathrm{~B}$ & 109.5 \\
\hline $\mathrm{C} 92 \mathrm{~B}-\mathrm{C} 93 \mathrm{~B}-\mathrm{H} 93 \mathrm{C}$ & 109.5 \\
\hline $\mathrm{H} 93 \mathrm{~A}-\mathrm{C} 93 \mathrm{~B}-\mathrm{H} 93 \mathrm{~B}$ & 109.5 \\
\hline $\mathrm{H} 93 \mathrm{~A}-\mathrm{C} 93 \mathrm{~B}-\mathrm{H} 93 \mathrm{C}$ & 109.5 \\
\hline $\mathrm{H} 93 \mathrm{~B}-\mathrm{C} 93 \mathrm{~B}-\mathrm{H} 93 \mathrm{C}$ & 109.5 \\
\hline $\mathrm{C} 46-\mathrm{O} 3-\mathrm{C} 81$ & $116.2(6)$ \\
\hline $\mathrm{C} 43-\mathrm{N} 3-\mathrm{Ir}$ & $112.4(5)$ \\
\hline $\mathrm{C} 47-\mathrm{N} 3-\mathrm{Ir}$ & $125.9(6)$ \\
\hline $\mathrm{C} 47-\mathrm{N} 3-\mathrm{C} 43$ & $121.7(7)$ \\
\hline $\mathrm{C} 38-\mathrm{C} 37-\mathrm{Ir}$ & $127.1(5)$ \\
\hline $\mathrm{C} 38-\mathrm{C} 37-\mathrm{C} 42$ & $119.1(6)$ \\
\hline $\mathrm{C} 42-\mathrm{C} 37-\mathrm{Ir}$ & $113.9(5)$ \\
\hline $\mathrm{C} 37-\mathrm{C} 38-\mathrm{H} 38$ & 120.3 \\
\hline $\mathrm{C} 39-\mathrm{C} 38-\mathrm{C} 37$ & $119.8(6)$ \\
\hline $\mathrm{C} 39-\mathrm{C} 38-\mathrm{H} 38$ & 119.9 \\
\hline $\mathrm{C} 38-\mathrm{C} 39-\mathrm{C} 40$ & $120.9(7)$ \\
\hline $\mathrm{C} 38-\mathrm{C} 39-\mathrm{C} 48$ & $130.2(8)$ \\
\hline $\mathrm{C} 40-\mathrm{C} 39-\mathrm{C} 48$ & $108.9(6)$ \\
\hline $\mathrm{C} 39-\mathrm{C} 40-\mathrm{C} 54$ & $110.1(6)$ \\
\hline $\mathrm{C} 41-\mathrm{C} 40-\mathrm{C} 39$ & $121.4(6)$ \\
\hline $\mathrm{C} 41-\mathrm{C} 40-\mathrm{C} 54$ & $128.4(7)$ \\
\hline $\mathrm{C} 40-\mathrm{C} 41-\mathrm{H} 41$ & 121.2 \\
\hline $\mathrm{C} 40-\mathrm{C} 41-\mathrm{C} 42$ & $117.6(6)$ \\
\hline $\mathrm{C} 42-\mathrm{C} 41-\mathrm{H} 41$ & 121.2 \\
\hline $\mathrm{C} 37-\mathrm{C} 42-\mathrm{C} 43$ & $117.0(5)$ \\
\hline $\mathrm{C} 41-\mathrm{C} 42-\mathrm{C} 37$ & $121.1(6)$ \\
\hline $\mathrm{C} 41-\mathrm{C} 42-\mathrm{C} 43$ & $121.9(6)$ \\
\hline $\mathrm{N} 3-\mathrm{C} 43-\mathrm{C} 42$ & $115.4(6)$ \\
\hline
\end{tabular}




\begin{tabular}{|c|c|c|c|}
\hline $\mathrm{C} 60-\mathrm{C} 59-\mathrm{H} 59 \mathrm{~A}$ & 108.5 & $\mathrm{~N} 3-\mathrm{C} 43-\mathrm{C} 44$ & $116.7(6)$ \\
\hline $\mathrm{C} 60-\mathrm{C} 59-\mathrm{H} 59 \mathrm{~B}$ & 109.3 & $\mathrm{C} 44-\mathrm{C} 43-\mathrm{C} 42$ & $127.8(6)$ \\
\hline $\mathrm{C} 59-\mathrm{C} 60-\mathrm{H} 60 \mathrm{~A}$ & 108.3 & $\mathrm{C} 43-\mathrm{C} 44-\mathrm{H} 44$ & 118.5 \\
\hline $\mathrm{C} 59-\mathrm{C} 60-\mathrm{H} 60 \mathrm{~B}$ & 108.0 & $\mathrm{C} 45-\mathrm{C} 44-\mathrm{C} 43$ & $122.4(6)$ \\
\hline $\mathrm{H} 60 \mathrm{~A}-\mathrm{C} 60-\mathrm{H} 60 \mathrm{~B}$ & 107.4 & $\mathrm{C} 45-\mathrm{C} 44-\mathrm{H} 44$ & 119.1 \\
\hline $\mathrm{C} 61-\mathrm{C} 60-\mathrm{C} 59$ & $115.7(7)$ & $\mathrm{C} 44-\mathrm{C} 45-\mathrm{H} 45$ & 120.8 \\
\hline $\mathrm{C} 61-\mathrm{C} 60-\mathrm{H} 60 \mathrm{~A}$ & 108.7 & $\mathrm{C} 44-\mathrm{C} 45-\mathrm{C} 46$ & $119.0(6)$ \\
\hline $\mathrm{C} 61-\mathrm{C} 60-\mathrm{H} 60 \mathrm{~B}$ & 108.4 & $\mathrm{C} 46-\mathrm{C} 45-\mathrm{H} 45$ & 120.3 \\
\hline $\mathrm{C} 60-\mathrm{C} 61-\mathrm{H} 61 \mathrm{~A}$ & 109.5 & $\mathrm{O} 3-\mathrm{C} 46-\mathrm{C} 45$ & $127.5(7)$ \\
\hline $\mathrm{C} 60-\mathrm{C} 61-\mathrm{H} 61 \mathrm{~B}$ & 109.5 & $\mathrm{O} 3-\mathrm{C} 46-\mathrm{C} 47$ & $114.7(8)$ \\
\hline $\mathrm{C} 60-\mathrm{C} 61-\mathrm{H} 61 \mathrm{C}$ & 109.5 & $\mathrm{C} 45-\mathrm{C} 46-\mathrm{C} 47$ & $117.8(7)$ \\
\hline $\mathrm{H} 61 \mathrm{~A}-\mathrm{C} 61-\mathrm{H} 61 \mathrm{~B}$ & 109.5 & $\mathrm{~N} 3-\mathrm{C} 47-\mathrm{C} 46$ & $122.3(8)$ \\
\hline $\mathrm{H} 61 \mathrm{~A}-\mathrm{C} 61-\mathrm{H} 61 \mathrm{C}$ & 109.5 & $\mathrm{~N} 3-\mathrm{C} 47-\mathrm{H} 47$ & 118.8 \\
\hline $\mathrm{H} 61 \mathrm{~B}-\mathrm{C} 61-\mathrm{H} 61 \mathrm{C}$ & 109.5 & $\mathrm{C} 46-\mathrm{C} 47-\mathrm{H} 47$ & 118.8 \\
\hline $\mathrm{C} 18-\mathrm{C} 62-\mathrm{H} 62 \mathrm{~A}$ & 108.3 & $\mathrm{C} 49-\mathrm{C} 48-\mathrm{C} 39$ & $130.7(7)$ \\
\hline $\mathrm{C} 18-\mathrm{C} 62-\mathrm{H} 62 \mathrm{~B}$ & 108.7 & $\mathrm{C} 53-\mathrm{C} 48-\mathrm{C} 39$ & $108.5(7)$ \\
\hline $\mathrm{H} 62 \mathrm{~A}-\mathrm{C} 62-\mathrm{H} 62 \mathrm{~B}$ & 107.5 & $\mathrm{C} 53-\mathrm{C} 48-\mathrm{C} 49$ & $120.8(7)$ \\
\hline $\mathrm{C} 63-\mathrm{C} 62-\mathrm{C} 18$ & $115.3(7)$ & $\mathrm{C} 48-\mathrm{C} 49-\mathrm{H} 49$ & 120.6 \\
\hline $\mathrm{C} 63-\mathrm{C} 62-\mathrm{H} 62 \mathrm{~A}$ & 108.0 & $\mathrm{C} 50-\mathrm{C} 49-\mathrm{C} 48$ & $118.8(7)$ \\
\hline $\mathrm{C} 63-\mathrm{C} 62-\mathrm{H} 62 \mathrm{~B}$ & 108.7 & $\mathrm{C} 50-\mathrm{C} 49-\mathrm{H} 49$ & 120.6 \\
\hline $\mathrm{C} 62-\mathrm{C} 63-\mathrm{H} 63 \mathrm{~A}$ & 108.9 & $\mathrm{C} 49-\mathrm{C} 50-\mathrm{H} 50$ & 119.6 \\
\hline C62-C63-H63B & 108.3 & $\mathrm{C} 51-\mathrm{C} 50-\mathrm{C} 49$ & $120.7(7)$ \\
\hline $\mathrm{H} 63 \mathrm{~A}-\mathrm{C} 63-\mathrm{H} 63 \mathrm{~B}$ & 107.6 & $\mathrm{C} 51-\mathrm{C} 50-\mathrm{H} 50$ & 119.7 \\
\hline $\mathrm{C} 64-\mathrm{C} 63-\mathrm{C} 62$ & $114.3(8)$ & $\mathrm{C} 50-\mathrm{C} 51-\mathrm{H} 51$ & 119.2 \\
\hline $\mathrm{C} 64-\mathrm{C} 63-\mathrm{H} 63 \mathrm{~A}$ & 109.2 & $\mathrm{C} 50-\mathrm{C} 51-\mathrm{C} 52$ & $121.2(8)$ \\
\hline C64-C63-H63B & 108.3 & $\mathrm{C} 52-\mathrm{C} 51-\mathrm{H} 51$ & 119.6 \\
\hline $\mathrm{C} 63-\mathrm{C} 64-\mathrm{H} 64 \mathrm{~A}$ & 107.8 & $\mathrm{C} 51-\mathrm{C} 52-\mathrm{H} 52$ & 120.7 \\
\hline C63-C64-H64B & 108.0 & $\mathrm{C} 51-\mathrm{C} 52-\mathrm{C} 53$ & $118.4(8)$ \\
\hline C63-C64-C65 & $116.8(7)$ & $\mathrm{C} 53-\mathrm{C} 52-\mathrm{H} 52$ & 120.8 \\
\hline $\mathrm{H} 64 \mathrm{~A}-\mathrm{C} 64-\mathrm{H} 64 \mathrm{~B}$ & 107.4 & $\mathrm{C} 48-\mathrm{C} 53-\mathrm{C} 52$ & $120.1(8)$ \\
\hline $\mathrm{C} 65-\mathrm{C} 64-\mathrm{H} 64 \mathrm{~A}$ & 107.9 & $\mathrm{C} 48-\mathrm{C} 53-\mathrm{C} 54$ & $111.9(7)$ \\
\hline C65-C64-H64B & 108.6 & $\mathrm{C} 52-\mathrm{C} 53-\mathrm{C} 54$ & $128.0(8)$ \\
\hline $\mathrm{C} 64-\mathrm{C} 65-\mathrm{H} 65 \mathrm{~A}$ & 108.6 & $\mathrm{C} 53-\mathrm{C} 54-\mathrm{C} 40$ & $100.5(6)$ \\
\hline C64-C65-H65B & 108.9 & $\mathrm{C} 53-\mathrm{C} 54-\mathrm{C} 82$ & $112.8(7)$ \\
\hline $\mathrm{H} 65 \mathrm{~A}-\mathrm{C} 65-\mathrm{H} 65 \mathrm{~B}$ & 107.6 & $\mathrm{C} 53-\mathrm{C} 54-\mathrm{C} 88$ & $109.7(7)$ \\
\hline C66-C65-C64 & $113.9(9)$ & $\mathrm{C} 82-\mathrm{C} 54-\mathrm{C} 40$ & $111.3(7)$ \\
\hline $\mathrm{C} 66-\mathrm{C} 65-\mathrm{H} 65 \mathrm{~A}$ & 108.2 & $\mathrm{C} 82-\mathrm{C} 54-\mathrm{C} 88$ & $110.8(6)$ \\
\hline C66- $665-\mathrm{H} 65 \mathrm{~B}$ & 109.4 & $\mathrm{C} 88-\mathrm{C} 54-\mathrm{C} 40$ & $111.4(6)$ \\
\hline C67-C66-H66A & 108.7 & $\mathrm{C} 76-\mathrm{C} 77-\mathrm{H} 77 \mathrm{C}$ & 107.3 \\
\hline C67-C66-H66B & 108.6 & $\mathrm{C} 76-\mathrm{C} 77-\mathrm{H} 77 \mathrm{D}$ & 106.7 \\
\hline C65-C66-C67 & $114.3(12)$ & $\mathrm{H} 77 \mathrm{C}-\mathrm{C} 77-\mathrm{H} 77 \mathrm{D}$ & 106.8 \\
\hline $\mathrm{C} 65-\mathrm{C} 66-\mathrm{H} 66 \mathrm{~A}$ & 109.4 & $\mathrm{C} 78-\mathrm{C} 77-\mathrm{C} 76$ & $120.8(7)$ \\
\hline C65-C66-H66B & 108.1 & $\mathrm{C} 78-\mathrm{C} 77-\mathrm{H} 77 \mathrm{C}$ & 107.6 \\
\hline $\mathrm{H} 66 \mathrm{~A}-\mathrm{C} 66-\mathrm{H} 66 \mathrm{~B}$ & 107.5 & $\mathrm{C} 78-\mathrm{C} 77-\mathrm{H} 77 \mathrm{D}$ & 106.9 \\
\hline $\mathrm{C} 36-\mathrm{C} 69-\mathrm{H} 69 \mathrm{~A}$ & 110.7 & $\mathrm{C} 77-\mathrm{C} 78-\mathrm{H} 78 \mathrm{C}$ & 106.9 \\
\hline $\mathrm{C} 36-\mathrm{C} 69-\mathrm{H} 69 \mathrm{~B}$ & 110.7 & $\mathrm{C} 77-\mathrm{C} 78-\mathrm{H} 78 \mathrm{D}$ & 110.4 \\
\hline $\mathrm{H} 69 \mathrm{~A}-\mathrm{C} 69-\mathrm{H} 69 \mathrm{~B}$ & 108.8 & $\mathrm{H} 78 \mathrm{C}-\mathrm{C} 78-\mathrm{H} 78 \mathrm{D}$ & 106.1 \\
\hline $\mathrm{C} 70-\mathrm{C} 69-\mathrm{C} 36$ & $105.3(7)$ & $\mathrm{C} 79-\mathrm{C} 78-\mathrm{C} 77$ & $121.9(7)$ \\
\hline
\end{tabular}




\begin{tabular}{|c|c|}
\hline $\mathrm{C} 70-\mathrm{C} 69-\mathrm{H} 69 \mathrm{~A}$ & 110.8 \\
\hline $\mathrm{C} 70-\mathrm{C} 69-\mathrm{H} 69 \mathrm{~B}$ & 110.5 \\
\hline $\mathrm{C} 69-\mathrm{C} 70-\mathrm{H} 70 \mathrm{~A}$ & 108.7 \\
\hline C69- $\mathrm{C} 70-\mathrm{H} 70 \mathrm{~B}$ & 108.9 \\
\hline $\mathrm{C} 69-\mathrm{C} 70-\mathrm{C} 71$ & $113.7(8)$ \\
\hline $\mathrm{H} 70 \mathrm{~A}-\mathrm{C} 70-\mathrm{H} 70 \mathrm{~B}$ & 107.6 \\
\hline $\mathrm{C} 71-\mathrm{C} 70-\mathrm{H} 70 \mathrm{~A}$ & 109.1 \\
\hline $\mathrm{C} 71-\mathrm{C} 70-\mathrm{H} 70 \mathrm{~B}$ & 108.7 \\
\hline $\mathrm{C} 70-\mathrm{C} 71-\mathrm{H} 71 \mathrm{~A}$ & 109.0 \\
\hline $\mathrm{C} 70-\mathrm{C} 71-\mathrm{H} 71 \mathrm{~B}$ & 108.6 \\
\hline $\mathrm{H} 71 \mathrm{~A}-\mathrm{C} 71-\mathrm{H} 71 \mathrm{~B}$ & 107.7 \\
\hline $\mathrm{C} 72-\mathrm{C} 71-\mathrm{C} 70$ & $114.1(8)$ \\
\hline $\mathrm{C} 72-\mathrm{C} 71-\mathrm{H} 71 \mathrm{~A}$ & 108.6 \\
\hline $\mathrm{C} 72-\mathrm{C} 71-\mathrm{H} 71 \mathrm{~B}$ & 108.6 \\
\hline $\mathrm{C} 71-\mathrm{C} 72-\mathrm{H} 72 \mathrm{~A}$ & 108.1 \\
\hline $\mathrm{C} 71-\mathrm{C} 72-\mathrm{H} 72 \mathrm{~B}$ & 108.1 \\
\hline $\mathrm{C} 71-\mathrm{C} 72-\mathrm{C} 73$ & $116.5(9)$ \\
\hline $\mathrm{H} 72 \mathrm{~A}-\mathrm{C} 72-\mathrm{H} 72 \mathrm{~B}$ & 107.4 \\
\hline $\mathrm{C} 73-\mathrm{C} 72-\mathrm{H} 72 \mathrm{~A}$ & 107.9 \\
\hline С73-C72-H72B & 108.6 \\
\hline $\mathrm{C} 72-\mathrm{C} 73-\mathrm{H} 73 \mathrm{~A}$ & 108.4 \\
\hline C72-C73-H73B & 109.2 \\
\hline $\mathrm{H} 73 \mathrm{~A}-\mathrm{C} 73-\mathrm{H} 73 \mathrm{~B}$ & 107.7 \\
\hline $\mathrm{C} 74-\mathrm{C} 73-\mathrm{C} 72$ & $113.7(10)$ \\
\hline $\mathrm{C} 74-\mathrm{C} 73-\mathrm{H} 73 \mathrm{~A}$ & 109.1 \\
\hline $\mathrm{C} 74-\mathrm{C} 73-\mathrm{H} 73 \mathrm{~B}$ & 108.6 \\
\hline $\mathrm{C} 73-\mathrm{C} 74-\mathrm{H} 74 \mathrm{~A}$ & 109.5 \\
\hline $\mathrm{C} 73-\mathrm{C} 74-\mathrm{H} 74 \mathrm{~B}$ & 109.5 \\
\hline $\mathrm{C} 73-\mathrm{C} 74-\mathrm{H} 74 \mathrm{C}$ & 109.5 \\
\hline $\mathrm{H} 74 \mathrm{~A}-\mathrm{C} 74-\mathrm{H} 74 \mathrm{~B}$ & 109.5 \\
\hline $\mathrm{H} 74 \mathrm{~A}-\mathrm{C} 74-\mathrm{H} 74 \mathrm{C}$ & 109.5 \\
\hline $\mathrm{H} 74 \mathrm{~B}-\mathrm{C} 74-\mathrm{H} 74 \mathrm{C}$ & 109.5 \\
\hline $\mathrm{C} 10-\mathrm{O} 1 \mathrm{~B}-\mathrm{C} 55 \mathrm{~B}$ & $109.7(8)$ \\
\hline $\mathrm{C} 2 \mathrm{~B}-\mathrm{C} 1 \mathrm{~B}-\mathrm{Ir}$ & $125.6(6)$ \\
\hline $\mathrm{C} 2 \mathrm{~B}-\mathrm{C} 1 \mathrm{~B}-\mathrm{C} 6 \mathrm{~B}$ & $115.7(7)$ \\
\hline $\mathrm{C} 6 \mathrm{~B}-\mathrm{C} 1 \mathrm{~B}-\mathrm{Ir}$ & $118.6(5)$ \\
\hline $\mathrm{C} 1 \mathrm{~B}-\mathrm{C} 2 \mathrm{~B}-\mathrm{H} 2 \mathrm{~B}$ & 119.2 \\
\hline $\mathrm{C} 3 \mathrm{~B}-\mathrm{C} 2 \mathrm{~B}-\mathrm{C} 1 \mathrm{~B}$ & $121.6(8)$ \\
\hline $\mathrm{C} 3 \mathrm{~B}-\mathrm{C} 2 \mathrm{~B}-\mathrm{H} 2 \mathrm{~B}$ & 119.2 \\
\hline $\mathrm{C} 2 \mathrm{~B}-\mathrm{C} 3 \mathrm{~B}-\mathrm{C} 4 \mathrm{~B}$ & $120.7(7)$ \\
\hline $\mathrm{C} 2 \mathrm{~B}-\mathrm{C} 3 \mathrm{~B}-\mathrm{C} 12 \mathrm{~B}$ & $131.1(7)$ \\
\hline $\mathrm{C} 4 \mathrm{~B}-\mathrm{C} 3 \mathrm{~B}-\mathrm{C} 12 \mathrm{~B}$ & $108.1(6)$ \\
\hline $\mathrm{C} 3 \mathrm{~B}-\mathrm{C} 4 \mathrm{~B}-\mathrm{C} 18 \mathrm{~B}$ & $111.2(6)$ \\
\hline $\mathrm{C} 5 \mathrm{~B}-\mathrm{C} 4 \mathrm{~B}-\mathrm{C} 3 \mathrm{~B}$ & $120.2(6)$ \\
\hline $\mathrm{C} 5 \mathrm{~B}-\mathrm{C} 4 \mathrm{~B}-\mathrm{C} 18 \mathrm{~B}$ & $128.5(6)$ \\
\hline $\mathrm{C} 4 \mathrm{~B}-\mathrm{C} 5 \mathrm{~B}-\mathrm{H} 5 \mathrm{~B}$ & 120.7 \\
\hline $\mathrm{C} 4 \mathrm{~B}-\mathrm{C} 5 \mathrm{~B}-\mathrm{C} 6 \mathrm{~B}$ & $119.0(6)$ \\
\hline & \\
\hline
\end{tabular}

C79-C78-H78C 104.9

$\mathrm{C} 79-\mathrm{C} 78-\mathrm{H} 78 \mathrm{D} \quad 105.5$

$\mathrm{C} 78-\mathrm{C} 79-\mathrm{H} 79 \mathrm{C} \quad 108.6$

$\mathrm{C} 78-\mathrm{C} 79-\mathrm{H} 79 \mathrm{D} \quad 109.0$

$\mathrm{C} 78-\mathrm{C} 79-\mathrm{C} 80 \quad 114.2(7)$

$\mathrm{H} 79 \mathrm{C}-\mathrm{C} 79-\mathrm{H} 79 \mathrm{D} \quad 107.6$

$\mathrm{C} 80-\mathrm{C} 79-\mathrm{H} 79 \mathrm{C} \quad 108.8$

$\mathrm{C} 80-\mathrm{C} 79-\mathrm{H} 79 \mathrm{D} \quad 108.4$

C79-C $80-\mathrm{H} 80 \mathrm{D} \quad 109.6$

$\mathrm{C} 79-\mathrm{C} 80-\mathrm{H} 80 \mathrm{E} \quad 109.2$

$\mathrm{C} 79-\mathrm{C} 80-\mathrm{H} 80 \mathrm{~F} \quad 109.7$

$\mathrm{H} 80 \mathrm{D}-\mathrm{C} 80-\mathrm{H} 80 \mathrm{E} \quad 109.5$

$\mathrm{H} 80 \mathrm{D}-\mathrm{C} 80-\mathrm{H} 80 \mathrm{~F} \quad 109.5$

$\mathrm{H} 80 \mathrm{E}-\mathrm{C} 80-\mathrm{H} 80 \mathrm{~F} \quad 109.4$

O3-C $81-\mathrm{H} 81 \mathrm{D} \quad 109.5$

$\mathrm{O} 3-\mathrm{C} 81-\mathrm{H} 81 \mathrm{E} \quad 109.5$

$\mathrm{O} 3-\mathrm{C} 81-\mathrm{H} 81 \mathrm{~F} \quad 109.5$

$\mathrm{H} 81 \mathrm{D}-\mathrm{C} 81-\mathrm{H} 81 \mathrm{E} \quad 109.5$

$\mathrm{H} 81 \mathrm{D}-\mathrm{C} 81-\mathrm{H} 81 \mathrm{~F} \quad 109.5$

$\mathrm{H} 81 \mathrm{E}-\mathrm{C} 81-\mathrm{H} 81 \mathrm{~F} \quad 109.5$

$\mathrm{C} 54-\mathrm{C} 82-\mathrm{H} 82 \mathrm{C} \quad 108.7$

$\mathrm{C} 54-\mathrm{C} 82-\mathrm{H} 82 \mathrm{D} \quad 108.6$

$\mathrm{C} 54-\mathrm{C} 82-\mathrm{C} 83 \quad 114.8$ (7)

$\mathrm{H} 82 \mathrm{C}-\mathrm{C} 82-\mathrm{H} 82 \mathrm{D} \quad 107.6$

$\mathrm{C} 83-\mathrm{C} 82-\mathrm{H} 82 \mathrm{C} \quad 108.4$

$\mathrm{C} 83-\mathrm{C} 82-\mathrm{H} 82 \mathrm{D} \quad 108.5$

$\mathrm{C} 82-\mathrm{C} 83-\mathrm{H} 83 \mathrm{C} \quad 108.1$

C82-C 83-H83D $\quad 108.4$

$\mathrm{H} 83 \mathrm{C}-\mathrm{C} 83-\mathrm{H} 83 \mathrm{D} \quad 107.4$

$\mathrm{C} 84-\mathrm{C} 83-\mathrm{C} 82 \quad 116.0(8)$

$\mathrm{C} 84-\mathrm{C} 83-\mathrm{H} 83 \mathrm{C} \quad 108.3$

C84-C83-H83D $\quad 108.4$

$\mathrm{C} 83-\mathrm{C} 84-\mathrm{H} 84 \mathrm{C} \quad 109.0$

$\mathrm{C} 83-\mathrm{C} 84-\mathrm{H} 84 \mathrm{D} \quad 109.1$

$\mathrm{C} 83-\mathrm{C} 84-\mathrm{C} 85 \quad 114.1$ (8)

$\mathrm{H} 84 \mathrm{C}-\mathrm{C} 84-\mathrm{H} 84 \mathrm{D} \quad 107.8$

$\mathrm{C} 85-\mathrm{C} 84-\mathrm{H} 84 \mathrm{C} \quad 112.9$

$\mathrm{C} 85-\mathrm{C} 84-\mathrm{H} 84 \mathrm{D} \quad 103.7$

$\mathrm{C} 84-\mathrm{C} 85-\mathrm{H} 85 \mathrm{C} \quad 107.8$

$\mathrm{C} 84-\mathrm{C} 85-\mathrm{H} 85 \mathrm{D} \quad 107.8$

$\mathrm{H} 85 \mathrm{C}-\mathrm{C} 85-\mathrm{H} 85 \mathrm{D} \quad 107.1$

$\mathrm{C} 86-\mathrm{C} 85-\mathrm{C} 84 \quad 118.0$ (9)

$\mathrm{C} 86-\mathrm{C} 85-\mathrm{H} 85 \mathrm{C} \quad 107.8$

$\mathrm{C} 86-\mathrm{C} 85-\mathrm{H} 85 \mathrm{D} \quad 107.8$

$\mathrm{C} 85-\mathrm{C} 86-\mathrm{H} 86 \mathrm{C}$

$\mathrm{C} 85-\mathrm{C} 86-\mathrm{H} 86 \mathrm{D} \quad 105.8$

$\mathrm{C} 85-\mathrm{C} 86-\mathrm{C} 87 \quad 112.4$ (9)

$\mathrm{H} 86 \mathrm{C}-\mathrm{C} 86-\mathrm{H} 86 \mathrm{D}$ 


$\begin{array}{ll}\text { C1B-C6B-C7 } & 111.1(6) \\ \text { C5B-C6B-C7 } & 125.5(6) \\ \text { C5B-C6B-C1B } & 122.7(6) \\ \text { C13B-C12B-C3B } & 130.6(7) \\ \text { C13B-C12B-C17B } & 120.2(7) \\ \text { C17B-C12B-C3B } & 109.2(6) \\ \text { C12B-C13B-H13B } & 120.6 \\ \text { C14B-C13B-C12B } & 118.7(8) \\ \text { C14B-C13B-H13B } & 120.6 \\ \text { C13B-C14B-H14B } & 119.5 \\ \text { C13B-C14B-C15B } & 121.0(9) \\ \text { C15B-C14B-H14B } & 119.5 \\ \text { C14B-C15B-H15B } & 119.2 \\ \text { C14B-C15B-C16B } & 120.7(8) \\ \text { C16B-C15B-H15B } & 119.4 \\ \text { C15B-C16B-H16B } & 120.9 \\ \text { C17B-C16B-C15B } & 118.3(7) \\ \text { C17B-C16B-H16B } & 120.8 \\ \text { C12B-C17B-C18B } & 110.9(6) \\ \text { C16B-C17B-C12B } & 121.0(6) \\ \text { C16B-C17B-C18B } & 128.0(6) \\ \text { C17B-C18B-C4B } & 100.6(6) \\ \text { C17B-C18B-C56B } & 110.6(7) \\ \text { C56B-C18B-C4B } & 112.5(7) \\ \text { C62B-C18B-C4B } & 112.0(6) \\ \text { C62B-C18B-C17B } & 112.0(7) \\ \text { C62B-C18B-C56B } & 109.0(7) \\ \text { O1B-C55B-H55D } & 109.5 \\ \text { O1B-C55B-H55E } & 109.5 \\ \text { O1B-C55B-H55F } & 109.5 \\ \text { H55D-C55B-H55E } & 109.5 \\ \text { H55D-C55B-H55F } & 109.5 \\ \text { H55E-C55B-H55F } & 109.5 \\ \text { C18B-C56B-H56C } & 108.2 \\ \text { C18B-C56B-H56D } & 108.2 \\ \text { H56C-C56B-H56D } & 107.4 \\ \text { C57B-C56B-C18B } & 116.6(7) \\ \text { C57B-C56B-H56C } & 108.3 \\ \text { C57B-C56B-H56D } & 107.9 \\ \text { C56B-C57B-H57C } & 109.0 \\ \text { C56B-C57B-H57D } & 108.7 \\ \text { H57C-C57B-H57D } & 107.7 \\ \text { C58B-C57B-C56B } & 112.7(8) \\ \text { C58B-C57B-H57C } & 109.6 \\ \text { C58B-C57B-H57D } & 109.1 \\ \text { C57B-C58B-H58C } & 108.4 \\ \text { C57B-C58B-H58D } & 107.3 \\ \text { H58C-C58B-H58D } & \\ & \end{array}$

\begin{tabular}{|c|c|}
\hline $\mathrm{C} 87-\mathrm{C} 86-\mathrm{H} 86 \mathrm{C}$ & 109.3 \\
\hline $\mathrm{C} 87-\mathrm{C} 86-\mathrm{H} 86 \mathrm{D}$ & 108.3 \\
\hline $\mathrm{C} 86-\mathrm{C} 87-\mathrm{H} 87 \mathrm{D}$ & 108.6 \\
\hline $\mathrm{C} 86-\mathrm{C} 87-\mathrm{H} 87 \mathrm{E}$ & 110.5 \\
\hline $\mathrm{C} 86-\mathrm{C} 87-\mathrm{H} 87 \mathrm{~F}$ & 109.4 \\
\hline $\mathrm{H} 87 \mathrm{D}-\mathrm{C} 87-\mathrm{H} 87 \mathrm{E}$ & 109.5 \\
\hline $\mathrm{H} 87 \mathrm{D}-\mathrm{C} 87-\mathrm{H} 87 \mathrm{~F}$ & 109.5 \\
\hline $\mathrm{H} 87 \mathrm{E}-\mathrm{C} 87-\mathrm{H} 87 \mathrm{~F}$ & 109.4 \\
\hline $\mathrm{C} 54-\mathrm{C} 88-\mathrm{H} 88 \mathrm{C}$ & 108.5 \\
\hline $\mathrm{C} 54-\mathrm{C} 88-\mathrm{H} 88 \mathrm{D}$ & 108.5 \\
\hline $\mathrm{C} 54-\mathrm{C} 88-\mathrm{C} 89$ & $115.0(7)$ \\
\hline $\mathrm{H} 88 \mathrm{C}-\mathrm{C} 88-\mathrm{H} 88 \mathrm{D}$ & 107.5 \\
\hline $\mathrm{C} 89-\mathrm{C} 88-\mathrm{H} 88 \mathrm{C}$ & 108.5 \\
\hline $\mathrm{C} 89-\mathrm{C} 88-\mathrm{H} 88 \mathrm{D}$ & 108.5 \\
\hline $\mathrm{C} 88-\mathrm{C} 89-\mathrm{H} 89 \mathrm{C}$ & 105.4 \\
\hline $\mathrm{C} 88-\mathrm{C} 89-\mathrm{H} 89 \mathrm{D}$ & 105.4 \\
\hline $\mathrm{C} 88-\mathrm{C} 89-\mathrm{C} 90$ & $127.4(7)$ \\
\hline $\mathrm{H} 89 \mathrm{C}-\mathrm{C} 89-\mathrm{H} 89 \mathrm{D}$ & 106.0 \\
\hline $\mathrm{C} 90-\mathrm{C} 89-\mathrm{H} 89 \mathrm{C}$ & 105.4 \\
\hline $\mathrm{C} 90-\mathrm{C} 89-\mathrm{H} 89 \mathrm{D}$ & 105.4 \\
\hline $\mathrm{C} 89-\mathrm{C} 90-\mathrm{H} 90 \mathrm{C}$ & 108.8 \\
\hline $\mathrm{C} 89-\mathrm{C} 90-\mathrm{H} 90 \mathrm{D}$ & 108.8 \\
\hline $\mathrm{H} 90 \mathrm{C}-\mathrm{C} 90-\mathrm{H} 90 \mathrm{D}$ & 107.7 \\
\hline $\mathrm{C} 91-\mathrm{C} 90-\mathrm{C} 89$ & $113.7(9)$ \\
\hline $\mathrm{C} 91-\mathrm{C} 90-\mathrm{H} 90 \mathrm{C}$ & 108.8 \\
\hline $\mathrm{C} 91-\mathrm{C} 90-\mathrm{H} 90 \mathrm{D}$ & 108.8 \\
\hline $\mathrm{C} 90-\mathrm{C} 91-\mathrm{H} 91 \mathrm{C}$ & 108.9 \\
\hline $\mathrm{C} 90-\mathrm{C} 91-\mathrm{H} 91 \mathrm{D}$ & 108.9 \\
\hline $\mathrm{C} 90-\mathrm{C} 91-\mathrm{C} 92$ & $113.2(10)$ \\
\hline $\mathrm{H} 91 \mathrm{C}-\mathrm{C} 91-\mathrm{H} 91 \mathrm{D}$ & 107.7 \\
\hline C92-C91-H91C & 108.9 \\
\hline $\mathrm{C} 92-\mathrm{C} 91-\mathrm{H} 91 \mathrm{D}$ & 108.9 \\
\hline C91-C92-H92C & 109.2 \\
\hline C91-C92-H92D & 109.2 \\
\hline $\mathrm{C} 91-\mathrm{C} 92-\mathrm{C} 93$ & $111.9(10)$ \\
\hline $\mathrm{H} 92 \mathrm{C}-\mathrm{C} 92-\mathrm{H} 92 \mathrm{D}$ & 107.9 \\
\hline C93-C92-H92C & 109.2 \\
\hline C93-C92-H92D & 109.2 \\
\hline C92-C93-H93D & 109.5 \\
\hline C92-C93-H93E & 109.5 \\
\hline $\mathrm{C} 92-\mathrm{C} 93-\mathrm{H} 93 \mathrm{~F}$ & 109.5 \\
\hline H93D-C93-H93E & 109.5 \\
\hline $\mathrm{H} 93 \mathrm{D}-\mathrm{C} 93-\mathrm{H} 93 \mathrm{~F}$ & 109.5 \\
\hline $\mathrm{H} 93 \mathrm{E}-\mathrm{C} 93-\mathrm{H} 93 \mathrm{~F}$ & 109.5 \\
\hline $\mathrm{H} 1 \mathrm{SA}-\mathrm{C} 1 \mathrm{~S}-\mathrm{H} 1 \mathrm{SB}$ & 109.5 \\
\hline $\mathrm{H} 1 \mathrm{SA}-\mathrm{C} 1 \mathrm{~S}-\mathrm{H} 1 \mathrm{SC}$ & 109.5 \\
\hline $\mathrm{H} 1 \mathrm{SB}-\mathrm{C} 1 \mathrm{~S}-\mathrm{H} 1 \mathrm{SC}$ & 109.5 \\
\hline $\mathrm{H} 1 \mathrm{SD}-\mathrm{C} 1 \mathrm{~S}-\mathrm{H} 1 \mathrm{SE}$ & 109.5 \\
\hline
\end{tabular}




\begin{tabular}{|c|c|}
\hline $\mathrm{C} 59 \mathrm{~B}-\mathrm{C} 58 \mathrm{~B}-\mathrm{C} 57 \mathrm{~B}$ & $114.5(7)$ \\
\hline $\mathrm{C} 59 \mathrm{~B}-\mathrm{C} 58 \mathrm{~B}-\mathrm{H} 58 \mathrm{C}$ & 107.8 \\
\hline $\mathrm{C} 59 \mathrm{~B}-\mathrm{C} 58 \mathrm{~B}-\mathrm{H} 58 \mathrm{D}$ & 109.1 \\
\hline $\mathrm{C} 58 \mathrm{~B}-\mathrm{C} 59 \mathrm{~B}-\mathrm{H} 59 \mathrm{C}$ & 107.1 \\
\hline $\mathrm{C} 58 \mathrm{~B}-\mathrm{C} 59 \mathrm{~B}-\mathrm{H} 59 \mathrm{D}$ & 107.8 \\
\hline $\mathrm{H} 59 \mathrm{C}-\mathrm{C} 59 \mathrm{~B}-\mathrm{H} 59 \mathrm{D}$ & 107.1 \\
\hline $\mathrm{C} 60 \mathrm{~B}-\mathrm{C} 59 \mathrm{~B}-\mathrm{C} 58 \mathrm{~B}$ & $119.4(8)$ \\
\hline $\mathrm{C} 60 \mathrm{~B}-\mathrm{C} 59 \mathrm{~B}-\mathrm{H} 59 \mathrm{C}$ & 107.1 \\
\hline $\mathrm{C} 60 \mathrm{~B}-\mathrm{C} 59 \mathrm{~B}-\mathrm{H} 59 \mathrm{D}$ & 107.7 \\
\hline $\mathrm{C} 59 \mathrm{~B}-\mathrm{C} 60 \mathrm{~B}-\mathrm{H} 60 \mathrm{C}$ & 108.0 \\
\hline $\mathrm{C} 59 \mathrm{~B}-\mathrm{C} 60 \mathrm{~B}-\mathrm{H} 60 \mathrm{D}$ & 109.1 \\
\hline $\mathrm{H} 60 \mathrm{C}-\mathrm{C} 60 \mathrm{~B}-\mathrm{H} 60 \mathrm{D}$ & 107.4 \\
\hline $\mathrm{C} 61 \mathrm{~B}-\mathrm{C} 60 \mathrm{~B}-\mathrm{C} 59 \mathrm{~B}$ & $116.3(11)$ \\
\hline $\mathrm{C} 61 \mathrm{~B}-\mathrm{C} 60 \mathrm{~B}-\mathrm{H} 60 \mathrm{C}$ & 108.4 \\
\hline $\mathrm{C} 61 \mathrm{~B}-\mathrm{C} 60 \mathrm{~B}-\mathrm{H} 60 \mathrm{D}$ & 107.2 \\
\hline $\mathrm{C} 60 \mathrm{~B}-\mathrm{C} 61 \mathrm{~B}-\mathrm{H} 61 \mathrm{D}$ & 109.5 \\
\hline C60B-C61B-H61E & 109.5 \\
\hline $\mathrm{C} 60 \mathrm{~B}-\mathrm{C} 61 \mathrm{~B}-\mathrm{H} 61 \mathrm{~F}$ & 109.5 \\
\hline $\mathrm{H} 61 \mathrm{D}-\mathrm{C} 61 \mathrm{~B}-\mathrm{H} 61 \mathrm{E}$ & 109.5 \\
\hline $\mathrm{H} 61 \mathrm{D}-\mathrm{C} 61 \mathrm{~B}-\mathrm{H} 61 \mathrm{~F}$ & 109.5 \\
\hline $\mathrm{H} 61 \mathrm{E}-\mathrm{C} 61 \mathrm{~B}-\mathrm{H} 61 \mathrm{~F}$ & 109.5 \\
\hline $\mathrm{C} 18 \mathrm{~B}-\mathrm{C} 62 \mathrm{~B}-\mathrm{H} 62 \mathrm{C}$ & 108.3 \\
\hline $\mathrm{C} 18 \mathrm{~B}-\mathrm{C} 62 \mathrm{~B}-\mathrm{H} 62 \mathrm{D}$ & 108.3 \\
\hline $\mathrm{H} 62 \mathrm{C}-\mathrm{C} 62 \mathrm{~B}-\mathrm{H} 62 \mathrm{D}$ & 107.4 \\
\hline $\mathrm{C} 63 \mathrm{~B}-\mathrm{C} 62 \mathrm{~B}-\mathrm{C} 18 \mathrm{~B}$ & $116.1(7)$ \\
\hline $\mathrm{C} 63 \mathrm{~B}-\mathrm{C} 62 \mathrm{~B}-\mathrm{H} 62 \mathrm{C}$ & 108.4 \\
\hline $\mathrm{C} 63 \mathrm{~B}-\mathrm{C} 62 \mathrm{~B}-\mathrm{H} 62 \mathrm{D}$ & 108.0 \\
\hline $\mathrm{C} 62 \mathrm{~B}-\mathrm{C} 63 \mathrm{~B}-\mathrm{H} 63 \mathrm{C}$ & 109.0 \\
\hline $\mathrm{C} 62 \mathrm{~B}-\mathrm{C} 63 \mathrm{~B}-\mathrm{H} 63 \mathrm{D}$ & 108.5 \\
\hline $\mathrm{C} 62 \mathrm{~B}-\mathrm{C} 63 \mathrm{~B}-\mathrm{C} 64 \mathrm{~B}$ & $114.3(7)$ \\
\hline $\mathrm{H} 63 \mathrm{C}-\mathrm{C} 63 \mathrm{~B}-\mathrm{H} 63 \mathrm{D}$ & 107.7 \\
\hline $\mathrm{C} 64 \mathrm{~B}-\mathrm{C} 63 \mathrm{~B}-\mathrm{H} 63 \mathrm{C}$ & 109.2 \\
\hline $\mathrm{C} 64 \mathrm{~B}-\mathrm{C} 63 \mathrm{~B}-\mathrm{H} 63 \mathrm{D}$ & 108.0 \\
\hline $\mathrm{C} 63 \mathrm{~B}-\mathrm{C} 64 \mathrm{~B}-\mathrm{H} 64 \mathrm{C}$ & 109.0 \\
\hline $\mathrm{C} 63 \mathrm{~B}-\mathrm{C} 64 \mathrm{~B}-\mathrm{H} 64 \mathrm{D}$ & 108.0 \\
\hline $\mathrm{H} 64 \mathrm{C}-\mathrm{C} 64 \mathrm{~B}-\mathrm{H} 64 \mathrm{D}$ & 107.6 \\
\hline $\mathrm{C} 65 \mathrm{~B}-\mathrm{C} 64 \mathrm{~B}-\mathrm{C} 63 \mathrm{~B}$ & $114.8(7)$ \\
\hline $\mathrm{C} 65 \mathrm{~B}-\mathrm{C} 64 \mathrm{~B}-\mathrm{H} 64 \mathrm{C}$ & 108.7 \\
\hline $\mathrm{C} 65 \mathrm{~B}-\mathrm{C} 64 \mathrm{~B}-\mathrm{H} 64 \mathrm{D}$ & 108.6 \\
\hline $\mathrm{C} 64 \mathrm{~B}-\mathrm{C} 65 \mathrm{~B}-\mathrm{H} 65 \mathrm{C}$ & 108.7 \\
\hline $\mathrm{C} 64 \mathrm{~B}-\mathrm{C} 65 \mathrm{~B}-\mathrm{H} 65 \mathrm{D}$ & 108.6 \\
\hline $\mathrm{H} 65 \mathrm{C}-\mathrm{C} 65 \mathrm{~B}-\mathrm{H} 65 \mathrm{D}$ & 107.5 \\
\hline $\mathrm{C} 66 \mathrm{~B}-\mathrm{C} 65 \mathrm{~B}-\mathrm{C} 64 \mathrm{~B}$ & $114.8(9)$ \\
\hline $\mathrm{C} 66 \mathrm{~B}-\mathrm{C} 65 \mathrm{~B}-\mathrm{H} 65 \mathrm{C}$ & 108.2 \\
\hline $\mathrm{C} 66 \mathrm{~B}-\mathrm{C} 65 \mathrm{~B}-\mathrm{H} 65 \mathrm{D}$ & 108.8 \\
\hline $\mathrm{C} 67-\mathrm{C} 66 \mathrm{~B}-\mathrm{C} 65 \mathrm{~B}$ & $111.3(12)$ \\
\hline $\mathrm{C} 67-\mathrm{C} 66 \mathrm{~B}-\mathrm{H} 66 \mathrm{C}$ & 109.4 \\
\hline $7-\mathrm{C} 66 \mathrm{~B}-$ & \\
\hline
\end{tabular}

\begin{tabular}{|c|c|}
\hline $\mathrm{H} 1 \mathrm{SD}-\mathrm{C} 1 \mathrm{~S}-\mathrm{H} 1 \mathrm{SF}$ & 109.5 \\
\hline $\mathrm{H} 1 \mathrm{SE}-\mathrm{C} 1 \mathrm{~S}-\mathrm{H} 1 \mathrm{SF}$ & 109.5 \\
\hline $\mathrm{C} 2 \mathrm{~S}-\mathrm{C} 1 \mathrm{~S}-\mathrm{H} 1 \mathrm{SA}$ & 109.5 \\
\hline $\mathrm{C} 2 \mathrm{~S}-\mathrm{C} 1 \mathrm{~S}-\mathrm{H} 1 \mathrm{SB}$ & 109.5 \\
\hline $\mathrm{C} 2 \mathrm{~S}-\mathrm{C} 1 \mathrm{~S}-\mathrm{H} 1 \mathrm{SC}$ & 109.5 \\
\hline $\mathrm{C} 2 \mathrm{~S}^{\prime}-\mathrm{C} 1 \mathrm{~S}-\mathrm{H} 1 \mathrm{SD}$ & 109.5 \\
\hline $\mathrm{C} 2 \mathrm{~S}^{\prime}-\mathrm{C} 1 \mathrm{~S}-\mathrm{H} 1 \mathrm{SE}$ & 109.5 \\
\hline $\mathrm{C} 2 \mathrm{~S}^{\prime}-\mathrm{C} 1 \mathrm{~S}-\mathrm{H} 1 \mathrm{SF}$ & 109.5 \\
\hline $\mathrm{C} 1 \mathrm{~S}-\mathrm{C} 2 \mathrm{~S}-\mathrm{H} 2 \mathrm{SA}$ & 110.0 \\
\hline $\mathrm{C} 1 \mathrm{~S}-\mathrm{C} 2 \mathrm{~S}-\mathrm{H} 2 \mathrm{SB}$ & 110.0 \\
\hline $\mathrm{C} 1 \mathrm{~S}-\mathrm{C} 2 \mathrm{~S}-\mathrm{C} 3 \mathrm{~S}$ & $108.7(8)$ \\
\hline $\mathrm{H} 2 \mathrm{SA}-\mathrm{C} 2 \mathrm{~S}-\mathrm{H} 2 \mathrm{SB}$ & 108.3 \\
\hline $\mathrm{C} 3 \mathrm{~S}-\mathrm{C} 2 \mathrm{~S}-\mathrm{H} 2 \mathrm{SA}$ & 110.0 \\
\hline $\mathrm{C} 3 \mathrm{~S}-\mathrm{C} 2 \mathrm{~S}-\mathrm{H} 2 \mathrm{SB}$ & 110.0 \\
\hline $\mathrm{C} 2 \mathrm{~S}-\mathrm{C} 3 \mathrm{~S}-\mathrm{H} 3 \mathrm{SA}$ & 109.3 \\
\hline $\mathrm{C} 2 \mathrm{~S}-\mathrm{C} 3 \mathrm{~S}-\mathrm{H} 3 \mathrm{SB}$ & 109.3 \\
\hline $\mathrm{H} 3 \mathrm{SA}-\mathrm{C} 3 \mathrm{~S}-\mathrm{H} 3 \mathrm{SB}$ & 108.0 \\
\hline $\mathrm{C} 4 \mathrm{~S}-\mathrm{C} 3 \mathrm{~S}-\mathrm{C} 2 \mathrm{~S}$ & $111.5(9)$ \\
\hline $\mathrm{C} 4 \mathrm{~S}-\mathrm{C} 3 \mathrm{~S}-\mathrm{H} 3 \mathrm{SA}$ & 109.3 \\
\hline $\mathrm{C} 4 \mathrm{~S}-\mathrm{C} 3 \mathrm{~S}-\mathrm{H} 3 \mathrm{SB}$ & 109.3 \\
\hline $\mathrm{C} 3 \mathrm{~S}-\mathrm{C} 4 \mathrm{~S}-\mathrm{H} 4 \mathrm{SA}$ & 112.8 \\
\hline $\mathrm{C} 3 \mathrm{~S}-\mathrm{C} 4 \mathrm{~S}-\mathrm{H} 4 \mathrm{SB}$ & 112.8 \\
\hline $\mathrm{C} 3 \mathrm{~S}-\mathrm{C} 4 \mathrm{~S}-\mathrm{C} 5 \mathrm{~S}$ & $94.5(9)$ \\
\hline $\mathrm{H} 4 \mathrm{SA}-\mathrm{C} 4 \mathrm{~S}-\mathrm{H} 4 \mathrm{SB}$ & 110.3 \\
\hline $\mathrm{C} 5 \mathrm{~S}-\mathrm{C} 4 \mathrm{~S}-\mathrm{H} 4 \mathrm{SA}$ & 112.8 \\
\hline $\mathrm{C} 5 \mathrm{~S}-\mathrm{C} 4 \mathrm{~S}-\mathrm{H} 4 \mathrm{SB}$ & 112.8 \\
\hline $\mathrm{C} 4 \mathrm{~S}-\mathrm{C} 5 \mathrm{~S}-\mathrm{H} 5 \mathrm{SA}$ & 109.5 \\
\hline $\mathrm{C} 4 \mathrm{~S}-\mathrm{C} 5 \mathrm{~S}-\mathrm{H} 5 \mathrm{SB}$ & 109.5 \\
\hline $\mathrm{C} 4 \mathrm{~S}-\mathrm{C} 5 \mathrm{~S}-\mathrm{H} 5 \mathrm{SC}$ & 109.5 \\
\hline $\mathrm{H} 5 \mathrm{SA}-\mathrm{C} 5 \mathrm{~S}-\mathrm{H} 5 \mathrm{SB}$ & 109.5 \\
\hline $\mathrm{H} 5 \mathrm{SA}-\mathrm{C} 5 \mathrm{~S}-\mathrm{H} 5 \mathrm{SC}$ & 109.5 \\
\hline $\mathrm{H} 5 \mathrm{SB}-\mathrm{C} 5 \mathrm{~S}-\mathrm{H} 5 \mathrm{SC}$ & 109.5 \\
\hline $\mathrm{C} 1 \mathrm{~S}-\mathrm{C} 2 \mathrm{~S}^{\prime}-\mathrm{H} 2 \mathrm{SC}$ & 103.4 \\
\hline $\mathrm{C} 1 \mathrm{~S}-\mathrm{C} 2 \mathrm{~S}^{\prime}-\mathrm{H} 2 \mathrm{SD}$ & 103.4 \\
\hline $\mathrm{H} 2 \mathrm{SC}-\mathrm{C} 2 \mathrm{~S}^{\prime}-\mathrm{H} 2 \mathrm{SD}$ & 105.2 \\
\hline $\mathrm{C} 3 \mathrm{~S}^{\prime}-\mathrm{C} 2 \mathrm{~S}^{\prime}-\mathrm{C} 1 \mathrm{~S}$ & $135.1(10)$ \\
\hline $\mathrm{C} 3 \mathrm{~S}^{\prime}-\mathrm{C} 2 \mathrm{~S}^{\prime}-\mathrm{H} 2 \mathrm{SC}$ & 103.4 \\
\hline $\mathrm{C} 3 \mathrm{~S}^{\prime}-\mathrm{C} 2 \mathrm{~S}^{\prime}-\mathrm{H} 2 \mathrm{SD}$ & 103.4 \\
\hline $\mathrm{C} 2 \mathrm{~S}^{\prime}-\mathrm{C} 3 \mathrm{~S}^{\prime}-\mathrm{H} 3 \mathrm{SC}$ & 108.1 \\
\hline $\mathrm{C} 2 \mathrm{~S}^{\prime}-\mathrm{C} 3 \mathrm{~S}^{\prime}-\mathrm{H} 3 \mathrm{SD}$ & 108.1 \\
\hline $\mathrm{C} 2 \mathrm{~S}^{\prime}-\mathrm{C} 3 \mathrm{~S}^{\prime}-\mathrm{C} 4 \mathrm{~S}^{\prime}$ & $116.7(10)$ \\
\hline $\mathrm{H} 3 \mathrm{SC}-\mathrm{C} 3 \mathrm{~S}^{\prime}-\mathrm{H} 3 \mathrm{SD}$ & 107.3 \\
\hline $\mathrm{C} 4 \mathrm{~S}^{\prime}-\mathrm{C} 3 \mathrm{~S}^{\prime}-\mathrm{H} 3 \mathrm{SC}$ & 108.1 \\
\hline $\mathrm{C} 4 \mathrm{~S}^{\prime}-\mathrm{C} 3 \mathrm{~S}^{\prime}-\mathrm{H} 3 \mathrm{SD}$ & 108.1 \\
\hline $\mathrm{C} 3 \mathrm{~S}^{\prime}-\mathrm{C} 4 \mathrm{~S}^{\prime}-\mathrm{H} 4 \mathrm{SC}$ & 106.3 \\
\hline $\mathrm{C} 3 \mathrm{~S}^{\prime}-\mathrm{C} 4 \mathrm{~S}^{\prime}-\mathrm{H} 4 \mathrm{SD}$ & 106.3 \\
\hline $\mathrm{C} 3 \mathrm{~S}^{\prime}-\mathrm{C} 4 \mathrm{~S}^{\prime}-\mathrm{C} 5 \mathrm{~S}^{\prime}$ & $123.9(12)$ \\
\hline $\mathrm{H} 4 \mathrm{SC}-\mathrm{C} 4 \mathrm{~S}^{\prime}-\mathrm{H} 4 \mathrm{SD}$ & 106.4 \\
\hline
\end{tabular}




\begin{tabular}{|c|c|}
\hline $\mathrm{C} 65 \mathrm{~B}-\mathrm{C} 66 \mathrm{~B}-\mathrm{H} 66 \mathrm{C}$ & 109.4 \\
\hline $\mathrm{C} 65 \mathrm{~B}-\mathrm{C} 66 \mathrm{~B}-\mathrm{H} 66 \mathrm{D}$ & 109.4 \\
\hline $\mathrm{H} 66 \mathrm{C}-\mathrm{C} 66 \mathrm{~B}-\mathrm{H} 66 \mathrm{D}$ & 108.0 \\
\hline $\mathrm{C} 36-\mathrm{C} 69 \mathrm{~B}-\mathrm{H} 69 \mathrm{C}$ & 105.9 \\
\hline $\mathrm{C} 36-\mathrm{C} 69 \mathrm{~B}-\mathrm{H} 69 \mathrm{D}$ & 105.5 \\
\hline $\mathrm{H} 69 \mathrm{C}-\mathrm{C} 69 \mathrm{~B}-\mathrm{H} 69 \mathrm{D}$ & 106.1 \\
\hline $\mathrm{C} 70 \mathrm{~B}-\mathrm{C} 69 \mathrm{~B}-\mathrm{C} 36$ & $126.6(7)$ \\
\hline $\mathrm{C} 70 \mathrm{~B}-\mathrm{C} 69 \mathrm{~B}-\mathrm{H} 69 \mathrm{C}$ & 105.9 \\
\hline $\mathrm{Ir}-\mathrm{N} 1-\mathrm{C} 7-\mathrm{C} 8$ & $178.8(3)$ \\
\hline $\mathrm{Ir}-\mathrm{N} 1-\mathrm{C} 7-\mathrm{C} 6$ & $8.9(5)$ \\
\hline $\mathrm{Ir}-\mathrm{N} 1-\mathrm{C} 7-\mathrm{C} 6 \mathrm{~B}$ & $-10.1(5)$ \\
\hline $\mathrm{Ir}-\mathrm{N} 1-\mathrm{C} 11-\mathrm{C} 10$ & $-179.0(3)$ \\
\hline $\mathrm{Ir}-\mathrm{N} 2-\mathrm{C} 25-\mathrm{C} 24$ & $1.9(3)$ \\
\hline $\mathrm{Ir}-\mathrm{N} 2-\mathrm{C} 25-\mathrm{C} 26$ & $179.9(2)$ \\
\hline $\mathrm{Ir}-\mathrm{N} 2-\mathrm{C} 29-\mathrm{C} 28$ & $-178.1(2)$ \\
\hline $\mathrm{Ir}-\mathrm{C} 19-\mathrm{C} 20-\mathrm{C} 21$ & $178.9(3)$ \\
\hline $\mathrm{Ir}-\mathrm{C} 19-\mathrm{C} 24-\mathrm{C} 23$ & $178.6(3)$ \\
\hline $\mathrm{Ir}-\mathrm{C} 19-\mathrm{C} 24-\mathrm{C} 25$ & $-2.0(4)$ \\
\hline $\mathrm{Ir}-\mathrm{C} 1-\mathrm{C} 2-\mathrm{C} 3$ & $-174.8(7)$ \\
\hline $\mathrm{Ir}-\mathrm{C} 1-\mathrm{C} 6-\mathrm{C} 7$ & $5.5(9)$ \\
\hline $\mathrm{Ir}-\mathrm{C} 1-\mathrm{C} 6-\mathrm{C} 5$ & $174.9(6)$ \\
\hline $\mathrm{Ir}-\mathrm{C} 1 \mathrm{~B}-\mathrm{C} 2 \mathrm{~B}-\mathrm{C} 3 \mathrm{~B}$ & $-178.6(6)$ \\
\hline $\mathrm{Ir}-\mathrm{C} 1 \mathrm{~B}-\mathrm{C} 6 \mathrm{~B}-\mathrm{C} 7$ & $-11.4(9)$ \\
\hline $\mathrm{Ir}-\mathrm{C} 1 \mathrm{~B}-\mathrm{C} 6 \mathrm{~B}-\mathrm{C} 5 \mathrm{~B}$ & $177.6(6)$ \\
\hline $\mathrm{Ir}-\mathrm{N} 3 \mathrm{~B}-\mathrm{C} 43 \mathrm{~B}-\mathrm{C} 42 \mathrm{~B}$ & $-2.4(10)$ \\
\hline $\mathrm{Ir}-\mathrm{N} 3 \mathrm{~B}-\mathrm{C} 43 \mathrm{~B}-\mathrm{C} 44 \mathrm{~B}$ & $177.0(6)$ \\
\hline $\mathrm{Ir}-\mathrm{N} 3 \mathrm{~B}-\mathrm{C} 47 \mathrm{~B}-\mathrm{C} 46 \mathrm{~B}$ & $-179.2(9)$ \\
\hline $\mathrm{Ir}-\mathrm{C} 37 \mathrm{~B}-\mathrm{C} 38 \mathrm{~B}-\mathrm{C} 39 \mathrm{~B}$ & $-174.0(6)$ \\
\hline $\mathrm{Ir}-\mathrm{C} 37 \mathrm{~B}-\mathrm{C} 42 \mathrm{~B}-\mathrm{C} 41 \mathrm{~B}$ & $176.4(6)$ \\
\hline $\mathrm{Ir}-\mathrm{C} 37 \mathrm{~B}-\mathrm{C} 42 \mathrm{~B}-\mathrm{C} 43 \mathrm{~B}$ & $2.3(9)$ \\
\hline $\mathrm{Ir}-\mathrm{N} 3-\mathrm{C} 43-\mathrm{C} 42$ & $1.6(10)$ \\
\hline $\mathrm{Ir}-\mathrm{N} 3-\mathrm{C} 43-\mathrm{C} 44$ & $178.8(5)$ \\
\hline $\mathrm{Ir}-\mathrm{N} 3-\mathrm{C} 47-\mathrm{C} 46$ & $-177.1(9)$ \\
\hline $\mathrm{Ir}-\mathrm{C} 37-\mathrm{C} 38-\mathrm{C} 39$ & $-178.1(6)$ \\
\hline $\mathrm{Ir}-\mathrm{C} 37-\mathrm{C} 42-\mathrm{C} 41$ & $176.3(6)$ \\
\hline $\mathrm{Ir}-\mathrm{C} 37-\mathrm{C} 42-\mathrm{C} 43$ & $-6.0(9)$ \\
\hline $\mathrm{O} 2-\mathrm{C} 28-\mathrm{C} 29-\mathrm{N} 2$ & $179.1(3)$ \\
\hline $\mathrm{N} 1-\mathrm{C} 7-\mathrm{C} 8-\mathrm{C} 9$ & $1.1(6)$ \\
\hline $\mathrm{N} 1-\mathrm{C} 7-\mathrm{C} 6-\mathrm{C} 1$ & $-9.8(9)$ \\
\hline $\mathrm{N} 1-\mathrm{C} 7-\mathrm{C} 6-\mathrm{C} 5$ & $-179.6(6)$ \\
\hline $\mathrm{N} 1-\mathrm{C} 7-\mathrm{C} 6 \mathrm{~B}-\mathrm{C} 1 \mathrm{~B}$ & $13.7(8)$ \\
\hline $\mathrm{N} 1-\mathrm{C} 7-\mathrm{C} 6 \mathrm{~B}-\mathrm{C} 5 \mathrm{~B}$ & $-175.6(6)$ \\
\hline $\mathrm{N} 2-\mathrm{C} 25-\mathrm{C} 26-\mathrm{C} 27$ & $-1.7(5)$ \\
\hline $\mathrm{C} 7-\mathrm{N} 1-\mathrm{C} 11-\mathrm{C} 10$ & $1.1(5)$ \\
\hline $\mathrm{C} 7-\mathrm{C} 8-\mathrm{C} 9-\mathrm{C} 10$ & $-0.7(6)$ \\
\hline $\mathrm{C} 8-\mathrm{C} 7-\mathrm{C} 6-\mathrm{C} 1$ & $-178.5(6)$ \\
\hline & \\
\hline
\end{tabular}

$\begin{array}{ll}\text { C5S'-C4S'-H4SC } & 106.3 \\ \text { C5S'-C4S'-H4SD } & 106.3 \\ \text { C4S'-C5S'-H5SD } & 109.5 \\ \text { C4S'-C5S'-H5SE } & 109.5 \\ \text { C4S'-C5S'-H5SF } & 109.5 \\ \text { H5SD-C5S'-H5SE } & 109.5 \\ \text { H5SD-C5S'-H5SF } & 109.5 \\ \text { H5SE-C5S'-H5SF } & 109.5\end{array}$

$\mathrm{C} 3 \mathrm{~B}-\mathrm{C} 4 \mathrm{~B}-\mathrm{C} 18 \mathrm{~B}-\mathrm{C} 17 \mathrm{~B} \quad 0.6(9)$

$\mathrm{C} 3 \mathrm{~B}-\mathrm{C} 4 \mathrm{~B}-\mathrm{C} 18 \mathrm{~B}-\mathrm{C} 56 \mathrm{~B} \quad 118.3(8)$

$\mathrm{C} 3 \mathrm{~B}-\mathrm{C} 4 \mathrm{~B}-\mathrm{C} 18 \mathrm{~B}-\mathrm{C} 62 \mathrm{~B} \quad-118.5(8)$

$\mathrm{C} 3 \mathrm{~B}-\mathrm{C} 12 \mathrm{~B}-\mathrm{C} 13 \mathrm{~B}-\mathrm{C} 14 \mathrm{~B} \quad-179.9(8)$

$\mathrm{C} 3 \mathrm{~B}-\mathrm{C} 12 \mathrm{~B}-\mathrm{C} 17 \mathrm{~B}-\mathrm{C} 16 \mathrm{~B} \quad-179.8(7)$

$\mathrm{C} 3 \mathrm{~B}-\mathrm{C} 12 \mathrm{~B}-\mathrm{C} 17 \mathrm{~B}-\mathrm{C} 18 \mathrm{~B} \quad-2.3(10)$

$\mathrm{C} 4 \mathrm{~B}-\mathrm{C} 3 \mathrm{~B}-\mathrm{C} 12 \mathrm{~B}-\mathrm{C} 13 \mathrm{~B} \quad-178.4(9)$

$\mathrm{C} 4 \mathrm{~B}-\mathrm{C} 3 \mathrm{~B}-\mathrm{C} 12 \mathrm{~B}-\mathrm{C} 17 \mathrm{~B} \quad 2.6(10)$

$\mathrm{C} 4 \mathrm{~B}-\mathrm{C} 5 \mathrm{~B}-\mathrm{C} 6 \mathrm{~B}-\mathrm{C} 7 \quad-169.5(7)$

$\mathrm{C} 4 \mathrm{~B}-\mathrm{C} 5 \mathrm{~B}-\mathrm{C} 6 \mathrm{~B}-\mathrm{C} 1 \mathrm{~B} \quad 0.2(12)$

$\mathrm{C} 4 \mathrm{~B}-\mathrm{C} 18 \mathrm{~B}-\mathrm{C} 56 \mathrm{~B}-\mathrm{C} 57 \mathrm{~B} \quad-63.7(9)$

$\mathrm{C} 4 \mathrm{~B}-\mathrm{C} 18 \mathrm{~B}-\mathrm{C} 62 \mathrm{~B}-\mathrm{C} 63 \mathrm{~B} \quad 63.4(9)$

$\mathrm{C} 5 \mathrm{~B}-\mathrm{C} 4 \mathrm{~B}-\mathrm{C} 18 \mathrm{~B}-\mathrm{C} 17 \mathrm{~B} \quad-179.2(8)$

$\mathrm{C} 5 \mathrm{~B}-\mathrm{C} 4 \mathrm{~B}-\mathrm{C} 18 \mathrm{~B}-\mathrm{C} 56 \mathrm{~B} \quad-61.5(11)$

$\mathrm{C} 5 \mathrm{~B}-\mathrm{C} 4 \mathrm{~B}-\mathrm{C} 18 \mathrm{~B}-\mathrm{C} 62 \mathrm{~B} \quad 61.7$ (11)

$\mathrm{C} 6 \mathrm{~B}-\mathrm{C} 7-\mathrm{C} 8-\mathrm{C} 9 \quad-169.2(5)$

$\mathrm{C} 6 \mathrm{~B}-\mathrm{C} 1 \mathrm{~B}-\mathrm{C} 2 \mathrm{~B}-\mathrm{C} 3 \mathrm{~B} \quad-0.6(12)$

$\mathrm{C} 12 \mathrm{~B}-\mathrm{C} 3 \mathrm{~B}-\mathrm{C} 4 \mathrm{~B}-\mathrm{C} 5 \mathrm{~B} \quad 177.8$ (7)

$\mathrm{C} 12 \mathrm{~B}-\mathrm{C} 3 \mathrm{~B}-\mathrm{C} 4 \mathrm{~B}-\mathrm{C} 18 \mathrm{~B} \quad-2.0(10)$

$\mathrm{C} 12 \mathrm{~B}-\mathrm{C} 13 \mathrm{~B}-\mathrm{C} 14 \mathrm{~B}-\mathrm{C} 15 \mathrm{~B} \quad 0.3(13)$

$\mathrm{C} 12 \mathrm{~B}-\mathrm{C} 17 \mathrm{~B}-\mathrm{C} 18 \mathrm{~B}-\mathrm{C} 4 \mathrm{~B} \quad 1.0(9)$

$\mathrm{C} 12 \mathrm{~B}-\mathrm{C} 17 \mathrm{~B}-\mathrm{C} 18 \mathrm{~B}-\mathrm{C} 56 \mathrm{~B}-118.0(7)$

$\mathrm{C} 12 \mathrm{~B}-\mathrm{C} 17 \mathrm{~B}-\mathrm{C} 18 \mathrm{~B}-\mathrm{C} 62 \mathrm{~B} \quad 120.2(8)$

$\mathrm{C} 13 \mathrm{~B}-\mathrm{C} 12 \mathrm{~B}-\mathrm{C} 17 \mathrm{~B}-\mathrm{C} 16 \mathrm{~B} \quad 1.1(13)$

$\mathrm{C} 13 \mathrm{~B}-\mathrm{C} 12 \mathrm{~B}-\mathrm{C} 17 \mathrm{~B}-\mathrm{C} 18 \mathrm{~B} \quad 178.7(8)$

$\mathrm{C} 13 \mathrm{~B}-\mathrm{C} 14 \mathrm{~B}-\mathrm{C} 15 \mathrm{~B}-\mathrm{C} 16 \mathrm{~B} \quad 0.4(13)$

$\mathrm{C} 14 \mathrm{~B}-\mathrm{C} 15 \mathrm{~B}-\mathrm{C} 16 \mathrm{~B}-\mathrm{C} 17 \mathrm{~B}-0.4(13)$

$\mathrm{C} 15 \mathrm{~B}-\mathrm{C} 16 \mathrm{~B}-\mathrm{C} 17 \mathrm{~B}-\mathrm{C} 12 \mathrm{~B} \quad-0.4(12)$

$\mathrm{C} 15 \mathrm{~B}-\mathrm{C} 16 \mathrm{~B}-\mathrm{C} 17 \mathrm{~B}-\mathrm{C} 18 \mathrm{~B} \quad-177.4(8)$

$\mathrm{C} 16 \mathrm{~B}-\mathrm{C} 17 \mathrm{~B}-\mathrm{C} 18 \mathrm{~B}-\mathrm{C} 4 \mathrm{~B} \quad 178.4(8)$

$\mathrm{C} 16 \mathrm{~B}-\mathrm{C} 17 \mathrm{~B}-\mathrm{C} 18 \mathrm{~B}-\mathrm{C} 56 \mathrm{~B} \quad 59.3$ (11)

$\mathrm{C} 16 \mathrm{~B}-\mathrm{C} 17 \mathrm{~B}-\mathrm{C} 18 \mathrm{~B}-\mathrm{C} 62 \mathrm{~B}-62.5$ (11)

$\mathrm{C} 17 \mathrm{~B}-\mathrm{C} 12 \mathrm{~B}-\mathrm{C} 13 \mathrm{~B}-\mathrm{C} 14 \mathrm{~B}-1.1(13)$

$\mathrm{C} 17 \mathrm{~B}-\mathrm{C} 18 \mathrm{~B}-\mathrm{C} 56 \mathrm{~B}-\mathrm{C} 57 \mathrm{~B} \quad 47.8(9)$

$\mathrm{C} 17 \mathrm{~B}-\mathrm{C} 18 \mathrm{~B}-\mathrm{C} 62 \mathrm{~B}-\mathrm{C} 63 \mathrm{~B}-48.7$ (9)

$\mathrm{C} 18 \mathrm{~B}-\mathrm{C} 4 \mathrm{~B}-\mathrm{C} 5 \mathrm{~B}-\mathrm{C} 6 \mathrm{~B} \quad-178.9(8)$

$\mathrm{C} 18 \mathrm{~B}-\mathrm{C} 56 \mathrm{~B}-\mathrm{C} 57 \mathrm{~B}-\mathrm{C} 58 \mathrm{~B} \quad-175.7$ (7)

$\mathrm{C} 18 \mathrm{~B}-\mathrm{C} 62 \mathrm{~B}-\mathrm{C} 63 \mathrm{~B}-\mathrm{C} 64 \mathrm{~B}-176.5(6)$

$\mathrm{C} 56 \mathrm{~B}-\mathrm{C} 18 \mathrm{~B}-\mathrm{C} 62 \mathrm{~B}-\mathrm{C} 63 \mathrm{~B}-171.4(7)$ 


\begin{tabular}{|c|c|}
\hline $\mathrm{C} 8-\mathrm{C} 7-\mathrm{C} 6 \mathrm{~B}-\mathrm{C} 1 \mathrm{~B}$ & $-175.7(6)$ \\
\hline $\mathrm{C} 8-\mathrm{C} 7-\mathrm{C} 6 \mathrm{~B}-\mathrm{C} 5 \mathrm{~B}$ & $-5.0(10)$ \\
\hline $\mathrm{C} 8-\mathrm{C} 9-\mathrm{C} 10-\mathrm{C} 11$ & $0.5(6)$ \\
\hline $\mathrm{C} 8-\mathrm{C} 9-\mathrm{C} 10-\mathrm{O} 1$ & $-171.1(6)$ \\
\hline $\mathrm{C} 8-\mathrm{C} 9-\mathrm{C} 10-\mathrm{O} 1 \mathrm{~B}$ & $166.1(6)$ \\
\hline $\mathrm{C} 9-\mathrm{C} 10-\mathrm{C} 11-\mathrm{N} 1$ & $-0.7(6)$ \\
\hline $\mathrm{C} 9-\mathrm{C} 10-\mathrm{O} 1-\mathrm{C} 55$ & $-0.5(12)$ \\
\hline $\mathrm{C} 9-\mathrm{C} 10-\mathrm{O} 1 \mathrm{~B}-\mathrm{C} 55 \mathrm{~B}$ & $19.3(12)$ \\
\hline $\mathrm{C} 11-\mathrm{N} 1-\mathrm{C} 7-\mathrm{C} 8$ & $-1.2(5)$ \\
\hline $\mathrm{C} 11-\mathrm{N} 1-\mathrm{C} 7-\mathrm{C} 6$ & $-171.1(4)$ \\
\hline $\mathrm{C} 11-\mathrm{N} 1-\mathrm{C} 7-\mathrm{C} 6 \mathrm{~B}$ & $169.9(4)$ \\
\hline $\mathrm{C} 11-\mathrm{C} 10-\mathrm{O} 1-\mathrm{C} 55$ & $-171.7(8)$ \\
\hline $\mathrm{C} 11-\mathrm{C} 10-\mathrm{O} 1 \mathrm{~B}-\mathrm{C} 55 \mathrm{~B}$ & $-173.8(8)$ \\
\hline $\mathrm{C} 19-\mathrm{C} 20-\mathrm{C} 21-\mathrm{C} 22$ & $2.2(5)$ \\
\hline $\mathrm{C} 19-\mathrm{C} 20-\mathrm{C} 21-\mathrm{C} 30$ & $-174.2(3)$ \\
\hline $\mathrm{C} 19-\mathrm{C} 24-\mathrm{C} 25-\mathrm{N} 2$ & $-0.1(4)$ \\
\hline $\mathrm{C} 19-\mathrm{C} 24-\mathrm{C} 25-\mathrm{C} 26$ & $-177.8(3)$ \\
\hline $\mathrm{C} 20-\mathrm{C} 19-\mathrm{C} 24-\mathrm{C} 23$ & $-2.1(5)$ \\
\hline $\mathrm{C} 20-\mathrm{C} 19-\mathrm{C} 24-\mathrm{C} 25$ & $177.3(3)$ \\
\hline $\mathrm{C} 20-\mathrm{C} 21-\mathrm{C} 22-\mathrm{C} 23$ & $-1.8(6)$ \\
\hline $\mathrm{C} 20-\mathrm{C} 21-\mathrm{C} 22-\mathrm{C} 36$ & $179.8(3)$ \\
\hline $\mathrm{C} 20-\mathrm{C} 21-\mathrm{C} 30-\mathrm{C} 31$ & $1.1(7)$ \\
\hline $\mathrm{C} 20-\mathrm{C} 21-\mathrm{C} 30-\mathrm{C} 35$ & $177.9(4)$ \\
\hline $\mathrm{C} 21-\mathrm{C} 22-\mathrm{C} 23-\mathrm{C} 24$ & $-0.6(6)$ \\
\hline $\mathrm{C} 21-\mathrm{C} 22-\mathrm{C} 36-\mathrm{C} 35$ & $3.6(4)$ \\
\hline $\mathrm{C} 21-\mathrm{C} 22-\mathrm{C} 36-\mathrm{C} 75$ & $123.4(4)$ \\
\hline $\mathrm{C} 21-\mathrm{C} 22-\mathrm{C} 36-\mathrm{C} 69$ & $-127.5(5)$ \\
\hline $\mathrm{C} 21-\mathrm{C} 22-\mathrm{C} 36-\mathrm{C} 69 \mathrm{~B}$ & $-102.8(5)$ \\
\hline $\mathrm{C} 21-\mathrm{C} 30-\mathrm{C} 31-\mathrm{C} 32$ & $175.4(4)$ \\
\hline $\mathrm{C} 21-\mathrm{C} 30-\mathrm{C} 35-\mathrm{C} 34$ & $-176.4(3)$ \\
\hline $\mathrm{C} 21-\mathrm{C} 30-\mathrm{C} 35-\mathrm{C} 36$ & $1.3(4)$ \\
\hline $\mathrm{C} 22-\mathrm{C} 21-\mathrm{C} 30-\mathrm{C} 31$ & $-175.7(4)$ \\
\hline $\mathrm{C} 22-\mathrm{C} 21-\mathrm{C} 30-\mathrm{C} 35$ & $1.1(4)$ \\
\hline $\mathrm{C} 22-\mathrm{C} 23-\mathrm{C} 24-\mathrm{C} 19$ & $2.6(6)$ \\
\hline $\mathrm{C} 22-\mathrm{C} 23-\mathrm{C} 24-\mathrm{C} 25$ & $-176.8(3)$ \\
\hline $\mathrm{C} 22-\mathrm{C} 36-\mathrm{C} 75-\mathrm{C} 76$ & $-54.9(5)$ \\
\hline $\mathrm{C} 22-\mathrm{C} 36-\mathrm{C} 69-\mathrm{C} 70$ & $66.2(9)$ \\
\hline $\mathrm{C} 22-\mathrm{C} 36-\mathrm{C} 69 \mathrm{~B}-\mathrm{C} 70 \mathrm{~B}$ & $44.6(11)$ \\
\hline $\mathrm{C} 23-\mathrm{C} 22-\mathrm{C} 36-\mathrm{C} 35$ & $-174.6(4)$ \\
\hline $\mathrm{C} 23-\mathrm{C} 22-\mathrm{C} 36-\mathrm{C} 75$ & $-54.8(5)$ \\
\hline $\mathrm{C} 23-\mathrm{C} 22-\mathrm{C} 36-\mathrm{C} 69$ & $54.3(7)$ \\
\hline $\mathrm{C} 23-\mathrm{C} 22-\mathrm{C} 36-\mathrm{C} 69 \mathrm{~B}$ & $79.0(6)$ \\
\hline $\mathrm{C} 23-\mathrm{C} 24-\mathrm{C} 25-\mathrm{N} 2$ & $179.3(3)$ \\
\hline $\mathrm{C} 23-\mathrm{C} 24-\mathrm{C} 25-\mathrm{C} 26$ & $1.6(5)$ \\
\hline $\mathrm{C} 24-\mathrm{C} 19-\mathrm{C} 20-\mathrm{C} 21$ & $-0.3(5)$ \\
\hline $\mathrm{C} 24-\mathrm{C} 25-\mathrm{C} 26-\mathrm{C} 27$ & $175.9(3)$ \\
\hline $\mathrm{C} 25-\mathrm{N} 2-\mathrm{C} 29-\mathrm{C} 28$ & $1.0(5)$ \\
\hline $\mathrm{C} 25-\mathrm{C} 26-\mathrm{C} 27-\mathrm{C} 28$ & $1.1(5)$ \\
\hline
\end{tabular}

\begin{tabular}{|c|c|}
\hline $56 \mathrm{~B}-\mathrm{C} 57 \mathrm{~B}-\mathrm{C} 58 \mathrm{~B}-\mathrm{C} 59 \mathrm{~B}$ & $160.5(8)$ \\
\hline $\mathrm{C} 57 \mathrm{~B}-\mathrm{C} 58 \mathrm{~B}-\mathrm{C} 59 \mathrm{~B}-\mathrm{C} 60 \mathrm{~B}$ & $60.9(12)$ \\
\hline $\mathrm{C} 58 \mathrm{~B}-\mathrm{C} 59 \mathrm{~B}-\mathrm{C} 60 \mathrm{~B}-\mathrm{C} 61 \mathrm{~B}$ & $74.1(13)$ \\
\hline $\mathrm{C} 62 \mathrm{~B}-\mathrm{C} 18 \mathrm{~B}-\mathrm{C} 56 \mathrm{~B}-\mathrm{C} 57 \mathrm{~B}$ & $171.4(7)$ \\
\hline $\mathrm{C} 62 \mathrm{~B}-\mathrm{C} 63 \mathrm{~B}-\mathrm{C} 64 \mathrm{~B}-\mathrm{C} 65 \mathrm{~B}$ & $-55.0(10)$ \\
\hline $\mathrm{C} 63 \mathrm{~B}-\mathrm{C} 64 \mathrm{~B}-\mathrm{C} 65 \mathrm{~B}-\mathrm{C} 66 \mathrm{~B}$ & $-63.7(11)$ \\
\hline $\mathrm{C} 64 \mathrm{~B}-\mathrm{C} 65 \mathrm{~B}-\mathrm{C} 66 \mathrm{~B}-\mathrm{C} 67$ & $178.1(8)$ \\
\hline $\mathrm{C} 69 \mathrm{~B}-\mathrm{C} 36-\mathrm{C} 75-\mathrm{C} 76$ & $176.9(5)$ \\
\hline $\mathrm{C} 69 \mathrm{~B}-\mathrm{C} 70 \mathrm{~B}-\mathrm{C} 71 \mathrm{~B}-\mathrm{C} 72 \mathrm{~B}$ & $165.1(10)$ \\
\hline $\mathrm{C} 70 \mathrm{~B}-\mathrm{C} 71 \mathrm{~B}-\mathrm{C} 72 \mathrm{~B}-\mathrm{C} 73 \mathrm{~B}$ & 171. \\
\hline $\mathrm{C} 71 \mathrm{~B}-\mathrm{C} 72 \mathrm{~B}-\mathrm{C} 73 \mathrm{~B}-\mathrm{C} 74 \mathrm{~B}$ & $-178.2(11)$ \\
\hline $\mathrm{C} 77 \mathrm{~B}-\mathrm{C} 78 \mathrm{~B}-\mathrm{C} 79 \mathrm{~B}-\mathrm{C} 80 \mathrm{~B}$ & $-173.0(7)$ \\
\hline $\mathrm{O} 3 \mathrm{~B}-\mathrm{C} 46 \mathrm{~B}-\mathrm{C} 47 \mathrm{~B}-\mathrm{N} 3 \mathrm{~B}$ & $-178.9(11)$ \\
\hline $\mathrm{N} 3 \mathrm{~B}-\mathrm{C} 43 \mathrm{~B}-\mathrm{C} 44 \mathrm{~B}-\mathrm{C} 45 \mathrm{~B}$ & $3.5(12)$ \\
\hline $\mathrm{C} 37 \mathrm{~B}-\mathrm{C} 38 \mathrm{~B}-\mathrm{C} 39 \mathrm{~B}-\mathrm{C} 40 \mathrm{~B}$ & $-2.5(12)$ \\
\hline $\mathrm{C} 37 \mathrm{~B}-\mathrm{C} 38 \mathrm{~B}-\mathrm{C} 39 \mathrm{~B}-\mathrm{C} 48 \mathrm{~B}$ & $172.1(8)$ \\
\hline $\mathrm{C} 37 \mathrm{~B}-\mathrm{C} 42 \mathrm{~B}-\mathrm{C} 43 \mathrm{~B}-\mathrm{N} 3 \mathrm{~B}$ & $0.2(11)$ \\
\hline $\mathrm{C} 37 \mathrm{~B}-\mathrm{C} 42 \mathrm{~B}-\mathrm{C} 43 \mathrm{~B}-\mathrm{C} 44 \mathrm{~B}$ & $-179.1(7)$ \\
\hline $\mathrm{C} 38 \mathrm{~B}-\mathrm{C} 37 \mathrm{~B}-\mathrm{C} 42 \mathrm{~B}-\mathrm{C} 41 \mathrm{~B}$ & $-0.7(12)$ \\
\hline $\mathrm{C} 38 \mathrm{~B}-\mathrm{C} 37 \mathrm{~B}-\mathrm{C} 42 \mathrm{~B}-\mathrm{C} 43 \mathrm{~B}$ & $-174.8(7)$ \\
\hline $\mathrm{C} 38 \mathrm{~B}-\mathrm{C} 39 \mathrm{~B}-\mathrm{C} 40 \mathrm{~B}-\mathrm{C} 41 \mathrm{~B}$ & $0.0(12)$ \\
\hline $\mathrm{C} 38 \mathrm{~B}-\mathrm{C} 39 \mathrm{~B}-\mathrm{C} 40 \mathrm{~B}-\mathrm{C} 54 \mathrm{~B}$ & $177.1(7)$ \\
\hline $\mathrm{C} 38 \mathrm{~B}-\mathrm{C} 39 \mathrm{~B}-\mathrm{C} 48 \mathrm{~B}-\mathrm{C} 49 \mathrm{~B}$ & $5.0(15)$ \\
\hline $\mathrm{C} 38 \mathrm{~B}-\mathrm{C} 39 \mathrm{~B}-\mathrm{C} 48 \mathrm{~B}-\mathrm{C} 53 \mathrm{~B}$ & $9(8)$ \\
\hline $\mathrm{C} 39 \mathrm{~B}-\mathrm{C} 40 \mathrm{~B}-\mathrm{C} 41 \mathrm{~B}-\mathrm{C} 42 \mathrm{~B}$ & $1.9(11)$ \\
\hline $\mathrm{C} 39 \mathrm{~B}-\mathrm{C} 40 \mathrm{~B}-\mathrm{C} 54 \mathrm{~B}-\mathrm{C} 53 \mathrm{~B}$ & $-3.2(8)$ \\
\hline $\mathrm{C} 39 \mathrm{~B}-\mathrm{C} 40 \mathrm{~B}-\mathrm{C} 54 \mathrm{~B}-\mathrm{C} 82 \mathrm{~B}$ & $115.0(8)$ \\
\hline $\mathrm{C} 39 \mathrm{~B}-\mathrm{C} 40 \mathrm{~B}-\mathrm{C} 54 \mathrm{~B}-\mathrm{C} 88 \mathrm{~B}$ & $4(7)$ \\
\hline $\mathrm{C} 39 \mathrm{~B}-\mathrm{C} 48 \mathrm{~B}-\mathrm{C} 49 \mathrm{~B}-\mathrm{C} 50 \mathrm{~B}$ & $-175.3(8)$ \\
\hline $\mathrm{C} 39 \mathrm{~B}-\mathrm{C} 48 \mathrm{~B}-\mathrm{C} 53 \mathrm{~B}-\mathrm{C} 52 \mathrm{~B}$ & $176.6(7)$ \\
\hline $\mathrm{C} 39 \mathrm{~B}-\mathrm{C} 48 \mathrm{~B}-\mathrm{C} 53 \mathrm{~B}-\mathrm{C} 54 \mathrm{~B}$ & $-3.5(9)$ \\
\hline $\mathrm{C} 40 \mathrm{~B}-\mathrm{C} 39 \mathrm{~B}-\mathrm{C} 48 \mathrm{~B}-\mathrm{C} 49 \mathrm{~B}$ & $-179.9(8)$ \\
\hline $\mathrm{C} 40 \mathrm{~B}-\mathrm{C} 39 \mathrm{~B}-\mathrm{C} 48 \mathrm{~B}-\mathrm{C} 53 \mathrm{~B}$ & $1.2(9)$ \\
\hline $\mathrm{C} 40 \mathrm{~B}-\mathrm{C} 41 \mathrm{~B}-\mathrm{C} 42 \mathrm{~B}-\mathrm{C} 37 \mathrm{~B}$ & $-1.6(12)$ \\
\hline $\mathrm{C} 40 \mathrm{~B}-\mathrm{C} 41 \mathrm{~B}-\mathrm{C} 42 \mathrm{~B}-\mathrm{C} 43 \mathrm{~B}$ & $172.1(7)$ \\
\hline $\mathrm{C} 40 \mathrm{~B}-\mathrm{C} 54 \mathrm{~B}-\mathrm{C} 82 \mathrm{~B}-\mathrm{C} 83 \mathrm{~B}$ & $-64.5(10)$ \\
\hline $\mathrm{C} 40 \mathrm{~B}-\mathrm{C} 54 \mathrm{~B}-\mathrm{C} 88 \mathrm{~B}-\mathrm{C} 89 \mathrm{~B}$ & $50.1(9)$ \\
\hline $\mathrm{C} 41 \mathrm{~B}-\mathrm{C} 40 \mathrm{~B}-\mathrm{C} 54 \mathrm{~B}-\mathrm{C} 53 \mathrm{~B}$ & $173.6(8)$ \\
\hline $\mathrm{C} 41 \mathrm{~B}-\mathrm{C} 40 \mathrm{~B}-\mathrm{C} 54 \mathrm{~B}-\mathrm{C} 82 \mathrm{~B}$ & $-68.2(10)$ \\
\hline $\mathrm{C} 41 \mathrm{~B}-\mathrm{C} 40 \mathrm{~B}-\mathrm{C} 54 \mathrm{~B}-\mathrm{C} 88 \mathrm{~B}$ & $54.4(10)$ \\
\hline $\mathrm{C} 41 \mathrm{~B}-\mathrm{C} 42 \mathrm{~B}-\mathrm{C} 43 \mathrm{~B}-\mathrm{N} 3 \mathrm{~B}$ & $-174.1(8)$ \\
\hline $\mathrm{C} 41 \mathrm{~B}-\mathrm{C} 42 \mathrm{~B}-\mathrm{C} 43 \mathrm{~B}-\mathrm{C} 44 \mathrm{~B}$ & $6.6(12)$ \\
\hline $\mathrm{C} 42 \mathrm{~B}-\mathrm{C} 37 \mathrm{~B}-\mathrm{C} 38 \mathrm{~B}-\mathrm{C} 39 \mathrm{~B}$ & $2.7(11)$ \\
\hline $\mathrm{C} 42 \mathrm{~B}-\mathrm{C} 43 \mathrm{~B}-\mathrm{C} 44 \mathrm{~B}-\mathrm{C} 45 \mathrm{~B}$ & $-177.2(7)$ \\
\hline $\mathrm{C} 43 \mathrm{~B}-\mathrm{N} 3 \mathrm{~B}-\mathrm{C} 47 \mathrm{~B}-\mathrm{C} 46 \mathrm{~B}$ & $0.4(19)$ \\
\hline $\mathrm{C} 43 \mathrm{~B}-\mathrm{C} 44 \mathrm{~B}-\mathrm{C} 45 \mathrm{~B}-\mathrm{C} 46 \mathrm{~B}$ & $-2.0(12)$ \\
\hline $\mathrm{C} 44 \mathrm{~B}-\mathrm{C} 45 \mathrm{~B}-\mathrm{C} 46 \mathrm{~B}-\mathrm{O} 3 \mathrm{~B}$ & $179.8(7)$ \\
\hline $\mathrm{C} 44 \mathrm{~B}-\mathrm{C} 45 \mathrm{~B}-\mathrm{C} 46 \mathrm{~B}-\mathrm{C} 47 \mathrm{~B}$ & $-0.3(13)$ \\
\hline
\end{tabular}




\begin{tabular}{|c|c|}
\hline $\mathrm{C} 26-\mathrm{C} 27-\mathrm{C} 28-\mathrm{O} 2$ & $179.8(3)$ \\
\hline $\mathrm{C} 26-\mathrm{C} 27-\mathrm{C} 28-\mathrm{C} 29$ & $0.5(5)$ \\
\hline $\mathrm{C} 27-\mathrm{C} 28-\mathrm{C} 29-\mathrm{N} 2$ & $-1.6(5)$ \\
\hline $\mathrm{C} 29-\mathrm{N} 2-\mathrm{C} 25-\mathrm{C} 24$ & $-177.3(3)$ \\
\hline $\mathrm{C} 29-\mathrm{N} 2-\mathrm{C} 25-\mathrm{C} 26$ & $0.7(5)$ \\
\hline $\mathrm{C} 30-\mathrm{C} 21-\mathrm{C} 22-\mathrm{C} 23$ & $175.4(3)$ \\
\hline $\mathrm{C} 30-\mathrm{C} 21-\mathrm{C} 22-\mathrm{C} 36$ & $-3.0(4)$ \\
\hline $\mathrm{C} 30-\mathrm{C} 31-\mathrm{C} 32-\mathrm{C} 33$ & $0.1(6)$ \\
\hline $\mathrm{C} 30-\mathrm{C} 35-\mathrm{C} 36-\mathrm{C} 22$ & $-2.9(4)$ \\
\hline $\mathrm{C} 30-\mathrm{C} 35-\mathrm{C} 36-\mathrm{C} 75$ & $-123.5(4)$ \\
\hline $\mathrm{C} 30-\mathrm{C} 35-\mathrm{C} 36-\mathrm{C} 69$ & $123.7(5)$ \\
\hline $\mathrm{C} 30-\mathrm{C} 35-\mathrm{C} 36-\mathrm{C} 69 \mathrm{~B}$ & $108.0(5)$ \\
\hline $\mathrm{C} 31-\mathrm{C} 30-\mathrm{C} 35-\mathrm{C} 34$ & $0.8(6)$ \\
\hline $\mathrm{C} 31-\mathrm{C} 30-\mathrm{C} 35-\mathrm{C} 36$ & $178.5(3)$ \\
\hline $\mathrm{C} 31-\mathrm{C} 32-\mathrm{C} 33-\mathrm{C} 34$ & $1.1(6)$ \\
\hline $\mathrm{C} 32-\mathrm{C} 33-\mathrm{C} 34-\mathrm{C} 35$ & $-1.3(6)$ \\
\hline $\mathrm{C} 33-\mathrm{C} 34-\mathrm{C} 35-\mathrm{C} 30$ & $0.4(6)$ \\
\hline $\mathrm{C} 33-\mathrm{C} 34-\mathrm{C} 35-\mathrm{C} 36$ & $-176.9(4)$ \\
\hline $\mathrm{C} 34-\mathrm{C} 35-\mathrm{C} 36-\mathrm{C} 22$ & $174.6(4)$ \\
\hline $\mathrm{C} 34-\mathrm{C} 35-\mathrm{C} 36-\mathrm{C} 75$ & $53.9(5)$ \\
\hline $\mathrm{C} 34-\mathrm{C} 35-\mathrm{C} 36-\mathrm{C} 69$ & $-58.8(7)$ \\
\hline $\mathrm{C} 34-\mathrm{C} 35-\mathrm{C} 36-\mathrm{C} 69 \mathrm{~B}$ & $-74.5(6)$ \\
\hline $\mathrm{C} 35-\mathrm{C} 30-\mathrm{C} 31-\mathrm{C} 32$ & $-1.0(5)$ \\
\hline $\mathrm{C} 35-\mathrm{C} 36-\mathrm{C} 75-\mathrm{C} 76$ & $58.0(5)$ \\
\hline $\mathrm{C} 35-\mathrm{C} 36-\mathrm{C} 69-\mathrm{C} 70$ & $-53.9(9)$ \\
\hline $\mathrm{C} 35-\mathrm{C} 36-\mathrm{C} 69 \mathrm{~B}-\mathrm{C} 70 \mathrm{~B}$ & $-60.7(10)$ \\
\hline $\mathrm{C} 36-\mathrm{C} 22-\mathrm{C} 23-\mathrm{C} 24$ & $177.5(4)$ \\
\hline $\mathrm{C} 36-\mathrm{C} 75-\mathrm{C} 76-\mathrm{C} 77 \mathrm{~B}$ & $178.7(6)$ \\
\hline $\mathrm{C} 36-\mathrm{C} 75-\mathrm{C} 76-\mathrm{C} 77$ & $-177.1(5)$ \\
\hline $\mathrm{C} 36-\mathrm{C} 69-\mathrm{C} 70-\mathrm{C} 71$ & $-176.9(8)$ \\
\hline $\mathrm{C} 36-\mathrm{C} 69 \mathrm{~B}-\mathrm{C} 70 \mathrm{~B}-\mathrm{C} 71 \mathrm{~B}$ & $165.7(8)$ \\
\hline $\mathrm{C} 68-\mathrm{O} 2-\mathrm{C} 28-\mathrm{C} 27$ & $10.9(6)$ \\
\hline $\mathrm{C} 68-\mathrm{O} 2-\mathrm{C} 28-\mathrm{C} 29$ & $-169.8(3)$ \\
\hline $\mathrm{C} 75-\mathrm{C} 36-\mathrm{C} 69-\mathrm{C} 70$ & $-174.6(7)$ \\
\hline $\mathrm{C} 75-\mathrm{C} 36-\mathrm{C} 69 \mathrm{~B}-\mathrm{C} 70 \mathrm{~B}$ & $175.3(8)$ \\
\hline $\mathrm{C} 75-\mathrm{C} 76-\mathrm{C} 77 \mathrm{~B}-\mathrm{C} 78 \mathrm{~B}$ & $-179.0(6)$ \\
\hline $\mathrm{C} 75-\mathrm{C} 76-\mathrm{C} 77-\mathrm{C} 78$ & $-48.0(11)$ \\
\hline $\mathrm{C} 76-\mathrm{C} 77 \mathrm{~B}-\mathrm{C} 78 \mathrm{~B}-\mathrm{C} 79 \mathrm{~B}$ & $162.1(7)$ \\
\hline $\mathrm{C} 76-\mathrm{C} 77-\mathrm{C} 78-\mathrm{C} 79$ & $-169.4(8)$ \\
\hline $\mathrm{O} 1-\mathrm{C} 10-\mathrm{C} 11-\mathrm{N} 1$ & $170.5(6)$ \\
\hline $\mathrm{C} 1-\mathrm{C} 2-\mathrm{C} 3-\mathrm{C} 4$ & $-2.1(13)$ \\
\hline $\mathrm{C} 1-\mathrm{C} 2-\mathrm{C} 3-\mathrm{C} 12$ & $177.5(8)$ \\
\hline $\mathrm{C} 2-\mathrm{C} 1-\mathrm{C} 6-\mathrm{C} 7$ & $-173.3(7)$ \\
\hline $\mathrm{C} 2-\mathrm{C} 1-\mathrm{C} 6-\mathrm{C} 5$ & $-3.9(13)$ \\
\hline $\mathrm{C} 2-\mathrm{C} 3-\mathrm{C} 4-\mathrm{C} 5$ & $0.4(13)$ \\
\hline $\mathrm{C} 2-\mathrm{C} 3-\mathrm{C} 4-\mathrm{C} 18$ & $179.1(7)$ \\
\hline $\mathrm{C} 2-\mathrm{C} 3-\mathrm{C} 12-\mathrm{C} 13$ & $0.9(16)$ \\
\hline $\mathrm{C} 2-\mathrm{C} 3-\mathrm{C} 12-\mathrm{C} 17$ & $-177.8(8)$ \\
\hline
\end{tabular}

\begin{tabular}{|c|c|}
\hline $\mathrm{C} 45 \mathrm{~B}-\mathrm{C} 46 \mathrm{~B}-\mathrm{C} 47 \mathrm{~B}-\mathrm{N} 3 \mathrm{~B}$ & $1.2(18)$ \\
\hline $\mathrm{C} 47 \mathrm{~B}-\mathrm{N} 3 \mathrm{~B}-\mathrm{C} 43 \mathrm{~B}-\mathrm{C} 42 \mathrm{~B}$ & $178.0(10)$ \\
\hline $\mathrm{C} 47 \mathrm{~B}-\mathrm{N} 3 \mathrm{~B}-\mathrm{C} 43 \mathrm{~B}-\mathrm{C} 44 \mathrm{~B}$ & $-2.7(14)$ \\
\hline $\mathrm{C} 48 \mathrm{~B}-\mathrm{C} 39 \mathrm{~B}-\mathrm{C} 40 \mathrm{~B}-\mathrm{C} 41 \mathrm{~B}$ & $-175.6(7)$ \\
\hline $\mathrm{C} 48 \mathrm{~B}-\mathrm{C} 39 \mathrm{~B}-\mathrm{C} 40 \mathrm{~B}-\mathrm{C} 54 \mathrm{~B}$ & $1.4(9)$ \\
\hline $\mathrm{C} 48 \mathrm{~B}-\mathrm{C} 49 \mathrm{~B}-\mathrm{C} 50 \mathrm{~B}-\mathrm{C} 51 \mathrm{~B}$ & $-2.0(12)$ \\
\hline $\mathrm{C} 48 \mathrm{~B}-\mathrm{C} 53 \mathrm{~B}-\mathrm{C} 54 \mathrm{~B}-\mathrm{C} 40 \mathrm{~B}$ & $4.0(8)$ \\
\hline $\mathrm{C} 48 \mathrm{~B}-\mathrm{C} 53 \mathrm{~B}-\mathrm{C} 54 \mathrm{~B}-\mathrm{C} 82 \mathrm{~B}$ & $-114.6(7)$ \\
\hline $\mathrm{C} 48 \mathrm{~B}-\mathrm{C} 53 \mathrm{~B}-\mathrm{C} 54 \mathrm{~B}-\mathrm{C} 88 \mathrm{~B}$ & $124.1(7)$ \\
\hline $\mathrm{C} 49 \mathrm{~B}-\mathrm{C} 48 \mathrm{~B}-\mathrm{C} 53 \mathrm{~B}-\mathrm{C} 52 \mathrm{~B}$ & $-2.4(12)$ \\
\hline $\mathrm{C} 49 \mathrm{~B}-\mathrm{C} 48 \mathrm{~B}-\mathrm{C} 53 \mathrm{~B}-\mathrm{C} 54 \mathrm{~B}$ & $177.5(7)$ \\
\hline $\mathrm{C} 49 \mathrm{~B}-\mathrm{C} 50 \mathrm{~B}-\mathrm{C} 51 \mathrm{~B}-\mathrm{C} 52 \mathrm{~B}$ & $-0.7(13)$ \\
\hline $\mathrm{C} 50 \mathrm{~B}-\mathrm{C} 51 \mathrm{~B}-\mathrm{C} 52 \mathrm{~B}-\mathrm{C} 53 \mathrm{~B}$ & $1.8(12)$ \\
\hline $\mathrm{C} 51 \mathrm{~B}-\mathrm{C} 52 \mathrm{~B}-\mathrm{C} 53 \mathrm{~B}-\mathrm{C} 48 \mathrm{~B}$ & $-0.2(11)$ \\
\hline $\mathrm{C} 51 \mathrm{~B}-\mathrm{C} 52 \mathrm{~B}-\mathrm{C} 53 \mathrm{~B}-\mathrm{C} 54 \mathrm{~B}$ & $179.9(8)$ \\
\hline $\mathrm{C} 52 \mathrm{~B}-\mathrm{C} 53 \mathrm{~B}-\mathrm{C} 54 \mathrm{~B}-\mathrm{C} 40 \mathrm{~B}$ & $-176.0(8)$ \\
\hline $\mathrm{C} 52 \mathrm{~B}-\mathrm{C} 53 \mathrm{~B}-\mathrm{C} 54 \mathrm{~B}-\mathrm{C} 82 \mathrm{~B}$ & $65.3(10)$ \\
\hline $\mathrm{C} 52 \mathrm{~B}-\mathrm{C} 53 \mathrm{~B}-\mathrm{C} 54 \mathrm{~B}-\mathrm{C} 88 \mathrm{~B}$ & $-55.9(11)$ \\
\hline $\mathrm{C} 53 \mathrm{~B}-\mathrm{C} 48 \mathrm{~B}-\mathrm{C} 49 \mathrm{~B}-\mathrm{C} 50 \mathrm{~B}$ & $3.5(12)$ \\
\hline $\mathrm{C} 53 \mathrm{~B}-\mathrm{C} 54 \mathrm{~B}-\mathrm{C} 82 \mathrm{~B}-\mathrm{C} 83 \mathrm{~B}$ & $46.7(10)$ \\
\hline $\mathrm{C} 53 \mathrm{~B}-\mathrm{C} 54 \mathrm{~B}-\mathrm{C} 88 \mathrm{~B}-\mathrm{C} 89 \mathrm{~B}$ & $-62.1(9)$ \\
\hline $\mathrm{C} 54 \mathrm{~B}-\mathrm{C} 40 \mathrm{~B}-\mathrm{C} 41 \mathrm{~B}-\mathrm{C} 42 \mathrm{~B}$ & $-174.6(7)$ \\
\hline $\mathrm{C} 54 \mathrm{~B}-\mathrm{C} 82 \mathrm{~B}-\mathrm{C} 83 \mathrm{~B}-\mathrm{C} 84 \mathrm{~B}$ & $169.2(8)$ \\
\hline $\mathrm{C} 54 \mathrm{~B}-\mathrm{C} 88 \mathrm{~B}-\mathrm{C} 89 \mathrm{~B}-\mathrm{C} 90 \mathrm{~B}$ & $173.3(6)$ \\
\hline $\mathrm{C} 81 \mathrm{~B}-\mathrm{O} 3 \mathrm{~B}-\mathrm{C} 46 \mathrm{~B}-\mathrm{C} 45 \mathrm{~B}$ & $5.1(12)$ \\
\hline $\mathrm{C} 81 \mathrm{~B}-\mathrm{O} 3 \mathrm{~B}-\mathrm{C} 46 \mathrm{~B}-\mathrm{C} 47 \mathrm{~B}$ & $-174.8(9)$ \\
\hline $\mathrm{C} 82 \mathrm{~B}-\mathrm{C} 54 \mathrm{~B}-\mathrm{C} 88 \mathrm{~B}-\mathrm{C} 89 \mathrm{~B}$ & $174.8(6)$ \\
\hline $\mathrm{C} 82 \mathrm{~B}-\mathrm{C} 83 \mathrm{~B}-\mathrm{C} 84 \mathrm{~B}-\mathrm{C} 85 \mathrm{~B}$ & $-172.4(10)$ \\
\hline $\mathrm{C} 83 \mathrm{~B}-\mathrm{C} 84 \mathrm{~B}-\mathrm{C} 85 \mathrm{~B}-\mathrm{C} 86 \mathrm{~B}$ & $175.2(10)$ \\
\hline $\mathrm{C} 84 \mathrm{~B}-\mathrm{C} 85 \mathrm{~B}-\mathrm{C} 86 \mathrm{~B}-\mathrm{C} 87 \mathrm{~B}$ & $-98.5(12)$ \\
\hline $\mathrm{C} 88 \mathrm{~B}-\mathrm{C} 54 \mathrm{~B}-\mathrm{C} 82 \mathrm{~B}-\mathrm{C} 83 \mathrm{~B}$ & $170.1(8)$ \\
\hline $\mathrm{C} 88 \mathrm{~B}-\mathrm{C} 89 \mathrm{~B}-\mathrm{C} 90 \mathrm{~B}-\mathrm{C} 91 \mathrm{~B}$ & $-178.8(6)$ \\
\hline $\mathrm{C} 89 \mathrm{~B}-\mathrm{C} 90 \mathrm{~B}-\mathrm{C} 91 \mathrm{~B}-\mathrm{C} 92 \mathrm{~B}$ & $177.5(8)$ \\
\hline $\mathrm{C} 90 \mathrm{~B}-\mathrm{C} 91 \mathrm{~B}-\mathrm{C} 92 \mathrm{~B}-\mathrm{C} 93 \mathrm{~B}$ & $-148.6(7)$ \\
\hline $\mathrm{O} 3-\mathrm{C} 46-\mathrm{C} 47-\mathrm{N} 3$ & $178.5(11)$ \\
\hline $\mathrm{N} 3-\mathrm{C} 43-\mathrm{C} 44-\mathrm{C} 45$ & $-0.9(11)$ \\
\hline $\mathrm{C} 37-\mathrm{C} 38-\mathrm{C} 39-\mathrm{C} 40$ & $1.7(11)$ \\
\hline $\mathrm{C} 37-\mathrm{C} 38-\mathrm{C} 39-\mathrm{C} 48$ & $-178.8(8)$ \\
\hline $\mathrm{C} 37-\mathrm{C} 42-\mathrm{C} 43-\mathrm{N} 3$ & $2.8(11)$ \\
\hline $\mathrm{C} 37-\mathrm{C} 42-\mathrm{C} 43-\mathrm{C} 44$ & $-174.1(7)$ \\
\hline $\mathrm{C} 38-\mathrm{C} 37-\mathrm{C} 42-\mathrm{C} 41$ & $-3.2(11)$ \\
\hline $\mathrm{C} 38-\mathrm{C} 37-\mathrm{C} 42-\mathrm{C} 43$ & $174.4(7)$ \\
\hline $\mathrm{C} 38-\mathrm{C} 39-\mathrm{C} 40-\mathrm{C} 41$ & $-3.0(11)$ \\
\hline $\mathrm{C} 38-\mathrm{C} 39-\mathrm{C} 40-\mathrm{C} 54$ & $177.7(7)$ \\
\hline $\mathrm{C} 38-\mathrm{C} 39-\mathrm{C} 48-\mathrm{C} 49$ & $-0.4(14)$ \\
\hline $\mathrm{C} 38-\mathrm{C} 39-\mathrm{C} 48-\mathrm{C} 53$ & $-179.3(8)$ \\
\hline $\mathrm{C} 39-\mathrm{C} 40-\mathrm{C} 41-\mathrm{C} 42$ & $1.1(11)$ \\
\hline $\mathrm{C} 39-\mathrm{C} 40-\mathrm{C} 54-\mathrm{C} 53$ & $2.7(8)$ \\
\hline
\end{tabular}




\begin{tabular}{|c|c|}
\hline $\mathrm{C} 3-\mathrm{C} 4-\mathrm{C} 5-\mathrm{C} 6$ & $-0.4(12)$ \\
\hline $\mathrm{C} 3-\mathrm{C} 4-\mathrm{C} 18-\mathrm{C} 17$ & $-0.8(9)$ \\
\hline $\mathrm{C} 3-\mathrm{C} 4-\mathrm{C} 18-\mathrm{C} 56$ & $118.5(8)$ \\
\hline $\mathrm{C} 3-\mathrm{C} 4-\mathrm{C} 18-\mathrm{C} 62$ & $-119.2(7)$ \\
\hline $\mathrm{C} 3-\mathrm{C} 12-\mathrm{C} 13-\mathrm{C} 14$ & $-179.5(8)$ \\
\hline $\mathrm{C} 3-\mathrm{C} 12-\mathrm{C} 17-\mathrm{C} 16$ & $178.2(7)$ \\
\hline $\mathrm{C} 3-\mathrm{C} 12-\mathrm{C} 17-\mathrm{C} 18$ & $-2.4(10)$ \\
\hline $\mathrm{C} 4-\mathrm{C} 3-\mathrm{C} 12-\mathrm{C} 13$ & $-179.5(9)$ \\
\hline $\mathrm{C} 4-\mathrm{C} 3-\mathrm{C} 12-\mathrm{C} 17$ & $1.8(10)$ \\
\hline $\mathrm{C} 4-\mathrm{C} 5-\mathrm{C} 6-\mathrm{C} 7$ & $171.9(6)$ \\
\hline $\mathrm{C} 4-\mathrm{C} 5-\mathrm{C} 6-\mathrm{C} 1$ & $2.3(12)$ \\
\hline $\mathrm{C} 4-\mathrm{C} 18-\mathrm{C} 56-\mathrm{C} 57$ & $-63.4(8)$ \\
\hline $\mathrm{C} 4-\mathrm{C} 18-\mathrm{C} 62-\mathrm{C} 63$ & $56.0(9)$ \\
\hline $\mathrm{C} 5-\mathrm{C} 4-\mathrm{C} 18-\mathrm{C} 17$ & $177.8(8)$ \\
\hline $\mathrm{C} 5-\mathrm{C} 4-\mathrm{C} 18-\mathrm{C} 56$ & $-62.9(11)$ \\
\hline $\mathrm{C} 5-\mathrm{C} 4-\mathrm{C} 18-\mathrm{C} 62$ & $59.4(11)$ \\
\hline $\mathrm{C} 6-\mathrm{C} 7-\mathrm{C} 8-\mathrm{C} 9$ & $168.9(5)$ \\
\hline $\mathrm{C} 6-\mathrm{C} 1-\mathrm{C} 2-\mathrm{C} 3$ & $3.8(13)$ \\
\hline $\mathrm{C} 12-\mathrm{C} 3-\mathrm{C} 4-\mathrm{C} 5$ & $-179.3(7)$ \\
\hline $\mathrm{C} 12-\mathrm{C} 3-\mathrm{C} 4-\mathrm{C} 18$ & $-0.6(10)$ \\
\hline $\mathrm{C} 12-\mathrm{C} 13-\mathrm{C} 14-\mathrm{C} 15$ & $2.8(13)$ \\
\hline $\mathrm{C} 12-\mathrm{C} 17-\mathrm{C} 18-\mathrm{C} 4$ & $2.0(9)$ \\
\hline $\mathrm{C} 12-\mathrm{C} 17-\mathrm{C} 18-\mathrm{C} 56$ & $-116.1(8)$ \\
\hline $\mathrm{C} 12-\mathrm{C} 17-\mathrm{C} 18-\mathrm{C} 62$ & $119.4(7)$ \\
\hline $\mathrm{C} 13-\mathrm{C} 12-\mathrm{C} 17-\mathrm{C} 16$ & $-0.7(13)$ \\
\hline $\mathrm{C} 13-\mathrm{C} 12-\mathrm{C} 17-\mathrm{C} 18$ & $178.7(7)$ \\
\hline $\mathrm{C} 13-\mathrm{C} 14-\mathrm{C} 15-\mathrm{C} 16$ & $-3.2(13)$ \\
\hline $\mathrm{C} 14-\mathrm{C} 15-\mathrm{C} 16-\mathrm{C} 17$ & $1.6(13)$ \\
\hline $\mathrm{C} 15-\mathrm{C} 16-\mathrm{C} 17-\mathrm{C} 12$ & $0.3(12)$ \\
\hline $\mathrm{C} 15-\mathrm{C} 16-\mathrm{C} 17-\mathrm{C} 18$ & $-178.9(8)$ \\
\hline $\mathrm{C} 16-\mathrm{C} 17-\mathrm{C} 18-\mathrm{C} 4$ & $-178.7(8)$ \\
\hline $\mathrm{C} 16-\mathrm{C} 17-\mathrm{C} 18-\mathrm{C} 56$ & $63.3(11)$ \\
\hline $\mathrm{C} 16-\mathrm{C} 17-\mathrm{C} 18-\mathrm{C} 62$ & $-61.3(11)$ \\
\hline $\mathrm{C} 17-\mathrm{C} 12-\mathrm{C} 13-\mathrm{C} 14$ & $-0.9(13)$ \\
\hline $\mathrm{C} 17-\mathrm{C} 18-\mathrm{C} 56-\mathrm{C} 57$ & $48.0(9)$ \\
\hline $\mathrm{C} 17-\mathrm{C} 18-\mathrm{C} 62-\mathrm{C} 63$ & $-54.9(9)$ \\
\hline $\mathrm{C} 18-\mathrm{C} 4-\mathrm{C} 5-\mathrm{C} 6$ & $-178.9(8)$ \\
\hline $\mathrm{C} 18-\mathrm{C} 56-\mathrm{C} 57-\mathrm{C} 58$ & $169.7(6)$ \\
\hline $\mathrm{C} 18-\mathrm{C} 62-\mathrm{C} 63-\mathrm{C} 64$ & $-174.2(6)$ \\
\hline $\mathrm{C} 56-\mathrm{C} 18-\mathrm{C} 62-\mathrm{C} 63$ & $179.0(7)$ \\
\hline $\mathrm{C} 56-\mathrm{C} 57-\mathrm{C} 58-\mathrm{C} 59$ & $-175.2(7)$ \\
\hline $\mathrm{C} 57-\mathrm{C} 58-\mathrm{C} 59-\mathrm{C} 60$ & $-64.6(10)$ \\
\hline $\mathrm{C} 58-\mathrm{C} 59-\mathrm{C} 60-\mathrm{C} 61$ & $89.6(9)$ \\
\hline $\mathrm{C} 62-\mathrm{C} 18-\mathrm{C} 56-\mathrm{C} 57$ & $173.8(6)$ \\
\hline $\mathrm{C} 62-\mathrm{C} 63-\mathrm{C} 64-\mathrm{C} 65$ & $-64.3(11)$ \\
\hline $\mathrm{C} 63-\mathrm{C} 64-\mathrm{C} 65-\mathrm{C} 66$ & $-63.7(12)$ \\
\hline $\mathrm{C} 64-\mathrm{C} 65-\mathrm{C} 66-\mathrm{C} 67$ & $-171.8(8)$ \\
\hline $\mathrm{C} 69-\mathrm{C} 36-\mathrm{C} 75-\mathrm{C} 76$ & $-174.7(5)$ \\
\hline
\end{tabular}

\begin{tabular}{|c|c|}
\hline $\mathrm{C} 39-\mathrm{C} 40-\mathrm{C} 54-\mathrm{C} 82$ & $122.3(7)$ \\
\hline $\mathrm{C} 39-\mathrm{C} 40-\mathrm{C} 54-\mathrm{C} 88$ & $-113.4(8)$ \\
\hline $\mathrm{C} 39-\mathrm{C} 48-\mathrm{C} 49-\mathrm{C} 50$ & $-179.0(8)$ \\
\hline $\mathrm{C} 39-\mathrm{C} 48-\mathrm{C} 53-\mathrm{C} 52$ & $179.6(7)$ \\
\hline $\mathrm{C} 39-\mathrm{C} 48-\mathrm{C} 53-\mathrm{C} 54$ & $1.5(9)$ \\
\hline $\mathrm{C} 40-\mathrm{C} 39-\mathrm{C} 48-\mathrm{C} 49$ & $179.2(8)$ \\
\hline $\mathrm{C} 40-\mathrm{C} 39-\mathrm{C} 48-\mathrm{C} 53$ & $0.3(9)$ \\
\hline $\mathrm{C} 40-\mathrm{C} 41-\mathrm{C} 42-\mathrm{C} 37$ & $2.0(11)$ \\
\hline $\mathrm{C} 40-\mathrm{C} 41-\mathrm{C} 42-\mathrm{C} 43$ & $-175.5(7)$ \\
\hline $\mathrm{C} 40-\mathrm{C} 54-\mathrm{C} 82-\mathrm{C} 83$ & $-62.8(9)$ \\
\hline $\mathrm{C} 40-\mathrm{C} 54-\mathrm{C} 88-\mathrm{C} 89$ & $61.0(10)$ \\
\hline $\mathrm{C} 41-\mathrm{C} 40-\mathrm{C} 54-\mathrm{C} 53$ & $-176.7(8)$ \\
\hline $\mathrm{C} 41-\mathrm{C} 40-\mathrm{C} 54-\mathrm{C} 82$ & $-57.0(11)$ \\
\hline $\mathrm{C} 41-\mathrm{C} 40-\mathrm{C} 54-\mathrm{C} 88$ & $67.2(11)$ \\
\hline $\mathrm{C} 41-\mathrm{C} 42-\mathrm{C} 43-\mathrm{N} 3$ & $-179.6(8)$ \\
\hline $\mathrm{C} 41-\mathrm{C} 42-\mathrm{C} 43-\mathrm{C} 44$ & $3.5(12)$ \\
\hline $\mathrm{C} 42-\mathrm{C} 37-\mathrm{C} 38-\mathrm{C} 39$ & $1.3(11)$ \\
\hline $\mathrm{C} 42-\mathrm{C} 43-\mathrm{C} 44-\mathrm{C} 45$ & $176.0(7)$ \\
\hline $\mathrm{C} 43-\mathrm{N} 3-\mathrm{C} 47-\mathrm{C} 46$ & $1(2)$ \\
\hline $\mathrm{C} 43-\mathrm{C} 44-\mathrm{C} 45-\mathrm{C} 46$ & $-0.3(11)$ \\
\hline $\mathrm{C} 44-\mathrm{C} 45-\mathrm{C} 46-\mathrm{O} 3$ & $-179.0(8)$ \\
\hline $\mathrm{C} 44-\mathrm{C} 45-\mathrm{C} 46-\mathrm{C} 47$ & $1.8(13)$ \\
\hline $\mathrm{C} 45-\mathrm{C} 46-\mathrm{C} 47-\mathrm{N} 3$ & $-2.1(19)$ \\
\hline $\mathrm{C} 47-\mathrm{N} 3-\mathrm{C} 43-\mathrm{C} 42$ & $-176.6(11)$ \\
\hline $\mathrm{C} 47-\mathrm{N} 3-\mathrm{C} 43-\mathrm{C} 44$ & $0.6(15)$ \\
\hline $\mathrm{C} 48-\mathrm{C} 39-\mathrm{C} 40-\mathrm{C} 41$ & $177.4(7)$ \\
\hline $\mathrm{C} 48-\mathrm{C} 39-\mathrm{C} 40-\mathrm{C} 54$ & $-2.0(9)$ \\
\hline $\mathrm{C} 48-\mathrm{C} 49-\mathrm{C} 50-\mathrm{C} 51$ & $1.1(12)$ \\
\hline $\mathrm{C} 48-\mathrm{C} 53-\mathrm{C} 54-\mathrm{C} 40$ & $-2.5(9)$ \\
\hline $\mathrm{C} 48-\mathrm{C} 53-\mathrm{C} 54-\mathrm{C} 82$ & $-121.1(7)$ \\
\hline $\mathrm{C} 48-\mathrm{C} 53-\mathrm{C} 54-\mathrm{C} 88$ & $114.9(7)$ \\
\hline $\mathrm{C} 49-\mathrm{C} 48-\mathrm{C} 53-\mathrm{C} 52$ & $0.6(12)$ \\
\hline $\mathrm{C} 49-\mathrm{C} 48-\mathrm{C} 53-\mathrm{C} 54$ & $-177.5(7)$ \\
\hline $\mathrm{C} 49-\mathrm{C} 50-\mathrm{C} 51-\mathrm{C} 52$ & $-2.2(13)$ \\
\hline $\mathrm{C} 50-\mathrm{C} 51-\mathrm{C} 52-\mathrm{C} 53$ & $2.5(14)$ \\
\hline $\mathrm{C} 51-\mathrm{C} 52-\mathrm{C} 53-\mathrm{C} 48$ & $-1.7(13)$ \\
\hline $\mathrm{C} 51-\mathrm{C} 52-\mathrm{C} 53-\mathrm{C} 54$ & $176.1(8)$ \\
\hline $\mathrm{C} 52-\mathrm{C} 53-\mathrm{C} 54-\mathrm{C} 40$ & $179.6(8)$ \\
\hline $\mathrm{C} 52-\mathrm{C} 53-\mathrm{C} 54-\mathrm{C} 82$ & $61.0(11)$ \\
\hline $\mathrm{C} 52-\mathrm{C} 53-\mathrm{C} 54-\mathrm{C} 88$ & $-63.0(11)$ \\
\hline $\mathrm{C} 53-\mathrm{C} 48-\mathrm{C} 49-\mathrm{C} 50$ & $-0.3(12)$ \\
\hline $\mathrm{C} 53-\mathrm{C} 54-\mathrm{C} 82-\mathrm{C} 83$ & $49.3(9)$ \\
\hline $\mathrm{C} 53-\mathrm{C} 54-\mathrm{C} 88-\mathrm{C} 89$ & $-49.3(9)$ \\
\hline $\mathrm{C} 54-\mathrm{C} 40-\mathrm{C} 41-\mathrm{C} 42$ & $-179.6(7)$ \\
\hline $\mathrm{C} 54-\mathrm{C} 82-\mathrm{C} 83-\mathrm{C} 84$ & $163.0(7)$ \\
\hline $\mathrm{C} 54-\mathrm{C} 88-\mathrm{C} 89-\mathrm{C} 90$ & $124.3(9)$ \\
\hline $\mathrm{C} 77-\mathrm{C} 78-\mathrm{C} 79-\mathrm{C} 80$ & $178.7(8)$ \\
\hline $\mathrm{C} 81-\mathrm{O} 3-\mathrm{C} 46-\mathrm{C} 45$ & $1.0(13)$ \\
\hline
\end{tabular}




$\begin{array}{ll}\mathrm{C} 69-\mathrm{C} 70-\mathrm{C} 71-\mathrm{C} 72 & -172.5(10) \\ \mathrm{C} 70-\mathrm{C} 71-\mathrm{C} 72-\mathrm{C} 73 & -174.8(10) \\ \mathrm{C} 71-\mathrm{C} 72-\mathrm{C} 73-\mathrm{C} 74 & -170.1(11) \\ \mathrm{O} 1 \mathrm{~B}-\mathrm{C} 10-\mathrm{C} 11-\mathrm{N} 1 & -169.6(5) \\ \mathrm{C} 1 \mathrm{~B}-\mathrm{C} 2 \mathrm{~B}-\mathrm{C} 3 \mathrm{~B}-\mathrm{C} 4 \mathrm{~B} & 2.0(13) \\ \mathrm{C} 1 \mathrm{~B}-\mathrm{C} 2 \mathrm{~B}-\mathrm{C} 3 \mathrm{~B}-\mathrm{C} 12 \mathrm{~B} & -178.3(9) \\ \mathrm{C} 2 \mathrm{~B}-\mathrm{C} 1 \mathrm{~B}-\mathrm{C} 6 \mathrm{~B}-\mathrm{C} 7 & 170.5(7) \\ \mathrm{C} 2 \mathrm{~B}-\mathrm{C} 1 \mathrm{~B}-\mathrm{C} 6 \mathrm{~B}-\mathrm{C} 5 \mathrm{~B} & -0.5(13) \\ \mathrm{C} 2 \mathrm{~B}-\mathrm{C} 3 \mathrm{~B}-\mathrm{C} 4 \mathrm{~B}-\mathrm{C} 5 \mathrm{~B} & -2.4(13) \\ \mathrm{C} 2 \mathrm{~B}-\mathrm{C} 3 \mathrm{~B}-\mathrm{C} 4 \mathrm{~B}-\mathrm{C} 18 \mathrm{~B} & 177.8(7) \\ \mathrm{C} 2 \mathrm{~B}-\mathrm{C} 3 \mathrm{~B}-\mathrm{C} 12 \mathrm{~B}-\mathrm{C} 13 \mathrm{~B} & 1.8(16) \\ \mathrm{C} 2 \mathrm{~B}-\mathrm{C} 3 \mathrm{~B}-\mathrm{C} 12 \mathrm{~B}-\mathrm{C} 17 \mathrm{~B} & -177.1(9) \\ \mathrm{C} 3 \mathrm{~B}-\mathrm{C} 4 \mathrm{~B}-\mathrm{C} 5 \mathrm{~B}-\mathrm{C} 6 \mathrm{~B} & 1.3(12)\end{array}$

$\begin{array}{ll}\mathrm{C} 81-\mathrm{O} 3-\mathrm{C} 46-\mathrm{C} 47 & -179.7(10) \\ \mathrm{C} 82-\mathrm{C} 54-\mathrm{C} 88-\mathrm{C} 89 & -174.5(7) \\ \mathrm{C} 82-\mathrm{C} 83-\mathrm{C} 84-\mathrm{C} 85 & -178.9(8) \\ \mathrm{C} 83-\mathrm{C} 84-\mathrm{C} 85-\mathrm{C} 86 & 166.6(9) \\ \mathrm{C} 84-\mathrm{C} 85-\mathrm{C} 86-\mathrm{C} 87 & 174.7(9) \\ \mathrm{C} 88-\mathrm{C} 54-\mathrm{C} 82-\mathrm{C} 83 & 172.6(7) \\ \mathrm{C} 88-\mathrm{C} 89-\mathrm{C} 90-\mathrm{C} 91 & 149.7(10) \\ \mathrm{C} 89-\mathrm{C} 90-\mathrm{C} 91-\mathrm{C} 92 & -146.9(10) \\ \mathrm{C} 90-\mathrm{C} 91-\mathrm{C} 92-\mathrm{C} 93 & -131.9(12) \\ \mathrm{C} 1 \mathrm{~S}-\mathrm{C} 2 \mathrm{~S}-\mathrm{C} 3 \mathrm{~S}-\mathrm{C} 4 \mathrm{~S} & -169.3(10) \\ \mathrm{C} 1 \mathrm{~S}-\mathrm{C} 2 \mathrm{~S}^{\prime}-\mathrm{C} 3 \mathrm{~S}^{\prime}-\mathrm{C} 4 \mathrm{~S}^{\prime} & -169.0(13) \\ \mathrm{C} 2 \mathrm{~S}-\mathrm{C} 3 \mathrm{~S}-\mathrm{C} 4 \mathrm{~S}-\mathrm{C} 5 \mathrm{~S} & -143.5(11) \\ \mathrm{C} 2 \mathrm{~S}^{\prime}-\mathrm{C} 3 \mathrm{~S}^{\prime}-\mathrm{C} 4 \mathrm{~S}^{\prime}-\mathrm{C} 5 \mathrm{~S}^{\prime} & -84.0(17)\end{array}$

Di- $\mu_{2}$-chlorido-bis $\{b i s[2-(5$-fluoropyridin-2-yl)-9,9-dihexyl-9H-fluoren-3-yl]iridium\} pentane 0.3 -solvate (II)

Crystal data

$\left[\mathrm{Ir}_{2}\left(\mathrm{C}_{30} \mathrm{H}_{35} \mathrm{FN}\right)_{4} \mathrm{Cl}_{2}\right] \cdot 0.3 \mathrm{C}_{5} \mathrm{H}_{12}$

$M_{r}=2191.30$

Triclinic, $P \overline{1}$

$a=12.2744(7) \AA$

$b=17.6132(10) \AA$

$c=25.3966(15) \AA$

$\alpha=105.119(2)^{\circ}$

$\beta=93.787(2)^{\circ}$

$\gamma=90.779(2)^{\circ}$

$V=5286.2(5) \AA^{3}$

$Z=2$

$F(000)=2249$

$D_{\mathrm{x}}=1.377 \mathrm{Mg} \mathrm{m}^{-3}$

Mo $K \alpha$ radiation, $\lambda=0.71073 \AA$

Cell parameters from 5349 reflections

$\theta=2.3-25.3^{\circ}$

$\mu=2.62 \mathrm{~mm}^{-1}$

$T=120 \mathrm{~K}$

Block, orange

$0.14 \times 0.1 \times 0.04 \mathrm{~mm}$

Data collection

Bruker SMART CCD 6000

diffractometer

Radiation source: sealed X-ray tube

Graphite monochromator

Detector resolution: 5.6 pixels $\mathrm{mm}^{-1}$

$\omega$ scans

Absorption correction: integration

(SADABS; Krause et al., 2015)

$T_{\text {min }}=0.735, T_{\max }=0.927$

\section{Refinement}

Refinement on $F^{2}$

Least-squares matrix: full

$R\left[F^{2}>2 \sigma\left(F^{2}\right)\right]=0.041$

$w R\left(F^{2}\right)=0.103$

$S=0.95$

18623 reflections

1342 parameters

2905 restraints

Primary atom site location: structure-invariant direct methods
48264 measured reflections

18623 independent reflections

12096 reflections with $I>2 \sigma(I)$

$R_{\text {int }}=0.065$

$\theta_{\max }=25.0^{\circ}, \theta_{\min }=1.2^{\circ}$

$h=-14 \rightarrow 14$

$k=-20 \rightarrow 20$

$l=-30 \rightarrow 30$

Secondary atom site location: difference Fourier map

Hydrogen site location: mixed

$\mathrm{H}$-atom parameters constrained

$w=1 /\left[\sigma^{2}\left(F_{0}^{2}\right)+(0.0472 P)^{2}\right]$

where $P=\left(F_{\mathrm{o}}^{2}+2 F_{\mathrm{c}}{ }^{2}\right) / 3$

$(\Delta / \sigma)_{\max }=0.002$

$\Delta \rho_{\min }=-0.76 \mathrm{e}^{-3}$
$\Delta \rho_{\max }=1.39 \mathrm{e} \AA^{-3}$ 


\section{Special details}

Experimental. The data collection nominally covered full sphere of reciprocal space, by a combination of 4 runs of 600 and 1 run of 50 narrow-frame $\omega$-scans (scan width $0.3^{\circ} \omega, 40$ s exposure), every run at a different $\varphi$ and/or $2 \theta$ angle. Crystal to detector distance $4.84 \mathrm{~cm}$.

Geometry. All esds (except the esd in the dihedral angle between two 1.s. planes) are estimated using the full covariance matrix. The cell esds are taken into account individually in the estimation of esds in distances, angles and torsion angles; correlations between esds in cell parameters are only used when they are defined by crystal symmetry. An approximate (isotropic) treatment of cell esds is used for estimating esds involving l.s. planes.

Refinement. Intense disorder of n-hexyl chains, overlapping with the pentane molecule of crystallisation which has the occupancy of 0.3 .

Fractional atomic coordinates and isotropic or equivalent isotropic displacement parameters $\left(\AA^{2}\right)$

\begin{tabular}{|c|c|c|c|c|c|}
\hline & $x$ & $y$ & $z$ & $U_{\text {iso }} * / U_{\text {eq }}$ & Occ. $(<1)$ \\
\hline Ir1 & $0.64404(2)$ & $0.36376(2)$ & $0.19910(2)$ & $0.03102(8)$ & \\
\hline $\operatorname{Ir} 2$ & $0.54578(2)$ & $0.24992(2)$ & $0.29310(2)$ & $0.03270(8)$ & \\
\hline $\mathrm{Cl1}$ & $0.66078(13)$ & $0.36958(9)$ & $0.29922(6)$ & $0.0346(4)$ & \\
\hline $\mathrm{Cl} 2$ & 0.54538 (13) & $0.23529(9)$ & $0.19162(6)$ & $0.0365(4)$ & \\
\hline $\mathrm{F} 1$ & 0.2878 & $0.4982(2)$ & $0.31979(15)$ & $0.0502(10)$ & \\
\hline $\mathrm{F} 2$ & 0.8500 & 0.0463 & $0.21338(17)$ & $0.0788(15)$ & \\
\hline F3 & 0.2160 & $0.4031(2)$ & $0.16942(16)$ & $0.0569(11)$ & \\
\hline F4 & $0.9910(4)$ & 0.2071 & $0.24544(18)$ & $0.0780(15)$ & \\
\hline N1 & 0.4023 & 0.3079 & 0.29679 (19) & $0.0318(12)$ & \\
\hline $\mathrm{N} 2$ & $0.6790(4)$ & 0.1836 & $0.2967(2)$ & $0.0368(13)$ & \\
\hline N3 & $0.5051(4)$ & 0.4258 & $0.20334(18)$ & $0.0293(11)$ & \\
\hline N4 & $0.7826(4)$ & 0.3022 & $0.1836(2)$ & $0.0381(13)$ & \\
\hline $\mathrm{C} 1$ & $0.4431(5)$ & $0.1584(4)$ & $0.2827(3)$ & $0.0372(15)$ & \\
\hline $\mathrm{C} 2$ & $0.4671(5)$ & $0.0790(4)$ & $0.2742(2)$ & $0.0361(14)$ & \\
\hline $\mathrm{H} 2$ & 0.541059 & 0.063919 & 0.275666 & $0.043^{*}$ & \\
\hline $\mathrm{C} 3$ & $0.3836(5)$ & $0.0219(4)$ & $0.2637(2)$ & $0.0344(14)$ & \\
\hline $\mathrm{C} 4$ & $0.2739(5)$ & $0.0420(4)$ & 0.2615 & 0.0373 (14) & \\
\hline $\mathrm{C} 5$ & $0.2476(5)$ & 0.1200 & 0.2713 & $0.0414(16)$ & \\
\hline H5 & 0.173341 & 0.134399 & 0.270668 & $0.050^{*}$ & \\
\hline C6 & $0.3312(5)$ & $0.1777(4)$ & $0.2822(3)$ & $0.0396(15)$ & \\
\hline $\mathrm{C} 7$ & $0.3116(5)$ & $0.2606(4)$ & $0.2912(3)$ & $0.0396(15)$ & \\
\hline $\mathrm{C} 8$ & $0.2095(6)$ & $0.2952(4)$ & 0.2956 & 0.0517 (19) & \\
\hline H8 & 0.145714 & 0.262595 & 0.292166 & $0.062 *$ & \\
\hline C9 & $0.1989(6)$ & $0.3752(4)$ & 0.3049 (3) & 0.0533 (19) & \\
\hline H9 & 0.129545 & 0.398611 & 0.307147 & $0.064 *$ & \\
\hline $\mathrm{C} 10$ & $0.2929(6)$ & $0.4186(4)$ & $0.3106(3)$ & $0.0413(15)$ & \\
\hline C11 & $0.3940(5)$ & 0.3859 & $0.3079(2)$ & $0.0345(14)$ & \\
\hline H11 & 0.458001 & 0.418823 & 0.314023 & $0.041 *$ & \\
\hline $\mathrm{C} 12$ & $0.3874(5)$ & -0.0641 & $0.2533(2)$ & $0.0374(14)$ & \\
\hline $\mathrm{C} 13$ & $0.4752(5)$ & -0.1121 & $0.2537(2)$ & $0.0392(15)$ & \\
\hline H13 & 0.547919 & -0.090953 & 0.259368 & $0.047^{*}$ & \\
\hline $\mathrm{C} 14$ & $0.4542(6)$ & $-0.1921(4)$ & 0.2454 & 0.0469 (17) & \\
\hline H14 & 0.513275 & -0.226119 & 0.246359 & $0.056^{*}$ & \\
\hline C15 & $0.3487(6)$ & $-0.2227(4)$ & $0.2359(3)$ & 0.0477 (17) & \\
\hline
\end{tabular}




\begin{tabular}{|c|c|c|c|c|}
\hline H15 & 0.335952 & -0.277579 & 0.230407 & $0.057^{*}$ \\
\hline $\mathrm{C} 16$ & $0.2621(6)$ & $-0.1752(4)$ & $0.2341(3)$ & $0.0486(18)$ \\
\hline H16 & 0.189630 & -0.196769 & 0.226386 & $0.058^{*}$ \\
\hline $\mathrm{C} 17$ & $0.2820(6)$ & $-0.0953(4)$ & $0.2437(3)$ & $0.0392(15)$ \\
\hline C19 & $0.5649(5)$ & $0.2663(4)$ & $0.3739(2)$ & $0.0361(15)$ \\
\hline $\mathrm{C} 20$ & $0.5102(5)$ & 0.3175 (4) & $0.4140(2)$ & $0.0386(15)$ \\
\hline $\mathrm{H} 20$ & 0.452150 & 0.346860 & 0.403475 & $0.046^{*}$ \\
\hline $\mathrm{C} 21$ & $0.5386(6)$ & $0.3266(4)$ & $0.4686(3)$ & $0.0422(16)$ \\
\hline $\mathrm{C} 22$ & $0.6223(6)$ & $0.2841(4)$ & $0.4854(3)$ & $0.0506(18)$ \\
\hline $\mathrm{C} 23$ & $0.6810(6)$ & $0.2344(4)$ & $0.4468(3)$ & $0.054(2)$ \\
\hline $\mathrm{H} 23$ & 0.739510 & 0.205897 & 0.457842 & $0.065^{*}$ \\
\hline $\mathrm{C} 24$ & $0.6537(6)$ & $0.2267(4)$ & $0.3920(3)$ & $0.0466(18)$ \\
\hline $\mathrm{C} 25$ & $0.7125(6)$ & $0.1782(4)$ & $0.3477(3)$ & 0.0504 (19) \\
\hline $\mathrm{C} 26$ & 0.7959 (7) & $0.1282(5)$ & $0.3542(3)$ & 0.079 \\
\hline $\mathrm{H} 26$ & 0.820772 & 0.125245 & 0.389775 & $0.095^{*}$ \\
\hline $\mathrm{C} 27$ & 0.8427 (7) & $0.0828(6)$ & $0.3091(3)$ & $0.081(3)$ \\
\hline $\mathrm{H} 27$ & 0.898635 & 0.047501 & 0.313146 & $0.097^{*}$ \\
\hline $\mathrm{C} 28$ & $0.8065(6)$ & $0.0897(5)$ & $0.2580(3)$ & $0.061(2)$ \\
\hline $\mathrm{C} 29$ & $0.7246(5)$ & $0.1386(4)$ & $0.2518(3)$ & $0.0452(17)$ \\
\hline $\mathrm{H} 29$ & 0.699135 & 0.141554 & 0.216303 & $0.054^{*}$ \\
\hline $\mathrm{C} 30$ & $0.4935(6)$ & $0.3753(4)$ & $0.5176(3)$ & $0.0455(16)$ \\
\hline $\mathrm{C} 31$ & 0.4090 (6) & 0.4299 (4) & $0.5229(3)$ & 0.0469 (17) \\
\hline H31 & 0.373580 & 0.442106 & 0.491830 & $0.056^{*}$ \\
\hline $\mathrm{C} 32$ & $0.3800(6)$ & $0.4650(4)$ & $0.5753(3)$ & 0.0509 (18) \\
\hline H32 & 0.324773 & 0.503000 & 0.580377 & $0.061^{*}$ \\
\hline $\mathrm{C} 33$ & $0.4295(6)$ & $0.4461(4)$ & $0.6200(3)$ & $0.0523(18)$ \\
\hline H33 & 0.404521 & 0.468694 & 0.655171 & $0.063 *$ \\
\hline $\mathrm{C} 34$ & $0.5137(6)$ & 0.3955 (4) & $0.6152(3)$ & $0.0532(19)$ \\
\hline H34 & 0.548834 & 0.384743 & 0.646842 & $0.064 *$ \\
\hline $\mathrm{C} 35$ & $0.5477(6)$ & 0.3597 (4) & $0.5634(3)$ & $0.0516(18)$ \\
\hline $\mathrm{C} 36$ & $0.6384(7)$ & $0.3026(4)$ & $0.5477(3)$ & $0.0606(19)$ \\
\hline $\mathrm{C} 37$ & $0.7103(5)$ & 0.4728 (4) & $0.2191(2)$ & $0.0333(14)$ \\
\hline C38 & $0.8207(5)$ & 0.4959 (4) & $0.2298(2)$ & $0.0333(14)$ \\
\hline H38 & 0.875149 & 0.457545 & 0.221596 & $0.040 *$ \\
\hline C39 & $0.8513(5)$ & $0.5744(4)$ & $0.2522(2)$ & $0.0321(13)$ \\
\hline $\mathrm{C} 40$ & $0.7728(5)$ & $0.6323(4)$ & $0.2649(2)$ & $0.0356(14)$ \\
\hline $\mathrm{C} 41$ & $0.6632(5)$ & $0.6111(4)$ & $0.2538(2)$ & $0.0353(14)$ \\
\hline H41 & 0.609309 & 0.649774 & 0.262333 & $0.042 *$ \\
\hline $\mathrm{C} 42$ & $0.6322(5)$ & $0.5325(4)$ & $0.2301(2)$ & $0.0323(13)$ \\
\hline $\mathrm{C} 43$ & $0.5184(5)$ & $0.5046(4)$ & $0.2168(2)$ & $0.0326(14)$ \\
\hline C44 & $0.4306(5)$ & $0.5511(4)$ & $0.2123(3)$ & $0.0402(16)$ \\
\hline H44 & 0.441343 & 0.606625 & 0.220849 & $0.048^{*}$ \\
\hline $\mathrm{C} 45$ & $0.3274(5)$ & $0.5182(4)$ & $0.1955(3)$ & $0.0457(17)$ \\
\hline H45 & 0.266492 & 0.549695 & 0.192006 & $0.055^{*}$ \\
\hline $\mathrm{C} 46$ & $0.3170(5)$ & 0.4387 (4) & $0.1842(3)$ & $0.0425(15)$ \\
\hline C47 & $0.4038(5)$ & $0.3922(4)$ & $0.1874(2)$ & $0.0388(15)$ \\
\hline $\mathrm{H} 47$ & 0.393614 & 0.336660 & 0.178452 & $0.047^{*}$ \\
\hline C48 & $0.9588(5)$ & $0.6130(4)$ & $0.2682(2)$ & $0.0326(13)$ \\
\hline
\end{tabular}




\begin{tabular}{|c|c|c|c|c|c|}
\hline C49 & $1.0619(5)$ & $0.5829(4)$ & 0.2650 & $0.0369(15)$ & \\
\hline H49 & 1.071729 & 0.528367 & 0.249895 & $0.044 *$ & \\
\hline $\mathrm{C} 50$ & $1.1525(5)$ & $0.6350(4)$ & $0.2846(3)$ & $0.0404(15)$ & \\
\hline H50 & 1.224306 & 0.615586 & 0.282469 & $0.049 *$ & \\
\hline C51 & $1.1375(5)$ & $0.7138(4)$ & $0.3069(3)$ & $0.0410(15)$ & \\
\hline H51 & 1.199287 & 0.748038 & 0.320514 & $0.049 *$ & \\
\hline C52 & $1.0339(5)$ & $0.7441(4)$ & $0.3097(3)$ & $0.0407(15)$ & \\
\hline H52 & 1.024465 & 0.798821 & 0.324577 & $0.049^{*}$ & \\
\hline $\mathrm{C} 53$ & $0.9449(5)$ & $0.6939(4)$ & $0.2907(2)$ & $0.0362(14)$ & \\
\hline $\mathrm{C} 54$ & $0.8250(5)$ & $0.7124(4)$ & 0.2907 & $0.0436(15)$ & \\
\hline $\mathrm{C} 55$ & $0.6405(5)$ & $0.3491(4)$ & $0.1176(2)$ & $0.0361(15)$ & \\
\hline C56 & $0.5656(5)$ & 0.3785 & $0.0855(2)$ & $0.0379(15)$ & \\
\hline H56 & 0.512315 & 0.413985 & 0.101879 & $0.045^{*}$ & \\
\hline C57 & $0.5692(5)$ & $0.3556(4)$ & $0.0291(2)$ & $0.0402(16)$ & \\
\hline C58 & $0.6478(6)$ & $0.3044(4)$ & $0.0041(3)$ & 0.0525 (19) & \\
\hline C59 & $0.7243(6)$ & 0.2768 & $0.0354(3)$ & $0.055(2)$ & \\
\hline H59 & 0.778854 & 0.242764 & 0.018764 & $0.066^{*}$ & \\
\hline $\mathrm{C} 60$ & $0.7212(5)$ & $0.2993(4)$ & 0.0923 & $0.0443(17)$ & \\
\hline C61 & $0.8003(6)$ & 0.2740 & 0.1290 & 0.0457 (17) & \\
\hline C62 & $0.8878(7)$ & $0.2270(5)$ & 0.1139 & $0.071(3)$ & \\
\hline H62 & 0.901631 & 0.209550 & 0.076366 & $0.085 *$ & \\
\hline C63 & $0.9553(7)$ & $0.2049(5)$ & $0.1521(3)$ & $0.073(3)$ & \\
\hline H63 & 1.015383 & 0.172193 & 0.141843 & $0.088^{*}$ & \\
\hline C64 & $0.9326(6)$ & $0.2316(5)$ & 0.2055 & $0.059(2)$ & \\
\hline C65 & $0.8486(5)$ & 0.2816 & $0.2216(3)$ & 0.0467 (17) & \\
\hline H65 & 0.837442 & 0.301309 & 0.259316 & $0.056^{*}$ & \\
\hline C66 & $0.4987(5)$ & $0.3764(4)$ & $-0.0136(2)$ & $0.0394(15)$ & \\
\hline C67 & 0.4109 (5) & $0.4254(4)$ & -0.0093 & $0.0417(16)$ & \\
\hline H67 & 0.387171 & 0.451617 & 0.025496 & $0.050 *$ & \\
\hline C68 & $0.3576(6)$ & $0.4355(4)$ & $-0.0573(3)$ & $0.0474(17)$ & \\
\hline H68 & 0.297780 & 0.469478 & -0.055589 & $0.057^{*}$ & \\
\hline C69 & $0.3930(6)$ & $0.3954(4)$ & $-0.1076(3)$ & $0.0492(18)$ & \\
\hline H69 & 0.356125 & 0.402447 & -0.139937 & $0.059 *$ & \\
\hline $\mathrm{C} 70$ & $0.4781(6)$ & $0.3465(4)$ & -0.1121 & $0.0498(18)$ & \\
\hline $\mathrm{H} 70$ & 0.499715 & 0.319041 & -0.147062 & $0.060^{*}$ & \\
\hline C71 & $0.5330(6)$ & $0.3373(4)$ & -0.0649 & $0.0451(16)$ & \\
\hline $\mathrm{C} 72$ & $0.6298(6)$ & $0.2863(4)$ & $-0.0584(3)$ & $0.0574(18)$ & \\
\hline $\mathrm{C} 82$ & $0.0930(10)$ & $-0.0673(7)$ & 0.4255 & $0.116(4)$ & \\
\hline $\mathrm{H} 82 \mathrm{C}$ & 0.027750 & -0.086675 & 0.439677 & $0.139 *$ & 0.67 \\
\hline H82D & 0.141652 & -0.111889 & 0.413186 & $0.139 *$ & 0.67 \\
\hline H82A & 0.020250 & -0.051112 & 0.414048 & $0.139 *$ & 0.33 \\
\hline H82B & 0.107566 & -0.119647 & 0.401457 & $0.139 *$ & 0.33 \\
\hline $\mathrm{C} 85$ & $0.7518(7)$ & $0.3415(5)$ & 0.5686 & $0.072(2)$ & \\
\hline H85A & 0.757472 & 0.352184 & 0.608996 & $0.087^{*}$ & \\
\hline H85B & 0.807278 & 0.302461 & 0.554753 & $0.087^{*}$ & \\
\hline C86 & 0.7833 (6) & $0.4178(5)$ & $0.5545(3)$ & $0.065(2)$ & \\
\hline H86A & 0.778654 & 0.408744 & 0.514209 & $0.079 *$ & \\
\hline H86B & 0.730941 & 0.458795 & 0.569434 & $0.079 *$ & \\
\hline
\end{tabular}




\begin{tabular}{|c|c|c|c|c|c|}
\hline $\mathrm{C} 87$ & $0.8986(6)$ & $0.4468(5)$ & 0.5775 & $0.076(2)$ & \\
\hline H87A & 0.904995 & 0.449300 & 0.616978 & $0.091^{*}$ & \\
\hline H87B & 0.950903 & 0.407959 & 0.559371 & $0.091 *$ & \\
\hline $\mathrm{C} 88$ & $0.9306(6)$ & $0.5260(6)$ & 0.5706 & 0.080 & \\
\hline $\mathrm{H} 88 \mathrm{~A}$ & 0.877039 & 0.564918 & 0.587337 & $0.096^{*}$ & \\
\hline H88B & 0.928573 & 0.523296 & 0.531089 & $0.096^{*}$ & \\
\hline $\mathrm{C} 89$ & 1.0455 (7) & $0.5534(6)$ & $0.5971(4)$ & 0.094 (3) & \\
\hline H89A & 1.048696 & 0.551810 & 0.635832 & $0.113^{*}$ & \\
\hline H89B & 1.099215 & 0.516193 & 0.578413 & $0.113^{*}$ & \\
\hline $\mathrm{C} 90$ & $1.0773(8)$ & $0.6353(7)$ & $0.5946(4)$ & 0.107 (4) & \\
\hline $\mathrm{H} 90 \mathrm{~A}$ & 1.071429 & 0.638003 & 0.556411 & $0.161^{*}$ & \\
\hline H90B & 1.152732 & 0.648095 & 0.609869 & $0.161^{*}$ & \\
\hline $\mathrm{H} 90 \mathrm{C}$ & 1.028379 & 0.673165 & 0.615750 & $0.161^{*}$ & \\
\hline C91 & $0.6265(8)$ & $0.2301(5)$ & $0.5705(3)$ & $0.082(3)$ & \\
\hline H91A & 0.686897 & 0.194476 & 0.558293 & $0.098^{*}$ & \\
\hline H91B & 0.634704 & 0.247767 & 0.610900 & $0.098^{*}$ & \\
\hline C92 & $0.5211(8)$ & $0.1849(5)$ & $0.5537(4)$ & 0.088 & \\
\hline H92A & 0.522596 & 0.157612 & 0.514382 & $0.105^{*}$ & 0.5 \\
\hline H92B & 0.462821 & 0.223804 & 0.556654 & $0.105^{*}$ & 0.5 \\
\hline $\mathrm{H} 92 \mathrm{C}$ & 0.508664 & 0.166734 & 0.513390 & $0.105^{*}$ & 0.5 \\
\hline H92D & 0.458686 & 0.216306 & 0.568849 & $0.105^{*}$ & 0.5 \\
\hline C97 & $0.7927(6)$ & $0.7465(4)$ & $0.3500(3)$ & $0.062(2)$ & \\
\hline H97A & 0.825250 & 0.800349 & 0.363692 & $0.075^{*}$ & \\
\hline H97B & 0.712328 & 0.751005 & 0.348991 & $0.075^{*}$ & \\
\hline C98 & $0.8263(6)$ & $0.7003(5)$ & $0.3903(3)$ & $0.068(2)$ & \\
\hline H98A & 0.906902 & 0.698104 & 0.393534 & $0.081^{*}$ & \\
\hline H98B & 0.796198 & 0.645730 & 0.376632 & $0.081^{*}$ & \\
\hline C99 & $0.7857(8)$ & $0.7372(7)$ & 0.4468 & $0.111(4)$ & \\
\hline H99A & 0.804330 & 0.794232 & 0.457215 & $0.133^{*}$ & \\
\hline H99B & 0.705112 & 0.730912 & 0.444528 & $0.133^{*}$ & \\
\hline $\mathrm{C} 100$ & $0.8326(9)$ & $0.7022(8)$ & $0.4909(3)$ & $0.126(5)$ & \\
\hline $\mathrm{H} 10 \mathrm{C}$ & 0.907227 & 0.684879 & 0.482090 & $0.151^{*}$ & 0.25 \\
\hline H10D & 0.787935 & 0.654701 & 0.490350 & $0.151^{*}$ & 0.25 \\
\hline $\mathrm{H} 10 \mathrm{~A}$ & 0.911814 & 0.715805 & 0.496234 & $0.151^{*}$ & 0.75 \\
\hline H10B & 0.825266 & 0.644221 & 0.476801 & $0.151^{*}$ & 0.75 \\
\hline $\mathrm{C} 103$ & $0.7993(6)$ & $0.7717(4)$ & $0.2570(3)$ & 0.067 (2) & \\
\hline $\mathrm{H} 10 \mathrm{E}$ & 0.719459 & 0.779000 & 0.255319 & $0.080^{*}$ & \\
\hline $\mathrm{H} 10 \mathrm{~F}$ & 0.834842 & 0.822967 & 0.276603 & $0.080^{*}$ & \\
\hline $\mathrm{C} 104$ & $0.8352(8)$ & $0.7492(5)$ & 0.1991 & 0.081 (3) & \\
\hline H10G & 0.795489 & 0.699281 & 0.179994 & $0.097^{*}$ & \\
\hline $\mathrm{H} 10 \mathrm{H}$ & 0.913409 & 0.736691 & 0.201994 & $0.097^{*}$ & \\
\hline C109 & 0.6047 (7) & 0.1989 (4) & $-0.0844(3)$ & 0.069 (2) & \\
\hline H10I & 0.597821 & 0.190047 & -0.124604 & $0.082^{*}$ & \\
\hline H10J & 0.667859 & 0.168997 & -0.075397 & $0.082^{*}$ & \\
\hline C110 & $0.5023(8)$ & $0.1651(4)$ & $-0.0672(3)$ & 0.078 & \\
\hline H11A & 0.439683 & 0.195456 & -0.076171 & $0.094^{*}$ & 0.6 \\
\hline H11B & 0.509556 & 0.174610 & -0.026970 & $0.094 *$ & 0.6 \\
\hline H11C & 0.435461 & 0.183672 & -0.083310 & $0.094 *$ & 0.4 \\
\hline
\end{tabular}




\begin{tabular}{|c|c|c|c|c|c|}
\hline H11D & 0.500549 & 0.179057 & -0.026943 & $0.094 *$ & 0.4 \\
\hline $\mathrm{C} 115$ & $0.7326(6)$ & $0.3095(5)$ & -0.0835 & $0.068(2)$ & \\
\hline H11E & 0.794181 & 0.278972 & -0.073624 & $0.081^{*}$ & \\
\hline $\mathrm{H} 11 \mathrm{~F}$ & 0.719449 & 0.292812 & -0.123838 & $0.081^{*}$ & \\
\hline C116 & $0.7680(6)$ & $0.3947(5)$ & -0.0675 & $0.072(2)$ & \\
\hline H11G & 0.775317 & 0.415974 & -0.027245 & $0.086^{*}$ & 0.6 \\
\hline $\mathrm{H} 11 \mathrm{H}$ & 0.716173 & 0.426711 & -0.083429 & $0.086^{*}$ & 0.6 \\
\hline H11I & 0.776472 & 0.410602 & -0.027028 & $0.086^{*}$ & 0.4 \\
\hline H11J & 0.705369 & 0.423726 & -0.078153 & $0.086^{*}$ & 0.4 \\
\hline $\mathrm{C} 18$ & $0.1972(7)$ & $-0.0316(5)$ & $0.2410(4)$ & $0.032(2)$ & 0.67 \\
\hline $\mathrm{C} 73$ & $0.1407(9)$ & $-0.0413(7)$ & $0.1833(4)$ & $0.046(3)$ & 0.67 \\
\hline $\mathrm{H} 73 \mathrm{~A}$ & 0.110500 & -0.095873 & 0.169647 & $0.055^{*}$ & 0.67 \\
\hline H73B & 0.078472 & -0.005603 & 0.186409 & $0.055^{*}$ & 0.67 \\
\hline $\mathrm{C} 74$ & $0.2141(10)$ & $-0.0245(7)$ & $0.1410(4)$ & 0.063 & 0.67 \\
\hline $\mathrm{H} 74 \mathrm{~A}$ & 0.196198 & -0.062957 & 0.105268 & $0.075^{*}$ & 0.67 \\
\hline H74B & 0.291419 & -0.030717 & 0.152489 & $0.075^{*}$ & 0.67 \\
\hline C75A & 0.1979 (11) & $0.0630(8)$ & $0.1348(6)$ & 0.078 (4) & 0.67 \\
\hline $\mathrm{H} 75 \mathrm{~A}$ & 0.192894 & 0.098528 & 0.171807 & $0.093^{*}$ & 0.67 \\
\hline H75B & 0.126896 & 0.063752 & 0.114093 & $0.093^{*}$ & 0.67 \\
\hline $\mathrm{C} 76$ & $0.2902(13)$ & $0.0991(8)$ & $0.1053(6)$ & $0.091(4)$ & 0.67 \\
\hline H76A & 0.361282 & 0.102279 & 0.126724 & $0.109^{*}$ & 0.67 \\
\hline H76B & 0.297749 & 0.064005 & 0.068421 & $0.109^{*}$ & 0.67 \\
\hline $\mathrm{C} 77$ & $0.2610(13)$ & $0.1803(8)$ & $0.1004(6)$ & $0.093(5)$ & 0.67 \\
\hline $\mathrm{H} 77 \mathrm{~A}$ & 0.260904 & 0.216387 & 0.137566 & $0.112 *$ & 0.67 \\
\hline H77B & 0.186135 & 0.177800 & 0.082838 & $0.112^{*}$ & 0.67 \\
\hline $\mathrm{C} 78$ & $0.3393(13)$ & $0.2139(10)$ & $0.0675(6)$ & $0.099(6)$ & 0.67 \\
\hline $\mathrm{H} 78 \mathrm{~A}$ & 0.316268 & 0.266339 & 0.065725 & $0.148^{*}$ & 0.67 \\
\hline H78B & 0.413315 & 0.217826 & 0.085110 & $0.148^{*}$ & 0.67 \\
\hline $\mathrm{H} 78 \mathrm{C}$ & 0.338498 & 0.179215 & 0.030347 & $0.148^{*}$ & 0.67 \\
\hline C79 & 0.1089 (7) & $-0.0342(6)$ & $0.2806(3)$ & $0.031(2)$ & 0.67 \\
\hline $\mathrm{H} 79 \mathrm{~A}$ & 0.061246 & -0.081563 & 0.264753 & $0.037^{*}$ & 0.67 \\
\hline H79B & 0.063206 & 0.012301 & 0.283291 & $0.037^{*}$ & 0.67 \\
\hline $\mathrm{C} 80$ & $0.1507(8)$ & $-0.0358(7)$ & $0.3389(4)$ & $0.044(3)$ & 0.67 \\
\hline $\mathrm{H} 80 \mathrm{~A}$ & 0.189702 & -0.085112 & 0.337086 & $0.052 *$ & 0.67 \\
\hline H80B & 0.203617 & 0.008758 & 0.353973 & $0.052 *$ & 0.67 \\
\hline C81 & $0.0589(9)$ & $-0.0303(8)$ & $0.3774(4)$ & $0.070(4)$ & 0.67 \\
\hline H81A & -0.007525 & -0.058240 & 0.356789 & $0.084 *$ & 0.67 \\
\hline H81B & 0.041511 & 0.025594 & 0.392399 & $0.084 *$ & 0.67 \\
\hline $\mathrm{C} 83$ & $0.1498(15)$ & $-0.0046(9)$ & $0.4675(6)$ & $0.112(6)$ & 0.67 \\
\hline $\mathrm{H} 83 \mathrm{~A}$ & 0.104971 & 0.042356 & 0.479226 & $0.134 *$ & 0.67 \\
\hline H83B & 0.221265 & 0.010699 & 0.456761 & $0.134^{*}$ & 0.67 \\
\hline $\mathrm{C} 84$ & $0.1615(19)$ & $-0.0535(17)$ & $0.5115(9)$ & $0.230(13)$ & 0.67 \\
\hline $\mathrm{H} 84 \mathrm{~A}$ & 0.199547 & -0.021065 & 0.545126 & $0.345^{*}$ & 0.67 \\
\hline H84B & 0.088734 & -0.069302 & 0.519359 & $0.345^{*}$ & 0.67 \\
\hline $\mathrm{H} 84 \mathrm{C}$ & 0.203336 & -0.100498 & 0.497221 & $0.345^{*}$ & 0.67 \\
\hline C93 & $0.4825(12)$ & $0.1203(8)$ & $0.5840(6)$ & $0.084(4)^{*}$ & 0.67 \\
\hline H93A & 0.473968 & 0.147361 & 0.622845 & $0.101^{*}$ & 0.67 \\
\hline H93B & 0.409658 & 0.098578 & 0.567413 & $0.101^{*}$ & 0.67 \\
\hline
\end{tabular}




\begin{tabular}{|c|c|c|c|c|c|}
\hline C94 & $0.5575(13)$ & $0.0529(9)$ & $0.5820(8)$ & $0.120(5)^{*}$ & 0.67 \\
\hline H94A & 0.619080 & 0.069783 & 0.610031 & $0.144 *$ & 0.67 \\
\hline H94B & 0.587816 & 0.037092 & 0.545700 & $0.144^{*}$ & 0.67 \\
\hline C95 & $0.4949(12)$ & $-0.0186(8)$ & $0.5925(7)$ & $0.096(4)^{*}$ & 0.67 \\
\hline H95A & 0.468081 & -0.005052 & 0.629582 & $0.115^{*}$ & 0.67 \\
\hline H95B & 0.431870 & -0.035707 & 0.565290 & $0.115^{*}$ & 0.67 \\
\hline C96 & $0.5779(12)$ & $-0.0827(9)$ & $0.5867(7)$ & $0.110(5)^{*}$ & 0.67 \\
\hline H96A & 0.614493 & -0.087680 & 0.552877 & $0.164 *$ & 0.67 \\
\hline H96B & 0.540716 & -0.132809 & 0.585495 & $0.164 *$ & 0.67 \\
\hline H96C & 0.632181 & -0.069131 & 0.618038 & $0.164 *$ & 0.67 \\
\hline C101 & $0.7860(13)$ & $0.7246(9)$ & $0.5482(5)$ & $0.115(5)$ & 0.75 \\
\hline $\mathrm{H} 10 \mathrm{~K}$ & 0.705661 & 0.728350 & 0.544291 & $0.139^{*}$ & 0.75 \\
\hline H10L & 0.803316 & 0.683814 & 0.567665 & $0.139^{*}$ & 0.75 \\
\hline C102 & $0.8367(15)$ & $0.8019(8)$ & $0.5797(5)$ & $0.139(7)$ & 0.75 \\
\hline $\mathrm{H} 10 \mathrm{M}$ & 0.808271 & 0.817139 & 0.616031 & $0.208^{*}$ & 0.75 \\
\hline $\mathrm{H} 10 \mathrm{~N}$ & 0.916130 & 0.797571 & 0.583533 & $0.208^{*}$ & 0.75 \\
\hline $\mathrm{H} 10 \mathrm{O}$ & 0.818814 & 0.841951 & 0.560240 & $0.208^{*}$ & 0.75 \\
\hline C105 & 0.794 & $0.8133(17)$ & $0.1725(10)$ & $0.111(7)$ & 0.67 \\
\hline $\mathrm{H} 10 \mathrm{P}$ & 0.713450 & 0.816360 & 0.173865 & $0.134 *$ & 0.67 \\
\hline H10Q & 0.826954 & 0.864942 & 0.192949 & $0.134 *$ & 0.67 \\
\hline C106 & $0.8227(18)$ & $0.7950(13)$ & $0.1140(8)$ & $0.143(7)^{*}$ & 0.67 \\
\hline H10R & 0.750720 & 0.788176 & 0.093022 & $0.171^{*}$ & 0.67 \\
\hline H10S & 0.853620 & 0.845663 & 0.110720 & $0.171^{*}$ & 0.67 \\
\hline $\mathrm{C} 107$ & $0.890(2)$ & $0.7356(15)$ & $0.0790(10)$ & $0.230(12)^{*}$ & 0.67 \\
\hline H10T & 0.867467 & 0.683541 & 0.083316 & $0.276^{*}$ & 0.67 \\
\hline H10U & 0.966718 & 0.745944 & 0.094436 & $0.276^{*}$ & 0.67 \\
\hline C108 & $0.890(2)$ & $0.7282(15)$ & $0.0180(9)$ & $0.203(10)^{*}$ & 0.67 \\
\hline $\mathrm{H} 10 \mathrm{~V}$ & 0.938834 & 0.686372 & 0.001511 & $0.305^{*}$ & 0.67 \\
\hline H10W & 0.815853 & 0.715352 & 0.001040 & $0.305^{*}$ & 0.67 \\
\hline $\mathrm{H} 10 \mathrm{X}$ & 0.915748 & 0.778160 & 0.012233 & $0.305^{*}$ & 0.67 \\
\hline C111 & $0.4737(16)$ & $0.0780(7)$ & $-0.0919(9)$ & $0.059(5)$ & 0.6 \\
\hline $\mathrm{H} 11 \mathrm{~K}$ & 0.530236 & 0.048138 & -0.076999 & $0.071^{*}$ & 0.6 \\
\hline H11L & 0.482060 & 0.066949 & -0.131667 & $0.071^{*}$ & 0.6 \\
\hline $\mathrm{C} 112$ & $0.3628(13)$ & $0.0421(8)$ & $-0.0852(7)$ & $0.071(4)$ & 0.6 \\
\hline $\mathrm{H} 11 \mathrm{M}$ & 0.346641 & 0.062715 & -0.046504 & $0.086^{*}$ & 0.6 \\
\hline $\mathrm{H} 11 \mathrm{~N}$ & 0.307286 & 0.062984 & -0.107385 & $0.086^{*}$ & 0.6 \\
\hline C113 & $0.345(2)$ & $-0.0456(9)$ & $-0.0997(7)$ & $0.081(6)$ & 0.6 \\
\hline $\mathrm{H} 11 \mathrm{O}$ & 0.380021 & -0.069343 & -0.133956 & $0.098^{*}$ & 0.6 \\
\hline H11P & 0.265705 & -0.058441 & -0.106427 & $0.098^{*}$ & 0.6 \\
\hline C114 & $0.3912(18)$ & $-0.0812(11)$ & $-0.0551(6)$ & $0.085(5)$ & 0.6 \\
\hline H11Q & 0.378859 & -0.138391 & -0.066555 & $0.128^{*}$ & 0.6 \\
\hline H11R & 0.354792 & -0.059350 & -0.021462 & $0.128^{*}$ & 0.6 \\
\hline H11S & 0.469777 & -0.068785 & -0.048369 & $0.128 *$ & 0.6 \\
\hline $\mathrm{C} 117$ & $0.8800(12)$ & $0.3923(9)$ & $-0.0922(9)$ & $0.064(5)$ & 0.6 \\
\hline $\mathrm{H} 11 \mathrm{~T}$ & 0.930692 & 0.359371 & -0.076474 & $0.077^{*}$ & 0.6 \\
\hline $\mathrm{H} 11 \mathrm{U}$ & 0.871775 & 0.370432 & -0.132406 & $0.077^{*}$ & 0.6 \\
\hline C118 & $0.9217(11)$ & $0.4759(9)$ & $-0.0777(8)$ & $0.068(5)$ & 0.6 \\
\hline $\mathrm{H} 11 \mathrm{~V}$ & 0.928766 & 0.496950 & -0.037468 & $0.081^{*}$ & 0.6 \\
\hline
\end{tabular}




\begin{tabular}{|c|c|c|c|c|c|}
\hline H11W & 0.869132 & 0.508208 & -0.092901 & $0.081^{*}$ & 0.6 \\
\hline C119 & $1.0319(12)$ & $0.4810(9)$ & $-0.1005(7)$ & $0.081(5)$ & 0.6 \\
\hline $\mathrm{H} 11 \mathrm{X}$ & 1.025756 & 0.456671 & -0.140440 & $0.097 *$ & 0.6 \\
\hline H11Y & 1.085543 & 0.451415 & -0.083521 & $0.097 *$ & 0.6 \\
\hline $\mathrm{C} 120$ & $1.0717(17)$ & $0.5660(10)$ & $-0.0893(8)$ & $0.088(5)$ & 0.6 \\
\hline H12A & 1.033368 & 0.590737 & -0.115241 & $0.132 *$ & 0.6 \\
\hline H12B & 1.150447 & 0.567901 & -0.093412 & $0.132 *$ & 0.6 \\
\hline $\mathrm{H} 12 \mathrm{C}$ & 1.057054 & 0.594365 & -0.051892 & $0.132 *$ & 0.6 \\
\hline C96B & $0.422(3)$ & $-0.0451(19)$ & $0.5954(18)$ & $0.160(18)^{*}$ & 0.33 \\
\hline H96D & 0.385303 & -0.077240 & 0.615511 & $0.240^{*}$ & 0.33 \\
\hline H96E & 0.422974 & -0.074291 & 0.556922 & $0.240^{*}$ & 0.33 \\
\hline $\mathrm{H} 96 \mathrm{~F}$ & 0.497369 & -0.032492 & 0.611182 & $0.240^{*}$ & 0.33 \\
\hline $\mathrm{C} 211$ & $0.514(3)$ & $0.0767(8)$ & $-0.0906(19)$ & $0.080(11)$ & 0.4 \\
\hline $\mathrm{H} 21 \mathrm{~A}$ & 0.501978 & 0.061528 & -0.130926 & $0.096^{*}$ & 0.4 \\
\hline $\mathrm{H} 21 \mathrm{~B}$ & 0.587921 & 0.060241 & -0.080354 & $0.096^{*}$ & 0.4 \\
\hline $\mathrm{C} 212$ & $0.427(2)$ & $0.0405(12)$ & $-0.0644(10)$ & $0.074(6)$ & 0.4 \\
\hline $\mathrm{H} 21 \mathrm{C}$ & 0.448640 & 0.047594 & -0.025221 & $0.089^{*}$ & 0.4 \\
\hline H21D & 0.357564 & 0.068251 & -0.067001 & $0.089^{*}$ & 0.4 \\
\hline $\mathrm{C} 213$ & $0.407(2)$ & $-0.0457(13)$ & $-0.0917(14)$ & $0.091(10)$ & 0.4 \\
\hline $\mathrm{H} 21 \mathrm{E}$ & 0.473320 & -0.074643 & -0.085155 & $0.109^{*}$ & 0.4 \\
\hline $\mathrm{H} 21 \mathrm{~F}$ & 0.393822 & -0.053597 & -0.131616 & $0.109^{*}$ & 0.4 \\
\hline $\mathrm{C} 214$ & $0.310(2)$ & $-0.0784(18)$ & $-0.0695(13)$ & $0.098(9)$ & 0.4 \\
\hline $\mathrm{H} 21 \mathrm{G}$ & 0.242674 & -0.070843 & -0.090358 & $0.147^{*}$ & 0.4 \\
\hline $\mathrm{H} 21 \mathrm{H}$ & 0.305539 & -0.050746 & -0.030895 & $0.147^{*}$ & 0.4 \\
\hline H21I & 0.318638 & -0.134704 & -0.073020 & $0.147^{*}$ & 0.4 \\
\hline C93B & $0.539(2)$ & $0.1162(14)$ & $0.5789(13)$ & $0.084(4)^{*}$ & 0.33 \\
\hline H93C & 0.560997 & 0.134687 & 0.618558 & $0.101^{*}$ & 0.33 \\
\hline H93D & 0.595574 & 0.081073 & 0.560567 & $0.101^{*}$ & 0.33 \\
\hline C94B & $0.424(2)$ & $0.0746(19)$ & $0.5681(14)$ & $0.120(5)^{*}$ & 0.33 \\
\hline $\mathrm{H} 94 \mathrm{C}$ & 0.373085 & 0.116767 & 0.564848 & $0.144^{*}$ & 0.33 \\
\hline H94D & 0.423957 & 0.039076 & 0.530810 & $0.144^{*}$ & 0.33 \\
\hline C95B & $0.364(2)$ & $0.0278(17)$ & $0.5994(12)$ & $0.096(4)^{*}$ & 0.33 \\
\hline $\mathrm{H} 95 \mathrm{C}$ & 0.362354 & 0.057840 & 0.638175 & $0.115^{*}$ & 0.33 \\
\hline H95D & 0.287637 & 0.015861 & 0.583680 & $0.115^{*}$ & 0.33 \\
\hline C201 & $0.839(3)$ & $0.756(2)$ & $0.5486(10)$ & $0.115(5)$ & 0.25 \\
\hline $\mathrm{H} 20 \mathrm{~A}$ & 0.838947 & 0.725519 & 0.576122 & $0.139^{*}$ & 0.25 \\
\hline $\mathrm{H} 20 \mathrm{~B}$ & 0.905632 & 0.790656 & 0.555732 & $0.139^{*}$ & 0.25 \\
\hline C202 & $0.741(3)$ & $0.803(3)$ & $0.5503(19)$ & $0.100(14)^{*}$ & 0.25 \\
\hline $\mathrm{H} 20 \mathrm{C}$ & 0.738816 & 0.839751 & 0.586583 & $0.150^{*}$ & 0.25 \\
\hline H20D & 0.741941 & 0.832647 & 0.522586 & $0.150^{*}$ & 0.25 \\
\hline $\mathrm{H} 20 \mathrm{E}$ & 0.675690 & 0.767934 & 0.542843 & $0.150^{*}$ & 0.25 \\
\hline $\mathrm{C} 217$ & $0.871(2)$ & $0.4282(12)$ & $-0.0873(17)$ & $0.070(8)$ & 0.4 \\
\hline $\mathrm{H} 21 \mathrm{~J}$ & 0.934923 & 0.405642 & -0.071781 & $0.084 *$ & 0.4 \\
\hline $\mathrm{H} 21 \mathrm{~K}$ & 0.868407 & 0.406015 & -0.127453 & $0.084 *$ & 0.4 \\
\hline $\mathrm{C} 218$ & $0.8967(16)$ & $0.5165(12)$ & $-0.0766(12)$ & $0.067(6)$ & 0.4 \\
\hline $\mathrm{H} 21 \mathrm{~L}$ & 0.911211 & 0.537771 & -0.036562 & $0.080^{*}$ & 0.4 \\
\hline $\mathrm{H} 21 \mathrm{M}$ & 0.829395 & 0.540899 & -0.087127 & $0.080^{*}$ & 0.4 \\
\hline C219 & $0.9906(15)$ & $0.5457(17)$ & $-0.1038(10)$ & $0.080(6)$ & 0.4 \\
\hline
\end{tabular}




\begin{tabular}{|c|c|c|c|c|c|}
\hline $\mathrm{H} 21 \mathrm{~N}$ & 0.972985 & 0.532488 & -0.143852 & $0.096^{*}$ & 0.4 \\
\hline $\mathrm{H} 21 \mathrm{O}$ & 0.998720 & 0.603649 & -0.090289 & $0.096^{*}$ & 0.4 \\
\hline $\mathrm{C} 220$ & $1.096(2)$ & $0.510(2)$ & $-0.0923(12)$ & $0.092(10)$ & 0.4 \\
\hline $\mathrm{H} 22 \mathrm{~A}$ & 1.153503 & 0.527465 & -0.111753 & $0.138^{*}$ & 0.4 \\
\hline $\mathrm{H} 22 \mathrm{~B}$ & 1.087759 & 0.452138 & -0.104578 & $0.138^{*}$ & 0.4 \\
\hline $\mathrm{H} 22 \mathrm{C}$ & 1.116432 & 0.525538 & -0.052862 & $0.138^{*}$ & 0.4 \\
\hline C205 & $0.831(6)$ & $0.800(4)$ & $0.159(3)$ & $0.111(7)$ & 0.33 \\
\hline $\mathrm{H} 20 \mathrm{~F}$ & 0.757340 & 0.789503 & 0.139110 & $0.134^{*}$ & 0.33 \\
\hline $\mathrm{H} 20 \mathrm{G}$ & 0.830100 & 0.855351 & 0.181608 & $0.134^{*}$ & 0.33 \\
\hline C206 & $0.909(4)$ & $0.802(3)$ & $0.1147(18)$ & $0.143(7)^{*}$ & 0.33 \\
\hline $\mathrm{H} 20 \mathrm{H}$ & 0.981569 & 0.801510 & 0.133730 & $0.171^{*}$ & 0.33 \\
\hline H20I & 0.897487 & 0.749500 & 0.089005 & $0.171^{*}$ & 0.33 \\
\hline C207 & $0.929(5)$ & $0.854(3)$ & $0.078(2)$ & $0.230(12)^{*}$ & 0.33 \\
\hline H20J & 0.860036 & 0.857961 & 0.055880 & $0.276^{*}$ & 0.33 \\
\hline $\mathrm{H} 20 \mathrm{~K}$ & 0.951809 & 0.907378 & 0.099785 & $0.276^{*}$ & 0.33 \\
\hline C208 & $1.015(4)$ & $0.821(3)$ & $0.040(2)$ & $0.203(10)^{*}$ & 0.33 \\
\hline $\mathrm{H} 20 \mathrm{~L}$ & 1.027096 & 0.854746 & 0.016332 & $0.305^{*}$ & 0.33 \\
\hline $\mathrm{H} 20 \mathrm{M}$ & 1.082702 & 0.817222 & 0.062086 & $0.305 *$ & 0.33 \\
\hline $\mathrm{H} 20 \mathrm{~N}$ & 0.991105 & 0.767900 & 0.018266 & $0.305^{*}$ & 0.33 \\
\hline C75B & $0.062(2)$ & $-0.0416(17)$ & $0.1128(9)$ & $0.081(8)^{*}$ & 0.33 \\
\hline $\mathrm{H} 75 \mathrm{C}$ & 0.003189 & -0.080944 & 0.111364 & $0.098^{*}$ & 0.33 \\
\hline H75D & 0.030889 & 0.010929 & 0.125062 & $0.098^{*}$ & 0.33 \\
\hline C78B & $0.065(2)$ & $-0.0100(18)$ & $-0.0212(11)$ & $0.099(10)^{*}$ & 0.33 \\
\hline H78D & 0.016386 & -0.003936 & -0.052037 & $0.148^{*}$ & 0.33 \\
\hline H78E & 0.092714 & 0.041892 & 0.000482 & $0.148^{*}$ & 0.33 \\
\hline $\mathrm{H} 78 \mathrm{~F}$ & 0.125829 & -0.042704 & -0.034972 & $0.148^{*}$ & 0.33 \\
\hline C76B & $0.093(2)$ & $-0.051(2)$ & $0.0573(9)$ & $0.105(11)^{*}$ & 0.33 \\
\hline $\mathrm{H} 76 \mathrm{C}$ & 0.128418 & -0.101906 & 0.045581 & $0.126^{*}$ & 0.33 \\
\hline H76D & 0.148233 & -0.009165 & 0.058092 & $0.126^{*}$ & 0.33 \\
\hline C77B & $0.0030(18)$ & $-0.0478(15)$ & $0.0134(9)$ & $0.065(7)^{*}$ & 0.33 \\
\hline $\mathrm{H} 77 \mathrm{C}$ & -0.024925 & -0.101109 & -0.006919 & $0.079^{*}$ & 0.33 \\
\hline H77D & -0.058388 & -0.015627 & 0.028907 & $0.079^{*}$ & 0.33 \\
\hline C73B & $0.1165(18)$ & $-0.0344(15)$ & $0.2154(9)$ & $0.055(6)^{*}$ & 0.33 \\
\hline $\mathrm{H} 73 \mathrm{C}$ & 0.075511 & 0.014831 & 0.223475 & $0.066^{*}$ & 0.33 \\
\hline H73D & 0.064392 & -0.077379 & 0.216273 & $0.066^{*}$ & 0.33 \\
\hline C74B & $0.149(2)$ & $-0.049(2)$ & $0.1570(9)$ & $0.068(9)^{*}$ & 0.33 \\
\hline $\mathrm{H} 74 \mathrm{C}$ & 0.176644 & -0.103082 & 0.146082 & $0.081^{*}$ & 0.33 \\
\hline H74D & 0.210155 & -0.012378 & 0.156911 & $0.081^{*}$ & 0.33 \\
\hline C79B & $0.1465(19)$ & $-0.0258(16)$ & $0.3146(9)$ & $0.044(7)^{*}$ & 0.33 \\
\hline H79C & 0.091717 & 0.015738 & 0.320414 & $0.053^{*}$ & 0.33 \\
\hline H79D & 0.106866 & -0.076752 & 0.309955 & $0.053^{*}$ & 0.33 \\
\hline C80B & $0.224(2)$ & $-0.0111(19)$ & $0.3657(10)$ & $0.090(9)^{*}$ & 0.33 \\
\hline H80C & 0.265158 & 0.038685 & 0.368447 & $0.108^{*}$ & 0.33 \\
\hline H80D & 0.277646 & -0.053432 & 0.358958 & $0.108^{*}$ & 0.33 \\
\hline C81B & $0.183(3)$ & $-0.006(2)$ & $0.4223(12)$ & $0.109(11)^{*}$ & 0.33 \\
\hline H81C & 0.154924 & 0.047647 & 0.436378 & $0.131^{*}$ & 0.33 \\
\hline H81D & 0.246982 & -0.010687 & 0.447107 & $0.131^{*}$ & 0.33 \\
\hline C18B & $0.2000(13)$ & $-0.0277(9)$ & $0.2614(8)$ & $0.040(7)^{*}$ & 0.33 \\
\hline
\end{tabular}




$\begin{array}{llllll}\text { C83B } & 0.097(5) & -0.070(3) & 0.4842(9) & 0.220(17) & 0.33 \\ \text { H83C } & 0.173621 & -0.065669 & 0.499617 & 0.264^{*} & 0.33 \\ \text { H83D } & 0.056737 & -0.025869 & 0.505862 & 0.264^{*} & 0.33 \\ \text { C84B } & 0.046(2) & -0.1468(19) & 0.4873(11) & 0.112(11) & 0.33 \\ \text { H84D } & 0.048349 & -0.148942 & 0.525555 & 0.167^{*} & 0.33 \\ \text { H84E } & -0.030222 & -0.150926 & 0.472325 & 0.167^{*} & 0.33 \\ \text { H84F } & 0.086362 & -0.190624 & 0.466095 & 0.167^{*} & 0.33 \\ \text { C1S } & 0.1557(19) & 0.3347(15) & 0.0220(10) & 0.055(7)^{*} & 0.3 \\ \text { H1SA } & 0.183661 & 0.367368 & -0.000338 & 0.083^{*} & 0.3 \\ \text { H1SB } & 0.101026 & 0.296424 & -0.000072 & 0.083^{*} & 0.3 \\ \text { H1SC } & 0.122049 & 0.368267 & 0.053413 & 0.083^{*} & 0.3 \\ \text { C2S } & 0.250(2) & 0.2911(17) & 0.0423(12) & 0.080(9)^{*} & 0.3 \\ \text { H2SA } & 0.284880 & 0.257675 & 0.010666 & 0.096^{*} & 0.3 \\ \text { H2SB } & 0.305946 & 0.329665 & 0.064261 & 0.096^{*} & 0.3 \\ \text { C3S } & 0.208(3) & 0.240(2) & 0.0769(15) & 0.107(12)^{*} & 0.3 \\ \text { H3SA } & 0.142194 & 0.208400 & 0.058969 & 0.128^{*} & 0.3 \\ \text { H3SB } & 0.191658 & 0.272115 & 0.113744 & 0.128^{*} & 0.3 \\ \text { C4S } & 0.308(4) & 0.189(3) & 0.079(2) & 0.13(2)^{*} & 0.3 \\ \text { H4SA } & 0.313263 & 0.146002 & 0.045551 & 0.155^{*} & 0.3 \\ \text { H4SB } & 0.378179 & 0.220241 & 0.088549 & 0.155^{*} & 0.3 \\ \text { C5S } & 0.268(4) & 0.161(3) & 0.1272(16) & 0.112(15)^{*} & 0.3 \\ \text { H5SA } & 0.320485 & 0.125298 & 0.137508 & 0.167^{*} & 0.3 \\ \text { H5SB } & 0.260611 & 0.206706 & 0.158421 & 0.167^{*} & 0.3 \\ \text { H5SC } & 0.196782 & 0.133710 & 0.116143 & 0.167^{*} & 0.3\end{array}$

Atomic displacement parameters $\left(\AA^{2}\right)$

\begin{tabular}{lllllll}
\hline & $U^{11}$ & $U^{22}$ & $U^{33}$ & $U^{12}$ & $U^{13}$ & $U^{23}$ \\
\hline Ir1 & $0.02894(15)$ & $0.03413(16)$ & $0.02566(14)$ & $0.00551(12)$ & $-0.00019(11)$ & $0.00046(11)$ \\
Ir2 & $0.03295(15)$ & $0.03058(15)$ & $0.03104(15)$ & $0.00763(12)$ & $0.00348(12)$ & $0.00119(12)$ \\
C11 & $0.0407(9)$ & $0.0321(9)$ & $0.0266(8)$ & $0.0021(7)$ & $-0.0021(7)$ & $0.0010(7)$ \\
C12 & $0.0359(9)$ & $0.0345(9)$ & $0.0322(8)$ & $0.0026(7)$ & $-0.0007(7)$ & $-0.0027(7)$ \\
F1 & $0.063(3)$ & $0.035(2)$ & $0.051(2)$ & $0.0159(19)$ & $0.007(2)$ & $0.0071(18)$ \\
F2 & $0.080(3)$ & $0.096(4)$ & $0.052(3)$ & $0.058(3)$ & $0.013(2)$ & $0.000(2)$ \\
F3 & $0.032(2)$ & $0.074(3)$ & $0.060(3)$ & $0.002(2)$ & $-0.0080(19)$ & $0.012(2)$ \\
F4 & $0.061(3)$ & $0.093(4)$ & $0.068(3)$ & $0.040(3)$ & $-0.022(2)$ & $0.004(3)$ \\
N1 & $0.037(3)$ & $0.028(3)$ & $0.028(3)$ & $0.010(2)$ & $0.006(2)$ & $0.002(2)$ \\
N2 & $0.034(3)$ & $0.040(3)$ & $0.032(3)$ & $0.013(2)$ & $0.004(2)$ & $0.003(2)$ \\
N3 & $0.030(3)$ & $0.036(3)$ & $0.018(2)$ & $0.007(2)$ & $0.001(2)$ & $-0.001(2)$ \\
N4 & $0.033(3)$ & $0.040(3)$ & $0.036(3)$ & $0.005(2)$ & $-0.001(2)$ & $0.002(2)$ \\
C1 & $0.033(3)$ & $0.045(3)$ & $0.034(3)$ & $0.012(3)$ & $0.008(3)$ & $0.010(3)$ \\
C2 & $0.036(3)$ & $0.040(3)$ & $0.035(3)$ & $0.009(3)$ & $0.009(3)$ & $0.014(3)$ \\
C3 & $0.038(3)$ & $0.037(3)$ & $0.029(3)$ & $0.011(2)$ & $0.011(3)$ & $0.008(3)$ \\
C4 & $0.038(3)$ & $0.038(3)$ & $0.038(4)$ & $0.009(3)$ & $0.007(3)$ & $0.012(3)$ \\
C5 & $0.032(3)$ & $0.038(3)$ & $0.057(4)$ & $0.011(3)$ & $0.014(3)$ & $0.014(3)$ \\
C6 & $0.037(3)$ & $0.038(3)$ & $0.046(4)$ & $0.012(3)$ & $0.010(3)$ & $0.012(3)$ \\
C7 & $0.035(3)$ & $0.035(3)$ & $0.046(4)$ & $0.008(3)$ & $0.008(3)$ & $0.005(3)$ \\
C8 & $0.036(4)$ & $0.042(4)$ & $0.071(5)$ & $0.010(3)$ & $0.008(4)$ & $0.003(4)$
\end{tabular}




\begin{tabular}{|c|c|c|c|c|c|c|}
\hline $\mathrm{C} 9$ & $0.044(4)$ & $0.046(4)$ & $0.066(5)$ & $0.016(3)$ & $0.002(4)$ & $0.006(4)$ \\
\hline $\mathrm{C} 10$ & $0.052(4)$ & $0.032(3)$ & $0.037(4)$ & $0.013(3)$ & $0.004(3)$ & $0.004(3)$ \\
\hline $\mathrm{C} 11$ & $0.043(3)$ & $0.029(3)$ & $0.031(3)$ & $0.007(3)$ & $0.003(3)$ & $0.005(3)$ \\
\hline $\mathrm{C} 12$ & $0.047(3)$ & $0.036(3)$ & $0.030(3)$ & $0.006(3)$ & $0.010(3)$ & $0.009(3)$ \\
\hline $\mathrm{C} 13$ & $0.042(4)$ & $0.038(3)$ & $0.035(4)$ & $0.009(3)$ & $0.012(3)$ & $0.003(3)$ \\
\hline C14 & $0.053(4)$ & $0.040(4)$ & $0.052(4)$ & $0.023(3)$ & $0.015(4)$ & $0.015(3)$ \\
\hline C15 & $0.062(4)$ & $0.031(4)$ & $0.054(4)$ & 0.009 (3) & $0.019(4)$ & $0.016(3)$ \\
\hline C16 & $0.052(4)$ & $0.037(3)$ & $0.061(5)$ & $0.006(3)$ & $0.017(4)$ & $0.017(4)$ \\
\hline $\mathrm{C} 17$ & $0.047(3)$ & $0.035(3)$ & 0.039 (4) & $0.007(3)$ & $0.016(3)$ & $0.013(3)$ \\
\hline C19 & $0.037(4)$ & $0.037(4)$ & 0.032 & $0.012(3)$ & $0.007(3)$ & $0.003(3)$ \\
\hline $\mathrm{C} 20$ & $0.050(4)$ & $0.031(4)$ & 0.035 & $0.010(3)$ & $0.010(3)$ & $0.007(3)$ \\
\hline $\mathrm{C} 21$ & 0.055 (4) & 0.039 (4) & $0.033(3)$ & 0.009 (3) & $0.012(3)$ & $0.008(3)$ \\
\hline $\mathrm{C} 22$ & $0.061(5)$ & $0.059(5)$ & $0.032(3)$ & $0.021(4)$ & $0.008(3)$ & $0.010(3)$ \\
\hline $\mathrm{C} 23$ & $0.059(5)$ & $0.064(5)$ & $0.037(3)$ & $0.024(4)$ & $0.000(3)$ & $0.009(3)$ \\
\hline $\mathrm{C} 24$ & $0.055(4)$ & $0.051(4)$ & $0.035(3)$ & $0.026(3)$ & $0.008(3)$ & $0.012(3)$ \\
\hline $\mathrm{C} 25$ & $0.057(5)$ & $0.057(5)$ & $0.036(3)$ & 0.029 (4) & $0.006(3)$ & $0.008(3)$ \\
\hline $\mathrm{C} 26$ & $0.077(6)$ & $0.109(7)$ & $0.044(4)$ & $0.059(5)$ & $-0.001(4)$ & $0.006(4)$ \\
\hline $\mathrm{C} 27$ & $0.071(6)$ & $0.115(8)$ & $0.055(4)$ & $0.064(6)$ & $0.011(4)$ & $0.016(5)$ \\
\hline $\mathrm{C} 28$ & $0.058(5)$ & $0.078(6)$ & $0.042(4)$ & $0.037(4)$ & $0.010(4)$ & $0.002(4)$ \\
\hline $\mathrm{C} 29$ & $0.041(4)$ & $0.052(4)$ & $0.036(3)$ & 0.015 & $0.006(3)$ & -0.003 \\
\hline $\mathrm{C} 30$ & $0.056(4)$ & $0.042(4)$ & 0.035 & $0.005(3)$ & $0.014(3)$ & $0.002(3)$ \\
\hline C31 & $0.064(5)$ & $0.037(4)$ & $0.039(3)$ & $0.006(3)$ & 0.015 & $0.007(3)$ \\
\hline $\mathrm{C} 32$ & $0.065(5)$ & $0.037(4)$ & $0.044(4)$ & $0.004(4)$ & $0.018(3)$ & $-0.004(3)$ \\
\hline C33 & $0.069(5)$ & $0.044(4)$ & $0.041(4)$ & $0.000(3)$ & $0.024(4)$ & $0.001(3)$ \\
\hline $\mathrm{C} 34$ & $0.074(5)$ & $0.048(4)$ & $0.035(3)$ & $0.001(4)$ & $0.014(4)$ & $0.003(3)$ \\
\hline $\mathrm{C} 35$ & 0.063 & $0.053(4)$ & $0.040(3)$ & $0.011(4)$ & 0.015 & $0.009(3)$ \\
\hline $\mathrm{C} 36$ & $0.089(5)$ & $0.065(5)$ & $0.030(3)$ & $0.031(4)$ & $0.015(4)$ & $0.013(3)$ \\
\hline C37 & $0.038(3)$ & 0.035 & $0.025(3)$ & $0.006(3)$ & $0.002(3)$ & $0.003(3)$ \\
\hline C38 & $0.036(3)$ & $0.038(3)$ & $0.026(3)$ & $0.011(3)$ & $0.004(3)$ & $0.007(3)$ \\
\hline C39 & $0.034(3)$ & $0.037(3)$ & $0.027(3)$ & $0.001(2)$ & 0.000 & $0.011(3)$ \\
\hline $\mathrm{C} 40$ & $0.039(3)$ & $0.037(3)$ & $0.030(3)$ & $0.005(3)$ & $-0.002(3)$ & $0.008(3)$ \\
\hline $\mathrm{C} 41$ & $0.034(3)$ & $0.037(3)$ & $0.033(3)$ & $0.008(3)$ & $0.000(3)$ & $0.006(3)$ \\
\hline C42 & 0.033 & $0.037(3)$ & $0.027(3)$ & $0.004(2)$ & $0.000(3)$ & $0.010(3)$ \\
\hline $\mathrm{C} 43$ & $0.035(3)$ & $0.038(3)$ & $0.022(3)$ & $0.007(3)$ & $0.001(3)$ & $0.002(3)$ \\
\hline $\mathrm{C} 44$ & $0.037(3)$ & $0.043(4)$ & $0.038(4)$ & $0.012(3)$ & $0.000(3)$ & $0.007(3)$ \\
\hline $\mathrm{C} 45$ & $0.033(3)$ & $0.058(4)$ & $0.042(4)$ & $0.016(3)$ & $-0.003(3)$ & $0.008(3)$ \\
\hline $\mathrm{C} 46$ & 0.034 & $0.061(4)$ & $0.031(3)$ & $0.005(3)$ & $-0.001(3)$ & $0.010(3)$ \\
\hline $\mathrm{C} 47$ & $0.033(3)$ & $0.049(4)$ & $0.028(3)$ & $0.007(3)$ & $0.000(3)$ & $0.000(3)$ \\
\hline $\mathrm{C} 48$ & $0.033(3)$ & $0.038(3)$ & $0.029(3)$ & $0.003(3)$ & $0.002(3)$ & $0.013(3)$ \\
\hline $\mathrm{C} 49$ & $0.035(3)$ & $0.037(4)$ & $0.042(4)$ & $0.003(3)$ & $0.003(3)$ & $0.016(3)$ \\
\hline $\mathrm{C} 50$ & $0.034(3)$ & $0.048(4)$ & $0.042(4)$ & -0.001 & $0.004(3)$ & $0.014(3)$ \\
\hline $\mathrm{C} 51$ & $0.039(3)$ & $0.047(4)$ & $0.040(4)$ & $-0.006(3)$ & $-0.006(3)$ & $0.020(3)$ \\
\hline $\mathrm{C} 52$ & $0.047(4)$ & $0.035(4)$ & 0.039 (4) & $0.003(3)$ & $-0.006(3)$ & $0.008(3)$ \\
\hline C53 & $0.040(3)$ & $0.039(3)$ & $0.031(3)$ & $0.005(3)$ & $0.003(3)$ & $0.012(3)$ \\
\hline $\mathrm{C} 54$ & $0.035(3)$ & $0.039(3)$ & $0.050(4)$ & $0.004(3)$ & $-0.006(3)$ & $0.002(3)$ \\
\hline C55 & $0.026(3)$ & $0.039(4)$ & $0.036(3)$ & $0.006(3)$ & $0.003(3)$ & -0.003 \\
\hline C56 & $0.036(4)$ & $0.046(4)$ & $0.027(3)$ & $0.008(3)$ & $0.004(3)$ & $0.001(3)$ \\
\hline $\mathrm{C} 57$ & $0.042(4)$ & $0.046(4)$ & $0.029(3)$ & $0.005(3)$ & $0.001(3)$ & $0.003(3)$ \\
\hline
\end{tabular}




\begin{tabular}{|c|c|c|c|c|c|c|}
\hline C58 & $0.060(5)$ & $0.065(5)$ & $0.030(3)$ & $0.021(4)$ & 0.011 & $0.005(3)$ \\
\hline C59 & $0.060(5)$ & $0.070(5)$ & $0.034(3)$ & $0.026(4)$ & $0.013(3)$ & $0.007(3)$ \\
\hline C60 & $0.038(4)$ & $0.055(4)$ & $0.035(3)$ & 0.018 (3) & $0.010(3)$ & $0.003(3)$ \\
\hline C61 & $0.044(4)$ & $0.053(4)$ & $0.037(3)$ & $0.019(3)$ & 0.009 (3) & $0.005(3)$ \\
\hline C62 & $0.070(5)$ & $0.091(6)$ & 0.049 (4) & $0.049(5)$ & $0.015(4)$ & $0.010(4)$ \\
\hline C63 & $0.054(5)$ & $0.095(7)$ & $0.062(4)$ & $0.044(5)$ & $0.000(4)$ & $0.002(5)$ \\
\hline C64 & $0.043(4)$ & $0.072(6)$ & $0.057(4)$ & $0.018(4)$ & $-0.014(4)$ & $0.010(4)$ \\
\hline C65 & $0.038(4)$ & $0.048(4)$ & $0.045(4)$ & $0.006(3)$ & $-0.011(3)$ & -0.001 \\
\hline C66 & $0.042(4)$ & $0.046(4)$ & 0.028 & -0.002 & $0.002(3)$ & $0.006(3)$ \\
\hline C67 & $0.043(4)$ & $0.050(4)$ & $0.031(3)$ & 0.001 & $0.001(3)$ & $0.008(3)$ \\
\hline C68 & $0.041(4)$ & $0.060(5)$ & $0.042(3)$ & $-0.005(3)$ & $-0.007(3)$ & $0.018(3)$ \\
\hline C69 & $0.056(4)$ & $0.059(5)$ & $0.033(3)$ & -0.009 & -0.009 & $0.015(3)$ \\
\hline $\mathrm{C} 70$ & $0.064(5)$ & $0.055(5)$ & $0.028(3)$ & -0.003 & $0.005(3)$ & $0.005(3)$ \\
\hline $\mathrm{C} 71$ & $0.053(4)$ & 0.048 (4) & $0.033(3)$ & 0.000 & 0.010 & $0.006(3)$ \\
\hline C72 & $0.068(5)$ & $0.071(5)$ & $0.031(3)$ & 0.017 (4) & $0.014(3)$ & $0.006(3)$ \\
\hline $\mathrm{C} 82$ & $0.127(10)$ & 0.159 (11) & $0.058(6)$ & $0.002(8)$ & $0.009(6)$ & $0.019(6)$ \\
\hline $\mathrm{C} 85$ & $0.083(5)$ & $0.098(6)$ & $0.032(4)$ & $0.035(4)$ & $0.001(4)$ & $0.010(4)$ \\
\hline $\mathrm{C} 86$ & $0.054(4)$ & $0.096(6)$ & $0.039(4)$ & $0.024(4)$ & $0.002(4)$ & $0.004(4)$ \\
\hline C87 & $0.055(5)$ & $0.126(7)$ & $0.038(4)$ & $0.035(4)$ & $0.002(4)$ & $0.006(5)$ \\
\hline $\mathrm{C} 88$ & $0.058(5)$ & $0.127(7)$ & $0.047(5)$ & $0.020(5)$ & $0.006(4)$ & $0.006(5)$ \\
\hline $\mathrm{C} 89$ & $0.055(5)$ & $0.149(9)$ & $0.066(6)$ & $0.029(5)$ & $-0.003(5)$ & $0.005(7)$ \\
\hline $\mathrm{C} 90$ & $0.069(7)$ & $0.170(10)$ & $0.075(7)$ & $0.001(7)$ & $0.010(6)$ & $0.015(8)$ \\
\hline C91 & $0.127(8)$ & $0.066(5)$ & $0.049(5)$ & $0.024(5)$ & -0.007 (5) & $0.010(4)$ \\
\hline C92 & $0.119(8)$ & $0.069(6)$ & $0.070(6)$ & $0.017(5)$ & $0.002(6)$ & $0.008(5)$ \\
\hline C97 & $0.043(4)$ & $0.056(5)$ & $0.067(4)$ & $0.005(4)$ & $0.008(4)$ & -0.021 \\
\hline C98 & $0.050(5)$ & $0.090(6)$ & $0.047(4)$ & $-0.006(4)$ & 0.010 & -0.012 \\
\hline C99 & $0.080(7)$ & $0.162(11)$ & $0.061(5)$ & $-0.017(7)$ & $0.025(5)$ & $-0.028(6)$ \\
\hline $\mathrm{C} 100$ & $0.089(8)$ & $0.222(13)$ & $0.043(5)$ & $-0.032(8)$ & $0.018(5)$ & $-0.011(6)$ \\
\hline C103 & $0.055(5)$ & $0.034(4)$ & $0.106(5)$ & $-0.002(4)$ & $-0.031(5)$ & $0.019(4)$ \\
\hline C104 & $0.107(7)$ & $0.053(5)$ & $0.085(5)$ & $-0.029(5)$ & $-0.045(5)$ & $0.037(5)$ \\
\hline C109 & $0.103(6)$ & $0.067(4)$ & $0.032(4)$ & $0.024(4)$ & $0.015(4)$ & 0.003 (4) \\
\hline $\mathrm{C} 110$ & $0.127(8)$ & $0.052(4)$ & $0.056(5)$ & $0.012(5)$ & $0.026(5)$ & $0.010(4)$ \\
\hline C115 & $0.063(5)$ & $0.105(6)$ & $0.030(4)$ & $0.013(4)$ & $0.013(4)$ & $0.007(4)$ \\
\hline C116 & $0.053(5)$ & $0.118(6)$ & $0.039(4)$ & $-0.005(5)$ & $0.014(4)$ & $0.009(5)$ \\
\hline C18 & $0.043(5)$ & $0.036(5)$ & $0.020(5)$ & $0.003(3)$ & $0.006(4)$ & $0.010(4)$ \\
\hline $\mathrm{C} 73$ & $0.066(7)$ & $0.048(7)$ & $0.024(5)$ & $0.005(5)$ & $-0.004(5)$ & $0.014(5)$ \\
\hline $\mathrm{C} 74$ & $0.072(8)$ & $0.082(8)$ & $0.039(6)$ & $0.032(7)$ & $0.012(6)$ & $0.023(6)$ \\
\hline $\mathrm{C} 75 \mathrm{~A}$ & $0.082(9)$ & $0.084(8)$ & $0.074(9)$ & $0.017(7)$ & $0.011(7)$ & $0.030(7)$ \\
\hline C76 & 0.107 (11) & $0.102(10)$ & $0.066(9)$ & $-0.011(9)$ & $0.016(9)$ & $0.024(9)$ \\
\hline $\mathrm{C} 77$ & $0.129(14)$ & $0.084(9)$ & $0.063(10)$ & $-0.060(9)$ & $-0.021(9)$ & $0.024(8)$ \\
\hline $\mathrm{C} 78$ & $0.126(14)$ & $0.108(12)$ & $0.041(8)$ & $-0.063(12)$ & $0.010(8)$ & $-0.016(7)$ \\
\hline C79 & $0.030(5)$ & $0.038(5)$ & $0.025(4)$ & $0.005(4)$ & -0.002 & $0.009(4)$ \\
\hline C80 & $0.044(6)$ & $0.067(8)$ & $0.018(5)$ & $0.027(6)$ & $0.007(4)$ & $0.007(5)$ \\
\hline C81 & $0.074(8)$ & $0.096(10)$ & $0.042(6)$ & $0.022(7)$ & $0.034(6)$ & $0.014(6)$ \\
\hline $\mathrm{C} 83$ & $0.147(16)$ & $0.106(12)$ & $0.092(10)$ & $-0.004(11)$ & $0.000(10)$ & $0.044(8)$ \\
\hline C84 & $0.21(3)$ & $0.43(3)$ & $0.131(14)$ & $0.20(2)$ & 0.069 (14) & $0.19(2)$ \\
\hline C101 & $0.099(12)$ & $0.157(13)$ & $0.073(6)$ & $-0.028(10)$ & $0.045(7)$ & $-0.009(7)$ \\
\hline C102 & $0.215(19)$ & $0.111(12)$ & $0.055(8)$ & $0.042(11)$ & $0.008(11)$ & $-0.039(8)$ \\
\hline
\end{tabular}




\begin{tabular}{lllllll} 
& & & & & \\
C105 & $0.15(3)$ & $0.079(13)$ & $0.116(16)$ & $-0.001(11)$ & $-0.030(11)$ & $0.055(14)$ \\
C111 & $0.090(13)$ & $0.054(6)$ & $0.033(8)$ & $0.030(6)$ & $0.013(10)$ & $0.007(7)$ \\
C112 & $0.097(11)$ & $0.048(7)$ & $0.059(10)$ & $0.013(8)$ & $0.006(9)$ & $-0.005(7)$ \\
C113 & $0.119(16)$ & $0.048(7)$ & $0.064(10)$ & $0.020(9)$ & $-0.015(13)$ & $-0.006(8)$ \\
C114 & $0.119(15)$ & $0.070(11)$ & $0.066(10)$ & $0.003(12)$ & $-0.002(11)$ & $0.019(9)$ \\
C117 & $0.056(8)$ & $0.098(12)$ & $0.044(10)$ & $0.008(8)$ & $0.006(7)$ & $0.025(11)$ \\
C118 & $0.039(8)$ & $0.109(12)$ & $0.070(11)$ & $0.000(8)$ & $-0.006(7)$ & $0.053(12)$ \\
C119 & $0.035(7)$ & $0.150(14)$ & $0.071(11)$ & $-0.017(10)$ & $-0.013(8)$ & $0.056(11)$ \\
C120 & $0.053(11)$ & $0.144(14)$ & $0.074(12)$ & $-0.018(11)$ & $0.006(10)$ & $0.043(13)$ \\
C211 & $0.10(2)$ & $0.058(8)$ & $0.08(2)$ & $0.018(11)$ & $0.010(18)$ & $0.013(12)$ \\
C212 & $0.084(17)$ & $0.049(9)$ & $0.081(17)$ & $0.004(11)$ & $-0.018(13)$ & $0.008(11)$ \\
C213 & $0.12(2)$ & $0.049(11)$ & $0.09(2)$ & $0.000(14)$ & $0.003(19)$ & $0.006(13)$ \\
C214 & $0.12(2)$ & $0.075(18)$ & $0.10(2)$ & $-0.025(17)$ & $-0.025(18)$ & $0.037(17)$ \\
C201 & $0.099(12)$ & $0.157(13)$ & $0.073(6)$ & $-0.028(10)$ & $0.045(7)$ & $-0.009(7)$ \\
C217 & $0.033(11)$ & $0.094(15)$ & $0.08(2)$ & $0.020(12)$ & $0.028(13)$ & $0.013(18)$ \\
C218 & $0.030(10)$ & $0.102(15)$ & $0.073(15)$ & $0.008(10)$ & $-0.003(10)$ & $0.032(15)$ \\
C219 & $0.050(12)$ & $0.116(19)$ & $0.070(15)$ & $-0.003(12)$ & $0.010(11)$ & $0.017(14)$ \\
C220 & $0.043(13)$ & $0.16(3)$ & $0.065(16)$ & $0.010(17)$ & $0.004(12)$ & $0.01(2)$ \\
C205 & $0.15(3)$ & $0.079(13)$ & $0.116(16)$ & $-0.001(11)$ & $-0.030(11)$ & $0.055(14)$ \\
C83B & $0.25(4)$ & $0.33(3)$ & $0.164(19)$ & $0.05(3)$ & $0.08(2)$ & $0.20(3)$ \\
C84B & $0.10(2)$ & $0.20(3)$ & $0.056(17)$ & $0.10(2)$ & $0.037(16)$ & $0.062(19)$ \\
& & & & & & \\
\hline
\end{tabular}

Geometric parameters $\left(\AA,{ }^{\circ}\right)$

\begin{tabular}{llll}
\hline Ir1-C11 & $2.5123(15)$ & $\mathrm{C} 116-\mathrm{H} 11 \mathrm{G}$ & 0.9900 \\
$\mathrm{Ir} 1-\mathrm{C} 12$ & $2.5088(16)$ & $\mathrm{C} 116-\mathrm{H} 11 \mathrm{H}$ & 0.9900 \\
$\mathrm{Ir} 1-\mathrm{N} 3$ & $2.031(5)$ & $\mathrm{C} 116-\mathrm{H} 11 \mathrm{I}$ & 0.9900 \\
$\mathrm{Ir} 1-\mathrm{N} 4$ & $2.034(5)$ & $\mathrm{C} 116-\mathrm{H} 11 \mathrm{~J}$ & 0.9900 \\
$\mathrm{Ir} 1-\mathrm{C} 37$ & $2.001(6)$ & $\mathrm{C} 116-\mathrm{C} 117$ & $1.545(11)$ \\
$\mathrm{Ir} 1-\mathrm{C} 55$ & $2.015(6)$ & $\mathrm{C} 116-\mathrm{C} 217$ & $1.555(13)$ \\
$\mathrm{I} 2-\mathrm{C} 11$ & $2.4854(16)$ & $\mathrm{C} 18-\mathrm{C} 73$ & $1.546(10)$ \\
$\mathrm{Ir} 2-\mathrm{C} 12$ & $2.5218(15)$ & $\mathrm{C} 18-\mathrm{C} 79$ & $1.536(9)$ \\
$\mathrm{Ir} 2-\mathrm{N} 1$ & $2.043(5)$ & $\mathrm{C} 18-\mathrm{C} 73 \mathrm{~B}$ & $1.14(2)$ \\
$\mathrm{I} 2-\mathrm{N} 2$ & $2.034(5)$ & $\mathrm{C} 18-\mathrm{C} 79 \mathrm{~B}$ & $1.99(2)$ \\
$\mathrm{Ir} 2-\mathrm{C} 1$ & $1.983(7)$ & $\mathrm{C} 18-\mathrm{C} 18 \mathrm{~B}$ & $0.504(19)$ \\
$\mathrm{Ir} 2-\mathrm{C} 19$ & $1.994(6)$ & $\mathrm{C} 73-\mathrm{H} 73 \mathrm{~A}$ & 0.9900 \\
$\mathrm{~F} 1-\mathrm{C} 10$ & $1.364(7)$ & $\mathrm{C} 73-\mathrm{H} 73 \mathrm{~B}$ & 0.9900 \\
$\mathrm{~F} 2-\mathrm{C} 28$ & $1.339(7)$ & $\mathrm{C} 73-\mathrm{C} 74$ & $1.529(11)$ \\
$\mathrm{F} 3-\mathrm{C} 46$ & $1.365(7)$ & $\mathrm{C} 73-\mathrm{C} 75 \mathrm{~B}$ & $1.97(2)$ \\
$\mathrm{F} 4-\mathrm{C} 64$ & $1.368(8)$ & $\mathrm{C} 73-\mathrm{C} 73 \mathrm{~B}$ & $0.86(2)$ \\
$\mathrm{N} 1-\mathrm{C} 7$ & $1.358(8)$ & $\mathrm{C} 73-\mathrm{C} 74 \mathrm{~B}$ & $0.66(2)$ \\
$\mathrm{N} 1-\mathrm{C} 11$ & $1.335(7)$ & $\mathrm{C} 73-\mathrm{C} 18 \mathrm{~B}$ & $2.02(2)$ \\
$\mathrm{N} 2-\mathrm{C} 25$ & $1.359(8)$ & $\mathrm{C} 74-\mathrm{H} 74 \mathrm{~A}$ & 0.9900 \\
$\mathrm{~N} 2-\mathrm{C} 29$ & $1.366(7)$ & $\mathrm{C} 74-\mathrm{H} 74 \mathrm{~B}$ & 0.9900 \\
$\mathrm{~N} 3-\mathrm{C} 43$ & $1.345(7)$ & $\mathrm{C} 74-\mathrm{C} 75 \mathrm{~A}$ & $1.604(16)$ \\
$\mathrm{N} 3-\mathrm{C} 47$ & $1.360(7)$ & $\mathrm{C} 74-\mathrm{C} 75 \mathrm{~B}$ & $1.94(3)$ \\
$\mathrm{N} 4-\mathrm{C} 61$ & $1.377(8)$ & $\mathrm{C} 74-\mathrm{C} 74 \mathrm{~B}$ & $1.06(3)$ \\
$\mathrm{N} 4-\mathrm{C} 65$ & $1.345(8)$ & $\mathrm{C} 75 \mathrm{~A}-\mathrm{H} 75 \mathrm{~A}$ & 0.9900 \\
& & &
\end{tabular}




\begin{tabular}{|c|c|c|c|}
\hline $\mathrm{C} 1-\mathrm{C} 2$ & $1.398(8)$ & $\mathrm{C} 75 \mathrm{~A}-\mathrm{H} 75 \mathrm{~B}$ & 0.9900 \\
\hline $\mathrm{C} 1-\mathrm{C} 6$ & $1.419(8)$ & $\mathrm{C} 75 \mathrm{~A}-\mathrm{C} 76$ & $1.609(18)$ \\
\hline $\mathrm{C} 2-\mathrm{H} 2$ & 0.9500 & $\mathrm{C} 76-\mathrm{H} 76 \mathrm{~A}$ & 0.9900 \\
\hline $\mathrm{C} 2-\mathrm{C} 3$ & $1.389(8)$ & $\mathrm{C} 76-\mathrm{H} 76 \mathrm{~B}$ & 0.9900 \\
\hline $\mathrm{C} 3-\mathrm{C} 4$ & $1.399(8)$ & $\mathrm{C} 76-\mathrm{C} 77$ & $1.513(12)$ \\
\hline $\mathrm{C} 3-\mathrm{C} 12$ & $1.470(8)$ & C77-H77A & 0.9900 \\
\hline $\mathrm{C} 4-\mathrm{C} 5$ & $1.377(8)$ & C77-H77B & 0.9900 \\
\hline $\mathrm{C} 4-\mathrm{C} 18$ & $1.545(10)$ & $\mathrm{C} 77-\mathrm{C} 78$ & $1.525(13)$ \\
\hline $\mathrm{C} 4-\mathrm{C} 18 \mathrm{~B}$ & $1.516(16)$ & C78-H78A & 0.9800 \\
\hline $\mathrm{C} 5-\mathrm{H} 5$ & 0.9500 & C78-H78B & 0.9800 \\
\hline $\mathrm{C} 5-\mathrm{C} 6$ & $1.396(9)$ & $\mathrm{C} 78-\mathrm{H} 78 \mathrm{C}$ & 0.9800 \\
\hline $\mathrm{C} 6-\mathrm{C} 7$ & $1.445(9)$ & $\mathrm{C} 79-\mathrm{H} 79 \mathrm{~A}$ & 0.9900 \\
\hline $\mathrm{C} 7-\mathrm{C} 8$ & $1.400(9)$ & C79-H79B & 0.9900 \\
\hline $\mathrm{C} 8-\mathrm{H} 8$ & 0.9500 & $\mathrm{C} 79-\mathrm{C} 80$ & $1.540(9)$ \\
\hline $\mathrm{C} 8-\mathrm{C} 9$ & $1.375(9)$ & $\mathrm{C} 79-\mathrm{C} 73 \mathrm{~B}$ & $1.66(2)$ \\
\hline C9-H9 & 0.9500 & $\mathrm{C} 79-\mathrm{C} 79 \mathrm{~B}$ & $0.93(2)$ \\
\hline $\mathrm{C} 9-\mathrm{C} 10$ & $1.354(9)$ & $\mathrm{C} 79-\mathrm{C} 18 \mathrm{~B}$ & $1.265(17)$ \\
\hline $\mathrm{C} 10-\mathrm{C} 11$ & $1.373(9)$ & $\mathrm{C} 80-\mathrm{H} 80 \mathrm{~A}$ & 0.9900 \\
\hline $\mathrm{C} 11-\mathrm{H} 11$ & 0.9500 & $\mathrm{C} 80-\mathrm{H} 80 \mathrm{~B}$ & 0.9900 \\
\hline $\mathrm{C} 12-\mathrm{C} 13$ & $1.379(8)$ & $\mathrm{C} 80-\mathrm{C} 81$ & $1.528(10)$ \\
\hline $\mathrm{C} 12-\mathrm{C} 17$ & $1.380(9)$ & $\mathrm{C} 80-\mathrm{C} 79 \mathrm{~B}$ & $0.68(2)$ \\
\hline $\mathrm{C} 13-\mathrm{H} 13$ & 0.9500 & $\mathrm{C} 80-\mathrm{C} 80 \mathrm{~B}$ & $1.10(3)$ \\
\hline $\mathrm{C} 13-\mathrm{C} 14$ & $1.387(9)$ & $\mathrm{C} 81-\mathrm{H} 81 \mathrm{~A}$ & 0.9900 \\
\hline C14-H14 & 0.9500 & C81-H81B & 0.9900 \\
\hline $\mathrm{C} 14-\mathrm{C} 15$ & $1.378(10)$ & $\mathrm{C} 81-\mathrm{C} 79 \mathrm{~B}$ & $2.00(2)$ \\
\hline $\mathrm{C} 15-\mathrm{H} 15$ & 0.9500 & $\mathrm{C} 81-\mathrm{C} 81 \mathrm{~B}$ & $1.82(4)$ \\
\hline $\mathrm{C} 15-\mathrm{C} 16$ & $1.368(9)$ & $\mathrm{C} 83-\mathrm{H} 83 \mathrm{~A}$ & 0.9900 \\
\hline $\mathrm{C} 16-\mathrm{H} 16$ & 0.9500 & $\mathrm{C} 83-\mathrm{H} 83 \mathrm{~B}$ & 0.9900 \\
\hline $\mathrm{C} 16-\mathrm{C} 17$ & $1.380(9)$ & $\mathrm{C} 83-\mathrm{C} 84$ & $1.579(12)$ \\
\hline $\mathrm{C} 17-\mathrm{C} 18$ & $1.551(10)$ & $\mathrm{C} 83-\mathrm{C} 81 \mathrm{~B}$ & $1.24(3)$ \\
\hline $\mathrm{C} 17-\mathrm{C} 18 \mathrm{~B}$ & $1.563(16)$ & $\mathrm{C} 83-\mathrm{C} 83 \mathrm{~B}$ & $1.49(4)$ \\
\hline $\mathrm{C} 19-\mathrm{C} 20$ & $1.388(8)$ & $\mathrm{C} 84-\mathrm{H} 84 \mathrm{~A}$ & 0.9800 \\
\hline $\mathrm{C} 19-\mathrm{C} 24$ & $1.417(9)$ & C $84-H 84 B$ & 0.9800 \\
\hline $\mathrm{C} 20-\mathrm{H} 20$ & 0.9500 & $\mathrm{C} 84-\mathrm{H} 84 \mathrm{C}$ & 0.9800 \\
\hline $\mathrm{C} 20-\mathrm{C} 21$ & $1.375(9)$ & $\mathrm{C} 84-\mathrm{C} 83 \mathrm{~B}$ & $1.01(5)$ \\
\hline $\mathrm{C} 21-\mathrm{C} 22$ & $1.388(9)$ & C93-H93A & 0.9900 \\
\hline $\mathrm{C} 21-\mathrm{C} 30$ & $1.461(8)$ & С93-H93B & 0.9900 \\
\hline $\mathrm{C} 22-\mathrm{C} 23$ & $1.383(9)$ & C93-C94 & $1.505(12)$ \\
\hline $\mathrm{C} 22-\mathrm{C} 36$ & $1.527(9)$ & $\mathrm{C} 94-\mathrm{H} 94 \mathrm{~A}$ & 0.9900 \\
\hline $\mathrm{C} 23-\mathrm{H} 23$ & 0.9500 & C94-H94B & 0.9900 \\
\hline $\mathrm{C} 23-\mathrm{C} 24$ & $1.382(9)$ & C94-C95 & $1.556(12)$ \\
\hline $\mathrm{C} 24-\mathrm{C} 25$ & $1.462(9)$ & C95-H95A & 0.9900 \\
\hline $\mathrm{C} 25-\mathrm{C} 26$ & $1.389(9)$ & C95-H95B & 0.9900 \\
\hline $\mathrm{C} 26-\mathrm{H} 26$ & 0.9500 & C95-C96 & $1.516(12)$ \\
\hline $\mathrm{C} 26-\mathrm{C} 27$ & $1.379(10)$ & C96-H96A & 0.9800 \\
\hline $\mathrm{C} 27-\mathrm{H} 27$ & 0.9500 & C96-H96B & 0.9800 \\
\hline $\mathrm{C} 27-\mathrm{C} 28$ & $1.382(10)$ & C96-H96C & 0.9800 \\
\hline $\mathrm{C} 28-\mathrm{C} 29$ & $1.359(9)$ & $\mathrm{C} 101-\mathrm{H} 10 \mathrm{~K}$ & 0.9900 \\
\hline
\end{tabular}




\begin{tabular}{|c|c|c|c|}
\hline $\mathrm{C} 29-\mathrm{H} 29$ & 0.9500 & $\mathrm{C} 101-\mathrm{H} 10 \mathrm{~L}$ & 0.9900 \\
\hline $\mathrm{C} 30-\mathrm{C} 31$ & $1.414(9)$ & $\mathrm{C} 101-\mathrm{C} 102$ & $1.492(12)$ \\
\hline $\mathrm{C} 30-\mathrm{C} 35$ & $1.393(10)$ & $\mathrm{C} 102-\mathrm{H} 10 \mathrm{M}$ & 0.9800 \\
\hline C31-H31 & 0.9500 & $\mathrm{C} 102-\mathrm{H} 10 \mathrm{~N}$ & 0.9800 \\
\hline C31-C32 & $1.386(9)$ & $\mathrm{C} 102-\mathrm{H} 10 \mathrm{O}$ & 0.9800 \\
\hline C32-H32 & 0.9500 & $\mathrm{C} 105-\mathrm{H} 10 \mathrm{P}$ & 0.9900 \\
\hline $\mathrm{C} 32-\mathrm{C} 33$ & $1.372(10)$ & $\mathrm{C} 105-\mathrm{H} 10 \mathrm{Q}$ & 0.9900 \\
\hline C33-H33 & 0.9500 & $\mathrm{C} 105-\mathrm{C} 106$ & $1.504(13)$ \\
\hline C33-C34 & $1.364(10)$ & $\mathrm{C} 106-\mathrm{H} 10 \mathrm{R}$ & 0.9900 \\
\hline C34-H34 & 0.9500 & C106-H10S & 0.9900 \\
\hline $\mathrm{C} 34-\mathrm{C} 35$ & $1.398(9)$ & $\mathrm{C} 106-\mathrm{C} 107$ & $1.484(11)$ \\
\hline $\mathrm{C} 35-\mathrm{C} 36$ & $1.513(10)$ & $\mathrm{C} 107-\mathrm{H} 10 \mathrm{~T}$ & 0.9900 \\
\hline C36-C85 & $1.542(9)$ & C107-H10U & 0.9900 \\
\hline C36-C91 & $1.542(9)$ & $\mathrm{C} 107-\mathrm{C} 108$ & $1.520(11)$ \\
\hline $\mathrm{C} 37-\mathrm{C} 38$ & $1.399(8)$ & $\mathrm{C} 108-\mathrm{H} 10 \mathrm{~V}$ & 0.9800 \\
\hline $\mathrm{C} 37-\mathrm{C} 42$ & $1.418(8)$ & $\mathrm{C} 108-\mathrm{H} 10 \mathrm{~W}$ & 0.9800 \\
\hline C $38-\mathrm{H} 38$ & 0.9500 & C108-H10X & 0.9800 \\
\hline C $38-$ C 39 & $1.387(8)$ & C111-H11K & 0.9900 \\
\hline $\mathrm{C} 39-\mathrm{C} 40$ & $1.403(8)$ & C111-H11L & 0.9900 \\
\hline C $39-\mathrm{C} 48$ & $1.460(8)$ & C111-C112 & $1.530(12)$ \\
\hline $\mathrm{C} 40-\mathrm{C} 41$ & $1.382(8)$ & $\mathrm{C} 112-\mathrm{H} 11 \mathrm{M}$ & 0.9900 \\
\hline $\mathrm{C} 40-\mathrm{C} 54$ & $1.506(9)$ & $\mathrm{C} 112-\mathrm{H} 11 \mathrm{~N}$ & 0.9900 \\
\hline $\mathrm{C} 41-\mathrm{H} 41$ & 0.9500 & $\mathrm{C} 112-\mathrm{C} 113$ & $1.501(12)$ \\
\hline $\mathrm{C} 41-\mathrm{C} 42$ & $1.394(8)$ & $\mathrm{C} 113-\mathrm{H} 11 \mathrm{O}$ & 0.9900 \\
\hline $\mathrm{C} 42-\mathrm{C} 43$ & $1.463(8)$ & C113-H11P & 0.9900 \\
\hline $\mathrm{C} 43-\mathrm{C} 44$ & $1.379(8)$ & $\mathrm{C} 113-\mathrm{C} 114$ & $1.514(12)$ \\
\hline $\mathrm{C} 44-\mathrm{H} 44$ & 0.9500 & C114-H11Q & 0.9800 \\
\hline C44-C45 & $1.379(9)$ & C114-H11R & 0.9800 \\
\hline $\mathrm{C} 45-\mathrm{H} 45$ & 0.9500 & $\mathrm{C} 114-\mathrm{H} 11 \mathrm{~S}$ & 0.9800 \\
\hline $\mathrm{C} 45-\mathrm{C} 46$ & $1.356(9)$ & $\mathrm{C} 117-\mathrm{H} 11 \mathrm{~T}$ & 0.9900 \\
\hline $\mathrm{C} 46-\mathrm{C} 47$ & $1.363(9)$ & C117- H11U & 0.9900 \\
\hline C47-H47 & 0.9500 & $\mathrm{C} 117-\mathrm{C} 118$ & $1.497(12)$ \\
\hline $\mathrm{C} 48-\mathrm{C} 49$ & $1.378(8)$ & $\mathrm{C} 118-\mathrm{H} 11 \mathrm{~V}$ & 0.9900 \\
\hline $\mathrm{C} 48-\mathrm{C} 53$ & $1.407(8)$ & C118-H11W & 0.9900 \\
\hline $\mathrm{C} 49-\mathrm{H} 49$ & 0.9500 & $\mathrm{C} 118-\mathrm{C} 119$ & $1.516(12)$ \\
\hline $\mathrm{C} 49-\mathrm{C} 50$ & $1.409(8)$ & C119-H11X & 0.9900 \\
\hline $\mathrm{C} 50-\mathrm{H} 50$ & 0.9500 & C119- H11Y & 0.9900 \\
\hline $\mathrm{C} 50-\mathrm{C} 51$ & $1.375(9)$ & $\mathrm{C} 119-\mathrm{C} 120$ & $1.517(13)$ \\
\hline C51-H51 & 0.9500 & $\mathrm{C} 120-\mathrm{H} 12 \mathrm{~A}$ & 0.9800 \\
\hline C51-C52 & $1.385(9)$ & $\mathrm{C} 120-\mathrm{H} 12 \mathrm{~B}$ & 0.9800 \\
\hline C52-H52 & 0.9500 & $\mathrm{C} 120-\mathrm{H} 12 \mathrm{C}$ & 0.9800 \\
\hline $\mathrm{C} 52-\mathrm{C} 53$ & $1.375(9)$ & $\mathrm{C} 96 \mathrm{~B}-\mathrm{H} 96 \mathrm{D}$ & 0.9800 \\
\hline C53-C54 & $1.512(9)$ & C96B-H96E & 0.9800 \\
\hline C54-C97 & $1.549(8)$ & C96B-H96F & 0.9800 \\
\hline $\mathrm{C} 54-\mathrm{C} 103$ & $1.535(8)$ & $\mathrm{C} 96 \mathrm{~B}-\mathrm{C} 95 \mathrm{~B}$ & $1.461(14)$ \\
\hline $\mathrm{C} 55-\mathrm{C} 56$ & $1.383(8)$ & $\mathrm{C} 211-\mathrm{H} 21 \mathrm{~A}$ & 0.9900 \\
\hline $\mathrm{C} 55-\mathrm{C} 60$ & $1.406(8)$ & $\mathrm{C} 211-\mathrm{H} 21 \mathrm{~B}$ & 0.9900 \\
\hline C56-H56 & 0.9500 & $\mathrm{C} 211-\mathrm{C} 212$ & $1.519(14)$ \\
\hline
\end{tabular}




\begin{tabular}{|c|c|c|c|}
\hline $\mathrm{C} 56-\mathrm{C} 57$ & $1.388(8)$ & $\mathrm{C} 212-\mathrm{H} 21 \mathrm{C}$ & 0.9900 \\
\hline $\mathrm{C} 57-\mathrm{C} 58$ & $1.398(9)$ & C212-H21D & 0.9900 \\
\hline C57-C66 & $1.465(9)$ & $\mathrm{C} 212-\mathrm{C} 213$ & $1.504(14)$ \\
\hline C58-C59 & $1.366(9)$ & $\mathrm{C} 213-\mathrm{H} 21 \mathrm{E}$ & 0.9900 \\
\hline $\mathrm{C} 58-\mathrm{C} 72$ & $1.535(9)$ & $\mathrm{C} 213-\mathrm{H} 21 \mathrm{~F}$ & 0.9900 \\
\hline C59-H59 & 0.9500 & $\mathrm{C} 213-\mathrm{C} 214$ & $1.522(14)$ \\
\hline C59-C60 & $1.398(9)$ & $\mathrm{C} 214-\mathrm{H} 21 \mathrm{G}$ & 0.9800 \\
\hline $\mathrm{C} 60-\mathrm{C} 61$ & $1.457(9)$ & $\mathrm{C} 214-\mathrm{H} 21 \mathrm{H}$ & 0.9800 \\
\hline C61-C62 & $1.379(9)$ & $\mathrm{C} 214-\mathrm{H} 21 \mathrm{I}$ & 0.9800 \\
\hline C62-H62 & 0.9500 & C93B-H93C & 0.9900 \\
\hline C62-C63 & $1.371(10)$ & C93B-H93D & 0.9900 \\
\hline C63-H63 & 0.9500 & C93B-C94B & $1.566(14)$ \\
\hline C63-C64 & $1.361(10)$ & C94B-H94C & 0.9900 \\
\hline C64-C65 & $1.378(9)$ & C94B-H94D & 0.9900 \\
\hline C65-H65 & 0.9500 & $\mathrm{C} 94 \mathrm{~B}-\mathrm{C} 95 \mathrm{~B}$ & $1.500(14)$ \\
\hline $\mathrm{C} 66-\mathrm{C} 67$ & $1.383(9)$ & $\mathrm{C} 95 \mathrm{~B}-\mathrm{H} 95 \mathrm{C}$ & 0.9900 \\
\hline $\mathrm{C} 66-\mathrm{C} 71$ & $1.401(8)$ & $\mathrm{C} 95 \mathrm{~B}-\mathrm{H} 95 \mathrm{D}$ & 0.9900 \\
\hline C67-H67 & 0.9500 & $\mathrm{C} 201-\mathrm{H} 20 \mathrm{~A}$ & 0.9900 \\
\hline C67-C68 & $1.400(9)$ & $\mathrm{C} 201-\mathrm{H} 20 \mathrm{~B}$ & 0.9900 \\
\hline C68-H68 & 0.9500 & C201-C202 & $1.46(2)$ \\
\hline C68-C69 & $1.388(9)$ & $\mathrm{C} 202-\mathrm{H} 20 \mathrm{C}$ & 0.9800 \\
\hline C69-H69 & 0.9500 & $\mathrm{C} 202-\mathrm{H} 20 \mathrm{D}$ & 0.9800 \\
\hline $\mathrm{C} 69-\mathrm{C} 70$ & $1.354(10)$ & $\mathrm{C} 202-\mathrm{H} 20 \mathrm{E}$ & 0.9800 \\
\hline $\mathrm{C} 70-\mathrm{H} 70$ & 0.9500 & $\mathrm{C} 217-\mathrm{H} 21 \mathrm{~J}$ & 0.9900 \\
\hline $\mathrm{C} 70-\mathrm{C} 71$ & $1.383(9)$ & $\mathrm{C} 217-\mathrm{H} 21 \mathrm{~K}$ & 0.9900 \\
\hline $\mathrm{C} 71-\mathrm{C} 72$ & $1.525(10)$ & $\mathrm{C} 217-\mathrm{C} 218$ & $1.530(13)$ \\
\hline $\mathrm{C} 72-\mathrm{C} 109$ & $1.526(8)$ & $\mathrm{C} 218-\mathrm{H} 21 \mathrm{~L}$ & 0.9900 \\
\hline $\mathrm{C} 72-\mathrm{C} 115$ & $1.548(8)$ & $\mathrm{C} 218-\mathrm{H} 21 \mathrm{M}$ & 0.9900 \\
\hline $\mathrm{C} 82-\mathrm{H} 82 \mathrm{C}$ & 0.9900 & $\mathrm{C} 218-\mathrm{C} 219$ & $1.533(13)$ \\
\hline $\mathrm{C} 82-\mathrm{H} 82 \mathrm{D}$ & 0.9900 & $\mathrm{C} 219-\mathrm{H} 21 \mathrm{~N}$ & 0.9900 \\
\hline $\mathrm{C} 82-\mathrm{H} 82 \mathrm{~A}$ & 0.9900 & $\mathrm{C} 219-\mathrm{H} 21 \mathrm{O}$ & 0.9900 \\
\hline $\mathrm{C} 82-\mathrm{H} 82 \mathrm{~B}$ & 0.9900 & $\mathrm{C} 219-\mathrm{C} 220$ & $1.496(14)$ \\
\hline $\mathrm{C} 82-\mathrm{C} 81$ & $1.565(11)$ & $\mathrm{C} 220-\mathrm{H} 22 \mathrm{~A}$ & 0.9800 \\
\hline $\mathrm{C} 82-\mathrm{C} 83$ & $1.453(12)$ & $\mathrm{C} 220-\mathrm{H} 22 \mathrm{~B}$ & 0.9800 \\
\hline $\mathrm{C} 82-\mathrm{C} 81 \mathrm{~B}$ & $1.57(3)$ & $\mathrm{C} 220-\mathrm{H} 22 \mathrm{C}$ & 0.9800 \\
\hline $\mathrm{C} 82-\mathrm{C} 83 \mathrm{~B}$ & $1.502(14)$ & $\mathrm{C} 205-\mathrm{H} 20 \mathrm{~F}$ & 0.9900 \\
\hline C $85-H 85 A$ & 0.9900 & $\mathrm{C} 205-\mathrm{H} 20 \mathrm{G}$ & 0.9900 \\
\hline C $85-H 85 B$ & 0.9900 & $\mathrm{C} 205-\mathrm{C} 206$ & $1.527(15)$ \\
\hline $\mathrm{C} 85-\mathrm{C} 86$ & $1.530(9)$ & $\mathrm{C} 206-\mathrm{H} 20 \mathrm{H}$ & 0.9900 \\
\hline C86-H86A & 0.9900 & C206-H20I & 0.9900 \\
\hline C $86-\mathrm{H} 86 \mathrm{~B}$ & 0.9900 & $\mathrm{C} 206-\mathrm{C} 207$ & $1.49(6)$ \\
\hline $\mathrm{C} 86-\mathrm{C} 87$ & $1.523(9)$ & C207-H20J & 0.9900 \\
\hline C87-H87A & 0.9900 & C207-H20K & 0.9900 \\
\hline C87-H87B & 0.9900 & C207-C208 & $1.49(2)$ \\
\hline C $87-\mathrm{C} 88$ & $1.500(9)$ & C208-H20L & 0.9800 \\
\hline C $88-\mathrm{H} 88 \mathrm{~A}$ & 0.9900 & $\mathrm{C} 208-\mathrm{H} 20 \mathrm{M}$ & 0.9800 \\
\hline C $88-\mathrm{H} 88 \mathrm{~B}$ & 0.9900 & $\mathrm{C} 208-\mathrm{H} 20 \mathrm{~N}$ & 0.9800 \\
\hline C88-C89 & $1.537(9)$ & $\mathrm{C} 75 \mathrm{~B}-\mathrm{H} 75 \mathrm{C}$ & 0.9900 \\
\hline
\end{tabular}




\begin{tabular}{|c|c|c|c|}
\hline C89-H89A & 0.9900 & $\mathrm{C} 75 \mathrm{~B}-\mathrm{H} 75 \mathrm{D}$ & 0.9900 \\
\hline С $89-\mathrm{H} 89 \mathrm{~B}$ & 0.9900 & $\mathrm{C} 75 \mathrm{~B}-\mathrm{C} 76 \mathrm{~B}$ & $1.450(18)$ \\
\hline C89-C90 & $1.509(10)$ & $\mathrm{C} 75 \mathrm{~B}-\mathrm{C} 74 \mathrm{~B}$ & $1.523(18)$ \\
\hline $\mathrm{C} 90-\mathrm{H} 90 \mathrm{~A}$ & 0.9800 & $\mathrm{C} 78 \mathrm{~B}-\mathrm{H} 78 \mathrm{D}$ & 0.9800 \\
\hline $\mathrm{C} 90-\mathrm{H} 90 \mathrm{~B}$ & 0.9800 & $\mathrm{C} 78 \mathrm{~B}-\mathrm{H} 78 \mathrm{E}$ & 0.9800 \\
\hline $\mathrm{C} 90-\mathrm{H} 90 \mathrm{C}$ & 0.9800 & $\mathrm{C} 78 \mathrm{~B}-\mathrm{H} 78 \mathrm{~F}$ & 0.9800 \\
\hline C91-H91A & 0.9900 & $\mathrm{C} 78 \mathrm{~B}-\mathrm{C} 77 \mathrm{~B}$ & $1.471(8)$ \\
\hline C91-H91B & 0.9900 & $\mathrm{C} 76 \mathrm{~B}-\mathrm{H} 76 \mathrm{C}$ & 0.9900 \\
\hline $\mathrm{C} 91-\mathrm{C} 92$ & $1.488(9)$ & $\mathrm{C} 76 \mathrm{~B}-\mathrm{H} 76 \mathrm{D}$ & 0.9900 \\
\hline C92-H92A & 0.9900 & $\mathrm{C} 76 \mathrm{~B}-\mathrm{C} 77 \mathrm{~B}$ & $1.525(13)$ \\
\hline C92-H92B & 0.9900 & $\mathrm{C} 77 \mathrm{~B}-\mathrm{H} 77 \mathrm{C}$ & 0.9900 \\
\hline $\mathrm{C} 92-\mathrm{H} 92 \mathrm{C}$ & 0.9900 & C77B-H77D & 0.9900 \\
\hline C92-H92D & 0.9900 & $\mathrm{C} 73 \mathrm{~B}-\mathrm{H} 73 \mathrm{C}$ & 0.9900 \\
\hline $\mathrm{C} 92-\mathrm{C} 93$ & $1.613(11)$ & $\mathrm{C} 73 \mathrm{~B}-\mathrm{H} 73 \mathrm{D}$ & 0.9900 \\
\hline C92-C93B & $1.522(13)$ & $\mathrm{C} 73 \mathrm{~B}-\mathrm{C} 74 \mathrm{~B}$ & $1.517(18)$ \\
\hline C97-H97A & 0.9900 & $\mathrm{C} 73 \mathrm{~B}-\mathrm{C} 18 \mathrm{~B}$ & $1.483(17)$ \\
\hline C97-H97B & 0.9900 & $\mathrm{C} 74 \mathrm{~B}-\mathrm{H} 74 \mathrm{C}$ & 0.9900 \\
\hline C97-C98 & $1.507(9)$ & $\mathrm{C} 74 \mathrm{~B}-\mathrm{H} 74 \mathrm{D}$ & 0.9900 \\
\hline C98-H98A & 0.9900 & $\mathrm{C} 79 \mathrm{~B}-\mathrm{H} 79 \mathrm{C}$ & 0.9900 \\
\hline C98-H98B & 0.9900 & C79B-H79D & 0.9900 \\
\hline C98-C99 & $1.532(8)$ & $\mathrm{C} 79 \mathrm{~B}-\mathrm{C} 80 \mathrm{~B}$ & $1.521(18)$ \\
\hline C99-H99A & 0.9900 & $\mathrm{C} 79 \mathrm{~B}-\mathrm{C} 18 \mathrm{~B}$ & $1.533(17)$ \\
\hline C99-H99B & 0.9900 & $\mathrm{C} 80 \mathrm{~B}-\mathrm{H} 80 \mathrm{C}$ & 0.9900 \\
\hline C99-C100 & $1.499(11)$ & $\mathrm{C} 80 \mathrm{~B}-\mathrm{H} 80 \mathrm{D}$ & 0.9900 \\
\hline $\mathrm{C} 100-\mathrm{H} 10 \mathrm{C}$ & 0.9900 & $\mathrm{C} 80 \mathrm{~B}-\mathrm{C} 81 \mathrm{~B}$ & $1.530(18)$ \\
\hline $\mathrm{C} 100-\mathrm{H} 10 \mathrm{D}$ & 0.9900 & $\mathrm{C} 81 \mathrm{~B}-\mathrm{H} 81 \mathrm{C}$ & 0.9900 \\
\hline $\mathrm{C} 100-\mathrm{H} 10 \mathrm{~A}$ & 0.9900 & $\mathrm{C} 81 \mathrm{~B}-\mathrm{H} 81 \mathrm{D}$ & 0.9900 \\
\hline $\mathrm{C} 100-\mathrm{H} 10 \mathrm{~B}$ & 0.9900 & $\mathrm{C} 83 \mathrm{~B}-\mathrm{H} 83 \mathrm{C}$ & 0.9900 \\
\hline $\mathrm{C} 100-\mathrm{C} 101$ & $1.555(12)$ & $\mathrm{C} 83 \mathrm{~B}-\mathrm{H} 83 \mathrm{D}$ & 0.9900 \\
\hline $\mathrm{C} 100-\mathrm{C} 201$ & $1.523(12)$ & $\mathrm{C} 83 \mathrm{~B}-\mathrm{C} 84 \mathrm{~B}$ & $1.501(15)$ \\
\hline $\mathrm{C} 103-\mathrm{H} 10 \mathrm{E}$ & 0.9900 & $\mathrm{C} 84 \mathrm{~B}-\mathrm{H} 84 \mathrm{D}$ & 0.9800 \\
\hline $\mathrm{C} 103-\mathrm{H} 10 \mathrm{~F}$ & 0.9900 & $\mathrm{C} 84 \mathrm{~B}-\mathrm{H} 84 \mathrm{E}$ & 0.9800 \\
\hline $\mathrm{C} 103-\mathrm{C} 104$ & $1.516(9)$ & $\mathrm{C} 84 \mathrm{~B}-\mathrm{H} 84 \mathrm{~F}$ & 0.9800 \\
\hline $\mathrm{C} 104-\mathrm{H} 10 \mathrm{G}$ & 0.9903 & C1S-H1SA & 0.9800 \\
\hline $\mathrm{C} 104-\mathrm{H} 10 \mathrm{H}$ & 0.9900 & $\mathrm{C} 1 \mathrm{~S}-\mathrm{H} 1 \mathrm{SB}$ & 0.9800 \\
\hline $\mathrm{C} 104-\mathrm{C} 105$ & $1.537(12)$ & $\mathrm{C} 1 \mathrm{~S}-\mathrm{H} 1 \mathrm{SC}$ & 0.9800 \\
\hline $\mathrm{C} 104-\mathrm{C} 205$ & $1.534(14)$ & $\mathrm{C} 1 \mathrm{~S}-\mathrm{C} 2 \mathrm{~S}$ & $1.53(2)$ \\
\hline C109-H10I & 0.9900 & $\mathrm{C} 2 \mathrm{~S}-\mathrm{H} 2 \mathrm{SA}$ & 0.9900 \\
\hline C109-H10J & 0.9900 & $\mathrm{C} 2 \mathrm{~S}-\mathrm{H} 2 \mathrm{SB}$ & 0.9900 \\
\hline $\mathrm{C} 109-\mathrm{C} 110$ & $1.520(9)$ & $\mathrm{C} 2 \mathrm{~S}-\mathrm{C} 3 \mathrm{~S}$ & $1.52(3)$ \\
\hline $\mathrm{C} 110-\mathrm{H} 11 \mathrm{~A}$ & 0.9900 & $\mathrm{C} 3 \mathrm{~S}-\mathrm{H} 3 \mathrm{SA}$ & 0.9900 \\
\hline $\mathrm{C} 110-\mathrm{H} 11 \mathrm{~B}$ & 0.9900 & $\mathrm{C} 3 \mathrm{~S}-\mathrm{H} 3 \mathrm{SB}$ & 0.9900 \\
\hline $\mathrm{C} 110-\mathrm{H} 11 \mathrm{C}$ & 0.9900 & $\mathrm{C} 3 \mathrm{~S}-\mathrm{C} 4 \mathrm{~S}$ & $1.55(3)$ \\
\hline C110-H11D & 0.9900 & $\mathrm{C} 4 \mathrm{~S}-\mathrm{H} 4 \mathrm{SA}$ & 0.9900 \\
\hline $\mathrm{C} 110-\mathrm{C} 111$ & $1.526(11)$ & $\mathrm{C} 4 \mathrm{~S}-\mathrm{H} 4 \mathrm{SB}$ & 0.9900 \\
\hline $\mathrm{C} 110-\mathrm{C} 211$ & $1.528(14)$ & $\mathrm{C} 4 \mathrm{~S}-\mathrm{C} 5 \mathrm{~S}$ & $1.54(3)$ \\
\hline $\mathrm{C} 115-\mathrm{H} 11 \mathrm{E}$ & 0.9900 & $\mathrm{C} 5 \mathrm{~S}-\mathrm{H} 5 \mathrm{SA}$ & 0.9800 \\
\hline $\mathrm{C} 115-\mathrm{H} 11 \mathrm{~F}$ & 0.9900 & $\mathrm{C} 5 \mathrm{~S}-\mathrm{H} 5 \mathrm{SB}$ & 0.9800 \\
\hline
\end{tabular}




\begin{tabular}{|c|c|c|c|}
\hline $\mathrm{C} 115-\mathrm{C} 116$ & $1.499(9)$ & $\mathrm{C} 5 \mathrm{~S}-\mathrm{H} 5 \mathrm{SC}$ & 0.9800 \\
\hline $\mathrm{Cl} 2-\mathrm{Ir} 1-\mathrm{Cl} 1$ & $83.11(5)$ & $\mathrm{C} 74 \mathrm{~B}-\mathrm{C} 74-\mathrm{C} 75 \mathrm{~A}$ & $116(2)$ \\
\hline $\mathrm{N} 3-\mathrm{Ir} 1-\mathrm{Cl1}$ & $94.59(13)$ & $\mathrm{C} 74 \mathrm{~B}-\mathrm{C} 74-\mathrm{C} 75 \mathrm{~B}$ & $51.4(14)$ \\
\hline $\mathrm{N} 3-\mathrm{Ir} 1-\mathrm{Cl} 2$ & $94.04(15)$ & $\mathrm{C} 74-\mathrm{C} 75 \mathrm{~A}-\mathrm{H} 75 \mathrm{~A}$ & 108.0 \\
\hline $\mathrm{N} 3-\mathrm{Ir} 1-\mathrm{N} 4$ & $171.84(19)$ & $\mathrm{C} 74-\mathrm{C} 75 \mathrm{~A}-\mathrm{H} 75 \mathrm{~B}$ & 108.0 \\
\hline $\mathrm{N} 4-\mathrm{Ir} 1-\mathrm{Cl} 1$ & $93.53(15)$ & $\mathrm{C} 74-\mathrm{C} 75 \mathrm{~A}-\mathrm{C} 76$ & $117.2(10)$ \\
\hline $\mathrm{N} 4-\mathrm{Ir} 1-\mathrm{Cl} 2$ & $87.70(15)$ & $\mathrm{H} 75 \mathrm{~A}-\mathrm{C} 75 \mathrm{~A}-\mathrm{H} 75 \mathrm{~B}$ & 107.2 \\
\hline C37-Ir1-Cl1 & $87.52(17)$ & $\mathrm{C} 76-\mathrm{C} 75 \mathrm{~A}-\mathrm{H} 75 \mathrm{~A}$ & 108.0 \\
\hline $\mathrm{C} 37-\mathrm{Ir} 1-\mathrm{Cl} 2$ & $168.90(17)$ & $\mathrm{C} 76-\mathrm{C} 75 \mathrm{~A}-\mathrm{H} 75 \mathrm{~B}$ & 108.0 \\
\hline $\mathrm{C} 37-\operatorname{Ir} 1-\mathrm{N} 3$ & $80.7(2)$ & $\mathrm{C} 75 \mathrm{~A}-\mathrm{C} 76-\mathrm{H} 76 \mathrm{~A}$ & 109.5 \\
\hline $\mathrm{C} 37-\mathrm{Ir} 1-\mathrm{N} 4$ & $98.9(2)$ & $\mathrm{C} 75 \mathrm{~A}-\mathrm{C} 76-\mathrm{H} 76 \mathrm{~B}$ & 109.5 \\
\hline $\mathrm{C} 37-\mathrm{Ir} 1-\mathrm{C} 55$ & $95.6(2)$ & $\mathrm{H} 76 \mathrm{~A}-\mathrm{C} 76-\mathrm{H} 76 \mathrm{~B}$ & 108.0 \\
\hline $\mathrm{C} 55-\mathrm{Ir} 1-\mathrm{Cl} 1$ & $173.97(18)$ & $\mathrm{C} 77-\mathrm{C} 76-\mathrm{C} 75 \mathrm{~A}$ & $110.9(12)$ \\
\hline $\mathrm{C} 55-\mathrm{Ir} 1-\mathrm{Cl} 2$ & $94.28(19)$ & $\mathrm{C} 77-\mathrm{C} 76-\mathrm{H} 76 \mathrm{~A}$ & 109.5 \\
\hline $\mathrm{C} 55-\operatorname{Ir} 1-\mathrm{N} 3$ & $91.0(2)$ & $\mathrm{C} 77-\mathrm{C} 76-\mathrm{H} 76 \mathrm{~B}$ & 109.5 \\
\hline $\mathrm{C} 55-\mathrm{Ir} 1-\mathrm{N} 4$ & $80.9(2)$ & $\mathrm{C} 76-\mathrm{C} 77-\mathrm{H} 77 \mathrm{~A}$ & 108.9 \\
\hline $\mathrm{C} 11-\mathrm{Ir} 2-\mathrm{Cl} 2$ & $83.39(5)$ & $\mathrm{C} 76-\mathrm{C} 77-\mathrm{H} 77 \mathrm{~B}$ & 108.9 \\
\hline $\mathrm{N} 1-\mathrm{Ir} 2-\mathrm{C} 11$ & $94.21(15)$ & $\mathrm{C} 76-\mathrm{C} 77-\mathrm{C} 78$ & $113.2(14)$ \\
\hline $\mathrm{N} 1-\mathrm{Ir} 2-\mathrm{Cl} 2$ & $91.21(14)$ & $\mathrm{H} 77 \mathrm{~A}-\mathrm{C} 77-\mathrm{H} 77 \mathrm{~B}$ & 107.7 \\
\hline $\mathrm{N} 2-\mathrm{Ir} 2-\mathrm{Cl} 1$ & $92.18(16)$ & $\mathrm{C} 78-\mathrm{C} 77-\mathrm{H} 77 \mathrm{~A}$ & 108.9 \\
\hline $\mathrm{N} 2-\mathrm{Ir} 2-\mathrm{Cl} 2$ & $94.92(14)$ & $\mathrm{C} 78-\mathrm{C} 77-\mathrm{H} 77 \mathrm{~B}$ & 108.9 \\
\hline $\mathrm{N} 2-\mathrm{Ir} 2-\mathrm{N} 1$ & $171.6(2)$ & $\mathrm{C} 77-\mathrm{C} 78-\mathrm{H} 78 \mathrm{~A}$ & 109.5 \\
\hline $\mathrm{C} 1-\mathrm{Ir} 2-\mathrm{Cl} 1$ & $173.83(18)$ & $\mathrm{C} 77-\mathrm{C} 78-\mathrm{H} 78 \mathrm{~B}$ & 109.5 \\
\hline $\mathrm{C} 1-\mathrm{Ir} 2-\mathrm{Cl} 2$ & $92.49(18)$ & $\mathrm{C} 77-\mathrm{C} 78-\mathrm{H} 78 \mathrm{C}$ & 109.5 \\
\hline $\mathrm{C} 1-\mathrm{Ir} 2-\mathrm{N} 1$ & $81.3(2)$ & $\mathrm{H} 78 \mathrm{~A}-\mathrm{C} 78-\mathrm{H} 78 \mathrm{~B}$ & 109.5 \\
\hline $\mathrm{C} 1-\mathrm{Ir} 2-\mathrm{N} 2$ & $92.7(2)$ & $\mathrm{H} 78 \mathrm{~A}-\mathrm{C} 78-\mathrm{H} 78 \mathrm{C}$ & 109.5 \\
\hline $\mathrm{C} 1-\mathrm{Ir} 2-\mathrm{C} 19$ & $93.4(3)$ & $\mathrm{H} 78 \mathrm{~B}-\mathrm{C} 78-\mathrm{H} 78 \mathrm{C}$ & 109.5 \\
\hline $\mathrm{C} 19-\mathrm{Ir} 2-\mathrm{Cl1}$ & $91.01(19)$ & $\mathrm{C} 18-\mathrm{C} 79-\mathrm{H} 79 \mathrm{~A}$ & 108.3 \\
\hline $\mathrm{C} 19-\mathrm{Ir} 2-\mathrm{Cl} 2$ & $173.02(19)$ & $\mathrm{C} 18-\mathrm{C} 79-\mathrm{H} 79 \mathrm{~B}$ & 108.3 \\
\hline $\mathrm{C} 19-\mathrm{Ir} 2-\mathrm{N} 1$ & $93.3(2)$ & $\mathrm{C} 18-\mathrm{C} 79-\mathrm{C} 80$ & $115.8(7)$ \\
\hline $\mathrm{C} 19-\mathrm{Ir} 2-\mathrm{N} 2$ & $81.1(2)$ & $\mathrm{C} 18-\mathrm{C} 79-\mathrm{C} 73 \mathrm{~B}$ & $41.6(8)$ \\
\hline $\mathrm{Ir} 2-\mathrm{Cl} 1-\mathrm{Ir} 1$ & $96.88(5)$ & $\mathrm{H} 79 \mathrm{~A}-\mathrm{C} 79-\mathrm{H} 79 \mathrm{~B}$ & 107.4 \\
\hline $\mathrm{Ir} 1-\mathrm{Cl} 2-\mathrm{Ir} 2$ & $96.04(5)$ & $\mathrm{C} 80-\mathrm{C} 79-\mathrm{H} 79 \mathrm{~A}$ & 108.3 \\
\hline $\mathrm{C} 7-\mathrm{N} 1-\mathrm{Ir} 2$ & $114.3(4)$ & $\mathrm{C} 80-\mathrm{C} 79-\mathrm{H} 79 \mathrm{~B}$ & 108.3 \\
\hline $\mathrm{C} 11-\mathrm{N} 1-\mathrm{Ir} 2$ & $125.1(4)$ & $\mathrm{C} 80-\mathrm{C} 79-\mathrm{C} 73 \mathrm{~B}$ & $157.3(10)$ \\
\hline $\mathrm{C} 11-\mathrm{N} 1-\mathrm{C} 7$ & $120.4(5)$ & $\mathrm{C} 73 \mathrm{~B}-\mathrm{C} 79-\mathrm{H} 79 \mathrm{~A}$ & 83.2 \\
\hline $\mathrm{C} 25-\mathrm{N} 2-\mathrm{Ir} 2$ & $114.9(4)$ & $\mathrm{C} 73 \mathrm{~B}-\mathrm{C} 79-\mathrm{H} 79 \mathrm{~B}$ & 85.6 \\
\hline $\mathrm{C} 25-\mathrm{N} 2-\mathrm{C} 29$ & $120.5(5)$ & $\mathrm{C} 79 \mathrm{~B}-\mathrm{C} 79-\mathrm{C} 18$ & $104.9(15)$ \\
\hline $\mathrm{C} 29-\mathrm{N} 2-\mathrm{Ir} 2$ & $124.0(4)$ & $\mathrm{C} 79 \mathrm{~B}-\mathrm{C} 79-\mathrm{H} 79 \mathrm{~A}$ & 122.0 \\
\hline $\mathrm{C} 43-\mathrm{N} 3-\mathrm{Ir} 1$ & $116.2(4)$ & $\mathrm{C} 79 \mathrm{~B}-\mathrm{C} 79-\mathrm{H} 79 \mathrm{~B}$ & 105.2 \\
\hline $\mathrm{C} 43-\mathrm{N} 3-\mathrm{C} 47$ & $119.6(5)$ & $\mathrm{C} 79 \mathrm{~B}-\mathrm{C} 79-\mathrm{C} 80$ & $14.3(16)$ \\
\hline $\mathrm{C} 47-\mathrm{N} 3-\mathrm{Ir} 1$ & $123.6(4)$ & $\mathrm{C} 79 \mathrm{~B}-\mathrm{C} 79-\mathrm{C} 73 \mathrm{~B}$ & $145.9(17)$ \\
\hline C61-N4-Ir1 & $115.1(4)$ & $\mathrm{C} 79 \mathrm{~B}-\mathrm{C} 79-\mathrm{C} 18 \mathrm{~B}$ & $87.4(15)$ \\
\hline $\mathrm{C} 65-\mathrm{N} 4-\mathrm{Ir} 1$ & $124.8(4)$ & $\mathrm{C} 18 \mathrm{~B}-\mathrm{C} 79-\mathrm{C} 18$ & $17.6(9)$ \\
\hline $\mathrm{C} 65-\mathrm{N} 4-\mathrm{C} 61$ & $119.7(6)$ & $\mathrm{C} 18 \mathrm{~B}-\mathrm{C} 79-\mathrm{H} 79 \mathrm{~A}$ & 119.9 \\
\hline $\mathrm{C} 2-\mathrm{C} 1-\mathrm{Ir} 2$ & $128.4(5)$ & $\mathrm{C} 18 \mathrm{~B}-\mathrm{C} 79-\mathrm{H} 79 \mathrm{~B}$ & 113.4 \\
\hline $\mathrm{C} 2-\mathrm{C} 1-\mathrm{C} 6$ & $117.3(6)$ & $\mathrm{C} 18 \mathrm{~B}-\mathrm{C} 79-\mathrm{C} 80$ & $98.6(11)$ \\
\hline
\end{tabular}




\begin{tabular}{|c|c|}
\hline $\mathrm{C} 6-\mathrm{C} 1-\mathrm{Ir} 2$ & $114.3(5)$ \\
\hline $\mathrm{C} 1-\mathrm{C} 2-\mathrm{H} 2$ & 119.8 \\
\hline $\mathrm{C} 3-\mathrm{C} 2-\mathrm{C} 1$ & $120.4(6)$ \\
\hline $\mathrm{C} 3-\mathrm{C} 2-\mathrm{H} 2$ & 119.8 \\
\hline $\mathrm{C} 2-\mathrm{C} 3-\mathrm{C} 4$ & $121.3(6)$ \\
\hline $\mathrm{C} 2-\mathrm{C} 3-\mathrm{C} 12$ & $130.8(6)$ \\
\hline $\mathrm{C} 4-\mathrm{C} 3-\mathrm{C} 12$ & $108.0(6)$ \\
\hline $\mathrm{C} 3-\mathrm{C} 4-\mathrm{C} 18$ & $111.6(6)$ \\
\hline $\mathrm{C} 3-\mathrm{C} 4-\mathrm{C} 18 \mathrm{~B}$ & $110.6(8)$ \\
\hline $\mathrm{C} 5-\mathrm{C} 4-\mathrm{C} 3$ & $119.6(6)$ \\
\hline $\mathrm{C} 5-\mathrm{C} 4-\mathrm{C} 18$ & $128.4(6)$ \\
\hline $\mathrm{C} 5-\mathrm{C} 4-\mathrm{C} 18 \mathrm{~B}$ & $128.1(8)$ \\
\hline $\mathrm{C} 18 \mathrm{~B}-\mathrm{C} 4-\mathrm{C} 18$ & $18.9(7)$ \\
\hline $\mathrm{C} 4-\mathrm{C} 5-\mathrm{H} 5$ & 120.3 \\
\hline $\mathrm{C} 4-\mathrm{C} 5-\mathrm{C} 6$ & $119.4(6)$ \\
\hline $\mathrm{C} 6-\mathrm{C} 5-\mathrm{H} 5$ & 120.3 \\
\hline $\mathrm{C} 1-\mathrm{C} 6-\mathrm{C} 7$ & $114.7(6)$ \\
\hline $\mathrm{C} 5-\mathrm{C} 6-\mathrm{C} 1$ & $122.0(6)$ \\
\hline $\mathrm{C} 5-\mathrm{C} 6-\mathrm{C} 7$ & $123.3(6)$ \\
\hline $\mathrm{N} 1-\mathrm{C} 7-\mathrm{C} 6$ & $115.5(5)$ \\
\hline $\mathrm{N} 1-\mathrm{C} 7-\mathrm{C} 8$ & $118.6(6)$ \\
\hline $\mathrm{C} 8-\mathrm{C} 7-\mathrm{C} 6$ & $125.9(6)$ \\
\hline $\mathrm{C} 7-\mathrm{C} 8-\mathrm{H} 8$ & 119.1 \\
\hline $\mathrm{C} 9-\mathrm{C} 8-\mathrm{C} 7$ & $121.8(7)$ \\
\hline $\mathrm{C} 9-\mathrm{C} 8-\mathrm{H} 8$ & 119.1 \\
\hline $\mathrm{C} 8-\mathrm{C} 9-\mathrm{H} 9$ & 121.9 \\
\hline $\mathrm{C} 10-\mathrm{C} 9-\mathrm{C} 8$ & $116.3(7)$ \\
\hline $\mathrm{C} 10-\mathrm{C} 9-\mathrm{H} 9$ & 121.9 \\
\hline $\mathrm{F} 1-\mathrm{C} 10-\mathrm{C} 11$ & $118.2(6)$ \\
\hline $\mathrm{C} 9-\mathrm{C} 10-\mathrm{F} 1$ & $119.0(6)$ \\
\hline $\mathrm{C} 9-\mathrm{C} 10-\mathrm{C} 11$ & $122.8(6)$ \\
\hline $\mathrm{N} 1-\mathrm{C} 11-\mathrm{C} 10$ & $120.0(6)$ \\
\hline $\mathrm{N} 1-\mathrm{C} 11-\mathrm{H} 11$ & 120.0 \\
\hline $\mathrm{C} 10-\mathrm{C} 11-\mathrm{H} 11$ & 120.0 \\
\hline $\mathrm{C} 13-\mathrm{C} 12-\mathrm{C} 3$ & $130.4(6)$ \\
\hline $\mathrm{C} 13-\mathrm{C} 12-\mathrm{C} 17$ & $120.8(6)$ \\
\hline $\mathrm{C} 17-\mathrm{C} 12-\mathrm{C} 3$ & $108.8(6)$ \\
\hline $\mathrm{C} 12-\mathrm{C} 13-\mathrm{H} 13$ & 120.9 \\
\hline $\mathrm{C} 12-\mathrm{C} 13-\mathrm{C} 14$ & $118.1(6)$ \\
\hline $\mathrm{C} 14-\mathrm{C} 13-\mathrm{H} 13$ & 120.9 \\
\hline $\mathrm{C} 13-\mathrm{C} 14-\mathrm{H} 14$ & 119.6 \\
\hline $\mathrm{C} 15-\mathrm{C} 14-\mathrm{C} 13$ & $120.7(6)$ \\
\hline $\mathrm{C} 15-\mathrm{C} 14-\mathrm{H} 14$ & 119.7 \\
\hline C14-C15-H15 & 119.5 \\
\hline $\mathrm{C} 16-\mathrm{C} 15-\mathrm{C} 14$ & $121.0(6)$ \\
\hline $\mathrm{C} 16-\mathrm{C} 15-\mathrm{H} 15$ & 119.5 \\
\hline $\mathrm{C} 15-\mathrm{C} 16-\mathrm{H} 16$ & 120.7 \\
\hline $\mathrm{C} 15-\mathrm{C} 16-\mathrm{C} 17$ & $118.7(7)$ \\
\hline
\end{tabular}

\begin{tabular}{|c|c|}
\hline $\mathrm{C} 18 \mathrm{~B}-\mathrm{C} 79-\mathrm{C} 73 \mathrm{~B}$ & $58.9(9)$ \\
\hline $\mathrm{C} 79-\mathrm{C} 80-\mathrm{H} 80 \mathrm{~A}$ & 109.0 \\
\hline $\mathrm{C} 79-\mathrm{C} 80-\mathrm{H} 80 \mathrm{~B}$ & 109.0 \\
\hline $\mathrm{H} 80 \mathrm{~A}-\mathrm{C} 80-\mathrm{H} 80 \mathrm{~B}$ & 107.8 \\
\hline $\mathrm{C} 81-\mathrm{C} 80-\mathrm{C} 79$ & $112.8(8)$ \\
\hline $\mathrm{C} 81-\mathrm{C} 80-\mathrm{H} 80 \mathrm{~A}$ & 109.0 \\
\hline $\mathrm{C} 81-\mathrm{C} 80-\mathrm{H} 80 \mathrm{~B}$ & 109.0 \\
\hline $\mathrm{C} 79 \mathrm{~B}-\mathrm{C} 80-\mathrm{C} 79$ & $20(2)$ \\
\hline $\mathrm{C} 79 \mathrm{~B}-\mathrm{C} 80-\mathrm{H} 80 \mathrm{~A}$ & 113.4 \\
\hline $\mathrm{C} 79 \mathrm{~B}-\mathrm{C} 80-\mathrm{H} 80 \mathrm{~B}$ & 89.8 \\
\hline $\mathrm{C} 79 \mathrm{~B}-\mathrm{C} 80-\mathrm{C} 81$ & $125(2)$ \\
\hline $\mathrm{C} 79 \mathrm{~B}-\mathrm{C} 80-\mathrm{C} 80 \mathrm{~B}$ & $115(3)$ \\
\hline $\mathrm{C} 80 \mathrm{~B}-\mathrm{C} 80-\mathrm{C} 79$ & $134.1(17)$ \\
\hline $\mathrm{C} 80 \mathrm{~B}-\mathrm{C} 80-\mathrm{H} 80 \mathrm{~A}$ & 81.0 \\
\hline $\mathrm{C} 80 \mathrm{~B}-\mathrm{C} 80-\mathrm{H} 80 \mathrm{~B}$ & 30.8 \\
\hline $\mathrm{C} 80 \mathrm{~B}-\mathrm{C} 80-\mathrm{C} 81$ & $104.9(16)$ \\
\hline $\mathrm{C} 82-\mathrm{C} 81-\mathrm{H} 81 \mathrm{~A}$ & 109.5 \\
\hline $\mathrm{C} 82-\mathrm{C} 81-\mathrm{H} 81 \mathrm{~B}$ & 109.5 \\
\hline $\mathrm{C} 82-\mathrm{C} 81-\mathrm{C} 79 \mathrm{~B}$ & $127.0(11)$ \\
\hline $\mathrm{C} 82-\mathrm{C} 81-\mathrm{C} 81 \mathrm{~B}$ & $54.4(11)$ \\
\hline $\mathrm{C} 80-\mathrm{C} 81-\mathrm{C} 82$ & $110.8(9)$ \\
\hline $\mathrm{C} 80-\mathrm{C} 81-\mathrm{H} 81 \mathrm{~A}$ & 109.5 \\
\hline $\mathrm{C} 80-\mathrm{C} 81-\mathrm{H} 81 \mathrm{~B}$ & 109.5 \\
\hline $\mathrm{C} 80-\mathrm{C} 81-\mathrm{C} 79 \mathrm{~B}$ & $16.3(8)$ \\
\hline $\mathrm{C} 80-\mathrm{C} 81-\mathrm{C} 81 \mathrm{~B}$ & $75.1(10)$ \\
\hline $\mathrm{H} 81 \mathrm{~A}-\mathrm{C} 81-\mathrm{H} 81 \mathrm{~B}$ & 108.1 \\
\hline $\mathrm{C} 79 \mathrm{~B}-\mathrm{C} 81-\mathrm{H} 81 \mathrm{~A}$ & 99.1 \\
\hline $\mathrm{C} 79 \mathrm{~B}-\mathrm{C} 81-\mathrm{H} 81 \mathrm{~B}$ & 102.3 \\
\hline $\mathrm{C} 81 \mathrm{~B}-\mathrm{C} 81-\mathrm{H} 81 \mathrm{~A}$ & 162.8 \\
\hline $\mathrm{C} 81 \mathrm{~B}-\mathrm{C} 81-\mathrm{H} 81 \mathrm{~B}$ & 85.0 \\
\hline $\mathrm{C} 81 \mathrm{~B}-\mathrm{C} 81-\mathrm{C} 79 \mathrm{~B}$ & $88.6(11)$ \\
\hline $\mathrm{C} 82-\mathrm{C} 83-\mathrm{H} 83 \mathrm{~A}$ & 112.7 \\
\hline $\mathrm{C} 82-\mathrm{C} 83-\mathrm{H} 83 \mathrm{~B}$ & 112.7 \\
\hline $\mathrm{C} 82-\mathrm{C} 83-\mathrm{C} 84$ & $95.0(13)$ \\
\hline $\mathrm{C} 82-\mathrm{C} 83-\mathrm{C} 83 \mathrm{~B}$ & $61.4(11)$ \\
\hline $\mathrm{H} 83 \mathrm{~A}-\mathrm{C} 83-\mathrm{H} 83 \mathrm{~B}$ & 110.2 \\
\hline $\mathrm{C} 84-\mathrm{C} 83-\mathrm{H} 83 \mathrm{~A}$ & 112.7 \\
\hline $\mathrm{C} 84-\mathrm{C} 83-\mathrm{H} 83 \mathrm{~B}$ & 112.7 \\
\hline $\mathrm{C} 81 \mathrm{~B}-\mathrm{C} 83-\mathrm{C} 82$ & $70.5(18)$ \\
\hline $\mathrm{C} 81 \mathrm{~B}-\mathrm{C} 83-\mathrm{H} 83 \mathrm{~A}$ & 108.4 \\
\hline $\mathrm{C} 81 \mathrm{~B}-\mathrm{C} 83-\mathrm{H} 83 \mathrm{~B}$ & 47.9 \\
\hline $\mathrm{C} 81 \mathrm{~B}-\mathrm{C} 83-\mathrm{C} 84$ & $139(3)$ \\
\hline $\mathrm{C} 81 \mathrm{~B}-\mathrm{C} 83-\mathrm{C} 83 \mathrm{~B}$ & $128(2)$ \\
\hline $\mathrm{C} 83 \mathrm{~B}-\mathrm{C} 83-\mathrm{H} 83 \mathrm{~A}$ & 107.7 \\
\hline $\mathrm{C} 83 \mathrm{~B}-\mathrm{C} 83-\mathrm{H} 83 \mathrm{~B}$ & 140.1 \\
\hline $\mathrm{C} 83 \mathrm{~B}-\mathrm{C} 83-\mathrm{C} 84$ & $38.1(18)$ \\
\hline $\mathrm{C} 83-\mathrm{C} 84-\mathrm{H} 84 \mathrm{~A}$ & 109.5 \\
\hline $\mathrm{C} 83-\mathrm{C} 84-\mathrm{H} 84 \mathrm{~B}$ & 109.5 \\
\hline
\end{tabular}


C17-C16- 116

C12-C17-C18

C12-C17-C18B

$\mathrm{C} 16-\mathrm{C} 17-\mathrm{C} 12$

$\mathrm{C} 16-\mathrm{C} 17-\mathrm{C} 18$

C16-C17-C18B

$\mathrm{C} 18-\mathrm{C} 17-\mathrm{C} 18 \mathrm{~B}$

$\mathrm{C} 20-\mathrm{C} 19-\mathrm{Ir} 2$

$\mathrm{C} 20-\mathrm{C} 19-\mathrm{C} 24$

C24-C19-Ir2

$\mathrm{C} 19-\mathrm{C} 20-\mathrm{H} 20$

$\mathrm{C} 21-\mathrm{C} 20-\mathrm{C} 19$

$\mathrm{C} 21-\mathrm{C} 20-\mathrm{H} 20$

$\mathrm{C} 20-\mathrm{C} 21-\mathrm{C} 22$

$\mathrm{C} 20-\mathrm{C} 21-\mathrm{C} 30$

$\mathrm{C} 22-\mathrm{C} 21-\mathrm{C} 30$

$\mathrm{C} 21-\mathrm{C} 22-\mathrm{C} 36$

$\mathrm{C} 23-\mathrm{C} 22-\mathrm{C} 21$

$\mathrm{C} 23-\mathrm{C} 22-\mathrm{C} 36$

$\mathrm{C} 22-\mathrm{C} 23-\mathrm{H} 23$

$\mathrm{C} 24-\mathrm{C} 23-\mathrm{C} 22$

$\mathrm{C} 24-\mathrm{C} 23-\mathrm{H} 23$

$\mathrm{C} 19-\mathrm{C} 24-\mathrm{C} 25$

$\mathrm{C} 23-\mathrm{C} 24-\mathrm{C} 19$

$\mathrm{C} 23-\mathrm{C} 24-\mathrm{C} 25$

$\mathrm{N} 2-\mathrm{C} 25-\mathrm{C} 24$

$\mathrm{N} 2-\mathrm{C} 25-\mathrm{C} 26$

$\mathrm{C} 26-\mathrm{C} 25-\mathrm{C} 24$

$\mathrm{C} 25-\mathrm{C} 26-\mathrm{H} 26$

$\mathrm{C} 27-\mathrm{C} 26-\mathrm{C} 25$

$\mathrm{C} 27-\mathrm{C} 26-\mathrm{H} 26$

$\mathrm{C} 26-\mathrm{C} 27-\mathrm{H} 27$

$\mathrm{C} 26-\mathrm{C} 27-\mathrm{C} 28$

$\mathrm{C} 28-\mathrm{C} 27-\mathrm{H} 27$

F2-C28-C27

$\mathrm{F} 2-\mathrm{C} 28-\mathrm{C} 29$

$\mathrm{C} 29-\mathrm{C} 28-\mathrm{C} 27$

$\mathrm{N} 2-\mathrm{C} 29-\mathrm{H} 29$

$\mathrm{C} 28-\mathrm{C} 29-\mathrm{N} 2$

$\mathrm{C} 28-\mathrm{C} 29-\mathrm{H} 29$

$\mathrm{C} 31-\mathrm{C} 30-\mathrm{C} 21$

$\mathrm{C} 35-\mathrm{C} 30-\mathrm{C} 21$

$\mathrm{C} 35-\mathrm{C} 30-\mathrm{C} 31$

$\mathrm{C} 30-\mathrm{C} 31-\mathrm{H} 31$

$\mathrm{C} 32-\mathrm{C} 31-\mathrm{C} 30$

$\mathrm{C} 32-\mathrm{C} 31-\mathrm{H} 31$

$\mathrm{C} 31-\mathrm{C} 32-\mathrm{H} 32$

$\mathrm{C} 33-\mathrm{C} 32-\mathrm{C} 31$
120.7

$111.9(6)$

$109.3(8)$

$120.7(6)$

$127.2(7)$

$127.8(8)$

$18.6(7)$

$128.1(5)$

$116.9(6)$

114.6 (4)

119.4

121.2 (6)

119.4

$120.9(6)$

$131.3(6)$

$107.8(6)$

$111.9(6)$

$119.7(6)$

128.4 (7)

120.4

$119.2(7)$

120.4

$114.0(6)$

$122.0(6)$

$124.0(6)$

$115.1(6)$

$119.6(6)$

$125.3(6)$

119.9

120.3 (7)

119.9

120.8

118.4 (7)

120.8

$119.9(6)$

$119.0(7)$

$121.0(7)$

120.0

120.1 (6)

120.0

$130.3(7)$

$108.6(6)$

$121.1(6)$

121.4

$117.2(7)$

121.4

119.3

$121.4(7)$
C83-C $84-\mathrm{H} 84 \mathrm{C}$

$\mathrm{H} 84 \mathrm{~A}-\mathrm{C} 84-\mathrm{H} 84 \mathrm{~B}$

$\mathrm{H} 84 \mathrm{~A}-\mathrm{C} 84-\mathrm{H} 84 \mathrm{C}$

H84B-C $84-\mathrm{H} 84 \mathrm{C}$

$\mathrm{C} 83 \mathrm{~B}-\mathrm{C} 84-\mathrm{C} 83$

$\mathrm{C} 83 \mathrm{~B}-\mathrm{C} 84-\mathrm{H} 84 \mathrm{~A}$

C83B-C $84-\mathrm{H} 84 \mathrm{~B}$

$\mathrm{C} 83 \mathrm{~B}-\mathrm{C} 84-\mathrm{H} 84 \mathrm{C}$

C92-C93-H93A

C92-C93-H93B

H93A-C93-H93B

C94-C93-C92

C94-C93-H93A

C94-C93-H93B

C93-C94-H94A

C93-C94-H94B

C93-C94-C95

H94A-C94-H94B

C95-C94-H94A

C95-C94-H94B

C94-C95-H95A

C94-C95-H95B

H95A-C95-H95B

C96-C95-C94

C96- C95-H95A

C96- C95-H95B

C95-C96-H96A

C95-C96-H96B

C95-C96- $\mathrm{H} 96 \mathrm{C}$

H96A-C96-H96B

$\mathrm{H} 96 \mathrm{~A}-\mathrm{C} 96-\mathrm{H} 96 \mathrm{C}$

H96B-C96-H96C

$\mathrm{C} 100-\mathrm{C} 101-\mathrm{H} 10 \mathrm{~K}$

$\mathrm{C} 100-\mathrm{C} 101-\mathrm{H} 10 \mathrm{~L}$

H10K-C101-H10L

$\mathrm{C} 102-\mathrm{C} 101-\mathrm{C} 100$

$\mathrm{C} 102-\mathrm{C} 101-\mathrm{H} 10 \mathrm{~K}$

C102-C101-H10L

C101-C102-H10M

C101-C102-H10N

C101-C102-H10O

$\mathrm{H} 10 \mathrm{M}-\mathrm{C} 102-\mathrm{H} 10 \mathrm{~N}$

$\mathrm{H} 10 \mathrm{M}-\mathrm{C} 102-\mathrm{H} 10 \mathrm{O}$

$\mathrm{H} 10 \mathrm{~N}-\mathrm{C} 102-\mathrm{H} 10 \mathrm{O}$

C104-C105-H10P

C104-C105-H10Q

H10P-C105-H10Q

C106-C105-C104
109.5

109.5

109.5

109.5

$66.1(19)$

154.4

53.9

95.3

108.3

108.3

107.4

115.8 (11)

108.3

108.3

109.5

109.5

110.7 (12)

108.1

109.5

109.5

110.7

110.7

108.8

$105.0(12)$

110.7

110.7

109.5

109.5

109.5

109.5

109.5

109.5

110.1

110.1

108.4

108.2 (10)

110.1

110.1

109.5

109.5

109.5

109.5

109.5

109.5

109.6

109.6

108.1

110.3 (15) 


\begin{tabular}{|c|c|}
\hline $\mathrm{C} 33-\mathrm{C} 32-\mathrm{H} 32$ & 119.3 \\
\hline $\mathrm{C} 32-\mathrm{C} 33-\mathrm{H} 33$ & 119.2 \\
\hline $\mathrm{C} 34-\mathrm{C} 33-\mathrm{C} 32$ & $121.5(7)$ \\
\hline $\mathrm{C} 34-\mathrm{C} 33-\mathrm{H} 33$ & 119.2 \\
\hline $\mathrm{C} 33-\mathrm{C} 34-\mathrm{H} 34$ & 120.3 \\
\hline $\mathrm{C} 33-\mathrm{C} 34-\mathrm{C} 35$ & $119.4(7)$ \\
\hline $\mathrm{C} 35-\mathrm{C} 34-\mathrm{H} 34$ & 120.3 \\
\hline $\mathrm{C} 30-\mathrm{C} 35-\mathrm{C} 34$ & $119.3(7)$ \\
\hline $\mathrm{C} 30-\mathrm{C} 35-\mathrm{C} 36$ & $111.5(6)$ \\
\hline $\mathrm{C} 34-\mathrm{C} 35-\mathrm{C} 36$ & $129.2(7)$ \\
\hline $\mathrm{C} 22-\mathrm{C} 36-\mathrm{C} 85$ & $111.2(6)$ \\
\hline $\mathrm{C} 22-\mathrm{C} 36-\mathrm{C} 91$ & $114.1(6)$ \\
\hline $\mathrm{C} 35-\mathrm{C} 36-\mathrm{C} 22$ & $100.0(6)$ \\
\hline $\mathrm{C} 35-\mathrm{C} 36-\mathrm{C} 85$ & $111.7(6)$ \\
\hline $\mathrm{C} 35-\mathrm{C} 36-\mathrm{C} 91$ & $112.2(7)$ \\
\hline $\mathrm{C} 85-\mathrm{C} 36-\mathrm{C} 91$ & $107.6(7)$ \\
\hline $\mathrm{C} 38-\mathrm{C} 37-\mathrm{Ir} 1$ & $128.2(5)$ \\
\hline $\mathrm{C} 38-\mathrm{C} 37-\mathrm{C} 42$ & $117.7(6)$ \\
\hline $\mathrm{C} 42-\mathrm{C} 37-\mathrm{Ir} 1$ & $113.6(4)$ \\
\hline $\mathrm{C} 37-\mathrm{C} 38-\mathrm{H} 38$ & 119.8 \\
\hline $\mathrm{C} 39-\mathrm{C} 38-\mathrm{C} 37$ & $120.4(6)$ \\
\hline $\mathrm{C} 39-\mathrm{C} 38-\mathrm{H} 38$ & 119.8 \\
\hline $\mathrm{C} 38-\mathrm{C} 39-\mathrm{C} 40$ & $121.1(6)$ \\
\hline $\mathrm{C} 38-\mathrm{C} 39-\mathrm{C} 48$ & $131.1(6)$ \\
\hline $\mathrm{C} 40-\mathrm{C} 39-\mathrm{C} 48$ & $107.8(5)$ \\
\hline $\mathrm{C} 39-\mathrm{C} 40-\mathrm{C} 54$ & $111.6(5)$ \\
\hline $\mathrm{C} 41-\mathrm{C} 40-\mathrm{C} 39$ & $119.6(6)$ \\
\hline $\mathrm{C} 41-\mathrm{C} 40-\mathrm{C} 54$ & $128.9(6)$ \\
\hline $\mathrm{C} 40-\mathrm{C} 41-\mathrm{H} 41$ & 120.2 \\
\hline $\mathrm{C} 40-\mathrm{C} 41-\mathrm{C} 42$ & $119.5(6)$ \\
\hline $\mathrm{C} 42-\mathrm{C} 41-\mathrm{H} 41$ & 120.2 \\
\hline $\mathrm{C} 37-\mathrm{C} 42-\mathrm{C} 43$ & $114.9(5)$ \\
\hline $\mathrm{C} 41-\mathrm{C} 42-\mathrm{C} 37$ & $121.6(6)$ \\
\hline $\mathrm{C} 41-\mathrm{C} 42-\mathrm{C} 43$ & $123.4(6)$ \\
\hline $\mathrm{N} 3-\mathrm{C} 43-\mathrm{C} 42$ & $113.8(5)$ \\
\hline $\mathrm{N} 3-\mathrm{C} 43-\mathrm{C} 44$ & $120.3(6)$ \\
\hline $\mathrm{C} 44-\mathrm{C} 43-\mathrm{C} 42$ & $125.6(6)$ \\
\hline $\mathrm{C} 43-\mathrm{C} 44-\mathrm{H} 44$ & 119.5 \\
\hline $\mathrm{C} 43-\mathrm{C} 44-\mathrm{C} 45$ & $121.0(6)$ \\
\hline $\mathrm{C} 45-\mathrm{C} 44-\mathrm{H} 44$ & 119.5 \\
\hline $\mathrm{C} 44-\mathrm{C} 45-\mathrm{H} 45$ & 121.6 \\
\hline $\mathrm{C} 46-\mathrm{C} 45-\mathrm{C} 44$ & $116.8(6)$ \\
\hline $\mathrm{C} 46-\mathrm{C} 45-\mathrm{H} 45$ & 121.6 \\
\hline $\mathrm{C} 45-\mathrm{C} 46-\mathrm{F} 3$ & $119.3(6)$ \\
\hline $\mathrm{C} 45-\mathrm{C} 46-\mathrm{C} 47$ & $122.5(7)$ \\
\hline $\mathrm{C} 47-\mathrm{C} 46-\mathrm{F} 3$ & $118.1(6)$ \\
\hline $\mathrm{N} 3-\mathrm{C} 47-\mathrm{C} 46$ & $119.7(6)$ \\
\hline $\mathrm{N} 3-\mathrm{C} 47-\mathrm{H} 47$ & 120.2 \\
\hline
\end{tabular}

\begin{tabular}{|c|c|}
\hline $\mathrm{C} 106-\mathrm{C} 105-\mathrm{H} 10 \mathrm{P}$ & 109.6 \\
\hline $\mathrm{C} 106-\mathrm{C} 105-\mathrm{H} 10 \mathrm{Q}$ & 109.6 \\
\hline $\mathrm{C} 105-\mathrm{C} 106-\mathrm{H} 10 \mathrm{R}$ & 103.4 \\
\hline $\mathrm{C} 105-\mathrm{C} 106-\mathrm{H} 10 \mathrm{~S}$ & 103.4 \\
\hline $\mathrm{H} 10 \mathrm{R}-\mathrm{C} 106-\mathrm{H} 10 \mathrm{~S}$ & 105.2 \\
\hline $\mathrm{C} 107-\mathrm{C} 106-\mathrm{C} 105$ & $135.1(19)$ \\
\hline $\mathrm{C} 107-\mathrm{C} 106-\mathrm{H} 10 \mathrm{R}$ & 103.4 \\
\hline $\mathrm{C} 107-\mathrm{C} 106-\mathrm{H} 10 \mathrm{~S}$ & 103.4 \\
\hline $\mathrm{C} 106-\mathrm{C} 107-\mathrm{H} 10 \mathrm{~T}$ & 107.2 \\
\hline $\mathrm{C} 106-\mathrm{C} 107-\mathrm{H} 10 \mathrm{U}$ & 107.2 \\
\hline $\mathrm{C} 106-\mathrm{C} 107-\mathrm{C} 108$ & $120.5(19)$ \\
\hline $\mathrm{H} 10 \mathrm{~T}-\mathrm{C} 107-\mathrm{H} 10 \mathrm{U}$ & 106.8 \\
\hline C108-C107-H10T & 107.2 \\
\hline $\mathrm{C} 108-\mathrm{C} 107-\mathrm{H} 10 \mathrm{U}$ & 107.2 \\
\hline $\mathrm{C} 107-\mathrm{C} 108-\mathrm{H} 10 \mathrm{~V}$ & 109.5 \\
\hline $\mathrm{C} 107-\mathrm{C} 108-\mathrm{H} 10 \mathrm{~W}$ & 109.5 \\
\hline $\mathrm{C} 107-\mathrm{C} 108-\mathrm{H} 10 \mathrm{X}$ & 109.5 \\
\hline $\mathrm{H} 10 \mathrm{~V}-\mathrm{C} 108-\mathrm{H} 10 \mathrm{~W}$ & 109.5 \\
\hline $\mathrm{H} 10 \mathrm{~V}-\mathrm{C} 108-\mathrm{H} 10 \mathrm{X}$ & 109.5 \\
\hline $\mathrm{H} 10 \mathrm{~W}-\mathrm{C} 108-\mathrm{H} 10 \mathrm{X}$ & 109.5 \\
\hline $\mathrm{C} 110-\mathrm{C} 111-\mathrm{H} 11 \mathrm{~K}$ & 106.9 \\
\hline $\mathrm{C} 110-\mathrm{C} 111-\mathrm{H} 11 \mathrm{~L}$ & 106.9 \\
\hline $\mathrm{C} 110-\mathrm{C} 111-\mathrm{C} 112$ & $121.6(11)$ \\
\hline $\mathrm{H} 11 \mathrm{~K}-\mathrm{C} 111-\mathrm{H} 11 \mathrm{~L}$ & 106.7 \\
\hline $\mathrm{C} 112-\mathrm{C} 111-\mathrm{H} 11 \mathrm{~K}$ & 106.9 \\
\hline $\mathrm{C} 112-\mathrm{C} 111-\mathrm{H} 11 \mathrm{~L}$ & 106.9 \\
\hline $\mathrm{C} 111-\mathrm{C} 112-\mathrm{H} 11 \mathrm{M}$ & 107.3 \\
\hline $\mathrm{C} 111-\mathrm{C} 112-\mathrm{H} 11 \mathrm{~N}$ & 107.3 \\
\hline $\mathrm{H} 11 \mathrm{M}-\mathrm{C} 112-\mathrm{H} 11 \mathrm{~N}$ & 106.9 \\
\hline $\mathrm{C} 113-\mathrm{C} 112-\mathrm{C} 111$ & $120.2(14)$ \\
\hline $\mathrm{C} 113-\mathrm{C} 112-\mathrm{H} 11 \mathrm{M}$ & 107.3 \\
\hline $\mathrm{C} 113-\mathrm{C} 112-\mathrm{H} 11 \mathrm{~N}$ & 107.3 \\
\hline $\mathrm{C} 112-\mathrm{C} 113-\mathrm{H} 11 \mathrm{O}$ & 109.1 \\
\hline $\mathrm{C} 112-\mathrm{C} 113-\mathrm{H} 11 \mathrm{P}$ & 109.1 \\
\hline $\mathrm{C} 112-\mathrm{C} 113-\mathrm{C} 114$ & $112.3(16)$ \\
\hline $\mathrm{H} 11 \mathrm{O}-\mathrm{C} 113-\mathrm{H} 11 \mathrm{P}$ & 107.9 \\
\hline $\mathrm{C} 114-\mathrm{C} 113-\mathrm{H} 11 \mathrm{O}$ & 109.1 \\
\hline $\mathrm{C} 114-\mathrm{C} 113-\mathrm{H} 11 \mathrm{P}$ & 109.1 \\
\hline $\mathrm{C} 113-\mathrm{C} 114-\mathrm{H} 11 \mathrm{Q}$ & 109.5 \\
\hline $\mathrm{C} 113-\mathrm{C} 114-\mathrm{H} 11 \mathrm{R}$ & 109.5 \\
\hline $\mathrm{C} 113-\mathrm{C} 114-\mathrm{H} 11 \mathrm{~S}$ & 109.5 \\
\hline $\mathrm{H} 11 \mathrm{Q}-\mathrm{C} 114-\mathrm{H} 11 \mathrm{R}$ & 109.5 \\
\hline $\mathrm{H} 11 \mathrm{Q}-\mathrm{C} 114-\mathrm{H} 11 \mathrm{~S}$ & 109.5 \\
\hline $\mathrm{H} 11 \mathrm{R}-\mathrm{C} 114-\mathrm{H} 11 \mathrm{~S}$ & 109.5 \\
\hline $\mathrm{C} 116-\mathrm{C} 117-\mathrm{H} 11 \mathrm{~T}$ & 110.7 \\
\hline $\mathrm{C} 116-\mathrm{C} 117-\mathrm{H} 11 \mathrm{U}$ & 110.7 \\
\hline $\mathrm{H} 11 \mathrm{~T}-\mathrm{C} 117-\mathrm{H} 11 \mathrm{U}$ & 108.8 \\
\hline $\mathrm{C} 118-\mathrm{C} 117-\mathrm{C} 116$ & $105.4(11)$ \\
\hline
\end{tabular}




\begin{tabular}{|c|c|}
\hline $\mathrm{C} 46-\mathrm{C} 47-\mathrm{H} 47$ & 120.2 \\
\hline $\mathrm{C} 49-\mathrm{C} 48-\mathrm{C} 39$ & $130.9(6)$ \\
\hline $\mathrm{C} 49-\mathrm{C} 48-\mathrm{C} 53$ & $120.5(6)$ \\
\hline $\mathrm{C} 53-\mathrm{C} 48-\mathrm{C} 39$ & $108.6(5)$ \\
\hline $\mathrm{C} 48-\mathrm{C} 49-\mathrm{H} 49$ & 120.8 \\
\hline $\mathrm{C} 48-\mathrm{C} 49-\mathrm{C} 50$ & $118.4(6)$ \\
\hline $\mathrm{C} 50-\mathrm{C} 49-\mathrm{H} 49$ & 120.8 \\
\hline $\mathrm{C} 49-\mathrm{C} 50-\mathrm{H} 50$ & 119.8 \\
\hline $\mathrm{C} 51-\mathrm{C} 50-\mathrm{C} 49$ & $120.4(6)$ \\
\hline $\mathrm{C} 51-\mathrm{C} 50-\mathrm{H} 50$ & 119.8 \\
\hline $\mathrm{C} 50-\mathrm{C} 51-\mathrm{H} 51$ & 119.4 \\
\hline $\mathrm{C} 50-\mathrm{C} 51-\mathrm{C} 52$ & $121.1(6)$ \\
\hline $\mathrm{C} 52-\mathrm{C} 51-\mathrm{H} 51$ & 119.4 \\
\hline $\mathrm{C} 51-\mathrm{C} 52-\mathrm{H} 52$ & 120.5 \\
\hline $\mathrm{C} 53-\mathrm{C} 52-\mathrm{C} 51$ & $119.0(6)$ \\
\hline C53-C52-H52 & 120.5 \\
\hline $\mathrm{C} 48-\mathrm{C} 53-\mathrm{C} 54$ & $110.7(6)$ \\
\hline $\mathrm{C} 52-\mathrm{C} 53-\mathrm{C} 48$ & $120.5(6)$ \\
\hline $\mathrm{C} 52-\mathrm{C} 53-\mathrm{C} 54$ & $128.7(6)$ \\
\hline $\mathrm{C} 40-\mathrm{C} 54-\mathrm{C} 53$ & $101.4(5)$ \\
\hline $\mathrm{C} 40-\mathrm{C} 54-\mathrm{C} 97$ & $112.0(6)$ \\
\hline $\mathrm{C} 40-\mathrm{C} 54-\mathrm{C} 103$ & $112.8(5)$ \\
\hline $\mathrm{C} 53-\mathrm{C} 54-\mathrm{C} 97$ & $110.3(5)$ \\
\hline $\mathrm{C} 53-\mathrm{C} 54-\mathrm{C} 103$ & $110.6(6)$ \\
\hline $\mathrm{C} 103-\mathrm{C} 54-\mathrm{C} 97$ & $109.5(6)$ \\
\hline C56-C55-Ir1 & $127.3(4)$ \\
\hline $\mathrm{C} 56-\mathrm{C} 55-\mathrm{C} 60$ & $119.0(6)$ \\
\hline $\mathrm{C} 60-\mathrm{C} 55-\mathrm{Ir} 1$ & $113.6(5)$ \\
\hline $\mathrm{C} 55-\mathrm{C} 56-\mathrm{H} 56$ & 120.4 \\
\hline $\mathrm{C} 55-\mathrm{C} 56-\mathrm{C} 57$ & $119.2(6)$ \\
\hline C57-C56-H56 & 120.4 \\
\hline $\mathrm{C} 56-\mathrm{C} 57-\mathrm{C} 58$ & $121.4(6)$ \\
\hline $\mathrm{C} 56-\mathrm{C} 57-\mathrm{C} 66$ & $129.9(6)$ \\
\hline $\mathrm{C} 58-\mathrm{C} 57-\mathrm{C} 66$ & $108.6(6)$ \\
\hline C57-C58-C72 & $110.4(6)$ \\
\hline $\mathrm{C} 59-\mathrm{C} 58-\mathrm{C} 57$ & $119.9(6)$ \\
\hline $\mathrm{C} 59-\mathrm{C} 58-\mathrm{C} 72$ & $129.7(6)$ \\
\hline $\mathrm{C} 58-\mathrm{C} 59-\mathrm{H} 59$ & 120.4 \\
\hline С $58-\mathrm{C} 59-\mathrm{C} 60$ & $119.1(6)$ \\
\hline $\mathrm{C} 60-\mathrm{C} 59-\mathrm{H} 59$ & 120.4 \\
\hline $\mathrm{C} 55-\mathrm{C} 60-\mathrm{C} 61$ & $115.7(6)$ \\
\hline $\mathrm{C} 59-\mathrm{C} 60-\mathrm{C} 55$ & $121.2(6)$ \\
\hline $\mathrm{C} 59-\mathrm{C} 60-\mathrm{C} 61$ & $123.0(6)$ \\
\hline $\mathrm{N} 4-\mathrm{C} 61-\mathrm{C} 60$ & $114.0(5)$ \\
\hline $\mathrm{N} 4-\mathrm{C} 61-\mathrm{C} 62$ & $119.8(6)$ \\
\hline $\mathrm{C} 62-\mathrm{C} 61-\mathrm{C} 60$ & $126.2(6)$ \\
\hline $\mathrm{C} 61-\mathrm{C} 62-\mathrm{H} 62$ & 119.4 \\
\hline C63-C62-C61 & $121.1(7)$ \\
\hline
\end{tabular}

\begin{tabular}{|c|c|}
\hline $\mathrm{C} 118-\mathrm{C} 117-\mathrm{H} 11 \mathrm{~T}$ & 110.7 \\
\hline $\mathrm{C} 118-\mathrm{C} 117-\mathrm{H} 11 \mathrm{U}$ & 110.7 \\
\hline $\mathrm{C} 117-\mathrm{C} 118-\mathrm{H} 11 \mathrm{~V}$ & 109.6 \\
\hline $\mathrm{C} 117-\mathrm{C} 118-\mathrm{H} 11 \mathrm{~W}$ & 109.6 \\
\hline $\mathrm{C} 117-\mathrm{C} 118-\mathrm{C} 119$ & $110.2(12)$ \\
\hline $\mathrm{H} 11 \mathrm{~V}-\mathrm{C} 118-\mathrm{H} 11 \mathrm{~W}$ & 108.1 \\
\hline $\mathrm{C} 119-\mathrm{C} 118-\mathrm{H} 11 \mathrm{~V}$ & 109.6 \\
\hline $\mathrm{C} 119-\mathrm{C} 118-\mathrm{H} 11 \mathrm{~W}$ & 109.6 \\
\hline $\mathrm{C} 118-\mathrm{C} 119-\mathrm{H} 11 \mathrm{X}$ & 109.5 \\
\hline $\mathrm{C} 118-\mathrm{C} 119-\mathrm{H} 11 \mathrm{Y}$ & 109.5 \\
\hline $\mathrm{C} 118-\mathrm{C} 119-\mathrm{C} 120$ & $110.7(15)$ \\
\hline $\mathrm{H} 11 \mathrm{X}-\mathrm{C} 119-\mathrm{H} 11 \mathrm{Y}$ & 108.1 \\
\hline $\mathrm{C} 120-\mathrm{C} 119-\mathrm{H} 11 \mathrm{X}$ & 109.5 \\
\hline $\mathrm{C} 120-\mathrm{C} 119-\mathrm{H} 11 \mathrm{Y}$ & 109.5 \\
\hline $\mathrm{C} 119-\mathrm{C} 120-\mathrm{H} 12 \mathrm{~A}$ & 109.5 \\
\hline $\mathrm{C} 119-\mathrm{C} 120-\mathrm{H} 12 \mathrm{~B}$ & 109.5 \\
\hline $\mathrm{C} 119-\mathrm{C} 120-\mathrm{H} 12 \mathrm{C}$ & 109.5 \\
\hline $\mathrm{H} 12 \mathrm{~A}-\mathrm{C} 120-\mathrm{H} 12 \mathrm{~B}$ & 109.5 \\
\hline $\mathrm{H} 12 \mathrm{~A}-\mathrm{C} 120-\mathrm{H} 12 \mathrm{C}$ & 109.5 \\
\hline $\mathrm{H} 12 \mathrm{~B}-\mathrm{C} 120-\mathrm{H} 12 \mathrm{C}$ & 109.5 \\
\hline $\mathrm{H} 96 \mathrm{D}-\mathrm{C} 96 \mathrm{~B}-\mathrm{H} 96 \mathrm{E}$ & 109.5 \\
\hline $\mathrm{H} 96 \mathrm{D}-\mathrm{C} 96 \mathrm{~B}-\mathrm{H} 96 \mathrm{~F}$ & 109.5 \\
\hline $\mathrm{H} 96 \mathrm{E}-\mathrm{C} 96 \mathrm{~B}-\mathrm{H} 96 \mathrm{~F}$ & 109.5 \\
\hline $\mathrm{C} 95 \mathrm{~B}-\mathrm{C} 96 \mathrm{~B}-\mathrm{H} 96 \mathrm{D}$ & 109.5 \\
\hline $\mathrm{C} 95 \mathrm{~B}-\mathrm{C} 96 \mathrm{~B}-\mathrm{H} 96 \mathrm{E}$ & 109.5 \\
\hline $\mathrm{C} 95 \mathrm{~B}-\mathrm{C} 96 \mathrm{~B}-\mathrm{H} 96 \mathrm{~F}$ & 109.5 \\
\hline $\mathrm{C} 110-\mathrm{C} 211-\mathrm{H} 21 \mathrm{~A}$ & 111.1 \\
\hline $\mathrm{C} 110-\mathrm{C} 211-\mathrm{H} 21 \mathrm{~B}$ & 111.1 \\
\hline $\mathrm{H} 21 \mathrm{~A}-\mathrm{C} 211-\mathrm{H} 21 \mathrm{~B}$ & 109.0 \\
\hline $\mathrm{C} 212-\mathrm{C} 211-\mathrm{C} 110$ & $103.4(16)$ \\
\hline $\mathrm{C} 212-\mathrm{C} 211-\mathrm{H} 21 \mathrm{~A}$ & 111.1 \\
\hline $\mathrm{C} 212-\mathrm{C} 211-\mathrm{H} 21 \mathrm{~B}$ & 111.1 \\
\hline $\mathrm{C} 211-\mathrm{C} 212-\mathrm{H} 21 \mathrm{C}$ & 109.3 \\
\hline $\mathrm{C} 211-\mathrm{C} 212-\mathrm{H} 21 \mathrm{D}$ & 109.3 \\
\hline $\mathrm{H} 21 \mathrm{C}-\mathrm{C} 212-\mathrm{H} 21 \mathrm{D}$ & 107.9 \\
\hline $\mathrm{C} 213-\mathrm{C} 212-\mathrm{C} 211$ & $111.8(17)$ \\
\hline $\mathrm{C} 213-\mathrm{C} 212-\mathrm{H} 21 \mathrm{C}$ & 109.3 \\
\hline $\mathrm{C} 213-\mathrm{C} 212-\mathrm{H} 21 \mathrm{D}$ & 109.3 \\
\hline $\mathrm{C} 212-\mathrm{C} 213-\mathrm{H} 21 \mathrm{E}$ & 109.5 \\
\hline $\mathrm{C} 212-\mathrm{C} 213-\mathrm{H} 21 \mathrm{~F}$ & 109.5 \\
\hline $\mathrm{C} 212-\mathrm{C} 213-\mathrm{C} 214$ & $111(2)$ \\
\hline $\mathrm{H} 21 \mathrm{E}-\mathrm{C} 213-\mathrm{H} 21 \mathrm{~F}$ & 108.1 \\
\hline $\mathrm{C} 214-\mathrm{C} 213-\mathrm{H} 21 \mathrm{E}$ & 109.5 \\
\hline $\mathrm{C} 214-\mathrm{C} 213-\mathrm{H} 21 \mathrm{~F}$ & 109.5 \\
\hline $\mathrm{C} 213-\mathrm{C} 214-\mathrm{H} 21 \mathrm{G}$ & 109.5 \\
\hline $\mathrm{C} 213-\mathrm{C} 214-\mathrm{H} 21 \mathrm{H}$ & 109.5 \\
\hline $\mathrm{C} 213-\mathrm{C} 214-\mathrm{H} 21 \mathrm{I}$ & 109.5 \\
\hline $\mathrm{H} 21 \mathrm{G}-\mathrm{C} 214-\mathrm{H} 21 \mathrm{H}$ & 109.5 \\
\hline
\end{tabular}




\begin{tabular}{|c|c|}
\hline $\mathrm{C} 63-\mathrm{C} 62-\mathrm{H} 62$ & 119.4 \\
\hline $\mathrm{C} 62-\mathrm{C} 63-\mathrm{H} 63$ & 121.3 \\
\hline $\mathrm{C} 64-\mathrm{C} 63-\mathrm{C} 62$ & $117.3(7)$ \\
\hline $\mathrm{C} 64-\mathrm{C} 63-\mathrm{H} 63$ & 121.3 \\
\hline $\mathrm{F} 4-\mathrm{C} 64-\mathrm{C} 65$ & $117.0(7)$ \\
\hline $\mathrm{C} 63-\mathrm{C} 64-\mathrm{F} 4$ & $120.6(7)$ \\
\hline $\mathrm{C} 63-\mathrm{C} 64-\mathrm{C} 65$ & $122.4(7)$ \\
\hline $\mathrm{N} 4-\mathrm{C} 65-\mathrm{C} 64$ & $119.6(6)$ \\
\hline $\mathrm{N} 4-\mathrm{C} 65-\mathrm{H} 65$ & 120.2 \\
\hline $\mathrm{C} 64-\mathrm{C} 65-\mathrm{H} 65$ & 120.2 \\
\hline C67-C66-C57 & $130.2(6)$ \\
\hline $\mathrm{C} 67-\mathrm{C} 66-\mathrm{C} 71$ & $120.7(6)$ \\
\hline $\mathrm{C} 71-\mathrm{C} 66-\mathrm{C} 57$ & $109.1(6)$ \\
\hline C66-C67-H67 & 120.8 \\
\hline C66-C67-C68 & $118.5(6)$ \\
\hline C68-C67-H67 & 120.8 \\
\hline C67-C68-H68 & 120.3 \\
\hline $\mathrm{C} 69-\mathrm{C} 68-\mathrm{C} 67$ & $119.4(7)$ \\
\hline $\mathrm{C} 69-\mathrm{C} 68-\mathrm{H} 68$ & 120.3 \\
\hline $\mathrm{C} 68-\mathrm{C} 69-\mathrm{H} 69$ & 118.8 \\
\hline $\mathrm{C} 70-\mathrm{C} 69-\mathrm{C} 68$ & $122.4(7)$ \\
\hline $\mathrm{C} 70-\mathrm{C} 69-\mathrm{H} 69$ & 118.8 \\
\hline $\mathrm{C} 69-\mathrm{C} 70-\mathrm{H} 70$ & 120.6 \\
\hline $\mathrm{C} 69-\mathrm{C} 70-\mathrm{C} 71$ & $118.8(6)$ \\
\hline $\mathrm{C} 71-\mathrm{C} 70-\mathrm{H} 70$ & 120.6 \\
\hline $\mathrm{C} 66-\mathrm{C} 71-\mathrm{C} 72$ & $110.3(6)$ \\
\hline $\mathrm{C} 70-\mathrm{C} 71-\mathrm{C} 66$ & $120.2(7)$ \\
\hline $\mathrm{C} 70-\mathrm{C} 71-\mathrm{C} 72$ & $129.5(6)$ \\
\hline $\mathrm{C} 58-\mathrm{C} 72-\mathrm{C} 115$ & $110.9(6)$ \\
\hline $\mathrm{C} 71-\mathrm{C} 72-\mathrm{C} 58$ & $101.5(5)$ \\
\hline $\mathrm{C} 71-\mathrm{C} 72-\mathrm{C} 109$ & $112.7(6)$ \\
\hline $\mathrm{C} 71-\mathrm{C} 72-\mathrm{C} 115$ & $112.5(6)$ \\
\hline $\mathrm{C} 109-\mathrm{C} 72-\mathrm{C} 58$ & $111.7(6)$ \\
\hline $\mathrm{C} 109-\mathrm{C} 72-\mathrm{C} 115$ & $107.6(6)$ \\
\hline $\mathrm{H} 82 \mathrm{C}-\mathrm{C} 82-\mathrm{H} 82 \mathrm{D}$ & 108.7 \\
\hline $\mathrm{H} 82 \mathrm{C}-\mathrm{C} 82-\mathrm{H} 82 \mathrm{~A}$ & 61.7 \\
\hline $\mathrm{H} 82 \mathrm{C}-\mathrm{C} 82-\mathrm{H} 82 \mathrm{~B}$ & 93.7 \\
\hline $\mathrm{H} 82 \mathrm{D}-\mathrm{C} 82-\mathrm{H} 82 \mathrm{~A}$ & 137.4 \\
\hline $\mathrm{H} 82 \mathrm{D}-\mathrm{C} 82-\mathrm{H} 82 \mathrm{~B}$ & 28.8 \\
\hline $\mathrm{H} 82 \mathrm{~A}-\mathrm{C} 82-\mathrm{H} 82 \mathrm{~B}$ & 108.7 \\
\hline $\mathrm{C} 81-\mathrm{C} 82-\mathrm{H} 82 \mathrm{C}$ & 110.6 \\
\hline $\mathrm{C} 81-\mathrm{C} 82-\mathrm{H} 82 \mathrm{D}$ & 110.6 \\
\hline $\mathrm{C} 81-\mathrm{C} 82-\mathrm{H} 82 \mathrm{~A}$ & 50.4 \\
\hline $\mathrm{C} 81-\mathrm{C} 82-\mathrm{H} 82 \mathrm{~B}$ & 94.7 \\
\hline $\mathrm{C} 81-\mathrm{C} 82-\mathrm{C} 81 \mathrm{~B}$ & $71.2(14)$ \\
\hline $\mathrm{C} 83-\mathrm{C} 82-\mathrm{H} 82 \mathrm{C}$ & 110.6 \\
\hline $\mathrm{C} 83-\mathrm{C} 82-\mathrm{H} 82 \mathrm{D}$ & 110.6 \\
\hline 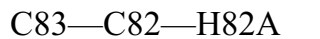 & \\
\hline
\end{tabular}

$\mathrm{H} 21 \mathrm{G}-\mathrm{C} 214-\mathrm{H} 21 \mathrm{I}$

$\mathrm{H} 21 \mathrm{H}-\mathrm{C} 214-\mathrm{H} 21 \mathrm{I} \quad 109.5$

$\mathrm{C} 92-\mathrm{C} 93 \mathrm{~B}-\mathrm{H} 93 \mathrm{C} \quad 111.5$

C92-C93B-H93D $\quad 111.5$

C92-C93B-C94B 101.4 (16)

H93C-C93B-H93D $\quad 109.3$

$\mathrm{C} 94 \mathrm{~B}-\mathrm{C} 93 \mathrm{~B}-\mathrm{H} 93 \mathrm{C} \quad 111.5$

$\mathrm{C} 94 \mathrm{~B}-\mathrm{C} 93 \mathrm{~B}-\mathrm{H} 93 \mathrm{D} \quad 111.5$

C93B-C94B-H94C $\quad 104.4$

C93B-C94B-H94D $\quad 104.4$

$\mathrm{H} 94 \mathrm{C}-\mathrm{C} 94 \mathrm{~B}-\mathrm{H} 94 \mathrm{D} \quad 105.6$

C95B-C94B-C93B 132 (3)

$\mathrm{C} 95 \mathrm{~B}-\mathrm{C} 94 \mathrm{~B}-\mathrm{H} 94 \mathrm{C} \quad 104.4$

C95B-C94B-H94D $\quad 104.4$

$\mathrm{C} 96 \mathrm{~B}-\mathrm{C} 95 \mathrm{~B}-\mathrm{C} 94 \mathrm{~B} \quad 107$ (2)

$\mathrm{C} 96 \mathrm{~B}-\mathrm{C} 95 \mathrm{~B}-\mathrm{H} 95 \mathrm{C} \quad 110.3$

$\mathrm{C} 96 \mathrm{~B}-\mathrm{C} 95 \mathrm{~B}-\mathrm{H} 95 \mathrm{D} \quad 110.3$

$\mathrm{C} 94 \mathrm{~B}-\mathrm{C} 95 \mathrm{~B}-\mathrm{H} 95 \mathrm{C} \quad 110.3$

C94B-C95B-H95D $\quad 110.3$

$\mathrm{H} 95 \mathrm{C}-\mathrm{C} 95 \mathrm{~B}-\mathrm{H} 95 \mathrm{D} \quad 108.5$

$\mathrm{C} 100-\mathrm{C} 201-\mathrm{H} 20 \mathrm{~A} \quad 110.8$

$\mathrm{C} 100-\mathrm{C} 201-\mathrm{H} 20 \mathrm{~B} \quad 110.8$

$\mathrm{H} 20 \mathrm{~A}-\mathrm{C} 201-\mathrm{H} 20 \mathrm{~B} \quad 108.9$

$\mathrm{C} 202-\mathrm{C} 201-\mathrm{C} 100 \quad 105$ (3)

$\mathrm{C} 202-\mathrm{C} 201-\mathrm{H} 20 \mathrm{~A} \quad 110.8$

$\mathrm{C} 202-\mathrm{C} 201-\mathrm{H} 20 \mathrm{~B} \quad 110.8$

$\mathrm{C} 201-\mathrm{C} 202-\mathrm{H} 20 \mathrm{C} \quad 109.5$

$\mathrm{C} 201-\mathrm{C} 202-\mathrm{H} 20 \mathrm{D} \quad 109.5$

$\mathrm{C} 201-\mathrm{C} 202-\mathrm{H} 20 \mathrm{E}$

$\mathrm{H} 20 \mathrm{C}-\mathrm{C} 202-\mathrm{H} 20 \mathrm{D} \quad 109.5$

$\mathrm{H} 20 \mathrm{C}-\mathrm{C} 202-\mathrm{H} 20 \mathrm{E} \quad 109.5$

$\mathrm{H} 20 \mathrm{D}-\mathrm{C} 202-\mathrm{H} 20 \mathrm{E} \quad 109.5$

C116-C217-H21J $\quad 106.6$

$\mathrm{C} 116-\mathrm{C} 217-\mathrm{H} 21 \mathrm{~K} \quad 106.6$

$\mathrm{H} 21 \mathrm{~J}-\mathrm{C} 217-\mathrm{H} 21 \mathrm{~K} \quad 106.5$

$\mathrm{C} 218-\mathrm{C} 217-\mathrm{C} 116 \quad 123.1$ (15)

$\mathrm{C} 218-\mathrm{C} 217-\mathrm{H} 21 \mathrm{~J} \quad 106.6$

$\mathrm{C} 218-\mathrm{C} 217-\mathrm{H} 21 \mathrm{~K} \quad 106.6$

C217-C218-H21L $\quad 107.2$

$\mathrm{C} 217-\mathrm{C} 218-\mathrm{H} 21 \mathrm{M} \quad 107.2$

$\mathrm{C} 217-\mathrm{C} 218-\mathrm{C} 219 \quad 120$ (2)

$\mathrm{H} 21 \mathrm{~L}-\mathrm{C} 218-\mathrm{H} 21 \mathrm{M} \quad 106.9$

C219-C218-H21L 107.2

$\mathrm{C} 219-\mathrm{C} 218-\mathrm{H} 21 \mathrm{M} \quad 107.2$

$\mathrm{C} 218-\mathrm{C} 219-\mathrm{H} 21 \mathrm{~N} \quad 109.3$

$\mathrm{C} 218-\mathrm{C} 219-\mathrm{H} 21 \mathrm{O} \quad 109.3$

$\mathrm{H} 21 \mathrm{~N}-\mathrm{C} 219-\mathrm{H} 21 \mathrm{O} \quad 107.9$

$\mathrm{C} 220-\mathrm{C} 219-\mathrm{C} 218 \quad 112(2)$ 


\begin{tabular}{|c|c|}
\hline $\mathrm{C} 83-\mathrm{C} 82-\mathrm{H} 82 \mathrm{~B}$ & 139.4 \\
\hline $\mathrm{C} 83-\mathrm{C} 82-\mathrm{C} 81$ & $105.7(11)$ \\
\hline $\mathrm{C} 83-\mathrm{C} 82-\mathrm{C} 81 \mathrm{~B}$ & $48.5(12)$ \\
\hline $\mathrm{C} 83-\mathrm{C} 82-\mathrm{C} 83 \mathrm{~B}$ & $60.4(16)$ \\
\hline $\mathrm{C} 81 \mathrm{~B}-\mathrm{C} 82-\mathrm{H} 82 \mathrm{C}$ & 155.7 \\
\hline $\mathrm{C} 81 \mathrm{~B}-\mathrm{C} 82-\mathrm{H} 82 \mathrm{D}$ & 92.3 \\
\hline $\mathrm{C} 81 \mathrm{~B}-\mathrm{C} 82-\mathrm{H} 82 \mathrm{~A}$ & 110.5 \\
\hline $\mathrm{C} 81 \mathrm{~B}-\mathrm{C} 82-\mathrm{H} 82 \mathrm{~B}$ & 110.5 \\
\hline $\mathrm{C} 83 \mathrm{~B}-\mathrm{C} 82-\mathrm{H} 82 \mathrm{C}$ & 60.6 \\
\hline $\mathrm{C} 83 \mathrm{~B}-\mathrm{C} 82-\mathrm{H} 82 \mathrm{D}$ & 96.0 \\
\hline $\mathrm{C} 83 \mathrm{~B}-\mathrm{C} 82-\mathrm{H} 82 \mathrm{~A}$ & 110.5 \\
\hline $\mathrm{C} 83 \mathrm{~B}-\mathrm{C} 82-\mathrm{H} 82 \mathrm{~B}$ & 110.5 \\
\hline $\mathrm{C} 83 \mathrm{~B}-\mathrm{C} 82-\mathrm{C} 81$ & $153(2)$ \\
\hline $\mathrm{C} 83 \mathrm{~B}-\mathrm{C} 82-\mathrm{C} 81 \mathrm{~B}$ & $106(2)$ \\
\hline $\mathrm{C} 36-\mathrm{C} 85-\mathrm{H} 85 \mathrm{~A}$ & 107.6 \\
\hline $\mathrm{C} 36-\mathrm{C} 85-\mathrm{H} 85 \mathrm{~B}$ & 107.6 \\
\hline $\mathrm{H} 85 \mathrm{~A}-\mathrm{C} 85-\mathrm{H} 85 \mathrm{~B}$ & 107.1 \\
\hline $\mathrm{C} 86-\mathrm{C} 85-\mathrm{C} 36$ & $118.8(6)$ \\
\hline $\mathrm{C} 86-\mathrm{C} 85-\mathrm{H} 85 \mathrm{~A}$ & 107.6 \\
\hline $\mathrm{C} 86-\mathrm{C} 85-\mathrm{H} 85 \mathrm{~B}$ & 107.6 \\
\hline $\mathrm{C} 85-\mathrm{C} 86-\mathrm{H} 86 \mathrm{~A}$ & 109.3 \\
\hline $\mathrm{C} 85-\mathrm{C} 86-\mathrm{H} 86 \mathrm{~B}$ & 109.3 \\
\hline $\mathrm{H} 86 \mathrm{~A}-\mathrm{C} 86-\mathrm{H} 86 \mathrm{~B}$ & 108.0 \\
\hline $\mathrm{C} 87-\mathrm{C} 86-\mathrm{C} 85$ & $111.5(7)$ \\
\hline $\mathrm{C} 87-\mathrm{C} 86-\mathrm{H} 86 \mathrm{~A}$ & 109.3 \\
\hline $\mathrm{C} 87-\mathrm{C} 86-\mathrm{H} 86 \mathrm{~B}$ & 109.3 \\
\hline $\mathrm{C} 86-\mathrm{C} 87-\mathrm{H} 87 \mathrm{~A}$ & 108.6 \\
\hline $\mathrm{C} 86-\mathrm{C} 87-\mathrm{H} 87 \mathrm{~B}$ & 108.6 \\
\hline $\mathrm{H} 87 \mathrm{~A}-\mathrm{C} 87-\mathrm{H} 87 \mathrm{~B}$ & 107.6 \\
\hline $\mathrm{C} 88-\mathrm{C} 87-\mathrm{C} 86$ & $114.5(7)$ \\
\hline $\mathrm{C} 88-\mathrm{C} 87-\mathrm{H} 87 \mathrm{~A}$ & 108.6 \\
\hline $\mathrm{C} 88-\mathrm{C} 87-\mathrm{H} 87 \mathrm{~B}$ & 108.6 \\
\hline $\mathrm{C} 87-\mathrm{C} 88-\mathrm{H} 88 \mathrm{~A}$ & 109.3 \\
\hline $\mathrm{C} 87-\mathrm{C} 88-\mathrm{H} 88 \mathrm{~B}$ & 109.3 \\
\hline C87-C88-C89 & $111.8(8)$ \\
\hline $\mathrm{H} 88 \mathrm{~A}-\mathrm{C} 88-\mathrm{H} 88 \mathrm{~B}$ & 107.9 \\
\hline $\mathrm{C} 89-\mathrm{C} 88-\mathrm{H} 88 \mathrm{~A}$ & 109.3 \\
\hline $\mathrm{C} 89-\mathrm{C} 88-\mathrm{H} 88 \mathrm{~B}$ & 109.3 \\
\hline $\mathrm{C} 88-\mathrm{C} 89-\mathrm{H} 89 \mathrm{~A}$ & 108.9 \\
\hline С $88-\mathrm{C} 89-\mathrm{H} 89 \mathrm{~B}$ & 108.9 \\
\hline $\mathrm{H} 89 \mathrm{~A}-\mathrm{C} 89-\mathrm{H} 89 \mathrm{~B}$ & 107.7 \\
\hline $\mathrm{C} 90-\mathrm{C} 89-\mathrm{C} 88$ & $113.3(8)$ \\
\hline $\mathrm{C} 90-\mathrm{C} 89-\mathrm{H} 89 \mathrm{~A}$ & 108.9 \\
\hline С90- C89-H89B & 108.9 \\
\hline $\mathrm{C} 89-\mathrm{C} 90-\mathrm{H} 90 \mathrm{~A}$ & 109.5 \\
\hline $\mathrm{C} 89-\mathrm{C} 90-\mathrm{H} 90 \mathrm{~B}$ & 109.5 \\
\hline $\mathrm{C} 89-\mathrm{C} 90-\mathrm{H} 90 \mathrm{C}$ & 109.5 \\
\hline & \\
\hline
\end{tabular}

\begin{tabular}{|c|c|}
\hline $\mathrm{C} 220-\mathrm{C} 219-\mathrm{H} 21 \mathrm{~N}$ & 109.3 \\
\hline $\mathrm{C} 220-\mathrm{C} 219-\mathrm{H} 21 \mathrm{O}$ & 109.3 \\
\hline $\mathrm{C} 219-\mathrm{C} 220-\mathrm{H} 22 \mathrm{~A}$ & 109.5 \\
\hline $\mathrm{C} 219-\mathrm{C} 220-\mathrm{H} 22 \mathrm{~B}$ & 109.5 \\
\hline $\mathrm{C} 219-\mathrm{C} 220-\mathrm{H} 22 \mathrm{C}$ & 109.5 \\
\hline $\mathrm{H} 22 \mathrm{~A}-\mathrm{C} 220-\mathrm{H} 22 \mathrm{~B}$ & 109.5 \\
\hline $\mathrm{H} 22 \mathrm{~A}-\mathrm{C} 220-\mathrm{H} 22 \mathrm{C}$ & 109.5 \\
\hline $\mathrm{H} 22 \mathrm{~B}-\mathrm{C} 220-\mathrm{H} 22 \mathrm{C}$ & 109.5 \\
\hline $\mathrm{C} 104-\mathrm{C} 205-\mathrm{H} 20 \mathrm{~F}$ & 105.3 \\
\hline $\mathrm{C} 104-\mathrm{C} 205-\mathrm{H} 20 \mathrm{G}$ & 105.3 \\
\hline $\mathrm{H} 20 \mathrm{~F}-\mathrm{C} 205-\mathrm{H} 20 \mathrm{G}$ & 106.0 \\
\hline $\mathrm{C} 206-\mathrm{C} 205-\mathrm{C} 104$ & $128(4)$ \\
\hline $\mathrm{C} 206-\mathrm{C} 205-\mathrm{H} 20 \mathrm{~F}$ & 105.3 \\
\hline $\mathrm{C} 206-\mathrm{C} 205-\mathrm{H} 20 \mathrm{G}$ & 105.3 \\
\hline $\mathrm{C} 205-\mathrm{C} 206-\mathrm{H} 20 \mathrm{H}$ & 103.1 \\
\hline $\mathrm{C} 205-\mathrm{C} 206-\mathrm{H} 20 \mathrm{I}$ & 103.1 \\
\hline $\mathrm{H} 20 \mathrm{H}-\mathrm{C} 206-\mathrm{H} 20 \mathrm{I}$ & 105.1 \\
\hline $\mathrm{C} 207-\mathrm{C} 206-\mathrm{C} 205$ & $136(4)$ \\
\hline $\mathrm{C} 207-\mathrm{C} 206-\mathrm{H} 20 \mathrm{H}$ & 103.1 \\
\hline $\mathrm{C} 207-\mathrm{C} 206-\mathrm{H} 20 \mathrm{I}$ & 103.1 \\
\hline $\mathrm{C} 206-\mathrm{C} 207-\mathrm{H} 20 \mathrm{~J}$ & 109.6 \\
\hline $\mathrm{C} 206-\mathrm{C} 207-\mathrm{H} 20 \mathrm{~K}$ & 109.6 \\
\hline $\mathrm{H} 20 \mathrm{~J}-\mathrm{C} 207-\mathrm{H} 20 \mathrm{~K}$ & 108.2 \\
\hline $\mathrm{C} 208-\mathrm{C} 207-\mathrm{C} 206$ & $110(5)$ \\
\hline $\mathrm{C} 208-\mathrm{C} 207-\mathrm{H} 20 \mathrm{~J}$ & 109.6 \\
\hline $\mathrm{C} 208-\mathrm{C} 207-\mathrm{H} 20 \mathrm{~K}$ & 109.6 \\
\hline $\mathrm{C} 207-\mathrm{C} 208-\mathrm{H} 20 \mathrm{~L}$ & 109.5 \\
\hline C207-C208-H20M & 109.5 \\
\hline $\mathrm{C} 207-\mathrm{C} 208-\mathrm{H} 20 \mathrm{~N}$ & 109.5 \\
\hline $\mathrm{H} 20 \mathrm{~L}-\mathrm{C} 208-\mathrm{H} 20 \mathrm{M}$ & 109.5 \\
\hline $\mathrm{H} 20 \mathrm{~L}-\mathrm{C} 208-\mathrm{H} 20 \mathrm{~N}$ & 109.5 \\
\hline $\mathrm{H} 20 \mathrm{M}-\mathrm{C} 208-\mathrm{H} 20 \mathrm{~N}$ & 109.5 \\
\hline $\mathrm{C} 73-\mathrm{C} 75 \mathrm{~B}-\mathrm{H} 75 \mathrm{C}$ & 100.2 \\
\hline $\mathrm{C} 73-\mathrm{C} 75 \mathrm{~B}-\mathrm{H} 75 \mathrm{D}$ & 96.7 \\
\hline $\mathrm{C} 74-\mathrm{C} 75 \mathrm{~B}-\mathrm{C} 73$ & $46.0(7)$ \\
\hline $\mathrm{C} 74-\mathrm{C} 75 \mathrm{~B}-\mathrm{H} 75 \mathrm{C}$ & 136.6 \\
\hline $\mathrm{C} 74-\mathrm{C} 75 \mathrm{~B}-\mathrm{H} 75 \mathrm{D}$ & 103.8 \\
\hline $\mathrm{H} 75 \mathrm{C}-\mathrm{C} 75 \mathrm{~B}-\mathrm{H} 75 \mathrm{D}$ & 106.9 \\
\hline $\mathrm{C} 76 \mathrm{~B}-\mathrm{C} 75 \mathrm{~B}-\mathrm{C} 73$ & $135.6(19)$ \\
\hline $\mathrm{C} 76 \mathrm{~B}-\mathrm{C} 75 \mathrm{~B}-\mathrm{C} 74$ & $91.5(16)$ \\
\hline $\mathrm{C} 76 \mathrm{~B}-\mathrm{C} 75 \mathrm{~B}-\mathrm{H} 75 \mathrm{C}$ & 107.4 \\
\hline $\mathrm{C} 76 \mathrm{~B}-\mathrm{C} 75 \mathrm{~B}-\mathrm{H} 75 \mathrm{D}$ & 107.4 \\
\hline $\mathrm{C} 76 \mathrm{~B}-\mathrm{C} 75 \mathrm{~B}-\mathrm{C} 74 \mathrm{~B}$ & $120(2)$ \\
\hline $\mathrm{C} 74 \mathrm{~B}-\mathrm{C} 75 \mathrm{~B}-\mathrm{C} 73$ & $16.0(11)$ \\
\hline $\mathrm{C} 74 \mathrm{~B}-\mathrm{C} 75 \mathrm{~B}-\mathrm{C} 74$ & $33.0(12)$ \\
\hline $\mathrm{C} 74 \mathrm{~B}-\mathrm{C} 75 \mathrm{~B}-\mathrm{H} 75 \mathrm{C}$ & 107.4 \\
\hline $\mathrm{C} 74 \mathrm{~B}-\mathrm{C} 75 \mathrm{~B}-\mathrm{H} 75 \mathrm{D}$ & 107.4 \\
\hline $\mathrm{H} 78 \mathrm{D}-\mathrm{C} 78 \mathrm{~B}-\mathrm{H} 78 \mathrm{E}$ & 109.5 \\
\hline
\end{tabular}


$\mathrm{H} 90 \mathrm{~A}-\mathrm{C} 90-\mathrm{H} 90 \mathrm{C}$

$\mathrm{H} 90 \mathrm{~B}-\mathrm{C} 90-\mathrm{H} 90 \mathrm{C}$

C36-C91-H91A

C36-C91-H91B

H91A-C91-H91B

C92-C91-C36

C92-C91-H91A

C92-C91-H91B

C91-C92-H92A

C91-C92-H92B

$\mathrm{C} 91-\mathrm{C} 92-\mathrm{H} 92 \mathrm{C}$

C91-C92-H92D

$\mathrm{C} 91-\mathrm{C} 92-\mathrm{C} 93$

C91-C92-C93B

$\mathrm{H} 92 \mathrm{~A}-\mathrm{C} 92-\mathrm{H} 92 \mathrm{~B}$

$\mathrm{H} 92 \mathrm{C}-\mathrm{C} 92-\mathrm{H} 92 \mathrm{D}$

C93-C92-H92A

C93- C92- $\mathrm{H} 92 \mathrm{~B}$

C93B-C92-H92C

C93B-C92-H92D

C54-C97-H97A

C54-C97-H97B

H97A-C97-H97B

C98-C97-C54

C98-C97-H97A

C98- C97-H97B

C97-C98-H98A

C97-C98-H98B

C97-C98-C99

H98A-C98-H98B

C99-C98-H98A

C99- C98-H98B

C98-C99-H99A

C98- C99-H99B

H99A-C99-H99B

C100-C99-C98

C100-C99-H99A

C100- C99-H99B

C99- $\mathrm{C} 100-\mathrm{H} 10 \mathrm{C}$

C99- $100-\mathrm{H} 10 \mathrm{D}$

C99- $100-\mathrm{H} 10 \mathrm{~A}$

C99- $100-\mathrm{H} 10 \mathrm{~B}$

C99- $100-\mathrm{C} 101$

$\mathrm{C} 99-\mathrm{C} 100-\mathrm{C} 201$

$\mathrm{H} 10 \mathrm{C}-\mathrm{C} 100-\mathrm{H} 10 \mathrm{D}$

$\mathrm{H} 10 \mathrm{~A}-\mathrm{C} 100-\mathrm{H} 10 \mathrm{~B}$

C101-C100-H10A

C101-C100-H10B
109.5

109.5

108.6

108.6

107.6

114.7 (7)

108.6

108.6

107.0

107.0

111.5

111.5

$121.4(9)$

101.2 (12)

106.7

109.3

107.0

107.0

111.5

111.5

108.2

108.2

107.4

116.2 (6)

108.2

108.2

109.3

109.3

111.7 (7)

107.9

109.3

109.3

108.8

108.8

107.7

114.0 (8)

108.8

108.8

108.4

108.4

107.4

107.4

119.7 (11)

$116(2)$

107.5

106.9

107.4

107.4
$\mathrm{H} 78 \mathrm{D}-\mathrm{C} 78 \mathrm{~B}-\mathrm{H} 78 \mathrm{~F}$

$\mathrm{H} 78 \mathrm{E}-\mathrm{C} 78 \mathrm{~B}-\mathrm{H} 78 \mathrm{~F}$

$\mathrm{C} 77 \mathrm{~B}-\mathrm{C} 78 \mathrm{~B}-\mathrm{H} 78 \mathrm{D}$

$\mathrm{C} 77 \mathrm{~B}-\mathrm{C} 78 \mathrm{~B}-\mathrm{H} 78 \mathrm{E}$

$\mathrm{C} 77 \mathrm{~B}-\mathrm{C} 78 \mathrm{~B}-\mathrm{H} 78 \mathrm{~F}$

$\mathrm{C} 75 \mathrm{~B}-\mathrm{C} 76 \mathrm{~B}-\mathrm{H} 76 \mathrm{C}$

$\mathrm{C} 75 \mathrm{~B}-\mathrm{C} 76 \mathrm{~B}-\mathrm{H} 76 \mathrm{D}$

$\mathrm{C} 75 \mathrm{~B}-\mathrm{C} 76 \mathrm{~B}-\mathrm{C} 77 \mathrm{~B}$

$\mathrm{H} 76 \mathrm{C}-\mathrm{C} 76 \mathrm{~B}-\mathrm{H} 76 \mathrm{D}$

$\mathrm{C} 77 \mathrm{~B}-\mathrm{C} 76 \mathrm{~B}-\mathrm{H} 76 \mathrm{C}$

$\mathrm{C} 77 \mathrm{~B}-\mathrm{C} 76 \mathrm{~B}-\mathrm{H} 76 \mathrm{D}$

$\mathrm{C} 78 \mathrm{~B}-\mathrm{C} 77 \mathrm{~B}-\mathrm{C} 76 \mathrm{~B}$

$\mathrm{C} 78 \mathrm{~B}-\mathrm{C} 77 \mathrm{~B}-\mathrm{H} 77 \mathrm{C}$

$\mathrm{C} 78 \mathrm{~B}-\mathrm{C} 77 \mathrm{~B}-\mathrm{H} 77 \mathrm{D}$

$\mathrm{C} 76 \mathrm{~B}-\mathrm{C} 77 \mathrm{~B}-\mathrm{H} 77 \mathrm{C}$

C76B-C77B-H77D

H77C $-\mathrm{C} 77 \mathrm{~B}-\mathrm{H} 77 \mathrm{D}$

C18-C73B-C79

$\mathrm{C} 18-\mathrm{C} 73 \mathrm{~B}-\mathrm{H} 73 \mathrm{C}$

C18-C73B-H73D

$\mathrm{C} 18-\mathrm{C} 73 \mathrm{~B}-\mathrm{C} 74 \mathrm{~B}$

$\mathrm{C} 18-\mathrm{C} 73 \mathrm{~B}-\mathrm{C} 18 \mathrm{~B}$

$\mathrm{C} 73-\mathrm{C} 73 \mathrm{~B}-\mathrm{C} 18$

$\mathrm{C} 73-\mathrm{C} 73 \mathrm{~B}-\mathrm{C} 79$

$\mathrm{C} 73-\mathrm{C} 73 \mathrm{~B}-\mathrm{H} 73 \mathrm{C}$

$\mathrm{C} 73-\mathrm{C} 73 \mathrm{~B}-\mathrm{H} 73 \mathrm{D}$

$\mathrm{C} 73-\mathrm{C} 73 \mathrm{~B}-\mathrm{C} 74 \mathrm{~B}$

$\mathrm{C} 73-\mathrm{C} 73 \mathrm{~B}-\mathrm{C} 18 \mathrm{~B}$

$\mathrm{C} 79-\mathrm{C} 73 \mathrm{~B}-\mathrm{H} 73 \mathrm{C}$

$\mathrm{C} 79-\mathrm{C} 73 \mathrm{~B}-\mathrm{H} 73 \mathrm{D}$

H73C-C73B-H73D

$\mathrm{C} 74 \mathrm{~B}-\mathrm{C} 73 \mathrm{~B}-\mathrm{C} 79$

$\mathrm{C} 74 \mathrm{~B}-\mathrm{C} 73 \mathrm{~B}-\mathrm{H} 73 \mathrm{C}$

C74B $-\mathrm{C} 73 \mathrm{~B}-\mathrm{H} 73 \mathrm{D}$

$\mathrm{C} 18 \mathrm{~B}-\mathrm{C} 73 \mathrm{~B}-\mathrm{C} 79$

$\mathrm{C} 18 \mathrm{~B}-\mathrm{C} 73 \mathrm{~B}-\mathrm{H} 73 \mathrm{C}$

$\mathrm{C} 18 \mathrm{~B}-\mathrm{C} 73 \mathrm{~B}-\mathrm{H} 73 \mathrm{D}$

$\mathrm{C} 18 \mathrm{~B}-\mathrm{C} 73 \mathrm{~B}-\mathrm{C} 74 \mathrm{~B}$

$\mathrm{C} 73-\mathrm{C} 74 \mathrm{~B}-\mathrm{C} 74$

$\mathrm{C} 73-\mathrm{C} 74 \mathrm{~B}-\mathrm{C} 75 \mathrm{~B}$

C73-C74B-C73B

$\mathrm{C} 73-\mathrm{C} 74 \mathrm{~B}-\mathrm{H} 74 \mathrm{C}$

C73-C74B-H74D

$\mathrm{C} 74-\mathrm{C} 74 \mathrm{~B}-\mathrm{C} 75 \mathrm{~B}$

$\mathrm{C} 74-\mathrm{C} 74 \mathrm{~B}-\mathrm{C} 73 \mathrm{~B}$

C74- C74B-H74C

C74-C74B-H74D

$\mathrm{C} 75 \mathrm{~B}-\mathrm{C} 74 \mathrm{~B}-\mathrm{H} 74 \mathrm{C}$
109.5

109.5

109.5

109.5

109.5

107.7

107.7

118 (2)

107.1

107.7

107.7

100.0 (17)

111.8

111.8

111.8

111.8

109.5

$63.1(10)$

114.9

115.8

104.7 (19)

$16.4(8)$

100 (2)

161 (2)

108.1

111.1

$5.2(16)$

116 (2)

87.5

72.7

106.8

165 (2)

107.0

107.0

46.9 (9)

107.0

107.0

121.2 (19)

124 (4)

125 (4)

7 (2)

107.2

101.6

$96(2)$

130 (3)

93.2

22.8

107.6 


\begin{tabular}{|c|c|c|c|}
\hline $\mathrm{C} 201-\mathrm{C} 100-\mathrm{H} 10 \mathrm{C}$ & 108.4 & $\mathrm{C} 75 \mathrm{~B}-\mathrm{C} 74 \mathrm{~B}-\mathrm{H} 74 \mathrm{D}$ & 107.6 \\
\hline $\mathrm{C} 201-\mathrm{C} 100-\mathrm{H} 10 \mathrm{D}$ & 108.4 & $\mathrm{C} 73 \mathrm{~B}-\mathrm{C} 74 \mathrm{~B}-\mathrm{C} 75 \mathrm{~B}$ & $119(2)$ \\
\hline $\mathrm{C} 54-\mathrm{C} 103-\mathrm{H} 10 \mathrm{E}$ & 108.4 & $\mathrm{C} 73 \mathrm{~B}-\mathrm{C} 74 \mathrm{~B}-\mathrm{H} 74 \mathrm{C}$ & 107.6 \\
\hline $\mathrm{C} 54-\mathrm{C} 103-\mathrm{H} 10 \mathrm{~F}$ & 108.4 & $\mathrm{C} 73 \mathrm{~B}-\mathrm{C} 74 \mathrm{~B}-\mathrm{H} 74 \mathrm{D}$ & 107.6 \\
\hline $\mathrm{H} 10 \mathrm{E}-\mathrm{C} 103-\mathrm{H} 10 \mathrm{~F}$ & 107.5 & $\mathrm{H} 74 \mathrm{C}-\mathrm{C} 74 \mathrm{~B}-\mathrm{H} 74 \mathrm{D}$ & 107.1 \\
\hline $\mathrm{C} 104-\mathrm{C} 103-\mathrm{C} 54$ & $115.5(6)$ & $\mathrm{C} 18-\mathrm{C} 79 \mathrm{~B}-\mathrm{C} 81$ & $165.1(15)$ \\
\hline $\mathrm{C} 104-\mathrm{C} 103-\mathrm{H} 10 \mathrm{E}$ & 108.4 & $\mathrm{C} 18-\mathrm{C} 79 \mathrm{~B}-\mathrm{H} 79 \mathrm{C}$ & 104.9 \\
\hline $\mathrm{C} 104-\mathrm{C} 103-\mathrm{H} 10 \mathrm{~F}$ & 108.4 & $\mathrm{C} 18-\mathrm{C} 79 \mathrm{~B}-\mathrm{H} 79 \mathrm{D}$ & 104.1 \\
\hline $\mathrm{C} 103-\mathrm{C} 104-\mathrm{H} 10 \mathrm{G}$ & 106.7 & $\mathrm{C} 79-\mathrm{C} 79 \mathrm{~B}-\mathrm{C} 18$ & $48.3(11)$ \\
\hline $\mathrm{C} 103-\mathrm{C} 104-\mathrm{H} 10 \mathrm{H}$ & 106.4 & $\mathrm{C} 79-\mathrm{C} 79 \mathrm{~B}-\mathrm{C} 81$ & $117.0(19)$ \\
\hline $\mathrm{C} 103-\mathrm{C} 104-\mathrm{C} 105$ & $106.2(10)$ & $\mathrm{C} 79-\mathrm{C} 79 \mathrm{~B}-\mathrm{H} 79 \mathrm{C}$ & 76.5 \\
\hline $\mathrm{C} 103-\mathrm{C} 104-\mathrm{C} 205$ & $127(2)$ & C79-C79B-H79D & 76.5 \\
\hline $\mathrm{H} 10 \mathrm{G}-\mathrm{C} 104-\mathrm{H} 10 \mathrm{H}$ & 106.5 & $\mathrm{C} 79-\mathrm{C} 79 \mathrm{~B}-\mathrm{C} 80 \mathrm{~B}$ & $171(2)$ \\
\hline $\mathrm{C} 105-\mathrm{C} 104-\mathrm{H} 10 \mathrm{G}$ & 107.7 & $\mathrm{C} 79-\mathrm{C} 79 \mathrm{~B}-\mathrm{C} 18 \mathrm{~B}$ & $55.5(13)$ \\
\hline $\mathrm{C} 105-\mathrm{C} 104-\mathrm{H} 10 \mathrm{H}$ & 122.5 & $\mathrm{C} 80-\mathrm{C} 79 \mathrm{~B}-\mathrm{C} 18$ & $151(3)$ \\
\hline $\mathrm{C} 205-\mathrm{C} 104-\mathrm{H} 10 \mathrm{G}$ & 106.0 & $\mathrm{C} 80-\mathrm{C} 79 \mathrm{~B}-\mathrm{C} 79$ & $146(4)$ \\
\hline $\mathrm{C} 205-\mathrm{C} 104-\mathrm{H} 10 \mathrm{H}$ & 103.3 & $\mathrm{C} 80-\mathrm{C} 79 \mathrm{~B}-\mathrm{C} 81$ & $38.8(18)$ \\
\hline $\mathrm{C} 72-\mathrm{C} 109-\mathrm{H} 10 \mathrm{I}$ & 108.3 & $\mathrm{C} 80-\mathrm{C} 79 \mathrm{~B}-\mathrm{H} 79 \mathrm{C}$ & 103.5 \\
\hline $\mathrm{C} 72-\mathrm{C} 109-\mathrm{H} 10 \mathrm{~J}$ & 108.3 & $\mathrm{C} 80-\mathrm{C} 79 \mathrm{~B}-\mathrm{H} 79 \mathrm{D}$ & 71.2 \\
\hline $\mathrm{H} 10 \mathrm{I}-\mathrm{C} 109-\mathrm{H} 10 \mathrm{~J}$ & 107.4 & $\mathrm{C} 80-\mathrm{C} 79 \mathrm{~B}-\mathrm{C} 80 \mathrm{~B}$ & $41(2)$ \\
\hline $\mathrm{C} 110-\mathrm{C} 109-\mathrm{C} 72$ & $115.9(6)$ & $\mathrm{C} 80-\mathrm{C} 79 \mathrm{~B}-\mathrm{C} 18 \mathrm{~B}$ & $146(3)$ \\
\hline $\mathrm{C} 110-\mathrm{C} 109-\mathrm{H} 10 \mathrm{I}$ & 108.3 & $\mathrm{C} 81-\mathrm{C} 79 \mathrm{~B}-\mathrm{H} 79 \mathrm{C}$ & 69.6 \\
\hline $\mathrm{C} 110-\mathrm{C} 109-\mathrm{H} 10 \mathrm{~J}$ & 108.3 & $\mathrm{C} 81-\mathrm{C} 79 \mathrm{~B}-\mathrm{H} 79 \mathrm{D}$ & 66.0 \\
\hline $\mathrm{C} 109-\mathrm{C} 110-\mathrm{H} 11 \mathrm{~A}$ & 107.8 & $\mathrm{H} 79 \mathrm{C}-\mathrm{C} 79 \mathrm{~B}-\mathrm{H} 79 \mathrm{D}$ & 107.4 \\
\hline $\mathrm{C} 109-\mathrm{C} 110-\mathrm{H} 11 \mathrm{~B}$ & 107.8 & $\mathrm{C} 80 \mathrm{~B}-\mathrm{C} 79 \mathrm{~B}-\mathrm{C} 18$ & $122.8(16)$ \\
\hline $\mathrm{C} 109-\mathrm{C} 110-\mathrm{H} 11 \mathrm{C}$ & 111.3 & $\mathrm{C} 80 \mathrm{~B}-\mathrm{C} 79 \mathrm{~B}-\mathrm{C} 81$ & $71.9(14)$ \\
\hline C109-C110-H11D & 111.3 & $\mathrm{C} 80 \mathrm{~B}-\mathrm{C} 79 \mathrm{~B}-\mathrm{H} 79 \mathrm{C}$ & 108.4 \\
\hline $\mathrm{C} 109-\mathrm{C} 110-\mathrm{C} 111$ & $118.0(9)$ & $\mathrm{C} 80 \mathrm{~B}-\mathrm{C} 79 \mathrm{~B}-\mathrm{H} 79 \mathrm{D}$ & 108.4 \\
\hline $\mathrm{C} 109-\mathrm{C} 110-\mathrm{C} 211$ & $102.1(11)$ & $\mathrm{C} 80 \mathrm{~B}-\mathrm{C} 79 \mathrm{~B}-\mathrm{C} 18 \mathrm{~B}$ & $115.6(18)$ \\
\hline $\mathrm{H} 11 \mathrm{~A}-\mathrm{C} 110-\mathrm{H} 11 \mathrm{~B}$ & 107.1 & $\mathrm{C} 18 \mathrm{~B}-\mathrm{C} 79 \mathrm{~B}-\mathrm{C} 18$ & $7.2(7)$ \\
\hline $\mathrm{H} 11 \mathrm{C}-\mathrm{C} 110-\mathrm{H} 11 \mathrm{D}$ & 109.2 & $\mathrm{C} 18 \mathrm{~B}-\mathrm{C} 79 \mathrm{~B}-\mathrm{C} 81$ & $172.2(18)$ \\
\hline $\mathrm{C} 111-\mathrm{C} 110-\mathrm{H} 11 \mathrm{~A}$ & 107.8 & $\mathrm{C} 18 \mathrm{~B}-\mathrm{C} 79 \mathrm{~B}-\mathrm{H} 79 \mathrm{C}$ & 108.4 \\
\hline $\mathrm{C} 111-\mathrm{C} 110-\mathrm{H} 11 \mathrm{~B}$ & 107.8 & $\mathrm{C} 18 \mathrm{~B}-\mathrm{C} 79 \mathrm{~B}-\mathrm{H} 79 \mathrm{D}$ & 108.4 \\
\hline $\mathrm{C} 211-\mathrm{C} 110-\mathrm{H} 11 \mathrm{C}$ & 111.3 & $\mathrm{C} 80-\mathrm{C} 80 \mathrm{~B}-\mathrm{C} 79 \mathrm{~B}$ & $24.1(11)$ \\
\hline $\mathrm{C} 211-\mathrm{C} 110-\mathrm{H} 11 \mathrm{D}$ & 111.3 & $\mathrm{C} 80-\mathrm{C} 80 \mathrm{~B}-\mathrm{H} 80 \mathrm{C}$ & 128.4 \\
\hline $\mathrm{C} 72-\mathrm{C} 115-\mathrm{H} 11 \mathrm{E}$ & 108.0 & $\mathrm{C} 80-\mathrm{C} 80 \mathrm{~B}-\mathrm{H} 80 \mathrm{D}$ & 105.3 \\
\hline $\mathrm{C} 72-\mathrm{C} 115-\mathrm{H} 11 \mathrm{~F}$ & 108.0 & $\mathrm{C} 80-\mathrm{C} 80 \mathrm{~B}-\mathrm{C} 81 \mathrm{~B}$ & $101(3)$ \\
\hline $\mathrm{H} 11 \mathrm{E}-\mathrm{C} 115-\mathrm{H} 11 \mathrm{~F}$ & 107.2 & $\mathrm{C} 79 \mathrm{~B}-\mathrm{C} 80 \mathrm{~B}-\mathrm{H} 80 \mathrm{C}$ & 106.8 \\
\hline $\mathrm{C} 116-\mathrm{C} 115-\mathrm{C} 72$ & $117.3(6)$ & $\mathrm{C} 79 \mathrm{~B}-\mathrm{C} 80 \mathrm{~B}-\mathrm{H} 80 \mathrm{D}$ & 106.8 \\
\hline $\mathrm{C} 116-\mathrm{C} 115-\mathrm{H} 11 \mathrm{E}$ & 108.0 & $\mathrm{C} 79 \mathrm{~B}-\mathrm{C} 80 \mathrm{~B}-\mathrm{C} 81 \mathrm{~B}$ & $122(2)$ \\
\hline $\mathrm{C} 116-\mathrm{C} 115-\mathrm{H} 11 \mathrm{~F}$ & 108.0 & $\mathrm{H} 80 \mathrm{C}-\mathrm{C} 80 \mathrm{~B}-\mathrm{H} 80 \mathrm{D}$ & 106.6 \\
\hline $\mathrm{C} 115-\mathrm{C} 116-\mathrm{H} 11 \mathrm{G}$ & 111.4 & $\mathrm{C} 81 \mathrm{~B}-\mathrm{C} 80 \mathrm{~B}-\mathrm{H} 80 \mathrm{C}$ & 106.8 \\
\hline $\mathrm{C} 115-\mathrm{C} 116-\mathrm{H} 11 \mathrm{H}$ & 111.4 & $\mathrm{C} 81 \mathrm{~B}-\mathrm{C} 80 \mathrm{~B}-\mathrm{H} 80 \mathrm{D}$ & 106.8 \\
\hline $\mathrm{C} 115-\mathrm{C} 116-\mathrm{H} 11 \mathrm{I}$ & 106.2 & $\mathrm{C} 82-\mathrm{C} 81 \mathrm{~B}-\mathrm{C} 81$ & $54.4(12)$ \\
\hline $\mathrm{C} 115-\mathrm{C} 116-\mathrm{H} 11 \mathrm{~J}$ & 106.2 & $\mathrm{C} 82-\mathrm{C} 81 \mathrm{~B}-\mathrm{H} 81 \mathrm{C}$ & 108.1 \\
\hline $\mathrm{C} 115-\mathrm{C} 116-\mathrm{C} 117$ & $101.9(8)$ & $\mathrm{C} 82-\mathrm{C} 81 \mathrm{~B}-\mathrm{H} 81 \mathrm{D}$ & 108.1 \\
\hline $\mathrm{C} 115-\mathrm{C} 116-\mathrm{C} 217$ & $124.3(11)$ & $\mathrm{C} 81-\mathrm{C} 81 \mathrm{~B}-\mathrm{H} 81 \mathrm{C}$ & 87.9 \\
\hline $\mathrm{H} 11 \mathrm{G}-\mathrm{C} 116-\mathrm{H} 11 \mathrm{H}$ & 109.2 & $\mathrm{C} 81-\mathrm{C} 81 \mathrm{~B}-\mathrm{H} 81 \mathrm{D}$ & 160.6 \\
\hline
\end{tabular}




\begin{tabular}{|c|c|}
\hline $\mathrm{H} 11 \mathrm{I}-\mathrm{C} 116-\mathrm{H} 11 \mathrm{~J}$ & 106.4 \\
\hline $\mathrm{C} 117-\mathrm{C} 116-\mathrm{H} 11 \mathrm{G}$ & 111.4 \\
\hline $\mathrm{C} 117-\mathrm{C} 116-\mathrm{H} 11 \mathrm{H}$ & 111.4 \\
\hline C217-C116-H11I & 106.2 \\
\hline $\mathrm{C} 217-\mathrm{C} 116-\mathrm{H} 11 \mathrm{~J}$ & 106.2 \\
\hline $\mathrm{C} 4-\mathrm{C} 18-\mathrm{C} 17$ & $98.5(6)$ \\
\hline $\mathrm{C} 4-\mathrm{C} 18-\mathrm{C} 73$ & $114.4(7)$ \\
\hline $\mathrm{C} 4-\mathrm{C} 18-\mathrm{C} 79 \mathrm{~B}$ & $94.0(9)$ \\
\hline $\mathrm{C} 17-\mathrm{C} 18-\mathrm{C} 79 \mathrm{~B}$ & $94.2(9)$ \\
\hline $\mathrm{C} 73-\mathrm{C} 18-\mathrm{C} 17$ & $113.7(8)$ \\
\hline $\mathrm{C} 73-\mathrm{C} 18-\mathrm{C} 79 \mathrm{~B}$ & $135.1(10)$ \\
\hline $\mathrm{C} 79-\mathrm{C} 18-\mathrm{C} 4$ & $112.2(7)$ \\
\hline $\mathrm{C} 79-\mathrm{C} 18-\mathrm{C} 17$ & $109.4(7)$ \\
\hline $\mathrm{C} 79-\mathrm{C} 18-\mathrm{C} 73$ & $108.4(7)$ \\
\hline $\mathrm{C} 79-\mathrm{C} 18-\mathrm{C} 79 \mathrm{~B}$ & $26.8(7)$ \\
\hline $\mathrm{C} 73 \mathrm{~B}-\mathrm{C} 18-\mathrm{C} 4$ & $125.1(15)$ \\
\hline $\mathrm{C} 73 \mathrm{~B}-\mathrm{C} 18-\mathrm{C} 17$ & $131.6(15)$ \\
\hline $\mathrm{C} 73 \mathrm{~B}-\mathrm{C} 18-\mathrm{C} 73$ & $33.5(11)$ \\
\hline $\mathrm{C} 73 \mathrm{~B}-\mathrm{C} 18-\mathrm{C} 79$ & $75.3(13)$ \\
\hline $\mathrm{C} 73 \mathrm{~B}-\mathrm{C} 18-\mathrm{C} 79 \mathrm{~B}$ & $101.8(14)$ \\
\hline $\mathrm{C} 18 \mathrm{~B}-\mathrm{C} 18-\mathrm{C} 4$ & $77(2)$ \\
\hline $\mathrm{C} 18 \mathrm{~B}-\mathrm{C} 18-\mathrm{C} 17$ & $82(2)$ \\
\hline $\mathrm{C} 18 \mathrm{~B}-\mathrm{C} 18-\mathrm{C} 73$ & $157(2)$ \\
\hline $\mathrm{C} 18 \mathrm{~B}-\mathrm{C} 18-\mathrm{C} 79$ & $49(2)$ \\
\hline $\mathrm{C} 18 \mathrm{~B}-\mathrm{C} 18-\mathrm{C} 73 \mathrm{~B}$ & $124(3)$ \\
\hline $\mathrm{C} 18 \mathrm{~B}-\mathrm{C} 18-\mathrm{C} 79 \mathrm{~B}$ & $22(2)$ \\
\hline $\mathrm{C} 18-\mathrm{C} 73-\mathrm{H} 73 \mathrm{~A}$ & 108.5 \\
\hline $\mathrm{C} 18-\mathrm{C} 73-\mathrm{H} 73 \mathrm{~B}$ & 108.5 \\
\hline $\mathrm{C} 18-\mathrm{C} 73-\mathrm{C} 75 \mathrm{~B}$ & $173.6(12)$ \\
\hline $\mathrm{C} 18-\mathrm{C} 73-\mathrm{C} 18 \mathrm{~B}$ & $5.5(6)$ \\
\hline $\mathrm{H} 73 \mathrm{~A}-\mathrm{C} 73-\mathrm{H} 73 \mathrm{~B}$ & 107.5 \\
\hline $\mathrm{C} 74-\mathrm{C} 73-\mathrm{C} 18$ & $115.2(9)$ \\
\hline $\mathrm{C} 74-\mathrm{C} 73-\mathrm{H} 73 \mathrm{~A}$ & 108.5 \\
\hline $\mathrm{C} 74-\mathrm{C} 73-\mathrm{H} 73 \mathrm{~B}$ & 108.5 \\
\hline $\mathrm{C} 74-\mathrm{C} 73-\mathrm{C} 75 \mathrm{~B}$ & $65.8(10)$ \\
\hline $\mathrm{C} 74-\mathrm{C} 73-\mathrm{C} 18 \mathrm{~B}$ & $120.3(9)$ \\
\hline $\mathrm{C} 75 \mathrm{~B}-\mathrm{C} 73-\mathrm{H} 73 \mathrm{~A}$ & 76.6 \\
\hline $\mathrm{C} 75 \mathrm{~B}-\mathrm{C} 73-\mathrm{H} 73 \mathrm{~B}$ & 65.7 \\
\hline $\mathrm{C} 75 \mathrm{~B}-\mathrm{C} 73-\mathrm{C} 18 \mathrm{~B}$ & $169.7(12)$ \\
\hline $\mathrm{C} 73 \mathrm{~B}-\mathrm{C} 73-\mathrm{C} 18$ & $46.7(16)$ \\
\hline $\mathrm{C} 73 \mathrm{~B}-\mathrm{C} 73-\mathrm{H} 73 \mathrm{~A}$ & 94.3 \\
\hline $\mathrm{C} 73 \mathrm{~B}-\mathrm{C} 73-\mathrm{H} 73 \mathrm{~B}$ & 71.1 \\
\hline $\mathrm{C} 73 \mathrm{~B}-\mathrm{C} 73-\mathrm{C} 74$ & $156(2)$ \\
\hline $\mathrm{C} 73 \mathrm{~B}-\mathrm{C} 73-\mathrm{C} 75 \mathrm{~B}$ & $130(2)$ \\
\hline $\mathrm{C} 73 \mathrm{~B}-\mathrm{C} 73-\mathrm{C} 18 \mathrm{~B}$ & $41.2(15)$ \\
\hline $\mathrm{C} 74 \mathrm{~B}-\mathrm{C} 73-\mathrm{C} 18$ & $144(3)$ \\
\hline $\mathrm{C} 74 \mathrm{~B}-\mathrm{C} 73-\mathrm{H} 73 \mathrm{~A}$ & 78.0 \\
\hline & \\
\hline
\end{tabular}

$\begin{array}{ll}\text { C83-C81B-C82 } & 61.1(14) \\ \text { C83-C81B-C81 } & 102(2) \\ \text { C83-C81B-C80B } & 177(3) \\ \text { C83-C81B-H81C } & 74.3 \\ \text { C83-C81B-H81D } & 71.9 \\ \text { C80B-C81B-C82 } & 117(3) \\ \text { C80B-C81B-C81 } & 77.2(19) \\ \text { C80B-C81B-H81C } & 108.1 \\ \text { C80B-C81B-H81D } & 108.1 \\ \text { H81C-C81B-H81D } & 107.3 \\ \text { C18-C18B-C4 } & 84(2) \\ \text { C18-C18B-C79 } & 113(3) \\ \text { C18-C18B-C73B } & 40(2) \\ \text { C79-C18B-C4 } & 133.6(13) \\ \text { C79-C18B-C73B } & 74.1(13) \\ \text { C73B-C18B-C4 } & 105.8(15) \\ \text { C82-C83B-H83C } & 109.8 \\ \text { C82-C83B-H83D } & 109.8 \\ \text { C83-C83B-C82 } & 58.2(9) \\ \text { C83-C83B-H83C } & 73.2 \\ \text { C83-C83B-H83D } & 80.7 \\ \text { C83-C83B-C84B } & 167(3) \\ \text { C84-C83B-C82 } & 125(4) \\ \text { C84-C83B-C83 } & 76(3) \\ \text { C84-C83B-H83C } & 21.0 \\ \text { C84-C83B-H83D } & 88.5 \\ \text { C84-C83B-C84B } & 112(3) \\ \text { H83C-C83B-H83D } & 108.3 \\ \text { C84B-C83B-C82 } & 109(2) \\ \text { C84B-C83B-H83C } & 109.8 \\ \text { C84B-C83B-H83D } & 109.8 \\ \text { C83B-C84B-H84D } & 109.5 \\ \text { C83B-C84B-H84E } & 109.5 \\ \text { C83B-C84B-H84F } & 109.5 \\ \text { H84D-C84B-H84E } & 109.5 \\ \text { H84D-C84B-H84F } & 109.5 \\ \text { H84E-C84B-H84F } & 109.5 \\ \text { H1SA-C1S-H1SB } & 109.5 \\ \text { H1SA-C1S-H1SC } & 109.5 \\ \text { H1SB-C1S-H1SC } & 109.5 \\ \text { C2S-C1S-H1SA } & 109.5 \\ \text { C2S-C1S-H1SB } & 109.5 \\ \text { C2S-C1S-H1SC } & 109.5 \\ \text { C1S-C2S-H2SA } & 109.7 \\ \text { C1S-C2S-H2SB } & 109.7 \\ \text { H2SA-C2S-H2SB } & 108.2 \\ \text { C3S-C2S-C1S } & 110(3) \\ \text { C3S-C2S-H2SA } & 109.7 \\ & \end{array}$




\begin{tabular}{|c|c|c|c|}
\hline $\mathrm{C} 74 \mathrm{~B}-\mathrm{C} 73-\mathrm{C} 74$ & $35(3)$ & $\mathrm{C} 3 \mathrm{~S}-\mathrm{C} 2 \mathrm{~S}-\mathrm{H} 2 \mathrm{SB}$ & 109.7 \\
\hline $\mathrm{C} 74 \mathrm{~B}-\mathrm{C} 73-\mathrm{C} 75 \mathrm{~B}$ & $39(3)$ & $\mathrm{C} 2 \mathrm{~S}-\mathrm{C} 3 \mathrm{~S}-\mathrm{H} 3 \mathrm{SA}$ & 111.9 \\
\hline $\mathrm{C} 74 \mathrm{~B}-\mathrm{C} 73-\mathrm{C} 73 \mathrm{~B}$ & $168(4)$ & $\mathrm{C} 2 \mathrm{~S}-\mathrm{C} 3 \mathrm{~S}-\mathrm{H} 3 \mathrm{SB}$ & 111.9 \\
\hline $\mathrm{C} 74 \mathrm{~B}-\mathrm{C} 73-\mathrm{C} 18 \mathrm{~B}$ & $150(3)$ & $\mathrm{C} 2 \mathrm{~S}-\mathrm{C} 3 \mathrm{~S}-\mathrm{C} 4 \mathrm{~S}$ & $99(3)$ \\
\hline $\mathrm{C} 18 \mathrm{~B}-\mathrm{C} 73-\mathrm{H} 73 \mathrm{~A}$ & 107.3 & $\mathrm{H} 3 \mathrm{SA}-\mathrm{C} 3 \mathrm{~S}-\mathrm{H} 3 \mathrm{SB}$ & 109.6 \\
\hline $\mathrm{C} 18 \mathrm{~B}-\mathrm{C} 73-\mathrm{H} 73 \mathrm{~B}$ & 104.1 & $\mathrm{C} 4 \mathrm{~S}-\mathrm{C} 3 \mathrm{~S}-\mathrm{H} 3 \mathrm{SA}$ & 111.9 \\
\hline $\mathrm{C} 73-\mathrm{C} 74-\mathrm{H} 74 \mathrm{~A}$ & 109.5 & $\mathrm{C} 4 \mathrm{~S}-\mathrm{C} 3 \mathrm{~S}-\mathrm{H} 3 \mathrm{SB}$ & 111.9 \\
\hline $\mathrm{C} 73-\mathrm{C} 74-\mathrm{H} 74 \mathrm{~B}$ & 109.5 & $\mathrm{C} 3 \mathrm{~S}-\mathrm{C} 4 \mathrm{~S}-\mathrm{H} 4 \mathrm{SA}$ & 113.2 \\
\hline $\mathrm{C} 73-\mathrm{C} 74-\mathrm{C} 75 \mathrm{~A}$ & $110.7(9)$ & $\mathrm{C} 3 \mathrm{~S}-\mathrm{C} 4 \mathrm{~S}-\mathrm{H} 4 \mathrm{SB}$ & 113.2 \\
\hline $\mathrm{C} 73-\mathrm{C} 74-\mathrm{C} 75 \mathrm{~B}$ & $68.2(9)$ & $\mathrm{H} 4 \mathrm{SA}-\mathrm{C} 4 \mathrm{~S}-\mathrm{H} 4 \mathrm{SB}$ & 110.6 \\
\hline $\mathrm{H} 74 \mathrm{~A}-\mathrm{C} 74-\mathrm{H} 74 \mathrm{~B}$ & 108.1 & $\mathrm{C} 5 \mathrm{~S}-\mathrm{C} 4 \mathrm{~S}-\mathrm{C} 3 \mathrm{~S}$ & $92(3)$ \\
\hline $\mathrm{C} 75 \mathrm{~A}-\mathrm{C} 74-\mathrm{H} 74 \mathrm{~A}$ & 109.5 & $\mathrm{C} 5 \mathrm{~S}-\mathrm{C} 4 \mathrm{~S}-\mathrm{H} 4 \mathrm{SA}$ & 113.2 \\
\hline $\mathrm{C} 75 \mathrm{~A}-\mathrm{C} 74-\mathrm{H} 74 \mathrm{~B}$ & 109.5 & $\mathrm{C} 5 \mathrm{~S}-\mathrm{C} 4 \mathrm{~S}-\mathrm{H} 4 \mathrm{SB}$ & 113.2 \\
\hline $\mathrm{C} 75 \mathrm{~A}-\mathrm{C} 74-\mathrm{C} 75 \mathrm{~B}$ & $84.4(11)$ & $\mathrm{C} 4 \mathrm{~S}-\mathrm{C} 5 \mathrm{~S}-\mathrm{H} 5 \mathrm{SA}$ & 109.5 \\
\hline $\mathrm{C} 75 \mathrm{~B}-\mathrm{C} 74-\mathrm{H} 74 \mathrm{~A}$ & 61.2 & $\mathrm{C} 4 \mathrm{~S}-\mathrm{C} 5 \mathrm{~S}-\mathrm{H} 5 \mathrm{SB}$ & 109.5 \\
\hline $\mathrm{C} 75 \mathrm{~B}-\mathrm{C} 74-\mathrm{H} 74 \mathrm{~B}$ & 165.3 & $\mathrm{C} 4 \mathrm{~S}-\mathrm{C} 5 \mathrm{~S}-\mathrm{H} 5 \mathrm{SC}$ & 109.5 \\
\hline $\mathrm{C} 74 \mathrm{~B}-\mathrm{C} 74-\mathrm{C} 73$ & $21.1(14)$ & $\mathrm{H} 5 \mathrm{SA}-\mathrm{C} 5 \mathrm{~S}-\mathrm{H} 5 \mathrm{SB}$ & 109.5 \\
\hline $\mathrm{C} 74 \mathrm{~B}-\mathrm{C} 74-\mathrm{H} 74 \mathrm{~A}$ & 88.9 & $\mathrm{H} 5 \mathrm{SA}-\mathrm{C} 5 \mathrm{~S}-\mathrm{H} 5 \mathrm{SC}$ & 109.5 \\
\hline $\mathrm{C} 74 \mathrm{~B}-\mathrm{C} 74-\mathrm{H} 74 \mathrm{~B}$ & 122.2 & $\mathrm{H} 5 \mathrm{SB}-\mathrm{C} 5 \mathrm{~S}-\mathrm{H} 5 \mathrm{SC}$ & 109.5 \\
\hline $\mathrm{Ir} 1-\mathrm{N} 3-\mathrm{C} 43-\mathrm{C} 42$ & $-4.3(6)$ & $\mathrm{C} 86-\mathrm{C} 87-\mathrm{C} 88-\mathrm{C} 89$ & $177.3(7)$ \\
\hline Ir $1-\mathrm{N} 3-\mathrm{C} 43-\mathrm{C} 44$ & $169.7(5)$ & $\mathrm{C} 87-\mathrm{C} 88-\mathrm{C} 89-\mathrm{C} 90$ & $-175.8(8)$ \\
\hline $\mathrm{Ir} 1-\mathrm{N} 3-\mathrm{C} 47-\mathrm{C} 46$ & $-170.6(5)$ & $\mathrm{C} 91-\mathrm{C} 36-\mathrm{C} 85-\mathrm{C} 86$ & $-177.0(7)$ \\
\hline Ir $1-\mathrm{N} 4-\mathrm{C} 61-\mathrm{C} 60$ & $5.1(8)$ & $\mathrm{C} 91-\mathrm{C} 92-\mathrm{C} 93-\mathrm{C} 94$ & $-59.9(17)$ \\
\hline Ir1-N4-C61-C62 & $-175.5(6)$ & C91-C92-C93B-C94B & $173(2)$ \\
\hline Ir1-N4-C65-C64 & $171.9(6)$ & $\mathrm{C} 92-\mathrm{C} 93-\mathrm{C} 94-\mathrm{C} 95$ & $-159.0(13)$ \\
\hline Ir1-C37-C $38-\mathrm{C} 39$ & $-168.8(4)$ & $\mathrm{C} 92-\mathrm{C} 93 \mathrm{~B}-\mathrm{C} 94 \mathrm{~B}-\mathrm{C} 95 \mathrm{~B}$ & $-149(3)$ \\
\hline $\mathrm{Ir} 1-\mathrm{C} 37-\mathrm{C} 42-\mathrm{C} 41$ & $168.5(5)$ & $\mathrm{C} 97-\mathrm{C} 54-\mathrm{C} 103-\mathrm{C} 104$ & $175.2(6)$ \\
\hline $\mathrm{Ir} 1-\mathrm{C} 37-\mathrm{C} 42-\mathrm{C} 43$ & $-9.1(7)$ & $\mathrm{C} 97-\mathrm{C} 98-\mathrm{C} 99-\mathrm{C} 100$ & $-170.0(8)$ \\
\hline $\mathrm{Ir} 1-\mathrm{C} 55-\mathrm{C} 56-\mathrm{C} 57$ & $-173.3(5)$ & $\mathrm{C} 98-\mathrm{C} 99-\mathrm{C} 100-\mathrm{C} 101$ & $-170.8(9)$ \\
\hline $\mathrm{Ir} 1-\mathrm{C} 55-\mathrm{C} 60-\mathrm{C} 59$ & $174.0(6)$ & $\mathrm{C} 98-\mathrm{C} 99-\mathrm{C} 100-\mathrm{C} 201$ & $153(2)$ \\
\hline $\mathrm{Ir} 1-\mathrm{C} 55-\mathrm{C} 60-\mathrm{C} 61$ & $-6.6(8)$ & $\mathrm{C} 99-\mathrm{C} 100-\mathrm{C} 101-\mathrm{C} 102$ & $-81.1(17)$ \\
\hline $\mathrm{Ir} 2-\mathrm{N} 1-\mathrm{C} 7-\mathrm{C} 6$ & $-1.5(7)$ & $\mathrm{C} 99-\mathrm{C} 100-\mathrm{C} 201-\mathrm{C} 202$ & $36(4)$ \\
\hline $\mathrm{Ir} 2-\mathrm{N} 1-\mathrm{C} 7-\mathrm{C} 8$ & $177.1(5)$ & $\mathrm{C} 103-\mathrm{C} 54-\mathrm{C} 97-\mathrm{C} 98$ & $-172.2(6)$ \\
\hline $\mathrm{Ir} 2-\mathrm{N} 1-\mathrm{C} 11-\mathrm{C} 10$ & $-178.4(4)$ & $\mathrm{C} 103-\mathrm{C} 104-\mathrm{C} 105-\mathrm{C} 106$ & $-178(2)$ \\
\hline $\mathrm{Ir} 2-\mathrm{N} 2-\mathrm{C} 25-\mathrm{C} 24$ & $-5.5(8)$ & $\mathrm{C} 103-\mathrm{C} 104-\mathrm{C} 205-\mathrm{C} 206$ & $147(6)$ \\
\hline Ir2-N2-C25-C26 & $173.7(7)$ & $\mathrm{C} 104-\mathrm{C} 105-\mathrm{C} 106-\mathrm{C} 107$ & $-8(6)$ \\
\hline $\mathrm{Ir} 2-\mathrm{N} 2-\mathrm{C} 29-\mathrm{C} 28$ & $-173.1(6)$ & $\mathrm{C} 104-\mathrm{C} 205-\mathrm{C} 206-\mathrm{C} 207$ & $-169(6)$ \\
\hline $\mathrm{Ir} 2-\mathrm{C} 1-\mathrm{C} 2-\mathrm{C} 3$ & $-175.3(5)$ & $\mathrm{C} 109-\mathrm{C} 72-\mathrm{C} 115-\mathrm{C} 116$ & $175.2(7)$ \\
\hline $\mathrm{Ir} 2-\mathrm{C} 1-\mathrm{C} 6-\mathrm{C} 5$ & $175.1(5)$ & $\mathrm{C} 109-\mathrm{C} 110-\mathrm{C} 111-\mathrm{C} 112$ & $-168.9(15)$ \\
\hline $\mathrm{Ir} 2-\mathrm{C} 1-\mathrm{C} 6-\mathrm{C} 7$ & $-1.9(8)$ & $\mathrm{C} 109-\mathrm{C} 110-\mathrm{C} 211-\mathrm{C} 212$ & $169(2)$ \\
\hline Ir $2-\mathrm{C} 19-\mathrm{C} 20-\mathrm{C} 21$ & $-174.9(5)$ & $\mathrm{C} 110-\mathrm{C} 111-\mathrm{C} 112-\mathrm{C} 113$ & $-167.5(17)$ \\
\hline $\mathrm{Ir} 2-\mathrm{C} 19-\mathrm{C} 24-\mathrm{C} 23$ & $177.3(6)$ & $\mathrm{C} 110-\mathrm{C} 211-\mathrm{C} 212-\mathrm{C} 213$ & $167(3)$ \\
\hline Ir2-C19-C24-C25 & $-2.4(8)$ & $\mathrm{C} 115-\mathrm{C} 72-\mathrm{C} 109-\mathrm{C} 110$ & $-177.0(7)$ \\
\hline $\mathrm{F} 1-\mathrm{C} 10-\mathrm{C} 11-\mathrm{N} 1$ & $-177.2(5)$ & $\mathrm{C} 115-\mathrm{C} 116-\mathrm{C} 117-\mathrm{C} 118$ & $-179.8(13)$ \\
\hline $\mathrm{F} 2-\mathrm{C} 28-\mathrm{C} 29-\mathrm{N} 2$ & $-179.9(7)$ & $\mathrm{C} 115-\mathrm{C} 116-\mathrm{C} 217-\mathrm{C} 218$ & $172(2)$ \\
\hline $\mathrm{F} 3-\mathrm{C} 46-\mathrm{C} 47-\mathrm{N} 3$ & $-178.3(5)$ & $\mathrm{C} 116-\mathrm{C} 117-\mathrm{C} 118-\mathrm{C} 119$ & $-179.5(14)$ \\
\hline $\mathrm{F} 4-\mathrm{C} 64-\mathrm{C} 65-\mathrm{N} 4$ & $-174.4(6)$ & $\mathrm{C} 116-\mathrm{C} 217-\mathrm{C} 218-\mathrm{C} 219$ & $-172(3)$ \\
\hline
\end{tabular}




\begin{tabular}{|c|c|c|c|}
\hline $\mathrm{N} 1-\mathrm{C} 7-\mathrm{C} 8-\mathrm{C} 9$ & $0.7(11)$ & $\mathrm{C} 18-\mathrm{C} 4-\mathrm{C} 5-\mathrm{C} 6$ & $-170.9(7)$ \\
\hline $\mathrm{N} 2-\mathrm{C} 25-\mathrm{C} 26-\mathrm{C} 27$ & $-1.6(14)$ & $\mathrm{C} 18-\mathrm{C} 4-\mathrm{C} 18 \mathrm{~B}-\mathrm{C} 79$ & $-116(3)$ \\
\hline $\mathrm{N} 3-\mathrm{C} 43-\mathrm{C} 44-\mathrm{C} 45$ & $1.8(10)$ & $\mathrm{C} 18-\mathrm{C} 4-\mathrm{C} 18 \mathrm{~B}-\mathrm{C} 73 \mathrm{~B}$ & $-33.6(19)$ \\
\hline $\mathrm{N} 4-\mathrm{C} 61-\mathrm{C} 62-\mathrm{C} 63$ & $2.8(13)$ & $\mathrm{C} 18-\mathrm{C} 73-\mathrm{C} 74-\mathrm{C} 75 \mathrm{~A}$ & $-98.3(11)$ \\
\hline $\mathrm{C} 1-\mathrm{C} 2-\mathrm{C} 3-\mathrm{C} 4$ & $-0.2(9)$ & $\mathrm{C} 18-\mathrm{C} 73-\mathrm{C} 74-\mathrm{C} 75 \mathrm{~B}$ & $-173.0(13)$ \\
\hline $\mathrm{C} 1-\mathrm{C} 2-\mathrm{C} 3-\mathrm{C} 12$ & $179.7(6)$ & $\mathrm{C} 18-\mathrm{C} 73-\mathrm{C} 74-\mathrm{C} 74 \mathrm{~B}$ & $153(6)$ \\
\hline $\mathrm{C} 1-\mathrm{C} 6-\mathrm{C} 7-\mathrm{N} 1$ & $2.2(9)$ & $\mathrm{C} 18-\mathrm{C} 73-\mathrm{C} 73 \mathrm{~B}-\mathrm{C} 79$ & $-24(7)$ \\
\hline $\mathrm{C} 1-\mathrm{C} 6-\mathrm{C} 7-\mathrm{C} 8$ & $-176.2(7)$ & $\mathrm{C} 18-\mathrm{C} 73-\mathrm{C} 73 \mathrm{~B}-\mathrm{C} 74 \mathrm{~B}$ & $-161(20)$ \\
\hline $\mathrm{C} 2-\mathrm{C} 1-\mathrm{C} 6-\mathrm{C} 5$ & $-2.9(10)$ & $\mathrm{C} 18-\mathrm{C} 73-\mathrm{C} 73 \mathrm{~B}-\mathrm{C} 18 \mathrm{~B}$ & $0.0(9)$ \\
\hline $\mathrm{C} 2-\mathrm{C} 1-\mathrm{C} 6-\mathrm{C} 7$ & $-180.0(6)$ & $\mathrm{C} 18-\mathrm{C} 73-\mathrm{C} 74 \mathrm{~B}-\mathrm{C} 74$ & $-44(9)$ \\
\hline $\mathrm{C} 2-\mathrm{C} 3-\mathrm{C} 4-\mathrm{C} 5$ & $-1.8(10)$ & $\mathrm{C} 18-\mathrm{C} 73-\mathrm{C} 74 \mathrm{~B}-\mathrm{C} 75 \mathrm{~B}$ & $-172(2)$ \\
\hline $\mathrm{C} 2-\mathrm{C} 3-\mathrm{C} 4-\mathrm{C} 18$ & $171.7(6)$ & $\mathrm{C} 18-\mathrm{C} 73-\mathrm{C} 74 \mathrm{~B}-\mathrm{C} 73 \mathrm{~B}$ & $156(25)$ \\
\hline $\mathrm{C} 2-\mathrm{C} 3-\mathrm{C} 4-\mathrm{C} 18 \mathrm{~B}$ & $-168.1(9)$ & $\mathrm{C} 18-\mathrm{C} 79-\mathrm{C} 80-\mathrm{C} 81$ & $174.4(9)$ \\
\hline $\mathrm{C} 2-\mathrm{C} 3-\mathrm{C} 12-\mathrm{C} 13$ & $3.5(11)$ & $\mathrm{C} 18-\mathrm{C} 79-\mathrm{C} 80-\mathrm{C} 79 \mathrm{~B}$ & $42(7)$ \\
\hline $\mathrm{C} 2-\mathrm{C} 3-\mathrm{C} 12-\mathrm{C} 17$ & $-178.5(6)$ & $\mathrm{C} 18-\mathrm{C} 79-\mathrm{C} 80-\mathrm{C} 80 \mathrm{~B}$ & $32(3)$ \\
\hline $\mathrm{C} 3-\mathrm{C} 4-\mathrm{C} 5-\mathrm{C} 6$ & $1.4(10)$ & $\mathrm{C} 18-\mathrm{C} 79-\mathrm{C} 73 \mathrm{~B}-\mathrm{C} 73$ & $27(8)$ \\
\hline $\mathrm{C} 3-\mathrm{C} 4-\mathrm{C} 18-\mathrm{C} 17$ & $10.8(8)$ & $\mathrm{C} 18-\mathrm{C} 79-\mathrm{C} 73 \mathrm{~B}-\mathrm{C} 74 \mathrm{~B}$ & $41(8)$ \\
\hline $\mathrm{C} 3-\mathrm{C} 4-\mathrm{C} 18-\mathrm{C} 73$ & $-110.1(8)$ & $\mathrm{C} 18-\mathrm{C} 79-\mathrm{C} 73 \mathrm{~B}-\mathrm{C} 18 \mathrm{~B}$ & $-3.4(11)$ \\
\hline $\mathrm{C} 3-\mathrm{C} 4-\mathrm{C} 18-\mathrm{C} 79$ & $125.9(7)$ & $\mathrm{C} 18-\mathrm{C} 79-\mathrm{C} 79 \mathrm{~B}-\mathrm{C} 80$ & $-141(6)$ \\
\hline $\mathrm{C} 3-\mathrm{C} 4-\mathrm{C} 18-\mathrm{C} 73 \mathrm{~B}$ & $-146.9(16)$ & $\mathrm{C} 18-\mathrm{C} 79-\mathrm{C} 79 \mathrm{~B}-\mathrm{C} 81$ & $-177.1(14)$ \\
\hline $\mathrm{C} 3-\mathrm{C} 4-\mathrm{C} 18-\mathrm{C} 79 \mathrm{~B}$ & $105.6(9)$ & $\mathrm{C} 18-\mathrm{C} 79-\mathrm{C} 79 \mathrm{~B}-\mathrm{C} 18 \mathrm{~B}$ & $0.6(9)$ \\
\hline $\mathrm{C} 3-\mathrm{C} 4-\mathrm{C} 18-\mathrm{C} 18 \mathrm{~B}$ & $91(2)$ & $\mathrm{C} 18-\mathrm{C} 79-\mathrm{C} 18 \mathrm{~B}-\mathrm{C} 4$ & $104(3)$ \\
\hline $\mathrm{C} 3-\mathrm{C} 4-\mathrm{C} 18 \mathrm{~B}-\mathrm{C} 18$ & $-97(2)$ & $\mathrm{C} 18-\mathrm{C} 79-\mathrm{C} 18 \mathrm{~B}-\mathrm{C} 73 \mathrm{~B}$ & $7(2)$ \\
\hline $\mathrm{C} 3-\mathrm{C} 4-\mathrm{C} 18 \mathrm{~B}-\mathrm{C} 79$ & $146.8(17)$ & $\mathrm{C} 18-\mathrm{C} 73 \mathrm{~B}-\mathrm{C} 74 \mathrm{~B}-\mathrm{C} 73$ & $-19(21)$ \\
\hline $\mathrm{C} 3-\mathrm{C} 4-\mathrm{C} 18 \mathrm{~B}-\mathrm{C} 73 \mathrm{~B}$ & $-130.3(12)$ & $\mathrm{C} 18-\mathrm{C} 73 \mathrm{~B}-\mathrm{C} 74 \mathrm{~B}-\mathrm{C} 74$ & $-42(5)$ \\
\hline $\mathrm{C} 3-\mathrm{C} 12-\mathrm{C} 13-\mathrm{C} 14$ & $176.3(6)$ & $\mathrm{C} 18-\mathrm{C} 73 \mathrm{~B}-\mathrm{C} 74 \mathrm{~B}-\mathrm{C} 75 \mathrm{~B}$ & $-169(2)$ \\
\hline $\mathrm{C} 3-\mathrm{C} 12-\mathrm{C} 17-\mathrm{C} 16$ & $-178.4(6)$ & $\mathrm{C} 18-\mathrm{C} 73 \mathrm{~B}-\mathrm{C} 18 \mathrm{~B}-\mathrm{C} 4$ & $59(3)$ \\
\hline $\mathrm{C} 3-\mathrm{C} 12-\mathrm{C} 17-\mathrm{C} 18$ & $6.0(8)$ & $\mathrm{C} 18-\mathrm{C} 73 \mathrm{~B}-\mathrm{C} 18 \mathrm{~B}-\mathrm{C} 79$ & $-169(3)$ \\
\hline $\mathrm{C} 3-\mathrm{C} 12-\mathrm{C} 17-\mathrm{C} 18 \mathrm{~B}$ & $-13.7(10)$ & $\mathrm{C} 18-\mathrm{C} 79 \mathrm{~B}-\mathrm{C} 80 \mathrm{~B}-\mathrm{C} 80$ & $-148(4)$ \\
\hline $\mathrm{C} 4-\mathrm{C} 3-\mathrm{C} 12-\mathrm{C} 13$ & $-176.6(6)$ & $\mathrm{C} 18-\mathrm{C} 79 \mathrm{~B}-\mathrm{C} 80 \mathrm{~B}-\mathrm{C} 81 \mathrm{~B}$ & $179(2)$ \\
\hline $\mathrm{C} 4-\mathrm{C} 3-\mathrm{C} 12-\mathrm{C} 17$ & $1.4(7)$ & $\mathrm{C} 73-\mathrm{C} 18-\mathrm{C} 79-\mathrm{C} 80$ & $172.7(9)$ \\
\hline $\mathrm{C} 4-\mathrm{C} 5-\mathrm{C} 6-\mathrm{C} 1$ & $1.0(10)$ & $\mathrm{C} 73-\mathrm{C} 18-\mathrm{C} 79-\mathrm{C} 73 \mathrm{~B}$ & $-5.0(15)$ \\
\hline $\mathrm{C} 4-\mathrm{C} 5-\mathrm{C} 6-\mathrm{C} 7$ & $177.8(6)$ & $\mathrm{C} 73-\mathrm{C} 18-\mathrm{C} 79-\mathrm{C} 79 \mathrm{~B}$ & $-177(2)$ \\
\hline $\mathrm{C} 4-\mathrm{C} 18-\mathrm{C} 73-\mathrm{C} 74$ & $42.8(12)$ & $\mathrm{C} 73-\mathrm{C} 18-\mathrm{C} 79-\mathrm{C} 18 \mathrm{~B}$ & $-175(3)$ \\
\hline $\mathrm{C} 4-\mathrm{C} 18-\mathrm{C} 73-\mathrm{C} 73 \mathrm{~B}$ & $-117(3)$ & $\mathrm{C} 73-\mathrm{C} 18-\mathrm{C} 73 \mathrm{~B}-\mathrm{C} 79$ & $171(3)$ \\
\hline $\mathrm{C} 4-\mathrm{C} 18-\mathrm{C} 73-\mathrm{C} 74 \mathrm{~B}$ & $69(7)$ & $\mathrm{C} 73-\mathrm{C} 18-\mathrm{C} 73 \mathrm{~B}-\mathrm{C} 74 \mathrm{~B}$ & $1.7(19)$ \\
\hline $\mathrm{C} 4-\mathrm{C} 18-\mathrm{C} 73-\mathrm{C} 18 \mathrm{~B}$ & $-117(6)$ & $\mathrm{C} 73-\mathrm{C} 18-\mathrm{C} 73 \mathrm{~B}-\mathrm{C} 18 \mathrm{~B}$ & $-180(3)$ \\
\hline $\mathrm{C} 4-\mathrm{C} 18-\mathrm{C} 79-\mathrm{C} 80$ & $-59.9(10)$ & $\mathrm{C} 73-\mathrm{C} 18-\mathrm{C} 18 \mathrm{~B}-\mathrm{C} 4$ & $-124(5)$ \\
\hline $\mathrm{C} 4-\mathrm{C} 18-\mathrm{C} 79-\mathrm{C} 73 \mathrm{~B}$ & $122.3(16)$ & $\mathrm{C} 73-\mathrm{C} 18-\mathrm{C} 18 \mathrm{~B}-\mathrm{C} 79$ & $11(7)$ \\
\hline $\mathrm{C} 4-\mathrm{C} 18-\mathrm{C} 79-\mathrm{C} 79 \mathrm{~B}$ & $-50(2)$ & $\mathrm{C} 73-\mathrm{C} 18-\mathrm{C} 18 \mathrm{~B}-\mathrm{C} 73 \mathrm{~B}$ & $0(4)$ \\
\hline $\mathrm{C} 4-\mathrm{C} 18-\mathrm{C} 79-\mathrm{C} 18 \mathrm{~B}$ & $-48(2)$ & $\mathrm{C} 73-\mathrm{C} 74-\mathrm{C} 75 \mathrm{~A}-\mathrm{C} 76$ & $163.6(11)$ \\
\hline $\mathrm{C} 4-\mathrm{C} 18-\mathrm{C} 73 \mathrm{~B}-\mathrm{C} 73$ & $82(3)$ & $\mathrm{C} 73-\mathrm{C} 74-\mathrm{C} 74 \mathrm{~B}-\mathrm{C} 75 \mathrm{~B}$ & $139(6)$ \\
\hline $\mathrm{C} 4-\mathrm{C} 18-\mathrm{C} 73 \mathrm{~B}-\mathrm{C} 79$ & $-107.0(12)$ & $\mathrm{C} 73-\mathrm{C} 74-\mathrm{C} 74 \mathrm{~B}-\mathrm{C} 73 \mathrm{~B}$ & $3(3)$ \\
\hline $\mathrm{C} 4-\mathrm{C} 18-\mathrm{C} 73 \mathrm{~B}-\mathrm{C} 74 \mathrm{~B}$ & $83(2)$ & $\mathrm{C} 73-\mathrm{C} 75 \mathrm{~B}-\mathrm{C} 76 \mathrm{~B}-\mathrm{C} 77 \mathrm{~B}$ & $179(2)$ \\
\hline $\mathrm{C} 4-\mathrm{C} 18-\mathrm{C} 73 \mathrm{~B}-\mathrm{C} 18 \mathrm{~B}$ & $-98(3)$ & $\mathrm{C} 73-\mathrm{C} 75 \mathrm{~B}-\mathrm{C} 74 \mathrm{~B}-\mathrm{C} 74$ & $-138(7)$ \\
\hline $\mathrm{C} 4-\mathrm{C} 18-\mathrm{C} 18 \mathrm{~B}-\mathrm{C} 79$ & $135.1(19)$ & $\mathrm{C} 73-\mathrm{C} 75 \mathrm{~B}-\mathrm{C} 74 \mathrm{~B}-\mathrm{C} 73 \mathrm{~B}$ & $4(3)$ \\
\hline $\mathrm{C} 4-\mathrm{C} 18-\mathrm{C} 18 \mathrm{~B}-\mathrm{C} 73 \mathrm{~B}$ & $124(2)$ & $\mathrm{C} 73-\mathrm{C} 73 \mathrm{~B}-\mathrm{C} 74 \mathrm{~B}-\mathrm{C} 74$ & $-22(19)$ \\
\hline $\mathrm{C} 5-\mathrm{C} 4-\mathrm{C} 18-\mathrm{C} 17$ & $-176.4(7)$ & $\mathrm{C} 73-\mathrm{C} 73 \mathrm{~B}-\mathrm{C} 74 \mathrm{~B}-\mathrm{C} 75 \mathrm{~B}$ & $-150(22)$ \\
\hline
\end{tabular}




\begin{tabular}{|c|c|}
\hline $\mathrm{C} 5-\mathrm{C} 4-\mathrm{C} 18-\mathrm{C} 73$ & $62.6(11)$ \\
\hline $\mathrm{C} 5-\mathrm{C} 4-\mathrm{C} 18-\mathrm{C} 79$ & $-61.4(10)$ \\
\hline $\mathrm{C} 5-\mathrm{C} 4-\mathrm{C} 18-\mathrm{C} 73 \mathrm{~B}$ & $25.8(18)$ \\
\hline $\mathrm{C} 5-\mathrm{C} 4-\mathrm{C} 18-\mathrm{C} 79 \mathrm{~B}$ & $-81.6(11)$ \\
\hline $\mathrm{C} 5-\mathrm{C} 4-\mathrm{C} 18-\mathrm{C} 18 \mathrm{~B}$ & $-97(2)$ \\
\hline $\mathrm{C} 5-\mathrm{C} 4-\mathrm{C} 18 \mathrm{~B}-\mathrm{C} 18$ & $98(2)$ \\
\hline $\mathrm{C} 5-\mathrm{C} 4-\mathrm{C} 18 \mathrm{~B}-\mathrm{C} 79$ & $-18(3)$ \\
\hline $\mathrm{C} 5-\mathrm{C} 4-\mathrm{C} 18 \mathrm{~B}-\mathrm{C} 73 \mathrm{~B}$ & $64.9(17)$ \\
\hline $\mathrm{C} 5-\mathrm{C} 6-\mathrm{C} 7-\mathrm{N} 1$ & $-174.8(6)$ \\
\hline $\mathrm{C} 5-\mathrm{C} 6-\mathrm{C} 7-\mathrm{C} 8$ & $6.8(11)$ \\
\hline $\mathrm{C} 6-\mathrm{C} 1-\mathrm{C} 2-\mathrm{C} 3$ & $2.5(9)$ \\
\hline $\mathrm{C} 6-\mathrm{C} 7-\mathrm{C} 8-\mathrm{C} 9$ & $179.1(7)$ \\
\hline $\mathrm{C} 7-\mathrm{N} 1-\mathrm{C} 11-\mathrm{C} 10$ & $-3.8(9)$ \\
\hline $\mathrm{C} 7-\mathrm{C} 8-\mathrm{C} 9-\mathrm{C} 10$ & $-1.4(11)$ \\
\hline $\mathrm{C} 8-\mathrm{C} 9-\mathrm{C} 10-\mathrm{F} 1$ & $179.8(6)$ \\
\hline $\mathrm{C} 8-\mathrm{C} 9-\mathrm{C} 10-\mathrm{C} 11$ & $-0.5(11)$ \\
\hline $\mathrm{C} 9-\mathrm{C} 10-\mathrm{C} 11-\mathrm{N} 1$ & $3.1(10)$ \\
\hline $\mathrm{C} 11-\mathrm{N} 1-\mathrm{C} 7-\mathrm{C} 6$ & $-176.6(6)$ \\
\hline $\mathrm{C} 11-\mathrm{N} 1-\mathrm{C} 7-\mathrm{C} 8$ & $2.0(9)$ \\
\hline $\mathrm{C} 12-\mathrm{C} 3-\mathrm{C} 4-\mathrm{C} 5$ & $178.3(6)$ \\
\hline $\mathrm{C} 12-\mathrm{C} 3-\mathrm{C} 4-\mathrm{C} 18$ & $-8.2(8)$ \\
\hline $\mathrm{C} 12-\mathrm{C} 3-\mathrm{C} 4-\mathrm{C} 18 \mathrm{~B}$ & $12.0(10)$ \\
\hline $\mathrm{C} 12-\mathrm{C} 13-\mathrm{C} 14-\mathrm{C} 15$ & $1.5(10)$ \\
\hline $\mathrm{C} 12-\mathrm{C} 17-\mathrm{C} 18-\mathrm{C} 4$ & $-10.0(8)$ \\
\hline $\mathrm{C} 12-\mathrm{C} 17-\mathrm{C} 18-\mathrm{C} 73$ & $111.5(8)$ \\
\hline $\mathrm{C} 12-\mathrm{C} 17-\mathrm{C} 18-\mathrm{C} 79$ & $-127.2(7)$ \\
\hline $\mathrm{C} 12-\mathrm{C} 17-\mathrm{C} 18-\mathrm{C} 73 \mathrm{~B}$ & $145.5(18)$ \\
\hline $\mathrm{C} 12-\mathrm{C} 17-\mathrm{C} 18-\mathrm{C} 79 \mathrm{~B}$ & $-104.6(9)$ \\
\hline $\mathrm{C} 12-\mathrm{C} 17-\mathrm{C} 18-\mathrm{C} 18 \mathrm{~B}$ & $-86(2)$ \\
\hline $\mathrm{C} 13-\mathrm{C} 12-\mathrm{C} 17-\mathrm{C} 16$ & $-0.2(10)$ \\
\hline $\mathrm{C} 13-\mathrm{C} 12-\mathrm{C} 17-\mathrm{C} 18$ & $-175.8(6)$ \\
\hline $\mathrm{C} 13-\mathrm{C} 12-\mathrm{C} 17-\mathrm{C} 18 \mathrm{~B}$ & $164.5(9)$ \\
\hline $\mathrm{C} 13-\mathrm{C} 14-\mathrm{C} 15-\mathrm{C} 16$ & $0.2(11)$ \\
\hline $\mathrm{C} 14-\mathrm{C} 15-\mathrm{C} 16-\mathrm{C} 17$ & $-1.9(11)$ \\
\hline $\mathrm{C} 15-\mathrm{C} 16-\mathrm{C} 17-\mathrm{C} 12$ & $2.0(10)$ \\
\hline $\mathrm{C} 15-\mathrm{C} 16-\mathrm{C} 17-\mathrm{C} 18$ & $176.8(7)$ \\
\hline $\mathrm{C} 15-\mathrm{C} 16-\mathrm{C} 17-\mathrm{C} 18 \mathrm{~B}$ & $-159.7(11)$ \\
\hline $\mathrm{C} 16-\mathrm{C} 17-\mathrm{C} 18-\mathrm{C} 4$ & $174.8(6)$ \\
\hline $\mathrm{C} 16-\mathrm{C} 17-\mathrm{C} 18-\mathrm{C} 73$ & $-63.7(10)$ \\
\hline $\mathrm{C} 16-\mathrm{C} 17-\mathrm{C} 18-\mathrm{C} 79$ & $57.6(10)$ \\
\hline $\mathrm{C} 16-\mathrm{C} 17-\mathrm{C} 18-\mathrm{C} 73 \mathrm{~B}$ & $-30(2)$ \\
\hline $\mathrm{C} 16-\mathrm{C} 17-\mathrm{C} 18-\mathrm{C} 79 \mathrm{~B}$ & $80.2(10)$ \\
\hline $\mathrm{C} 16-\mathrm{C} 17-\mathrm{C} 18-\mathrm{C} 18 \mathrm{~B}$ & $99(2)$ \\
\hline $\mathrm{C} 17-\mathrm{C} 12-\mathrm{C} 13-\mathrm{C} 14$ & $-1.5(9)$ \\
\hline $\mathrm{C} 17-\mathrm{C} 18-\mathrm{C} 73-\mathrm{C} 74$ & $-69.3(11)$ \\
\hline $\mathrm{C} 17-\mathrm{C} 18-\mathrm{C} 73-\mathrm{C} 73 \mathrm{~B}$ & $131(3)$ \\
\hline $\mathrm{C} 17-\mathrm{C} 18-\mathrm{C} 73-\mathrm{C} 74 \mathrm{~B}$ & $-43(7)$ \\
\hline $\mathrm{C} 17-\mathrm{C} 18-\mathrm{C} 73-\mathrm{C} 18 \mathrm{~B}$ & $131(6)$ \\
\hline
\end{tabular}

\begin{tabular}{|c|c|}
\hline $\mathrm{C} 73-\mathrm{C} 73 \mathrm{~B}-\mathrm{C} 18 \mathrm{~B}-\mathrm{C} 4$ & $59(3)$ \\
\hline $\mathrm{C} 73-\mathrm{C} 73 \mathrm{~B}-\mathrm{C} 18 \mathrm{~B}-\mathrm{C} 18$ & $0(3)$ \\
\hline $\mathrm{C} 73-\mathrm{C} 73 \mathrm{~B}-\mathrm{C} 18 \mathrm{~B}-\mathrm{C} 79$ & $-169(3)$ \\
\hline $\mathrm{C} 74-\mathrm{C} 73-\mathrm{C} 73 \mathrm{~B}-\mathrm{C} 18$ & $-48(6)$ \\
\hline $\mathrm{C} 74-\mathrm{C} 73-\mathrm{C} 73 \mathrm{~B}-\mathrm{C} 79$ & $-73(11)$ \\
\hline $\mathrm{C} 74-\mathrm{C} 73-\mathrm{C} 73 \mathrm{~B}-\mathrm{C} 74 \mathrm{~B}$ & $151(24)$ \\
\hline $\mathrm{C} 74-\mathrm{C} 73-\mathrm{C} 73 \mathrm{~B}-\mathrm{C} 18 \mathrm{~B}$ & $-48(6)$ \\
\hline $\mathrm{C} 74-\mathrm{C} 73-\mathrm{C} 74 \mathrm{~B}-\mathrm{C} 75 \mathrm{~B}$ & $-127(8)$ \\
\hline $\mathrm{C} 74-\mathrm{C} 73-\mathrm{C} 74 \mathrm{~B}-\mathrm{C} 73 \mathrm{~B}$ & $-159(17)$ \\
\hline $\mathrm{C} 74-\mathrm{C} 75 \mathrm{~A}-\mathrm{C} 76-\mathrm{C} 77$ & $177.6(12)$ \\
\hline $\mathrm{C} 74-\mathrm{C} 75 \mathrm{~B}-\mathrm{C} 76 \mathrm{~B}-\mathrm{C} 77 \mathrm{~B}$ & $-166(2)$ \\
\hline $\mathrm{C} 74-\mathrm{C} 75 \mathrm{~B}-\mathrm{C} 74 \mathrm{~B}-\mathrm{C} 73$ & $138(7)$ \\
\hline $\mathrm{C} 74-\mathrm{C} 75 \mathrm{~B}-\mathrm{C} 74 \mathrm{~B}-\mathrm{C} 73 \mathrm{~B}$ & $142(4)$ \\
\hline $\mathrm{C} 75 \mathrm{~A}-\mathrm{C} 74-\mathrm{C} 74 \mathrm{~B}-\mathrm{C} 73$ & $-80(5)$ \\
\hline $\mathrm{C} 75 \mathrm{~A}-\mathrm{C} 74-\mathrm{C} 74 \mathrm{~B}-\mathrm{C} 75 \mathrm{~B}$ & $58(2)$ \\
\hline $\mathrm{C} 75 \mathrm{~A}-\mathrm{C} 74-\mathrm{C} 74 \mathrm{~B}-\mathrm{C} 73 \mathrm{~B}$ & $-77(4)$ \\
\hline $\mathrm{C} 75 \mathrm{~A}-\mathrm{C} 76-\mathrm{C} 77-\mathrm{C} 78$ & $-173.9(12)$ \\
\hline $\mathrm{C} 79-\mathrm{C} 18-\mathrm{C} 73-\mathrm{C} 74$ & $168.9(9)$ \\
\hline $\mathrm{C} 79-\mathrm{C} 18-\mathrm{C} 73-\mathrm{C} 73 \mathrm{~B}$ & $9(3)$ \\
\hline $\mathrm{C} 79-\mathrm{C} 18-\mathrm{C} 73-\mathrm{C} 74 \mathrm{~B}$ & $-165(6)$ \\
\hline $\mathrm{C} 79-\mathrm{C} 18-\mathrm{C} 73-\mathrm{C} 18 \mathrm{~B}$ & $9(5)$ \\
\hline $\mathrm{C} 79-\mathrm{C} 18-\mathrm{C} 73 \mathrm{~B}-\mathrm{C} 73$ & $-171(3)$ \\
\hline $\mathrm{C} 79-\mathrm{C} 18-\mathrm{C} 73 \mathrm{~B}-\mathrm{C} 74 \mathrm{~B}$ & $-170(2)$ \\
\hline $\mathrm{C} 79-\mathrm{C} 18-\mathrm{C} 73 \mathrm{~B}-\mathrm{C} 18 \mathrm{~B}$ & $9(3)$ \\
\hline $\mathrm{C} 79-\mathrm{C} 18-\mathrm{C} 18 \mathrm{~B}-\mathrm{C} 4$ & $-135.1(19)$ \\
\hline $\mathrm{C} 79-\mathrm{C} 18-\mathrm{C} 18 \mathrm{~B}-\mathrm{C} 73 \mathrm{~B}$ & $-11(3)$ \\
\hline $\mathrm{C} 79-\mathrm{C} 80-\mathrm{C} 81-\mathrm{C} 82$ & $155.5(9)$ \\
\hline $\mathrm{C} 79-\mathrm{C} 80-\mathrm{C} 81-\mathrm{C} 79 \mathrm{~B}$ & $-18(3)$ \\
\hline $\mathrm{C} 79-\mathrm{C} 80-\mathrm{C} 81-\mathrm{C} 81 \mathrm{~B}$ & $-162.7(15)$ \\
\hline $\mathrm{C} 79-\mathrm{C} 80-\mathrm{C} 79 \mathrm{~B}-\mathrm{C} 18$ & $-103(11)$ \\
\hline $\mathrm{C} 79-\mathrm{C} 80-\mathrm{C} 79 \mathrm{~B}-\mathrm{C} 81$ & $56(8)$ \\
\hline $\mathrm{C} 79-\mathrm{C} 80-\mathrm{C} 79 \mathrm{~B}-\mathrm{C} 80 \mathrm{~B}$ & $-172(6)$ \\
\hline $\mathrm{C} 79-\mathrm{C} 80-\mathrm{C} 79 \mathrm{~B}-\mathrm{C} 18 \mathrm{~B}$ & $-114(11)$ \\
\hline $\mathrm{C} 79-\mathrm{C} 80-\mathrm{C} 80 \mathrm{~B}-\mathrm{C} 79 \mathrm{~B}$ & $4(3)$ \\
\hline $\mathrm{C} 79-\mathrm{C} 80-\mathrm{C} 80 \mathrm{~B}-\mathrm{C} 81 \mathrm{~B}$ & $155.8(18)$ \\
\hline $\mathrm{C} 79-\mathrm{C} 73 \mathrm{~B}-\mathrm{C} 74 \mathrm{~B}-\mathrm{C} 73$ & $-56(23)$ \\
\hline $\mathrm{C} 79-\mathrm{C} 73 \mathrm{~B}-\mathrm{C} 74 \mathrm{~B}-\mathrm{C} 74$ & $-79(10)$ \\
\hline $\mathrm{C} 79-\mathrm{C} 73 \mathrm{~B}-\mathrm{C} 74 \mathrm{~B}-\mathrm{C} 75 \mathrm{~B}$ & $154(7)$ \\
\hline $\mathrm{C} 79-\mathrm{C} 73 \mathrm{~B}-\mathrm{C} 18 \mathrm{~B}-\mathrm{C} 4$ & $-131.6(13)$ \\
\hline $\mathrm{C} 79-\mathrm{C} 73 \mathrm{~B}-\mathrm{C} 18 \mathrm{~B}-\mathrm{C} 18$ & $169(3)$ \\
\hline $\mathrm{C} 80-\mathrm{C} 79-\mathrm{C} 73 \mathrm{~B}-\mathrm{C} 18$ & $-5(4)$ \\
\hline $\mathrm{C} 80-\mathrm{C} 79-\mathrm{C} 73 \mathrm{~B}-\mathrm{C} 73$ & $22(11)$ \\
\hline $\mathrm{C} 80-\mathrm{C} 79-\mathrm{C} 73 \mathrm{~B}-\mathrm{C} 74 \mathrm{~B}$ & $35(10)$ \\
\hline $\mathrm{C} 80-\mathrm{C} 79-\mathrm{C} 73 \mathrm{~B}-\mathrm{C} 18 \mathrm{~B}$ & $-9(3)$ \\
\hline $\mathrm{C} 80-\mathrm{C} 79-\mathrm{C} 79 \mathrm{~B}-\mathrm{C} 18$ & $141(6)$ \\
\hline $\mathrm{C} 80-\mathrm{C} 79-\mathrm{C} 79 \mathrm{~B}-\mathrm{C} 81$ & $-36(5)$ \\
\hline $\mathrm{C} 80-\mathrm{C} 79-\mathrm{C} 79 \mathrm{~B}-\mathrm{C} 18 \mathrm{~B}$ & $142(7)$ \\
\hline $\mathrm{C} 80-\mathrm{C} 79-\mathrm{C} 18 \mathrm{~B}-\mathrm{C} 4$ & $-86(2)$ \\
\hline
\end{tabular}




\begin{tabular}{|c|c|}
\hline $\mathrm{C} 17-\mathrm{C} 18-\mathrm{C} 79-\mathrm{C} 80$ & $48.3(10)$ \\
\hline $\mathrm{C} 17-\mathrm{C} 18-\mathrm{C} 79-\mathrm{C} 73 \mathrm{~B}$ & $-129.4(16)$ \\
\hline $\mathrm{C} 17-\mathrm{C} 18-\mathrm{C} 79-\mathrm{C} 79 \mathrm{~B}$ & $58(2)$ \\
\hline $\mathrm{C} 17-\mathrm{C} 18-\mathrm{C} 79-\mathrm{C} 18 \mathrm{~B}$ & $60(2)$ \\
\hline $\mathrm{C} 17-\mathrm{C} 18-\mathrm{C} 73 \mathrm{~B}-\mathrm{C} 73$ & $-68(3)$ \\
\hline $\mathrm{C} 17-\mathrm{C} 18-\mathrm{C} 73 \mathrm{~B}-\mathrm{C} 79$ & $103.1(14)$ \\
\hline $\mathrm{C} 17-\mathrm{C} 18-\mathrm{C} 73 \mathrm{~B}-\mathrm{C} 74 \mathrm{~B}$ & $-67(3)$ \\
\hline $\mathrm{C} 17-\mathrm{C} 18-\mathrm{C} 73 \mathrm{~B}-\mathrm{C} 18 \mathrm{~B}$ & $112(3)$ \\
\hline $\mathrm{C} 17-\mathrm{C} 18-\mathrm{C} 18 \mathrm{~B}-\mathrm{C} 4$ & $100.6(7)$ \\
\hline $\mathrm{C} 17-\mathrm{C} 18-\mathrm{C} 18 \mathrm{~B}-\mathrm{C} 79$ & $-124.3(18)$ \\
\hline $\mathrm{C} 17-\mathrm{C} 18-\mathrm{C} 18 \mathrm{~B}-\mathrm{C} 73 \mathrm{~B}$ & $-135(2)$ \\
\hline $\mathrm{C} 19-\mathrm{C} 20-\mathrm{C} 21-\mathrm{C} 22$ & $-0.6(11)$ \\
\hline $\mathrm{C} 19-\mathrm{C} 20-\mathrm{C} 21-\mathrm{C} 30$ & $-179.3(7)$ \\
\hline $\mathrm{C} 19-\mathrm{C} 24-\mathrm{C} 25-\mathrm{N} 2$ & $5.2(10)$ \\
\hline $\mathrm{C} 19-\mathrm{C} 24-\mathrm{C} 25-\mathrm{C} 26$ & $-173.9(8)$ \\
\hline $\mathrm{C} 20-\mathrm{C} 19-\mathrm{C} 24-\mathrm{C} 23$ & $3.9(11)$ \\
\hline $\mathrm{C} 20-\mathrm{C} 19-\mathrm{C} 24-\mathrm{C} 25$ & $-175.8(6)$ \\
\hline $\mathrm{C} 20-\mathrm{C} 21-\mathrm{C} 22-\mathrm{C} 23$ & $2.6(12)$ \\
\hline $\mathrm{C} 20-\mathrm{C} 21-\mathrm{C} 22-\mathrm{C} 36$ & $-179.1(7)$ \\
\hline $\mathrm{C} 20-\mathrm{C} 21-\mathrm{C} 30-\mathrm{C} 31$ & $-2.5(13)$ \\
\hline $\mathrm{C} 20-\mathrm{C} 21-\mathrm{C} 30-\mathrm{C} 35$ & $176.7(8)$ \\
\hline $\mathrm{C} 21-\mathrm{C} 22-\mathrm{C} 23-\mathrm{C} 24$ & $-1.3(12)$ \\
\hline $\mathrm{C} 21-\mathrm{C} 22-\mathrm{C} 36-\mathrm{C} 35$ & $2.1(8)$ \\
\hline $\mathrm{C} 21-\mathrm{C} 22-\mathrm{C} 36-\mathrm{C} 85$ & $-116.0(7)$ \\
\hline $\mathrm{C} 21-\mathrm{C} 22-\mathrm{C} 36-\mathrm{C} 91$ & $122.1(8)$ \\
\hline $\mathrm{C} 21-\mathrm{C} 30-\mathrm{C} 31-\mathrm{C} 32$ & $176.9(7)$ \\
\hline $\mathrm{C} 21-\mathrm{C} 30-\mathrm{C} 35-\mathrm{C} 34$ & $-175.7(7)$ \\
\hline $\mathrm{C} 21-\mathrm{C} 30-\mathrm{C} 35-\mathrm{C} 36$ & $3.6(9)$ \\
\hline $\mathrm{C} 22-\mathrm{C} 21-\mathrm{C} 30-\mathrm{C} 31$ & $178.7(7)$ \\
\hline $\mathrm{C} 22-\mathrm{C} 21-\mathrm{C} 30-\mathrm{C} 35$ & $-2.1(9)$ \\
\hline $\mathrm{C} 22-\mathrm{C} 23-\mathrm{C} 24-\mathrm{C} 19$ & $-2.0(12)$ \\
\hline $\mathrm{C} 22-\mathrm{C} 23-\mathrm{C} 24-\mathrm{C} 25$ & $177.7(7)$ \\
\hline $\mathrm{C} 22-\mathrm{C} 36-\mathrm{C} 85-\mathrm{C} 86$ & $57.4(9)$ \\
\hline $\mathrm{C} 22-\mathrm{C} 36-\mathrm{C} 91-\mathrm{C} 92$ & $-55.7(10)$ \\
\hline $\mathrm{C} 23-\mathrm{C} 22-\mathrm{C} 36-\mathrm{C} 35$ & $-179.9(8)$ \\
\hline $\mathrm{C} 23-\mathrm{C} 22-\mathrm{C} 36-\mathrm{C} 85$ & $62.0(11)$ \\
\hline $\mathrm{C} 23-\mathrm{C} 22-\mathrm{C} 36-\mathrm{C} 91$ & $-59.9(11)$ \\
\hline $\mathrm{C} 23-\mathrm{C} 24-\mathrm{C} 25-\mathrm{N} 2$ & $-174.5(7)$ \\
\hline $\mathrm{C} 23-\mathrm{C} 24-\mathrm{C} 25-\mathrm{C} 26$ & $6.4(14)$ \\
\hline $\mathrm{C} 24-\mathrm{C} 19-\mathrm{C} 20-\mathrm{C} 21$ & $-2.6(10)$ \\
\hline $\mathrm{C} 24-\mathrm{C} 25-\mathrm{C} 26-\mathrm{C} 27$ & $177.4(9)$ \\
\hline $\mathrm{C} 25-\mathrm{N} 2-\mathrm{C} 29-\mathrm{C} 28$ & $-2.0(11)$ \\
\hline $\mathrm{C} 25-\mathrm{C} 26-\mathrm{C} 27-\mathrm{C} 28$ & $1.5(16)$ \\
\hline $\mathrm{C} 26-\mathrm{C} 27-\mathrm{C} 28-\mathrm{F} 2$ & $-179.8(9)$ \\
\hline $\mathrm{C} 26-\mathrm{C} 27-\mathrm{C} 28-\mathrm{C} 29$ & $-1.6(15)$ \\
\hline $\mathrm{C} 27-\mathrm{C} 28-\mathrm{C} 29-\mathrm{N} 2$ & $1.9(13)$ \\
\hline $\mathrm{C} 29-\mathrm{N} 2-\mathrm{C} 25-\mathrm{C} 24$ & $-177.3(6)$ \\
\hline $\mathrm{C} 29-\mathrm{N} 2-\mathrm{C} 25-\mathrm{C} 26$ & $1.9(11)$ \\
\hline
\end{tabular}

\begin{tabular}{|c|c|}
\hline $\mathrm{C} 80-\mathrm{C} 79-\mathrm{C} 18 \mathrm{~B}-\mathrm{C} 18$ & $169(2)$ \\
\hline $\mathrm{C} 80-\mathrm{C} 79-\mathrm{C} 18 \mathrm{~B}-\mathrm{C} 73 \mathrm{~B}$ & $176.6(13)$ \\
\hline $\mathrm{C} 80-\mathrm{C} 81-\mathrm{C} 81 \mathrm{~B}-\mathrm{C} 82$ & $-130.0(14)$ \\
\hline $\mathrm{C} 80-\mathrm{C} 81-\mathrm{C} 81 \mathrm{~B}-\mathrm{C} 83$ & $-171(2)$ \\
\hline $\mathrm{C} 80-\mathrm{C} 81-\mathrm{C} 81 \mathrm{~B}-\mathrm{C} 80 \mathrm{~B}$ & $6.7(17)$ \\
\hline $\mathrm{C} 80-\mathrm{C} 79 \mathrm{~B}-\mathrm{C} 80 \mathrm{~B}-\mathrm{C} 81 \mathrm{~B}$ & $-33(4)$ \\
\hline $\mathrm{C} 80-\mathrm{C} 80 \mathrm{~B}-\mathrm{C} 81 \mathrm{~B}-\mathrm{C} 82$ & $29(4)$ \\
\hline $\mathrm{C} 80-\mathrm{C} 80 \mathrm{~B}-\mathrm{C} 81 \mathrm{~B}-\mathrm{C} 81$ & $-9(2)$ \\
\hline $\mathrm{C} 81-\mathrm{C} 82-\mathrm{C} 83-\mathrm{C} 84$ & $173.9(12)$ \\
\hline $\mathrm{C} 81-\mathrm{C} 82-\mathrm{C} 83-\mathrm{C} 81 \mathrm{~B}$ & $-46(2)$ \\
\hline $\mathrm{C} 81-\mathrm{C} 82-\mathrm{C} 83-\mathrm{C} 83 \mathrm{~B}$ & $155(3)$ \\
\hline $\mathrm{C} 81-\mathrm{C} 82-\mathrm{C} 81 \mathrm{~B}-\mathrm{C} 83$ & $133(2)$ \\
\hline $\mathrm{C} 81-\mathrm{C} 82-\mathrm{C} 81 \mathrm{~B}-\mathrm{C} 80 \mathrm{~B}$ & $-48(2)$ \\
\hline $\mathrm{C} 81-\mathrm{C} 82-\mathrm{C} 83 \mathrm{~B}-\mathrm{C} 83$ & $-64(5)$ \\
\hline $\mathrm{C} 81-\mathrm{C} 82-\mathrm{C} 83 \mathrm{~B}-\mathrm{C} 84$ & $-102(8)$ \\
\hline $\mathrm{C} 81-\mathrm{C} 82-\mathrm{C} 83 \mathrm{~B}-\mathrm{C} 84 \mathrm{~B}$ & $121(4)$ \\
\hline $\mathrm{C} 81-\mathrm{C} 80-\mathrm{C} 79 \mathrm{~B}-\mathrm{C} 18$ & $-160(4)$ \\
\hline $\mathrm{C} 81-\mathrm{C} 80-\mathrm{C} 79 \mathrm{~B}-\mathrm{C} 79$ & $-56(8)$ \\
\hline $\mathrm{C} 81-\mathrm{C} 80-\mathrm{C} 79 \mathrm{~B}-\mathrm{C} 80 \mathrm{~B}$ & $132(4)$ \\
\hline $\mathrm{C} 81-\mathrm{C} 80-\mathrm{C} 79 \mathrm{~B}-\mathrm{C} 18 \mathrm{~B}$ & $-170(4)$ \\
\hline $\mathrm{C} 81-\mathrm{C} 80-\mathrm{C} 80 \mathrm{~B}-\mathrm{C} 79 \mathrm{~B}$ & $-141(4)$ \\
\hline $\mathrm{C} 81-\mathrm{C} 80-\mathrm{C} 80 \mathrm{~B}-\mathrm{C} 81 \mathrm{~B}$ & $11(3)$ \\
\hline $\mathrm{C} 81-\mathrm{C} 79 \mathrm{~B}-\mathrm{C} 80 \mathrm{~B}-\mathrm{C} 80$ & $29(3)$ \\
\hline $\mathrm{C} 81-\mathrm{C} 79 \mathrm{~B}-\mathrm{C} 80 \mathrm{~B}-\mathrm{C} 81 \mathrm{~B}$ & $-4(3)$ \\
\hline $\mathrm{C} 83-\mathrm{C} 82-\mathrm{C} 81-\mathrm{C} 80$ & $86.9(14)$ \\
\hline $\mathrm{C} 83-\mathrm{C} 82-\mathrm{C} 81-\mathrm{C} 79 \mathrm{~B}$ & $89.3(17)$ \\
\hline $\mathrm{C} 83-\mathrm{C} 82-\mathrm{C} 81-\mathrm{C} 81 \mathrm{~B}$ & $34.5(16)$ \\
\hline $\mathrm{C} 83-\mathrm{C} 82-\mathrm{C} 81 \mathrm{~B}-\mathrm{C} 81$ & $-133(2)$ \\
\hline $\mathrm{C} 83-\mathrm{C} 82-\mathrm{C} 81 \mathrm{~B}-\mathrm{C} 80 \mathrm{~B}$ & $178(4)$ \\
\hline $\mathrm{C} 83-\mathrm{C} 82-\mathrm{C} 83 \mathrm{~B}-\mathrm{C} 84$ & $-38(5)$ \\
\hline $\mathrm{C} 83-\mathrm{C} 82-\mathrm{C} 83 \mathrm{~B}-\mathrm{C} 84 \mathrm{~B}$ & $-175(5)$ \\
\hline $\mathrm{C} 83-\mathrm{C} 84-\mathrm{C} 83 \mathrm{~B}-\mathrm{C} 82$ & $33(4)$ \\
\hline $\mathrm{C} 83-\mathrm{C} 84-\mathrm{C} 83 \mathrm{~B}-\mathrm{C} 84 \mathrm{~B}$ & $168(5)$ \\
\hline $\mathrm{C} 84-\mathrm{C} 83-\mathrm{C} 81 \mathrm{~B}-\mathrm{C} 82$ & $75(3)$ \\
\hline $\mathrm{C} 84-\mathrm{C} 83-\mathrm{C} 81 \mathrm{~B}-\mathrm{C} 81$ & $112(3)$ \\
\hline $\mathrm{C} 84-\mathrm{C} 83-\mathrm{C} 83 \mathrm{~B}-\mathrm{C} 82$ & $-149(5)$ \\
\hline $\mathrm{C} 84-\mathrm{C} 83-\mathrm{C} 83 \mathrm{~B}-\mathrm{C} 84 \mathrm{~B}$ & $-127(24)$ \\
\hline $\mathrm{C} 93-\mathrm{C} 94-\mathrm{C} 95-\mathrm{C} 96$ & $177.5(14)$ \\
\hline $\mathrm{C} 105-\mathrm{C} 106-\mathrm{C} 107-\mathrm{C} 108$ & $172(3)$ \\
\hline $\mathrm{C} 111-\mathrm{C} 112-\mathrm{C} 113-\mathrm{C} 114$ & $80(3)$ \\
\hline $\mathrm{C} 117-\mathrm{C} 118-\mathrm{C} 119-\mathrm{C} 120$ & $176.3(16)$ \\
\hline $\mathrm{C} 211-\mathrm{C} 212-\mathrm{C} 213-\mathrm{C} 214$ & $-173(3)$ \\
\hline $\mathrm{C} 93 \mathrm{~B}-\mathrm{C} 94 \mathrm{~B}-\mathrm{C} 95 \mathrm{~B}-\mathrm{C} 96 \mathrm{~B}$ & $-68(5)$ \\
\hline $\mathrm{C} 217-\mathrm{C} 218-\mathrm{C} 219-\mathrm{C} 220$ & $-55(4)$ \\
\hline $\mathrm{C} 205-\mathrm{C} 206-\mathrm{C} 207-\mathrm{C} 208$ & $-176(7)$ \\
\hline $\mathrm{C} 75 \mathrm{~B}-\mathrm{C} 73-\mathrm{C} 74-\mathrm{C} 75 \mathrm{~A}$ & $74.7(13)$ \\
\hline $\mathrm{C} 75 \mathrm{~B}-\mathrm{C} 73-\mathrm{C} 74-\mathrm{C} 74 \mathrm{~B}$ & $-34(5)$ \\
\hline $\mathrm{C} 75 \mathrm{~B}-\mathrm{C} 73-\mathrm{C} 73 \mathrm{~B}-\mathrm{C} 18$ & $-172.5(15)$ \\
\hline
\end{tabular}




\begin{tabular}{|c|c|}
\hline $\mathrm{C} 30-\mathrm{C} 21-\mathrm{C} 22-\mathrm{C} 23$ & $-178.4(7)$ \\
\hline $\mathrm{C} 30-\mathrm{C} 21-\mathrm{C} 22-\mathrm{C} 36$ & $-0.2(9)$ \\
\hline $\mathrm{C} 30-\mathrm{C} 31-\mathrm{C} 32-\mathrm{C} 33$ & $-1.5(11)$ \\
\hline $\mathrm{C} 30-\mathrm{C} 35-\mathrm{C} 36-\mathrm{C} 22$ & $-3.4(8)$ \\
\hline $\mathrm{C} 30-\mathrm{C} 35-\mathrm{C} 36-\mathrm{C} 85$ & $114.4(7)$ \\
\hline $\mathrm{C} 30-\mathrm{C} 35-\mathrm{C} 36-\mathrm{C} 91$ & $-124.7(7)$ \\
\hline $\mathrm{C} 31-\mathrm{C} 30-\mathrm{C} 35-\mathrm{C} 34$ & $3.7(11)$ \\
\hline $\mathrm{C} 31-\mathrm{C} 30-\mathrm{C} 35-\mathrm{C} 36$ & $-177.1(7)$ \\
\hline $\mathrm{C} 31-\mathrm{C} 32-\mathrm{C} 33-\mathrm{C} 34$ & $4.0(12)$ \\
\hline $\mathrm{C} 32-\mathrm{C} 33-\mathrm{C} 34-\mathrm{C} 35$ & $-2.5(11)$ \\
\hline $\mathrm{C} 33-\mathrm{C} 34-\mathrm{C} 35-\mathrm{C} 30$ & $-1.3(11)$ \\
\hline $\mathrm{C} 33-\mathrm{C} 34-\mathrm{C} 35-\mathrm{C} 36$ & $179.6(7)$ \\
\hline $\mathrm{C} 34-\mathrm{C} 35-\mathrm{C} 36-\mathrm{C} 22$ & $175.7(8)$ \\
\hline $\mathrm{C} 34-\mathrm{C} 35-\mathrm{C} 36-\mathrm{C} 85$ & $-66.5(10)$ \\
\hline $\mathrm{C} 34-\mathrm{C} 35-\mathrm{C} 36-\mathrm{C} 91$ & $54.4(11)$ \\
\hline $\mathrm{C} 35-\mathrm{C} 30-\mathrm{C} 31-\mathrm{C} 32$ & $-2.3(11)$ \\
\hline $\mathrm{C} 35-\mathrm{C} 36-\mathrm{C} 85-\mathrm{C} 86$ & $-53.4(9)$ \\
\hline $\mathrm{C} 35-\mathrm{C} 36-\mathrm{C} 91-\mathrm{C} 92$ & $57.2(9)$ \\
\hline $\mathrm{C} 36-\mathrm{C} 22-\mathrm{C} 23-\mathrm{C} 24$ & $-179.2(8)$ \\
\hline $\mathrm{C} 36-\mathrm{C} 85-\mathrm{C} 86-\mathrm{C} 87$ & $-178.8(6)$ \\
\hline $\mathrm{C} 36-\mathrm{C} 91-\mathrm{C} 92-\mathrm{C} 93$ & $-165.7(9)$ \\
\hline $\mathrm{C} 36-\mathrm{C} 91-\mathrm{C} 92-\mathrm{C} 93 \mathrm{~B}$ & $176.1(15)$ \\
\hline $\mathrm{C} 37-\mathrm{C} 38-\mathrm{C} 39-\mathrm{C} 40$ & $0.4(9)$ \\
\hline $\mathrm{C} 37-\mathrm{C} 38-\mathrm{C} 39-\mathrm{C} 48$ & $178.2(6)$ \\
\hline $\mathrm{C} 37-\mathrm{C} 42-\mathrm{C} 43-\mathrm{N} 3$ & $8.8(8)$ \\
\hline $\mathrm{C} 37-\mathrm{C} 42-\mathrm{C} 43-\mathrm{C} 44$ & $-164.9(6)$ \\
\hline $\mathrm{C} 38-\mathrm{C} 37-\mathrm{C} 42-\mathrm{C} 41$ & $-3.8(9)$ \\
\hline $\mathrm{C} 38-\mathrm{C} 37-\mathrm{C} 42-\mathrm{C} 43$ & $178.5(5)$ \\
\hline $\mathrm{C} 38-\mathrm{C} 39-\mathrm{C} 40-\mathrm{C} 41$ & $-1.6(9)$ \\
\hline $\mathrm{C} 38-\mathrm{C} 39-\mathrm{C} 40-\mathrm{C} 54$ & $177.9(5)$ \\
\hline $\mathrm{C} 38-\mathrm{C} 39-\mathrm{C} 48-\mathrm{C} 49$ & $1.3(11)$ \\
\hline $\mathrm{C} 38-\mathrm{C} 39-\mathrm{C} 48-\mathrm{C} 53$ & $-178.0(6)$ \\
\hline $\mathrm{C} 39-\mathrm{C} 40-\mathrm{C} 41-\mathrm{C} 42$ & $0.0(9)$ \\
\hline $\mathrm{C} 39-\mathrm{C} 40-\mathrm{C} 54-\mathrm{C} 53$ & $0.6(7)$ \\
\hline $\mathrm{C} 39-\mathrm{C} 40-\mathrm{C} 54-\mathrm{C} 97$ & $-117.0(6)$ \\
\hline $\mathrm{C} 39-\mathrm{C} 40-\mathrm{C} 54-\mathrm{C} 103$ & $118.9(6)$ \\
\hline $\mathrm{C} 39-\mathrm{C} 48-\mathrm{C} 49-\mathrm{C} 50$ & $-179.2(6)$ \\
\hline $\mathrm{C} 39-\mathrm{C} 48-\mathrm{C} 53-\mathrm{C} 52$ & $179.4(5)$ \\
\hline $\mathrm{C} 39-\mathrm{C} 48-\mathrm{C} 53-\mathrm{C} 54$ & $0.3(7)$ \\
\hline $\mathrm{C} 40-\mathrm{C} 39-\mathrm{C} 48-\mathrm{C} 49$ & $179.3(6)$ \\
\hline $\mathrm{C} 40-\mathrm{C} 39-\mathrm{C} 48-\mathrm{C} 53$ & $0.1(7)$ \\
\hline $\mathrm{C} 40-\mathrm{C} 41-\mathrm{C} 42-\mathrm{C} 37$ & $2.7(9)$ \\
\hline $\mathrm{C} 40-\mathrm{C} 41-\mathrm{C} 42-\mathrm{C} 43$ & $-179.8(6)$ \\
\hline $\mathrm{C} 40-\mathrm{C} 54-\mathrm{C} 97-\mathrm{C} 98$ & $61.8(8)$ \\
\hline $\mathrm{C} 40-\mathrm{C} 54-\mathrm{C} 103-\mathrm{C} 104$ & $-59.3(8)$ \\
\hline $\mathrm{C} 41-\mathrm{C} 40-\mathrm{C} 54-\mathrm{C} 53$ & $180.0(6)$ \\
\hline $\mathrm{C} 41-\mathrm{C} 40-\mathrm{C} 54-\mathrm{C} 97$ & $62.4(9)$ \\
\hline $\mathrm{C} 41-\mathrm{C} 40-\mathrm{C} 54-\mathrm{C} 103$ & $-61.7(9)$ \\
\hline
\end{tabular}

\begin{tabular}{|c|c|}
\hline $\mathrm{C} 75 \mathrm{~B}-\mathrm{C} 73-\mathrm{C} 73 \mathrm{~B}-\mathrm{C} 79$ & $163(7)$ \\
\hline $\mathrm{C} 75 \mathrm{~B}-\mathrm{C} 73-\mathrm{C} 73 \mathrm{~B}-\mathrm{C} 74 \mathrm{~B}$ & $26(20)$ \\
\hline $\mathrm{C} 75 \mathrm{~B}-\mathrm{C} 73-\mathrm{C} 73 \mathrm{~B}-\mathrm{C} 18 \mathrm{~B}$ & $-172.5(15)$ \\
\hline $\mathrm{C} 75 \mathrm{~B}-\mathrm{C} 73-\mathrm{C} 74 \mathrm{~B}-\mathrm{C} 74$ & $127(8)$ \\
\hline $\mathrm{C} 75 \mathrm{~B}-\mathrm{C} 73-\mathrm{C} 74 \mathrm{~B}-\mathrm{C} 73 \mathrm{~B}$ & $-32(24)$ \\
\hline $\mathrm{C} 75 \mathrm{~B}-\mathrm{C} 74-\mathrm{C} 75 \mathrm{~A}-\mathrm{C} 76$ & $-132.3(13)$ \\
\hline $\mathrm{C} 75 \mathrm{~B}-\mathrm{C} 74-\mathrm{C} 74 \mathrm{~B}-\mathrm{C} 73$ & $-139(6)$ \\
\hline $\mathrm{C} 75 \mathrm{~B}-\mathrm{C} 74-\mathrm{C} 74 \mathrm{~B}-\mathrm{C} 73 \mathrm{~B}$ & $-136(5)$ \\
\hline $\mathrm{C} 75 \mathrm{~B}-\mathrm{C} 76 \mathrm{~B}-\mathrm{C} 77 \mathrm{~B}-\mathrm{C} 78 \mathrm{~B}$ & $146(3)$ \\
\hline $\mathrm{C} 76 \mathrm{~B}-\mathrm{C} 75 \mathrm{~B}-\mathrm{C} 74 \mathrm{~B}-\mathrm{C} 73$ & $172(5)$ \\
\hline $\mathrm{C} 76 \mathrm{~B}-\mathrm{C} 75 \mathrm{~B}-\mathrm{C} 74 \mathrm{~B}-\mathrm{C} 74$ & $34(4)$ \\
\hline $\mathrm{C} 76 \mathrm{~B}-\mathrm{C} 75 \mathrm{~B}-\mathrm{C} 74 \mathrm{~B}-\mathrm{C} 73 \mathrm{~B}$ & $176(3)$ \\
\hline $\mathrm{C} 73 \mathrm{~B}-\mathrm{C} 18-\mathrm{C} 73-\mathrm{C} 74$ & $160(3)$ \\
\hline $\mathrm{C} 73 \mathrm{~B}-\mathrm{C} 18-\mathrm{C} 73-\mathrm{C} 74 \mathrm{~B}$ & $-173(7)$ \\
\hline $\mathrm{C} 73 \mathrm{~B}-\mathrm{C} 18-\mathrm{C} 73-\mathrm{C} 18 \mathrm{~B}$ & $0(6)$ \\
\hline $\mathrm{C} 73 \mathrm{~B}-\mathrm{C} 18-\mathrm{C} 79-\mathrm{C} 80$ & $177.7(16)$ \\
\hline $\mathrm{C} 73 \mathrm{~B}-\mathrm{C} 18-\mathrm{C} 79-\mathrm{C} 79 \mathrm{~B}$ & $-172(2)$ \\
\hline $\mathrm{C} 73 \mathrm{~B}-\mathrm{C} 18-\mathrm{C} 79-\mathrm{C} 18 \mathrm{~B}$ & $-170(3)$ \\
\hline $\mathrm{C} 73 \mathrm{~B}-\mathrm{C} 18-\mathrm{C} 18 \mathrm{~B}-\mathrm{C} 4$ & $-124(2)$ \\
\hline $\mathrm{C} 73 \mathrm{~B}-\mathrm{C} 18-\mathrm{C} 18 \mathrm{~B}-\mathrm{C} 79$ & $11(3)$ \\
\hline $\mathrm{C} 73 \mathrm{~B}-\mathrm{C} 73-\mathrm{C} 74-\mathrm{C} 75 \mathrm{~A}$ & $-61(5)$ \\
\hline $\mathrm{C} 73 \mathrm{~B}-\mathrm{C} 73-\mathrm{C} 74-\mathrm{C} 75 \mathrm{~B}$ & $-136(5)$ \\
\hline $\mathrm{C} 73 \mathrm{~B}-\mathrm{C} 73-\mathrm{C} 74-\mathrm{C} 74 \mathrm{~B}$ & $-170(8)$ \\
\hline $\mathrm{C} 73 \mathrm{~B}-\mathrm{C} 73-\mathrm{C} 74 \mathrm{~B}-\mathrm{C} 74$ & $159(17)$ \\
\hline $\mathrm{C} 73 \mathrm{~B}-\mathrm{C} 73-\mathrm{C} 74 \mathrm{~B}-\mathrm{C} 75 \mathrm{~B}$ & $32(24)$ \\
\hline $\mathrm{C} 73 \mathrm{~B}-\mathrm{C} 79-\mathrm{C} 80-\mathrm{C} 81$ & $178(3)$ \\
\hline $\mathrm{C} 73 \mathrm{~B}-\mathrm{C} 79-\mathrm{C} 80-\mathrm{C} 79 \mathrm{~B}$ & $46(8)$ \\
\hline $\mathrm{C} 73 \mathrm{~B}-\mathrm{C} 79-\mathrm{C} 80-\mathrm{C} 80 \mathrm{~B}$ & $36(4)$ \\
\hline $\mathrm{C} 73 \mathrm{~B}-\mathrm{C} 79-\mathrm{C} 79 \mathrm{~B}-\mathrm{C} 18$ & $-9(3)$ \\
\hline $\mathrm{C} 73 \mathrm{~B}-\mathrm{C} 79-\mathrm{C} 79 \mathrm{~B}-\mathrm{C} 80$ & $-150(5)$ \\
\hline $\mathrm{C} 73 \mathrm{~B}-\mathrm{C} 79-\mathrm{C} 79 \mathrm{~B}-\mathrm{C} 81$ & $174(2)$ \\
\hline $\mathrm{C} 73 \mathrm{~B}-\mathrm{C} 79-\mathrm{C} 79 \mathrm{~B}-\mathrm{C} 18 \mathrm{~B}$ & $-8(3)$ \\
\hline $\mathrm{C} 73 \mathrm{~B}-\mathrm{C} 79-\mathrm{C} 18 \mathrm{~B}-\mathrm{C} 4$ & $97(2)$ \\
\hline $\mathrm{C} 73 \mathrm{~B}-\mathrm{C} 79-\mathrm{C} 18 \mathrm{~B}-\mathrm{C} 18$ & $-7(2)$ \\
\hline $\mathrm{C} 74 \mathrm{~B}-\mathrm{C} 73-\mathrm{C} 74-\mathrm{C} 75 \mathrm{~A}$ & $108(5)$ \\
\hline $\mathrm{C} 74 \mathrm{~B}-\mathrm{C} 73-\mathrm{C} 74-\mathrm{C} 75 \mathrm{~B}$ & $34(5)$ \\
\hline $\mathrm{C} 74 \mathrm{~B}-\mathrm{C} 73-\mathrm{C} 73 \mathrm{~B}-\mathrm{C} 18$ & $161(20)$ \\
\hline $\mathrm{C} 74 \mathrm{~B}-\mathrm{C} 73-\mathrm{C} 73 \mathrm{~B}-\mathrm{C} 79$ & $137(19)$ \\
\hline $\mathrm{C} 74 \mathrm{~B}-\mathrm{C} 73-\mathrm{C} 73 \mathrm{~B}-\mathrm{C} 18 \mathrm{~B}$ & $161(20)$ \\
\hline $\mathrm{C} 74 \mathrm{~B}-\mathrm{C} 74-\mathrm{C} 75 \mathrm{~A}-\mathrm{C} 76$ & $-174.1(19)$ \\
\hline $\mathrm{C} 74 \mathrm{~B}-\mathrm{C} 75 \mathrm{~B}-\mathrm{C} 76 \mathrm{~B}-\mathrm{C} 77 \mathrm{~B}$ & $176(3)$ \\
\hline $\mathrm{C} 74 \mathrm{~B}-\mathrm{C} 73 \mathrm{~B}-\mathrm{C} 18 \mathrm{~B}-\mathrm{C} 4$ & $61(3)$ \\
\hline $\mathrm{C} 74 \mathrm{~B}-\mathrm{C} 73 \mathrm{~B}-\mathrm{C} 18 \mathrm{~B}-\mathrm{C} 18$ & $2(3)$ \\
\hline $\mathrm{C} 74 \mathrm{~B}-\mathrm{C} 73 \mathrm{~B}-\mathrm{C} 18 \mathrm{~B}-\mathrm{C} 79$ & $-167(3)$ \\
\hline $\mathrm{C} 79 \mathrm{~B}-\mathrm{C} 18-\mathrm{C} 73-\mathrm{C} 74$ & $167.2(13)$ \\
\hline $\mathrm{C} 79 \mathrm{~B}-\mathrm{C} 18-\mathrm{C} 73-\mathrm{C} 73 \mathrm{~B}$ & $7(3)$ \\
\hline $\mathrm{C} 79 \mathrm{~B}-\mathrm{C} 18-\mathrm{C} 73-\mathrm{C} 74 \mathrm{~B}$ & $-166(6)$ \\
\hline $\mathrm{C} 79 \mathrm{~B}-\mathrm{C} 18-\mathrm{C} 73-\mathrm{C} 18 \mathrm{~B}$ & $7(5)$ \\
\hline
\end{tabular}




\begin{tabular}{|c|c|}
\hline $\mathrm{C} 41-\mathrm{C} 42-\mathrm{C} 43-\mathrm{N} 3$ & $-168.8(5)$ \\
\hline $\mathrm{C} 41-\mathrm{C} 42-\mathrm{C} 43-\mathrm{C} 44$ & $17.5(10)$ \\
\hline $\mathrm{C} 42-\mathrm{C} 37-\mathrm{C} 38-\mathrm{C} 39$ & $2.2(9)$ \\
\hline $\mathrm{C} 42-\mathrm{C} 43-\mathrm{C} 44-\mathrm{C} 45$ & $175.1(6)$ \\
\hline $\mathrm{C} 43-\mathrm{N} 3-\mathrm{C} 47-\mathrm{C} 46$ & $1.1(9)$ \\
\hline $\mathrm{C} 43-\mathrm{C} 44-\mathrm{C} 45-\mathrm{C} 46$ & $0.5(10)$ \\
\hline $\mathrm{C} 44-\mathrm{C} 45-\mathrm{C} 46-\mathrm{F} 3$ & $177.5(6)$ \\
\hline $\mathrm{C} 44-\mathrm{C} 45-\mathrm{C} 46-\mathrm{C} 47$ & $-2.0(10)$ \\
\hline $\mathrm{C} 45-\mathrm{C} 46-\mathrm{C} 47-\mathrm{N} 3$ & $1.2(10)$ \\
\hline $\mathrm{C} 47-\mathrm{N} 3-\mathrm{C} 43-\mathrm{C} 42$ & $-176.7(5)$ \\
\hline $\mathrm{C} 47-\mathrm{N} 3-\mathrm{C} 43-\mathrm{C} 44$ & $-2.6(9)$ \\
\hline $\mathrm{C} 48-\mathrm{C} 39-\mathrm{C} 40-\mathrm{C} 41$ & $-179.9(5)$ \\
\hline $\mathrm{C} 48-\mathrm{C} 39-\mathrm{C} 40-\mathrm{C} 54$ & $-0.4(7)$ \\
\hline $\mathrm{C} 48-\mathrm{C} 49-\mathrm{C} 50-\mathrm{C} 51$ & $0.6(9)$ \\
\hline $\mathrm{C} 48-\mathrm{C} 53-\mathrm{C} 54-\mathrm{C} 40$ & $-0.6(7)$ \\
\hline $\mathrm{C} 48-\mathrm{C} 53-\mathrm{C} 54-\mathrm{C} 97$ & $118.3(6)$ \\
\hline $\mathrm{C} 48-\mathrm{C} 53-\mathrm{C} 54-\mathrm{C} 103$ & $-120.5(6)$ \\
\hline $\mathrm{C} 49-\mathrm{C} 48-\mathrm{C} 53-\mathrm{C} 52$ & $0.0(9)$ \\
\hline $\mathrm{C} 49-\mathrm{C} 48-\mathrm{C} 53-\mathrm{C} 54$ & $-179.0(5)$ \\
\hline $\mathrm{C} 49-\mathrm{C} 50-\mathrm{C} 51-\mathrm{C} 52$ & $-1.1(10)$ \\
\hline $\mathrm{C} 50-\mathrm{C} 51-\mathrm{C} 52-\mathrm{C} 53$ & $1.1(10)$ \\
\hline $\mathrm{C} 51-\mathrm{C} 52-\mathrm{C} 53-\mathrm{C} 48$ & $-0.5(9)$ \\
\hline $\mathrm{C} 51-\mathrm{C} 52-\mathrm{C} 53-\mathrm{C} 54$ & $178.3(6)$ \\
\hline $\mathrm{C} 52-\mathrm{C} 53-\mathrm{C} 54-\mathrm{C} 40$ & $-179.5(6)$ \\
\hline $\mathrm{C} 52-\mathrm{C} 53-\mathrm{C} 54-\mathrm{C} 97$ & $-60.6(9)$ \\
\hline $\mathrm{C} 52-\mathrm{C} 53-\mathrm{C} 54-\mathrm{C} 103$ & $60.6(9)$ \\
\hline $\mathrm{C} 53-\mathrm{C} 48-\mathrm{C} 49-\mathrm{C} 50$ & $0.0(9)$ \\
\hline $\mathrm{C} 53-\mathrm{C} 54-\mathrm{C} 97-\mathrm{C} 98$ & $-50.3(8)$ \\
\hline $\mathrm{C} 53-\mathrm{C} 54-\mathrm{C} 103-\mathrm{C} 104$ & $53.5(8)$ \\
\hline $\mathrm{C} 54-\mathrm{C} 40-\mathrm{C} 41-\mathrm{C} 42$ & $-179.3(6)$ \\
\hline $\mathrm{C} 54-\mathrm{C} 97-\mathrm{C} 98-\mathrm{C} 99$ & $-177.4(7)$ \\
\hline $\mathrm{C} 54-\mathrm{C} 103-\mathrm{C} 104-\mathrm{C} 105$ & $175.9(18)$ \\
\hline $\mathrm{C} 54-\mathrm{C} 103-\mathrm{C} 104-\mathrm{C} 205$ & $-173(4)$ \\
\hline $\mathrm{C} 55-\mathrm{C} 56-\mathrm{C} 57-\mathrm{C} 58$ & $-0.9(11)$ \\
\hline $\mathrm{C} 55-\mathrm{C} 56-\mathrm{C} 57-\mathrm{C} 66$ & $178.4(7)$ \\
\hline $\mathrm{C} 55-\mathrm{C} 60-\mathrm{C} 61-\mathrm{N} 4$ & $1.0(10)$ \\
\hline $\mathrm{C} 55-\mathrm{C} 60-\mathrm{C} 61-\mathrm{C} 62$ & $-178.3(8)$ \\
\hline $\mathrm{C} 56-\mathrm{C} 55-\mathrm{C} 60-\mathrm{C} 59$ & $-2.4(11)$ \\
\hline $\mathrm{C} 56-\mathrm{C} 55-\mathrm{C} 60-\mathrm{C} 61$ & $177.0(6)$ \\
\hline $\mathrm{C} 56-\mathrm{C} 57-\mathrm{C} 58-\mathrm{C} 59$ & $-1.0(12)$ \\
\hline $\mathrm{C} 56-\mathrm{C} 57-\mathrm{C} 58-\mathrm{C} 72$ & $177.5(6)$ \\
\hline $\mathrm{C} 56-\mathrm{C} 57-\mathrm{C} 66-\mathrm{C} 67$ & $1.3(13)$ \\
\hline $\mathrm{C} 56-\mathrm{C} 57-\mathrm{C} 66-\mathrm{C} 71$ & $-178.7(7)$ \\
\hline $\mathrm{C} 57-\mathrm{C} 58-\mathrm{C} 59-\mathrm{C} 60$ & $1.2(12)$ \\
\hline $\mathrm{C} 57-\mathrm{C} 58-\mathrm{C} 72-\mathrm{C} 71$ & $2.4(8)$ \\
\hline $\mathrm{C} 57-\mathrm{C} 58-\mathrm{C} 72-\mathrm{C} 109$ & $-118.0(7)$ \\
\hline $\mathrm{C} 57-\mathrm{C} 58-\mathrm{C} 72-\mathrm{C} 115$ & $122.1(7)$ \\
\hline $\mathrm{C} 57-\mathrm{C} 66-\mathrm{C} 67-\mathrm{C} 68$ & $179.5(7)$ \\
\hline
\end{tabular}

\begin{tabular}{|c|c|}
\hline $\mathrm{C} 79 \mathrm{~B}-\mathrm{C} 18-\mathrm{C} 79-\mathrm{C} 80$ & $-10(2)$ \\
\hline $\mathrm{C} 79 \mathrm{~B}-\mathrm{C} 18-\mathrm{C} 79-\mathrm{C} 73 \mathrm{~B}$ & $172(2)$ \\
\hline $\mathrm{C} 79 \mathrm{~B}-\mathrm{C} 18-\mathrm{C} 79-\mathrm{C} 18 \mathrm{~B}$ & $2(3)$ \\
\hline $\mathrm{C} 79 \mathrm{~B}-\mathrm{C} 18-\mathrm{C} 73 \mathrm{~B}-\mathrm{C} 73$ & $-175(2)$ \\
\hline $\mathrm{C} 79 \mathrm{~B}-\mathrm{C} 18-\mathrm{C} 73 \mathrm{~B}-\mathrm{C} 79$ & $-3.5(11)$ \\
\hline $\mathrm{C} 79 \mathrm{~B}-\mathrm{C} 18-\mathrm{C} 73 \mathrm{~B}-\mathrm{C} 74 \mathrm{~B}$ & $-173(2)$ \\
\hline $\mathrm{C} 79 \mathrm{~B}-\mathrm{C} 18-\mathrm{C} 73 \mathrm{~B}-\mathrm{C} 18 \mathrm{~B}$ & $5(3)$ \\
\hline $\mathrm{C} 79 \mathrm{~B}-\mathrm{C} 18-\mathrm{C} 18 \mathrm{~B}-\mathrm{C} 4$ & $-137(5)$ \\
\hline $\mathrm{C} 79 \mathrm{~B}-\mathrm{C} 18-\mathrm{C} 18 \mathrm{~B}-\mathrm{C} 79$ & $-2(4)$ \\
\hline $\mathrm{C} 79 \mathrm{~B}-\mathrm{C} 18-\mathrm{C} 18 \mathrm{~B}-\mathrm{C} 73 \mathrm{~B}$ & $-14(7)$ \\
\hline $\mathrm{C} 79 \mathrm{~B}-\mathrm{C} 79-\mathrm{C} 80-\mathrm{C} 81$ & $132(7)$ \\
\hline $\mathrm{C} 79 \mathrm{~B}-\mathrm{C} 79-\mathrm{C} 80-\mathrm{C} 80 \mathrm{~B}$ & $-11(7)$ \\
\hline $\mathrm{C} 79 \mathrm{~B}-\mathrm{C} 79-\mathrm{C} 73 \mathrm{~B}-\mathrm{C} 18$ & $13(4)$ \\
\hline $\mathrm{C} 79 \mathrm{~B}-\mathrm{C} 79-\mathrm{C} 73 \mathrm{~B}-\mathrm{C} 73$ & $40(10)$ \\
\hline $\mathrm{C} 79 \mathrm{~B}-\mathrm{C} 79-\mathrm{C} 73 \mathrm{~B}-\mathrm{C} 74 \mathrm{~B}$ & $54(10)$ \\
\hline $\mathrm{C} 79 \mathrm{~B}-\mathrm{C} 79-\mathrm{C} 73 \mathrm{~B}-\mathrm{C} 18 \mathrm{~B}$ & $10(4)$ \\
\hline $\mathrm{C} 79 \mathrm{~B}-\mathrm{C} 79-\mathrm{C} 18 \mathrm{~B}-\mathrm{C} 4$ & $-78(3)$ \\
\hline $\mathrm{C} 79 \mathrm{~B}-\mathrm{C} 79-\mathrm{C} 18 \mathrm{~B}-\mathrm{C} 18$ & $178(3)$ \\
\hline $\mathrm{C} 79 \mathrm{~B}-\mathrm{C} 79-\mathrm{C} 18 \mathrm{~B}-\mathrm{C} 73 \mathrm{~B}$ & $-174(2)$ \\
\hline $\mathrm{C} 79 \mathrm{~B}-\mathrm{C} 80-\mathrm{C} 81-\mathrm{C} 82$ & $173(3)$ \\
\hline $\mathrm{C} 79 \mathrm{~B}-\mathrm{C} 80-\mathrm{C} 81-\mathrm{C} 81 \mathrm{~B}$ & $-145(4)$ \\
\hline $\mathrm{C} 79 \mathrm{~B}-\mathrm{C} 80-\mathrm{C} 80 \mathrm{~B}-\mathrm{C} 81 \mathrm{~B}$ & $152(3)$ \\
\hline $\mathrm{C} 79 \mathrm{~B}-\mathrm{C} 81-\mathrm{C} 81 \mathrm{~B}-\mathrm{C} 82$ & $-139.2(14)$ \\
\hline $\mathrm{C} 79 \mathrm{~B}-\mathrm{C} 81-\mathrm{C} 81 \mathrm{~B}-\mathrm{C} 83$ & $-180(2)$ \\
\hline $\mathrm{C} 79 \mathrm{~B}-\mathrm{C} 81-\mathrm{C} 81 \mathrm{~B}-\mathrm{C} 80 \mathrm{~B}$ & $-2.5(19)$ \\
\hline $\mathrm{C} 79 \mathrm{~B}-\mathrm{C} 80 \mathrm{~B}-\mathrm{C} 81 \mathrm{~B}-\mathrm{C} 82$ & $42(4)$ \\
\hline $\mathrm{C} 79 \mathrm{~B}-\mathrm{C} 80 \mathrm{~B}-\mathrm{C} 81 \mathrm{~B}-\mathrm{C} 81$ & $4(3)$ \\
\hline $\mathrm{C} 80 \mathrm{~B}-\mathrm{C} 80-\mathrm{C} 81-\mathrm{C} 82$ & $-51(2)$ \\
\hline $\mathrm{C} 80 \mathrm{~B}-\mathrm{C} 80-\mathrm{C} 81-\mathrm{C} 79 \mathrm{~B}$ & $136(4)$ \\
\hline $\mathrm{C} 80 \mathrm{~B}-\mathrm{C} 80-\mathrm{C} 81-\mathrm{C} 81 \mathrm{~B}$ & $-9(2)$ \\
\hline $\mathrm{C} 80 \mathrm{~B}-\mathrm{C} 80-\mathrm{C} 79 \mathrm{~B}-\mathrm{C} 18$ & $68(7)$ \\
\hline $\mathrm{C} 80 \mathrm{~B}-\mathrm{C} 80-\mathrm{C} 79 \mathrm{~B}-\mathrm{C} 79$ & $172(6)$ \\
\hline $\mathrm{C} 80 \mathrm{~B}-\mathrm{C} 80-\mathrm{C} 79 \mathrm{~B}-\mathrm{C} 81$ & $-132(4)$ \\
\hline $\mathrm{C} 80 \mathrm{~B}-\mathrm{C} 80-\mathrm{C} 79 \mathrm{~B}-\mathrm{C} 18 \mathrm{~B}$ & $58(7)$ \\
\hline $\mathrm{C} 81 \mathrm{~B}-\mathrm{C} 82-\mathrm{C} 81-\mathrm{C} 80$ & $52.4(15)$ \\
\hline $\mathrm{C} 81 \mathrm{~B}-\mathrm{C} 82-\mathrm{C} 81-\mathrm{C} 79 \mathrm{~B}$ & $54.8(18)$ \\
\hline $\mathrm{C} 81 \mathrm{~B}-\mathrm{C} 82-\mathrm{C} 83-\mathrm{C} 84$ & $-140(3)$ \\
\hline $\mathrm{C} 81 \mathrm{~B}-\mathrm{C} 82-\mathrm{C} 83-\mathrm{C} 83 \mathrm{~B}$ & $-159(4)$ \\
\hline $\mathrm{C} 81 \mathrm{~B}-\mathrm{C} 82-\mathrm{C} 83 \mathrm{~B}-\mathrm{C} 83$ & $16(3)$ \\
\hline $\mathrm{C} 81 \mathrm{~B}-\mathrm{C} 82-\mathrm{C} 83 \mathrm{~B}-\mathrm{C} 84$ & $-22(7)$ \\
\hline $\mathrm{C} 81 \mathrm{~B}-\mathrm{C} 82-\mathrm{C} 83 \mathrm{~B}-\mathrm{C} 84 \mathrm{~B}$ & $-159(3)$ \\
\hline $\mathrm{C} 81 \mathrm{~B}-\mathrm{C} 83-\mathrm{C} 84-\mathrm{C} 83 \mathrm{~B}$ & $-93(5)$ \\
\hline $\mathrm{C} 81 \mathrm{~B}-\mathrm{C} 83-\mathrm{C} 83 \mathrm{~B}-\mathrm{C} 82$ & $-25(4)$ \\
\hline $\mathrm{C} 81 \mathrm{~B}-\mathrm{C} 83-\mathrm{C} 83 \mathrm{~B}-\mathrm{C} 84$ & $124(4)$ \\
\hline $\mathrm{C} 81 \mathrm{~B}-\mathrm{C} 83-\mathrm{C} 83 \mathrm{~B}-\mathrm{C} 84 \mathrm{~B}$ & $-3(23)$ \\
\hline $\mathrm{C} 18 \mathrm{~B}-\mathrm{C} 4-\mathrm{C} 5-\mathrm{C} 6$ & $165.0(11)$ \\
\hline $\mathrm{C} 18 \mathrm{~B}-\mathrm{C} 4-\mathrm{C} 18-\mathrm{C} 17$ & $-80(2)$ \\
\hline $\mathrm{C} 18 \mathrm{~B}-\mathrm{C} 4-\mathrm{C} 18-\mathrm{C} 73$ & $159(2)$ \\
\hline
\end{tabular}




\begin{tabular}{|c|c|}
\hline $\mathrm{C} 57-\mathrm{C} 66-\mathrm{C} 71-\mathrm{C} 70$ & $179.1(6)$ \\
\hline $\mathrm{C} 57-\mathrm{C} 66-\mathrm{C} 71-\mathrm{C} 72$ & $1.0(8)$ \\
\hline $\mathrm{C} 58-\mathrm{C} 57-\mathrm{C} 66-\mathrm{C} 67$ & $-179.3(7)$ \\
\hline $\mathrm{C} 58-\mathrm{C} 57-\mathrm{C} 66-\mathrm{C} 71$ & $0.6(8)$ \\
\hline $\mathrm{C} 58-\mathrm{C} 59-\mathrm{C} 60-\mathrm{C} 55$ & $0.5(12)$ \\
\hline $\mathrm{C} 58-\mathrm{C} 59-\mathrm{C} 60-\mathrm{C} 61$ & $-178.9(7)$ \\
\hline $\mathrm{C} 58-\mathrm{C} 72-\mathrm{C} 109-\mathrm{C} 110$ & $61.2(9)$ \\
\hline $\mathrm{C} 58-\mathrm{C} 72-\mathrm{C} 115-\mathrm{C} 116$ & $-62.4(9)$ \\
\hline $\mathrm{C} 59-\mathrm{C} 58-\mathrm{C} 72-\mathrm{C} 71$ & $-179.3(8)$ \\
\hline $\mathrm{C} 59-\mathrm{C} 58-\mathrm{C} 72-\mathrm{C} 109$ & $60.4(11)$ \\
\hline $\mathrm{C} 59-\mathrm{C} 58-\mathrm{C} 72-\mathrm{C} 115$ & $-59.6(11)$ \\
\hline $\mathrm{C} 59-\mathrm{C} 60-\mathrm{C} 61-\mathrm{N} 4$ & $-179.6(7)$ \\
\hline $\mathrm{C} 59-\mathrm{C} 60-\mathrm{C} 61-\mathrm{C} 62$ & $1.1(13)$ \\
\hline $\mathrm{C} 60-\mathrm{C} 55-\mathrm{C} 56-\mathrm{C} 57$ & $2.6(10)$ \\
\hline $\mathrm{C} 60-\mathrm{C} 61-\mathrm{C} 62-\mathrm{C} 63$ & $-177.9(8)$ \\
\hline $\mathrm{C} 61-\mathrm{N} 4-\mathrm{C} 65-\mathrm{C} 64$ & $-0.7(10)$ \\
\hline $\mathrm{C} 61-\mathrm{C} 62-\mathrm{C} 63-\mathrm{C} 64$ & $-0.4(14)$ \\
\hline $\mathrm{C} 62-\mathrm{C} 63-\mathrm{C} 64-\mathrm{F} 4$ & $174.9(8)$ \\
\hline $\mathrm{C} 62-\mathrm{C} 63-\mathrm{C} 64-\mathrm{C} 65$ & $-2.7(14)$ \\
\hline $\mathrm{C} 63-\mathrm{C} 64-\mathrm{C} 65-\mathrm{N} 4$ & $3.3(13)$ \\
\hline $\mathrm{C} 65-\mathrm{N} 4-\mathrm{C} 61-\mathrm{C} 60$ & $178.4(6)$ \\
\hline $\mathrm{C} 65-\mathrm{N} 4-\mathrm{C} 61-\mathrm{C} 62$ & $-2.2(11)$ \\
\hline $\mathrm{C} 66-\mathrm{C} 57-\mathrm{C} 58-\mathrm{C} 59$ & $179.6(7)$ \\
\hline $\mathrm{C} 66-\mathrm{C} 57-\mathrm{C} 58-\mathrm{C} 72$ & $-1.9(9)$ \\
\hline $\mathrm{C} 66-\mathrm{C} 67-\mathrm{C} 68-\mathrm{C} 69$ & $1.0(10)$ \\
\hline $\mathrm{C} 66-\mathrm{C} 71-\mathrm{C} 72-\mathrm{C} 58$ & $-2.0(8)$ \\
\hline $\mathrm{C} 66-\mathrm{C} 71-\mathrm{C} 72-\mathrm{C} 109$ & $117.6(7)$ \\
\hline $\mathrm{C} 66-\mathrm{C} 71-\mathrm{C} 72-\mathrm{C} 115$ & $-120.5(6)$ \\
\hline $\mathrm{C} 67-\mathrm{C} 66-\mathrm{C} 71-\mathrm{C} 70$ & $-1.0(11)$ \\
\hline $\mathrm{C} 67-\mathrm{C} 66-\mathrm{C} 71-\mathrm{C} 72$ & $-179.1(6)$ \\
\hline $\mathrm{C} 67-\mathrm{C} 68-\mathrm{C} 69-\mathrm{C} 70$ & $-0.2(11)$ \\
\hline $\mathrm{C} 68-\mathrm{C} 69-\mathrm{C} 70-\mathrm{C} 71$ & $-1.2(11)$ \\
\hline $\mathrm{C} 69-\mathrm{C} 70-\mathrm{C} 71-\mathrm{C} 66$ & $1.8(11)$ \\
\hline $\mathrm{C} 69-\mathrm{C} 70-\mathrm{C} 71-\mathrm{C} 72$ & $179.5(7)$ \\
\hline $\mathrm{C} 70-\mathrm{C} 71-\mathrm{C} 72-\mathrm{C} 58$ & $-179.9(7)$ \\
\hline $\mathrm{C} 70-\mathrm{C} 71-\mathrm{C} 72-\mathrm{C} 109$ & $-60.3(10)$ \\
\hline $\mathrm{C} 70-\mathrm{C} 71-\mathrm{C} 72-\mathrm{C} 115$ & $61.6(10)$ \\
\hline $\mathrm{C} 71-\mathrm{C} 66-\mathrm{C} 67-\mathrm{C} 68$ & $-0.4(10)$ \\
\hline $\mathrm{C} 71-\mathrm{C} 72-\mathrm{C} 109-\mathrm{C} 110$ & $-52.4(9)$ \\
\hline $\mathrm{C} 71-\mathrm{C} 72-\mathrm{C} 115-\mathrm{C} 116$ & $50.5(9)$ \\
\hline $\mathrm{C} 72-\mathrm{C} 58-\mathrm{C} 59-\mathrm{C} 60$ & $-177.0(8)$ \\
\hline $\mathrm{C} 72-\mathrm{C} 109-\mathrm{C} 110-\mathrm{C} 111$ & $-179.9(13)$ \\
\hline $\mathrm{C} 72-\mathrm{C} 109-\mathrm{C} 110-\mathrm{C} 211$ & $-169(2)$ \\
\hline $\mathrm{C} 72-\mathrm{C} 115-\mathrm{C} 116-\mathrm{C} 117$ & $171.1(11)$ \\
\hline $\mathrm{C} 72-\mathrm{C} 115-\mathrm{C} 116-\mathrm{C} 217$ & $178(2)$ \\
\hline $\mathrm{C} 82-\mathrm{C} 81-\mathrm{C} 81 \mathrm{~B}-\mathrm{C} 83$ & $-40.6(17)$ \\
\hline $\mathrm{C} 82-\mathrm{C} 81-\mathrm{C} 81 \mathrm{~B}-\mathrm{C} 80 \mathrm{~B}$ & $137(2)$ \\
\hline $\mathrm{C} 82-\mathrm{C} 83-\mathrm{C} 84-\mathrm{C} 83 \mathrm{~B}$ & $-27(4)$ \\
\hline
\end{tabular}

\begin{tabular}{|c|c|}
\hline $\mathrm{C} 18 \mathrm{~B}-\mathrm{C} 4-\mathrm{C} 18-\mathrm{C} 79$ & $35(2)$ \\
\hline $\mathrm{C} 18 \mathrm{~B}-\mathrm{C} 4-\mathrm{C} 18-\mathrm{C} 73 \mathrm{~B}$ & $122(3)$ \\
\hline $\mathrm{C} 18 \mathrm{~B}-\mathrm{C} 4-\mathrm{C} 18-\mathrm{C} 79 \mathrm{~B}$ & $15(2)$ \\
\hline $\mathrm{C} 18 \mathrm{~B}-\mathrm{C} 17-\mathrm{C} 18-\mathrm{C} 4$ & $76(2)$ \\
\hline $\mathrm{C} 18 \mathrm{~B}-\mathrm{C} 17-\mathrm{C} 18-\mathrm{C} 73$ & $-163(2)$ \\
\hline $\mathrm{C} 18 \mathrm{~B}-\mathrm{C} 17-\mathrm{C} 18-\mathrm{C} 79$ & $-42(2)$ \\
\hline $\mathrm{C} 18 \mathrm{~B}-\mathrm{C} 17-\mathrm{C} 18-\mathrm{C} 73 \mathrm{~B}$ & $-129(3)$ \\
\hline $\mathrm{C} 18 \mathrm{~B}-\mathrm{C} 17-\mathrm{C} 18-\mathrm{C} 79 \mathrm{~B}$ & $-19(2)$ \\
\hline $\mathrm{C} 18 \mathrm{~B}-\mathrm{C} 18-\mathrm{C} 73-\mathrm{C} 74$ & $160(5)$ \\
\hline $\mathrm{C} 18 \mathrm{~B}-\mathrm{C} 18-\mathrm{C} 73-\mathrm{C} 73 \mathrm{~B}$ & $0(6)$ \\
\hline $\mathrm{C} 18 \mathrm{~B}-\mathrm{C} 18-\mathrm{C} 73-\mathrm{C} 74 \mathrm{~B}$ & $-174(8)$ \\
\hline $\mathrm{C} 18 \mathrm{~B}-\mathrm{C} 18-\mathrm{C} 79-\mathrm{C} 80$ & $-12(3)$ \\
\hline $\mathrm{C} 18 \mathrm{~B}-\mathrm{C} 18-\mathrm{C} 79-\mathrm{C} 73 \mathrm{~B}$ & $170(3)$ \\
\hline $\mathrm{C} 18 \mathrm{~B}-\mathrm{C} 18-\mathrm{C} 79-\mathrm{C} 79 \mathrm{~B}$ & $-2(3)$ \\
\hline $\mathrm{C} 18 \mathrm{~B}-\mathrm{C} 18-\mathrm{C} 73 \mathrm{~B}-\mathrm{C} 73$ & $180(3)$ \\
\hline $\mathrm{C} 18 \mathrm{~B}-\mathrm{C} 18-\mathrm{C} 73 \mathrm{~B}-\mathrm{C} 79$ & $-9(3)$ \\
\hline $\mathrm{C} 18 \mathrm{~B}-\mathrm{C} 18-\mathrm{C} 73 \mathrm{~B}-\mathrm{C} 74 \mathrm{~B}$ & $-178(3)$ \\
\hline $\mathrm{C} 18 \mathrm{~B}-\mathrm{C} 73-\mathrm{C} 74-\mathrm{C} 75 \mathrm{~A}$ & $-96.1(12)$ \\
\hline $\mathrm{C} 18 \mathrm{~B}-\mathrm{C} 73-\mathrm{C} 74-\mathrm{C} 75 \mathrm{~B}$ & $-170.8(13)$ \\
\hline $\mathrm{C} 18 \mathrm{~B}-\mathrm{C} 73-\mathrm{C} 74-\mathrm{C} 74 \mathrm{~B}$ & $155(6)$ \\
\hline $\mathrm{C} 18 \mathrm{~B}-\mathrm{C} 73-\mathrm{C} 73 \mathrm{~B}-\mathrm{C} 18$ & $0.0(9)$ \\
\hline $\mathrm{C} 18 \mathrm{~B}-\mathrm{C} 73-\mathrm{C} 73 \mathrm{~B}-\mathrm{C} 79$ & $-24(6)$ \\
\hline $\mathrm{C} 18 \mathrm{~B}-\mathrm{C} 73-\mathrm{C} 73 \mathrm{~B}-\mathrm{C} 74 \mathrm{~B}$ & $-161(20)$ \\
\hline $\mathrm{C} 18 \mathrm{~B}-\mathrm{C} 73-\mathrm{C} 74 \mathrm{~B}-\mathrm{C} 74$ & $-46(10)$ \\
\hline $\mathrm{C} 18 \mathrm{~B}-\mathrm{C} 73-\mathrm{C} 74 \mathrm{~B}-\mathrm{C} 75 \mathrm{~B}$ & $-173(3)$ \\
\hline $\mathrm{C} 18 \mathrm{~B}-\mathrm{C} 73-\mathrm{C} 74 \mathrm{~B}-\mathrm{C} 73 \mathrm{~B}$ & $155(26)$ \\
\hline $\mathrm{C} 18 \mathrm{~B}-\mathrm{C} 79-\mathrm{C} 80-\mathrm{C} 81$ & $170.8(11)$ \\
\hline $\mathrm{C} 18 \mathrm{~B}-\mathrm{C} 79-\mathrm{C} 80-\mathrm{C} 79 \mathrm{~B}$ & $39(7)$ \\
\hline $\mathrm{C} 18 \mathrm{~B}-\mathrm{C} 79-\mathrm{C} 80-\mathrm{C} 80 \mathrm{~B}$ & $28(3)$ \\
\hline $\mathrm{C} 18 \mathrm{~B}-\mathrm{C} 79-\mathrm{C} 73 \mathrm{~B}-\mathrm{C} 18$ & $3.4(11)$ \\
\hline $\mathrm{C} 18 \mathrm{~B}-\mathrm{C} 79-\mathrm{C} 73 \mathrm{~B}-\mathrm{C} 73$ & $31(8)$ \\
\hline $\mathrm{C} 18 \mathrm{~B}-\mathrm{C} 79-\mathrm{C} 73 \mathrm{~B}-\mathrm{C} 74 \mathrm{~B}$ & $44(8)$ \\
\hline $\mathrm{C} 18 \mathrm{~B}-\mathrm{C} 79-\mathrm{C} 79 \mathrm{~B}-\mathrm{C} 18$ & $-0.6(9)$ \\
\hline $\mathrm{C} 18 \mathrm{~B}-\mathrm{C} 79-\mathrm{C} 79 \mathrm{~B}-\mathrm{C} 80$ & $-142(7)$ \\
\hline $\mathrm{C} 18 \mathrm{~B}-\mathrm{C} 79-\mathrm{C} 79 \mathrm{~B}-\mathrm{C} 81$ & $-177.7(19)$ \\
\hline $\mathrm{C} 18 \mathrm{~B}-\mathrm{C} 73 \mathrm{~B}-\mathrm{C} 74 \mathrm{~B}-\mathrm{C} 73$ & $-20(21)$ \\
\hline $\mathrm{C} 18 \mathrm{~B}-\mathrm{C} 73 \mathrm{~B}-\mathrm{C} 74 \mathrm{~B}-\mathrm{C} 74$ & $-42(5)$ \\
\hline $\mathrm{C} 18 \mathrm{~B}-\mathrm{C} 73 \mathrm{~B}-\mathrm{C} 74 \mathrm{~B}-\mathrm{C} 75 \mathrm{~B}$ & $-170(2)$ \\
\hline $\mathrm{C} 18 \mathrm{~B}-\mathrm{C} 79 \mathrm{~B}-\mathrm{C} 80 \mathrm{~B}-\mathrm{C} 80$ & $-148(4)$ \\
\hline $\mathrm{C} 18 \mathrm{~B}-\mathrm{C} 79 \mathrm{~B}-\mathrm{C} 80 \mathrm{~B}-\mathrm{C} 81 \mathrm{~B}$ & $179(3)$ \\
\hline $\mathrm{C} 83 \mathrm{~B}-\mathrm{C} 82-\mathrm{C} 81-\mathrm{C} 80$ & $141(4)$ \\
\hline $\mathrm{C} 83 \mathrm{~B}-\mathrm{C} 82-\mathrm{C} 81-\mathrm{C} 79 \mathrm{~B}$ & $144(4)$ \\
\hline $\mathrm{C} 83 \mathrm{~B}-\mathrm{C} 82-\mathrm{C} 81-\mathrm{C} 81 \mathrm{~B}$ & $89(5)$ \\
\hline $\mathrm{C} 83 \mathrm{~B}-\mathrm{C} 82-\mathrm{C} 83-\mathrm{C} 84$ & $19(3)$ \\
\hline $\mathrm{C} 83 \mathrm{~B}-\mathrm{C} 82-\mathrm{C} 83-\mathrm{C} 81 \mathrm{~B}$ & $159(4)$ \\
\hline $\mathrm{C} 83 \mathrm{~B}-\mathrm{C} 82-\mathrm{C} 81 \mathrm{~B}-\mathrm{C} 81$ & $-152(3)$ \\
\hline $\mathrm{C} 83 \mathrm{~B}-\mathrm{C} 82-\mathrm{C} 81 \mathrm{~B}-\mathrm{C} 83$ & $-19(3)$ \\
\hline $\mathrm{C} 83 \mathrm{~B}-\mathrm{C} 82-\mathrm{C} 81 \mathrm{~B}-\mathrm{C} 80 \mathrm{~B}$ & $159(3)$ \\
\hline
\end{tabular}




$\begin{array}{llll}\mathrm{C} 82-\mathrm{C} 83-\mathrm{C} 81 \mathrm{~B}-\mathrm{C} 81 & 37.3(14) & \mathrm{C} 83 \mathrm{~B}-\mathrm{C} 83-\mathrm{C} 81 \mathrm{~B}-\mathrm{C} 82 & 23(4) \\ \mathrm{C} 82-\mathrm{C} 83-\mathrm{C} 83 \mathrm{~B}-\mathrm{C} 84 & 149(5) & \mathrm{C} 83 \mathrm{~B}-\mathrm{C} 83-\mathrm{C} 81 \mathrm{~B}-\mathrm{C} 81 & 61(4) \\ \mathrm{C} 82-\mathrm{C} 83-\mathrm{C} 83 \mathrm{~B}-\mathrm{C} 84 \mathrm{~B} & 22(20) & \mathrm{C} 1 \mathrm{~S}-\mathrm{C} 2 \mathrm{~S}-\mathrm{C} 3 \mathrm{~S}-\mathrm{C} 4 \mathrm{~S} & 166(3) \\ \mathrm{C} 85-\mathrm{C} 36-\mathrm{C} 91-\mathrm{C} 92 & -179.6(7) & \mathrm{C} 2 \mathrm{~S}-\mathrm{C} 3 \mathrm{~S}-\mathrm{C} 4 \mathrm{~S}-\mathrm{C} 5 \mathrm{~S} & 164(4) \\ \mathrm{C} 85-\mathrm{C} 86-\mathrm{C} 87-\mathrm{C} 88 & -173.5(7) & & \end{array}$

Di- $\mu_{2}$-cyanato-bis $\{$ bis[9,9-dihexyl-2-(5-methoxypyridin-2-yl)-9H-fluoren-1-yl]iridium\} pentane monosolvate (III)

Crystal data

$\left[\mathrm{Ir}_{2}\left(\mathrm{C}_{31} \mathrm{H}_{38} \mathrm{NO}\right)_{4}(\mathrm{NCO})_{2}\right] \cdot \mathrm{C}_{5} \mathrm{H}_{12}$

$M_{r}=2303.07$

Monoclinic, $P 2_{1} / c$

$a=38.976(6) \AA$

$b=21.615(3) \AA$

$c=28.759(4) \AA$

$\beta=108.920(3)^{\circ}$

$V=22920(6) \AA^{3}$

$Z=8$

\section{Data collection}

Bruker SMART CCD 6000 area detector diffractometer

Radiation source: sealed X-ray tube

Graphite monochromator

Detector resolution: 5.6 pixels $\mathrm{mm}^{-1}$

$\omega$ scans

Absorption correction: multi-scan

(SADABS; Krause et al., 2015)

$T_{\min }=0.809, T_{\max }=1.000$

Refinement

Refinement on $F^{2}$

Least-squares matrix: full

$R\left[F^{2}>2 \sigma\left(F^{2}\right)\right]=0.077$

$w R\left(F^{2}\right)=0.185$

$S=0.97$

40676 reflections

2479 parameters

2600 restraints

Primary atom site location: dual
$F(000)=9552$

$D_{\mathrm{x}}=1.335 \mathrm{Mg} \mathrm{m}^{-3}$

Mo $K \alpha$ radiation, $\lambda=0.71073 \AA$

Cell parameters from 5377 reflections

$\theta=2.2-25.0^{\circ}$

$\mu=2.38 \mathrm{~mm}^{-1}$

$T=120 \mathrm{~K}$

Plate, yellow

$0.2 \times 0.1 \times 0.01 \mathrm{~mm}$

95765 measured reflections

40676 independent reflections

19348 reflections with $I>2 \sigma(I)$

$R_{\text {int }}=0.143$

$\theta_{\text {max }}=25.3^{\circ}, \theta_{\text {min }}=1.2^{\circ}$

$h=-42 \rightarrow 46$

$k=-23 \rightarrow 25$

$l=-34 \rightarrow 26$

Secondary atom site location: difference Fourier map

Hydrogen site location: inferred from

neighbouring sites

$\mathrm{H}$-atom parameters constrained

$w=1 /\left[\sigma^{2}\left(F_{\mathrm{o}}^{2}\right)+(0.064 P)^{2}\right]$

where $P=\left(F_{\mathrm{o}}^{2}+2 F_{\mathrm{c}}^{2}\right) / 3$

$(\Delta / \sigma)_{\max }=0.002$

$\Delta \rho_{\max }=2.80$ e $\AA^{-3}$

$\Delta \rho_{\min }=-2.20$ e $\AA^{-3}$

\section{Special details}

Experimental. The data collection nominally covered a full sphere of reciprocal space by a combination of 2 sets of 600 and $344 \omega$ scans, each set at different $\varphi$ angles and each scan (60 s exposure) covering $-0.3^{\circ}$ in $\omega$. The crystal to detector distance was $5.84 \mathrm{~cm}$.

Geometry. All esds (except the esd in the dihedral angle between two 1.s. planes) are estimated using the full covariance matrix. The cell esds are taken into account individually in the estimation of esds in distances, angles and torsion angles; correlations between esds in cell parameters are only used when they are defined by crystal symmetry. An approximate (isotropic) treatment of cell esds is used for estimating esds involving l.s. planes.

Refinement. Extensive disorder of n-hexyl chains. Disordered solvent (presumably pentane) could not be modelled at atomic resolution and was masked. 
Fractional atomic coordinates and isotropic or equivalent isotropic displacement parameters $\left(\AA^{2}\right)$

\begin{tabular}{|c|c|c|c|c|c|}
\hline & $x$ & $y$ & $z$ & $U_{\text {iso }} * / U_{\text {eq }}$ & Occ. $(<1)$ \\
\hline Ir1 & $0.38516(2)$ & $0.33601(2)$ & $0.61355(2)$ & $0.02057(11)$ & \\
\hline $\mathrm{Ir} 2$ & $0.42702(2)$ & 0.33949 (2) & $0.73765(2)$ & $0.02300(12)$ & \\
\hline $\mathrm{O} 1$ & $0.4959(3)$ & $0.3018(5)$ & $0.6592(4)$ & $0.070(3)$ & \\
\hline $\mathrm{O} 2$ & $0.3151(3)$ & $0.3372(8)$ & $0.6939(4)$ & $0.141(7)$ & \\
\hline $\mathrm{O} 3$ & $0.4252(2)$ & $0.0935(4)$ & 0.6313 & $0.040(2)$ & \\
\hline $\mathrm{O} 4$ & $0.3555(2)$ & $0.5731(4)$ & $0.6482(3)$ & $0.042(2)$ & \\
\hline $\mathrm{O} 5$ & $0.3651(2)$ & $0.1113(4)$ & $0.7197(3)$ & $0.040(2)$ & \\
\hline O6 & $0.4769(2)$ & $0.5511(4)$ & $0.6824(3)$ & $0.044(2)$ & \\
\hline N1 & $0.3782(2)$ & $0.2425(4)$ & $0.6066(3)$ & $0.021(2)$ & \\
\hline N01 & $0.4409(2)$ & $0.3274(4)$ & $0.6679(3)$ & $0.02057(11)$ & \\
\hline N02 & $0.3720(2)$ & $0.3438(4)$ & 0.6830 & $0.02057(11)$ & \\
\hline N2 & $0.3876(2)$ & $0.4302(4)$ & 0.6089 & $0.021(2)$ & \\
\hline N3 & $0.4246(2)$ & $0.2455(4)$ & $0.7482(3)$ & $0.020(2)^{*}$ & \\
\hline N4 & $0.4344(2)$ & $0.4315(4)$ & $0.7348(3)$ & $0.027(2)$ & \\
\hline $\mathrm{C} 01$ & $0.4667(3)$ & $0.3152(6)$ & $0.6639(4)$ & $0.035(3)$ & \\
\hline $\mathrm{C} 1$ & $0.3339(3)$ & $0.3342(5)$ & $0.5705(3)$ & $0.012(2)^{*}$ & \\
\hline $\mathrm{C} 02$ & $0.3442(3)$ & $0.3383(7)$ & $0.6887(4)$ & $0.050(4)$ & \\
\hline $\mathrm{C} 2$ & 0.3103 & $0.3846(6)$ & $0.5509(4)$ & $0.026(3)$ & \\
\hline $\mathrm{H} 2 \mathrm{~A}$ & 0.319531 & 0.425605 & 0.555252 & $0.032 *$ & \\
\hline $\mathrm{C} 3$ & $0.2743(3)$ & $0.3747(5)$ & $0.5259(4)$ & $0.023(2)$ & \\
\hline $\mathrm{C} 4$ & 0.2600 & $0.3155(5)$ & $0.5171(4)$ & $0.025(2)$ & \\
\hline $\mathrm{C} 5$ & $0.2817(3)$ & $0.2643(5)$ & $0.5347(4)$ & $0.030(3)$ & \\
\hline H5A & 0.272013 & 0.223609 & 0.529200 & $0.036^{*}$ & \\
\hline C6 & $0.3187(3)$ & $0.2745(5)$ & $0.5609(4)$ & $0.023(2)$ & \\
\hline $\mathrm{C} 7$ & $0.3442(3)$ & $0.2234(5)$ & $0.5784(4)$ & $0.027(2)$ & \\
\hline $\mathrm{C} 8$ & $0.3381(3)$ & $0.1608(5)$ & $0.5688(4)$ & $0.029(3)$ & \\
\hline H8 & 0.314557 & 0.147633 & 0.549232 & $0.035^{*}$ & \\
\hline C9 & $0.3640(3)$ & $0.1181(6)$ & $0.5859(4)$ & $0.034(3)$ & \\
\hline H9 & 0.358851 & 0.075481 & 0.579018 & $0.041 *$ & \\
\hline C10 & 0.3988 (3) & $0.1374(5)$ & $0.6142(4)$ & $0.030(3)$ & \\
\hline C11 & $0.4048(3)$ & $0.1995(5)$ & $0.6239(4)$ & $0.025(2)$ & \\
\hline H11 & 0.428345 & 0.212814 & 0.643230 & $0.030 *$ & \\
\hline $\mathrm{C} 12$ & 0.2450 & $0.4199(6)$ & $0.5032(4)$ & $0.028(3)$ & \\
\hline $\mathrm{C} 13$ & $0.2458(3)$ & $0.4825(6)$ & $0.5018(4)$ & $0.031(3)$ & \\
\hline $\mathrm{H} 13$ & 0.267775 & 0.503955 & 0.517626 & $0.038^{*}$ & \\
\hline $\mathrm{C} 14$ & 0.2148 & $0.5150(6)$ & $0.4773(4)$ & $0.038(3)$ & \\
\hline H14 & 0.215184 & 0.558913 & 0.476601 & $0.046 *$ & \\
\hline $\mathrm{C} 15$ & $0.1827(3)$ & $0.4825(7)$ & $0.4533(4)$ & $0.043(3)$ & \\
\hline H15 & 0.161443 & 0.504231 & 0.434982 & $0.052 *$ & \\
\hline $\mathrm{C} 16$ & $0.1822(3)$ & $0.4197(6)$ & $0.4567(4)$ & 0.037 (3) & \\
\hline H16 & 0.160202 & 0.398011 & 0.441766 & $0.044 *$ & \\
\hline $\mathrm{C} 17$ & $0.2132(3)$ & $0.3871(6)$ & $0.4812(4)$ & $0.029(2)$ & \\
\hline $\mathrm{C} 18$ & 0.2193 & $0.3170(6)$ & $0.4879(4)$ & $0.033(3)$ & \\
\hline C19 & $0.4062(3)$ & $0.3423(5)$ & $0.5594(4)$ & $0.022(2)$ & \\
\hline $\mathrm{C} 20$ & 0.4160 & $0.2960(5)$ & $0.5326(4)$ & $0.023(3)$ & \\
\hline
\end{tabular}




\begin{tabular}{|c|c|c|c|c|}
\hline H6 & 0.409484 & 0.254396 & 0.536381 & $0.027^{*}$ \\
\hline $\mathrm{C} 21$ & 0.4349 (3) & $0.3087(5)$ & $0.5007(4)$ & $0.024(2)$ \\
\hline $\mathrm{C} 22$ & $0.4436(3)$ & $0.3688(5)$ & $0.4922(4)$ & 0.025 (3) \\
\hline $\mathrm{C} 23$ & $0.4341(3)$ & $0.4173(5)$ & $0.5167(4)$ & $0.021(2)$ \\
\hline $\mathrm{H} 23$ & 0.439600 & 0.458763 & 0.510747 & $0.025^{*}$ \\
\hline $\mathrm{C} 24$ & $0.4161(3)$ & $0.4038(5)$ & $0.5507(4)$ & $0.022(2)$ \\
\hline $\mathrm{C} 25$ & 0.4033 (3) & $0.4528(5)$ & $0.5764(4)$ & $0.029(3)$ \\
\hline $\mathrm{C} 26$ & $0.4040(3)$ & $0.5161(5)$ & 0.5699 (4) & $0.030(3)$ \\
\hline $\mathrm{H} 26$ & 0.415868 & 0.532235 & 0.548451 & $0.036^{*}$ \\
\hline $\mathrm{C} 27$ & 0.3879 (3) & $0.5563(5)$ & $0.5937(4)$ & $0.032(3)$ \\
\hline $\mathrm{H} 27$ & 0.388224 & 0.599631 & 0.588221 & $0.038^{*}$ \\
\hline $\mathrm{C} 28$ & $0.3712(3)$ & $0.5325(6)$ & $0.6258(4)$ & $0.032(3)$ \\
\hline $\mathrm{C} 29$ & 0.3724 (3) & $0.4701(5)$ & $0.6338(4)$ & $0.026(3)$ \\
\hline $\mathrm{H} 29$ & 0.362348 & 0.453780 & 0.657298 & $0.031 *$ \\
\hline $\mathrm{C} 30$ & 0.4487 (3) & $0.2650(5)$ & $0.4701(4)$ & 0.027 (3) \\
\hline C31 & $0.4461(3)$ & $0.2019(5)$ & $0.4650(4)$ & $0.030(3)$ \\
\hline H31 & 0.434090 & 0.177821 & 0.482657 & $0.036^{*}$ \\
\hline $\mathrm{C} 32$ & 0.4618 (3) & $0.1746(5)$ & $0.4332(4)$ & 0.033 (3) \\
\hline H32 & 0.460880 & 0.130949 & 0.429288 & $0.039^{*}$ \\
\hline $\mathrm{C} 33$ & $0.4786(3)$ & $0.2094(6)$ & $0.4073(4)$ & $0.031(3)$ \\
\hline H33 & 0.488986 & 0.189617 & 0.385555 & $0.037^{*}$ \\
\hline $\mathrm{C} 34$ & 0.4808 (3) & $0.2727(6)$ & $0.4122(4)$ & $0.030(3)$ \\
\hline H34 & 0.492352 & 0.296213 & 0.393675 & $0.036^{*}$ \\
\hline $\mathrm{C} 35$ & 0.4659 (3) & $0.3024(5)$ & $0.4444(4)$ & $0.024(2)$ \\
\hline $\mathrm{C} 36$ & 0.4645 (3) & $0.3702(5)$ & $0.4548(4)$ & $0.028(2)$ \\
\hline $\mathrm{C} 37$ & 0.4775 (3) & $0.3258(5)$ & $0.7796(4)$ & $0.023(2)$ \\
\hline C38 & $0.5062(3)$ & $0.3675(5)$ & $0.7951(4)$ & $0.024(3)$ \\
\hline $\mathrm{H} 38$ & 0.501422 & 0.410380 & 0.789104 & $0.029^{*}$ \\
\hline C39 & $0.5422(3)$ & $0.3485(5)$ & $0.8195(4)$ & $0.027(3)$ \\
\hline $\mathrm{C} 40$ & $0.5496(3)$ & $0.2858(6)$ & $0.8324(4)$ & $0.032(3)$ \\
\hline $\mathrm{C} 41$ & $0.5223(3)$ & $0.2417(5)$ & $0.8182(4)$ & $0.026(3)$ \\
\hline H41 & 0.527385 & 0.199251 & 0.825901 & $0.031^{*}$ \\
\hline $\mathrm{C} 42$ & 0.4867 (3) & $0.2619(5)$ & 0.7919 (4) & $0.024(2)$ \\
\hline $\mathrm{C} 43$ & 0.4565 (3) & $0.2191(5)$ & $0.7741(4)$ & 0.025 (3) \\
\hline $\mathrm{C} 44$ & $0.4576(3)$ & $0.1557(5)$ & $0.7816(4)$ & $0.032(3)$ \\
\hline $\mathrm{H} 44$ & 0.479939 & 0.136736 & 0.799669 & $0.039^{*}$ \\
\hline $\mathrm{C} 45$ & $0.4273(3)$ & $0.1200(6)$ & 0.7635 (4) & $0.035(3)$ \\
\hline H45 & 0.428322 & 0.076415 & 0.768276 & $0.042 *$ \\
\hline $\mathrm{C} 46$ & 0.3947 (3) & $0.1492(6)$ & $0.7380(4)$ & 0.035 (3) \\
\hline $\mathrm{C} 47$ & $0.3936(3)$ & $0.2125(5)$ & $0.7302(4)$ & $0.028(3)$ \\
\hline H47 & 0.371445 & 0.232429 & 0.712609 & $0.033^{*}$ \\
\hline C48 & $0.5759(3)$ & $0.3822(5)$ & 0.8353 (4) & $0.031(3)$ \\
\hline C49 & $0.5827(3)$ & $0.4438(6)$ & $0.8293(4)$ & $0.034(3)$ \\
\hline H49 & 0.563422 & 0.471465 & 0.814045 & $0.041^{*}$ \\
\hline C50 & $0.6191(3)$ & $0.4650(6)$ & $0.8465(5)$ & 0.055 (4) \\
\hline $\mathrm{H} 50$ & 0.624485 & 0.507184 & 0.842608 & $0.066^{*}$ \\
\hline C51 & 0.6465 (4) & $0.4239(6)$ & $0.8689(6)$ & 0.060 (4) \\
\hline H51 & 0.670914 & 0.437927 & 0.880239 & $0.072 *$ \\
\hline
\end{tabular}




\begin{tabular}{|c|c|c|c|c|}
\hline C52 & $0.6391(4)$ & $0.3629(7)$ & $0.8749(6)$ & $0.063(5)$ \\
\hline H52 & 0.658272 & 0.335363 & 0.890861 & $0.076^{*}$ \\
\hline $\mathrm{C} 53$ & $0.6042(3)$ & $0.3418(6)$ & $0.8581(4)$ & $0.036(3)$ \\
\hline C54 & $0.5897(3)$ & $0.2771(6)$ & $0.8609(4)$ & $0.040(3)$ \\
\hline C55 & $0.4075(3)$ & $0.3666(5)$ & 0.7903 (4) & $0.022(2)$ \\
\hline C56 & $0.3929(3)$ & $0.3280(5)$ & $0.8201(4)$ & $0.026(3)$ \\
\hline H56 & 0.395740 & 0.284313 & 0.820199 & $0.032 *$ \\
\hline $\mathrm{C} 57$ & $0.3743(3)$ & $0.3558(5)$ & $0.8488(4)$ & $0.026(2)$ \\
\hline C58 & $0.3706(3)$ & $0.4198(5)$ & $0.8511(4)$ & 0.028 \\
\hline C59 & $0.3866(3)$ & $0.4582(5)$ & $0.8253(4)$ & 0.028 \\
\hline H59 & 0.385557 & 0.501956 & 0.828033 & $0.034 *$ \\
\hline C60 & 0.4043 & $0.4309(5)$ & $0.7948(4)$ & $0.021(2)$ \\
\hline C61 & 0.4215 & $0.4666(5)$ & $0.7648(4)$ & $0.021(3)$ \\
\hline C62 & $0.4264(3)$ & $0.5304(5)$ & $0.7668(4)$ & $0.032(3)$ \\
\hline H62 & 0.416723 & 0.554598 & 0.787151 & $0.038^{*}$ \\
\hline C63 & $0.4452(3)$ & $0.5591(6)$ & $0.7395(4)$ & $0.043(3)$ \\
\hline H63 & 0.449153 & 0.602494 & 0.741357 & $0.051^{*}$ \\
\hline C64 & $0.4582(3)$ & $0.5223(6)$ & $0.7093(4)$ & $0.031(3)$ \\
\hline C65 & 0.4528 & $0.4596(6)$ & $0.7087(4)$ & $0.029(3)$ \\
\hline H65 & 0.462556 & 0.434767 & 0.688790 & $0.035^{*}$ \\
\hline C66 & $0.3560(3)$ & $0.3267(5)$ & $0.8805(4)$ & $0.025(2)$ \\
\hline C67 & $0.3531(3)$ & $0.2654(6)$ & $0.8927(4)$ & 0.035 \\
\hline H67 & 0.364012 & 0.233725 & 0.879416 & $0.042 *$ \\
\hline C68 & $0.3342(3)$ & $0.2509(6)$ & $0.9242(4)$ & 0.039 \\
\hline H68 & 0.332529 & 0.209112 & 0.933372 & $0.047^{*}$ \\
\hline C69 & 0.3175 & $0.2976(7)$ & $0.9427(5)$ & 0.048 \\
\hline H69 & 0.303643 & 0.287515 & 0.963388 & $0.058^{*}$ \\
\hline $\mathrm{C} 70$ & 0.3213 & $0.3579(6)$ & $0.9310(4)$ & $0.036(3)$ \\
\hline $\mathrm{H} 70$ & 0.310604 & 0.389505 & 0.944664 & $0.043^{*}$ \\
\hline C71 & $0.3402(3)$ & $0.3740(5)$ & $0.8999(4)$ & $0.032(3)$ \\
\hline $\mathrm{C} 72$ & $0.3479(3)$ & $0.4374(5)$ & $0.8832(4)$ & $0.035(3)$ \\
\hline $\mathrm{C} 73$ & $0.1960(3)$ & $0.2896(6)$ & $0.5167(4)$ & $0.033(3)$ \\
\hline $\mathrm{H} 73 \mathrm{C}$ & 0.170150 & 0.297601 & 0.498015 & $0.040^{*}$ \\
\hline H73D & 0.199420 & 0.244137 & 0.518381 & $0.040^{*}$ \\
\hline $\mathrm{C} 74$ & $0.2036(3)$ & $0.3139(7)$ & $0.5688(4)$ & 0.048 \\
\hline $\mathrm{H} 74 \mathrm{C}$ & 0.201648 & 0.359560 & 0.567557 & $0.057^{*}$ \\
\hline H74D & 0.228908 & 0.303286 & 0.588354 & $0.057^{*}$ \\
\hline $\mathrm{C} 75$ & $0.1782(4)$ & $0.2884(7)$ & $0.5950(5)$ & $0.072(5)$ \\
\hline $\mathrm{H} 75 \mathrm{C}$ & 0.181587 & 0.313572 & 0.624928 & $0.086^{*}$ \\
\hline H75D & 0.152960 & 0.295194 & 0.573250 & $0.086^{*}$ \\
\hline $\mathrm{C} 76$ & $0.1817(6)$ & $0.2208(8)$ & $0.6103(9)$ & $0.157(10)$ \\
\hline $\mathrm{H} 76 \mathrm{C}$ & 0.204210 & 0.214748 & 0.638329 & $0.189^{*}$ \\
\hline H76D & 0.183170 & 0.194874 & 0.582628 & $0.189 *$ \\
\hline C77 & $0.1479(5)$ & $0.2005(10)$ & $0.6255(7)$ & $0.122(8)$ \\
\hline $\mathrm{H} 77 \mathrm{C}$ & 0.125591 & 0.206671 & 0.597138 & $0.147^{*}$ \\
\hline H77D & 0.149981 & 0.155861 & 0.633496 & $0.147^{*}$ \\
\hline $\mathrm{C} 78$ & $0.1446(7)$ & $0.2367(12)$ & $0.6697(8)$ & 0.198 \\
\hline H78D & 0.123265 & 0.222216 & 0.677484 & $0.297^{*}$ \\
\hline
\end{tabular}




\begin{tabular}{|c|c|c|c|c|}
\hline $\mathrm{H} 78 \mathrm{E}$ & 0.166406 & 0.230056 & 0.698125 & $0.297 *$ \\
\hline $\mathrm{H} 78 \mathrm{~F}$ & 0.142004 & 0.280890 & 0.661750 & $0.297 *$ \\
\hline $\mathrm{C} 79$ & $0.2109(3)$ & $0.2835(6)$ & $0.4389(4)$ & $0.042(3)$ \\
\hline H79E & 0.184288 & 0.283915 & 0.422418 & $0.050 *$ \\
\hline $\mathrm{H} 79 \mathrm{~F}$ & 0.218372 & 0.239698 & 0.445514 & $0.050 *$ \\
\hline $\mathrm{C} 80$ & $0.2289(3)$ & $0.3098(6)$ & $0.4034(4)$ & $0.040(3)$ \\
\hline H80E & 0.216566 & 0.348830 & 0.389450 & $0.048^{*}$ \\
\hline $\mathrm{H} 80 \mathrm{~F}$ & 0.254458 & 0.319814 & 0.422036 & $0.048^{*}$ \\
\hline C81 & $0.2281(4)$ & $0.2663(7)$ & $0.3616(4)$ & 0.052 (4) \\
\hline $\mathrm{H} 81 \mathrm{E}$ & 0.240266 & 0.227244 & 0.375729 & $0.063 *$ \\
\hline $\mathrm{H} 81 \mathrm{~F}$ & 0.202488 & 0.256419 & 0.343096 & $0.063 *$ \\
\hline C82 & 0.2460 (4) & $0.2913(7)$ & $0.3258(4)$ & 0.054 (4) \\
\hline $\mathrm{H} 82 \mathrm{C}$ & 0.231280 & 0.326169 & 0.307432 & $0.065^{*}$ \\
\hline H82D & 0.270162 & 0.307724 & 0.344755 & $0.065^{*}$ \\
\hline $\mathrm{C} 83$ & $0.2504(5)$ & $0.2441(9)$ & $0.2894(6)$ & $0.100(7)$ \\
\hline $\mathrm{H} 83 \mathrm{C}$ & 0.226178 & 0.228285 & 0.270012 & $0.120^{*}$ \\
\hline H83D & 0.264676 & 0.208837 & 0.307788 & $0.120^{*}$ \\
\hline C84 & $0.2691(6)$ & $0.2685(10)$ & $0.2538(7)$ & $0.144(10$ \\
\hline H84D & 0.270905 & 0.235213 & 0.231572 & $0.216^{*}$ \\
\hline $\mathrm{H} 84 \mathrm{E}$ & 0.254900 & 0.302725 & 0.234568 & $0.216^{*}$ \\
\hline $\mathrm{H} 84 \mathrm{~F}$ & 0.293471 & 0.283240 & 0.272416 & $0.216^{*}$ \\
\hline $\mathrm{C} 85$ & 0.5027 (3) & $0.3980(5)$ & $0.4776(3)$ & $0.028(3)$ \\
\hline $\mathrm{H} 85 \mathrm{C}$ & 0.516771 & 0.389722 & 0.455177 & $0.034 *$ \\
\hline H85D & 0.500227 & 0.443443 & 0.479497 & $0.034 *$ \\
\hline $\mathrm{C} 86$ & $0.5242(3)$ & $0.3747(5)$ & $0.5280(4)$ & 0.034 (3) \\
\hline H86C & 0.526489 & 0.329201 & 0.526450 & $0.041 *$ \\
\hline H86D & 0.510524 & 0.383782 & 0.550800 & $0.041 *$ \\
\hline C87 & $0.5618(3)$ & $0.4023(6)$ & 0.5489 (4) & 0.038 \\
\hline $\mathrm{H} 87 \mathrm{C}$ & 0.559815 & 0.447867 & 0.546091 & $0.046^{*}$ \\
\hline H87D & 0.576577 & 0.388285 & 0.528610 & $0.046^{*}$ \\
\hline $\mathrm{C} 88$ & $0.5818(3)$ & $0.3856(6)$ & $0.6025(4)$ & 0.050 \\
\hline $\mathrm{H} 88 \mathrm{C}$ & 0.584510 & 0.340033 & 0.605265 & $0.059^{*}$ \\
\hline H88D & 0.606497 & 0.403643 & 0.612178 & $0.059^{*}$ \\
\hline C89 & $0.5631(3)$ & $0.4078(7)$ & $0.6384(4)$ & $0.054(4)$ \\
\hline $\mathrm{H} 89 \mathrm{C}$ & 0.539562 & 0.386184 & 0.631107 & $0.065^{*}$ \\
\hline H89D & 0.558119 & 0.452599 & 0.633319 & $0.065^{*}$ \\
\hline $\mathrm{C} 90$ & 0.5855 (4) & $0.3965(8)$ & $0.6920(4)$ & $0.094(6)$ \\
\hline H90D & 0.572168 & 0.411654 & 0.713345 & $0.142 *$ \\
\hline H90E & 0.590105 & 0.352071 & 0.697517 & $0.142 *$ \\
\hline $\mathrm{H} 90 \mathrm{~F}$ & 0.608673 & 0.418522 & 0.699730 & $0.142 *$ \\
\hline C91 & 0.4428 (3) & $0.4065(5)$ & $0.4086(4)$ & 0.029 (3) \\
\hline H91C & 0.442119 & 0.450476 & 0.417804 & $0.035^{*}$ \\
\hline H91D & 0.456025 & 0.404264 & 0.384476 & $0.035^{*}$ \\
\hline C92 & $0.4042(3)$ & $0.3848(5)$ & $0.3836(4)$ & $0.028(3)$ \\
\hline $\mathrm{H} 92 \mathrm{C}$ & 0.393735 & 0.371239 & 0.409003 & $0.034^{*}$ \\
\hline H92D & 0.404566 & 0.348629 & 0.362656 & $0.034^{*}$ \\
\hline C93 & $0.3797(3)$ & $0.4353(6)$ & $0.3517(4)$ & $0.036(3)$ \\
\hline $\mathrm{H} 93 \mathrm{C}$ & 0.379264 & 0.471119 & 0.372911 & $0.043 *$ \\
\hline
\end{tabular}




\begin{tabular}{|c|c|c|c|c|c|}
\hline H93D & 0.390644 & 0.449337 & 0.326877 & $0.043^{*}$ & \\
\hline C94 & $0.3420(3)$ & $0.4155(6)$ & $0.3261(4)$ & $0.044(3)$ & \\
\hline H94C & 0.331507 & 0.397733 & 0.350161 & $0.053 *$ & \\
\hline H94D & 0.341898 & 0.383016 & 0.301769 & $0.053 *$ & \\
\hline C95 & 0.3189 (4) & $0.4700(8)$ & $0.2999(6)$ & $0.084(6)$ & \\
\hline $\mathrm{H} 95 \mathrm{C}$ & 0.328718 & 0.485478 & 0.274350 & $0.101 *$ & \\
\hline H95D & 0.321233 & 0.503772 & 0.323999 & $0.101 *$ & \\
\hline C96 & $0.2786(4)$ & $0.4556(9)$ & $0.2756(6)$ & $0.109(7)$ & \\
\hline H96D & 0.265883 & 0.493027 & 0.259785 & $0.163^{*}$ & \\
\hline H96E & 0.275786 & 0.423128 & 0.250898 & $0.163^{*}$ & \\
\hline $\mathrm{H} 96 \mathrm{~F}$ & 0.268285 & 0.441462 & 0.300652 & $0.163 *$ & \\
\hline C97 & $0.6066(4)$ & $0.2289(6)$ & $0.8358(4)$ & $0.055(4)$ & \\
\hline H97C & 0.632833 & 0.226021 & 0.854616 & $0.067 *$ & \\
\hline H97D & 0.595695 & 0.188182 & 0.838195 & $0.067^{*}$ & \\
\hline C98 & $0.6027(3)$ & $0.2405(6)$ & $0.7820(5)$ & $0.056(4)$ & \\
\hline H98C & 0.610266 & 0.283276 & 0.777728 & $0.067 *$ & \\
\hline H98D & 0.577021 & 0.235324 & 0.761252 & $0.067^{*}$ & \\
\hline C99 & $0.6267(4)$ & $0.1938(7)$ & $0.7668(5)$ & $0.082(5)$ & \\
\hline H99C & 0.652385 & 0.202749 & 0.785456 & $0.099 *$ & \\
\hline H99D & 0.621149 & 0.151795 & 0.776016 & $0.099 *$ & \\
\hline $\mathrm{C} 100$ & $0.6220(5)$ & $0.1945(8)$ & $0.7137(5)$ & $0.097(6)$ & \\
\hline $\mathrm{H} 10 \mathrm{C}$ & 0.627966 & 0.236303 & 0.704412 & $0.116^{*}$ & 0.33 \\
\hline H10D & 0.596320 & 0.185974 & 0.694858 & $0.116^{*}$ & 0.33 \\
\hline $\mathrm{H} 10 \mathrm{~A}$ & 0.622913 & 0.237833 & 0.702924 & $0.116^{*}$ & 0.67 \\
\hline H10B & 0.597879 & 0.177499 & 0.695209 & $0.116^{*}$ & 0.67 \\
\hline C103 & $0.5956(4)$ & $0.2555(6)$ & $0.9141(4)$ & $0.052(4)$ & \\
\hline $\mathrm{H} 10 \mathrm{E}$ & 0.583165 & 0.215212 & 0.912559 & $0.063^{*}$ & \\
\hline $\mathrm{H} 10 \mathrm{~F}$ & 0.621831 & 0.248223 & 0.930167 & $0.063^{*}$ & \\
\hline C104 & 0.5826 & $0.2984(6)$ & $0.9466(4)$ & $0.046(4)$ & \\
\hline H10G & 0.558503 & 0.314314 & 0.926592 & $0.056^{*}$ & 0.67 \\
\hline $\mathrm{H} 10 \mathrm{H}$ & 0.599279 & 0.334293 & 0.954575 & $0.056^{*}$ & 0.67 \\
\hline H10I & 0.555942 & 0.303870 & 0.934343 & $0.056^{*}$ & 0.33 \\
\hline $\mathrm{H} 10 \mathrm{~J}$ & 0.594617 & 0.339326 & 0.950407 & $0.056^{*}$ & 0.33 \\
\hline C109 & $0.3133(3)$ & $0.4708(6)$ & $0.8524(4)$ & $0.042(3)$ & \\
\hline $\mathrm{H} 10 \mathrm{~K}$ & 0.298911 & 0.481493 & 0.873958 & $0.050^{*}$ & \\
\hline H10L & 0.320306 & 0.510050 & 0.840095 & $0.050^{*}$ & \\
\hline C110 & $0.2896(3)$ & $0.4341(6)$ & $0.8089(4)$ & $0.061(4)$ & \\
\hline H11A & 0.280861 & 0.396325 & 0.821047 & $0.073 *$ & \\
\hline H11B & 0.304155 & 0.421086 & 0.788155 & $0.073 *$ & \\
\hline C111 & $0.2567(4)$ & $0.4718(8)$ & $0.7776(6)$ & $0.118(8)$ & \\
\hline $\mathrm{H} 11 \mathrm{C}$ & 0.245933 & 0.492981 & 0.800012 & $0.142^{*}$ & \\
\hline H11D & 0.265614 & 0.504174 & 0.759984 & $0.142 *$ & \\
\hline $\mathrm{C} 112$ & $0.2267(6)$ & $0.4354(13)$ & $0.7397(8)$ & $0.210(13)$ & \\
\hline H11E & 0.205597 & 0.462628 & 0.724495 & $0.252 *$ & \\
\hline $\mathrm{H} 11 \mathrm{~F}$ & 0.218583 & 0.400530 & 0.755902 & $0.252 *$ & \\
\hline C113 & $0.2417(6)$ & $0.4106(12)$ & $0.7000(9)$ & $0.230(13)$ & \\
\hline $\mathrm{H} 11 \mathrm{G}$ & 0.223062 & 0.383475 & 0.677653 & $0.275^{*}$ & \\
\hline $\mathrm{H} 11 \mathrm{H}$ & 0.263079 & 0.384456 & 0.716279 & $0.275^{*}$ & \\
\hline
\end{tabular}




\begin{tabular}{|c|c|c|c|c|c|}
\hline $\mathrm{C} 114$ & $0.2530(7)$ & $0.4593(14)$ & $0.6684(11)$ & $0.291(16)$ & \\
\hline H11I & 0.262070 & 0.438292 & 0.644490 & $0.437^{*}$ & \\
\hline H11J & 0.231935 & 0.484719 & 0.650897 & $0.437^{*}$ & \\
\hline $\mathrm{H} 11 \mathrm{~K}$ & 0.272089 & 0.485704 & 0.689657 & $0.437^{*}$ & \\
\hline C115 & 0.3677 (3) & $0.4801(5)$ & $0.9259(4)$ & $0.037(3)$ & \\
\hline H11L & 0.373074 & 0.519351 & 0.911854 & $0.045^{*}$ & \\
\hline $\mathrm{H} 11 \mathrm{M}$ & 0.350874 & 0.490022 & 0.944233 & $0.045^{*}$ & \\
\hline C116 & $0.4026(3)$ & $0.4568(5)$ & $0.9622(4)$ & $0.036(3)$ & \\
\hline $\mathrm{H} 11 \mathrm{~N}$ & 0.397506 & 0.417836 & 0.976914 & $0.043^{*}$ & \\
\hline $\mathrm{H} 11 \mathrm{O}$ & 0.419741 & 0.446949 & 0.944320 & $0.043^{*}$ & \\
\hline C117 & $0.4207(3)$ & $0.5014(6)$ & $1.0031(4)$ & $0.048(4)$ & \\
\hline H11P & 0.404597 & 0.507529 & 1.023229 & $0.058^{*}$ & \\
\hline H11Q & 0.423133 & 0.541867 & 0.988341 & $0.058^{*}$ & \\
\hline C118 & 0.4577 (3) & $0.4818(5)$ & $1.0367(4)$ & $0.038(3)$ & \\
\hline H11R & 0.473739 & 0.474366 & 1.016740 & $0.046^{*}$ & \\
\hline H11S & 0.455283 & 0.442278 & 1.052824 & $0.046^{*}$ & \\
\hline C119 & $0.4751(3)$ & $0.5294(6)$ & $1.0758(4)$ & $0.057(4)$ & \\
\hline $\mathrm{H} 11 \mathrm{~T}$ & 0.474975 & 0.569891 & 1.059664 & $0.068^{*}$ & \\
\hline H11U & 0.460067 & 0.533681 & 1.097612 & $0.068^{*}$ & \\
\hline $\mathrm{C} 120$ & $0.5134(3)$ & $0.5145(6)$ & 1.1069 (4) & $0.055(4)$ & \\
\hline $\mathrm{H} 12 \mathrm{~A}$ & 0.522671 & 0.547401 & 1.131227 & $0.082^{*}$ & \\
\hline H12B & 0.513773 & 0.475080 & 1.123905 & $0.082 *$ & \\
\hline $\mathrm{H} 12 \mathrm{C}$ & 0.528705 & 0.511351 & 1.085893 & $0.082 *$ & \\
\hline C121 & 0.4607 (3) & $0.1149(6)$ & $0.6556(5)$ & $0.047(3)$ & \\
\hline H12D & 0.461178 & 0.137570 & 0.685346 & $0.071^{*}$ & \\
\hline $\mathrm{H} 12 \mathrm{E}$ & 0.468319 & 0.142498 & 0.633721 & $0.071^{*}$ & \\
\hline $\mathrm{H} 12 \mathrm{~F}$ & 0.477357 & 0.079633 & 0.664707 & $0.071^{*}$ & \\
\hline $\mathrm{C} 122$ & 0.3334 (3) & $0.5470(6)$ & $0.6746(5)$ & $0.049(4)$ & \\
\hline $\mathrm{H} 12 \mathrm{G}$ & 0.348528 & 0.522124 & 0.702108 & $0.074^{*}$ & \\
\hline $\mathrm{H} 12 \mathrm{H}$ & 0.314619 & 0.520684 & 0.652488 & $0.074^{*}$ & \\
\hline H12I & 0.321831 & 0.580353 & 0.687276 & $0.074^{*}$ & \\
\hline $\mathrm{C} 123$ & 0.3310 (4) & $0.1408(7)$ & $0.6956(5)$ & $0.056(4)$ & \\
\hline H12J & 0.324223 & 0.166598 & 0.719353 & $0.083^{*}$ & \\
\hline $\mathrm{H} 12 \mathrm{~K}$ & 0.312306 & 0.109282 & 0.682379 & $0.083^{*}$ & \\
\hline $\mathrm{H} 12 \mathrm{~L}$ & 0.333081 & 0.166817 & 0.668770 & $0.083^{*}$ & \\
\hline C124 & 0.4945 (4) & $0.5122(6)$ & 0.6565 (4) & $0.049(4)$ & \\
\hline $\mathrm{H} 12 \mathrm{M}$ & 0.511817 & 0.484933 & 0.679973 & $0.074 *$ & \\
\hline $\mathrm{H} 12 \mathrm{~N}$ & 0.507490 & 0.538027 & 0.639688 & $0.074^{*}$ & \\
\hline $\mathrm{H} 12 \mathrm{O}$ & 0.476363 & 0.487119 & 0.632333 & $0.074 *$ & \\
\hline C101 & $0.6512(7)$ & $0.1569(16)$ & $0.7016(10)$ & $0.083(7)^{*}$ & 0.67 \\
\hline $\mathrm{H} 10 \mathrm{M}$ & 0.674900 & 0.177415 & 0.716952 & $0.099 *$ & 0.67 \\
\hline $\mathrm{H} 10 \mathrm{~N}$ & 0.652503 & 0.115522 & 0.716800 & $0.099 *$ & 0.67 \\
\hline $\mathrm{C} 102$ & $0.6455(8)$ & $0.1487(13)$ & $0.6477(8)$ & 0.107 (11) & 0.67 \\
\hline $\mathrm{H} 10 \mathrm{O}$ & 0.665416 & 0.124107 & 0.643460 & $0.161^{*}$ & 0.67 \\
\hline H10P & 0.622452 & 0.127252 & 0.632116 & $0.161^{*}$ & 0.67 \\
\hline H10Q & 0.644899 & 0.189286 & 0.632269 & $0.161^{*}$ & 0.67 \\
\hline $\mathrm{C} 105$ & $0.5792(6)$ & $0.2745(9)$ & $0.9948(6)$ & $0.052(6)$ & 0.67 \\
\hline H10R & 0.603385 & 0.260907 & 1.016175 & $0.062^{*}$ & 0.67 \\
\hline
\end{tabular}




\begin{tabular}{|c|c|c|c|c|c|}
\hline H10S & 0.563124 & 0.237767 & 0.987618 & $0.062 *$ & 0.67 \\
\hline C106 & $0.5642(8)$ & $0.3214(8)$ & $1.0229(7)$ & $0.050(6)$ & 0.67 \\
\hline H10T & 0.541035 & 0.337942 & 1.000563 & $0.060 *$ & 0.67 \\
\hline H10U & 0.581436 & 0.356353 & 1.032884 & $0.060 *$ & 0.67 \\
\hline C107 & $0.5576(8)$ & $0.2953(10)$ & $1.0688(8)$ & $0.100(11)$ & 0.67 \\
\hline H10V & 0.580955 & 0.281672 & 1.092523 & $0.120^{*}$ & 0.67 \\
\hline H10W & 0.541539 & 0.258726 & 1.059487 & $0.120^{*}$ & 0.67 \\
\hline C108 & $0.5401(8)$ & $0.3432(13)$ & $1.0936(10)$ & $0.101(9)^{*}$ & 0.67 \\
\hline $\mathrm{H} 10 \mathrm{X}$ & 0.536352 & 0.324727 & 1.122721 & $0.151 *$ & 0.67 \\
\hline $\mathrm{H} 10 \mathrm{Y}$ & 0.556119 & 0.379209 & 1.103523 & $0.151 *$ & 0.67 \\
\hline $\mathrm{H}$ & 0.516712 & 0.356269 & 1.070495 & $0.151^{*}$ & 0.67 \\
\hline $\mathrm{C} 10 \mathrm{~A}$ & $0.5952(14)$ & $0.260(2)$ & $0.9941(11)$ & $0.055(16)^{*}$ & 0.33 \\
\hline H10Z & 0.583319 & 0.219343 & 0.988111 & $0.066^{*}$ & 0.33 \\
\hline HA & 0.621712 & 0.253841 & 1.003701 & $0.066^{*}$ & 0.33 \\
\hline $\mathrm{C} 10 \mathrm{~B}$ & $0.5864(10)$ & $0.292(3)$ & $1.0362(16)$ & $0.075(17)^{*}$ & 0.33 \\
\hline H10 & 0.601464 & 0.329661 & 1.045261 & $0.090 *$ & 0.33 \\
\hline HB & 0.593842 & 0.263848 & 1.064987 & $0.090 *$ & 0.33 \\
\hline $\mathrm{C} 10 \mathrm{C}$ & $0.5466(10)$ & $0.310(2)$ & $1.0268(17)$ & $0.052(15)^{*}$ & 0.33 \\
\hline $\mathrm{H} 7$ & 0.539043 & 0.334868 & 0.996126 & $0.062 *$ & 0.33 \\
\hline $\mathrm{HC}$ & 0.532278 & 0.271614 & 1.019867 & $0.062^{*}$ & 0.33 \\
\hline C10D & $0.5346(17)$ & $0.346(3)$ & 1.0646 (19) & $0.101(9)^{*}$ & 0.33 \\
\hline H12 & 0.508353 & 0.353388 & 1.051711 & $0.151^{*}$ & 0.33 \\
\hline HD & 0.540420 & 0.322377 & 1.095181 & $0.151^{*}$ & 0.33 \\
\hline $\mathrm{HE}$ & 0.547237 & 0.386121 & 1.071257 & $0.151^{*}$ & 0.33 \\
\hline C125 & $0.6868(12)$ & $0.145(3)$ & $0.714(2)$ & $0.10(2)^{*}$ & 0.33 \\
\hline $\mathrm{H} 12 \mathrm{P}$ & 0.694063 & 0.108423 & 0.699209 & $0.155^{*}$ & 0.33 \\
\hline H12Q & 0.695513 & 0.182350 & 0.702221 & $0.155^{*}$ & 0.33 \\
\hline $\mathrm{H} 12 \mathrm{R}$ & 0.697399 & 0.142506 & 0.749889 & $0.155^{*}$ & 0.33 \\
\hline C126 & $0.6460(13)$ & $0.147(3)$ & $0.700(2)$ & $0.083(7)^{*}$ & 0.33 \\
\hline $\mathrm{H} 12 \mathrm{~S}$ & 0.639028 & 0.106706 & 0.710476 & $0.099 *$ & 0.33 \\
\hline $\mathrm{H} 12 \mathrm{~T}$ & 0.637166 & 0.146041 & 0.663417 & $0.099 *$ & 0.33 \\
\hline Ir3 & $0.09363(2)$ & $0.64428(3)$ & $0.16694(2)$ & $0.04359(17)$ & \\
\hline $\operatorname{Ir} 4$ & $0.13022(2)$ & $0.60856(3)$ & $0.28941(2)$ & $0.04372(17)$ & \\
\hline $\mathrm{O} 1^{\prime}$ & $0.2040(4)$ & $0.6189(11)$ & $0.2151(6)$ & $0.212(10)$ & \\
\hline $\mathrm{O} 2^{\prime}$ & 0.0184 (3) & $0.6253(6)$ & $0.2385(4)$ & 0.098 (4) & \\
\hline O3' & $0.0587(3)$ & $0.3980(5)$ & $0.1665(4)$ & $0.073(3)$ & \\
\hline $\mathrm{O} 4^{\prime}$ & 0.1683 (3) & $0.8598(6)$ & $0.2213(4)$ & 0.097 (4) & \\
\hline $\mathrm{O}^{\prime}$ & $0.1744(3)$ & $0.3953(6)$ & $0.2268(4)$ & 0.088 (4) & \\
\hline O6' & $0.0751(3)$ & $0.8402(5)$ & $0.2827(4)$ & $0.081(4)$ & \\
\hline N01' & $0.1484(3)$ & $0.6288(5)$ & 0.2228 & $0.042(3)$ & \\
\hline $\mathrm{N} 1^{\prime}$ & $0.0876(3)$ & $0.5542(5)$ & $0.1441(3)$ & $0.043(3)$ & \\
\hline $\mathrm{N} 2^{\prime}$ & 0.0994 (3) & $0.7329(6)$ & $0.1783(3)$ & $0.054(3)$ & \\
\hline N02' & $0.0771(3)$ & $0.6190(5)$ & $0.2328(3)$ & $0.043(3)$ & \\
\hline N3' & 0.1339 (3) & $0.5140(5)$ & $0.2814(4)$ & $0.048(3)$ & \\
\hline N4' & 0.1317 (3) & $0.6942(5)$ & $0.3046(3)$ & $0.046(2)$ & \\
\hline $\mathrm{C} 01^{\prime}$ & $0.1748(4)$ & $0.6244(9)$ & $0.2196(6)$ & $0.084(6)$ & \\
\hline $\mathrm{C} 1^{\prime}$ & $0.1104(3)$ & $0.6535(6)$ & $0.1081(4)$ & $0.041(3)$ & \\
\hline $\mathrm{C} 02^{\prime}$ & 0.0479 (4) & $0.6216(8)$ & $0.2351(5)$ & $0.065(5)$ & \\
\hline
\end{tabular}




\begin{tabular}{|c|c|c|c|c|}
\hline $\mathrm{C} 2^{\prime}$ & 0.1221 & $0.7059(6)$ & 0.0894 & 0.040 \\
\hline $\mathrm{H} 2^{\prime}$ & 0.121706 & 0.744816 & 0.104569 & $0.048^{*}$ \\
\hline $\mathrm{C} 3^{\prime}$ & $0.1340(3)$ & $0.7028(6)$ & $0.0502(4)$ & $0.038(3)$ \\
\hline $\mathrm{C} 4^{\prime}$ & $0.1372(4)$ & $0.6457(7)$ & $0.0292(5)$ & $0.052(4)$ \\
\hline $\mathrm{C} 5^{\prime}$ & $0.1262(4)$ & $0.5920(6)$ & $0.0456(4)$ & $0.050(4)$ \\
\hline $\mathrm{H} 5^{\prime}$ & 0.126855 & 0.553599 & 0.029785 & $0.060^{*}$ \\
\hline $\mathrm{C} 6^{\prime}$ & 0.1138 (3) & $0.5954(6)$ & $0.0869(5)$ & $0.043(3)$ \\
\hline $\mathrm{C} 7^{\prime}$ & $0.1005(3)$ & $0.5421(6)$ & $0.1062(4)$ & $0.041(3)$ \\
\hline $\mathrm{C} 8^{\prime}$ & 0.0984 (4) & $0.4825(7)$ & $0.0881(5)$ & $0.057(4)$ \\
\hline $\mathrm{H} 8^{\prime}$ & 0.106535 & 0.474131 & 0.060931 & $0.068^{*}$ \\
\hline $\mathrm{C} 9^{\prime}$ & 0.0847 (4) & $0.4351(7)$ & $0.1086(5)$ & $0.058(4)$ \\
\hline H9' & 0.084220 & 0.393902 & 0.096907 & $0.070^{*}$ \\
\hline $\mathrm{C} 10^{\prime}$ & 0.0715 & $0.4489(7)$ & $0.1474(5)$ & $0.056(4)$ \\
\hline $\mathrm{C} 11^{\prime}$ & 0.0739 (3) & $0.5091(7)$ & 0.1645 (4) & $0.050(4)$ \\
\hline H11' & 0.065695 & 0.518575 & 0.191378 & $0.060 *$ \\
\hline $\mathrm{C} 12^{\prime}$ & $0.1474(3)$ & $0.7518(6)$ & 0.0250 & 0.038 \\
\hline $\mathrm{C} 13^{\prime}$ & $0.1492(3)$ & $0.8147(6)$ & 0.0308 (4) & $0.043(3)$ \\
\hline H13' & 0.143303 & 0.833652 & 0.057058 & $0.052 *$ \\
\hline $\mathrm{C} 14^{\prime}$ & $0.1601(3)$ & $0.8510(7)$ & $-0.0026(4)$ & $0.044(3)$ \\
\hline H14' & 0.159504 & 0.894812 & -0.000737 & $0.053^{*}$ \\
\hline $\mathrm{C} 15^{\prime}$ & $0.1716(4)$ & $0.8237(7)$ & $-0.0377(5)$ & $0.058(4)$ \\
\hline H15' & 0.182010 & 0.848220 & -0.057085 & $0.070 *$ \\
\hline $\mathrm{C} 16^{\prime}$ & $0.1682(4)$ & $0.7618(7)$ & $-0.0452(5)$ & $0.055(4)$ \\
\hline H16' & 0.173045 & 0.744094 & -0.072676 & $0.066^{*}$ \\
\hline $\mathrm{C} 17^{\prime}$ & 0.1577 (4) & $0.7241(7)$ & $-0.0132(5)$ & 0.050 \\
\hline C18' & $0.1512(4)$ & $0.6538(7)$ & $-0.0148(5)$ & $0.055(3)$ \\
\hline $\mathrm{C} 19^{\prime}$ & $0.0431(3)$ & $0.6709(6)$ & $0.1287(4)$ & $0.036(3)^{*}$ \\
\hline $\mathrm{C} 20^{\prime}$ & $0.0135(4)$ & $0.6339(6)$ & 0.1067 (4) & $0.043(3)$ \\
\hline $\mathrm{H} 20^{\prime}$ & 0.015730 & 0.590199 & 0.105674 & $0.052 *$ \\
\hline $\mathrm{C} 21^{\prime}$ & $-0.0204(4)$ & $0.6635(7)$ & $0.0857(5)$ & 0.049 (3) \\
\hline $\mathrm{C} 22^{\prime}$ & $-0.0257(4)$ & $0.7259(7)$ & $0.0856(5)$ & $0.047(3)$ \\
\hline $\mathrm{C} 23^{\prime}$ & $0.0046(4)$ & $0.7628(6)$ & $0.1074(5)$ & $0.048(3)$ \\
\hline $\mathrm{H} 23^{\prime}$ & 0.002183 & 0.806579 & 0.107053 & $0.058^{*}$ \\
\hline C24' & 0.0384 (4) & $0.7358(7)$ & 0.1298 (4) & $0.046(3)$ \\
\hline $\mathrm{C} 25^{\prime}$ & 0.0707 (4) & $0.7708(7)$ & $0.1550(5)$ & $0.049(3)$ \\
\hline $\mathrm{C} 26^{\prime}$ & 0.0727 (4) & $0.8367(8)$ & $0.1547(5)$ & $0.065(4)$ \\
\hline $\mathrm{H} 26^{\prime}$ & 0.051592 & 0.860560 & 0.139199 & $0.078^{*}$ \\
\hline C27' & 0.1067 (5) & $0.8665(8)$ & 0.1779 (5) & 0.069 (4) \\
\hline $\mathrm{H} 27^{\prime}$ & 0.109255 & 0.910201 & 0.178406 & $0.083^{*}$ \\
\hline $\mathrm{C} 28^{\prime}$ & 0.1359 (4) & $0.8270(9)$ & $0.2000(5)$ & $0.064(4)$ \\
\hline $\mathrm{C} 29^{\prime}$ & 0.1319 (4) & $0.7650(8)$ & $0.2005(5)$ & $0.065(4)$ \\
\hline $\mathrm{H} 29^{\prime}$ & 0.152711 & 0.741243 & 0.217261 & $0.078^{*}$ \\
\hline $\mathrm{C} 30^{\prime}$ & $-0.0558(4)$ & $0.6327(7)$ & $0.0623(5)$ & $0.050(3)$ \\
\hline $\mathrm{C} 31^{\prime}$ & $-0.0676(4)$ & $0.5709(7)$ & $0.0559(5)$ & $0.052(4)$ \\
\hline $\mathrm{H} 31^{\prime}$ & -0.050438 & 0.538236 & 0.065771 & $0.062 *$ \\
\hline C32' & -0.1037 (4) & $0.5576(7)$ & $0.0356(5)$ & $0.062(4)$ \\
\hline $\mathrm{H} 32^{\prime}$ & -0.111650 & 0.515859 & 0.033242 & $0.075^{*}$ \\
\hline C33' & -0.1283 & $0.6039(7)$ & $0.0187(6)$ & $0.068(5)$ \\
\hline
\end{tabular}




\begin{tabular}{|c|c|c|c|c|}
\hline H33' & -0.152979 & 0.593490 & 0.002664 & $0.081^{*}$ \\
\hline C34' & -0.1184 & $0.6650(7)$ & $0.0241(5)$ & 0.058 \\
\hline H34' & -0.136183 & 0.696237 & 0.011861 & $0.070 *$ \\
\hline $\mathrm{C} 35^{\prime}$ & $-0.0828(4)$ & $0.6813(7)$ & $0.0471(5)$ & 0.054 \\
\hline $\mathrm{C} 36^{\prime}$ & -0.0652 & $0.7432(7)$ & $0.0602(5)$ & 0.053 \\
\hline $\mathrm{C} 37^{\prime}$ & $0.1097(3)$ & $0.5805(6)$ & $0.3408(4)$ & 0.041 \\
\hline C $38^{\prime}$ & $0.0962(4)$ & $0.6175(7)$ & $0.3724(4)$ & $0.047(3)$ \\
\hline $\mathrm{H} 38^{\prime}$ & 0.098965 & 0.661171 & 0.373480 & $0.057^{*}$ \\
\hline $\mathrm{C} 39^{\prime}$ & $0.0790(3)$ & $0.5875(6)$ & $0.4016(4)$ & 0.036 \\
\hline $\mathrm{C} 40^{\prime}$ & $0.0744(3)$ & $0.5241(6)$ & $0.4016(4)$ & 0.040 \\
\hline $\mathrm{C} 41^{\prime}$ & $0.0883(3)$ & $0.4869(6)$ & $0.3731(5)$ & 0.046 \\
\hline $\mathrm{H} 41^{\prime}$ & 0.085680 & 0.443254 & 0.373605 & $0.055^{*}$ \\
\hline C42' & 0.1066 (4) & $0.5148(6)$ & $0.3426(5)$ & 0.044 \\
\hline C43' & $0.1226(3)$ & $0.4799(7)$ & $0.3120(4)$ & 0.045 \\
\hline C44' & $0.1285(4)$ & $0.4153(7)$ & $0.3148(5)$ & 0.062 \\
\hline $\mathrm{H} 44^{\prime}$ & 0.120651 & 0.390550 & 0.336739 & $0.074 *$ \\
\hline C45' & 0.1461 (4) & $0.3887(8)$ & $0.2848(6)$ & 0.071 \\
\hline $\mathrm{H} 45^{\prime}$ & 0.150110 & 0.345344 & 0.285295 & $0.085^{*}$ \\
\hline $\mathrm{C} 46^{\prime}$ & $0.1577(4)$ & $0.4264(9)$ & $0.2542(6)$ & 0.069 \\
\hline $\mathrm{C} 47^{\prime}$ & $0.1511(3)$ & $0.4868(7)$ & $0.2524(5)$ & 0.053 \\
\hline H47' & 0.158646 & 0.511731 & 0.230323 & $0.064^{*}$ \\
\hline C48' & 0.0619 & $0.6140(6)$ & $0.4360(5)$ & 0.038 \\
\hline $\mathrm{C} 49^{\prime}$ & $0.0622(3)$ & $0.6745(6)$ & $0.4526(4)$ & 0.038 \\
\hline $\mathrm{H} 49^{\prime}$ & 0.072829 & 0.706994 & 0.439653 & $0.045^{*}$ \\
\hline $\mathrm{C} 50^{\prime}$ & $0.0460(4)$ & $0.6862(6)$ & $0.4900(5)$ & $0.047(3)$ \\
\hline $\mathrm{H} 50^{\prime}$ & 0.046531 & 0.726655 & 0.503156 & $0.056^{*}$ \\
\hline $\mathrm{C} 51^{\prime}$ & $0.0302(3)$ & $0.6402(7)$ & $0.5061(5)$ & 0.045 \\
\hline H51' & 0.019064 & 0.649182 & 0.530251 & $0.055^{*}$ \\
\hline C52' & $0.0290(4)$ & $0.5793(7)$ & $0.4895(5)$ & 0.051 \\
\hline H52' & 0.017332 & 0.547509 & 0.501442 & $0.061 *$ \\
\hline C53' & $0.0457(3)$ & $0.5676(6)$ & $0.4547(5)$ & 0.044 \\
\hline C5 $54^{\prime}$ & $0.0515(4)$ & $0.5045(7)$ & $0.4334(5)$ & 0.056 \\
\hline C55' & $0.1810(3)$ & $0.6147(6)$ & $0.3346(4)$ & 0.032 \\
\hline C56' & $0.2065(3)$ & $0.5677(6)$ & $0.3539(4)$ & 0.033 \\
\hline H56 & 0.199440 & 0.525536 & 0.348596 & $0.040^{*}$ \\
\hline $\mathrm{C} 57^{\prime}$ & $0.2419(3)$ & $0.5826(6)$ & $0.3808(4)$ & 0.033 \\
\hline C58' & $0.2535(4)$ & $0.6437(6)$ & $0.3899(4)$ & 0.036 \\
\hline $\mathrm{C} 59^{\prime}$ & 0.2285 & $0.6912(6)$ & $0.3730(4)$ & 0.034 \\
\hline H59' & 0.235776 & 0.733131 & 0.379816 & $0.040^{*}$ \\
\hline $\mathrm{C} 60^{\prime}$ & 0.1925 (4) & $0.6767(6)$ & $0.3457(4)$ & 0.041 \\
\hline C61' & 0.1635 & $0.7237(7)$ & $0.3287(4)$ & 0.041 \\
\hline C62' & $0.1677(4)$ & $0.7873(7)$ & $0.3350(5)$ & 0.053 \\
\hline H62' & 0.191123 & 0.804698 & 0.349736 & $0.063 *$ \\
\hline C63' & $0.1370(4)$ & $0.8256(7)$ & $0.3194(4)$ & 0.052 \\
\hline H63' & 0.139532 & 0.869191 & 0.323494 & $0.063^{*}$ \\
\hline C64' & $0.1051(4)$ & $0.8011(8)$ & $0.2991(5)$ & 0.054 \\
\hline $\mathrm{C} 65^{\prime}$ & $0.1021(4)$ & $0.7383(7)$ & $0.2906(5)$ & 0.055 \\
\hline H65' & 0.078498 & 0.722484 & 0.274142 & $0.066^{*}$ \\
\hline
\end{tabular}




\begin{tabular}{|c|c|c|c|c|c|}
\hline C66' & $0.2727(3)$ & $0.5424(6)$ & $0.4035(4)$ & $0.037(3)$ & \\
\hline C67' & $0.2754(3)$ & $0.4781(6)$ & $0.4058(4)$ & $0.037(3)$ & \\
\hline H67' & 0.254728 & 0.453062 & 0.390844 & $0.044^{*}$ & \\
\hline C68' & $0.3084(3)$ & $0.4514(6)$ & $0.4300(4)$ & $0.043(3)$ & \\
\hline H68' & 0.310106 & 0.407640 & 0.432985 & $0.051^{*}$ & \\
\hline C69' & $0.3392(4)$ & $0.4872(7)$ & $0.4501(5)$ & $0.052(4)$ & \\
\hline H69' & 0.361923 & 0.467683 & 0.465394 & $0.063^{*}$ & \\
\hline $\mathrm{C} 70^{\prime}$ & $0.3372(4)$ & $0.5515(7)$ & $0.4480(5)$ & $0.050(3)$ & \\
\hline $\mathrm{H} 70^{\prime}$ & 0.358217 & 0.576119 & 0.462390 & $0.059^{*}$ & \\
\hline C71' & $0.3040(3)$ & $0.5788(6)$ & $0.4246(5)$ & $0.041(3)$ & \\
\hline $\mathrm{C} 72^{\prime}$ & $0.2942(4)$ & $0.6467(7)$ & $0.4168(5)$ & $0.049(3)$ & \\
\hline C73' & $0.1214(4)$ & $0.6329(7)$ & $-0.0652(5)$ & $0.058(4)$ & \\
\hline H73A & 0.132400 & 0.635029 & -0.091694 & $0.070 *$ & \\
\hline H73B & 0.114966 & 0.589199 & -0.061993 & $0.070 *$ & \\
\hline C74' & $0.0876(4)$ & $0.6701(7)$ & $-0.0803(5)$ & $0.071(4)$ & \\
\hline H74A & 0.091132 & 0.704897 & -0.100566 & $0.085 *$ & \\
\hline H74B & 0.084606 & 0.688158 & -0.050171 & $0.085^{*}$ & \\
\hline $\mathrm{C} 75^{\prime}$ & $0.0520(5)$ & $0.6383(9)$ & $-0.1087(6)$ & $0.102(6)$ & \\
\hline $\mathrm{H} 75 \mathrm{~A}$ & 0.033153 & 0.670464 & -0.121181 & $0.122 *$ & \\
\hline H75B & 0.055200 & 0.617100 & -0.137501 & $0.122 *$ & \\
\hline $\mathrm{C} 76^{\prime}$ & $0.0386(4)$ & $0.5922(8)$ & $-0.0797(6)$ & $0.073(5)$ & \\
\hline H76A & 0.059662 & 0.569354 & -0.057669 & $0.088 *$ & \\
\hline H76B & 0.022797 & 0.561957 & -0.102629 & $0.088^{*}$ & \\
\hline C77' & $0.0178(5)$ & $0.6212(9)$ & $-0.0492(6)$ & $0.090(6)$ & \\
\hline H77A & 0.032671 & 0.654120 & -0.028109 & $0.108^{*}$ & \\
\hline H77B & -0.004689 & 0.640419 & -0.071109 & $0.108^{*}$ & \\
\hline $\mathrm{C} 78^{\prime}$ & $0.0085(4)$ & $0.5737(9)$ & $-0.0178(6)$ & $0.099(7)$ & \\
\hline H78A & -0.005023 & 0.593208 & 0.001703 & $0.148 *$ & \\
\hline H78B & -0.006537 & 0.541521 & -0.038757 & $0.148^{*}$ & \\
\hline $\mathrm{H} 78 \mathrm{C}$ & 0.030761 & 0.555200 & 0.004172 & $0.148^{*}$ & \\
\hline C79' & $0.1849(4)$ & $0.6186(8)$ & $-0.0091(6)$ & $0.083(5)$ & \\
\hline H79C & 0.196653 & 0.638508 & -0.030993 & $0.100 *$ & 0.4 \\
\hline H79D & 0.177281 & 0.576875 & -0.022715 & $0.100 *$ & 0.4 \\
\hline H79A & 0.190294 & 0.620036 & -0.040406 & $0.100 *$ & 0.6 \\
\hline H79B & 0.181051 & 0.574808 & -0.002029 & $0.100 *$ & 0.6 \\
\hline C81' & $0.2500(7)$ & $0.6057(14)$ & 0.0270 & $0.239(16)^{*}$ & \\
\hline $\mathrm{H} 81 \mathrm{C}$ & 0.253887 & 0.560375 & 0.027794 & $0.287^{*}$ & 0.4 \\
\hline H81D & 0.268015 & 0.620849 & 0.057723 & $0.287^{*}$ & 0.4 \\
\hline H81A & 0.241987 & 0.562203 & 0.019875 & $0.287^{*}$ & 0.6 \\
\hline H81B & 0.268868 & 0.606034 & 0.059756 & $0.287^{*}$ & 0.6 \\
\hline $\mathrm{C} 82^{\prime}$ & $0.2690(8)$ & $0.6260(16)$ & $-0.0126(12)$ & $0.268(18)^{*}$ & \\
\hline $\mathrm{H} 82 \mathrm{~A}$ & 0.252349 & 0.612817 & -0.044984 & $0.322 *$ & \\
\hline H82B & 0.268856 & 0.671795 & -0.012813 & $0.322^{*}$ & \\
\hline C83' & $0.3074(7)$ & $0.6066(12)$ & $-0.0116(9)$ & $0.175(11)^{*}$ & \\
\hline H83A & 0.326549 & 0.626325 & 0.015908 & $0.210^{*}$ & \\
\hline H83B & 0.310582 & 0.561151 & -0.009633 & $0.210^{*}$ & \\
\hline C84' & $0.3071(8)$ & $0.6317(14)$ & $-0.0612(10)$ & $0.253(17)^{*}$ & \\
\hline H84A & 0.330397 & 0.622668 & -0.065928 & $0.380 *$ & \\
\hline
\end{tabular}




\begin{tabular}{|c|c|c|c|c|}
\hline H84B & 0.287488 & 0.612068 & -0.087380 & $0.380^{*}$ \\
\hline $\mathrm{H} 84 \mathrm{C}$ & 0.303290 & 0.676566 & -0.062104 & $0.380^{*}$ \\
\hline $\mathrm{C} 85^{\prime}$ & $-0.0692(4)$ & $0.7833(6)$ & $0.0155(5)$ & $0.056(4)$ \\
\hline $\mathrm{H} 85 \mathrm{~A}$ & -0.056872 & 0.823201 & 0.026767 & $0.067^{*}$ \\
\hline H85B & -0.095340 & 0.792287 & -0.000479 & $0.067^{*}$ \\
\hline $\mathrm{C} 86^{\prime}$ & $-0.0544(4)$ & $0.7562(7)$ & $-0.0232(5)$ & $0.061(4)$ \\
\hline H86A & -0.028567 & 0.745271 & -0.006826 & $0.074^{*}$ \\
\hline H86B & -0.067557 & 0.717250 & -0.035567 & $0.074^{*}$ \\
\hline C87' & $-0.0567(4)$ & $0.7969(6)$ & $-0.0666(5)$ & $0.051(4)$ \\
\hline H87A & -0.082608 & 0.805658 & -0.084347 & $0.061^{*}$ \\
\hline H87B & -0.044732 & 0.836750 & -0.054329 & $0.061^{*}$ \\
\hline C88' & $-0.0399(4)$ & $0.7703(8)$ & $-0.1026(5)$ & $0.073(5)$ \\
\hline H88A & -0.054685 & 0.734529 & -0.119227 & $0.087^{*}$ \\
\hline H88B & -0.015473 & 0.754637 & -0.083979 & $0.087^{*}$ \\
\hline C89' & $-0.0364(4)$ & $0.8146(7)$ & $-0.1416(6)$ & $0.073(5)$ \\
\hline H89A & -0.061006 & 0.826718 & -0.162747 & $0.088^{*}$ \\
\hline H89B & -0.023902 & 0.852467 & -0.125249 & $0.088^{*}$ \\
\hline $\mathrm{C} 90^{\prime}$ & $-0.0160(5)$ & $0.7889(8)$ & $-0.1736(6)$ & $0.098(6)$ \\
\hline $\mathrm{H} 90 \mathrm{~A}$ & -0.014920 & 0.820166 & -0.197798 & $0.147^{*}$ \\
\hline H90B & 0.008635 & 0.777813 & -0.153223 & $0.147^{*}$ \\
\hline $\mathrm{H} 90 \mathrm{C}$ & -0.028529 & 0.752023 & -0.190782 & $0.147^{*}$ \\
\hline C91' & -0.0809 (4) & $0.7770(7)$ & $0.0955(5)$ & $0.064(4)$ \\
\hline H91A & -0.067032 & 0.815671 & 0.106641 & $0.076^{*}$ \\
\hline H91B & -0.106328 & 0.788664 & 0.077556 & $0.076^{*}$ \\
\hline C92' & $-0.0801(5)$ & $0.7393(10)$ & $0.1402(7)$ & $0.118(8)$ \\
\hline H92A & -0.092219 & 0.699342 & 0.128345 & $0.142 *$ \\
\hline H92B & -0.054392 & 0.729899 & 0.158538 & $0.142 *$ \\
\hline C93' & $-0.0958(6)$ & 0.7639 (11) & $0.1739(7)$ & $0.129(8)$ \\
\hline H93A & -0.120791 & 0.777119 & 0.155284 & $0.155^{*}$ \\
\hline H93B & -0.082007 & 0.801447 & 0.188640 & $0.155^{*}$ \\
\hline C94' & $-0.0975(8)$ & $0.7226(14)$ & $0.2147(9)$ & $0.172(11)$ \\
\hline $\mathrm{H} 94 \mathrm{~A}$ & -0.101088 & 0.748268 & 0.241328 & $0.207^{*}$ \\
\hline H94B & -0.074125 & 0.700604 & 0.228352 & $0.207^{*}$ \\
\hline C95' & $-0.1300(8)$ & $0.6722(12)$ & $0.1973(9)$ & $0.165(11)$ \\
\hline H95A & -0.152512 & 0.692104 & 0.176109 & $0.198 *$ \\
\hline H95B & -0.123354 & 0.638729 & 0.178230 & $0.198 *$ \\
\hline C96' & $-0.1358(7)$ & $0.6456(15)$ & $0.2434(10)$ & $0.247(18)$ \\
\hline H96A & -0.155341 & 0.614920 & 0.233893 & $0.370^{*}$ \\
\hline H96B & -0.113389 & 0.625924 & 0.263960 & $0.370^{*}$ \\
\hline $\mathrm{H} 96 \mathrm{C}$ & -0.142432 & 0.679089 & 0.261847 & $0.370^{*}$ \\
\hline C97' & $0.0734(4)$ & $0.4608(7)$ & $0.4761(5)$ & $0.058(4)$ \\
\hline H97A & 0.057277 & 0.447664 & 0.494740 & $0.069^{*}$ \\
\hline H97B & 0.080437 & 0.423299 & 0.461677 & $0.069^{*}$ \\
\hline C98' & $0.1071(4)$ & $0.4896(7)$ & $0.5114(5)$ & $0.056(4)$ \\
\hline H98A & 0.100108 & 0.524470 & 0.528839 & $0.067^{*}$ \\
\hline H98B & 0.122402 & 0.506311 & 0.492812 & $0.067 *$ \\
\hline C99' & $0.1288(5)$ & $0.4422(7)$ & $0.5490(6)$ & $0.084(5)$ \\
\hline H99A & 0.112715 & 0.422571 & 0.565162 & $0.101 *$ \\
\hline
\end{tabular}




\begin{tabular}{|c|c|c|c|c|c|}
\hline Н99B & 0.137678 & 0.409408 & 0.531683 & $0.101 *$ & \\
\hline C200 & $0.1607(5)$ & $0.4711(10)$ & $0.5876(6)$ & $0.111(7)$ & \\
\hline $\mathrm{H} 20 \mathrm{~A}$ & 0.172863 & 0.501394 & 0.572325 & $0.134^{*}$ & \\
\hline $\mathrm{H} 20 \mathrm{~B}$ & 0.178543 & 0.438915 & 0.604213 & $0.134^{*}$ & \\
\hline C201 & $0.1463(7)$ & $0.5033(11)$ & $0.6245(8)$ & $0.159(10)$ & \\
\hline $\mathrm{H} 20 \mathrm{C}$ & 0.127715 & 0.533865 & 0.606795 & $0.190^{*}$ & \\
\hline H20D & 0.166385 & 0.526252 & 0.648233 & $0.190 *$ & \\
\hline C202 & $0.1283(8)$ & $0.4561(13)$ & $0.6551(9)$ & $0.220(16)$ & \\
\hline H20E & 0.119606 & 0.480016 & 0.678008 & $0.329 *$ & \\
\hline $\mathrm{H} 20 \mathrm{~F}$ & 0.107887 & 0.433944 & 0.631972 & $0.329 *$ & \\
\hline H20G & 0.146609 & 0.426321 & 0.673467 & $0.329 *$ & \\
\hline C203 & $0.0151(4)$ & $0.4751(7)$ & $0.4020(5)$ & $0.066(4)$ & \\
\hline $\mathrm{H} 20 \mathrm{H}$ & 0.019640 & 0.432047 & 0.393719 & $0.079 *$ & 0.6 \\
\hline H20I & -0.001396 & 0.473295 & 0.421923 & $0.079 *$ & 0.6 \\
\hline $\mathrm{H} 20 \mathrm{~J}$ & 0.021755 & 0.438555 & 0.385993 & $0.079 *$ & 0.4 \\
\hline $\mathrm{H} 20 \mathrm{~K}$ & 0.003966 & 0.458592 & 0.425924 & $0.079 *$ & 0.4 \\
\hline C206 & $-0.0490(6)$ & $0.5164(10)$ & $0.2748(6)$ & $0.138(9)$ & \\
\hline $\mathrm{H} 20 \mathrm{~N}$ & -0.071651 & 0.495533 & 0.274638 & $0.166^{*}$ & 0.4 \\
\hline $\mathrm{H} 20 \mathrm{O}$ & -0.050645 & 0.561483 & 0.279690 & $0.166^{*}$ & 0.4 \\
\hline H20L & -0.075823 & 0.518494 & 0.263123 & $0.166^{*}$ & 0.6 \\
\hline H20M & -0.040177 & 0.558883 & 0.284729 & $0.166^{*}$ & 0.6 \\
\hline C207 & $-0.0378(5)$ & $0.5005(8)$ & $0.2303(6)$ & $0.109(7)$ & \\
\hline $\mathrm{H} 20 \mathrm{P}$ & -0.039181 & 0.455103 & 0.225222 & $0.131^{*}$ & \\
\hline H20Q & -0.012332 & 0.513365 & 0.236423 & $0.131^{*}$ & \\
\hline C208 & $-0.0625(6)$ & $0.5329(10)$ & $0.1836(6)$ & $0.165(11)$ & \\
\hline H20R & -0.054640 & 0.521824 & 0.155576 & $0.247^{*}$ & \\
\hline $\mathrm{H} 20 \mathrm{~S}$ & -0.087607 & 0.519596 & 0.177246 & $0.247 *$ & \\
\hline $\mathrm{H} 20 \mathrm{~T}$ & -0.060785 & 0.577799 & 0.188435 & $0.247^{*}$ & \\
\hline C209 & $0.3024(4)$ & $0.6806(6)$ & $0.4668(4)$ & $0.051(4)$ & \\
\hline H20U & 0.328834 & 0.678712 & 0.483984 & $0.061 *$ & \\
\hline $\mathrm{H} 20 \mathrm{~V}$ & 0.295841 & 0.724709 & 0.459990 & $0.061 *$ & \\
\hline $\mathrm{C} 210$ & $0.2834(4)$ & $0.6566(7)$ & $0.5013(5)$ & $0.058(4)$ & \\
\hline H21A & 0.256842 & 0.657941 & 0.484329 & $0.070^{*}$ & \\
\hline H21B & 0.290251 & 0.612781 & 0.509073 & $0.070^{*}$ & \\
\hline C211 & $0.2919(4)$ & $0.6921(7)$ & $0.5486(5)$ & $0.069(5)$ & \\
\hline $\mathrm{H} 21 \mathrm{C}$ & 0.279230 & 0.732454 & 0.541171 & $0.083^{*}$ & 0.5 \\
\hline H21D & 0.318237 & 0.700890 & 0.560310 & $0.083 *$ & 0.5 \\
\hline $\mathrm{H} 21 \mathrm{E}$ & 0.291352 & 0.737063 & 0.541755 & $0.083^{*}$ & 0.5 \\
\hline $\mathrm{H} 21 \mathrm{~F}$ & 0.316538 & 0.681306 & 0.570413 & $0.083^{*}$ & 0.5 \\
\hline $\mathrm{C} 215$ & $0.3146(4)$ & $0.6794(7)$ & $0.3854(5)$ & $0.063(4)$ & \\
\hline $\mathrm{H} 21 \mathrm{G}$ & 0.307904 & 0.723716 & 0.382548 & $0.075^{*}$ & \\
\hline $\mathrm{H} 21 \mathrm{H}$ & 0.340979 & 0.676631 & 0.402950 & $0.075^{*}$ & \\
\hline $\mathrm{C} 216$ & $0.3070(5)$ & $0.6533(9)$ & $0.3346(6)$ & $0.101(7)$ & \\
\hline $\mathrm{H} 21 \mathrm{I}$ & 0.280509 & 0.655697 & 0.317435 & $0.121^{*}$ & 0.5 \\
\hline $\mathrm{H} 21 \mathrm{~J}$ & 0.313707 & 0.609004 & 0.337738 & $0.121 *$ & 0.5 \\
\hline $\mathrm{H} 21 \mathrm{~K}$ & 0.284141 & 0.630336 & 0.330003 & $0.121 *$ & 0.5 \\
\hline $\mathrm{H} 21 \mathrm{~L}$ & 0.325823 & 0.620917 & 0.340246 & $0.121 *$ & 0.5 \\
\hline $\mathrm{C} 221$ & $0.0431(4)$ & $0.4146(8)$ & $0.2036(6)$ & $0.093(6)$ & \\
\hline
\end{tabular}




\begin{tabular}{|c|c|c|c|c|c|}
\hline $\mathrm{H} 22 \mathrm{~A}$ & 0.062546 & 0.424561 & 0.234159 & $0.139^{*}$ & \\
\hline $\mathrm{H} 22 \mathrm{~B}$ & 0.028835 & 0.379881 & 0.209302 & $0.139^{*}$ & \\
\hline $\mathrm{H} 22 \mathrm{C}$ & 0.027395 & 0.450747 & 0.192668 & $0.139^{*}$ & \\
\hline $\mathrm{C} 222$ & $0.2012(5)$ & $0.8206(10)$ & $0.2354(6)$ & $0.110(7)$ & \\
\hline H22D & 0.205608 & 0.804389 & 0.268603 & $0.165^{*}$ & \\
\hline $\mathrm{H} 22 \mathrm{E}$ & 0.222086 & 0.845421 & 0.234623 & $0.165^{*}$ & \\
\hline $\mathrm{H} 22 \mathrm{~F}$ & 0.197755 & 0.786141 & 0.212170 & $0.165^{*}$ & \\
\hline $\mathrm{C} 223$ & $0.1877(5)$ & $0.4334(9)$ & $0.1955(6)$ & $0.097(6)$ & \\
\hline $\mathrm{H} 22 \mathrm{G}$ & 0.205696 & 0.462526 & 0.215653 & $0.146^{*}$ & \\
\hline $\mathrm{H} 22 \mathrm{H}$ & 0.167508 & 0.456423 & 0.172823 & $0.146^{*}$ & \\
\hline H22I & 0.198997 & 0.407212 & 0.176732 & $0.146^{*}$ & \\
\hline $\mathrm{C} 224$ & 0.0389 (4) & $0.8137(9)$ & $0.2602(6)$ & $0.107(7)$ & \\
\hline H22J & 0.032774 & 0.787487 & 0.284144 & $0.160^{*}$ & \\
\hline $\mathrm{H} 22 \mathrm{~K}$ & 0.038591 & 0.788867 & 0.231565 & $0.160^{*}$ & \\
\hline $\mathrm{H} 22 \mathrm{~L}$ & 0.021072 & 0.847209 & 0.249735 & $0.160^{*}$ & \\
\hline $\mathrm{C} 21 \mathrm{~B}$ & $0.2822(8)$ & $0.6626(16)$ & $0.5914(10)$ & $0.076(8)^{*}$ & 0.5 \\
\hline $\mathrm{H} 21 \mathrm{M}$ & 0.292260 & 0.620143 & 0.595948 & $0.091^{*}$ & 0.5 \\
\hline $\mathrm{H} 21 \mathrm{~N}$ & 0.295187 & 0.686058 & 0.621591 & $0.091^{*}$ & 0.5 \\
\hline $\mathrm{C} 21 \mathrm{C}$ & $0.2431(9)$ & $0.658(2)$ & $0.5887(15)$ & $0.16(2)^{*}$ & 0.5 \\
\hline $\mathrm{H} 21 \mathrm{O}$ & 0.229629 & 0.638052 & 0.557170 & $0.188^{*}$ & 0.5 \\
\hline $\mathrm{H} 21 \mathrm{P}$ & 0.233611 & 0.700859 & 0.587443 & $0.188^{*}$ & 0.5 \\
\hline C21D & $0.2342(9)$ & $0.6237(16)$ & $0.6298(12)$ & $0.086(11)^{*}$ & 0.5 \\
\hline H21Q & 0.207896 & 0.624419 & 0.623348 & $0.128^{*}$ & 0.5 \\
\hline $\mathrm{H} 21 \mathrm{R}$ & 0.242432 & 0.580732 & 0.630913 & $0.128^{*}$ & 0.5 \\
\hline $\mathrm{H} 21 \mathrm{~S}$ & 0.246432 & 0.643829 & 0.661327 & $0.128^{*}$ & 0.5 \\
\hline $\mathrm{C} 80^{\prime}$ & $0.2166(5)$ & $0.6437(11)$ & $0.0313(8)$ & $0.048(7)^{*}$ & 0.6 \\
\hline H80A & 0.213491 & 0.637174 & 0.063775 & $0.058^{*}$ & 0.6 \\
\hline H80B & 0.219787 & 0.688451 & 0.026683 & $0.058^{*}$ & 0.6 \\
\hline C204 & $-0.0039(6)$ & $0.5106(10)$ & $0.3540(7)$ & $0.062(8)^{*}$ & 0.7 \\
\hline $\mathrm{H} 20 \mathrm{~W}$ & -0.010593 & 0.552373 & 0.362262 & $0.075^{*}$ & 0.7 \\
\hline $\mathrm{H} 20 \mathrm{X}$ & 0.013236 & 0.515570 & 0.335337 & $0.075^{*}$ & 0.7 \\
\hline C205 & $-0.0381(7)$ & $0.4774(13)$ & $0.3217(8)$ & $0.118(11)$ & 0.7 \\
\hline $\mathrm{H} 20 \mathrm{Y}$ & -0.032699 & 0.434190 & 0.314910 & $0.141^{*}$ & 0.7 \\
\hline $\mathrm{HF}$ & -0.057340 & 0.477381 & 0.337199 & $0.141^{*}$ & 0.7 \\
\hline $\mathrm{C} 217$ & $0.3262(11)$ & $0.684(2)$ & $0.3023(11)$ & $0.136(13)^{*}$ & 0.5 \\
\hline $\mathrm{H} 21 \mathrm{~T}$ & 0.351997 & 0.671619 & 0.313205 & $0.163^{*}$ & 0.5 \\
\hline $\mathrm{H} 21 \mathrm{U}$ & 0.325210 & 0.729772 & 0.305601 & $0.163^{*}$ & 0.5 \\
\hline C218 & $0.3081(9)$ & $0.6658(18)$ & $0.2483(12)$ & $0.096(9)^{*}$ & 0.5 \\
\hline $\mathrm{H} 21 \mathrm{~V}$ & 0.301258 & 0.621700 & 0.247999 & $0.116^{*}$ & 0.5 \\
\hline $\mathrm{H} 21 \mathrm{~W}$ & 0.326952 & 0.668372 & 0.232143 & $0.116^{*}$ & 0.5 \\
\hline C219 & $0.2745(10)$ & $0.701(2)$ & $0.2150(14)$ & $0.135(12)^{*}$ & 0.5 \\
\hline $\mathrm{H} 21 \mathrm{X}$ & 0.252287 & 0.687181 & 0.221694 & $0.162^{*}$ & 0.5 \\
\hline $\mathrm{H} 21 \mathrm{Y}$ & 0.277406 & 0.745799 & 0.219941 & $0.162 *$ & 0.5 \\
\hline $\mathrm{C} 220$ & $0.2731(15)$ & $0.683(3)$ & $0.1636(16)$ & $0.22(2)^{*}$ & 0.5 \\
\hline $\mathrm{H} 22 \mathrm{M}$ & 0.252310 & 0.702889 & 0.139630 & $0.324 *$ & 0.5 \\
\hline $\mathrm{H} 22 \mathrm{~N}$ & 0.295517 & 0.695880 & 0.158009 & $0.324 *$ & 0.5 \\
\hline $\mathrm{H} 22 \mathrm{O}$ & 0.270579 & 0.637686 & 0.159749 & $0.324 *$ & 0.5 \\
\hline C20B & $-0.0156(12)$ & $0.507(2)$ & $0.3623(11)$ & $0.060(18)^{*}$ & 0.3 \\
\hline
\end{tabular}




$\begin{array}{llllll}\text { H20Z } & -0.012967 & 0.552024 & 0.365823 & 0.072^{*} & 0.3 \\ \text { HG } & -0.039164 & 0.494767 & 0.365790 & 0.072^{*} & 0.3 \\ \text { C20C } & -0.0145(10) & 0.487(2) & 0.3112(12) & 0.059(15)^{*} & 0.3 \\ \text { H20 } & -0.015117 & 0.441142 & 0.307936 & 0.071^{*} & 0.3 \\ \text { HH } & 0.007664 & 0.502562 & 0.305446 & 0.071^{*} & 0.3 \\ \text { C21E } & 0.3026(13) & 0.6737(18) & 0.2821(8) & 0.136(13)^{*} & 0.5 \\ \text { H21Z } & 0.326131 & 0.692338 & 0.283679 & 0.163^{*} & 0.5 \\ \text { HI } & 0.284892 & 0.708095 & 0.275005 & 0.163^{*} & 0.5 \\ \text { C21F } & 0.2912(11) & 0.6324(16) & 0.2344(12) & 0.096(9)^{*} & 0.5 \\ \text { H21 } & 0.315575 & 0.614089 & 0.240588 & 0.116^{*} & 0.5 \\ \text { HJ } & 0.278450 & 0.600936 & 0.247706 & 0.116^{*} & 0.5 \\ \text { C21G } & 0.2762(12) & 0.6099(18) & 0.1815(11) & 0.135(12)^{*} & 0.5 \\ \text { H1 } & 0.296352 & 0.613337 & 0.167772 & 0.162^{*} & 0.5 \\ \text { HK } & 0.257840 & 0.640832 & 0.163852 & 0.162^{*} & 0.5 \\ \text { C21H } & 0.2589(14) & 0.547(2) & 0.1653(17) & 0.22(2)^{*} & 0.5 \\ \text { H2 } & 0.251421 & 0.543513 & 0.129368 & 0.324^{*} & 0.5 \\ \text { HL } & 0.276532 & 0.514020 & 0.180011 & 0.324^{*} & 0.5 \\ \text { HM } & 0.237625 & 0.541797 & 0.176051 & 0.324^{*} & 0.5 \\ \text { C80B } & 0.2148(7) & 0.609(2) & 0.0411(10) & 0.098(18)^{*} & 0.4 \\ \text { H80C } & 0.210945 & 0.570240 & 0.056942 & 0.118^{*} & 0.4 \\ \text { H80D } & 0.215689 & 0.644174 & 0.063516 & 0.118^{*} & 0.4 \\ \text { C212 } & 0.2643(8) & 0.6770(16) & 0.5742(12) & 0.076(8)^{*} & 0.5 \\ \text { H3 } & 0.239576 & 0.679526 & 0.550157 & 0.091^{*} & 0.5 \\ \text { HN } & 0.268245 & 0.634054 & 0.586866 & 0.091^{*} & 0.5 \\ \text { C213 } & 0.2673(11) & 0.7203(18) & 0.6159(13) & 0.127(16)^{*} & 0.5 \\ \text { H4 } & 0.258447 & 0.761697 & 0.602594 & 0.153^{*} & 0.5 \\ \text { HO } & 0.293103 & 0.724428 & 0.636240 & 0.153^{*} & 0.5 \\ \text { C214 } & 0.2455(12) & 0.698(2) & 0.6483(15) & 0.153(19)^{*} & 0.5 \\ \text { H5 } & 0.248164 & 0.727636 & 0.675050 & 0.230^{*} & 0.5 \\ \text { HP } & 0.219825 & 0.694687 & 0.628490 & 0.230^{*} & 0.5 \\ \text { HQ } & 0.254473 & 0.657426 & 0.662128 & 0.230^{*} & 0.5\end{array}$

Atomic displacement parameters $\left(\AA^{2}\right)$

\begin{tabular}{lllllll}
\hline & $U^{11}$ & $U^{22}$ & $U^{33}$ & $U^{12}$ & $U^{13}$ & $U^{23}$ \\
\hline Ir1 & $0.0230(3)$ & $0.0237(3)$ & $0.0155(2)$ & $-0.0003(2)$ & $0.00689(18)$ & $0.0022(2)$ \\
Ir2 & $0.0266(3)$ & $0.0262(3)$ & $0.0161(2)$ & $-0.0034(2)$ & $0.00675(19)$ & $0.0027(2)$ \\
O1 & $0.036(6)$ & $0.092(9)$ & $0.088(8)$ & $0.000(6)$ & $0.028(6)$ & $-0.004(7)$ \\
O2 & $0.039(4)$ & $0.31(2)$ & $0.086(9)$ & $0.001(6)$ & $0.041(5)$ & $0.028(11)$ \\
O3 & $0.044(5)$ & $0.029(5)$ & $0.049(6)$ & $0.017(4)$ & $0.021(4)$ & $0.016(4)$ \\
O4 & $0.049(6)$ & $0.032(5)$ & $0.051(6)$ & $0.000(4)$ & $0.025(4)$ & $-0.009(4)$ \\
O5 & $0.049(5)$ & $0.035(5)$ & $0.041(5)$ & $-0.021(4)$ & $0.022(4)$ & $-0.005(4)$ \\
O6 & $0.054(6)$ & $0.048(6)$ & $0.034(5)$ & $-0.014(5)$ & $0.019(4)$ & $0.001(4)$ \\
N1 & $0.034(5)$ & $0.012(5)$ & $0.018(5)$ & $0.000(4)$ & $0.012(4)$ & $0.004(4)$ \\
N01 & $0.0230(3)$ & $0.0237(3)$ & $0.0155(2)$ & $-0.0003(2)$ & $0.00689(18)$ & $0.0022(2)$ \\
N02 & $0.0230(3)$ & $0.0237(3)$ & $0.0155(2)$ & $-0.0003(2)$ & $0.00689(18)$ & $0.0022(2)$ \\
N2 & $0.026(6)$ & $0.018(5)$ & $0.018(5)$ & $-0.004(4)$ & $0.003(4)$ & $-0.004(4)$ \\
N4 & $0.019(6)$ & $0.037(6)$ & $0.024(5)$ & $-0.005(4)$ & $0.007(4)$ & $0.001(4)$
\end{tabular}




\begin{tabular}{|c|c|c|c|c|c|c|}
\hline $\mathrm{C} 01$ & $0.028(6)$ & $0.071(9)$ & $0.006(6)$ & $-0.020(5)$ & $0.003(5)$ & $-0.001(6)$ \\
\hline $\mathrm{C} 02$ & $0.028(3)$ & $0.096(12)$ & $0.030(7)$ & -0.010 & $0.015(4)$ & $-0.005(8)$ \\
\hline $\mathrm{C} 2$ & $0.020(6)$ & $0.039(8)$ & $0.021(6)$ & $0.000(5)$ & $0.007(4)$ & $0.007(5)$ \\
\hline $\mathrm{C} 3$ & $0.018(5)$ & $0.033(6)$ & $0.018(6)$ & -0.001 & $0.003(4)$ & $0.002(5)$ \\
\hline $\mathrm{C} 4$ & $0.025(5)$ & $0.031(5)$ & $0.021(6)$ & $-0.002(4)$ & 0.009 (4) & $0.002(5)$ \\
\hline $\mathrm{C} 5$ & $0.028(6)$ & $0.028(6)$ & $0.031(7)$ & $-0.008(5)$ & $0.004(5)$ & $0.000(5)$ \\
\hline C6 & $0.029(6)$ & $0.021(6)$ & $0.019(6)$ & $-0.003(5)$ & 0.009 (4) & -0.004 \\
\hline $\mathrm{C} 7$ & $0.031(6)$ & $0.023(5)$ & $0.032(7)$ & $0.005(4)$ & $0.019(5)$ & $0.006(5)$ \\
\hline $\mathrm{C} 8$ & $0.036(7)$ & $0.030(5)$ & $0.019(6)$ & $-0.006(5)$ & $0.004(5)$ & $-0.007(5)$ \\
\hline C9 & $0.053(7)$ & $0.029(7)$ & $0.021(6)$ & $0.002(5)$ & $0.013(5)$ & $0.005(5)$ \\
\hline $\mathrm{C} 10$ & $0.052(7)$ & $0.014(5)$ & $0.025(7)$ & $0.010(5)$ & $0.015(5)$ & $0.006(5)$ \\
\hline C11 & $0.030(7)$ & $0.021(5)$ & $0.028(6)$ & $0.001(4)$ & $0.017(5)$ & $0.008(5)$ \\
\hline $\mathrm{C} 12$ & $0.021(6)$ & $0.035(5)$ & $0.028(6)$ & $0.003(4)$ & $0.007(5)$ & $0.003(5)$ \\
\hline C13 & $0.025(6)$ & $0.037(6)$ & $0.030(7)$ & $0.006(5)$ & $0.006(5)$ & $-0.001(5)$ \\
\hline C14 & $0.026(7)$ & $0.046(8)$ & $0.043(8)$ & $0.012(5)$ & $0.013(5)$ & $0.015(6)$ \\
\hline C15 & $0.032(7)$ & $0.061(7)$ & $0.031(7)$ & $0.009(6)$ & $0.002(5)$ & $0.002(6)$ \\
\hline $\mathrm{C} 16$ & $0.017(6)$ & $0.058(7)$ & $0.034(7)$ & $0.004(5)$ & 0.007 (5) & $-0.010(6)$ \\
\hline $\mathrm{C} 17$ & $0.021(6)$ & $0.041(6)$ & $0.025(6)$ & $0.002(4)$ & 0.007 (4) & -0.002 \\
\hline C18 & $0.020(6)$ & $0.042(6)$ & $0.037(6)$ & $-0.002(5)$ & $0.010(5)$ & $0.000(5)$ \\
\hline C19 & $0.018(6)$ & $0.023(5)$ & $0.026(6)$ & $0.003(5)$ & 0.009 (4) & $0.005(5)$ \\
\hline $\mathrm{C} 20$ & $0.028(7)$ & $0.024(6)$ & $0.016(6)$ & $0.002(5)$ & $0.008(5)$ & $0.004(5)$ \\
\hline $\mathrm{C} 21$ & $0.030(7)$ & $0.026(5)$ & $0.016(6)$ & $0.003(5)$ & $0.007(5)$ & -0.002 \\
\hline $\mathrm{C} 22$ & $0.036(7)$ & $0.026(5)$ & $0.015(6)$ & $-0.008(5)$ & $0.011(5)$ & $0.000(4)$ \\
\hline $\mathrm{C} 23$ & $0.023(7)$ & $0.012(5)$ & $0.028(6)$ & $-0.007(5)$ & $0.008(5)$ & -0.001 \\
\hline $\mathrm{C} 24$ & $0.025(7)$ & $0.024(5)$ & $0.016(6)$ & $0.002(5)$ & $0.004(4)$ & $0.000(4)$ \\
\hline $\mathrm{C} 25$ & $0.041(8)$ & $0.027(5)$ & $0.023(6)$ & $-0.007(5)$ & $0.015(5)$ & $-0.007(5)$ \\
\hline $\mathrm{C} 26$ & $0.044(8)$ & $0.023(5)$ & $0.025(7)$ & $-0.004(5)$ & $0.014(5)$ & -0.004 \\
\hline $\mathrm{C} 27$ & $0.042(8)$ & $0.015(6)$ & $0.038(7)$ & $-0.004(5)$ & $0.013(5)$ & -0.009 \\
\hline $\mathrm{C} 28$ & $0.036(8)$ & $0.030(6)$ & $0.031(7)$ & $-0.009(5)$ & $0.012(5)$ & -0.012 \\
\hline $\mathrm{C} 29$ & $0.024(7)$ & $0.029(5)$ & $0.024(6)$ & $-0.009(5)$ & $0.007(5)$ & -0.008 \\
\hline $\mathrm{C} 30$ & $0.029(7)$ & $0.031(5)$ & $0.026(6)$ & $-0.001(5)$ & $0.015(5)$ & $-0.001(5)$ \\
\hline $\mathrm{C} 31$ & $0.044(8)$ & $0.030(6)$ & $0.019(6)$ & $-0.003(6)$ & $0.013(5)$ & $0.004(5)$ \\
\hline C32 & $0.049(8)$ & $0.031(7)$ & $0.021(6)$ & $-0.006(6)$ & $0.017(5)$ & -0.007 \\
\hline $\mathrm{C} 33$ & $0.037(8)$ & $0.042(6)$ & $0.018(6)$ & $0.002(6)$ & 0.015 & $0.001(5)$ \\
\hline $\mathrm{C} 34$ & $0.028(7)$ & $0.040(6)$ & $0.023(6)$ & $-0.009(6)$ & $0.011(5)$ & $-0.009(5)$ \\
\hline $\mathrm{C} 35$ & $0.026(7)$ & $0.032(5)$ & $0.008(5)$ & $0.001(5)$ & -0.001 & $0.002(4)$ \\
\hline C36 & $0.030(6)$ & $0.032(6)$ & $0.022(6)$ & $-0.004(5)$ & $0.011(4)$ & $-0.006(5)$ \\
\hline C37 & $0.028(6)$ & $0.029(6)$ & $0.014(5)$ & $0.002(4)$ & $0.008(4)$ & -0.003 \\
\hline C38 & $0.031(6)$ & $0.019(6)$ & $0.019(6)$ & $0.003(4)$ & $0.003(5)$ & -0.005 \\
\hline C39 & $0.027(5)$ & $0.025(6)$ & $0.025(6)$ & $0.000(4)$ & $0.004(4)$ & -0.003 \\
\hline $\mathrm{C} 40$ & $0.032(6)$ & $0.031(6)$ & $0.031(7)$ & $-0.002(4)$ & $0.006(5)$ & $0.004(5)$ \\
\hline $\mathrm{C} 41$ & $0.032(6)$ & $0.018(6)$ & $0.030(7)$ & $0.001(4)$ & $0.014(5)$ & $0.004(5)$ \\
\hline $\mathrm{C} 42$ & $0.028(6)$ & $0.030(5)$ & $0.021(6)$ & $0.000(4)$ & $0.017(4)$ & $0.002(5)$ \\
\hline $\mathrm{C} 43$ & $0.033(7)$ & $0.026(5)$ & $0.021(6)$ & $-0.001(5)$ & $0.014(5)$ & $-0.011(5)$ \\
\hline C44 & 0.039 & $0.030(6)$ & $0.032(7)$ & $0.002(5)$ & $0.017(5)$ & $-0.004(6)$ \\
\hline $\mathrm{C} 45$ & $0.052(7)$ & $0.021(7)$ & $0.044(8)$ & $-0.007(5)$ & $0.031(6)$ & $0.003(5)$ \\
\hline $\mathrm{C} 46$ & $0.045(6)$ & $0.036(6)$ & $0.033(7)$ & $-0.016(5)$ & $0.025(5)$ & $-0.006(5)$ \\
\hline $\mathrm{C} 47$ & $0.030(8)$ & $0.037(6)$ & $0.019(6)$ & $-0.007(5)$ & $0.012(5)$ & $-0.001(5)$ \\
\hline
\end{tabular}




\begin{tabular}{|c|c|c|c|c|c|c|}
\hline $\mathrm{C} 48$ & $0.027(6)$ & $0.030(6)$ & $0.039(7)$ & $-0.002(4)$ & $0.015(5)$ & $-0.002(5)$ \\
\hline C49 & $0.037(7)$ & $0.031(6)$ & $0.030(7)$ & $-0.005(5)$ & $0.003(5)$ & $0.000(6)$ \\
\hline $\mathrm{C} 50$ & $0.033(7)$ & $0.022(7)$ & 0.093 & $-0.009(5)$ & $-0.002(7)$ & $-0.012(7)$ \\
\hline C51 & $0.030(8)$ & $0.039(7)$ & $0.101(12)$ & $-0.006(6)$ & $0.007(7)$ & $-0.004(8)$ \\
\hline $\mathrm{C} 52$ & $0.022(7)$ & $0.040(7)$ & $0.120(14)$ & $0.005(6)$ & $0.015(7)$ & $0.001(8)$ \\
\hline $\mathrm{C} 53$ & $0.023(5)$ & $0.040(6)$ & $0.041(7)$ & $-0.002(5)$ & $0.005(5)$ & $-0.006(6)$ \\
\hline $\mathrm{C} 54$ & $0.027(6)$ & $0.038(6)$ & $0.050(7)$ & $0.009(5)$ & $0.005(5)$ & $0.005(6)$ \\
\hline C55 & $0.023(7)$ & $0.019(5)$ & $0.022(6)$ & $-0.004(5)$ & $0.004(5)$ & $0.002(4)$ \\
\hline C56 & $0.029(7)$ & $0.025(6)$ & $0.021(6)$ & $-0.003(5)$ & $0.003(5)$ & $0.004(5)$ \\
\hline $\mathrm{C} 57$ & $0.023(7)$ & $0.029(5)$ & $0.024(6)$ & $-0.003(5)$ & $0.006(5)$ & $0.000(5)$ \\
\hline $\mathrm{C} 58$ & $0.035(7)$ & $0.026(5)$ & $0.020(6)$ & $-0.010(5)$ & 0.007 (5) & $-0.005(5)$ \\
\hline C59 & $0.038(8)$ & $0.019(6)$ & $0.028(7)$ & $-0.007(5)$ & $0.012(5)$ & $-0.003(5)$ \\
\hline $\mathrm{C} 60$ & $0.028(7)$ & $0.022(5)$ & $0.012(6)$ & $-0.002(5)$ & $0.004(4)$ & $0.006(5)$ \\
\hline C62 & $0.032(8)$ & $0.034(8)$ & $0.027(7)$ & $0.001(6)$ & $0.008(5)$ & $-0.011(6)$ \\
\hline C63 & $0.046(9)$ & $0.042(8)$ & $0.041(8)$ & $-0.004(7)$ & $0.015(6)$ & $-0.003(6)$ \\
\hline C64 & $0.036(8)$ & $0.036(6)$ & $0.022(6)$ & $-0.008(6)$ & $0.010(5)$ & $0.007(5)$ \\
\hline C65 & $0.023(7)$ & $0.039(6)$ & $0.028(7)$ & $-0.002(5)$ & $0.010(5)$ & $0.001(5)$ \\
\hline C66 & $0.027(7)$ & $0.032(5)$ & $0.011(5)$ & $-0.001(5)$ & $0.001(4)$ & -0.001 \\
\hline C67 & $0.039(8)$ & $0.035(6)$ & $0.033(7)$ & $0.010(6)$ & $0.016(6)$ & $0.008(6)$ \\
\hline C68 & $0.037(8)$ & $0.043(7)$ & $0.040(8)$ & $-0.001(6)$ & $0.015(6)$ & $0.011(6)$ \\
\hline C69 & $0.038(9)$ & $0.062(8)$ & $0.052(9)$ & $-0.009(7)$ & $0.027(7)$ & $0.001(7)$ \\
\hline $\mathrm{C} 70$ & $0.045(8)$ & $0.039(7)$ & $0.026(7)$ & $-0.011(6)$ & $0.015(6)$ & $-0.017(5)$ \\
\hline C71 & $0.045(8)$ & $0.038(6)$ & $0.013(6)$ & $-0.013(5)$ & $0.011(5)$ & $-0.010(5)$ \\
\hline C72 & $0.049(7)$ & $0.033(6)$ & $0.024(6)$ & $-0.003(5)$ & 0.015 & $-0.007(5)$ \\
\hline C73 & $0.015(6)$ & $0.041(8)$ & $0.042(6)$ & $-0.008(6)$ & $0.007(5)$ & $0.008(6)$ \\
\hline C74 & $0.035(8)$ & $0.073(10)$ & $0.035(7)$ & $0.008(7)$ & $0.011(6)$ & $0.009(6)$ \\
\hline C75 & $0.069(12)$ & $0.100(12)$ & $0.055(10)$ & $-0.017(10)$ & $0.032(8)$ & $-0.004(9)$ \\
\hline C76 & $0.19(2)$ & 0.089 (14) & $0.24(3)$ & $-0.046(16)$ & $0.14(2)$ & $-0.006(16)$ \\
\hline C77 & $0.089(16)$ & $0.16(2)$ & $0.106(16)$ & $-0.038(14)$ & $0.011(12)$ & $0.038(14)$ \\
\hline $\mathrm{C} 78$ & $0.27(3)$ & $0.25(3)$ & $0.12(2)$ & $0.07(3)$ & $0.12(2)$ & $0.072(19)$ \\
\hline C79 & $0.043(9)$ & $0.046(8)$ & $0.032(6)$ & $0.001(7)$ & $0.007(5)$ & $0.000(5)$ \\
\hline $\mathrm{C} 80$ & $0.029(8)$ & $0.061(9)$ & $0.021(6)$ & $0.005(7)$ & $-0.002(5)$ & $-0.004(6)$ \\
\hline $\mathrm{C} 81$ & $0.050(10)$ & $0.068(10)$ & $0.033(7)$ & $0.002(8)$ & $0.005(6)$ & $-0.008(6)$ \\
\hline $\mathrm{C} 82$ & $0.058(10)$ & $0.073(11)$ & $0.029(7)$ & $0.005(8)$ & $0.011(6)$ & $-0.005(7)$ \\
\hline $\mathrm{C} 83$ & $0.107(16)$ & $0.121(16)$ & $0.092(14)$ & $-0.053(13)$ & $0.061(11)$ & $-0.062(11)$ \\
\hline $\mathrm{C} 84$ & $0.17(2)$ & $0.16(2)$ & 0.15 (2) & $-0.073(18)$ & $0.124(18)$ & $-0.070(16)$ \\
\hline $\mathrm{C} 85$ & $0.038(6)$ & $0.026(7)$ & $0.025(6)$ & $-0.009(5)$ & $0.018(4)$ & $0.000(5)$ \\
\hline $\mathrm{C} 86$ & $0.047(7)$ & $0.020(7)$ & $0.032(6)$ & $-0.005(5)$ & $0.009(5)$ & $0.000(5)$ \\
\hline $\mathrm{C} 87$ & $0.042(7)$ & $0.043(8)$ & $0.029(6)$ & $-0.005(6)$ & $0.011(5)$ & $-0.010(6)$ \\
\hline $\mathrm{C} 88$ & $0.055(9)$ & $0.046(9)$ & $0.044(7)$ & $-0.010(7)$ & $0.011(6)$ & $-0.002(7)$ \\
\hline C89 & $0.064(11)$ & $0.060(10)$ & $0.046(7)$ & $0.002(8)$ & $0.025(7)$ & $0.013(7)$ \\
\hline $\mathrm{C} 90$ & $0.125(16)$ & $0.109(16)$ & $0.043(8)$ & $-0.002(13)$ & $0.017(9)$ & $0.023(10)$ \\
\hline C91 & $0.042(6)$ & $0.022(6)$ & $0.026(6)$ & $-0.007(5)$ & $0.014(5)$ & -0.003 \\
\hline C92 & $0.035(6)$ & $0.026(7)$ & $0.030(7)$ & $0.005(5)$ & $0.019(5)$ & $0.003(5)$ \\
\hline C93 & $0.045(7)$ & $0.039(8)$ & $0.028(7)$ & $0.011(6)$ & $0.021(5)$ & $0.006(5)$ \\
\hline C94 & $0.054(7)$ & $0.060(9)$ & $0.016(6)$ & $0.000(6)$ & $0.006(5)$ & $-0.007(6)$ \\
\hline C95 & $0.069(10)$ & $0.116(15)$ & $0.064(11)$ & $0.032(10)$ & $0.016(8)$ & $0.034(10)$ \\
\hline C96 & $0.072(11)$ & $0.124(18)$ & $0.114(16)$ & $0.040(12)$ & $0.008(10)$ & $0.000(13)$ \\
\hline
\end{tabular}




\begin{tabular}{|c|c|c|c|c|c|c|}
\hline C97 & $0.044(9)$ & $0.044(8)$ & $0.074(8)$ & $0.001(7)$ & $0.013(7)$ & $-0.014(7)$ \\
\hline C98 & $0.039(9)$ & $0.054(10)$ & $0.079(8)$ & $-0.007(7)$ & $0.025(7)$ & $-0.016(8)$ \\
\hline C99 & $0.115(15)$ & $0.051(11)$ & $0.098(11)$ & $-0.002(10)$ & 0.058 (11) & $-0.032(10)$ \\
\hline $\mathrm{C} 100$ & $0.133(18)$ & $0.085(15)$ & $0.088(11)$ & $-0.016(13)$ & $0.058(12)$ & $-0.021(11)$ \\
\hline $\mathrm{C} 103$ & $0.049(10)$ & $0.051(9)$ & $0.047(7)$ & $0.005(7)$ & $0.002(6)$ & $0.009(6)$ \\
\hline C104 & $0.043(9)$ & $0.055(9)$ & $0.035(7)$ & $0.003(7)$ & $0.002(6)$ & $0.010(6)$ \\
\hline C109 & $0.039(7)$ & $0.045(8)$ & $0.045(8)$ & $-0.002(6)$ & $0.019(5)$ & $-0.002(6)$ \\
\hline $\mathrm{C} 110$ & $0.051(10)$ & 0.068 (11) & $0.050(9)$ & $0.001(8)$ & $-0.001(6)$ & $0.009(7)$ \\
\hline C111 & $0.079(14)$ & 0.128 (19) & $0.106(15)$ & 0.007 (11) & $-0.027(10)$ & $0.036(12)$ \\
\hline $\mathrm{C} 112$ & $0.10(2)$ & $0.38(4)$ & $0.102(17)$ & $-0.06(2)$ & $-0.031(11)$ & $0.004(18)$ \\
\hline $\mathrm{C} 113$ & $0.15(2)$ & $0.36(3)$ & $0.18(2)$ & $-0.18(2)$ & $0.052(18)$ & $-0.05(2)$ \\
\hline C114 & $0.22(3)$ & 0.37 (4) & $0.33(3)$ & $-0.21(3)$ & $0.16(3)$ & $-0.04(3)$ \\
\hline C115 & $0.054(8)$ & $0.034(7)$ & $0.027(6)$ & $0.003(6)$ & $0.018(5)$ & $-0.001(5)$ \\
\hline C116 & $0.049(8)$ & $0.034(8)$ & $0.025(6)$ & $-0.006(6)$ & $0.013(5)$ & $-0.003(5)$ \\
\hline C117 & $0.061(9)$ & $0.045(9)$ & $0.029(7)$ & $-0.001(7)$ & $0.002(5)$ & $0.001(6)$ \\
\hline C118 & $0.050(8)$ & $0.039(8)$ & $0.023(6)$ & $-0.015(6)$ & $0.009(5)$ & $-0.002(5)$ \\
\hline C119 & $0.058(9)$ & $0.049(9)$ & $0.051(9)$ & $-0.016(7)$ & $0.000(6)$ & $-0.005(7)$ \\
\hline $\mathrm{C} 120$ & $0.053(9)$ & $0.056(10)$ & $0.050(9)$ & $-0.021(8)$ & $0.010(6)$ & $0.012(7)$ \\
\hline $\mathrm{C} 121$ & $0.050(7)$ & $0.047(9)$ & $0.049(9)$ & $0.014(7)$ & $0.022(6)$ & $0.008(7)$ \\
\hline $\mathrm{C} 122$ & $0.041(9)$ & $0.046(9)$ & $0.072(10)$ & $-0.005(7)$ & $0.033(7)$ & $-0.014(7)$ \\
\hline $\mathrm{C} 123$ & $0.050(8)$ & $0.072(11)$ & $0.042(9)$ & $-0.024(7)$ & $0.011(6)$ & $-0.001(7)$ \\
\hline C124 & 0.078 & $0.045(9)$ & $0.039(8)$ & $-0.015(7)$ & $0.039(7)$ & $0.004(7)$ \\
\hline C102 & $0.14(3)$ & $0.11(3)$ & $0.09(2)$ & $0.05(2)$ & $0.06(2)$ & $0.023(19)$ \\
\hline C105 & $0.043(17)$ & $0.064(15)$ & 0.048 & $-0.010(12)$ & $0.013(10)$ & $-0.004(10)$ \\
\hline C106 & $0.076(18)$ & $0.033(13)$ & $0.047(12)$ & $-0.026(12)$ & $0.030(12)$ & $0.001(9)$ \\
\hline $\mathrm{C} 107$ & $0.22(3)$ & 0.050 (17) & $0.046(15)$ & $-0.018(19)$ & $0.068(18)$ & $-0.007(12)$ \\
\hline Ir3 & $0.0371(4)$ & 0.0704 (4) & $0.0235(3)$ & $-0.0001(3)$ & $0.0102(2)$ & $0.0080(3)$ \\
\hline Ir4 & $0.0394(4)$ & $0.0702(4)$ & $0.0224(3)$ & $0.0159(3)$ & $0.0112(2)$ & $0.0049(3)$ \\
\hline $\mathrm{O} 1^{\prime}$ & $0.045(9)$ & $0.47(3)$ & $0.136(14)$ & $0.020(13)$ & $0.052(9)$ & $0.001(17)$ \\
\hline $\mathrm{O} 2^{\prime}$ & $0.036(7)$ & $0.166(13)$ & $0.096(9)$ & $-0.004(7)$ & $0.026(6)$ & $-0.006(8)$ \\
\hline O3' & $0.073(8)$ & $0.082(8)$ & $0.063(7)$ & $-0.033(6)$ & $0.021(6)$ & $0.015(6)$ \\
\hline $\mathrm{O} 4^{\prime}$ & $0.083(8)$ & $0.146(12)$ & $0.061(8)$ & $-0.060(8)$ & $0.021(6)$ & $-0.028(7)$ \\
\hline O5' & $0.081(9)$ & $0.121(10)$ & $0.068(8)$ & $0.032(8)$ & $0.031(6)$ & $-0.031(7)$ \\
\hline O6' & $0.090(7)$ & $0.090(9)$ & $0.074(8)$ & $0.054(7)$ & $0.043(6)$ & $0.034(7)$ \\
\hline N01' & $0.022(6)$ & $0.084(9)$ & $0.019(5)$ & $0.006(6)$ & $0.004(4)$ & $-0.009(5)$ \\
\hline $\mathrm{N} 1^{\prime}$ & $0.041(7)$ & $0.058(7)$ & $0.022(5)$ & $-0.011(5)$ & $0.000(4)$ & $0.015(5)$ \\
\hline $\mathrm{N} 2^{\prime}$ & $0.034(6)$ & $0.118(9)$ & $0.014(6)$ & $0.007(5)$ & $0.013(4)$ & $-0.002(5)$ \\
\hline N02' & $0.029(6)$ & 0.083 (9) & $0.021(5)$ & $0.017(6)$ & $0.013(5)$ & $0.022(5)$ \\
\hline N3' & $0.045(7)$ & $0.071(7)$ & $0.027(6)$ & $0.018(6)$ & $0.010(5)$ & $-0.004(5)$ \\
\hline N4' & $0.048(4)$ & $0.071(4)$ & $0.026(5)$ & $0.012(3)$ & $0.022(3)$ & $0.026(4)$ \\
\hline $\mathrm{C}^{\prime \prime} 1^{\prime}$ & $0.027(8)$ & 0.161 (19) & $0.067(11)$ & $0.001(9)$ & $0.020(8)$ & $0.000(11)$ \\
\hline $\mathrm{C} 1^{\prime}$ & $0.039(8)$ & $0.055(7)$ & $0.029(7)$ & $0.010(7)$ & $0.011(6)$ & $0.014(5)$ \\
\hline $\mathrm{C} 02^{\prime}$ & $0.038(8)$ & $0.119(15)$ & $0.038(9)$ & $0.012(9)$ & $0.014(7)$ & $0.014(9)$ \\
\hline $\mathrm{C} 2^{\prime}$ & $0.035(8)$ & $0.054(8)$ & $0.037(7)$ & $-0.001(7)$ & $0.018(6)$ & $0.002(6)$ \\
\hline $\mathrm{C} 3^{\prime}$ & $0.038(8)$ & $0.053(7)$ & $0.023(6)$ & $0.001(6)$ & $0.011(5)$ & $0.003(5)$ \\
\hline $\mathrm{C} 4^{\prime}$ & $0.071(10)$ & $0.051(7)$ & $0.039(7)$ & $-0.016(7)$ & $0.027(7)$ & $-0.004(6)$ \\
\hline $\mathrm{C} 5^{\prime}$ & $0.074(11)$ & $0.049(8)$ & $0.031(7)$ & $-0.004(7)$ & $0.023(7)$ & $-0.001(6)$ \\
\hline $\mathrm{C} 6^{\prime}$ & $0.047(9)$ & $0.046(7)$ & $0.037(7)$ & $-0.004(7)$ & $0.016(6)$ & $0.010(6)$ \\
\hline
\end{tabular}




\begin{tabular}{|c|c|c|c|c|c|c|}
\hline$C 7^{\prime}$ & $0.043(8)$ & $0.052(7)$ & $0.021(6)$ & $-0.002(6)$ & $0.001(5)$ & $0.020(5)$ \\
\hline $\mathrm{C} 8^{\prime}$ & $0.066(11)$ & $0.047(7)$ & $0.065(10)$ & $0.005(8)$ & $0.032(8)$ & $0.018(7)$ \\
\hline $\mathrm{C} 9^{\prime}$ & $0.051(10)$ & $0.059(9)$ & $0.059(9)$ & $-0.006(8)$ & $0.011(7)$ & $0.017(7)$ \\
\hline $\mathrm{C} 10^{\prime}$ & $0.040(9)$ & $0.063(8)$ & $0.060(9)$ & $-0.005(7)$ & $0.010(7)$ & $0.024(7)$ \\
\hline C11' & $0.047(9)$ & $0.072(8)$ & $0.025(7)$ & $-0.010(8)$ & $0.003(6)$ & $0.023(6)$ \\
\hline $\mathrm{C} 12^{\prime}$ & $0.038(8)$ & $0.047(6)$ & $0.034(7)$ & $0.002(6)$ & $0.019(6)$ & $0.001(6)$ \\
\hline $\mathrm{C} 13^{\prime}$ & $0.055(9)$ & $0.047(6)$ & $0.035(7)$ & $-0.005(7)$ & $0.025(6)$ & $-0.004(6)$ \\
\hline $\mathrm{C} 14^{\prime}$ & $0.048(9)$ & $0.057(8)$ & $0.030(7)$ & $-0.019(7)$ & $0.014(6)$ & $-0.003(6)$ \\
\hline C15' & $0.066(11)$ & $0.065(8)$ & $0.056(9)$ & $-0.039(8)$ & $0.035(8)$ & $-0.017(7)$ \\
\hline $\mathrm{C} 16^{\prime}$ & $0.070(11)$ & $0.058(8)$ & $0.048(9)$ & $-0.022(8)$ & $0.035(8)$ & $-0.009(7)$ \\
\hline $\mathrm{C} 17^{\prime}$ & $0.053(10)$ & $0.059(7)$ & $0.048(8)$ & $-0.011(7)$ & $0.034(7)$ & $-0.011(6)$ \\
\hline $\mathrm{C} 18^{\prime}$ & $0.079(9)$ & $0.050(7)$ & $0.052(8)$ & $-0.006(7)$ & $0.042(6)$ & $-0.009(7)$ \\
\hline $\mathrm{C} 20^{\prime}$ & $0.054(8)$ & $0.049(9)$ & $0.030(7)$ & $0.012(7)$ & $0.019(6)$ & $0.000(6)$ \\
\hline $\mathrm{C} 21^{\prime}$ & $0.045(7)$ & $0.052(7)$ & $0.047(8)$ & $0.003(5)$ & $0.013(6)$ & $-0.005(7)$ \\
\hline $\mathrm{C} 22^{\prime}$ & $0.048(7)$ & $0.054(7)$ & $0.039(8)$ & $0.004(5)$ & $0.013(6)$ & $0.011(6)$ \\
\hline $\mathrm{C} 23^{\prime}$ & $0.059(7)$ & $0.040(8)$ & $0.044(8)$ & $0.008(6)$ & $0.014(6)$ & $-0.005(6)$ \\
\hline C24' & $0.037(7)$ & $0.081(10)$ & $0.022(7)$ & $-0.008(6)$ & $0.014(5)$ & $0.001(6)$ \\
\hline $\mathrm{C} 25^{\prime}$ & $0.050(7)$ & $0.075(8)$ & $0.026(7)$ & $-0.014(6)$ & $0.020(5)$ & $-0.007(6)$ \\
\hline $\mathrm{C} 26^{\prime}$ & $0.077(10)$ & $0.079(8)$ & $0.039(9)$ & $-0.014(8)$ & $0.018(7)$ & $-0.006(8)$ \\
\hline $\mathrm{C} 27^{\prime}$ & $0.086(10)$ & $0.068(10)$ & $0.056(10)$ & $-0.029(7)$ & $0.025(8)$ & $-0.006(8)$ \\
\hline $\mathrm{C} 28^{\prime}$ & $0.063(8)$ & $0.101(10)$ & $0.033(8)$ & $-0.023(7)$ & $0.022(7)$ & $-0.003(8)$ \\
\hline $\mathrm{C} 29^{\prime}$ & $0.052(9)$ & $0.098(10)$ & $0.047(9)$ & $-0.001(8)$ & $0.019(7)$ & $-0.002(9)$ \\
\hline $\mathrm{C} 30^{\prime}$ & $0.049(7)$ & $0.051(7)$ & $0.045(8)$ & $0.000(5)$ & $0.009(6)$ & $0.012(7)$ \\
\hline $\mathrm{C} 31^{\prime}$ & $0.057(8)$ & $0.045(7)$ & $0.052(9)$ & $0.008(6)$ & $0.016(7)$ & $0.017(7)$ \\
\hline $\mathrm{C} 32^{\prime}$ & $0.065(9)$ & $0.048(9)$ & $0.066(11)$ & $-0.003(7)$ & $0.011(8)$ & $0.013(8)$ \\
\hline C33' & $0.051(9)$ & $0.054(8)$ & $0.084(12)$ & $-0.007(6)$ & $0.001(8)$ & $0.023(8)$ \\
\hline C34' & $0.040(7)$ & $0.050(7)$ & $0.081(11)$ & $0.009(6)$ & $0.014(7)$ & $0.016(8)$ \\
\hline $\mathrm{C} 35^{\prime}$ & $0.042(7)$ & $0.054(7)$ & $0.066(10)$ & $0.001(5)$ & $0.015(6)$ & $0.011(7)$ \\
\hline $\mathrm{C} 36^{\prime}$ & $0.048(7)$ & $0.046(7)$ & $0.063(9)$ & $0.008(6)$ & $0.017(6)$ & $0.018(6)$ \\
\hline $\mathrm{C} 37^{\prime}$ & $0.037(8)$ & $0.054(7)$ & $0.031(7)$ & $0.012(6)$ & $0.011(5)$ & $0.002(6)$ \\
\hline C $38^{\prime}$ & $0.055(10)$ & $0.059(8)$ & $0.032(8)$ & $0.007(7)$ & $0.020(6)$ & $0.003(6)$ \\
\hline $\mathrm{C} 39^{\prime}$ & $0.030(8)$ & $0.045(6)$ & $0.032(7)$ & $0.000(6)$ & $0.008(5)$ & -0.008 \\
\hline $\mathrm{C} 40^{\prime}$ & $0.046(8)$ & $0.040(6)$ & $0.031(7)$ & $-0.006(6)$ & $0.008(6)$ & -0.004 \\
\hline $\mathrm{C} 41^{\prime}$ & $0.048(9)$ & $0.043(8)$ & $0.040(8)$ & $-0.004(7)$ & $0.007(6)$ & $-0.010(6)$ \\
\hline $\mathrm{C} 42^{\prime}$ & $0.047(9)$ & $0.052(7)$ & $0.033(7)$ & $-0.001(7)$ & $0.012(6)$ & $-0.007(6)$ \\
\hline C43' & $0.038(8)$ & $0.068(8)$ & $0.025(7)$ & $0.022(7)$ & $0.003(5)$ & $0.002(6)$ \\
\hline $\mathrm{C} 44^{\prime}$ & $0.061(11)$ & $0.062(8)$ & $0.060(10)$ & $0.019(8)$ & $0.017(7)$ & $-0.014(7)$ \\
\hline $\mathrm{C} 45^{\prime}$ & $0.061(11)$ & $0.081(11)$ & $0.073(12)$ & $0.003(9)$ & $0.025(8)$ & $-0.033(8)$ \\
\hline $\mathrm{C} 46^{\prime}$ & $0.051(11)$ & $0.093(10)$ & $0.063(11)$ & $0.027(9)$ & $0.018(8)$ & $-0.017(8)$ \\
\hline $\mathrm{C} 47^{\prime}$ & $0.029(8)$ & $0.089(9)$ & $0.035(8)$ & $0.021(8)$ & $0.001(6)$ & $-0.019(7)$ \\
\hline C48' & $0.032(8)$ & $0.041(6)$ & $0.045(8)$ & $-0.003(6)$ & $0.021(6)$ & $-0.002(6)$ \\
\hline C49' & $0.035(8)$ & $0.039(7)$ & $0.044(8)$ & $0.004(6)$ & $0.019(6)$ & $0.004(6)$ \\
\hline $\mathrm{C} 50^{\prime}$ & $0.051(10)$ & $0.049(8)$ & $0.044(8)$ & $0.008(7)$ & $0.021(7)$ & $0.003(6)$ \\
\hline $\mathrm{C} 51^{\prime}$ & $0.044(9)$ & $0.067(8)$ & $0.034(8)$ & $-0.005(7)$ & $0.025(6)$ & $0.000(6)$ \\
\hline $\mathrm{C} 52^{\prime}$ & $0.063(10)$ & $0.059(8)$ & $0.040(8)$ & $0.007(8)$ & $0.029(7)$ & $0.015(7)$ \\
\hline C53' & $0.039(9)$ & $0.053(7)$ & $0.045(8)$ & $-0.008(6)$ & $0.018(6)$ & $0.006(6)$ \\
\hline C5 $54^{\prime}$ & $0.062(9)$ & $0.052(7)$ & $0.057(9)$ & $-0.010(7)$ & $0.024(6)$ & $0.004(6)$ \\
\hline $\mathrm{C} 55^{\prime}$ & $0.032(7)$ & $0.039(6)$ & $0.030(6)$ & $0.009(5)$ & $0.017(5)$ & $0.008(5)$ \\
\hline
\end{tabular}




\begin{tabular}{|c|c|c|c|c|c|c|}
\hline C56' & $0.037(6)$ & $0.037(7)$ & $0.024(6)$ & $0.006(5)$ & $0.007(5)$ & $-0.002(5)$ \\
\hline C57' & $0.039(6)$ & $0.046(6)$ & $0.015(6)$ & $-0.003(5)$ & 0.009 (4) & $0.009(5)$ \\
\hline C58 & $0.048(6)$ & $0.042(6)$ & $0.026(6)$ & $-0.001(5)$ & $0.021(5)$ & $0.014(5)$ \\
\hline C59' & $0.052(6)$ & $0.033(7)$ & $0.023(6)$ & $-0.001(5)$ & $0.022(5)$ & $-0.001(5)$ \\
\hline $\mathrm{C} 60^{\prime}$ & $0.050(5)$ & $0.049(5)$ & $0.026(6)$ & $0.010(4)$ & $0.015(4)$ & $0.003(5)$ \\
\hline C61' & $0.053(4)$ & $0.057(4)$ & $0.021(5)$ & $0.016(3)$ & $0.025(4)$ & $0.013(4)$ \\
\hline C62' & $0.068(6)$ & $0.056(4)$ & $0.044(7)$ & $0.019(4)$ & $0.031(5)$ & $0.012(4)$ \\
\hline C63' & $0.072(6)$ & $0.064(6)$ & $0.033(7)$ & $0.024(4)$ & $0.033(5)$ & $0.015(5)$ \\
\hline C64' & $0.070(5)$ & $0.076(5)$ & $0.030(7)$ & $0.022(5)$ & $0.034(5)$ & $0.018(5)$ \\
\hline $\mathrm{C} 65^{\prime}$ & $0.059(5)$ & $0.077(6)$ & $0.031(7)$ & $0.022(4)$ & $0.018(5)$ & $0.014(5)$ \\
\hline C66' & $0.039(6)$ & $0.047(6)$ & $0.026(7)$ & $0.001(5)$ & $0.012(5)$ & $0.009(5)$ \\
\hline C67' & $0.034(7)$ & $0.046(6)$ & $0.034(7)$ & $0.007(6)$ & $0.018(5)$ & $-0.001(6)$ \\
\hline C68' & $0.047(7)$ & $0.053(8)$ & $0.039(8)$ & $0.016(6)$ & $0.029(6)$ & $0.025(6)$ \\
\hline C69' & $0.038(8)$ & $0.081(8)$ & $0.037(8)$ & $0.017(7)$ & $0.012(6)$ & $0.013(7)$ \\
\hline $\mathrm{C} 70^{\prime}$ & $0.034(7)$ & $0.076(8)$ & $0.039(8)$ & $0.014(6)$ & $0.013(6)$ & $0.010(7)$ \\
\hline $\mathrm{C} 71^{\prime}$ & $0.033(6)$ & $0.051(7)$ & $0.043(8)$ & $-0.001(5)$ & $0.017(5)$ & $0.010(6)$ \\
\hline $\mathrm{C} 72^{\prime}$ & $0.046(7)$ & $0.052(7)$ & $0.048(7)$ & $-0.001(6)$ & $0.013(6)$ & $0.004(6)$ \\
\hline $\mathrm{C} 73^{\prime}$ & $0.095(10)$ & $0.049(9)$ & $0.038(7)$ & $-0.020(7)$ & $0.031(7)$ & $0.003(6)$ \\
\hline $\mathrm{C} 74^{\prime}$ & $0.104(11)$ & $0.043(10)$ & $0.060(10)$ & $-0.018(7)$ & $0.020(8)$ & $-0.007(8)$ \\
\hline $\mathrm{C} 75^{\prime}$ & $0.098(12)$ & $0.106(16)$ & $0.075(13)$ & $-0.023(10)$ & $-0.007(9)$ & $0.041(10)$ \\
\hline $\mathrm{C} 76^{\prime}$ & $0.069(12)$ & $0.077(12)$ & $0.069(11)$ & $-0.005(9)$ & $0.018(8)$ & $0.009(9)$ \\
\hline $\mathrm{C} 77^{\prime}$ & $0.083(15)$ & $0.107(16)$ & $0.081(14)$ & 0.008 (11) & $0.030(10)$ & $-0.006(10)$ \\
\hline $\mathrm{C} 78^{\prime}$ & $0.053(12)$ & $0.154(19)$ & $0.086(14)$ & $-0.006(12)$ & $0.017(9)$ & $0.015(12)$ \\
\hline $\mathrm{C} 79^{\prime}$ & $0.088(12)$ & $0.075(12)$ & $0.098(14)$ & $0.007(9)$ & $0.046(10)$ & $-0.004(11)$ \\
\hline $\mathrm{C} 85^{\prime}$ & $0.060(10)$ & $0.049(9)$ & $0.052(8)$ & $-0.006(7)$ & $0.008(7)$ & $0.004(6)$ \\
\hline $\mathrm{C} 86^{\prime}$ & $0.052(10)$ & $0.083(12)$ & $0.041(8)$ & $-0.007(8)$ & $0.004(7)$ & $0.015(7)$ \\
\hline C87' & $0.043(9)$ & $0.051(10)$ & $0.049(8)$ & $-0.009(7)$ & $0.002(6)$ & $0.014(7)$ \\
\hline C $88^{\prime}$ & $0.079(13)$ & $0.085(13)$ & $0.051(10)$ & $0.008(10)$ & $0.015(8)$ & $0.026(8)$ \\
\hline C89' & $0.083(13)$ & $0.074(12)$ & $0.060(11)$ & $-0.012(9)$ & $0.021(8)$ & $0.011(8)$ \\
\hline $\mathrm{C} 90^{\prime}$ & $0.127(18)$ & $0.088(15)$ & $0.095(15)$ & $0.010(12)$ & $0.060(12)$ & $0.033(11)$ \\
\hline C91' & $0.071(11)$ & $0.049(10)$ & $0.071(10)$ & $0.016(8)$ & $0.023(8)$ & $0.002(7)$ \\
\hline C92' & $0.128(19)$ & $0.15(2)$ & $0.094(15)$ & $0.037(15)$ & $0.062(13)$ & $0.036(13)$ \\
\hline C93' & $0.136(19)$ & $0.18(2)$ & 0.077 (14) & $0.066(15)$ & $0.037(12)$ & $0.030(13)$ \\
\hline C94' & $0.22(3)$ & $0.22(3)$ & $0.10(2)$ & 0.037 (19) & $0.07(2)$ & $0.041(17)$ \\
\hline C95' & $0.21(3)$ & $0.16(3)$ & $0.13(2)$ & 0.085 (16) & $0.06(2)$ & $0.068(19)$ \\
\hline C96' & $0.21(3)$ & $0.39(5)$ & $0.16(3)$ & $-0.05(3)$ & $0.07(2)$ & $0.07(3)$ \\
\hline C97' & $0.074(10)$ & $0.051(9)$ & $0.064(9)$ & $0.002(7)$ & $0.044(6)$ & $0.013(7)$ \\
\hline C98' & $0.068(10)$ & $0.062(10)$ & $0.050(9)$ & $0.014(7)$ & $0.038(6)$ & $0.011(7)$ \\
\hline C99' & $0.114(15)$ & $0.051(11)$ & $0.077(11)$ & $0.022(9)$ & $0.017(8)$ & $0.019(8)$ \\
\hline C200 & $0.112(16)$ & $0.14(2)$ & $0.066(12)$ & $0.042(13)$ & $0.009(9)$ & $-0.006(11)$ \\
\hline C201 & $0.25(3)$ & $0.13(2)$ & $0.086(16)$ & 0.085 (19) & $0.051(16)$ & $0.001(13)$ \\
\hline C202 & $0.34(4)$ & $0.20(3)$ & $0.20(3)$ & $0.09(3)$ & $0.19(3)$ & $0.01(2)$ \\
\hline C203 & $0.072(10)$ & $0.059(11)$ & $0.075(11)$ & $-0.019(8)$ & $0.034(7)$ & -0.013 \\
\hline C206 & $0.20(2)$ & 0.14 (2) & $0.049(11)$ & 0.015 (17) & $0.003(13)$ & -0.018 \\
\hline C207 & $0.15(2)$ & $0.085(15)$ & $0.077(12)$ & 0.008 (13) & $0.013(12)$ & -0.007 \\
\hline C208 & $0.24(3)$ & $0.19(3)$ & $0.037(11)$ & $0.04(2)$ & $0.017(14)$ & $-0.003(13)$ \\
\hline C209 & 0.047 (9) & $0.058(9)$ & $0.039(7)$ & $-0.013(7)$ & $0.001(6)$ & $0.004(6)$ \\
\hline $\mathrm{C} 210$ & $0.066(11)$ & $0.053(10)$ & $0.053(8)$ & $-0.002(8)$ & $0.017(7)$ & $-0.001(7)$ \\
\hline
\end{tabular}




\begin{tabular}{lllllll}
\hline & & & & & \\
$\mathrm{C} 211$ & $0.071(12)$ & $0.071(12)$ & $0.059(9)$ & $-0.008(9)$ & $0.015(8)$ & $-0.006(8)$ \\
$\mathrm{C} 215$ & $0.068(10)$ & $0.048(10)$ & $0.082(10)$ & $-0.006(8)$ & $0.038(9)$ & $0.017(8)$ \\
$\mathrm{C} 216$ & $0.125(17)$ & $0.122(17)$ & $0.090(11)$ & $0.003(13)$ & $0.082(12)$ & $0.007(11)$ \\
$\mathrm{C} 221$ & $0.087(14)$ & $0.128(17)$ & $0.067(12)$ & $-0.016(12)$ & $0.032(9)$ & $0.037(11)$ \\
$\mathrm{C} 222$ & $0.069(11)$ & $0.22(2)$ & $0.051(11)$ & $-0.054(11)$ & $0.027(9)$ & $-0.024(12)$ \\
$\mathrm{C} 223$ & $0.089(15)$ & $0.139(18)$ & $0.070(13)$ & $0.040(12)$ & $0.034(10)$ & $-0.014(11)$ \\
$\mathrm{C} 224$ & $0.064(9)$ & $0.17(2)$ & $0.099(15)$ & $0.062(11)$ & $0.039(10)$ & $0.051(13)$ \\
$\mathrm{C} 205$ & $0.07(2)$ & $0.16(3)$ & $0.105(19)$ & $-0.03(2)$ & $0.000(16)$ & $0.033(18)$ \\
\hline
\end{tabular}

Geometric parameters $\left(\AA,{ }^{\circ}\right)$

\begin{tabular}{|c|c|c|c|}
\hline $\mathrm{Ir} 1-\mathrm{N} 1$ & $2.041(8)$ & $\mathrm{Ir} 4-\mathrm{C} 55^{\prime}$ & $1.990(12)$ \\
\hline Ir $1-\mathrm{N} 01$ & $2.237(9)$ & $\mathrm{O} 1^{\prime}-\mathrm{C} 01^{\prime}$ & $1.191(17)$ \\
\hline Ir1-N02 & $2.222(8)$ & $\mathrm{O} 2^{\prime}-\mathrm{C} 02^{\prime}$ & $1.185(16)$ \\
\hline $\mathrm{Ir} 1-\mathrm{N} 2$ & $2.045(9)$ & $\mathrm{O}^{\prime}-\mathrm{C} 10^{\prime}$ & $1.393(16)$ \\
\hline $\mathrm{Ir} 1-\mathrm{C} 1$ & $1.981(9)$ & $\mathrm{O}^{\prime}-\mathrm{C} 221$ & $1.433(17)$ \\
\hline $\mathrm{Ir} 1-\mathrm{C} 19$ & $1.986(10)$ & $\mathrm{O} 4^{\prime}-\mathrm{C} 28^{\prime}$ & $1.404(18)$ \\
\hline Ir2-N01 & $2.255(8)$ & $\mathrm{O} 4{ }^{\prime}-\mathrm{C} 222$ & $1.48(2)$ \\
\hline Ir2-N02 & $2.211(8)$ & $\mathrm{O}^{\prime}-\mathrm{C} 46^{\prime}$ & $1.353(16)$ \\
\hline Ir2-N3 & $2.061(9)$ & $\mathrm{O} 5^{\prime}-\mathrm{C} 223$ & 1.435 (19) \\
\hline Ir2-N4 & $2.015(9)$ & $\mathrm{O} 6^{\prime}-\mathrm{C} 64^{\prime}$ & $1.394(16)$ \\
\hline Ir2-C37 & 1.969 (11) & $\mathrm{O}^{\prime}-\mathrm{C} 224$ & $1.464(19)$ \\
\hline Ir $2-\mathrm{C} 55$ & $1.990(11)$ & $\mathrm{N} 01^{\prime}-\mathrm{C} 01^{\prime}$ & $1.068(16)$ \\
\hline $\mathrm{O} 1-\mathrm{C} 01$ & $1.222(14)$ & $\mathrm{N} 1^{\prime}-\mathrm{C}^{\prime}$ & $1.365(15)$ \\
\hline $\mathrm{O} 2-\mathrm{C} 02$ & $1.190(14)$ & $\mathrm{N} 1{ }^{\prime}-\mathrm{C} 11^{\prime}$ & $1.334(15)$ \\
\hline $\mathrm{O} 3-\mathrm{C} 10$ & $1.370(13)$ & $\mathrm{N} 2^{\prime}-\mathrm{C} 25^{\prime}$ & $1.373(17)$ \\
\hline $\mathrm{O} 3-\mathrm{C} 121$ & $1.411(14)$ & $\mathrm{N} 2^{\prime}-\mathrm{C} 29^{\prime}$ & $1.404(17)$ \\
\hline $\mathrm{O} 4-\mathrm{C} 28$ & $1.346(13)$ & $\mathrm{N} 02^{\prime}-\mathrm{C} 02^{\prime}$ & $1.162(16)$ \\
\hline $\mathrm{O} 4-\mathrm{C} 122$ & $1.439(13)$ & $\mathrm{N} 3^{\prime}-\mathrm{C} 43^{\prime}$ & $1.328(16)$ \\
\hline $\mathrm{O} 5-\mathrm{C} 46$ & $1.372(13)$ & $\mathrm{N} 3^{\prime}-\mathrm{C} 47^{\prime}$ & $1.362(14)$ \\
\hline $\mathrm{O} 5-\mathrm{C} 123$ & $1.436(14)$ & $\mathrm{N} 4^{\prime}-\mathrm{C} 61^{\prime}$ & $1.365(16)$ \\
\hline $\mathrm{O} 6-\mathrm{C} 64$ & $1.370(12)$ & $\mathrm{N} 4^{\prime}-\mathrm{C} 65^{\prime}$ & $1.450(16)$ \\
\hline $\mathrm{O} 6-\mathrm{C} 124$ & $1.438(13)$ & $\mathrm{C} 1^{\prime}-\mathrm{C} 2^{\prime}$ & $1.392(16)$ \\
\hline $\mathrm{N} 1-\mathrm{C} 7$ & $1.373(13)$ & $\mathrm{C} 1^{\prime}-\mathrm{C}^{\prime}$ & $1.420(17)$ \\
\hline $\mathrm{N} 1-\mathrm{C} 11$ & $1.361(13)$ & $\mathrm{C} 2^{\prime}-\mathrm{H} 2^{\prime}$ & 0.9500 \\
\hline $\mathrm{N} 01-\mathrm{C} 01$ & $1.082(13)$ & $\mathrm{C} 2^{\prime}-\mathrm{C} 3^{\prime}$ & $1.352(15)$ \\
\hline $\mathrm{N} 02-\mathrm{C} 02$ & $1.155(13)$ & $\mathrm{C} 3^{\prime}-\mathrm{C} 4^{\prime}$ & $1.398(17)$ \\
\hline $\mathrm{N} 2-\mathrm{C} 25$ & $1.363(13)$ & $\mathrm{C} 3^{\prime}-\mathrm{C} 12^{\prime}$ & $1.471(16)$ \\
\hline $\mathrm{N} 2-\mathrm{C} 29$ & $1.373(13)$ & $\mathrm{C} 4^{\prime}-\mathrm{C}^{\prime}$ & $1.374(17)$ \\
\hline $\mathrm{N} 3-\mathrm{C} 43$ & $1.351(13)$ & $\mathrm{C} 4^{\prime}-\mathrm{C} 18^{\prime}$ & $1.540(17)$ \\
\hline $\mathrm{N} 3-\mathrm{C} 47$ & $1.353(13)$ & $\mathrm{C} 5^{\prime}-\mathrm{H} 5^{\prime}$ & 0.9500 \\
\hline $\mathrm{N} 4-\mathrm{C} 61$ & $1.363(13)$ & $\mathrm{C} 5^{\prime}-\mathrm{C}^{\prime}$ & $1.421(16)$ \\
\hline $\mathrm{N} 4-\mathrm{C} 65$ & $1.339(13)$ & $\mathrm{C} 6^{\prime}-\mathrm{C} 7^{\prime}$ & $1.448(16)$ \\
\hline $\mathrm{C} 1-\mathrm{C} 2$ & $1.418(14)$ & $\mathrm{C} 7^{\prime}-\mathrm{C} 8^{\prime}$ & $1.384(18)$ \\
\hline $\mathrm{C} 1-\mathrm{C} 6$ & $1.411(14)$ & $\mathrm{C} 8^{\prime}-\mathrm{H}^{\prime}$ & 0.9500 \\
\hline $\mathrm{C} 2-\mathrm{H} 2 \mathrm{~A}$ & 0.9500 & $\mathrm{C} 8^{\prime}-\mathrm{C} 9^{\prime}$ & $1.374(17)$ \\
\hline $\mathrm{C} 2-\mathrm{C} 3$ & $1.370(14)$ & $\mathrm{C} 9^{\prime}-\mathrm{H} 9^{\prime}$ & 0.9500 \\
\hline $\mathrm{C} 3-\mathrm{C} 4$ & $1.386(15)$ & $\mathrm{C} 9^{\prime}-\mathrm{C} 10^{\prime}$ & 1.404 (19) \\
\hline
\end{tabular}




\begin{tabular}{|c|c|c|c|}
\hline $\mathrm{C} 3-\mathrm{C} 12$ & $1.482(15)$ & $\mathrm{C} 10^{\prime}-\mathrm{C} 11^{\prime}$ & $1.384(19)$ \\
\hline $\mathrm{C} 4-\mathrm{C} 5$ & $1.387(15)$ & $\mathrm{C} 11^{\prime}-\mathrm{H} 11^{\prime}$ & 0.9500 \\
\hline $\mathrm{C} 4-\mathrm{C} 18$ & $1.537(15)$ & $\mathrm{C} 12^{\prime}-\mathrm{C} 13^{\prime}$ & $1.369(16)$ \\
\hline $\mathrm{C} 5-\mathrm{H} 5 \mathrm{~A}$ & 0.9500 & $\mathrm{C} 12^{\prime}-\mathrm{C} 17^{\prime}$ & $1.419(16)$ \\
\hline $\mathrm{C} 5-\mathrm{C} 6$ & $1.410(14)$ & $\mathrm{C}^{\prime} 3^{\prime}-\mathrm{H} 13^{\prime}$ & 0.9500 \\
\hline $\mathrm{C} 6-\mathrm{C} 7$ & $1.463(15)$ & $\mathrm{C} 13^{\prime}-\mathrm{C} 14^{\prime}$ & $1.410(16)$ \\
\hline $\mathrm{C} 7-\mathrm{C} 8$ & $1.386(15)$ & C14'-H14' & 0.9500 \\
\hline $\mathrm{C} 8-\mathrm{H} 8$ & 0.9500 & $\mathrm{C} 14^{\prime}-\mathrm{C} 15^{\prime}$ & $1.363(17)$ \\
\hline $\mathrm{C} 8-\mathrm{C} 9$ & $1.338(15)$ & $\mathrm{C} 15^{\prime}-\mathrm{H} 15^{\prime}$ & 0.9500 \\
\hline C9-H9 & 0.9500 & $\mathrm{C} 15^{\prime}-\mathrm{C} 16^{\prime}$ & $1.354(18)$ \\
\hline $\mathrm{C} 9-\mathrm{C} 10$ & 1.399 (16) & $\mathrm{C} 16^{\prime}-\mathrm{H} 16^{\prime}$ & 0.9500 \\
\hline $\mathrm{C} 10-\mathrm{C} 11$ & $1.376(15)$ & $\mathrm{C} 16^{\prime}-\mathrm{C} 17^{\prime}$ & $1.388(17)$ \\
\hline C11-H11 & 0.9500 & $\mathrm{C} 17^{\prime}-\mathrm{C} 18^{\prime}$ & $1.539(18)$ \\
\hline $\mathrm{C} 12-\mathrm{C} 13$ & $1.354(15)$ & $\mathrm{C} 18^{\prime}-\mathrm{C} 73^{\prime}$ & 1.601 (19) \\
\hline $\mathrm{C} 12-\mathrm{C} 17$ & $1.391(15)$ & $\mathrm{C} 18^{\prime}-\mathrm{C} 79^{\prime}$ & $1.480(19)$ \\
\hline $\mathrm{C} 13-\mathrm{H} 13$ & 0.9500 & $\mathrm{C} 19^{\prime}-\mathrm{C} 20^{\prime}$ & $1.379(16)$ \\
\hline $\mathrm{C} 13-\mathrm{C} 14$ & $1.377(15)$ & $\mathrm{C} 19^{\prime}-\mathrm{C} 24^{\prime}$ & 1.417 (17) \\
\hline C14-H14 & 0.9500 & $\mathrm{C} 20^{\prime}-\mathrm{H} 20^{\prime}$ & 0.9500 \\
\hline $\mathrm{C} 14-\mathrm{C} 15$ & $1.406(16)$ & $\mathrm{C} 20^{\prime}-\mathrm{C} 21^{\prime}$ & $1.415(17)$ \\
\hline C15-H15 & 0.9500 & $\mathrm{C} 21^{\prime}-\mathrm{C} 22^{\prime}$ & $1.364(18)$ \\
\hline $\mathrm{C} 15-\mathrm{C} 16$ & $1.361(17)$ & $\mathrm{C} 21^{\prime}-\mathrm{C} 30^{\prime}$ & $1.483(18)$ \\
\hline C16-H16 & 0.9500 & $\mathrm{C} 22^{\prime}-\mathrm{C} 23^{\prime}$ & $1.395(17)$ \\
\hline $\mathrm{C} 16-\mathrm{C} 17$ & 1.379 (15) & $\mathrm{C} 22^{\prime}-\mathrm{C} 36^{\prime}$ & $1.522(18)$ \\
\hline $\mathrm{C} 17-\mathrm{C} 18$ & $1.536(16)$ & $\mathrm{C} 23^{\prime}-\mathrm{H} 23^{\prime}$ & 0.9500 \\
\hline $\mathrm{C} 18-\mathrm{C} 73$ & $1.533(11)$ & $\mathrm{C} 23^{\prime}-\mathrm{C} 24^{\prime}$ & $1.394(17)$ \\
\hline $\mathrm{C} 18-\mathrm{C} 79$ & $1.523(15)$ & $\mathrm{C} 24^{\prime}-\mathrm{C} 25^{\prime}$ & $1.446(17)$ \\
\hline $\mathrm{C} 19-\mathrm{C} 20$ & $1.390(14)$ & $\mathrm{C} 25^{\prime}-\mathrm{C} 26^{\prime}$ & $1.426(19)$ \\
\hline $\mathrm{C} 19-\mathrm{C} 24$ & $1.429(15)$ & $\mathrm{C} 26^{\prime}-\mathrm{H} 26^{\prime}$ & 0.9500 \\
\hline $\mathrm{C} 20-\mathrm{H} 6$ & 0.9500 & $\mathrm{C} 26^{\prime}-\mathrm{C} 27^{\prime}$ & 1.429 (19) \\
\hline $\mathrm{C} 20-\mathrm{C} 21$ & $1.379(14)$ & $\mathrm{C} 27^{\prime}-\mathrm{H} 27^{\prime}$ & 0.9500 \\
\hline $\mathrm{C} 21-\mathrm{C} 22$ & $1.383(14)$ & $\mathrm{C} 27^{\prime}-\mathrm{C} 28^{\prime}$ & $1.40(2)$ \\
\hline $\mathrm{C} 21-\mathrm{C} 30$ & $1.502(14)$ & $\mathrm{C} 28^{\prime}-\mathrm{C} 29^{\prime}$ & $1.35(2)$ \\
\hline $\mathrm{C} 22-\mathrm{C} 23$ & $1.379(14)$ & $\mathrm{C} 29^{\prime}-\mathrm{H} 29^{\prime}$ & 0.9500 \\
\hline $\mathrm{C} 22-\mathrm{C} 36$ & $1.546(14)$ & $\mathrm{C} 30^{\prime}-\mathrm{C} 31^{\prime}$ & $1.404(18)$ \\
\hline $\mathrm{C} 23-\mathrm{H} 23$ & 0.9500 & $\mathrm{C} 30^{\prime}-\mathrm{C} 35^{\prime}$ & $1.450(18)$ \\
\hline $\mathrm{C} 23-\mathrm{C} 24$ & 1.407 (13) & $\mathrm{C} 31^{\prime}-\mathrm{H} 31^{\prime}$ & 0.9500 \\
\hline $\mathrm{C} 24-\mathrm{C} 25$ & $1.469(14)$ & $\mathrm{C} 31^{\prime}-\mathrm{C} 32^{\prime}$ & $1.367(18)$ \\
\hline $\mathrm{C} 25-\mathrm{C} 26$ & $1.382(15)$ & $\mathrm{C} 32^{\prime}-\mathrm{H} 32^{\prime}$ & 0.9500 \\
\hline $\mathrm{C} 26-\mathrm{H} 26$ & 0.9500 & $\mathrm{C} 32^{\prime}-\mathrm{C} 33^{\prime}$ & $1.360(18)$ \\
\hline $\mathrm{C} 26-\mathrm{C} 27$ & $1.376(14)$ & $\mathrm{C} 33^{\prime}-\mathrm{H} 33^{\prime}$ & 0.9500 \\
\hline C27-H27 & 0.9500 & C $33^{\prime}-\mathrm{C} 34^{\prime}$ & $1.371(18)$ \\
\hline $\mathrm{C} 27-\mathrm{C} 28$ & $1.392(15)$ & C $34^{\prime}-\mathrm{H} 34^{\prime}$ & 0.9500 \\
\hline $\mathrm{C} 28-\mathrm{C} 29$ & $1.367(15)$ & $\mathrm{C} 34^{\prime}-\mathrm{C} 35^{\prime}$ & $1.374(18)$ \\
\hline С29-H29 & 0.9500 & $\mathrm{C} 35^{\prime}-\mathrm{C} 36^{\prime}$ & 1.495 (19) \\
\hline $\mathrm{C} 30-\mathrm{C} 31$ & $1.373(15)$ & $\mathrm{C} 36^{\prime}-\mathrm{C} 85^{\prime}$ & $1.516(17)$ \\
\hline $\mathrm{C} 30-\mathrm{C} 35$ & $1.405(14)$ & $\mathrm{C} 36^{\prime}-\mathrm{C} 91^{\prime}$ & $1.534(18)$ \\
\hline C $31-\mathrm{H} 31$ & 0.9500 & $\mathrm{C} 37^{\prime}-\mathrm{C} 38^{\prime}$ & $1.432(16)$ \\
\hline $\mathrm{C} 31-\mathrm{C} 32$ & $1.388(14)$ & $\mathrm{C} 37^{\prime}-\mathrm{C} 42^{\prime}$ & $1.427(18)$ \\
\hline
\end{tabular}




\begin{tabular}{|c|c|c|c|}
\hline $\mathrm{C} 32-\mathrm{H} 32$ & 0.9500 & $\mathrm{C} 38^{\prime}-\mathrm{H} 38^{\prime}$ & 0.9500 \\
\hline $\mathrm{C} 32-\mathrm{C} 33$ & $1.365(14)$ & $\mathrm{C} 38^{\prime}-\mathrm{C} 39^{\prime}$ & $1.392(16)$ \\
\hline $\mathrm{C} 33-\mathrm{H} 33$ & 0.9500 & $\mathrm{C} 39^{\prime}-\mathrm{C} 40^{\prime}$ & $1.383(17)$ \\
\hline $\mathrm{C} 33-\mathrm{C} 34$ & $1.374(15)$ & $\mathrm{C} 39^{\prime}-\mathrm{C} 48^{\prime}$ & $1.478(16)$ \\
\hline C34-H34 & 0.9500 & $\mathrm{C} 40^{\prime}-\mathrm{C} 41^{\prime}$ & $1.377(16)$ \\
\hline $\mathrm{C} 34-\mathrm{C} 35$ & $1.398(14)$ & $\mathrm{C} 40^{\prime}-\mathrm{C} 54^{\prime}$ & $1.530(17)$ \\
\hline $\mathrm{C} 35-\mathrm{C} 36$ & $1.498(15)$ & $\mathrm{C} 41^{\prime}-\mathrm{H} 41^{\prime}$ & 0.9500 \\
\hline $\mathrm{C} 36-\mathrm{C} 85$ & $1.541(10)$ & $\mathrm{C} 41^{\prime}-\mathrm{C} 42^{\prime}$ & $1.430(17)$ \\
\hline C36-C91 & $1.538(15)$ & $\mathrm{C} 42^{\prime}-\mathrm{C} 43^{\prime}$ & $1.444(17)$ \\
\hline C37-C38 & $1.394(14)$ & $\mathrm{C} 43^{\prime}-\mathrm{C} 44^{\prime}$ & $1.414(18)$ \\
\hline $\mathrm{C} 37-\mathrm{C} 42$ & $1.443(15)$ & $\mathrm{C} 44^{\prime}-\mathrm{H} 44^{\prime}$ & 0.9500 \\
\hline C38-H38 & 0.9500 & $\mathrm{C} 44^{\prime}-\mathrm{C} 45^{\prime}$ & $1.390(18)$ \\
\hline C38-C39 & $1.411(14)$ & $\mathrm{C} 45^{\prime}-\mathrm{H} 45^{\prime}$ & 0.9500 \\
\hline $\mathrm{C} 39-\mathrm{C} 40$ & $1.409(15)$ & $\mathrm{C} 45^{\prime}-\mathrm{C} 46^{\prime}$ & $1.38(2)$ \\
\hline $\mathrm{C} 39-\mathrm{C} 48$ & $1.439(15)$ & $\mathrm{C} 46^{\prime}-\mathrm{C} 47^{\prime}$ & $1.33(2)$ \\
\hline $\mathrm{C} 40-\mathrm{C} 41$ & $1.386(15)$ & $\mathrm{C} 47^{\prime}-\mathrm{H} 47^{\prime}$ & 0.9500 \\
\hline $\mathrm{C} 40-\mathrm{C} 54$ & $1.525(15)$ & $\mathrm{C} 48^{\prime}-\mathrm{C} 49^{\prime}$ & $1.390(16)$ \\
\hline $\mathrm{C} 41-\mathrm{H} 41$ & 0.9500 & $\mathrm{C} 48^{\prime}-\mathrm{C} 53^{\prime}$ & $1.382(16)$ \\
\hline $\mathrm{C} 41-\mathrm{C} 42$ & $1.418(14)$ & $\mathrm{C} 49^{\prime}-\mathrm{H} 49^{\prime}$ & 0.9500 \\
\hline $\mathrm{C} 42-\mathrm{C} 43$ & $1.453(15)$ & $\mathrm{C} 49^{\prime}-\mathrm{C} 50^{\prime}$ & $1.435(16)$ \\
\hline $\mathrm{C} 43-\mathrm{C} 44$ & $1.384(15)$ & $\mathrm{C} 50^{\prime}-\mathrm{H} 50^{\prime}$ & 0.9500 \\
\hline $\mathrm{C} 44-\mathrm{H} 44$ & 0.9500 & $\mathrm{C} 50^{\prime}-\mathrm{C} 51^{\prime}$ & $1.331(17)$ \\
\hline $\mathrm{C} 44-\mathrm{C} 45$ & $1.367(15)$ & $\mathrm{C} 51^{\prime}-\mathrm{H} 51^{\prime}$ & 0.9500 \\
\hline $\mathrm{C} 45-\mathrm{H} 45$ & 0.9500 & $\mathrm{C} 51^{\prime}-\mathrm{C} 52^{\prime}$ & $1.395(18)$ \\
\hline $\mathrm{C} 45-\mathrm{C} 46$ & $1.395(16)$ & C52'-H52' & 0.9500 \\
\hline $\mathrm{C} 46-\mathrm{C} 47$ & $1.386(16)$ & $\mathrm{C} 52^{\prime}-\mathrm{C} 53^{\prime}$ & $1.382(16)$ \\
\hline $\mathrm{C} 47-\mathrm{H} 47$ & 0.9500 & $\mathrm{C} 53^{\prime}-\mathrm{C} 54^{\prime}$ & $1.542(18)$ \\
\hline C48-C49 & $1.378(15)$ & C54'-C97' & $1.564(18)$ \\
\hline $\mathrm{C} 48-\mathrm{C} 53$ & $1.394(16)$ & $\mathrm{C} 54^{\prime}-\mathrm{C} 203$ & $1.551(12)$ \\
\hline $\mathrm{C} 49-\mathrm{H} 49$ & 0.9500 & $\mathrm{C} 55^{\prime}-\mathrm{C} 56^{\prime}$ & $1.404(15)$ \\
\hline $\mathrm{C} 49-\mathrm{C} 50$ & $1.417(16)$ & $\mathrm{C} 55^{\prime}-\mathrm{C} 60^{\prime}$ & $1.417(17)$ \\
\hline $\mathrm{C} 50-\mathrm{H} 50$ & 0.9500 & $\mathrm{C} 56^{\prime}-\mathrm{H} 56^{\prime}$ & 0.9500 \\
\hline $\mathrm{C} 50-\mathrm{C} 51$ & $1.380(17)$ & $\mathrm{C} 56^{\prime}-\mathrm{C} 57^{\prime}$ & $1.383(15)$ \\
\hline C51-H51 & 0.9500 & $\mathrm{C} 57^{\prime}-\mathrm{C} 58^{\prime}$ & $1.394(16)$ \\
\hline C51-C52 & $1.371(18)$ & $\mathrm{C} 57^{\prime}-\mathrm{C} 66^{\prime}$ & $1.453(16)$ \\
\hline C52-H52 & 0.9500 & $\mathrm{C} 58^{\prime}-\mathrm{C} 59^{\prime}$ & $1.390(16)$ \\
\hline $\mathrm{C} 52-\mathrm{C} 53$ & $1.366(16)$ & $\mathrm{C} 58^{\prime}-\mathrm{C} 72^{\prime}$ & $1.525(17)$ \\
\hline C53-C54 & $1.520(16)$ & $\mathrm{C} 59^{\prime}-\mathrm{H} 59^{\prime}$ & 0.9500 \\
\hline C54-C97 & $1.531(11)$ & $\mathrm{C} 59^{\prime}-\mathrm{C} 60^{\prime}$ & $1.405(16)$ \\
\hline $\mathrm{C} 54-\mathrm{C} 103$ & $1.543(11)$ & $\mathrm{C} 60^{\prime}-\mathrm{C} 61^{\prime}$ & $1.479(17)$ \\
\hline $\mathrm{C} 55-\mathrm{C} 56$ & $1.439(14)$ & $\mathrm{C} 61^{\prime}-\mathrm{C} 62^{\prime}$ & $1.390(18)$ \\
\hline $\mathrm{C} 55-\mathrm{C} 60$ & $1.406(14)$ & $\mathrm{C} 62^{\prime}-\mathrm{H} 62^{\prime}$ & 0.9500 \\
\hline C56-H56 & 0.9500 & $\mathrm{C} 62^{\prime}-\mathrm{C} 63^{\prime}$ & $1.403(17)$ \\
\hline C56-C57 & $1.397(14)$ & C63'-H63' & 0.9500 \\
\hline C57-C58 & $1.396(15)$ & $\mathrm{C} 63^{\prime}-\mathrm{C} 64^{\prime}$ & $1.305(19)$ \\
\hline C57-C66 & $1.470(14)$ & $\mathrm{C} 64^{\prime}-\mathrm{C} 65^{\prime}$ & $1.377(19)$ \\
\hline C58-C59 & $1.389(14)$ & $\mathrm{C} 65^{\prime}-\mathrm{H} 65^{\prime}$ & 0.9500 \\
\hline $\mathrm{C} 58-\mathrm{C} 72$ & $1.521(15)$ & $\mathrm{C} 66^{\prime}-\mathrm{C} 67^{\prime}$ & $1.394(16)$ \\
\hline
\end{tabular}




\begin{tabular}{|c|c|c|c|}
\hline $\mathrm{C} 59-\mathrm{H} 59$ & 0.9500 & $\mathrm{C} 66^{\prime}-\mathrm{C} 71^{\prime}$ & $1.413(17)$ \\
\hline $\mathrm{C} 59-\mathrm{C} 60$ & $1.409(14)$ & C67'-H67' & 0.9500 \\
\hline $\mathrm{C} 60-\mathrm{C} 61$ & $1.471(14)$ & C67'-C68' & $1.374(15)$ \\
\hline C61-C62 & $1.390(15)$ & C68'-H68' & 0.9500 \\
\hline C62-H62 & 0.9500 & $\mathrm{C} 68^{\prime}-\mathrm{C} 69^{\prime}$ & $1.389(17)$ \\
\hline C62-C63 & $1.382(15)$ & C69'-H69' & 0.9500 \\
\hline C63-H63 & 0.9500 & $\mathrm{C} 69^{\prime}-\mathrm{C} 70^{\prime}$ & $1.392(18)$ \\
\hline C63-C64 & $1.389(16)$ & $\mathrm{C} 70^{\prime}-\mathrm{H} 70^{\prime}$ & 0.9500 \\
\hline C64-C65 & $1.371(15)$ & $\mathrm{C} 70^{\prime}-\mathrm{C} 71^{\prime}$ & $1.382(16)$ \\
\hline C65-H65 & 0.9500 & $\mathrm{C} 71^{\prime}-\mathrm{C} 72^{\prime}$ & $1.515(18)$ \\
\hline C66-C67 & $1.383(15)$ & $\mathrm{C} 72^{\prime}-\mathrm{C} 209$ & $1.551(17)$ \\
\hline $\mathrm{C} 66-\mathrm{C} 71$ & $1.399(15)$ & $\mathrm{C} 72^{\prime}-\mathrm{C} 215$ & $1.551(17)$ \\
\hline C67-H67 & 0.9500 & $\mathrm{C} 73^{\prime}-\mathrm{H} 73 \mathrm{~A}$ & 0.9900 \\
\hline C67-C68 & $1.376(15)$ & $\mathrm{C} 73^{\prime}-\mathrm{H} 73 \mathrm{~B}$ & 0.9900 \\
\hline C68-H68 & 0.9500 & $\mathrm{C} 73^{\prime}-\mathrm{C} 74^{\prime}$ & $1.482(19)$ \\
\hline C68-C69 & $1.396(17)$ & $\mathrm{C} 74^{\prime}-\mathrm{H} 74 \mathrm{~A}$ & 0.9900 \\
\hline C69-H69 & 0.9500 & C74'-H74B & 0.9900 \\
\hline C69-C70 & $1.366(17)$ & $\mathrm{C} 74^{\prime}-\mathrm{C} 75^{\prime}$ & $1.52(2)$ \\
\hline $\mathrm{C} 70-\mathrm{H} 70$ & 0.9500 & $\mathrm{C} 75^{\prime}-\mathrm{H} 75 \mathrm{~A}$ & 0.9900 \\
\hline $\mathrm{C} 70-\mathrm{C} 71$ & $1.374(15)$ & $\mathrm{C} 75^{\prime}-\mathrm{H} 75 \mathrm{~B}$ & 0.9900 \\
\hline $\mathrm{C} 71-\mathrm{C} 72$ & $1.515(15)$ & $\mathrm{C} 75^{\prime}-\mathrm{C} 76^{\prime}$ & 1.497 (19) \\
\hline $\mathrm{C} 72-\mathrm{C} 109$ & $1.532(11)$ & $\mathrm{C} 76^{\prime}-\mathrm{H} 76 \mathrm{~A}$ & 0.9900 \\
\hline $\mathrm{C} 72-\mathrm{C} 115$ & $1.529(11)$ & $\mathrm{C} 76^{\prime}-\mathrm{H} 76 \mathrm{~B}$ & 0.9900 \\
\hline $\mathrm{C} 73-\mathrm{H} 73 \mathrm{C}$ & 0.9900 & $\mathrm{C} 76^{\prime}-\mathrm{C} 77^{\prime}$ & $1.512(16)$ \\
\hline C73-H73D & 0.9900 & $\mathrm{C} 77^{\prime}-\mathrm{H} 77 \mathrm{~A}$ & 0.9900 \\
\hline $\mathrm{C} 73-\mathrm{C} 74$ & $1.523(11)$ & C77'-H77B & 0.9900 \\
\hline $\mathrm{C} 74-\mathrm{H} 74 \mathrm{C}$ & 0.9900 & $\mathrm{C} 77^{\prime}-\mathrm{C} 78^{\prime}$ & $1.487(16)$ \\
\hline C74-H74D & 0.9900 & $\mathrm{C} 78^{\prime}-\mathrm{H} 78 \mathrm{~A}$ & 0.9800 \\
\hline $\mathrm{C} 74-\mathrm{C} 75$ & $1.531(12)$ & $\mathrm{C} 78^{\prime}-\mathrm{H} 78 \mathrm{~B}$ & 0.9800 \\
\hline $\mathrm{C} 75-\mathrm{H} 75 \mathrm{C}$ & 0.9900 & $\mathrm{C} 78^{\prime}-\mathrm{H} 78 \mathrm{C}$ & 0.9800 \\
\hline C75-H75D & 0.9900 & $\mathrm{C} 79^{\prime}-\mathrm{H} 79 \mathrm{C}$ & 0.9900 \\
\hline $\mathrm{C} 75-\mathrm{C} 76$ & $1.518(14)$ & $\mathrm{C} 79^{\prime}-\mathrm{H} 79 \mathrm{D}$ & 0.9900 \\
\hline $\mathrm{C} 76-\mathrm{H} 76 \mathrm{C}$ & 0.9900 & $\mathrm{C} 79^{\prime}-\mathrm{H} 79 \mathrm{~A}$ & 0.9900 \\
\hline C76-H76D & 0.9900 & $\mathrm{C} 79^{\prime}-\mathrm{H} 79 \mathrm{~B}$ & 0.9900 \\
\hline $\mathrm{C} 76-\mathrm{C} 77$ & $1.576(14)$ & $\mathrm{C} 79^{\prime}-\mathrm{C} 80^{\prime}$ & $1.498(16)$ \\
\hline C77-H77C & 0.9900 & $\mathrm{C} 79^{\prime}-\mathrm{C} 80 \mathrm{~B}$ & $1.548(19)$ \\
\hline C77-H77D & 0.9900 & $\mathrm{C} 81^{\prime}-\mathrm{H} 81 \mathrm{C}$ & 0.9900 \\
\hline C77-C78 & $1.534(15)$ & $\mathrm{C} 81^{\prime}-\mathrm{H} 81 \mathrm{D}$ & 0.9900 \\
\hline C78-H78D & 0.9800 & $\mathrm{C} 81^{\prime}-\mathrm{H} 81 \mathrm{~A}$ & 0.9900 \\
\hline C78-H78E & 0.9800 & $\mathrm{C} 81^{\prime}-\mathrm{H} 81 \mathrm{~B}$ & 0.9900 \\
\hline $\mathrm{C} 78-\mathrm{H} 78 \mathrm{~F}$ & 0.9800 & $\mathrm{C} 81^{\prime}-\mathrm{C} 82^{\prime}$ & $1.608(18)$ \\
\hline C79-H79E & 0.9900 & $\mathrm{C} 81^{\prime}-\mathrm{C} 80^{\prime}$ & $1.576(18)$ \\
\hline C79-H79F & 0.9900 & $\mathrm{C} 81^{\prime}-\mathrm{C} 80 \mathrm{~B}$ & $1.550(19)$ \\
\hline $\mathrm{C} 79-\mathrm{C} 80$ & $1.523(15)$ & $\mathrm{C} 82^{\prime}-\mathrm{H} 82 \mathrm{~A}$ & 0.9900 \\
\hline $\mathrm{C} 80-\mathrm{H} 80 \mathrm{E}$ & 0.9900 & $\mathrm{C} 82^{\prime}-\mathrm{H} 82 \mathrm{~B}$ & 0.9900 \\
\hline $\mathrm{C} 80-\mathrm{H} 80 \mathrm{~F}$ & 0.9900 & $\mathrm{C} 82^{\prime}-\mathrm{C} 83^{\prime}$ & $1.546(18)$ \\
\hline $\mathrm{C} 80-\mathrm{C} 81$ & $1.519(16)$ & $\mathrm{C} 83^{\prime}-\mathrm{H} 83 \mathrm{~A}$ & 0.9900 \\
\hline C81-H81E & 0.9900 & $\mathrm{C} 83^{\prime}-\mathrm{H} 83 \mathrm{~B}$ & 0.9900 \\
\hline
\end{tabular}




\begin{tabular}{|c|c|c|c|}
\hline $\mathrm{C} 81-\mathrm{H} 81 \mathrm{~F}$ & 0.9900 & $\mathrm{C} 83^{\prime}-\mathrm{C} 84^{\prime}$ & $1.521(17)$ \\
\hline $\mathrm{C} 81-\mathrm{C} 82$ & $1.516(17)$ & $\mathrm{C} 84^{\prime}-\mathrm{H} 84 \mathrm{~A}$ & 0.9800 \\
\hline $\mathrm{C} 82-\mathrm{H} 82 \mathrm{C}$ & 0.9900 & $\mathrm{C} 84^{\prime}-\mathrm{H} 84 \mathrm{~B}$ & 0.9800 \\
\hline $\mathrm{C} 82-\mathrm{H} 82 \mathrm{D}$ & 0.9900 & $\mathrm{C} 84^{\prime}-\mathrm{H} 84 \mathrm{C}$ & 0.9800 \\
\hline $\mathrm{C} 82-\mathrm{C} 83$ & $1.512(18)$ & $\mathrm{C} 85^{\prime}-\mathrm{H} 85 \mathrm{~A}$ & 0.9900 \\
\hline $\mathrm{C} 83-\mathrm{H} 83 \mathrm{C}$ & 0.9900 & $\mathrm{C} 85^{\prime}-\mathrm{H} 85 \mathrm{~B}$ & 0.9900 \\
\hline $\mathrm{C} 83-\mathrm{H} 83 \mathrm{D}$ & 0.9900 & $\mathrm{C} 85^{\prime}-\mathrm{C} 86^{\prime}$ & $1.528(18)$ \\
\hline $\mathrm{C} 83-\mathrm{C} 84$ & $1.53(2)$ & $\mathrm{C} 86^{\prime}-\mathrm{H} 86 \mathrm{~A}$ & 0.9900 \\
\hline C $84-H 84 D$ & 0.9800 & $\mathrm{C} 86^{\prime}-\mathrm{H} 86 \mathrm{~B}$ & 0.9900 \\
\hline C84-H84E & 0.9800 & $\mathrm{C} 86^{\prime}-\mathrm{C} 87^{\prime}$ & $1.507(16)$ \\
\hline $\mathrm{C} 84-\mathrm{H} 84 \mathrm{~F}$ & 0.9800 & $\mathrm{C} 87^{\prime}-\mathrm{H} 87 \mathrm{~A}$ & 0.9900 \\
\hline $\mathrm{C} 85-\mathrm{H} 85 \mathrm{C}$ & 0.9900 & $\mathrm{C} 87^{\prime}-\mathrm{H} 87 \mathrm{~B}$ & 0.9900 \\
\hline C $85-H 85 D$ & 0.9900 & $\mathrm{C} 87^{\prime}-\mathrm{C} 88^{\prime}$ & $1.506(18)$ \\
\hline $\mathrm{C} 85-\mathrm{C} 86$ & $1.506(10)$ & $\mathrm{C} 88^{\prime}-\mathrm{H} 88 \mathrm{~A}$ & 0.9900 \\
\hline $\mathrm{C} 86-\mathrm{H} 86 \mathrm{C}$ & 0.9900 & $\mathrm{C} 88^{\prime}-\mathrm{H} 88 \mathrm{~B}$ & 0.9900 \\
\hline C86-H86D & 0.9900 & $\mathrm{C} 88^{\prime}-\mathrm{C} 89^{\prime}$ & $1.513(18)$ \\
\hline $\mathrm{C} 86-\mathrm{C} 87$ & $1.515(10)$ & $\mathrm{C} 89^{\prime}-\mathrm{H} 89 \mathrm{~A}$ & 0.9900 \\
\hline C87-H87C & 0.9900 & C89'-H89B & 0.9900 \\
\hline C87-H87D & 0.9900 & $\mathrm{C} 89^{\prime}-\mathrm{C} 90^{\prime}$ & 1.504 (19) \\
\hline C87-C88 & $1.529(11)$ & $\mathrm{C} 90^{\prime}-\mathrm{H} 90 \mathrm{~A}$ & 0.9800 \\
\hline C $88-\mathrm{H} 88 \mathrm{C}$ & 0.9900 & C90'-H90B & 0.9800 \\
\hline C88-H88D & 0.9900 & $\mathrm{C} 90^{\prime}-\mathrm{H} 90 \mathrm{C}$ & 0.9800 \\
\hline $\mathrm{C} 88-\mathrm{C} 89$ & $1.520(11)$ & C91'-H91A & 0.9900 \\
\hline C89-H89C & 0.9900 & C91'-H91B & 0.9900 \\
\hline C89-H89D & 0.9900 & C $91^{\prime}-\mathrm{C} 92^{\prime}$ & $1.51(2)$ \\
\hline $\mathrm{C} 89-\mathrm{C} 90$ & $1.527(11)$ & $\mathrm{C} 92^{\prime}-\mathrm{H} 92 \mathrm{~A}$ & 0.9900 \\
\hline C90-H90D & 0.9800 & С92'-H92B & 0.9900 \\
\hline C90-H90E & 0.9800 & C92'-C93' & $1.41(2)$ \\
\hline C90-H90F & 0.9800 & C93'-H93A & 0.9900 \\
\hline C91-H91C & 0.9900 & C93'-H93B & 0.9900 \\
\hline C91-H91D & 0.9900 & C93'-C94' & $1.49(3)$ \\
\hline C91-C92 & $1.516(14)$ & C94'-H94A & 0.9900 \\
\hline C92- $\mathrm{H} 92 \mathrm{C}$ & 0.9900 & C94'-H94B & 0.9900 \\
\hline C92-H92D & 0.9900 & C94'-C95' & $1.62(3)$ \\
\hline $\mathrm{C} 92-\mathrm{C} 93$ & $1.541(14)$ & $\mathrm{C} 95^{\prime}-\mathrm{H} 95 \mathrm{~A}$ & 0.9900 \\
\hline C93-H93C & 0.9900 & C95'-H95B & 0.9900 \\
\hline C93-H93D & 0.9900 & $\mathrm{C} 95^{\prime}-\mathrm{C} 96^{\prime}$ & $1.53(3)$ \\
\hline C93-C94 & $1.478(15)$ & $\mathrm{C} 96^{\prime}-\mathrm{H} 96 \mathrm{~A}$ & 0.9800 \\
\hline C94-H94C & 0.9900 & $\mathrm{C} 96^{\prime}-\mathrm{H} 96 \mathrm{~B}$ & 0.9800 \\
\hline C94-H94D & 0.9900 & $\mathrm{C} 96^{\prime}-\mathrm{H} 96 \mathrm{C}$ & 0.9800 \\
\hline C94-C95 & $1.525(18)$ & C97'-H97A & 0.9900 \\
\hline C95-H95C & 0.9900 & C97'-H97B & 0.9900 \\
\hline C95-H95D & 0.9900 & C97'-C98' & $1.510(18)$ \\
\hline C95-C96 & $1.53(2)$ & C98'-H98A & 0.9900 \\
\hline C96-H96D & 0.9800 & C98'-H98B & 0.9900 \\
\hline C96-H96E & 0.9800 & C98'-C99' & $1.531(18)$ \\
\hline C96-H96F & 0.9800 & C99'-H99A & 0.9900 \\
\hline C97-H97C & 0.9900 & C99'-H99B & 0.9900 \\
\hline
\end{tabular}




\begin{tabular}{|c|c|c|c|}
\hline C97-H97D & 0.9900 & $\mathrm{C} 99^{\prime}-\mathrm{C} 200$ & $1.51(2)$ \\
\hline C97-C98 & $1.526(11)$ & $\mathrm{C} 200-\mathrm{H} 20 \mathrm{~A}$ & 0.9900 \\
\hline C98-H98C & 0.9900 & $\mathrm{C} 200-\mathrm{H} 20 \mathrm{~B}$ & 0.9900 \\
\hline C98-H98D & 0.9900 & $\mathrm{C} 200-\mathrm{C} 201$ & $1.52(2)$ \\
\hline C98-C99 & $1.534(11)$ & $\mathrm{C} 201-\mathrm{H} 20 \mathrm{C}$ & 0.9900 \\
\hline C99-H99C & 0.9900 & $\mathrm{C} 201-\mathrm{H} 20 \mathrm{D}$ & 0.9900 \\
\hline C99-H99D & 0.9900 & C201-C202 & $1.65(3)$ \\
\hline $\mathrm{C} 99-\mathrm{C} 100$ & $1.478(12)$ & $\mathrm{C} 202-\mathrm{H} 20 \mathrm{E}$ & 0.9800 \\
\hline $\mathrm{C} 100-\mathrm{H} 10 \mathrm{C}$ & 0.9900 & $\mathrm{C} 202-\mathrm{H} 20 \mathrm{~F}$ & 0.9800 \\
\hline $\mathrm{C} 100-\mathrm{H} 10 \mathrm{D}$ & 0.9900 & $\mathrm{C} 202-\mathrm{H} 20 \mathrm{G}$ & 0.9800 \\
\hline $\mathrm{C} 100-\mathrm{H} 10 \mathrm{~A}$ & 0.9900 & $\mathrm{C} 203-\mathrm{H} 20 \mathrm{H}$ & 0.9900 \\
\hline $\mathrm{C} 100-\mathrm{H} 10 \mathrm{~B}$ & 0.9900 & C203-H20I & 0.9900 \\
\hline $\mathrm{C} 100-\mathrm{C} 101$ & $1.526(17)$ & $\mathrm{C} 203-\mathrm{H} 20 \mathrm{~J}$ & 0.9900 \\
\hline $\mathrm{C} 100-\mathrm{C} 126$ & $1.526(12)$ & $\mathrm{C} 203-\mathrm{H} 20 \mathrm{~K}$ & 0.9900 \\
\hline C103-H10E & 0.9900 & C203-C204 & $1.543(12)$ \\
\hline $\mathrm{C} 103-\mathrm{H} 10 \mathrm{~F}$ & 0.9900 & $\mathrm{C} 203-\mathrm{C} 20 \mathrm{~B}$ & $1.520(19)$ \\
\hline $\mathrm{C} 103-\mathrm{C} 104$ & $1.515(11)$ & $\mathrm{C} 206-\mathrm{H} 20 \mathrm{~N}$ & 0.9900 \\
\hline $\mathrm{C} 104-\mathrm{H} 10 \mathrm{G}$ & 0.9900 & $\mathrm{C} 206-\mathrm{H} 20 \mathrm{O}$ & 0.9900 \\
\hline $\mathrm{C} 104-\mathrm{H} 10 \mathrm{H}$ & 0.9900 & C206-H20L & 0.9900 \\
\hline C104-H10I & 0.9900 & $\mathrm{C} 206-\mathrm{H} 20 \mathrm{M}$ & 0.9900 \\
\hline C104-H10J & 0.9900 & C206-C207 & $1.519(14)$ \\
\hline $\mathrm{C} 104-\mathrm{C} 105$ & $1.526(11)$ & $\mathrm{C} 206-\mathrm{C} 205$ & $1.529(12)$ \\
\hline $\mathrm{C} 104-\mathrm{C} 10 \mathrm{~A}$ & $1.534(19)$ & $\mathrm{C} 206-\mathrm{C} 20 \mathrm{C}$ & $1.550(13)$ \\
\hline $\mathrm{C} 109-\mathrm{H} 10 \mathrm{~K}$ & 0.9900 & C207-H20P & 0.9900 \\
\hline C109-H10L & 0.9900 & C207-H20Q & 0.9900 \\
\hline $\mathrm{C} 109-\mathrm{C} 110$ & $1.517(11)$ & C207-C208 & $1.543(14)$ \\
\hline C110-H11A & 0.9900 & $\mathrm{C} 208-\mathrm{H} 20 \mathrm{R}$ & 0.9800 \\
\hline C110-H11B & 0.9900 & $\mathrm{C} 208-\mathrm{H} 20 \mathrm{~S}$ & 0.9800 \\
\hline $\mathrm{C} 110-\mathrm{C} 111$ & $1.537(12)$ & $\mathrm{C} 208-\mathrm{H} 20 \mathrm{~T}$ & 0.9800 \\
\hline $\mathrm{C} 111-\mathrm{H} 11 \mathrm{C}$ & 0.9900 & $\mathrm{C} 209-\mathrm{H} 20 \mathrm{U}$ & 0.9900 \\
\hline C111-H11D & 0.9900 & $\mathrm{C} 209-\mathrm{H} 20 \mathrm{~V}$ & 0.9900 \\
\hline $\mathrm{C} 111-\mathrm{C} 112$ & $1.534(11)$ & $\mathrm{C} 209-\mathrm{C} 210$ & $1.511(17)$ \\
\hline $\mathrm{C} 112-\mathrm{H} 11 \mathrm{E}$ & 0.9900 & $\mathrm{C} 210-\mathrm{H} 21 \mathrm{~A}$ & 0.9900 \\
\hline $\mathrm{C} 112-\mathrm{H} 11 \mathrm{~F}$ & 0.9900 & $\mathrm{C} 210-\mathrm{H} 21 \mathrm{~B}$ & 0.9900 \\
\hline $\mathrm{C} 112-\mathrm{C} 113$ & $1.539(11)$ & $\mathrm{C} 210-\mathrm{C} 211$ & $1.503(18)$ \\
\hline $\mathrm{C} 113-\mathrm{H} 11 \mathrm{G}$ & 0.9900 & $\mathrm{C} 211-\mathrm{H} 21 \mathrm{C}$ & 0.9900 \\
\hline $\mathrm{C} 113-\mathrm{H} 11 \mathrm{H}$ & 0.9900 & $\mathrm{C} 211-\mathrm{H} 21 \mathrm{D}$ & 0.9900 \\
\hline $\mathrm{C} 113-\mathrm{C} 114$ & $1.545(12)$ & $\mathrm{C} 211-\mathrm{H} 21 \mathrm{E}$ & 0.9900 \\
\hline C114-H11I & 0.9800 & $\mathrm{C} 211-\mathrm{H} 21 \mathrm{~F}$ & 0.9900 \\
\hline C114-H11J & 0.9800 & $\mathrm{C} 211-\mathrm{C} 21 \mathrm{~B}$ & $1.538(18)$ \\
\hline $\mathrm{C} 114-\mathrm{H} 11 \mathrm{~K}$ & 0.9800 & $\mathrm{C} 211-\mathrm{C} 212$ & $1.521(18)$ \\
\hline $\mathrm{C} 115-\mathrm{H} 11 \mathrm{~L}$ & 0.9900 & $\mathrm{C} 215-\mathrm{H} 21 \mathrm{G}$ & 0.9900 \\
\hline $\mathrm{C} 115-\mathrm{H} 11 \mathrm{M}$ & 0.9900 & $\mathrm{C} 215-\mathrm{H} 21 \mathrm{H}$ & 0.9900 \\
\hline $\mathrm{C} 115-\mathrm{C} 116$ & $1.508(11)$ & $\mathrm{C} 215-\mathrm{C} 216$ & $1.50(2)$ \\
\hline C116-H11N & 0.9900 & $\mathrm{C} 216-\mathrm{H} 21 \mathrm{I}$ & 0.9900 \\
\hline C116- $\mathrm{H} 11 \mathrm{O}$ & 0.9900 & $\mathrm{C} 216-\mathrm{H} 21 \mathrm{~J}$ & 0.9900 \\
\hline $\mathrm{C} 116-\mathrm{C} 117$ & $1.507(11)$ & $\mathrm{C} 216-\mathrm{H} 21 \mathrm{~K}$ & 0.9900 \\
\hline C117-H11P & 0.9900 & C216-H21L & 0.9900 \\
\hline
\end{tabular}




\begin{tabular}{|c|c|c|c|}
\hline C117-H11Q & 0.9900 & $\mathrm{C} 216-\mathrm{C} 217$ & $1.525(14)$ \\
\hline $\mathrm{C} 117-\mathrm{C} 118$ & $1.514(11)$ & $\mathrm{C} 216-\mathrm{C} 21 \mathrm{E}$ & $1.529(14)$ \\
\hline C118-H11R & 0.9900 & $\mathrm{C} 221-\mathrm{H} 22 \mathrm{~A}$ & 0.9800 \\
\hline C118-H11S & 0.9900 & $\mathrm{C} 221-\mathrm{H} 22 \mathrm{~B}$ & 0.9800 \\
\hline C118-C119 & $1.514(11)$ & $\mathrm{C} 221-\mathrm{H} 22 \mathrm{C}$ & 0.9800 \\
\hline C119-H11T & 0.9900 & $\mathrm{C} 222-\mathrm{H} 22 \mathrm{D}$ & 0.9800 \\
\hline C119-H11U & 0.9900 & $\mathrm{C} 222-\mathrm{H} 22 \mathrm{E}$ & 0.9800 \\
\hline $\mathrm{C} 119-\mathrm{C} 120$ & $1.507(11)$ & $\mathrm{C} 222-\mathrm{H} 22 \mathrm{~F}$ & 0.9800 \\
\hline $\mathrm{C} 120-\mathrm{H} 12 \mathrm{~A}$ & 0.9800 & $\mathrm{C} 223-\mathrm{H} 22 \mathrm{G}$ & 0.9800 \\
\hline $\mathrm{C} 120-\mathrm{H} 12 \mathrm{~B}$ & 0.9800 & $\mathrm{C} 223-\mathrm{H} 22 \mathrm{H}$ & 0.9800 \\
\hline $\mathrm{C} 120-\mathrm{H} 12 \mathrm{C}$ & 0.9800 & $\mathrm{C} 223-\mathrm{H} 22 \mathrm{I}$ & 0.9800 \\
\hline $\mathrm{C} 121-\mathrm{H} 12 \mathrm{D}$ & 0.9800 & $\mathrm{C} 224-\mathrm{H} 22 \mathrm{~J}$ & 0.9800 \\
\hline $\mathrm{C} 121-\mathrm{H} 12 \mathrm{E}$ & 0.9800 & $\mathrm{C} 224-\mathrm{H} 22 \mathrm{~K}$ & 0.9800 \\
\hline $\mathrm{C} 121-\mathrm{H} 12 \mathrm{~F}$ & 0.9800 & $\mathrm{C} 224-\mathrm{H} 22 \mathrm{~L}$ & 0.9800 \\
\hline $\mathrm{C} 122-\mathrm{H} 12 \mathrm{G}$ & 0.9800 & $\mathrm{C} 21 \mathrm{~B}-\mathrm{H} 21 \mathrm{M}$ & 0.9900 \\
\hline $\mathrm{C} 122-\mathrm{H} 12 \mathrm{H}$ & 0.9800 & $\mathrm{C} 21 \mathrm{~B}-\mathrm{H} 21 \mathrm{~N}$ & 0.9900 \\
\hline $\mathrm{C} 122-\mathrm{H} 12 \mathrm{I}$ & 0.9800 & $\mathrm{C} 21 \mathrm{~B}-\mathrm{C} 21 \mathrm{C}$ & 1.502 (19) \\
\hline $\mathrm{C} 123-\mathrm{H} 12 \mathrm{~J}$ & 0.9800 & $\mathrm{C} 21 \mathrm{C}-\mathrm{H} 21 \mathrm{O}$ & 0.9900 \\
\hline $\mathrm{C} 123-\mathrm{H} 12 \mathrm{~K}$ & 0.9800 & $\mathrm{C} 21 \mathrm{C}-\mathrm{H} 21 \mathrm{P}$ & 0.9900 \\
\hline $\mathrm{C} 123-\mathrm{H} 12 \mathrm{~L}$ & 0.9800 & $\mathrm{C} 21 \mathrm{C}-\mathrm{C} 21 \mathrm{D}$ & $1.530(19)$ \\
\hline $\mathrm{C} 124-\mathrm{H} 12 \mathrm{M}$ & 0.9800 & $\mathrm{C} 21 \mathrm{D}-\mathrm{H} 21 \mathrm{Q}$ & 0.9800 \\
\hline $\mathrm{C} 124-\mathrm{H} 12 \mathrm{~N}$ & 0.9800 & $\mathrm{C} 21 \mathrm{D}-\mathrm{H} 21 \mathrm{R}$ & 0.9800 \\
\hline $\mathrm{C} 124-\mathrm{H} 12 \mathrm{O}$ & 0.9800 & $\mathrm{C} 21 \mathrm{D}-\mathrm{H} 21 \mathrm{~S}$ & 0.9800 \\
\hline $\mathrm{C} 101-\mathrm{H} 10 \mathrm{M}$ & 0.9900 & $\mathrm{C} 80^{\prime}-\mathrm{H} 80 \mathrm{~A}$ & 0.9900 \\
\hline $\mathrm{C} 101-\mathrm{H} 10 \mathrm{~N}$ & 0.9900 & $\mathrm{C} 80^{\prime}-\mathrm{H} 80 \mathrm{~B}$ & 0.9900 \\
\hline $\mathrm{C} 101-\mathrm{C} 102$ & 1.505 (19) & $\mathrm{C} 204-\mathrm{H} 20 \mathrm{~W}$ & 0.9900 \\
\hline $\mathrm{C} 102-\mathrm{H} 10 \mathrm{O}$ & 0.9800 & $\mathrm{C} 204-\mathrm{H} 20 \mathrm{X}$ & 0.9900 \\
\hline $\mathrm{C} 102-\mathrm{H} 10 \mathrm{P}$ & 0.9800 & $\mathrm{C} 204-\mathrm{C} 205$ & $1.533(12)$ \\
\hline C102-H10Q & 0.9800 & $\mathrm{C} 205-\mathrm{H} 20 \mathrm{Y}$ & 0.9900 \\
\hline C105-H10R & 0.9900 & C205-HF & 0.9900 \\
\hline $\mathrm{C} 105-\mathrm{H} 10 \mathrm{~S}$ & 0.9900 & $\mathrm{C} 217-\mathrm{H} 21 \mathrm{~T}$ & 0.9900 \\
\hline $\mathrm{C} 105-\mathrm{C} 106$ & $1.526(11)$ & $\mathrm{C} 217-\mathrm{H} 21 \mathrm{U}$ & 0.9900 \\
\hline $\mathrm{C} 106-\mathrm{H} 10 \mathrm{~T}$ & 0.9900 & $\mathrm{C} 217-\mathrm{C} 218$ & $1.536(14)$ \\
\hline $\mathrm{C} 106-\mathrm{H} 10 \mathrm{U}$ & 0.9900 & $\mathrm{C} 218-\mathrm{H} 21 \mathrm{~V}$ & 0.9900 \\
\hline $\mathrm{C} 106-\mathrm{C} 107$ & $1.531(16)$ & $\mathrm{C} 218-\mathrm{H} 21 \mathrm{~W}$ & 0.9900 \\
\hline C107-H10V & 0.9900 & $\mathrm{C} 218-\mathrm{C} 219$ & $1.541(19)$ \\
\hline C107-H10W & 0.9900 & $\mathrm{C} 219-\mathrm{H} 21 \mathrm{X}$ & 0.9900 \\
\hline $\mathrm{C} 107-\mathrm{C} 108$ & $1.536(18)$ & $\mathrm{C} 219-\mathrm{H} 21 \mathrm{Y}$ & 0.9900 \\
\hline $\mathrm{C} 108-\mathrm{H} 10 \mathrm{X}$ & 0.9800 & $\mathrm{C} 219-\mathrm{C} 220$ & $1.513(19)$ \\
\hline $\mathrm{C} 108-\mathrm{H} 10 \mathrm{Y}$ & 0.9800 & $\mathrm{C} 220-\mathrm{H} 22 \mathrm{M}$ & 0.9800 \\
\hline $\mathrm{C} 108-\mathrm{H}$ & 0.9800 & $\mathrm{C} 220-\mathrm{H} 22 \mathrm{~N}$ & 0.9800 \\
\hline $\mathrm{C} 10 \mathrm{~A}-\mathrm{H} 10 \mathrm{Z}$ & 0.9900 & $\mathrm{C} 220-\mathrm{H} 22 \mathrm{O}$ & 0.9800 \\
\hline $\mathrm{C} 10 \mathrm{~A}-\mathrm{HA}$ & 0.9900 & $\mathrm{C} 20 \mathrm{~B}-\mathrm{H} 20 \mathrm{Z}$ & 0.9900 \\
\hline $\mathrm{C} 10 \mathrm{~A}-\mathrm{C} 10 \mathrm{~B}$ & $1.52(2)$ & $\mathrm{C} 20 \mathrm{~B}-\mathrm{HG}$ & 0.9900 \\
\hline $\mathrm{C} 10 \mathrm{~B}-\mathrm{H} 10$ & 0.9900 & $\mathrm{C} 20 \mathrm{~B}-\mathrm{C} 20 \mathrm{C}$ & $1.55(2)$ \\
\hline $\mathrm{C} 10 \mathrm{~B}-\mathrm{HB}$ & 0.9900 & $\mathrm{C} 20 \mathrm{C}-\mathrm{H} 20$ & 0.9900 \\
\hline $\mathrm{C} 10 \mathrm{~B}-\mathrm{C} 10 \mathrm{C}$ & $1.54(2)$ & $\mathrm{C} 20 \mathrm{C}-\mathrm{HH}$ & 0.9900 \\
\hline $\mathrm{C} 10 \mathrm{C}-\mathrm{H} 7$ & 0.9900 & $\mathrm{C} 21 \mathrm{E}-\mathrm{H} 21 \mathrm{Z}$ & 0.9900 \\
\hline
\end{tabular}




\begin{tabular}{|c|c|c|c|}
\hline $\mathrm{C} 10 \mathrm{C}-\mathrm{HC}$ & 0.9900 & $\mathrm{C} 21 \mathrm{E}-\mathrm{HI}$ & 0.9900 \\
\hline $\mathrm{C} 10 \mathrm{C}-\mathrm{C} 10 \mathrm{D}$ & $1.530(12)$ & $\mathrm{C} 21 \mathrm{E}-\mathrm{C} 21 \mathrm{~F}$ & $1.574(19)$ \\
\hline $\mathrm{C} 10 \mathrm{D}-\mathrm{H} 12$ & 0.9800 & $\mathrm{C} 21 \mathrm{~F}-\mathrm{H} 21$ & 0.9900 \\
\hline $\mathrm{C} 10 \mathrm{D}-\mathrm{HD}$ & 0.9800 & $\mathrm{C} 21 \mathrm{~F}-\mathrm{HJ}$ & 0.9900 \\
\hline $\mathrm{C} 10 \mathrm{D}-\mathrm{HE}$ & 0.9800 & $\mathrm{C} 21 \mathrm{~F}-\mathrm{C} 21 \mathrm{G}$ & $1.523(14)$ \\
\hline $\mathrm{C} 125-\mathrm{H} 12 \mathrm{P}$ & 0.9800 & $\mathrm{C} 21 \mathrm{G}-\mathrm{H} 1$ & 0.9900 \\
\hline $\mathrm{C} 125-\mathrm{H} 12 \mathrm{Q}$ & 0.9800 & $\mathrm{C} 21 \mathrm{G}-\mathrm{HK}$ & 0.9900 \\
\hline $\mathrm{C} 125-\mathrm{H} 12 \mathrm{R}$ & 0.9800 & $\mathrm{C} 21 \mathrm{G}-\mathrm{C} 21 \mathrm{H}$ & $1.528(14)$ \\
\hline $\mathrm{C} 125-\mathrm{C} 126$ & $1.511(14)$ & $\mathrm{C} 21 \mathrm{H}-\mathrm{H} 2$ & 0.9800 \\
\hline $\mathrm{C} 126-\mathrm{H} 12 \mathrm{~S}$ & 0.9900 & $\mathrm{C} 21 \mathrm{H}-\mathrm{HL}$ & 0.9800 \\
\hline $\mathrm{C} 126-\mathrm{H} 12 \mathrm{~T}$ & 0.9900 & $\mathrm{C} 21 \mathrm{H}-\mathrm{HM}$ & 0.9800 \\
\hline Ir3-N01' & $2.243(10)$ & $\mathrm{C} 80 \mathrm{~B}-\mathrm{H} 80 \mathrm{C}$ & 0.9900 \\
\hline $\mathrm{Ir} 3-\mathrm{N} 1^{\prime}$ & $2.043(11)$ & $\mathrm{C} 80 \mathrm{~B}-\mathrm{H} 80 \mathrm{D}$ & 0.9900 \\
\hline $\mathrm{Ir} 3-\mathrm{N} 2^{\prime}$ & $1.943(13)$ & $\mathrm{C} 212-\mathrm{H} 3$ & 0.9900 \\
\hline Ir $3-\mathrm{N} 02^{\prime}$ & $2.257(9)$ & $\mathrm{C} 212-\mathrm{HN}$ & 0.9900 \\
\hline $\mathrm{Ir} 3-\mathrm{C} 1^{\prime}$ & $2.013(12)$ & $\mathrm{C} 212-\mathrm{C} 213$ & $1.496(19)$ \\
\hline $\mathrm{Ir} 3-\mathrm{C} 19^{\prime}$ & $2.002(12)$ & $\mathrm{C} 213-\mathrm{H} 4$ & 0.9900 \\
\hline Ir4-N01' & $2.289(10)$ & $\mathrm{C} 213-\mathrm{HO}$ & 0.9900 \\
\hline $\mathrm{Ir} 4-\mathrm{N} 02^{\prime}$ & $2.192(10)$ & $\mathrm{C} 213-\mathrm{C} 214$ & $1.528(19)$ \\
\hline $\mathrm{Ir} 4-\mathrm{N} 33^{\prime}$ & $2.067(11)$ & $\mathrm{C} 214-\mathrm{H} 5$ & 0.9800 \\
\hline $\mathrm{Ir} 4-\mathrm{N} 4{ }^{\prime}$ & $1.899(12)$ & C214-HP & 0.9800 \\
\hline $\mathrm{Ir} 4-\mathrm{C} 37^{\prime}$ & $1.990(12)$ & $\mathrm{C} 214-\mathrm{HQ}$ & 0.9800 \\
\hline N1-Ir1-N01 & $93.1(3)$ & $\mathrm{C} 37^{\prime}-\mathrm{Ir} 4-\mathrm{N} 02^{\prime}$ & $94.1(4)$ \\
\hline N1-Ir1-N02 & $95.8(3)$ & $\mathrm{C} 37^{\prime}-\mathrm{Ir} 4-\mathrm{N} 3^{\prime}$ & $80.8(5)$ \\
\hline $\mathrm{N} 1-\mathrm{Ir} 1-\mathrm{N} 2$ & $171.0(3)$ & $\mathrm{C} 55^{\prime}-\mathrm{Ir} 4-\mathrm{N} 01^{\prime}$ & $91.2(4)$ \\
\hline N02-Ir1-N01 & $80.4(3)$ & $\mathrm{C} 55^{\prime}-\mathrm{Ir} 4-\mathrm{N} 02^{\prime}$ & $168.2(4)$ \\
\hline N2-Ir1-N01 & $94.2(3)$ & $\mathrm{C} 55^{\prime}-\mathrm{Ir} 4-\mathrm{N} 3^{\prime}$ & $92.8(5)$ \\
\hline $\mathrm{N} 2-\mathrm{Ir} 1-\mathrm{N} 02$ & $90.7(3)$ & $\mathrm{C} 55^{\prime}-\mathrm{Ir} 4-\mathrm{C} 37^{\prime}$ & $95.2(5)$ \\
\hline $\mathrm{C} 1-\mathrm{Ir} 1-\mathrm{N} 1$ & $81.0(4)$ & $\mathrm{C} 10^{\prime}-\mathrm{O}^{\prime}-\mathrm{C} 221$ & $112.8(13)$ \\
\hline $\mathrm{C} 1-\mathrm{Ir} 1-\mathrm{N} 01$ & $172.1(3)$ & $\mathrm{C} 28^{\prime}-\mathrm{O} 4^{\prime}-\mathrm{C} 222$ & $114.0(15)$ \\
\hline $\mathrm{C} 1-\mathrm{Ir} 1-\mathrm{N} 02$ & $94.8(3)$ & $\mathrm{C} 46^{\prime}-\mathrm{O} 5^{\prime}-\mathrm{C} 223$ & $114.9(15)$ \\
\hline $\mathrm{C} 1-\mathrm{Ir} 1-\mathrm{N} 2$ & $92.2(4)$ & $\mathrm{C} 64^{\prime}-\mathrm{O} 6^{\prime}-\mathrm{C} 224$ & $119.7(14)$ \\
\hline $\mathrm{C} 1-\mathrm{Ir} 1-\mathrm{C} 19$ & $95.8(4)$ & $\mathrm{Ir} 3-\mathrm{N} 01^{\prime}-\mathrm{Ir} 4$ & $98.4(4)$ \\
\hline $\mathrm{C} 19-\mathrm{Ir} 1-\mathrm{N} 1$ & $93.8(4)$ & $\mathrm{C} 01^{\prime}-\mathrm{N} 01^{\prime}-\mathrm{Ir} 3$ & $132.2(12)$ \\
\hline $\mathrm{C} 19-\mathrm{Ir} 1-\mathrm{N} 01$ & $89.9(4)$ & $\mathrm{C} 01^{\prime}-\mathrm{N} 01^{\prime}-\mathrm{Ir} 4$ & $128.7(12)$ \\
\hline $\mathrm{C} 19-\mathrm{Ir} 1-\mathrm{N} 02$ & $166.7(4)$ & $\mathrm{C} 7^{\prime}-\mathrm{N} 1^{\prime}-\mathrm{Ir} 3$ & $113.9(8)$ \\
\hline $\mathrm{C} 19-\mathrm{Ir} 1-\mathrm{N} 2$ & $80.9(4)$ & $\mathrm{C} 11^{\prime}-\mathrm{N} 1^{\prime}-\mathrm{Ir} 3$ & $125.2(10)$ \\
\hline N02-Ir2-N01 & $80.2(3)$ & $\mathrm{C} 11^{\prime}-\mathrm{N} 1^{\prime}-\mathrm{C} 7^{\prime}$ & $120.9(12)$ \\
\hline N3-Ir2-N01 & $93.0(3)$ & $\mathrm{C} 25^{\prime}-\mathrm{N} 2^{\prime}-\mathrm{Ir} 3$ & $118.4(10)$ \\
\hline N3-Ir2-N02 & $93.8(3)$ & $\mathrm{C} 25^{\prime}-\mathrm{N} 2^{\prime}-\mathrm{C} 29^{\prime}$ & $113.4(14)$ \\
\hline N4-Ir2-N01 & $90.0(3)$ & $\mathrm{C} 29^{\prime}-\mathrm{N} 2^{\prime}-\mathrm{Ir} 3$ & $127.0(11)$ \\
\hline $\mathrm{N} 4-\mathrm{Ir} 2-\mathrm{N} 02$ & $92.7(3)$ & $\mathrm{Ir} 4-\mathrm{N} 02^{\prime}-\mathrm{Ir} 3$ & $100.9(4)$ \\
\hline $\mathrm{N} 4-\mathrm{Ir} 2-\mathrm{N} 3$ & $173.2(3)$ & $\mathrm{C} 02^{\prime}-\mathrm{N} 02^{\prime}-\mathrm{Ir} 3$ & $125.9(10)$ \\
\hline $\mathrm{C} 37-\mathrm{Ir} 2-\mathrm{N} 01$ & $92.7(4)$ & $\mathrm{C} 02^{\prime}-\mathrm{N} 02^{\prime}-\mathrm{Ir} 4$ & $132.1(10)$ \\
\hline C37-Ir2- N02 & $171.1(4)$ & $\mathrm{C} 43^{\prime}-\mathrm{N} 3^{\prime}-\mathrm{Ir} 4$ & $115.2(9)$ \\
\hline $\mathrm{C} 37-\mathrm{Ir} 2-\mathrm{N} 3$ & $81.2(4)$ & $\mathrm{C} 43^{\prime}-\mathrm{N} 3^{\prime}-\mathrm{C} 47^{\prime}$ & $120.1(13)$ \\
\hline $\mathrm{C} 37-\mathrm{Ir} 2-\mathrm{N} 4$ & $92.6(4)$ & $\mathrm{C} 47^{\prime}-\mathrm{N} 3^{\prime}-\mathrm{Ir} 4$ & $124.1(10)$ \\
\hline
\end{tabular}




$$
\begin{aligned}
& \text { C37-Ir2-C55 } \\
& \text { C55-Ir2-N01 } \\
& \mathrm{C} 55-\mathrm{Ir} 2-\mathrm{N} 02 \\
& \text { C55-Ir2-N3 } \\
& \text { C55-Ir2-N4 } \\
& \mathrm{C} 10-\mathrm{O} 3-\mathrm{C} 121 \\
& \mathrm{C} 28-\mathrm{O} 4-\mathrm{C} 122 \\
& \mathrm{C} 46-\mathrm{O} 5-\mathrm{C} 123 \\
& \text { C64-O6- } 124 \\
& \text { C7-N1-Ir1 } \\
& \text { C11-N1- Ir1 } \\
& \mathrm{C} 11-\mathrm{N} 1-\mathrm{C} 7 \\
& \text { Ir1-N01-Ir2 } \\
& \text { C01-N01-Ir1 } \\
& \mathrm{C} 01-\mathrm{N} 01-\mathrm{Ir} 2 \\
& \text { Ir2-N02-Ir1 } \\
& \mathrm{C} 02-\mathrm{N} 02-\mathrm{Ir} 1 \\
& \mathrm{C} 02-\mathrm{N} 02-\mathrm{Ir} 2 \\
& \mathrm{C} 25-\mathrm{N} 2-\mathrm{Ir} 1 \\
& \mathrm{C} 25-\mathrm{N} 2-\mathrm{C} 29 \\
& \text { C29-N2-Ir1 } \\
& \text { C43-N3-Ir2 } \\
& \mathrm{C} 43-\mathrm{N} 3-\mathrm{C} 47 \\
& \text { C47-N3-Ir2 } \\
& \text { C61-N4-Ir2 } \\
& \text { C65-N4-Ir2 } \\
& \text { C65-N4-C61 } \\
& \mathrm{N} 01-\mathrm{C} 01-\mathrm{O} 1 \\
& \mathrm{C} 2-\mathrm{C} 1-\mathrm{Ir} 1 \\
& \text { C6- C1-Ir1 } \\
& \mathrm{C} 6-\mathrm{C} 1-\mathrm{C} 2 \\
& \mathrm{~N} 02-\mathrm{C} 02-\mathrm{O} 2 \\
& \mathrm{C} 1-\mathrm{C} 2-\mathrm{H} 2 \mathrm{~A} \\
& \mathrm{C} 3-\mathrm{C} 2-\mathrm{C} 1 \\
& \mathrm{C} 3-\mathrm{C} 2-\mathrm{H} 2 \mathrm{~A} \\
& \mathrm{C} 2-\mathrm{C} 3-\mathrm{C} 4 \\
& \mathrm{C} 2-\mathrm{C} 3-\mathrm{C} 12 \\
& \mathrm{C} 4-\mathrm{C} 3-\mathrm{C} 12 \\
& \mathrm{C} 3-\mathrm{C} 4-\mathrm{C} 5 \\
& \text { C3-C4-C18 } \\
& \mathrm{C} 5-\mathrm{C} 4-\mathrm{C} 18 \\
& \mathrm{C} 4-\mathrm{C} 5-\mathrm{H} 5 \mathrm{~A} \\
& \mathrm{C} 4-\mathrm{C} 5-\mathrm{C} 6 \\
& \mathrm{C} 6-\mathrm{C} 5-\mathrm{H} 5 \mathrm{~A} \\
& \mathrm{C} 1-\mathrm{C} 6-\mathrm{C} 7 \\
& \mathrm{C} 5-\mathrm{C} 6-\mathrm{C} 1 \\
& \mathrm{C} 5-\mathrm{C} 6-\mathrm{C} 7 \\
& \mathrm{~N} 1-\mathrm{C} 7-\mathrm{C} 6
\end{aligned}
$$

\begin{tabular}{|c|c|}
\hline $\mathrm{C} 61^{\prime}-\mathrm{N} 4^{\prime}-\mathrm{Ir} 4$ & $121.8(9)$ \\
\hline $\mathrm{C} 61^{\prime}-\mathrm{N} 4^{\prime}-\mathrm{C} 65^{\prime}$ & $110.1(12)$ \\
\hline $\mathrm{C} 65^{\prime}-\mathrm{N} 4^{\prime}-\mathrm{Ir} 4$ & $127.9(10)$ \\
\hline $\mathrm{N} 01^{\prime}-\mathrm{C} 01^{\prime}-\mathrm{O}^{\prime}$ & $179(2)$ \\
\hline $\mathrm{C} 2^{\prime}-\mathrm{C} 1^{\prime}-\mathrm{Ir} 3$ & $129.9(10)$ \\
\hline $\mathrm{C} 2^{\prime}-\mathrm{C} 1^{\prime}-\mathrm{C} 6^{\prime}$ & $117.7(11)$ \\
\hline $\mathrm{C} 6^{\prime}-\mathrm{C} 1^{\prime}-\mathrm{Ir} 3$ & $112.0(9)$ \\
\hline $\mathrm{N} 02^{\prime}-\mathrm{C} 02^{\prime}-\mathrm{O} 2^{\prime}$ & $178(2)$ \\
\hline $\mathrm{C} 1^{\prime}-\mathrm{C} 2^{\prime}-\mathrm{H} 2^{\prime}$ & 119.2 \\
\hline $\mathrm{C} 3^{\prime}-\mathrm{C} 2^{\prime}-\mathrm{C} 1^{\prime}$ & $121.7(13)$ \\
\hline $\mathrm{C} 3^{\prime}-\mathrm{C} 2^{\prime}-\mathrm{H} 2^{\prime}$ & 119.2 \\
\hline $\mathrm{C} 2^{\prime}-\mathrm{C} 3^{\prime}-\mathrm{C} 4^{\prime}$ & $120.5(13)$ \\
\hline $\mathrm{C} 2^{\prime}-\mathrm{C} 3^{\prime}-\mathrm{C} 12^{\prime}$ & $130.3(13)$ \\
\hline $\mathrm{C} 4^{\prime}-\mathrm{C} 3^{\prime}-\mathrm{C} 12^{\prime}$ & $109.1(11)$ \\
\hline $\mathrm{C} 3^{\prime}-\mathrm{C} 4^{\prime}-\mathrm{C} 18^{\prime}$ & $111.1(12)$ \\
\hline $\mathrm{C} 5^{\prime}-\mathrm{C} 4^{\prime}-\mathrm{C} 3^{\prime}$ & $121.1(12)$ \\
\hline $\mathrm{C} 5^{\prime}-\mathrm{C} 4^{\prime}-\mathrm{C} 18^{\prime}$ & $127.6(13)$ \\
\hline $\mathrm{C} 4^{\prime}-\mathrm{C} 5^{\prime}-\mathrm{H} 5^{\prime}$ & 121.0 \\
\hline $\mathrm{C} 4^{\prime}-\mathrm{C} 5^{\prime}-\mathrm{C} 6^{\prime}$ & $118.1(13)$ \\
\hline $\mathrm{C} 6^{\prime}-\mathrm{C} 5^{\prime}-\mathrm{H} 5^{\prime}$ & 121.0 \\
\hline $\mathrm{C} 1^{\prime}-\mathrm{C} 6^{\prime}-\mathrm{C} 5^{\prime}$ & $120.7(12)$ \\
\hline $\mathrm{C} 1^{\prime}-\mathrm{C} 6^{\prime}-\mathrm{C} 7^{\prime}$ & $116.3(12)$ \\
\hline $\mathrm{C} 5^{\prime}-\mathrm{C} 6^{\prime}-\mathrm{C} 7^{\prime}$ & $122.7(13)$ \\
\hline $\mathrm{N} 1^{\prime}-\mathrm{C} 7^{\prime}-\mathrm{C} 6^{\prime}$ & $115.2(12)$ \\
\hline $\mathrm{N} 1^{\prime}-\mathrm{C} 7^{\prime}-\mathrm{C} 8^{\prime}$ & $119.2(12)$ \\
\hline $\mathrm{C} 8^{\prime}-\mathrm{C} 7^{\prime}-\mathrm{C} 6^{\prime}$ & $125.5(13)$ \\
\hline $\mathrm{C} 7^{\prime}-\mathrm{C} 8^{\prime}-\mathrm{H} 8^{\prime}$ & 119.5 \\
\hline $\mathrm{C} 9^{\prime}-\mathrm{C} 8^{\prime}-\mathrm{C} 7^{\prime}$ & $121.0(14)$ \\
\hline $\mathrm{C} 9^{\prime}-\mathrm{C} 8^{\prime}-\mathrm{H} 8^{\prime}$ & 119.5 \\
\hline $\mathrm{C} 8^{\prime}-\mathrm{C} 9^{\prime}-\mathrm{H} 9^{\prime}$ & 120.7 \\
\hline $\mathrm{C} 8^{\prime}-\mathrm{C} 9^{\prime}-\mathrm{C} 10^{\prime}$ & $118.5(15)$ \\
\hline $\mathrm{C} 10^{\prime}-\mathrm{C} 9^{\prime}-\mathrm{H} 9^{\prime}$ & 120.7 \\
\hline $\mathrm{O}^{\prime}-\mathrm{C} 10^{\prime}-\mathrm{C} 9^{\prime}$ & $114.6(15)$ \\
\hline $\mathrm{C} 11^{\prime}-\mathrm{C} 10^{\prime}-\mathrm{O}^{\prime}$ & $126.6(14)$ \\
\hline $\mathrm{C} 11^{\prime}-\mathrm{C} 10^{\prime}-\mathrm{C} 9^{\prime}$ & $118.8(14)$ \\
\hline $\mathrm{N} 1^{\prime}-\mathrm{C} 11^{\prime}-\mathrm{C} 10^{\prime}$ & $121.5(14)$ \\
\hline $\mathrm{N} 1^{\prime}-\mathrm{C} 11^{\prime}-\mathrm{H} 11^{\prime}$ & 119.2 \\
\hline $\mathrm{C} 10^{\prime}-\mathrm{C} 11^{\prime}-\mathrm{H} 11^{\prime}$ & 119.2 \\
\hline $\mathrm{C} 13^{\prime}-\mathrm{C} 12^{\prime}-\mathrm{C} 3^{\prime}$ & $131.9(11)$ \\
\hline $\mathrm{C} 13^{\prime}-\mathrm{C} 12^{\prime}-\mathrm{C} 17^{\prime}$ & $119.8(12)$ \\
\hline $\mathrm{C} 17^{\prime}-\mathrm{C} 12^{\prime}-\mathrm{C} 3^{\prime}$ & $108.2(12)$ \\
\hline $\mathrm{C} 12^{\prime}-\mathrm{C} 13^{\prime}-\mathrm{H} 13^{\prime}$ & 120.5 \\
\hline $\mathrm{C} 12^{\prime}-\mathrm{C} 13^{\prime}-\mathrm{C} 14^{\prime}$ & $119.0(12)$ \\
\hline $\mathrm{C} 14^{\prime}-\mathrm{C} 13^{\prime}-\mathrm{H} 13^{\prime}$ & 120.5 \\
\hline $\mathrm{C} 13^{\prime}-\mathrm{C} 14^{\prime}-\mathrm{H} 14^{\prime}$ & 119.7 \\
\hline $\mathrm{C} 15^{\prime}-\mathrm{C} 14^{\prime}-\mathrm{C} 13^{\prime}$ & $120.6(14)$ \\
\hline $\mathrm{C} 15^{\prime}-\mathrm{C} 14^{\prime}-\mathrm{H} 14^{\prime}$ & 119.7 \\
\hline $\mathrm{C} 14^{\prime}-\mathrm{C} 15^{\prime}-\mathrm{H} 15^{\prime}$ & 119.8 \\
\hline
\end{tabular}

$97.5(4)$
$166.4(4)$
$90.4(4)$
$97.5(4)$
$80.5(4)$
$116.9(10)$
$116.0(9)$
$116.9(10)$
$117.2(9)$
$115.4(7)$
$125.4(8)$
$119.0(9)$
$98.8(4)$
$132.2(8)$
$128.6(8)$
$100.6(3)$
$128.4(9)$
$129.3(9)$
$116.3(7)$
$119.9(9)$
$123.6(7)$
$114.9(7)$
$122.4(10)$
$122.7(8)$
$116.0(7)$
$125.4(8)$
$118.5(10)$
$179.6(17)$
$128.8(8)$
$114.5(7)$
$116.7(10)$
$175.3(19)$
119.6
$120.7(11)$
119.6
$121.5(11)$
$129.8(11)$
$108.7(10)$
$120.6(11)$
$111.4(10)$
$128.0(11)$
121.0
$117.9(11)$
121.0
$115.4(10)$
$122.6(10)$
$122.0(10)$
$113.2(10)$




\begin{tabular}{|c|c|c|c|}
\hline $\mathrm{N} 1-\mathrm{C} 7-\mathrm{C} 8$ & $118.9(10)$ & $\mathrm{C} 16^{\prime}-\mathrm{C} 15^{\prime}-\mathrm{C} 14^{\prime}$ & $120.4(13)$ \\
\hline $\mathrm{C} 8-\mathrm{C} 7-\mathrm{C} 6$ & $127.9(11)$ & $\mathrm{C} 16^{\prime}-\mathrm{C} 15^{\prime}-\mathrm{H} 15^{\prime}$ & 119.8 \\
\hline $\mathrm{C} 7-\mathrm{C} 8-\mathrm{H} 8$ & 118.7 & $\mathrm{C} 15^{\prime}-\mathrm{C} 16^{\prime}-\mathrm{H} 16^{\prime}$ & 119.7 \\
\hline $\mathrm{C} 9-\mathrm{C} 8-\mathrm{C} 7$ & $122.5(12)$ & $\mathrm{C} 15^{\prime}-\mathrm{C} 16^{\prime}-\mathrm{C} 17^{\prime}$ & $120.6(13)$ \\
\hline $\mathrm{C} 9-\mathrm{C} 8-\mathrm{H} 8$ & 118.7 & $\mathrm{C} 17^{\prime}-\mathrm{C} 16^{\prime}-\mathrm{H} 16^{\prime}$ & 119.7 \\
\hline $\mathrm{C} 8-\mathrm{C} 9-\mathrm{H} 9$ & 120.6 & $\mathrm{C} 12^{\prime}-\mathrm{C} 17^{\prime}-\mathrm{C} 18^{\prime}$ & $110.7(11)$ \\
\hline $\mathrm{C} 8-\mathrm{C} 9-\mathrm{C} 10$ & $118.8(12)$ & $\mathrm{C} 16^{\prime}-\mathrm{C} 17^{\prime}-\mathrm{C} 12^{\prime}$ & $119.0(13)$ \\
\hline $\mathrm{C} 10-\mathrm{C} 9-\mathrm{H} 9$ & 120.6 & $\mathrm{C} 16^{\prime}-\mathrm{C} 17^{\prime}-\mathrm{C} 18^{\prime}$ & $129.9(12)$ \\
\hline $\mathrm{O} 3-\mathrm{C} 10-\mathrm{C} 9$ & $118.5(11)$ & $\mathrm{C} 4^{\prime}-\mathrm{C} 18^{\prime}-\mathrm{C} 73^{\prime}$ & $111.5(12)$ \\
\hline $\mathrm{O} 3-\mathrm{C} 10-\mathrm{C} 11$ & $122.9(12)$ & $\mathrm{C} 17^{\prime}-\mathrm{C} 18^{\prime}-\mathrm{C} 4^{\prime}$ & $100.8(10)$ \\
\hline $\mathrm{C} 11-\mathrm{C} 10-\mathrm{C} 9$ & $118.6(11)$ & $\mathrm{C} 17^{\prime}-\mathrm{C} 18^{\prime}-\mathrm{C} 73^{\prime}$ & $111.6(12)$ \\
\hline $\mathrm{N} 1-\mathrm{C} 11-\mathrm{C} 10$ & 122.1 & $\mathrm{C} 79^{\prime}-\mathrm{C} 18^{\prime}-\mathrm{C} 4^{\prime}$ & $112.8(13)$ \\
\hline $\mathrm{N} 1-\mathrm{C} 11-\mathrm{H} 11$ & 118.9 & $\mathrm{C} 79^{\prime}-\mathrm{C} 18^{\prime}-\mathrm{C} 17^{\prime}$ & $111.9(13)$ \\
\hline $\mathrm{C} 10-\mathrm{C} 11-\mathrm{H} 11$ & 118.9 & $\mathrm{C} 79^{\prime}-\mathrm{C} 18^{\prime}-\mathrm{C} 73^{\prime}$ & $108.2(12)$ \\
\hline $\mathrm{C} 13-\mathrm{C} 12-\mathrm{C} 3$ & $130.5(11)$ & $\mathrm{C} 20^{\prime}-\mathrm{C} 19^{\prime}-\mathrm{Ir} 3$ & $127.9(10)$ \\
\hline $\mathrm{C} 13-\mathrm{C} 12-\mathrm{C} 17$ & $121.5(11)$ & $\mathrm{C} 20^{\prime}-\mathrm{C} 19^{\prime}-\mathrm{C} 24^{\prime}$ & $119.3(12)$ \\
\hline $\mathrm{C} 17-\mathrm{C} 12-\mathrm{C} 3$ & $108.0(11)$ & $\mathrm{C} 24^{\prime}-\mathrm{C} 19^{\prime}-\mathrm{Ir} 3$ & $112.5(9)$ \\
\hline $\mathrm{C} 12-\mathrm{C} 13-\mathrm{H} 13$ & 120.0 & $\mathrm{C} 19^{\prime}-\mathrm{C} 20^{\prime}-\mathrm{H} 20^{\prime}$ & 121.2 \\
\hline $\mathrm{C} 12-\mathrm{C} 13-\mathrm{C} 14$ & $120.0(12)$ & $\mathrm{C} 19^{\prime}-\mathrm{C} 20^{\prime}-\mathrm{C} 21^{\prime}$ & $117.5(13)$ \\
\hline $\mathrm{C} 14-\mathrm{C} 13-\mathrm{H} 13$ & 120.0 & $\mathrm{C} 21^{\prime}-\mathrm{C} 20^{\prime}-\mathrm{H} 20^{\prime}$ & 121.2 \\
\hline $\mathrm{C} 13-\mathrm{C} 14-\mathrm{H} 14$ & 120.3 & $\mathrm{C} 20^{\prime}-\mathrm{C} 21^{\prime}-\mathrm{C} 30^{\prime}$ & $126.4(14)$ \\
\hline $\mathrm{C} 13-\mathrm{C} 14-\mathrm{C} 15$ & $119.3(13)$ & $\mathrm{C} 22^{\prime}-\mathrm{C} 21^{\prime}-\mathrm{C} 20^{\prime}$ & $124.4(14)$ \\
\hline $\mathrm{C} 15-\mathrm{C} 14-\mathrm{H} 14$ & 120.3 & $\mathrm{C} 22^{\prime}-\mathrm{C} 21^{\prime}-\mathrm{C} 30^{\prime}$ & $109.1(13)$ \\
\hline $\mathrm{C} 14-\mathrm{C} 15-\mathrm{H} 15$ & 120.2 & $\mathrm{C} 21^{\prime}-\mathrm{C} 22^{\prime}-\mathrm{C} 23^{\prime}$ & $117.5(13)$ \\
\hline $\mathrm{C} 16-\mathrm{C} 15-\mathrm{C} 14$ & $119.6(12)$ & $\mathrm{C} 21^{\prime}-\mathrm{C} 22^{\prime}-\mathrm{C} 36^{\prime}$ & $111.8(13)$ \\
\hline $\mathrm{C} 16-\mathrm{C} 15-\mathrm{H} 15$ & 120.2 & $\mathrm{C} 23^{\prime}-\mathrm{C} 22^{\prime}-\mathrm{C} 36^{\prime}$ & $130.7(14)$ \\
\hline $\mathrm{C} 15-\mathrm{C} 16-\mathrm{H} 16$ & 119.4 & $\mathrm{C} 22^{\prime}-\mathrm{C} 23^{\prime}-\mathrm{H} 23^{\prime}$ & 119.9 \\
\hline $\mathrm{C} 15-\mathrm{C} 16-\mathrm{C} 17$ & $121.1(12)$ & $\mathrm{C} 24^{\prime}-\mathrm{C} 23^{\prime}-\mathrm{C} 22^{\prime}$ & $120.2(14)$ \\
\hline $\mathrm{C} 17-\mathrm{C} 16-\mathrm{H} 16$ & 119.4 & $\mathrm{C} 24^{\prime}-\mathrm{C} 23^{\prime}-\mathrm{H} 23^{\prime}$ & 119.9 \\
\hline $\mathrm{C} 12-\mathrm{C} 17-\mathrm{C} 18$ & $111.6(10)$ & $\mathrm{C} 19^{\prime}-\mathrm{C} 24^{\prime}-\mathrm{C} 25^{\prime}$ & $115.6(13)$ \\
\hline $\mathrm{C} 16-\mathrm{C} 17-\mathrm{C} 12$ & $118.4(12)$ & $\mathrm{C} 23^{\prime}-\mathrm{C} 24^{\prime}-\mathrm{C} 19^{\prime}$ & $121.0(13)$ \\
\hline $\mathrm{C} 16-\mathrm{C} 17-\mathrm{C} 18$ & $130.0(11)$ & $\mathrm{C} 23^{\prime}-\mathrm{C} 24^{\prime}-\mathrm{C} 25^{\prime}$ & $123.5(15)$ \\
\hline $\mathrm{C} 17-\mathrm{C} 18-\mathrm{C} 4$ & $100.2(9)$ & $\mathrm{N} 2{ }^{\prime}-\mathrm{C} 25^{\prime}-\mathrm{C} 24^{\prime}$ & $111.7(14)$ \\
\hline $\mathrm{C} 73-\mathrm{C} 18-\mathrm{C} 4$ & $112.6(9)$ & $\mathrm{N} 2{ }^{\prime}-\mathrm{C} 25^{\prime}-\mathrm{C} 26^{\prime}$ & $124.3(14)$ \\
\hline $\mathrm{C} 73-\mathrm{C} 18-\mathrm{C} 17$ & $110.8(9)$ & $\mathrm{C} 26^{\prime}-\mathrm{C} 25^{\prime}-\mathrm{C} 24^{\prime}$ & $124.0(15)$ \\
\hline $\mathrm{C} 79-\mathrm{C} 18-\mathrm{C} 4$ & $111.7(9)$ & $\mathrm{C} 25^{\prime}-\mathrm{C} 26^{\prime}-\mathrm{H} 26^{\prime}$ & 120.3 \\
\hline $\mathrm{C} 79-\mathrm{C} 18-\mathrm{C} 17$ & $112.1(10)$ & $\mathrm{C} 25^{\prime}-\mathrm{C} 26^{\prime}-\mathrm{C} 27^{\prime}$ & $119.3(16)$ \\
\hline $\mathrm{C} 79-\mathrm{C} 18-\mathrm{C} 73$ & $109.2(10)$ & $\mathrm{C} 27^{\prime}-\mathrm{C} 26^{\prime}-\mathrm{H} 26^{\prime}$ & 120.3 \\
\hline $\mathrm{C} 20-\mathrm{C} 19-\mathrm{Ir} 1$ & $130.1(8)$ & $\mathrm{C} 26^{\prime}-\mathrm{C} 27^{\prime}-\mathrm{H} 27^{\prime}$ & 122.2 \\
\hline $\mathrm{C} 20-\mathrm{C} 19-\mathrm{C} 24$ & $115.7(9)$ & $\mathrm{C} 28^{\prime}-\mathrm{C} 27^{\prime}-\mathrm{C} 26^{\prime}$ & $115.6(16)$ \\
\hline $\mathrm{C} 24-\mathrm{C} 19-\mathrm{Ir} 1$ & $113.9(8)$ & $\mathrm{C} 28^{\prime}-\mathrm{C} 27^{\prime}-\mathrm{H} 27^{\prime}$ & 122.2 \\
\hline $\mathrm{C} 19-\mathrm{C} 20-\mathrm{H} 6$ & 119.1 & $\mathrm{C} 27^{\prime}-\mathrm{C} 28^{\prime}-\mathrm{O} 4^{\prime}$ & $112.0(17)$ \\
\hline $\mathrm{C} 21-\mathrm{C} 20-\mathrm{C} 19$ & $121.9(11)$ & $\mathrm{C} 29^{\prime}-\mathrm{C} 28^{\prime}-\mathrm{O} 4^{\prime}$ & $126.1(17)$ \\
\hline $\mathrm{C} 21-\mathrm{C} 20-\mathrm{H} 6$ & 119.1 & $\mathrm{C} 29^{\prime}-\mathrm{C} 28^{\prime}-\mathrm{C} 27^{\prime}$ & $121.9(16)$ \\
\hline $\mathrm{C} 20-\mathrm{C} 21-\mathrm{C} 22$ & $121.3(10)$ & $\mathrm{N} 2^{\prime}-\mathrm{C} 29^{\prime}-\mathrm{H} 29^{\prime}$ & 117.3 \\
\hline $\mathrm{C} 20-\mathrm{C} 21-\mathrm{C} 30$ & $129.3(11)$ & $\mathrm{C} 28^{\prime}-\mathrm{C} 29^{\prime}-\mathrm{N} 2^{\prime}$ & $125.3(16)$ \\
\hline $\mathrm{C} 22-\mathrm{C} 21-\mathrm{C} 30$ & $109.4(9)$ & $\mathrm{C} 28^{\prime}-\mathrm{C} 29^{\prime}-\mathrm{H} 29^{\prime}$ & 117.3 \\
\hline $\mathrm{C} 21-\mathrm{C} 22-\mathrm{C} 36$ & $110.8(10)$ & $\mathrm{C} 31^{\prime}-\mathrm{C} 30^{\prime}-\mathrm{C} 21^{\prime}$ & $134.6(14)$ \\
\hline
\end{tabular}




\begin{tabular}{|c|c|}
\hline $\mathrm{C} 23-\mathrm{C} 22-\mathrm{C} 21$ & $120.1(10)$ \\
\hline $\mathrm{C} 23-\mathrm{C} 22-\mathrm{C} 36$ & $129.1(10)$ \\
\hline $\mathrm{C} 22-\mathrm{C} 23-\mathrm{H} 23$ & 120.8 \\
\hline $\mathrm{C} 22-\mathrm{C} 23-\mathrm{C} 24$ & $118.4(10)$ \\
\hline $\mathrm{C} 24-\mathrm{C} 23-\mathrm{H} 23$ & 120.8 \\
\hline $\mathrm{C} 19-\mathrm{C} 24-\mathrm{C} 25$ & $115.3(9)$ \\
\hline $\mathrm{C} 23-\mathrm{C} 24-\mathrm{C} 19$ & $122.6(10)$ \\
\hline $\mathrm{C} 23-\mathrm{C} 24-\mathrm{C} 25$ & $121.9(10)$ \\
\hline $\mathrm{N} 2-\mathrm{C} 25-\mathrm{C} 24$ & $112.8(10)$ \\
\hline $\mathrm{N} 2-\mathrm{C} 25-\mathrm{C} 26$ & $118.7(10)$ \\
\hline $\mathrm{C} 26-\mathrm{C} 25-\mathrm{C} 24$ & $128.4(10)$ \\
\hline $\mathrm{C} 25-\mathrm{C} 26-\mathrm{H} 26$ & 119.1 \\
\hline $\mathrm{C} 27-\mathrm{C} 26-\mathrm{C} 25$ & $121.7(11)$ \\
\hline $\mathrm{C} 27-\mathrm{C} 26-\mathrm{H} 26$ & 119.1 \\
\hline $\mathrm{C} 26-\mathrm{C} 27-\mathrm{H} 27$ & 120.6 \\
\hline $\mathrm{C} 26-\mathrm{C} 27-\mathrm{C} 28$ & $118.9(11)$ \\
\hline $\mathrm{C} 28-\mathrm{C} 27-\mathrm{H} 27$ & 120.6 \\
\hline $\mathrm{O} 4-\mathrm{C} 28-\mathrm{C} 27$ & $117.3(11)$ \\
\hline $\mathrm{O} 4-\mathrm{C} 28-\mathrm{C} 29$ & $124.1(11)$ \\
\hline $\mathrm{C} 29-\mathrm{C} 28-\mathrm{C} 27$ & $118.6(11)$ \\
\hline $\mathrm{N} 2-\mathrm{C} 29-\mathrm{H} 29$ & 119.0 \\
\hline $\mathrm{C} 28-\mathrm{C} 29-\mathrm{N} 2$ & $121.9(10)$ \\
\hline $\mathrm{C} 28-\mathrm{C} 29-\mathrm{H} 29$ & 119.0 \\
\hline $\mathrm{C} 31-\mathrm{C} 30-\mathrm{C} 21$ & $131.3(10)$ \\
\hline $\mathrm{C} 31-\mathrm{C} 30-\mathrm{C} 35$ & $123.1(10)$ \\
\hline $\mathrm{C} 35-\mathrm{C} 30-\mathrm{C} 21$ & $105.6(10)$ \\
\hline $\mathrm{C} 30-\mathrm{C} 31-\mathrm{H} 31$ & 121.3 \\
\hline $\mathrm{C} 30-\mathrm{C} 31-\mathrm{C} 32$ & $117.4(10)$ \\
\hline $\mathrm{C} 32-\mathrm{C} 31-\mathrm{H} 31$ & 121.3 \\
\hline $\mathrm{C} 31-\mathrm{C} 32-\mathrm{H} 32$ & 119.4 \\
\hline $\mathrm{C} 33-\mathrm{C} 32-\mathrm{C} 31$ & $121.2(11)$ \\
\hline $\mathrm{C} 33-\mathrm{C} 32-\mathrm{H} 32$ & 119.4 \\
\hline $\mathrm{C} 32-\mathrm{C} 33-\mathrm{H} 33$ & 119.4 \\
\hline $\mathrm{C} 32-\mathrm{C} 33-\mathrm{C} 34$ & $121.1(11)$ \\
\hline $\mathrm{C} 34-\mathrm{C} 33-\mathrm{H} 33$ & 119.4 \\
\hline $\mathrm{C} 33-\mathrm{C} 34-\mathrm{H} 34$ & 120.0 \\
\hline $\mathrm{C} 33-\mathrm{C} 34-\mathrm{C} 35$ & $120.1(11)$ \\
\hline $\mathrm{C} 35-\mathrm{C} 34-\mathrm{H} 34$ & 120.0 \\
\hline $\mathrm{C} 30-\mathrm{C} 35-\mathrm{C} 36$ & $113.8(10)$ \\
\hline $\mathrm{C} 34-\mathrm{C} 35-\mathrm{C} 30$ & 117.1 \\
\hline $\mathrm{C} 34-\mathrm{C} 35-\mathrm{C} 36$ & $129.1(10)$ \\
\hline $\mathrm{C} 35-\mathrm{C} 36-\mathrm{C} 22$ & $100.4(9)$ \\
\hline $\mathrm{C} 35-\mathrm{C} 36-\mathrm{C} 85$ & $111.9(9)$ \\
\hline $\mathrm{C} 35-\mathrm{C} 36-\mathrm{C} 91$ & $111.9(9)$ \\
\hline $\mathrm{C} 85-\mathrm{C} 36-\mathrm{C} 22$ & $111.4(8)$ \\
\hline $\mathrm{C} 91-\mathrm{C} 36-\mathrm{C} 22$ & $110.1(9)$ \\
\hline $\mathrm{C} 91-\mathrm{C} 36-\mathrm{C} 85$ & $110.7(9)$ \\
\hline & \\
\hline
\end{tabular}

\begin{tabular}{|c|c|}
\hline $\mathrm{C} 31^{\prime}-\mathrm{C} 30^{\prime}-\mathrm{C} 35^{\prime}$ & $118.4(13)$ \\
\hline $\mathrm{C} 35^{\prime}-\mathrm{C} 30^{\prime}-\mathrm{C} 21^{\prime}$ & $106.7(13)$ \\
\hline $\mathrm{C} 30^{\prime}-\mathrm{C} 31^{\prime}-\mathrm{H} 31^{\prime}$ & 119.9 \\
\hline $\mathrm{C} 32^{\prime}-\mathrm{C} 31^{\prime}-\mathrm{C} 30^{\prime}$ & $120.1(14)$ \\
\hline $\mathrm{C} 32^{\prime}-\mathrm{C} 31^{\prime}-\mathrm{H} 31^{\prime}$ & 119.9 \\
\hline $\mathrm{C} 31^{\prime}-\mathrm{C} 32^{\prime}-\mathrm{H} 32^{\prime}$ & 119.8 \\
\hline $\mathrm{C} 33^{\prime}-\mathrm{C} 32^{\prime}-\mathrm{C} 31^{\prime}$ & $120.4(15)$ \\
\hline $\mathrm{C} 33^{\prime}-\mathrm{C} 32^{\prime}-\mathrm{H} 32^{\prime}$ & 119.8 \\
\hline $\mathrm{C} 32^{\prime}-\mathrm{C} 33^{\prime}-\mathrm{H} 33^{\prime}$ & 119.1 \\
\hline $\mathrm{C} 32^{\prime}-\mathrm{C} 33^{\prime}-\mathrm{C} 34^{\prime}$ & $121.9(15)$ \\
\hline $\mathrm{C} 34^{\prime}-\mathrm{C} 33^{\prime}-\mathrm{H} 33^{\prime}$ & 119.1 \\
\hline $\mathrm{C} 33^{\prime}-\mathrm{C} 34^{\prime}-\mathrm{H} 34^{\prime}$ & 119.9 \\
\hline $\mathrm{C} 33^{\prime}-\mathrm{C} 34^{\prime}-\mathrm{C} 35^{\prime}$ & $120.2(14)$ \\
\hline $\mathrm{C} 35^{\prime}-\mathrm{C} 34^{\prime}-\mathrm{H} 34^{\prime}$ & 119.9 \\
\hline $\mathrm{C} 30^{\prime}-\mathrm{C} 35^{\prime}-\mathrm{C} 36^{\prime}$ & $110.1(13)$ \\
\hline $\mathrm{C} 34^{\prime}-\mathrm{C} 35^{\prime}-\mathrm{C} 30^{\prime}$ & $118.6(14)$ \\
\hline $\mathrm{C} 34^{\prime}-\mathrm{C} 35^{\prime}-\mathrm{C} 36^{\prime}$ & $131.3(14)$ \\
\hline $\mathrm{C} 22^{\prime}-\mathrm{C} 36^{\prime}-\mathrm{C} 91^{\prime}$ & $111.1(12)$ \\
\hline $\mathrm{C} 35^{\prime}-\mathrm{C} 36^{\prime}-\mathrm{C} 22^{\prime}$ & $102.2(12)$ \\
\hline $\mathrm{C} 35^{\prime}-\mathrm{C} 36^{\prime}-\mathrm{C} 85^{\prime}$ & $112.7(12)$ \\
\hline $\mathrm{C} 35^{\prime}-\mathrm{C} 36^{\prime}-\mathrm{C} 91^{\prime}$ & $110.1(12)$ \\
\hline $\mathrm{C} 85^{\prime}-\mathrm{C} 36^{\prime}-\mathrm{C} 22^{\prime}$ & $111.0(11)$ \\
\hline $\mathrm{C} 85^{\prime}-\mathrm{C} 36^{\prime}-\mathrm{C} 91^{\prime}$ & $109.6(12)$ \\
\hline $\mathrm{C} 38^{\prime}-\mathrm{C} 37^{\prime}-\mathrm{Ir} 4$ & $128.3(10)$ \\
\hline $\mathrm{C} 42^{\prime}-\mathrm{C} 37^{\prime}-\mathrm{Ir} 4$ & $112.9(9)$ \\
\hline $\mathrm{C} 42^{\prime}-\mathrm{C} 37^{\prime}-\mathrm{C} 38^{\prime}$ & $118.7(12)$ \\
\hline $\mathrm{C} 37^{\prime}-\mathrm{C} 38^{\prime}-\mathrm{H} 38^{\prime}$ & 121.0 \\
\hline $\mathrm{C} 39^{\prime}-\mathrm{C} 38^{\prime}-\mathrm{C} 37^{\prime}$ & $118.1(13)$ \\
\hline $\mathrm{C} 39^{\prime}-\mathrm{C} 38^{\prime}-\mathrm{H} 38^{\prime}$ & 121.0 \\
\hline $\mathrm{C} 38^{\prime}-\mathrm{C} 39^{\prime}-\mathrm{C} 48^{\prime}$ & $129.3(12)$ \\
\hline $\mathrm{C} 40^{\prime}-\mathrm{C} 39^{\prime}-\mathrm{C} 38^{\prime}$ & $123.3(12)$ \\
\hline $\mathrm{C} 40^{\prime}-\mathrm{C} 39^{\prime}-\mathrm{C} 48^{\prime}$ & $107.4(11)$ \\
\hline $\mathrm{C} 39^{\prime}-\mathrm{C} 40^{\prime}-\mathrm{C} 54^{\prime}$ & $112.0(12)$ \\
\hline $\mathrm{C} 41^{\prime}-\mathrm{C} 40^{\prime}-\mathrm{C} 39^{\prime}$ & $120.2(12)$ \\
\hline $\mathrm{C} 41^{\prime}-\mathrm{C} 40^{\prime}-\mathrm{C} 54^{\prime}$ & $127.7(13)$ \\
\hline $\mathrm{C} 40^{\prime}-\mathrm{C} 41^{\prime}-\mathrm{H} 41^{\prime}$ & 120.4 \\
\hline $\mathrm{C} 40^{\prime}-\mathrm{C} 41^{\prime}-\mathrm{C} 42^{\prime}$ & $119.2(13)$ \\
\hline $\mathrm{C} 42^{\prime}-\mathrm{C} 41^{\prime}-\mathrm{H} 41^{\prime}$ & 120.4 \\
\hline $\mathrm{C} 37^{\prime}-\mathrm{C} 42^{\prime}-\mathrm{C} 41^{\prime}$ & $120.4(12)$ \\
\hline $\mathrm{C} 37^{\prime}-\mathrm{C} 42^{\prime}-\mathrm{C} 43^{\prime}$ & $116.1(12)$ \\
\hline $\mathrm{C} 41^{\prime}-\mathrm{C} 42^{\prime}-\mathrm{C} 43^{\prime}$ & $123.5(13)$ \\
\hline $\mathrm{N} 3{ }^{\prime}-\mathrm{C} 43^{\prime}-\mathrm{C} 42^{\prime}$ & $114.5(13)$ \\
\hline $\mathrm{N} 3^{\prime}-\mathrm{C} 43^{\prime}-\mathrm{C} 44^{\prime}$ & $120.2(13)$ \\
\hline $\mathrm{C} 44^{\prime}-\mathrm{C} 43^{\prime}-\mathrm{C} 42^{\prime}$ & $125.2(14)$ \\
\hline $\mathrm{C} 43^{\prime}-\mathrm{C} 44^{\prime}-\mathrm{H} 44^{\prime}$ & 120.7 \\
\hline $\mathrm{C} 45^{\prime}-\mathrm{C} 44^{\prime}-\mathrm{C} 43^{\prime}$ & $118.5(16)$ \\
\hline $\mathrm{C} 45^{\prime}-\mathrm{C} 44^{\prime}-\mathrm{H} 44^{\prime}$ & 120.7 \\
\hline $\mathrm{C} 44^{\prime}-\mathrm{C} 45^{\prime}-\mathrm{H} 45^{\prime}$ & 120.6 \\
\hline
\end{tabular}




\begin{tabular}{|c|c|}
\hline $\mathrm{C} 38-\mathrm{C} 37-\mathrm{C} 42$ & $115.6(10)$ \\
\hline $\mathrm{C} 42-\mathrm{C} 37-\mathrm{Ir} 2$ & $114.4(8)$ \\
\hline $\mathrm{C} 37-\mathrm{C} 38-\mathrm{H} 38$ & 118.8 \\
\hline $\mathrm{C} 37-\mathrm{C} 38-\mathrm{C} 39$ & $122.5(11)$ \\
\hline $\mathrm{C} 39-\mathrm{C} 38-\mathrm{H} 38$ & 118.8 \\
\hline $\mathrm{C} 38-\mathrm{C} 39-\mathrm{C} 48$ & $131.8(11)$ \\
\hline $\mathrm{C} 40-\mathrm{C} 39-\mathrm{C} 38$ & $119.6(11)$ \\
\hline $\mathrm{C} 40-\mathrm{C} 39-\mathrm{C} 48$ & $108.6(10)$ \\
\hline $\mathrm{C} 39-\mathrm{C} 40-\mathrm{C} 54$ & $110.2(10)$ \\
\hline $\mathrm{C} 41-\mathrm{C} 40-\mathrm{C} 39$ & $120.9(11)$ \\
\hline $\mathrm{C} 41-\mathrm{C} 40-\mathrm{C} 54$ & $128.9(11)$ \\
\hline $\mathrm{C} 40-\mathrm{C} 41-\mathrm{H} 41$ & 121.0 \\
\hline $\mathrm{C} 40-\mathrm{C} 41-\mathrm{C} 42$ & $118.1(11)$ \\
\hline $\mathrm{C} 42-\mathrm{C} 41-\mathrm{H} 41$ & 121.0 \\
\hline $\mathrm{C} 37-\mathrm{C} 42-\mathrm{C} 43$ & $114.6(10)$ \\
\hline $\mathrm{C} 41-\mathrm{C} 42-\mathrm{C} 37$ & $123.1(10)$ \\
\hline $\mathrm{C} 41-\mathrm{C} 42-\mathrm{C} 43$ & $122.3(11)$ \\
\hline $\mathrm{N} 3-\mathrm{C} 43-\mathrm{C} 42$ & $114.8(10)$ \\
\hline $\mathrm{N} 3-\mathrm{C} 43-\mathrm{C} 44$ & $118.8(11)$ \\
\hline $\mathrm{C} 44-\mathrm{C} 43-\mathrm{C} 42$ & $126.4(11)$ \\
\hline $\mathrm{C} 43-\mathrm{C} 44-\mathrm{H} 44$ & 119.3 \\
\hline $\mathrm{C} 45-\mathrm{C} 44-\mathrm{C} 43$ & $121.3(12)$ \\
\hline $\mathrm{C} 45-\mathrm{C} 44-\mathrm{H} 44$ & 119.3 \\
\hline $\mathrm{C} 44-\mathrm{C} 45-\mathrm{H} 45$ & 120.9 \\
\hline $\mathrm{C} 44-\mathrm{C} 45-\mathrm{C} 46$ & $118.1(12)$ \\
\hline $\mathrm{C} 46-\mathrm{C} 45-\mathrm{H} 45$ & 120.9 \\
\hline $\mathrm{O} 5-\mathrm{C} 46-\mathrm{C} 45$ & $116.1(11)$ \\
\hline $\mathrm{O} 5-\mathrm{C} 46-\mathrm{C} 47$ & $123.3(12)$ \\
\hline $\mathrm{C} 47-\mathrm{C} 46-\mathrm{C} 45$ & $120.6(11)$ \\
\hline $\mathrm{N} 3-\mathrm{C} 47-\mathrm{C} 46$ & $118.8(11)$ \\
\hline $\mathrm{N} 3-\mathrm{C} 47-\mathrm{H} 47$ & 120.6 \\
\hline $\mathrm{C} 46-\mathrm{C} 47-\mathrm{H} 47$ & 120.6 \\
\hline $\mathrm{C} 49-\mathrm{C} 48-\mathrm{C} 39$ & $130.0(12)$ \\
\hline $\mathrm{C} 49-\mathrm{C} 48-\mathrm{C} 53$ & $120.6(11)$ \\
\hline $\mathrm{C} 53-\mathrm{C} 48-\mathrm{C} 39$ & $109.4(11)$ \\
\hline $\mathrm{C} 48-\mathrm{C} 49-\mathrm{H} 49$ & 120.6 \\
\hline $\mathrm{C} 48-\mathrm{C} 49-\mathrm{C} 50$ & $118.8(12)$ \\
\hline $\mathrm{C} 50-\mathrm{C} 49-\mathrm{H} 49$ & 120.6 \\
\hline $\mathrm{C} 49-\mathrm{C} 50-\mathrm{H} 50$ & 120.3 \\
\hline $\mathrm{C} 51-\mathrm{C} 50-\mathrm{C} 49$ & $119.3(13)$ \\
\hline $\mathrm{C} 51-\mathrm{C} 50-\mathrm{H} 50$ & 120.3 \\
\hline $\mathrm{C} 50-\mathrm{C} 51-\mathrm{H} 51$ & 119.5 \\
\hline $\mathrm{C} 52-\mathrm{C} 51-\mathrm{C} 50$ & $121.0(13)$ \\
\hline $\mathrm{C} 52-\mathrm{C} 51-\mathrm{H} 51$ & 119.5 \\
\hline $\mathrm{C} 51-\mathrm{C} 52-\mathrm{H} 52$ & 119.9 \\
\hline $\mathrm{C} 53-\mathrm{C} 52-\mathrm{C} 51$ & $120.2(14)$ \\
\hline $\mathrm{C} 53-\mathrm{C} 52-\mathrm{H} 52$ & 119.9 \\
\hline $\mathrm{C} 48-\mathrm{C} 53-\mathrm{C} 54$ & $110.6(10)$ \\
\hline
\end{tabular}

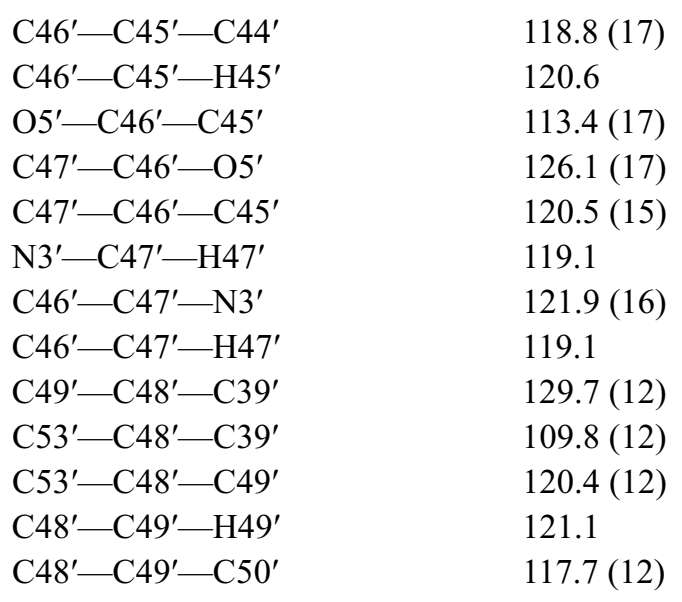




\begin{tabular}{|c|c|}
\hline $\mathrm{C} 52-\mathrm{C} 53-\mathrm{C} 48$ & $120.1(13)$ \\
\hline $\mathrm{C} 52-\mathrm{C} 53-\mathrm{C} 54$ & $129.3(12)$ \\
\hline $\mathrm{C} 40-\mathrm{C} 54-\mathrm{C} 97$ & $110.8(10)$ \\
\hline $\mathrm{C} 40-\mathrm{C} 54-\mathrm{C} 103$ & $111.5(10)$ \\
\hline $\mathrm{C} 53-\mathrm{C} 54-\mathrm{C} 40$ & $101.0(9)$ \\
\hline $\mathrm{C} 53-\mathrm{C} 54-\mathrm{C} 97$ & $112.2(10)$ \\
\hline $\mathrm{C} 53-\mathrm{C} 54-\mathrm{C} 103$ & $113.0(10)$ \\
\hline $\mathrm{C} 97-\mathrm{C} 54-\mathrm{C} 103$ & $108.2(10)$ \\
\hline $\mathrm{C} 56-\mathrm{C} 55-\mathrm{Ir} 2$ & $127.3(8)$ \\
\hline $\mathrm{C} 60-\mathrm{C} 55-\mathrm{Ir} 2$ & $115.4(8)$ \\
\hline $\mathrm{C} 60-\mathrm{C} 55-\mathrm{C} 56$ & $117.0(10)$ \\
\hline $\mathrm{C} 55-\mathrm{C} 56-\mathrm{H} 56$ & 120.6 \\
\hline $\mathrm{C} 57-\mathrm{C} 56-\mathrm{C} 55$ & $118.9(11)$ \\
\hline $\mathrm{C} 57-\mathrm{C} 56-\mathrm{H} 56$ & 120.6 \\
\hline $\mathrm{C} 56-\mathrm{C} 57-\mathrm{C} 66$ & $129.2(11)$ \\
\hline $\mathrm{C} 58-\mathrm{C} 57-\mathrm{C} 56$ & $122.4(11)$ \\
\hline $\mathrm{C} 58-\mathrm{C} 57-\mathrm{C} 66$ & $108.4(10)$ \\
\hline $\mathrm{C} 57-\mathrm{C} 58-\mathrm{C} 72$ & $111.5(10)$ \\
\hline $\mathrm{C} 59-\mathrm{C} 58-\mathrm{C} 57$ & $119.8(11)$ \\
\hline $\mathrm{C} 59-\mathrm{C} 58-\mathrm{C} 72$ & $128.7(11)$ \\
\hline $\mathrm{C} 58-\mathrm{C} 59-\mathrm{H} 59$ & 120.8 \\
\hline $\mathrm{C} 58-\mathrm{C} 59-\mathrm{C} 60$ & $118.5(10)$ \\
\hline $\mathrm{C} 60-\mathrm{C} 59-\mathrm{H} 59$ & 120.8 \\
\hline $\mathrm{C} 55-\mathrm{C} 60-\mathrm{C} 59$ & 123.3 \\
\hline $\mathrm{C} 55-\mathrm{C} 60-\mathrm{C} 61$ & $113.2(9)$ \\
\hline $\mathrm{C} 59-\mathrm{C} 60-\mathrm{C} 61$ & $123.5(10)$ \\
\hline $\mathrm{N} 4-\mathrm{C} 61-\mathrm{C} 60$ & $114.2(10)$ \\
\hline $\mathrm{N} 4-\mathrm{C} 61-\mathrm{C} 62$ & $120.2(10)$ \\
\hline C62-C61-C60 & $125.4(10)$ \\
\hline C61-C62-H62 & 119.6 \\
\hline $\mathrm{C} 63-\mathrm{C} 62-\mathrm{C} 61$ & $120.9(11)$ \\
\hline $\mathrm{C} 63-\mathrm{C} 62-\mathrm{H} 62$ & 119.6 \\
\hline C62-C63-H63 & 121.1 \\
\hline $\mathrm{C} 62-\mathrm{C} 63-\mathrm{C} 64$ & $117.8(12)$ \\
\hline $\mathrm{C} 64-\mathrm{C} 63-\mathrm{H} 63$ & 121.1 \\
\hline $\mathrm{O} 6-\mathrm{C} 64-\mathrm{C} 63$ & $117.4(11)$ \\
\hline $\mathrm{O} 6-\mathrm{C} 64-\mathrm{C} 65$ & $123.4(11)$ \\
\hline $\mathrm{C} 65-\mathrm{C} 64-\mathrm{C} 63$ & $119.2(11)$ \\
\hline $\mathrm{N} 4-\mathrm{C} 65-\mathrm{C} 64$ & $123.3(11)$ \\
\hline $\mathrm{N} 4-\mathrm{C} 65-\mathrm{H} 65$ & 118.3 \\
\hline C64-C65-H65 & 118.3 \\
\hline $\mathrm{C} 67-\mathrm{C} 66-\mathrm{C} 57$ & $131.5(11)$ \\
\hline $\mathrm{C} 67-\mathrm{C} 66-\mathrm{C} 71$ & $121.0(10)$ \\
\hline $\mathrm{C} 71-\mathrm{C} 66-\mathrm{C} 57$ & $107.5(10)$ \\
\hline $\mathrm{C} 66-\mathrm{C} 67-\mathrm{H} 67$ & 120.3 \\
\hline $\mathrm{C} 68-\mathrm{C} 67-\mathrm{C} 66$ & $119.3(12)$ \\
\hline C68-C67-H67 & 120.3 \\
\hline $\mathrm{C} 67-\mathrm{C} 68$ & \\
\hline
\end{tabular}

\begin{tabular}{|c|c|}
\hline $\mathrm{C} 59^{\prime}-\mathrm{C} 60^{\prime}-\mathrm{C} 55^{\prime}$ & $121.4(12)$ \\
\hline $\mathrm{C} 59^{\prime}-\mathrm{C} 60^{\prime}-\mathrm{C} 61^{\prime}$ & $123.4(13)$ \\
\hline $\mathrm{N} 4^{\prime}-\mathrm{C} 61^{\prime}-\mathrm{C} 60^{\prime}$ & $108.5(12)$ \\
\hline $\mathrm{N} 4^{\prime}-\mathrm{C} 61^{\prime}-\mathrm{C} 62^{\prime}$ & $125.3(13)$ \\
\hline $\mathrm{C} 62^{\prime}-\mathrm{C} 61^{\prime}-\mathrm{C} 60^{\prime}$ & $126.2(14)$ \\
\hline $\mathrm{C} 61^{\prime}-\mathrm{C} 62^{\prime}-\mathrm{H} 62^{\prime}$ & 120.4 \\
\hline $\mathrm{C} 61^{\prime}-\mathrm{C} 62^{\prime}-\mathrm{C} 63^{\prime}$ & $119.3(15)$ \\
\hline $\mathrm{C} 63^{\prime}-\mathrm{C} 62^{\prime}-\mathrm{H} 62^{\prime}$ & 120.4 \\
\hline $\mathrm{C} 62^{\prime}-\mathrm{C} 63^{\prime}-\mathrm{H} 63^{\prime}$ & 120.2 \\
\hline $\mathrm{C} 64^{\prime}-\mathrm{C} 63^{\prime}-\mathrm{C} 62^{\prime}$ & $119.6(16)$ \\
\hline C64'-C63'-H63' & 120.2 \\
\hline $\mathrm{C} 63^{\prime}-\mathrm{C} 64^{\prime}-\mathrm{O}^{\prime}$ & $118.6(16)$ \\
\hline $\mathrm{C} 63^{\prime}-\mathrm{C} 64^{\prime}-\mathrm{C} 65^{\prime}$ & $119.6(16)$ \\
\hline $\mathrm{C} 65^{\prime}-\mathrm{C} 64^{\prime}-\mathrm{O}^{\prime}$ & $121.6(15)$ \\
\hline $\mathrm{N} 4^{\prime}-\mathrm{C} 65^{\prime}-\mathrm{H} 65^{\prime}$ & 117.1 \\
\hline $\mathrm{C} 64^{\prime}-\mathrm{C} 65^{\prime}-\mathrm{N} 4^{\prime}$ & $125.7(15)$ \\
\hline $\mathrm{C} 64^{\prime}-\mathrm{C} 65^{\prime}-\mathrm{H} 65^{\prime}$ & 117.1 \\
\hline $\mathrm{C} 67^{\prime}-\mathrm{C} 66^{\prime}-\mathrm{C} 57^{\prime}$ & $131.0(12)$ \\
\hline $\mathrm{C} 67^{\prime}-\mathrm{C} 66^{\prime}-\mathrm{C} 71^{\prime}$ & $119.6(12)$ \\
\hline $\mathrm{C} 71^{\prime}-\mathrm{C} 66^{\prime}-\mathrm{C} 57^{\prime}$ & $109.4(12)$ \\
\hline $\mathrm{C} 66^{\prime}-\mathrm{C} 67^{\prime}-\mathrm{H} 67^{\prime}$ & 120.4 \\
\hline $\mathrm{C} 68^{\prime}-\mathrm{C} 67^{\prime}-\mathrm{C} 66^{\prime}$ & $119.1(13)$ \\
\hline $\mathrm{C} 68^{\prime}-\mathrm{C} 67^{\prime}-\mathrm{H} 67^{\prime}$ & 120.4 \\
\hline $\mathrm{C} 67^{\prime}-\mathrm{C} 68^{\prime}-\mathrm{H} 68^{\prime}$ & 119.4 \\
\hline $\mathrm{C} 67^{\prime}-\mathrm{C} 68^{\prime}-\mathrm{C} 69^{\prime}$ & $121.2(14)$ \\
\hline $\mathrm{C} 69^{\prime}-\mathrm{C} 68^{\prime}-\mathrm{H} 68^{\prime}$ & 119.4 \\
\hline $\mathrm{C} 68^{\prime}-\mathrm{C} 69^{\prime}-\mathrm{H} 69^{\prime}$ & 119.7 \\
\hline $\mathrm{C} 68^{\prime}-\mathrm{C} 69^{\prime}-\mathrm{C} 70^{\prime}$ & $120.7(13)$ \\
\hline $\mathrm{C} 70^{\prime}-\mathrm{C} 69^{\prime}-\mathrm{H} 69^{\prime}$ & 119.7 \\
\hline $\mathrm{C} 69^{\prime}-\mathrm{C} 70^{\prime}-\mathrm{H} 70^{\prime}$ & 120.8 \\
\hline $\mathrm{C} 71^{\prime}-\mathrm{C} 70^{\prime}-\mathrm{C} 69^{\prime}$ & $118.5(14)$ \\
\hline $\mathrm{C} 71^{\prime}-\mathrm{C} 70^{\prime}-\mathrm{H} 70^{\prime}$ & 120.8 \\
\hline $\mathrm{C} 66^{\prime}-\mathrm{C} 71^{\prime}-\mathrm{C} 72^{\prime}$ & $109.6(11)$ \\
\hline $\mathrm{C} 70^{\prime}-\mathrm{C} 71^{\prime}-\mathrm{C} 66^{\prime}$ & $120.9(14)$ \\
\hline $\mathrm{C} 70^{\prime}-\mathrm{C} 71^{\prime}-\mathrm{C} 72^{\prime}$ & $129.5(13)$ \\
\hline $\mathrm{C} 58^{\prime}-\mathrm{C} 72^{\prime}-\mathrm{C} 209$ & $110.9(11)$ \\
\hline $\mathrm{C} 58^{\prime}-\mathrm{C} 72^{\prime}-\mathrm{C} 215$ & $111.5(11)$ \\
\hline $\mathrm{C} 71^{\prime}-\mathrm{C} 72^{\prime}-\mathrm{C} 58^{\prime}$ & $101.8(11)$ \\
\hline $\mathrm{C} 71^{\prime}-\mathrm{C} 72^{\prime}-\mathrm{C} 209$ & $110.7(11)$ \\
\hline $\mathrm{C} 71^{\prime}-\mathrm{C} 72^{\prime}-\mathrm{C} 215$ & $112.1(11)$ \\
\hline $\mathrm{C} 215-\mathrm{C} 72^{\prime}-\mathrm{C} 209$ & $109.6(12)$ \\
\hline $\mathrm{C} 18^{\prime}-\mathrm{C} 73^{\prime}-\mathrm{H} 73 \mathrm{~A}$ & 108.4 \\
\hline $\mathrm{C} 18^{\prime}-\mathrm{C} 73^{\prime}-\mathrm{H} 73 \mathrm{~B}$ & 108.4 \\
\hline $\mathrm{H} 73 \mathrm{~A}-\mathrm{C} 73^{\prime}-\mathrm{H} 73 \mathrm{~B}$ & 107.5 \\
\hline $\mathrm{C} 74^{\prime}-\mathrm{C} 73^{\prime}-\mathrm{C} 18^{\prime}$ & $115.3(12)$ \\
\hline $\mathrm{C} 74^{\prime}-\mathrm{C} 73^{\prime}-\mathrm{H} 73 \mathrm{~A}$ & 108.4 \\
\hline $\mathrm{C} 74^{\prime}-\mathrm{C} 73^{\prime}-\mathrm{H} 73 \mathrm{~B}$ & 108.4 \\
\hline $\mathrm{C} 73^{\prime}-\mathrm{C} 74^{\prime}-\mathrm{H} 74 \mathrm{~A}$ & 107.6 \\
\hline
\end{tabular}




\begin{tabular}{|c|c|}
\hline $\mathrm{C} 67-\mathrm{C} 68-\mathrm{C} 69$ & $120.1(12)$ \\
\hline $\mathrm{C} 69-\mathrm{C} 68-\mathrm{H} 68$ & 120.0 \\
\hline C68-C69-H69 & 120.2 \\
\hline $\mathrm{C} 70-\mathrm{C} 69-\mathrm{C} 68$ & $119.6(12)$ \\
\hline $\mathrm{C} 70-\mathrm{C} 69-\mathrm{H} 69$ & 120.2 \\
\hline $\mathrm{C} 69-\mathrm{C} 70-\mathrm{H} 70$ & 119.1 \\
\hline $\mathrm{C} 69-\mathrm{C} 70-\mathrm{C} 71$ & $121.7(12)$ \\
\hline $\mathrm{C} 71-\mathrm{C} 70-\mathrm{H} 70$ & 119.1 \\
\hline $\mathrm{C} 66-\mathrm{C} 71-\mathrm{C} 72$ & $112.1(10)$ \\
\hline $\mathrm{C} 70-\mathrm{C} 71-\mathrm{C} 66$ & $118.2(11)$ \\
\hline $\mathrm{C} 70-\mathrm{C} 71-\mathrm{C} 72$ & $129.7(11)$ \\
\hline $\mathrm{C} 58-\mathrm{C} 72-\mathrm{C} 109$ & $110.0(9)$ \\
\hline $\mathrm{C} 58-\mathrm{C} 72-\mathrm{C} 115$ & $113.3(10)$ \\
\hline $\mathrm{C} 71-\mathrm{C} 72-\mathrm{C} 58$ & $100.4(9)$ \\
\hline $\mathrm{C} 71-\mathrm{C} 72-\mathrm{C} 109$ & $112.5(10)$ \\
\hline $\mathrm{C} 71-\mathrm{C} 72-\mathrm{C} 115$ & $113.1(9)$ \\
\hline $\mathrm{C} 115-\mathrm{C} 72-\mathrm{C} 109$ & $107.5(9)$ \\
\hline $\mathrm{C} 18-\mathrm{C} 73-\mathrm{H} 73 \mathrm{C}$ & 108.3 \\
\hline $\mathrm{C} 18-\mathrm{C} 73-\mathrm{H} 73 \mathrm{D}$ & 108.3 \\
\hline $\mathrm{H} 73 \mathrm{C}-\mathrm{C} 73-\mathrm{H} 73 \mathrm{D}$ & 107.4 \\
\hline $\mathrm{C} 74-\mathrm{C} 73-\mathrm{C} 18$ & $115.9(10)$ \\
\hline $\mathrm{C} 74-\mathrm{C} 73-\mathrm{H} 73 \mathrm{C}$ & 108.3 \\
\hline $\mathrm{C} 74-\mathrm{C} 73-\mathrm{H} 73 \mathrm{D}$ & 108.3 \\
\hline $\mathrm{C} 73-\mathrm{C} 74-\mathrm{H} 74 \mathrm{C}$ & 108.7 \\
\hline $\mathrm{C} 73-\mathrm{C} 74-\mathrm{H} 74 \mathrm{D}$ & 108.7 \\
\hline $\mathrm{C} 73-\mathrm{C} 74-\mathrm{C} 75$ & $114.1(11)$ \\
\hline $\mathrm{H} 74 \mathrm{C}-\mathrm{C} 74-\mathrm{H} 74 \mathrm{D}$ & 107.6 \\
\hline $\mathrm{C} 75-\mathrm{C} 74-\mathrm{H} 74 \mathrm{C}$ & 108.7 \\
\hline $\mathrm{C} 75-\mathrm{C} 74-\mathrm{H} 74 \mathrm{D}$ & 108.7 \\
\hline $\mathrm{C} 74-\mathrm{C} 75-\mathrm{H} 75 \mathrm{C}$ & 107.7 \\
\hline $\mathrm{C} 74-\mathrm{C} 75-\mathrm{H} 75 \mathrm{D}$ & 107.7 \\
\hline $\mathrm{H} 75 \mathrm{C}-\mathrm{C} 75-\mathrm{H} 75 \mathrm{D}$ & 107.1 \\
\hline $\mathrm{C} 76-\mathrm{C} 75-\mathrm{C} 74$ & 118.5 \\
\hline $\mathrm{C} 76-\mathrm{C} 75-\mathrm{H} 75 \mathrm{C}$ & 107.7 \\
\hline $\mathrm{C} 76-\mathrm{C} 75-\mathrm{H} 75 \mathrm{D}$ & 107.7 \\
\hline $\mathrm{C} 75-\mathrm{C} 76-\mathrm{H} 76 \mathrm{C}$ & 109.7 \\
\hline $\mathrm{C} 75-\mathrm{C} 76-\mathrm{H} 76 \mathrm{D}$ & 109.7 \\
\hline $\mathrm{C} 75-\mathrm{C} 76-\mathrm{C} 77$ & $110.0(16)$ \\
\hline $\mathrm{H} 76 \mathrm{C}-\mathrm{C} 76-\mathrm{H} 76 \mathrm{D}$ & 108.2 \\
\hline $\mathrm{C} 77-\mathrm{C} 76-\mathrm{H} 76 \mathrm{C}$ & 109.7 \\
\hline $\mathrm{C} 77-\mathrm{C} 76-\mathrm{H} 76 \mathrm{D}$ & 109.7 \\
\hline $\mathrm{C} 76-\mathrm{C} 77-\mathrm{H} 77 \mathrm{C}$ & 109.0 \\
\hline C76-C77-H77D & 109.0 \\
\hline $\mathrm{H} 77 \mathrm{C}-\mathrm{C} 77-\mathrm{H} 77 \mathrm{D}$ & 107.8 \\
\hline $\mathrm{C} 78-\mathrm{C} 77-\mathrm{C} 76$ & $113.0(18)$ \\
\hline $\mathrm{C} 78-\mathrm{C} 77-\mathrm{H} 77 \mathrm{C}$ & 109.0 \\
\hline C78-C77-H77D & 109.0 \\
\hline C78 & \\
\hline
\end{tabular}

\begin{tabular}{|c|c|}
\hline $\mathrm{C} 73^{\prime}-\mathrm{C} 74^{\prime}-\mathrm{H} 74 \mathrm{~B}$ & 107.6 \\
\hline $\mathrm{C} 73^{\prime}-\mathrm{C} 74^{\prime}-\mathrm{C} 75^{\prime}$ & $118.8(14)$ \\
\hline $\mathrm{H} 74 \mathrm{~A}-\mathrm{C} 74^{\prime}-\mathrm{H} 74 \mathrm{~B}$ & 107.1 \\
\hline $\mathrm{C} 75^{\prime}-\mathrm{C} 74^{\prime}-\mathrm{H} 74 \mathrm{~A}$ & 107.6 \\
\hline $\mathrm{C} 75^{\prime}-\mathrm{C} 74^{\prime}-\mathrm{H} 74 \mathrm{~B}$ & 107.6 \\
\hline $\mathrm{C} 74^{\prime}-\mathrm{C} 75^{\prime}-\mathrm{H} 75 \mathrm{~A}$ & 108.5 \\
\hline $\mathrm{C} 74^{\prime}-\mathrm{C} 75^{\prime}-\mathrm{H} 75 \mathrm{~B}$ & 108.5 \\
\hline $\mathrm{H} 75 \mathrm{~A}-\mathrm{C} 75^{\prime}-\mathrm{H} 75 \mathrm{~B}$ & 107.5 \\
\hline $\mathrm{C} 76^{\prime}-\mathrm{C} 75^{\prime}-\mathrm{C} 74^{\prime}$ & $114.9(14)$ \\
\hline $\mathrm{C} 76^{\prime}-\mathrm{C} 75^{\prime}-\mathrm{H} 75 \mathrm{~A}$ & 108.5 \\
\hline $\mathrm{C} 76^{\prime}-\mathrm{C} 75^{\prime}-\mathrm{H} 75 \mathrm{~B}$ & 108.5 \\
\hline $\mathrm{C} 75^{\prime}-\mathrm{C} 76^{\prime}-\mathrm{H} 76 \mathrm{~A}$ & 108.9 \\
\hline $\mathrm{C} 75^{\prime}-\mathrm{C} 76^{\prime}-\mathrm{H} 76 \mathrm{~B}$ & 108.9 \\
\hline $\mathrm{C} 75^{\prime}-\mathrm{C} 76^{\prime}-\mathrm{C} 77^{\prime}$ & $113.4(16)$ \\
\hline $\mathrm{H} 76 \mathrm{~A}-\mathrm{C} 76^{\prime}-\mathrm{H} 76 \mathrm{~B}$ & 107.7 \\
\hline $\mathrm{C} 77^{\prime}-\mathrm{C} 76^{\prime}-\mathrm{H} 76 \mathrm{~A}$ & 108.9 \\
\hline $\mathrm{C} 77^{\prime}-\mathrm{C} 76^{\prime}-\mathrm{H} 76 \mathrm{~B}$ & 108.9 \\
\hline $\mathrm{C} 76^{\prime}-\mathrm{C} 77^{\prime}-\mathrm{H} 77 \mathrm{~A}$ & 109.6 \\
\hline $\mathrm{C} 76^{\prime}-\mathrm{C} 77^{\prime}-\mathrm{H} 77 \mathrm{~B}$ & 109.6 \\
\hline $\mathrm{H} 77 \mathrm{~A}-\mathrm{C} 77^{\prime}-\mathrm{H} 77 \mathrm{~B}$ & 108.1 \\
\hline $\mathrm{C} 78^{\prime}-\mathrm{C} 77^{\prime}-\mathrm{C} 76^{\prime}$ & $110.4(16)$ \\
\hline $\mathrm{C} 78^{\prime}-\mathrm{C} 77^{\prime}-\mathrm{H} 77 \mathrm{~A}$ & 109.6 \\
\hline $\mathrm{C} 78^{\prime}-\mathrm{C} 77^{\prime}-\mathrm{H} 77 \mathrm{~B}$ & 109.6 \\
\hline $\mathrm{C} 77^{\prime}-\mathrm{C} 78^{\prime}-\mathrm{H} 78 \mathrm{~A}$ & 109.5 \\
\hline $\mathrm{C} 77^{\prime}-\mathrm{C} 78^{\prime}-\mathrm{H} 78 \mathrm{~B}$ & 109.5 \\
\hline $\mathrm{C} 77^{\prime}-\mathrm{C} 78^{\prime}-\mathrm{H} 78 \mathrm{C}$ & 109.5 \\
\hline $\mathrm{H} 78 \mathrm{~A}-\mathrm{C} 78^{\prime}-\mathrm{H} 78 \mathrm{~B}$ & 109.5 \\
\hline $\mathrm{H} 78 \mathrm{~A}-\mathrm{C} 78^{\prime}-\mathrm{H} 78 \mathrm{C}$ & 109.5 \\
\hline $\mathrm{H} 78 \mathrm{~B}-\mathrm{C} 78^{\prime}-\mathrm{H} 78 \mathrm{C}$ & 109.5 \\
\hline $\mathrm{C} 18^{\prime}-\mathrm{C} 79^{\prime}-\mathrm{H} 79 \mathrm{C}$ & 106.6 \\
\hline $\mathrm{C} 18^{\prime}-\mathrm{C} 79^{\prime}-\mathrm{H} 79 \mathrm{D}$ & 106.6 \\
\hline $\mathrm{C} 18^{\prime}-\mathrm{C} 79^{\prime}-\mathrm{H} 79 \mathrm{~A}$ & 109.1 \\
\hline $\mathrm{C} 18^{\prime}-\mathrm{C} 79^{\prime}-\mathrm{H} 79 \mathrm{~B}$ & 109.1 \\
\hline $\mathrm{C} 18^{\prime}-\mathrm{C} 79^{\prime}-\mathrm{C} 80^{\prime}$ & $112.3(15)$ \\
\hline $\mathrm{C} 18^{\prime}-\mathrm{C} 79^{\prime}-\mathrm{C} 80 \mathrm{~B}$ & $123.1(19)$ \\
\hline $\mathrm{H} 79 \mathrm{C}-\mathrm{C} 79^{\prime}-\mathrm{H} 79 \mathrm{D}$ & 106.5 \\
\hline $\mathrm{H} 79 \mathrm{~A}-\mathrm{C} 79^{\prime}-\mathrm{H} 79 \mathrm{~B}$ & 107.9 \\
\hline $\mathrm{C} 80^{\prime}-\mathrm{C} 79^{\prime}-\mathrm{H} 79 \mathrm{~A}$ & 109.1 \\
\hline $\mathrm{C} 80^{\prime}-\mathrm{C} 79^{\prime}-\mathrm{H} 79 \mathrm{~B}$ & 109.1 \\
\hline $\mathrm{C} 80 \mathrm{~B}-\mathrm{C} 79^{\prime}-\mathrm{H} 79 \mathrm{C}$ & 106.6 \\
\hline $\mathrm{C} 80 \mathrm{~B}-\mathrm{C} 79^{\prime}-\mathrm{H} 79 \mathrm{D}$ & 106.6 \\
\hline $\mathrm{H} 81 \mathrm{C}-\mathrm{C} 81^{\prime}-\mathrm{H} 81 \mathrm{D}$ & 104.3 \\
\hline $\mathrm{H} 81 \mathrm{~A}-\mathrm{C} 81^{\prime}-\mathrm{H} 81 \mathrm{~B}$ & 106.9 \\
\hline $\mathrm{C} 82^{\prime}-\mathrm{C} 81^{\prime}-\mathrm{H} 81 \mathrm{C}$ & 100.6 \\
\hline $\mathrm{C} 82^{\prime}-\mathrm{C} 81^{\prime}-\mathrm{H} 81 \mathrm{D}$ & 100.6 \\
\hline $\mathrm{C} 82^{\prime}-\mathrm{C} 81^{\prime}-\mathrm{H} 81 \mathrm{~A}$ & 107.4 \\
\hline $\mathrm{C} 82^{\prime}-\mathrm{C} 81^{\prime}-\mathrm{H} 81 \mathrm{~B}$ & 107.4 \\
\hline $\mathrm{C} 80^{\prime}-\mathrm{C} 81^{\prime}-\mathrm{H} 81 \mathrm{~A}$ & 107.4 \\
\hline
\end{tabular}


C77-C78-H78E

C77-C78-H78F

H78D-C78-H78E

$\mathrm{H} 78 \mathrm{D}-\mathrm{C} 78-\mathrm{H} 78 \mathrm{~F}$

H78E-C78-H78F

C18-C79-H79E

C18-C79-H79F

$\mathrm{C} 18-\mathrm{C} 79-\mathrm{C} 80$

H79E-C79-H79F

C80-C79-H79E

C80-C79-H79F

C79-C80-H80E

C79- $880-\mathrm{H} 80 \mathrm{~F}$

H80E-C80-H80F

$\mathrm{C} 81-\mathrm{C} 80-\mathrm{C} 79$

$\mathrm{C} 81-\mathrm{C} 80-\mathrm{H} 80 \mathrm{E}$

$\mathrm{C} 81-\mathrm{C} 80-\mathrm{H} 80 \mathrm{~F}$

C80-C81-H81E

$\mathrm{C} 80-\mathrm{C} 81-\mathrm{H} 81 \mathrm{~F}$

H81E-C81-H81F

$\mathrm{C} 82-\mathrm{C} 81-\mathrm{C} 80$

$\mathrm{C} 82-\mathrm{C} 81-\mathrm{H} 81 \mathrm{E}$

C82-C81-H81F

$\mathrm{C} 81-\mathrm{C} 82-\mathrm{H} 82 \mathrm{C}$

C81-C82-H82D

$\mathrm{H} 82 \mathrm{C}-\mathrm{C} 82-\mathrm{H} 82 \mathrm{D}$

$\mathrm{C} 83-\mathrm{C} 82-\mathrm{C} 81$

$\mathrm{C} 83-\mathrm{C} 82-\mathrm{H} 82 \mathrm{C}$

$\mathrm{C} 83-\mathrm{C} 82-\mathrm{H} 82 \mathrm{D}$

$\mathrm{C} 82-\mathrm{C} 83-\mathrm{H} 83 \mathrm{C}$

$\mathrm{C} 82-\mathrm{C} 83-\mathrm{H} 83 \mathrm{D}$

$\mathrm{C} 82-\mathrm{C} 83-\mathrm{C} 84$

$\mathrm{H} 83 \mathrm{C}-\mathrm{C} 83-\mathrm{H} 83 \mathrm{D}$

$\mathrm{C} 84-\mathrm{C} 83-\mathrm{H} 83 \mathrm{C}$

C84-C83-H83D

C83-C84-H84D

C83-C84-H84E

C83-C84-H84F

H84D-C84-H84E

H84D-C84-H84F

$\mathrm{H} 84 \mathrm{E}-\mathrm{C} 84-\mathrm{H} 84 \mathrm{~F}$

$\mathrm{C} 36-\mathrm{C} 85-\mathrm{H} 85 \mathrm{C}$

C36-C85-H85D

$\mathrm{H} 85 \mathrm{C}-\mathrm{C} 85-\mathrm{H} 85 \mathrm{D}$

$\mathrm{C} 86-\mathrm{C} 85-\mathrm{C} 36$

$\mathrm{C} 86-\mathrm{C} 85-\mathrm{H} 85 \mathrm{C}$

C86-C85-H85D

C85-C86-H86C
109.5

109.5

109.5

109.5

109.5

108.3

108.3

115.8 (10)

107.4

108.3

108.3

108.8

108.8

107.7

113.8 (11)

108.8

108.8

108.5

108.5

107.5

115.0 (12)

108.5

108.5

108.7

108.7

107.6

114.4 (13)

108.7

108.7

108.6

108.6

114.8 (15)

107.6

108.6

108.6

109.5

109.5

109.5

109.5

109.5

109.5

108.3

108.3

107.4

116.1 (9)

108.3

108.3

108.6
C80'-C81'- $\mathrm{H} 81 \mathrm{~B}$

$\mathrm{C} 80^{\prime}-\mathrm{C} 81^{\prime}-\mathrm{C} 82^{\prime}$

$\mathrm{C} 80 \mathrm{~B}-\mathrm{C} 81^{\prime}-\mathrm{H} 81 \mathrm{C}$

$\mathrm{C} 80 \mathrm{~B}-\mathrm{C} 81^{\prime}-\mathrm{H} 81 \mathrm{D}$

$\mathrm{C} 80 \mathrm{~B}-\mathrm{C} 81^{\prime}-\mathrm{C} 82^{\prime}$

$\mathrm{C} 81^{\prime}-\mathrm{C} 82^{\prime}-\mathrm{H} 82 \mathrm{~A}$

$\mathrm{C} 81^{\prime}-\mathrm{C} 82^{\prime}-\mathrm{H} 82 \mathrm{~B}$

$\mathrm{H} 82 \mathrm{~A}-\mathrm{C} 82^{\prime}-\mathrm{H} 82 \mathrm{~B}$

$\mathrm{C} 83^{\prime}-\mathrm{C} 82^{\prime}-\mathrm{C} 81^{\prime}$

$\mathrm{C} 83^{\prime}-\mathrm{C} 82^{\prime}-\mathrm{H} 82 \mathrm{~A}$

C83'-C $82^{\prime}-\mathrm{H} 82 \mathrm{~B}$

$\mathrm{C} 82^{\prime}-\mathrm{C} 83^{\prime}-\mathrm{H} 83 \mathrm{~A}$

$\mathrm{C} 82^{\prime}-\mathrm{C} 83^{\prime}-\mathrm{H} 83 \mathrm{~B}$

H83A-C $83^{\prime}-\mathrm{H} 83 \mathrm{~B}$

$\mathrm{C} 84^{\prime}-\mathrm{C} 83^{\prime}-\mathrm{C} 82^{\prime}$

$\mathrm{C} 84^{\prime}-\mathrm{C} 83^{\prime}-\mathrm{H} 83 \mathrm{~A}$

C84'-C83'-H83B

C83'-C84'-H84A

C83'-C84'-H84B

C83'- $84^{\prime}-\mathrm{H} 84 \mathrm{C}$

$\mathrm{H} 84 \mathrm{~A}-\mathrm{C} 84^{\prime}-\mathrm{H} 84 \mathrm{~B}$

$\mathrm{H} 84 \mathrm{~A}-\mathrm{C} 84^{\prime}-\mathrm{H} 84 \mathrm{C}$

$\mathrm{H} 84 \mathrm{~B}-\mathrm{C} 84^{\prime}-\mathrm{H} 84 \mathrm{C}$

$\mathrm{C} 36^{\prime}-\mathrm{C} 85^{\prime}-\mathrm{H} 85 \mathrm{~A}$

$\mathrm{C} 36^{\prime}-\mathrm{C} 85^{\prime}-\mathrm{H} 85 \mathrm{~B}$

$\mathrm{C} 36^{\prime}-\mathrm{C} 85^{\prime}-\mathrm{C} 86^{\prime}$

$\mathrm{H} 85 \mathrm{~A}-\mathrm{C} 85^{\prime}-\mathrm{H} 85 \mathrm{~B}$

$\mathrm{C} 86^{\prime}-\mathrm{C} 85^{\prime}-\mathrm{H} 85 \mathrm{~A}$

$\mathrm{C} 86^{\prime}-\mathrm{C} 85^{\prime}-\mathrm{H} 85 \mathrm{~B}$

$\mathrm{C} 85^{\prime}-\mathrm{C} 86^{\prime}-\mathrm{H} 86 \mathrm{~A}$

$\mathrm{C} 85^{\prime}-\mathrm{C} 86^{\prime}-\mathrm{H} 86 \mathrm{~B}$

H86A-C86'-H86B

$\mathrm{C} 87^{\prime}-\mathrm{C}^{\prime} 6^{\prime}-\mathrm{C}^{\prime} 5^{\prime}$

$\mathrm{C} 87^{\prime}-\mathrm{C} 86^{\prime}-\mathrm{H} 86 \mathrm{~A}$

$\mathrm{C} 87^{\prime}-\mathrm{C} 86^{\prime}-\mathrm{H} 86 \mathrm{~B}$

C86'-C87'-H87A

C86'-C87'-H87B

H87A-C87'-H87B

$\mathrm{C} 88^{\prime}-\mathrm{C} 87^{\prime}-\mathrm{C}^{\prime} 6^{\prime}$

C88'-C87'-H87A

C88'-C87'-H87B

$\mathrm{C} 87^{\prime}-\mathrm{C} 88^{\prime}-\mathrm{H} 88 \mathrm{~A}$

$\mathrm{C} 87^{\prime}-\mathrm{C} 88^{\prime}-\mathrm{H} 88 \mathrm{~B}$

C87'-C88'-C89'

$\mathrm{H} 88 \mathrm{~A}-\mathrm{C} 88^{\prime}-\mathrm{H} 88 \mathrm{~B}$

C89'-C88'-H88A

C89'-C88'-H88B

C88'-C89'-H89A
107.4

$120(2)$

100.6

100.6

145 (3)

106.0

106.0

106.3

125 (2)

106.0

106.0

111.8

111.8

109.5

$100(2)$

111.8

111.8

109.5

109.5

109.5

109.5

109.5

109.5

108.3

108.3

115.9 (12)

107.4

108.3

108.3

108.2

108.2

107.4

116.3 (13)

108.2

108.2

108.5

108.5

107.5

115.1 (13)

108.5

108.5

108.4

108.4

115.6 (14)

107.4

108.4

108.4

108.7 


\begin{tabular}{|c|c|}
\hline $\mathrm{C} 85-\mathrm{C} 86-\mathrm{H} 86 \mathrm{D}$ & 108.6 \\
\hline $\mathrm{C} 85-\mathrm{C} 86-\mathrm{C} 87$ & $114.6(9)$ \\
\hline $\mathrm{H} 86 \mathrm{C}-\mathrm{C} 86-\mathrm{H} 86 \mathrm{D}$ & 107.6 \\
\hline $\mathrm{C} 87-\mathrm{C} 86-\mathrm{H} 86 \mathrm{C}$ & 108.6 \\
\hline $\mathrm{C} 87-\mathrm{C} 86-\mathrm{H} 86 \mathrm{D}$ & 108.6 \\
\hline $\mathrm{C} 86-\mathrm{C} 87-\mathrm{H} 87 \mathrm{C}$ & 108.6 \\
\hline $\mathrm{C} 86-\mathrm{C} 87-\mathrm{H} 87 \mathrm{D}$ & 108.6 \\
\hline $\mathrm{C} 86-\mathrm{C} 87-\mathrm{C} 88$ & $114.8(10)$ \\
\hline $\mathrm{H} 87 \mathrm{C}-\mathrm{C} 87-\mathrm{H} 87 \mathrm{D}$ & 107.5 \\
\hline $\mathrm{C} 88-\mathrm{C} 87-\mathrm{H} 87 \mathrm{C}$ & 108.6 \\
\hline $\mathrm{C} 88-\mathrm{C} 87-\mathrm{H} 87 \mathrm{D}$ & 108.6 \\
\hline $\mathrm{C} 87-\mathrm{C} 88-\mathrm{H} 88 \mathrm{C}$ & 108.7 \\
\hline $\mathrm{C} 87-\mathrm{C} 88-\mathrm{H} 88 \mathrm{D}$ & 108.7 \\
\hline $\mathrm{H} 88 \mathrm{C}-\mathrm{C} 88-\mathrm{H} 88 \mathrm{D}$ & 107.6 \\
\hline $\mathrm{C} 89-\mathrm{C} 88-\mathrm{C} 87$ & $114.1(10)$ \\
\hline $\mathrm{C} 89-\mathrm{C} 88-\mathrm{H} 88 \mathrm{C}$ & 108.7 \\
\hline $\mathrm{C} 89-\mathrm{C} 88-\mathrm{H} 88 \mathrm{D}$ & 108.7 \\
\hline $\mathrm{C} 88-\mathrm{C} 89-\mathrm{H} 89 \mathrm{C}$ & 109.0 \\
\hline $\mathrm{C} 88-\mathrm{C} 89-\mathrm{H} 89 \mathrm{D}$ & 109.0 \\
\hline $\mathrm{C} 88-\mathrm{C} 89-\mathrm{C} 90$ & $113.1(12)$ \\
\hline $\mathrm{H} 89 \mathrm{C}-\mathrm{C} 89-\mathrm{H} 89 \mathrm{D}$ & 107.8 \\
\hline С $90-\mathrm{C} 89-\mathrm{H} 89 \mathrm{C}$ & 109.0 \\
\hline $\mathrm{C} 90-\mathrm{C} 89-\mathrm{H} 89 \mathrm{D}$ & 109.0 \\
\hline $\mathrm{C} 89-\mathrm{C} 90-\mathrm{H} 90 \mathrm{D}$ & 109.5 \\
\hline $\mathrm{C} 89-\mathrm{C} 90-\mathrm{H} 90 \mathrm{E}$ & 109.5 \\
\hline $\mathrm{C} 89-\mathrm{C} 90-\mathrm{H} 90 \mathrm{~F}$ & 109.5 \\
\hline H90D-C90-H90E & 109.5 \\
\hline $\mathrm{H} 90 \mathrm{D}-\mathrm{C} 90-\mathrm{H} 90 \mathrm{~F}$ & 109.5 \\
\hline H90E-C90-H90F & 109.5 \\
\hline $\mathrm{C} 36-\mathrm{C} 91-\mathrm{H} 91 \mathrm{C}$ & 108.3 \\
\hline C36-C91-H91D & 108.3 \\
\hline $\mathrm{H} 91 \mathrm{C}-\mathrm{C} 91-\mathrm{H} 91 \mathrm{D}$ & 107.4 \\
\hline $\mathrm{C} 92-\mathrm{C} 91-\mathrm{C} 36$ & $115.8(9)$ \\
\hline C92-C91-H91C & 108.3 \\
\hline C92-C91-H91D & 108.3 \\
\hline C91-C92-H92C & 109.0 \\
\hline C91-C92-H92D & 109.0 \\
\hline $\mathrm{C} 91-\mathrm{C} 92-\mathrm{C} 93$ & $113.0(9)$ \\
\hline $\mathrm{H} 92 \mathrm{C}-\mathrm{C} 92-\mathrm{H} 92 \mathrm{D}$ & 107.8 \\
\hline C93-C92-H92C & 109.0 \\
\hline C93-C92-H92D & 109.0 \\
\hline $\mathrm{C} 92-\mathrm{C} 93-\mathrm{H} 93 \mathrm{C}$ & 108.6 \\
\hline C92-C93-H93D & 108.6 \\
\hline $\mathrm{H} 93 \mathrm{C}-\mathrm{C} 93-\mathrm{H} 93 \mathrm{D}$ & 107.6 \\
\hline C94-C93-C92 & $114.6(10)$ \\
\hline $\mathrm{C} 94-\mathrm{C} 93-\mathrm{H} 93 \mathrm{C}$ & 108.6 \\
\hline C94-C93-H93D & 108.6 \\
\hline $\mathrm{CO2}$ & \\
\hline
\end{tabular}

\begin{tabular}{|c|c|}
\hline $\mathrm{C} 88^{\prime}-\mathrm{C} 89^{\prime}-\mathrm{H} 89 \mathrm{~B}$ & 108.7 \\
\hline $\mathrm{H} 89 \mathrm{~A}-\mathrm{C} 89^{\prime}-\mathrm{H} 89 \mathrm{~B}$ & 107.6 \\
\hline $\mathrm{C} 90^{\prime}-\mathrm{C} 89^{\prime}-\mathrm{C} 88^{\prime}$ & $114.4(14)$ \\
\hline $\mathrm{C} 90^{\prime}-\mathrm{C} 89^{\prime}-\mathrm{H} 89 \mathrm{~A}$ & 108.7 \\
\hline $\mathrm{C} 90^{\prime}-\mathrm{C} 89^{\prime}-\mathrm{H} 89 \mathrm{~B}$ & 108.7 \\
\hline $\mathrm{C} 89^{\prime}-\mathrm{C} 90^{\prime}-\mathrm{H} 90 \mathrm{~A}$ & 109.5 \\
\hline $\mathrm{C} 89^{\prime}-\mathrm{C} 90^{\prime}-\mathrm{H} 90 \mathrm{~B}$ & 109.5 \\
\hline $\mathrm{C} 89^{\prime}-\mathrm{C} 90^{\prime}-\mathrm{H} 90 \mathrm{C}$ & 109.5 \\
\hline $\mathrm{H} 90 \mathrm{~A}-\mathrm{C} 90^{\prime}-\mathrm{H} 90 \mathrm{~B}$ & 109.5 \\
\hline $\mathrm{H} 90 \mathrm{~A}-\mathrm{C} 90^{\prime}-\mathrm{H} 90 \mathrm{C}$ & 109.5 \\
\hline $\mathrm{H} 90 \mathrm{~B}-\mathrm{C} 90^{\prime}-\mathrm{H} 90 \mathrm{C}$ & 109.5 \\
\hline $\mathrm{C} 36^{\prime}-\mathrm{C} 91^{\prime}-\mathrm{H} 91 \mathrm{~A}$ & 108.8 \\
\hline $\mathrm{C} 36^{\prime}-\mathrm{C} 91^{\prime}-\mathrm{H} 91 \mathrm{~B}$ & 108.8 \\
\hline $\mathrm{H} 91 \mathrm{~A}-\mathrm{C} 91^{\prime}-\mathrm{H} 91 \mathrm{~B}$ & 107.7 \\
\hline $\mathrm{C} 92^{\prime}-\mathrm{C} 91^{\prime}-\mathrm{C} 36^{\prime}$ & $113.7(13)$ \\
\hline $\mathrm{C} 92^{\prime}-\mathrm{C} 91^{\prime}-\mathrm{H} 91 \mathrm{~A}$ & 108.8 \\
\hline C92'-C91'-H91B & 108.8 \\
\hline $\mathrm{C} 91^{\prime}-\mathrm{C} 92^{\prime}-\mathrm{H} 92 \mathrm{~A}$ & 107.5 \\
\hline C91'- C92'-H92B & 107.5 \\
\hline $\mathrm{H} 92 \mathrm{~A}-\mathrm{C} 92^{\prime}-\mathrm{H} 92 \mathrm{~B}$ & 107.0 \\
\hline $\mathrm{C} 93^{\prime}-\mathrm{C} 92^{\prime}-\mathrm{C} 91^{\prime}$ & $119.1(19)$ \\
\hline $\mathrm{C} 93^{\prime}-\mathrm{C} 92^{\prime}-\mathrm{H} 92 \mathrm{~A}$ & 107.5 \\
\hline C93'-C92'-H92B & 107.5 \\
\hline C92'-C93'-H93A & 108.0 \\
\hline C92'-C93'-H93B & 108.0 \\
\hline $\mathrm{C} 92^{\prime}-\mathrm{C} 93^{\prime}-\mathrm{C} 94^{\prime}$ & $117(2)$ \\
\hline $\mathrm{H} 93 \mathrm{~A}-\mathrm{C} 93^{\prime}-\mathrm{H} 93 \mathrm{~B}$ & 107.3 \\
\hline C94'- C93'-H93A & 108.0 \\
\hline C94'- C93'-H93B & 108.0 \\
\hline C93'-C94'-H94A & 109.0 \\
\hline C93'-C94'-H94B & 109.0 \\
\hline $\mathrm{C} 93^{\prime}-\mathrm{C} 94^{\prime}-\mathrm{C} 95^{\prime}$ & $113(2)$ \\
\hline H94A-C94'-H94B & 107.8 \\
\hline $\mathrm{C} 95^{\prime}-\mathrm{C} 94^{\prime}-\mathrm{H} 94 \mathrm{~A}$ & 109.0 \\
\hline C95'- C94'-H94B & 109.0 \\
\hline $\mathrm{C} 94^{\prime}-\mathrm{C} 95^{\prime}-\mathrm{H} 95 \mathrm{~A}$ & 110.2 \\
\hline C94'-C95'-H95B & 110.2 \\
\hline $\mathrm{H} 95 \mathrm{~A}-\mathrm{C} 95^{\prime}-\mathrm{H} 95 \mathrm{~B}$ & 108.5 \\
\hline $\mathrm{C} 96^{\prime}-\mathrm{C} 95^{\prime}-\mathrm{C} 94^{\prime}$ & $108(2)$ \\
\hline $\mathrm{C} 96^{\prime}-\mathrm{C} 95^{\prime}-\mathrm{H} 95 \mathrm{~A}$ & 110.2 \\
\hline C96'-C95'-H95B & 110.2 \\
\hline $\mathrm{C} 95^{\prime}-\mathrm{C} 96^{\prime}-\mathrm{H} 96 \mathrm{~A}$ & 109.5 \\
\hline $\mathrm{C} 95^{\prime}-\mathrm{C} 96^{\prime}-\mathrm{H} 96 \mathrm{~B}$ & 109.5 \\
\hline $\mathrm{C} 95^{\prime}-\mathrm{C} 96^{\prime}-\mathrm{H} 96 \mathrm{C}$ & 109.5 \\
\hline H96A-C96'-H96B & 109.5 \\
\hline $\mathrm{H} 96 \mathrm{~A}-\mathrm{C} 96^{\prime}-\mathrm{H} 96 \mathrm{C}$ & 109.5 \\
\hline $\mathrm{H} 96 \mathrm{~B}-\mathrm{C} 96^{\prime}-\mathrm{H} 96 \mathrm{C}$ & 109.5 \\
\hline $\mathrm{C} 54^{\prime}-\mathrm{C} 97^{\prime}-\mathrm{H} 97 \mathrm{~A}$ & 108.7 \\
\hline
\end{tabular}




\begin{tabular}{|c|c|c|c|}
\hline C93-C94-H94D & 109.5 & C54'-C97'-H97B & 108.7 \\
\hline $\mathrm{C} 93-\mathrm{C} 94-\mathrm{C} 95$ & $110.9(12)$ & H97A-C97'-H97B & 107.6 \\
\hline $\mathrm{H} 94 \mathrm{C}-\mathrm{C} 94-\mathrm{H} 94 \mathrm{D}$ & 108.1 & $\mathrm{C} 98^{\prime}-\mathrm{C} 97^{\prime}-\mathrm{C} 54^{\prime}$ & $114.4(12)$ \\
\hline $\mathrm{C} 95-\mathrm{C} 94-\mathrm{H} 94 \mathrm{C}$ & 109.5 & C98'-C97'-H97A & 108.7 \\
\hline C95-C94-H94D & 109.5 & C98'-C97'-H97B & 108.7 \\
\hline C94-C95-H95C & 108.5 & C97'-C98'-H98A & 109.4 \\
\hline $\mathrm{C} 94-\mathrm{C} 95-\mathrm{H} 95 \mathrm{D}$ & 108.5 & C97'-C98'-H98B & 109.4 \\
\hline C94-C95-C96 & $115.0(15)$ & C97'-C98'-C99' & $111.1(13)$ \\
\hline $\mathrm{H} 95 \mathrm{C}-\mathrm{C} 95-\mathrm{H} 95 \mathrm{D}$ & 107.5 & H98A-C98'-H98B & 108.0 \\
\hline $\mathrm{C} 96-\mathrm{C} 95-\mathrm{H} 95 \mathrm{C}$ & 108.5 & C99'-C98'-H98A & 109.4 \\
\hline C96-C95-H95D & 108.5 & C99'- C98'-H98B & 109.4 \\
\hline C95-C96-H96D & 109.5 & C98'-C99'-H99A & 109.2 \\
\hline C95-C96-H96E & 109.5 & C98'-C99'-H99B & 109.2 \\
\hline $\mathrm{C} 95-\mathrm{C} 96-\mathrm{H} 96 \mathrm{~F}$ & 109.5 & H99A-C99'-H99B & 107.9 \\
\hline $\mathrm{H} 96 \mathrm{D}-\mathrm{C} 96-\mathrm{H} 96 \mathrm{E}$ & 109.5 & $\mathrm{C} 200-\mathrm{C} 99^{\prime}-\mathrm{C} 98^{\prime}$ & $112.2(15)$ \\
\hline $\mathrm{H} 96 \mathrm{D}-\mathrm{C} 96-\mathrm{H} 96 \mathrm{~F}$ & 109.5 & $\mathrm{C} 200-\mathrm{C} 99^{\prime}-\mathrm{H} 99 \mathrm{~A}$ & 109.2 \\
\hline H96E-C96-H96F & 109.5 & $\mathrm{C} 200-\mathrm{C} 99^{\prime}-\mathrm{H} 99 \mathrm{~B}$ & 109.2 \\
\hline $\mathrm{C} 54-\mathrm{C} 97-\mathrm{H} 97 \mathrm{C}$ & 108.1 & $\mathrm{C} 99^{\prime}-\mathrm{C} 200-\mathrm{H} 20 \mathrm{~A}$ & 110.2 \\
\hline C54-C97-H97D & 108.1 & $\mathrm{C} 99^{\prime}-\mathrm{C} 200-\mathrm{H} 20 \mathrm{~B}$ & 110.2 \\
\hline $\mathrm{H} 97 \mathrm{C}-\mathrm{C} 97-\mathrm{H} 97 \mathrm{D}$ & 107.3 & $\mathrm{C} 99^{\prime}-\mathrm{C} 200-\mathrm{C} 201$ & $107.5(18)$ \\
\hline C98-C97-C54 & $116.6(10)$ & $\mathrm{H} 20 \mathrm{~A}-\mathrm{C} 200-\mathrm{H} 20 \mathrm{~B}$ & 108.5 \\
\hline C98- $997-\mathrm{H} 97 \mathrm{C}$ & 108.1 & $\mathrm{C} 201-\mathrm{C} 200-\mathrm{H} 20 \mathrm{~A}$ & 110.2 \\
\hline C98-C97-H97D & 108.1 & $\mathrm{C} 201-\mathrm{C} 200-\mathrm{H} 20 \mathrm{~B}$ & 110.2 \\
\hline C97-C98-H98C & 110.1 & $\mathrm{C} 200-\mathrm{C} 201-\mathrm{H} 20 \mathrm{C}$ & 108.7 \\
\hline C97-C98-H98D & 110.1 & $\mathrm{C} 200-\mathrm{C} 201-\mathrm{H} 20 \mathrm{D}$ & 108.7 \\
\hline C97-C98-C99 & $108.1(11)$ & $\mathrm{C} 200-\mathrm{C} 201-\mathrm{C} 202$ & $114(2)$ \\
\hline $\mathrm{H} 98 \mathrm{C}-\mathrm{C} 98-\mathrm{H} 98 \mathrm{D}$ & 108.4 & $\mathrm{H} 20 \mathrm{C}-\mathrm{C} 201-\mathrm{H} 20 \mathrm{D}$ & 107.6 \\
\hline C99- $998-\mathrm{H} 98 \mathrm{C}$ & 110.1 & $\mathrm{C} 202-\mathrm{C} 201-\mathrm{H} 20 \mathrm{C}$ & 108.7 \\
\hline C99-C98-H98D & 110.1 & $\mathrm{C} 202-\mathrm{C} 201-\mathrm{H} 20 \mathrm{D}$ & 108.7 \\
\hline С98- C99-H99C & 108.8 & $\mathrm{C} 201-\mathrm{C} 202-\mathrm{H} 20 \mathrm{E}$ & 109.5 \\
\hline C98-C99-H99D & 108.8 & $\mathrm{C} 201-\mathrm{C} 202-\mathrm{H} 20 \mathrm{~F}$ & 109.5 \\
\hline $\mathrm{H} 99 \mathrm{C}-\mathrm{C} 99-\mathrm{H} 99 \mathrm{D}$ & 107.7 & $\mathrm{C} 201-\mathrm{C} 202-\mathrm{H} 20 \mathrm{G}$ & 109.5 \\
\hline $\mathrm{C} 100-\mathrm{C} 99-\mathrm{C} 98$ & $114.0(13)$ & $\mathrm{H} 20 \mathrm{E}-\mathrm{C} 202-\mathrm{H} 20 \mathrm{~F}$ & 109.5 \\
\hline $\mathrm{C} 100-\mathrm{C} 99-\mathrm{H} 99 \mathrm{C}$ & 108.8 & $\mathrm{H} 20 \mathrm{E}-\mathrm{C} 202-\mathrm{H} 20 \mathrm{G}$ & 109.5 \\
\hline $\mathrm{C} 100-\mathrm{C} 99-\mathrm{H} 99 \mathrm{D}$ & 108.8 & $\mathrm{H} 20 \mathrm{~F}-\mathrm{C} 202-\mathrm{H} 20 \mathrm{G}$ & 109.5 \\
\hline $\mathrm{C} 99-\mathrm{C} 100-\mathrm{H} 10 \mathrm{C}$ & 109.1 & $\mathrm{C} 54^{\prime}-\mathrm{C} 203-\mathrm{H} 20 \mathrm{H}$ & 108.9 \\
\hline $\mathrm{C} 99-\mathrm{C} 100-\mathrm{H} 10 \mathrm{D}$ & 109.1 & $\mathrm{C} 54^{\prime}-\mathrm{C} 203-\mathrm{H} 20 \mathrm{I}$ & 108.9 \\
\hline $\mathrm{C} 99-\mathrm{C} 100-\mathrm{H} 10 \mathrm{~A}$ & 109.1 & $\mathrm{C} 54^{\prime}-\mathrm{C} 203-\mathrm{H} 20 \mathrm{~J}$ & 105.6 \\
\hline $\mathrm{C} 99-\mathrm{C} 100-\mathrm{H} 10 \mathrm{~B}$ & 109.1 & $\mathrm{C} 54^{\prime}-\mathrm{C} 203-\mathrm{H} 20 \mathrm{~K}$ & 105.6 \\
\hline $\mathrm{C} 99-\mathrm{C} 100-\mathrm{C} 101$ & $112.3(16)$ & $\mathrm{H} 20 \mathrm{H}-\mathrm{C} 203-\mathrm{H} 20 \mathrm{I}$ & 107.7 \\
\hline $\mathrm{C} 99-\mathrm{C} 100-\mathrm{C} 126$ & $113(3)$ & $\mathrm{H} 20 \mathrm{~J}-\mathrm{C} 203-\mathrm{H} 20 \mathrm{~K}$ & 106.1 \\
\hline $\mathrm{H} 10 \mathrm{C}-\mathrm{C} 100-\mathrm{H} 10 \mathrm{D}$ & 107.8 & $\mathrm{C} 204-\mathrm{C} 203-\mathrm{C} 54^{\prime}$ & $113.5(13)$ \\
\hline $\mathrm{H} 10 \mathrm{~A}-\mathrm{C} 100-\mathrm{H} 10 \mathrm{~B}$ & 107.9 & $\mathrm{C} 204-\mathrm{C} 203-\mathrm{H} 20 \mathrm{H}$ & 108.9 \\
\hline $\mathrm{C} 101-\mathrm{C} 100-\mathrm{H} 10 \mathrm{~A}$ & 109.2 & $\mathrm{C} 204-\mathrm{C} 203-\mathrm{H} 20 \mathrm{I}$ & 108.9 \\
\hline $\mathrm{C} 101-\mathrm{C} 100-\mathrm{H} 10 \mathrm{~B}$ & 109.1 & $\mathrm{C} 20 \mathrm{~B}-\mathrm{C} 203-\mathrm{C} 54^{\prime}$ & $127(3)$ \\
\hline $\mathrm{C} 126-\mathrm{C} 100-\mathrm{H} 10 \mathrm{C}$ & 109.1 & $\mathrm{C} 20 \mathrm{~B}-\mathrm{C} 203-\mathrm{H} 20 \mathrm{~J}$ & 105.6 \\
\hline $\mathrm{C} 126-\mathrm{C} 100-\mathrm{H} 10 \mathrm{D}$ & 109.1 & $\mathrm{C} 20 \mathrm{~B}-\mathrm{C} 203-\mathrm{H} 20 \mathrm{~K}$ & 105.6 \\
\hline $\mathrm{C} 54-\mathrm{C} 103-\mathrm{H} 10 \mathrm{E}$ & 108.0 & $\mathrm{H} 20 \mathrm{~N}-\mathrm{C} 206-\mathrm{H} 20 \mathrm{O}$ & 110.5 \\
\hline
\end{tabular}




\begin{tabular}{|c|c|}
\hline $\mathrm{C} 54-\mathrm{C} 103-\mathrm{H} 10 \mathrm{~F}$ & 108.0 \\
\hline $\mathrm{H} 10 \mathrm{E}-\mathrm{C} 103-\mathrm{H} 10 \mathrm{~F}$ & 107.3 \\
\hline $\mathrm{C} 104-\mathrm{C} 103-\mathrm{C} 54$ & $117.1(10)$ \\
\hline $\mathrm{C} 104-\mathrm{C} 103-\mathrm{H} 10 \mathrm{E}$ & 108.0 \\
\hline $\mathrm{C} 104-\mathrm{C} 103-\mathrm{H} 10 \mathrm{~F}$ & 108.0 \\
\hline $\mathrm{C} 103-\mathrm{C} 104-\mathrm{H} 10 \mathrm{G}$ & 107.3 \\
\hline $\mathrm{C} 103-\mathrm{C} 104-\mathrm{H} 10 \mathrm{H}$ & 107.3 \\
\hline $\mathrm{C} 103-\mathrm{C} 104-\mathrm{H} 10 \mathrm{I}$ & 112.1 \\
\hline $\mathrm{C} 103-\mathrm{C} 104-\mathrm{H} 10 \mathrm{~J}$ & 112.1 \\
\hline $\mathrm{C} 103-\mathrm{C} 104-\mathrm{C} 105$ & $119.9(12)$ \\
\hline $\mathrm{C} 103-\mathrm{C} 104-\mathrm{C} 10 \mathrm{~A}$ & $98.5(17)$ \\
\hline $\mathrm{H} 10 \mathrm{G}-\mathrm{C} 104-\mathrm{H} 10 \mathrm{H}$ & 106.9 \\
\hline $\mathrm{H} 10 \mathrm{I}-\mathrm{C} 104-\mathrm{H} 10 \mathrm{~J}$ & 109.7 \\
\hline $\mathrm{C} 105-\mathrm{C} 104-\mathrm{H} 10 \mathrm{G}$ & 107.3 \\
\hline $\mathrm{C} 105-\mathrm{C} 104-\mathrm{H} 10 \mathrm{H}$ & 107.3 \\
\hline $\mathrm{C} 10 \mathrm{~A}-\mathrm{C} 104-\mathrm{H} 10 \mathrm{I}$ & 112.1 \\
\hline $\mathrm{C} 10 \mathrm{~A}-\mathrm{C} 104-\mathrm{H} 10 \mathrm{~J}$ & 112.1 \\
\hline $\mathrm{C} 72-\mathrm{C} 109-\mathrm{H} 10 \mathrm{~K}$ & 108.6 \\
\hline $\mathrm{C} 72-\mathrm{C} 109-\mathrm{H} 10 \mathrm{~L}$ & 108.6 \\
\hline $\mathrm{H} 10 \mathrm{~K}-\mathrm{C} 109-\mathrm{H} 10 \mathrm{~L}$ & 107.6 \\
\hline $\mathrm{C} 110-\mathrm{C} 109-\mathrm{C} 72$ & $114.7(10)$ \\
\hline $\mathrm{C} 110-\mathrm{C} 109-\mathrm{H} 10 \mathrm{~K}$ & 108.6 \\
\hline $\mathrm{C} 110-\mathrm{C} 109-\mathrm{H} 10 \mathrm{~L}$ & 108.6 \\
\hline $\mathrm{C} 109-\mathrm{C} 110-\mathrm{H} 11 \mathrm{~A}$ & 109.2 \\
\hline $\mathrm{C} 109-\mathrm{C} 110-\mathrm{H} 11 \mathrm{~B}$ & 109.2 \\
\hline $\mathrm{C} 109-\mathrm{C} 110-\mathrm{C} 111$ & $112.1(12)$ \\
\hline $\mathrm{H} 11 \mathrm{~A}-\mathrm{C} 110-\mathrm{H} 11 \mathrm{~B}$ & 107.9 \\
\hline $\mathrm{C} 111-\mathrm{C} 110-\mathrm{H} 11 \mathrm{~A}$ & 109.2 \\
\hline $\mathrm{C} 111-\mathrm{C} 110-\mathrm{H} 11 \mathrm{~B}$ & 109.2 \\
\hline $\mathrm{C} 110-\mathrm{C} 111-\mathrm{H} 11 \mathrm{C}$ & 108.2 \\
\hline $\mathrm{C} 110-\mathrm{C} 111-\mathrm{H} 11 \mathrm{D}$ & 108.2 \\
\hline $\mathrm{H} 11 \mathrm{C}-\mathrm{C} 111-\mathrm{H} 11 \mathrm{D}$ & 107.4 \\
\hline $\mathrm{C} 112-\mathrm{C} 111-\mathrm{C} 110$ & $116.3(16)$ \\
\hline $\mathrm{C} 112-\mathrm{C} 111-\mathrm{H} 11 \mathrm{C}$ & 108.2 \\
\hline $\mathrm{C} 112-\mathrm{C} 111-\mathrm{H} 11 \mathrm{D}$ & 108.2 \\
\hline $\mathrm{C} 111-\mathrm{C} 112-\mathrm{H} 11 \mathrm{E}$ & 110.0 \\
\hline $\mathrm{C} 111-\mathrm{C} 112-\mathrm{H} 11 \mathrm{~F}$ & 110.0 \\
\hline $\mathrm{C} 111-\mathrm{C} 112-\mathrm{C} 113$ & $109(2)$ \\
\hline $\mathrm{H} 11 \mathrm{E}-\mathrm{C} 112-\mathrm{H} 11 \mathrm{~F}$ & 108.3 \\
\hline $\mathrm{C} 113-\mathrm{C} 112-\mathrm{H} 11 \mathrm{E}$ & 110.0 \\
\hline $\mathrm{C} 113-\mathrm{C} 112-\mathrm{H} 11 \mathrm{~F}$ & 110.0 \\
\hline $\mathrm{C} 112-\mathrm{C} 113-\mathrm{H} 11 \mathrm{G}$ & 108.1 \\
\hline $\mathrm{C} 112-\mathrm{C} 113-\mathrm{H} 11 \mathrm{H}$ & 108.1 \\
\hline $\mathrm{C} 112-\mathrm{C} 113-\mathrm{C} 114$ & $117(3)$ \\
\hline $\mathrm{H} 11 \mathrm{G}-\mathrm{C} 113-\mathrm{H} 11 \mathrm{H}$ & 107.3 \\
\hline $\mathrm{C} 114-\mathrm{C} 113-\mathrm{H} 11 \mathrm{G}$ & 108.1 \\
\hline $\mathrm{C} 114-\mathrm{C} 113-\mathrm{H} 11 \mathrm{H}$ & 108.1 \\
\hline & \\
\hline
\end{tabular}

$\mathrm{H} 20 \mathrm{~L}-\mathrm{C} 206-\mathrm{H} 20 \mathrm{M}$

$\mathrm{C} 207-\mathrm{C} 206-\mathrm{H} 20 \mathrm{~N} \quad 113.1$

$\mathrm{C} 207-\mathrm{C} 206-\mathrm{H} 20 \mathrm{O} \quad 113.1$

$\mathrm{C} 207-\mathrm{C} 206-\mathrm{H} 20 \mathrm{~L} \quad 106.6$

$\mathrm{C} 207-\mathrm{C} 206-\mathrm{H} 20 \mathrm{M} \quad 106.6$

$\mathrm{C} 207-\mathrm{C} 206-\mathrm{C} 205$

$\mathrm{C} 207-\mathrm{C} 206-\mathrm{C} 20 \mathrm{C} \quad 92.9(19)$

$\mathrm{C} 205-\mathrm{C} 206-\mathrm{H} 20 \mathrm{~L} \quad 106.6$

$\mathrm{C} 205-\mathrm{C} 206-\mathrm{H} 20 \mathrm{M} \quad 106.6$

$\mathrm{C} 20 \mathrm{C}-\mathrm{C} 206-\mathrm{H} 20 \mathrm{~N}$

$\mathrm{C} 20 \mathrm{C}-\mathrm{C} 206-\mathrm{H} 20 \mathrm{O} \quad 113.1$

C206-C207-H20P $\quad 109.4$

C206-C207-H20Q $\quad 109.4$

$\mathrm{C} 206-\mathrm{C} 207-\mathrm{C} 208 \quad 111.2(16)$

$\mathrm{H} 20 \mathrm{P}-\mathrm{C} 207-\mathrm{H} 20 \mathrm{Q} \quad 108.0$

$\mathrm{C} 208-\mathrm{C} 207-\mathrm{H} 20 \mathrm{P} \quad 109.4$

C208-C207-H20Q 109.4

C207-C208-H20R $\quad 109.5$

$\mathrm{C} 207-\mathrm{C} 208-\mathrm{H} 20 \mathrm{~S} \quad 109.5$

$\mathrm{C} 207-\mathrm{C} 208-\mathrm{H} 20 \mathrm{~T} \quad 109.5$

$\mathrm{H} 20 \mathrm{R}-\mathrm{C} 208-\mathrm{H} 20 \mathrm{~S} \quad 109.5$

$\mathrm{H} 20 \mathrm{R}-\mathrm{C} 208-\mathrm{H} 20 \mathrm{~T} \quad 109.5$

$\mathrm{H} 20 \mathrm{~S}-\mathrm{C} 208-\mathrm{H} 20 \mathrm{~T} \quad 109.5$

$\mathrm{C} 72^{\prime}-\mathrm{C} 209-\mathrm{H} 20 \mathrm{U} \quad 108.1$

$\mathrm{C} 72^{\prime}-\mathrm{C} 209-\mathrm{H} 20 \mathrm{~V} \quad 108.1$

$\mathrm{H} 20 \mathrm{U}-\mathrm{C} 209-\mathrm{H} 20 \mathrm{~V} \quad 107.3$

$\mathrm{C} 210-\mathrm{C} 209-\mathrm{C} 72^{\prime} \quad 116.8$ (11)

$\mathrm{C} 210-\mathrm{C} 209-\mathrm{H} 20 \mathrm{U} \quad 108.1$

$\mathrm{C} 210-\mathrm{C} 209-\mathrm{H} 20 \mathrm{~V} \quad 108.1$

$\mathrm{C} 209-\mathrm{C} 210-\mathrm{H} 21 \mathrm{~A} \quad 108.7$

$\mathrm{C} 209-\mathrm{C} 210-\mathrm{H} 21 \mathrm{~B} \quad 108.7$

$\mathrm{H} 21 \mathrm{~A}-\mathrm{C} 210-\mathrm{H} 21 \mathrm{~B} \quad 107.6$

$\mathrm{C} 211-\mathrm{C} 210-\mathrm{C} 209 \quad 114.1$ (12)

$\mathrm{C} 211-\mathrm{C} 210-\mathrm{H} 21 \mathrm{~A} \quad 108.7$

$\mathrm{C} 211-\mathrm{C} 210-\mathrm{H} 21 \mathrm{~B} \quad 108.7$

$\mathrm{C} 210-\mathrm{C} 211-\mathrm{H} 21 \mathrm{C} \quad 107.8$

$\mathrm{C} 210-\mathrm{C} 211-\mathrm{H} 21 \mathrm{D} \quad 107.8$

$\mathrm{C} 210-\mathrm{C} 211-\mathrm{H} 21 \mathrm{E} \quad 109.6$

$\mathrm{C} 210-\mathrm{C} 211-\mathrm{H} 21 \mathrm{~F} \quad 109.6$

$\mathrm{C} 210-\mathrm{C} 211-\mathrm{C} 21 \mathrm{~B} \quad 118.2(18)$

$\mathrm{C} 210-\mathrm{C} 211-\mathrm{C} 212$

$\mathrm{H} 21 \mathrm{C}-\mathrm{C} 211-\mathrm{H} 21 \mathrm{D} \quad 107.1$

$\mathrm{H} 21 \mathrm{E}-\mathrm{C} 211-\mathrm{H} 21 \mathrm{~F} \quad 108.1$

$\mathrm{C} 21 \mathrm{~B}-\mathrm{C} 211-\mathrm{H} 21 \mathrm{C} \quad 107.8$

$\mathrm{C} 21 \mathrm{~B}-\mathrm{C} 211-\mathrm{H} 21 \mathrm{D} \quad 107.8$

$\mathrm{C} 212-\mathrm{C} 211-\mathrm{H} 21 \mathrm{E} \quad 109.6$

$\mathrm{C} 212-\mathrm{C} 211-\mathrm{H} 21 \mathrm{~F} \quad 109.6$

C72'-C215-H21G $\quad 108.6$ 
C113-C114-H11J

C113-C114-H11K

H11I-C114-H11J

H11I-C114-H11K

$\mathrm{H} 11 \mathrm{~J}-\mathrm{C} 114-\mathrm{H} 11 \mathrm{~K}$

C72-C115-H11L

C72-C115-H11M

H11L-C115-H11M

$\mathrm{C} 116-\mathrm{C} 115-\mathrm{C} 72$

C116-C115-H11L

C116-C115-H11M

C115-C116-H11N

C115-C116- H11O

$\mathrm{H} 11 \mathrm{~N}-\mathrm{C} 116-\mathrm{H} 11 \mathrm{O}$

C117-C116-C115

C117-C116- H11N

C117-C116- $\mathrm{H} 11 \mathrm{O}$

C116-C117-H11P

C116-C117-H11Q

C116-C117-C118

$\mathrm{H} 11 \mathrm{P}-\mathrm{C} 117-\mathrm{H} 11 \mathrm{Q}$

C118-C117-H11P

C118-C117-H11Q

C117-C118-H11R

C117-C118-H11S

C117-C118-C119

H11R-C118-H11S

C119-C118-H11R

C119-C118-H11S

C118-C119-H11T

C118-C119-H11U

$\mathrm{H} 11 \mathrm{~T}-\mathrm{C} 119-\mathrm{H} 11 \mathrm{U}$

$\mathrm{C} 120-\mathrm{C} 119-\mathrm{C} 118$

C120-C119-H11T

C120-C119-H11U

C119-C120-H12A

C119-C120-H12B

C119-C120-H12C

$\mathrm{H} 12 \mathrm{~A}-\mathrm{C} 12 \mathrm{O}-\mathrm{H} 12 \mathrm{~B}$

$\mathrm{H} 12 \mathrm{~A}-\mathrm{C} 120-\mathrm{H} 12 \mathrm{C}$

$\mathrm{H} 12 \mathrm{~B}-\mathrm{C} 12 \mathrm{O}-\mathrm{H} 12 \mathrm{C}$

O3-C121-H12D

O3-C121-H12E

O3-C121- 12 F

$\mathrm{H} 12 \mathrm{D}-\mathrm{C} 121-\mathrm{H} 12 \mathrm{E}$

H12D-C121-H12F

$\mathrm{H} 12 \mathrm{E}-\mathrm{C} 121-\mathrm{H} 12 \mathrm{~F}$

O4-C122-H12G
109.5

109.5

109.5

109.5

109.5

107.9

107.9

107.2

$117.5(9)$

107.9

107.9

108.6

108.6

107.6

114.5 (9)

108.6

108.6

108.5

108.5

115.2 (10)

107.5

108.5

108.5

109.0

109.0

112.8 (10)

107.8

109.0

109.0

108.6

108.6

107.6

114.5 (11)

108.6

108.6

109.5

109.5

109.5

109.5

109.5

109.5

109.5

109.5

109.5

109.5

109.5

109.5

109.5
C72'-C215-H $21 \mathrm{H}$

$\mathrm{H} 21 \mathrm{G}-\mathrm{C} 215-\mathrm{H} 21 \mathrm{H}$

$\mathrm{C} 216-\mathrm{C} 215-\mathrm{C} 72^{\prime}$

$\mathrm{C} 216-\mathrm{C} 215-\mathrm{H} 21 \mathrm{G}$

$\mathrm{C} 216-\mathrm{C} 215-\mathrm{H} 21 \mathrm{H}$

C215- C216- $21 \mathrm{I}$

$\mathrm{C} 215-\mathrm{C} 216-\mathrm{H} 21 \mathrm{~J}$

C215-C216- $\mathrm{H} 21 \mathrm{~K}$

$\mathrm{C} 215-\mathrm{C} 216-\mathrm{H} 21 \mathrm{~L}$

$\mathrm{C} 215-\mathrm{C} 216-\mathrm{C} 217$

$\mathrm{C} 215-\mathrm{C} 216-\mathrm{C} 21 \mathrm{E}$

$\mathrm{H} 21 \mathrm{I}-\mathrm{C} 216-\mathrm{H} 21 \mathrm{~J}$

$\mathrm{H} 21 \mathrm{~K}-\mathrm{C} 216-\mathrm{H} 21 \mathrm{~L}$

$\mathrm{C} 217-\mathrm{C} 216-\mathrm{H} 21 \mathrm{I}$

$\mathrm{C} 217-\mathrm{C} 216-\mathrm{H} 21 \mathrm{~J}$

$\mathrm{C} 21 \mathrm{E}-\mathrm{C} 216-\mathrm{H} 21 \mathrm{~K}$

$\mathrm{C} 21 \mathrm{E}-\mathrm{C} 216-\mathrm{H} 21 \mathrm{~L}$

$\mathrm{O} 3{ }^{\prime}-\mathrm{C} 221-\mathrm{H} 22 \mathrm{~A}$

$\mathrm{O} 3{ }^{\prime}-\mathrm{C} 221-\mathrm{H} 22 \mathrm{~B}$

$\mathrm{O} 3{ }^{\prime}-\mathrm{C} 221-\mathrm{H} 22 \mathrm{C}$

$\mathrm{H} 22 \mathrm{~A}-\mathrm{C} 221-\mathrm{H} 22 \mathrm{~B}$

$\mathrm{H} 22 \mathrm{~A}-\mathrm{C} 221-\mathrm{H} 22 \mathrm{C}$

$\mathrm{H} 22 \mathrm{~B}-\mathrm{C} 221-\mathrm{H} 22 \mathrm{C}$

$\mathrm{O} 4^{\prime}-\mathrm{C} 222-\mathrm{H} 22 \mathrm{D}$

$\mathrm{O} 4^{\prime}-\mathrm{C} 222-\mathrm{H} 22 \mathrm{E}$

$\mathrm{O} 4$ '- $2222-\mathrm{H} 22 \mathrm{~F}$

$\mathrm{H} 22 \mathrm{D}-\mathrm{C} 222-\mathrm{H} 22 \mathrm{E}$

$\mathrm{H} 22 \mathrm{D}-\mathrm{C} 222-\mathrm{H} 22 \mathrm{~F}$

$\mathrm{H} 22 \mathrm{E}-\mathrm{C} 222-\mathrm{H} 22 \mathrm{~F}$

O5'-C223-H22G

$\mathrm{O} 5{ }^{\prime}-\mathrm{C} 223-\mathrm{H} 22 \mathrm{H}$

$\mathrm{O} 5$ '- $2223-\mathrm{H} 22 \mathrm{I}$

$\mathrm{H} 22 \mathrm{G}-\mathrm{C} 223-\mathrm{H} 22 \mathrm{H}$

$\mathrm{H} 22 \mathrm{G}-\mathrm{C} 223-\mathrm{H} 22 \mathrm{I}$

$\mathrm{H} 22 \mathrm{H}-\mathrm{C} 223-\mathrm{H} 22 \mathrm{I}$

$\mathrm{O} 6^{\prime}-\mathrm{C} 224-\mathrm{H} 22 \mathrm{~J}$

$\mathrm{O} 6^{\prime}-\mathrm{C} 224-\mathrm{H} 22 \mathrm{~K}$

O6'-C224-H22L

$\mathrm{H} 22 \mathrm{~J}-\mathrm{C} 224-\mathrm{H} 22 \mathrm{~K}$

$\mathrm{H} 22 \mathrm{~J}-\mathrm{C} 224-\mathrm{H} 22 \mathrm{~L}$

$\mathrm{H} 22 \mathrm{~K}-\mathrm{C} 224-\mathrm{H} 22 \mathrm{~L}$

$\mathrm{C} 211-\mathrm{C} 21 \mathrm{~B}-\mathrm{H} 21 \mathrm{M}$

$\mathrm{C} 211-\mathrm{C} 21 \mathrm{~B}-\mathrm{H} 21 \mathrm{~N}$

$\mathrm{H} 21 \mathrm{M}-\mathrm{C} 21 \mathrm{~B}-\mathrm{H} 21 \mathrm{~N}$

$\mathrm{C} 21 \mathrm{C}-\mathrm{C} 21 \mathrm{~B}-\mathrm{C} 211$

$\mathrm{C} 21 \mathrm{C}-\mathrm{C} 21 \mathrm{~B}-\mathrm{H} 21 \mathrm{M}$

$\mathrm{C} 21 \mathrm{C}-\mathrm{C} 21 \mathrm{~B}-\mathrm{H} 21 \mathrm{~N}$

$\mathrm{C} 21 \mathrm{~B}-\mathrm{C} 21 \mathrm{C}-\mathrm{H} 21 \mathrm{O}$
108.6

107.6

114.7 (13)

108.6

108.6

108.1

108.1

101.8

101.8

117 (2)

141 (2)

107.3

104.7

108.1

108.1

101.8

101.8

109.5

109.5

109.5

109.5

109.5

109.5

109.5

109.5

109.5

109.5

109.5

109.5

109.5

109.5

109.5

109.5

109.5

109.5

109.5

109.5

109.5

109.5

109.5

109.5

107.5

107.5

107.0

119 (2)

107.5

107.5

107.8 


$\begin{array}{ll}\text { O4-C122-H12H } & 109.5 \\ \text { O4-C122-H12I } & 109.5 \\ \text { H12G-C122-H12H } & 109.5 \\ \text { H12G-C122-H12I } & 109.5 \\ \text { H12H-C122-H12I } & 109.5 \\ \text { O5-C123-H12J } & 109.5 \\ \text { O5-C123-H12K } & 109.5 \\ \text { O5-C123-H12L } & 109.5 \\ \text { H12J-C123-H12K } & 109.5 \\ \text { H12J-C123-H12L } & 109.5 \\ \text { H12K-C123-H12L } & 109.5 \\ \text { O6-C124-H12M } & 109.5 \\ \text { O6-C124-H12N } & 109.5 \\ \text { O6-C124-H12O } & 109.5 \\ \text { H12M-C124-H12N } & 109.5 \\ \text { H12M-C124-H12O } & 109.5 \\ \text { H12N-C124-H12O } & 109.5 \\ \text { C100-C101-H10M } & 108.5 \\ \text { C100-C101-H10N } & 108.5 \\ \text { H10M-C101-H10N } & 107.5 \\ \text { C102-C101-C100 } & 115(2) \\ \text { C102-C101-H10M } & 108.5 \\ \text { C102-C101-H10N } & 108.5 \\ \text { C101-C102-H10O } & 109.5 \\ \text { C101-C102-H10P } & 109.5 \\ \text { C101-C102-H10Q } & 109.5 \\ \text { H10O-C102-H10P } & 109.5 \\ \text { H10O-C102-H10Q } & 109.5 \\ \text { H10P-C102-H10Q } & 109.5 \\ \text { C104-C105-H10R } & 108.6 \\ \text { C104-C105-H10S } & 108.6 \\ \text { C104-C105-C106 } & 114.8(14) \\ \text { H10R-C105-H10S } & 107.6 \\ \text { C106-C105-H10R } & 108.6 \\ \text { C106-C105-H10S } & 108.6 \\ \text { C105-C106-H10T } & 108.7 \\ \text { C105-C106-H10U } & 108.7 \\ \text { C105-C106-C107 } & 114.4(15) \\ \text { H10T-C106-H10U } & 107.6 \\ \text { C107-C106-H10T } & 108.7 \\ \text { C107-C106-H10U } & 108.7 \\ \text { C106-C107-H10V } & 109.2 \\ \text { C106-C107-H10W } & 109.2 \\ \text { C106-C107-C108 } & 112(2) \\ \text { H10V-C107-H10W } & 107.9 \\ \text { C108-C107-H10V } & 109.2 \\ \text { C108-C107-H10W } & 109.2 \\ \text { C107-C108-H10X } & \\ & \\ & \end{array}$

\begin{tabular}{|c|c|}
\hline $\mathrm{C} 21 \mathrm{~B}-\mathrm{C} 21 \mathrm{C}-\mathrm{H} 21 \mathrm{P}$ & 107.8 \\
\hline $\mathrm{C} 21 \mathrm{~B}-\mathrm{C} 21 \mathrm{C}-\mathrm{C} 21 \mathrm{D}$ & $118(3)$ \\
\hline $\mathrm{H} 21 \mathrm{O}-\mathrm{C} 21 \mathrm{C}-\mathrm{H} 21 \mathrm{P}$ & 107.1 \\
\hline $\mathrm{C} 21 \mathrm{D}-\mathrm{C} 21 \mathrm{C}-\mathrm{H} 21 \mathrm{O}$ & 107.8 \\
\hline $\mathrm{C} 21 \mathrm{D}-\mathrm{C} 21 \mathrm{C}-\mathrm{H} 21 \mathrm{P}$ & 107.8 \\
\hline $\mathrm{C} 21 \mathrm{C}-\mathrm{C} 21 \mathrm{D}-\mathrm{H} 21 \mathrm{Q}$ & 109.5 \\
\hline $\mathrm{C} 21 \mathrm{C}-\mathrm{C} 21 \mathrm{D}-\mathrm{H} 21 \mathrm{R}$ & 109.5 \\
\hline $\mathrm{C} 21 \mathrm{C}-\mathrm{C} 21 \mathrm{D}-\mathrm{H} 21 \mathrm{~S}$ & 109.5 \\
\hline $\mathrm{H} 21 \mathrm{Q}-\mathrm{C} 21 \mathrm{D}-\mathrm{H} 21 \mathrm{R}$ & 109.5 \\
\hline $\mathrm{H} 21 \mathrm{Q}-\mathrm{C} 21 \mathrm{D}-\mathrm{H} 21 \mathrm{~S}$ & 109.5 \\
\hline $\mathrm{H} 21 \mathrm{R}-\mathrm{C} 21 \mathrm{D}-\mathrm{H} 21 \mathrm{~S}$ & 109.5 \\
\hline $\mathrm{C} 79^{\prime}-\mathrm{C} 80^{\prime}-\mathrm{C} 81^{\prime}$ & $104.2(18)$ \\
\hline $\mathrm{C} 79^{\prime}-\mathrm{C} 80^{\prime}-\mathrm{H} 80 \mathrm{~A}$ & 110.9 \\
\hline $\mathrm{C} 79^{\prime}-\mathrm{C} 80^{\prime}-\mathrm{H} 80 \mathrm{~B}$ & 110.9 \\
\hline $\mathrm{C} 81^{\prime}-\mathrm{C} 80^{\prime}-\mathrm{H} 80 \mathrm{~A}$ & 110.9 \\
\hline $\mathrm{C} 81^{\prime}-\mathrm{C} 80^{\prime}-\mathrm{H} 80 \mathrm{~B}$ & 110.9 \\
\hline $\mathrm{H} 80 \mathrm{~A}-\mathrm{C} 80^{\prime}-\mathrm{H} 80 \mathrm{~B}$ & 108.9 \\
\hline $\mathrm{C} 203-\mathrm{C} 204-\mathrm{H} 20 \mathrm{~W}$ & 109.1 \\
\hline $\mathrm{C} 203-\mathrm{C} 204-\mathrm{H} 20 \mathrm{X}$ & 109.1 \\
\hline $\mathrm{H} 20 \mathrm{~W}-\mathrm{C} 204-\mathrm{H} 20 \mathrm{X}$ & 107.9 \\
\hline $\mathrm{C} 205-\mathrm{C} 204-\mathrm{C} 203$ & $112.4(15)$ \\
\hline $\mathrm{C} 205-\mathrm{C} 204-\mathrm{H} 20 \mathrm{~W}$ & 109.1 \\
\hline $\mathrm{C} 205-\mathrm{C} 204-\mathrm{H} 20 \mathrm{X}$ & 109.1 \\
\hline $\mathrm{C} 206-\mathrm{C} 205-\mathrm{C} 204$ & $102.5(15)$ \\
\hline $\mathrm{C} 206-\mathrm{C} 205-\mathrm{H} 20 \mathrm{Y}$ & 111.3 \\
\hline $\mathrm{C} 206-\mathrm{C} 205-\mathrm{HF}$ & 111.3 \\
\hline $\mathrm{C} 204-\mathrm{C} 205-\mathrm{H} 20 \mathrm{Y}$ & 111.3 \\
\hline $\mathrm{C} 204-\mathrm{C} 205-\mathrm{HF}$ & 111.3 \\
\hline $\mathrm{H} 20 \mathrm{Y}-\mathrm{C} 205-\mathrm{HF}$ & 109.2 \\
\hline $\mathrm{C} 216-\mathrm{C} 217-\mathrm{H} 21 \mathrm{~T}$ & 109.6 \\
\hline $\mathrm{C} 216-\mathrm{C} 217-\mathrm{H} 21 \mathrm{U}$ & 109.6 \\
\hline $\mathrm{C} 216-\mathrm{C} 217-\mathrm{C} 218$ & $110(3)$ \\
\hline $\mathrm{H} 21 \mathrm{~T}-\mathrm{C} 217-\mathrm{H} 21 \mathrm{U}$ & 108.1 \\
\hline $\mathrm{C} 218-\mathrm{C} 217-\mathrm{H} 21 \mathrm{~T}$ & 109.6 \\
\hline $\mathrm{C} 218-\mathrm{C} 217-\mathrm{H} 21 \mathrm{U}$ & 109.6 \\
\hline $\mathrm{C} 217-\mathrm{C} 218-\mathrm{H} 21 \mathrm{~V}$ & 107.0 \\
\hline $\mathrm{C} 217-\mathrm{C} 218-\mathrm{H} 21 \mathrm{~W}$ & 107.0 \\
\hline $\mathrm{C} 217-\mathrm{C} 218-\mathrm{C} 219$ & $121(4)$ \\
\hline $\mathrm{H} 21 \mathrm{~V}-\mathrm{C} 218-\mathrm{H} 21 \mathrm{~W}$ & 106.7 \\
\hline $\mathrm{C} 219-\mathrm{C} 218-\mathrm{H} 21 \mathrm{~V}$ & 107.0 \\
\hline $\mathrm{C} 219-\mathrm{C} 218-\mathrm{H} 21 \mathrm{~W}$ & 107.0 \\
\hline $\mathrm{C} 218-\mathrm{C} 219-\mathrm{H} 21 \mathrm{X}$ & 111.0 \\
\hline $\mathrm{C} 218-\mathrm{C} 219-\mathrm{H} 21 \mathrm{Y}$ & 111.0 \\
\hline $\mathrm{H} 21 \mathrm{X}-\mathrm{C} 219-\mathrm{H} 21 \mathrm{Y}$ & 109.0 \\
\hline $\mathrm{C} 220-\mathrm{C} 219-\mathrm{C} 218$ & $104(3)$ \\
\hline $\mathrm{C} 220-\mathrm{C} 219-\mathrm{H} 21 \mathrm{X}$ & 111.0 \\
\hline $\mathrm{C} 220-\mathrm{C} 219-\mathrm{H} 21 \mathrm{Y}$ & 111.0 \\
\hline $\mathrm{C} 219-\mathrm{C} 220-\mathrm{H} 22 \mathrm{M}$ & 109.5 \\
\hline
\end{tabular}


C107-C108-H10Y

C107- $\mathrm{C} 108-\mathrm{H}$

$\mathrm{H} 10 \mathrm{X}-\mathrm{C} 108-\mathrm{H} 10 \mathrm{Y}$

$\mathrm{H} 10 \mathrm{X}-\mathrm{C} 108-\mathrm{H}$

$\mathrm{H} 10 \mathrm{Y}-\mathrm{C} 108-\mathrm{H}$

$\mathrm{C} 104-\mathrm{C} 10 \mathrm{~A}-\mathrm{H} 10 \mathrm{Z}$

C104-C10A-HA

H10Z-C10A-HA

$\mathrm{C} 10 \mathrm{~B}-\mathrm{C} 10 \mathrm{~A}-\mathrm{C} 104$

$\mathrm{C} 10 \mathrm{~B}-\mathrm{C} 10 \mathrm{~A}-\mathrm{H} 10 \mathrm{Z}$

$\mathrm{C} 10 \mathrm{~B}-\mathrm{C} 10 \mathrm{~A}-\mathrm{HA}$

$\mathrm{C} 10 \mathrm{~A}-\mathrm{C} 10 \mathrm{~B}-\mathrm{H} 10$

C10A-C10B-HB

$\mathrm{C} 10 \mathrm{~A}-\mathrm{C} 10 \mathrm{~B}-\mathrm{C} 10 \mathrm{C}$

$\mathrm{H} 10-\mathrm{C} 10 \mathrm{~B}-\mathrm{HB}$

$\mathrm{C} 10 \mathrm{C}-\mathrm{C} 10 \mathrm{~B}-\mathrm{H} 10$

$\mathrm{C} 10 \mathrm{C}-\mathrm{C} 10 \mathrm{~B}-\mathrm{HB}$

$\mathrm{C} 10 \mathrm{~B}-\mathrm{C} 10 \mathrm{C}-\mathrm{H} 7$

$\mathrm{C} 10 \mathrm{~B}-\mathrm{C} 10 \mathrm{C}-\mathrm{HC}$

$\mathrm{H} 7-\mathrm{C} 10 \mathrm{C}-\mathrm{HC}$

$\mathrm{C} 10 \mathrm{D}-\mathrm{C} 10 \mathrm{C}-\mathrm{C} 10 \mathrm{~B}$

$\mathrm{C} 10 \mathrm{D}-\mathrm{C} 10 \mathrm{C}-\mathrm{H} 7$

$\mathrm{C} 10 \mathrm{D}-\mathrm{C} 10 \mathrm{C}-\mathrm{HC}$

$\mathrm{C} 10 \mathrm{C}-\mathrm{C} 10 \mathrm{D}-\mathrm{H} 12$

C10C-C10D-HD

C10C-C10D-HE

H12-C10D-HD

H12-C10D-HE

$\mathrm{HD}-\mathrm{C} 10 \mathrm{D}-\mathrm{HE}$

$\mathrm{H} 12 \mathrm{P}-\mathrm{C} 125-\mathrm{H} 12 \mathrm{Q}$

$\mathrm{H} 12 \mathrm{P}-\mathrm{C} 125-\mathrm{H} 12 \mathrm{R}$

$\mathrm{H} 12 \mathrm{Q}-\mathrm{C} 125-\mathrm{H} 12 \mathrm{R}$

$\mathrm{C} 126-\mathrm{C} 125-\mathrm{H} 12 \mathrm{P}$

$\mathrm{C} 126-\mathrm{C} 125-\mathrm{H} 12 \mathrm{Q}$

$\mathrm{C} 126-\mathrm{C} 125-\mathrm{H} 12 \mathrm{R}$

$\mathrm{C} 100-\mathrm{C} 126-\mathrm{H} 12 \mathrm{~S}$

$\mathrm{C} 100-\mathrm{C} 126-\mathrm{H} 12 \mathrm{~T}$

$\mathrm{C} 125-\mathrm{C} 126-\mathrm{C} 100$

$\mathrm{C} 125-\mathrm{C} 126-\mathrm{H} 12 \mathrm{~S}$

$\mathrm{C} 125-\mathrm{C} 126-\mathrm{H} 12 \mathrm{~T}$

$\mathrm{H} 12 \mathrm{~S}-\mathrm{C} 126-\mathrm{H} 12 \mathrm{~T}$

N01'-Ir3-N02'

$\mathrm{N} 1^{\prime}-\mathrm{Ir} 3-\mathrm{N} 01^{\prime}$

$\mathrm{N} 1^{\prime}-\mathrm{Ir} 3-\mathrm{N} 02^{\prime}$

$\mathrm{N} 2^{\prime}-\mathrm{Ir} 3-\mathrm{N} 01^{\prime}$

$\mathrm{N} 2^{\prime}-\mathrm{Ir} 3-\mathrm{N} 1^{\prime}$

$\mathrm{N} 2^{\prime}-\mathrm{Ir} 3-\mathrm{N} 02^{\prime}$

$\mathrm{N} 2^{\prime}-\mathrm{Ir} 3-\mathrm{C}^{\prime}$
109.5

109.5

109.5

109.5

109.5

109.1

109.1

107.9

112 (3)

109.1

109.1

108.1

108.1

117 (4)

107.3

108.1

108.1

106.8

106.8

106.6

122 (4)

106.8

106.8

109.5

109.5

109.5

109.5

109.5

109.5

109.5

109.5

109.5

109.5

109.5

109.5

105.0

105.0

129 (4)

105.0

105.0

105.9

80.0 (4)

$94.1(4)$

90.3 (4)

89.7 (4)

$171.0(4)$

98.4 (4)

$89.6(5)$
$\mathrm{C} 219-\mathrm{C} 220-\mathrm{H} 22 \mathrm{~N}$

$\mathrm{C} 219-\mathrm{C} 220-\mathrm{H} 22 \mathrm{O}$

$\mathrm{H} 22 \mathrm{M}-\mathrm{C} 22 \mathrm{O}-\mathrm{H} 22 \mathrm{~N}$

$\mathrm{H} 22 \mathrm{M}-\mathrm{C} 22 \mathrm{O}-\mathrm{H} 22 \mathrm{O}$

$\mathrm{H} 22 \mathrm{~N}-\mathrm{C} 220-\mathrm{H} 22 \mathrm{O}$

$\mathrm{C} 203-\mathrm{C} 20 \mathrm{~B}-\mathrm{H} 20 \mathrm{Z}$

C203-C20B-HG

$\mathrm{C} 203-\mathrm{C} 20 \mathrm{~B}-\mathrm{C} 20 \mathrm{C}$

$\mathrm{H} 20 \mathrm{Z}-\mathrm{C} 20 \mathrm{~B}-\mathrm{HG}$

$\mathrm{C} 20 \mathrm{C}-\mathrm{C} 20 \mathrm{~B}-\mathrm{H} 20 \mathrm{Z}$

$\mathrm{C} 20 \mathrm{C}-\mathrm{C} 20 \mathrm{~B}-\mathrm{HG}$

$\mathrm{C} 206-\mathrm{C} 20 \mathrm{C}-\mathrm{H} 20$

$\mathrm{C} 206-\mathrm{C} 20 \mathrm{C}-\mathrm{HH}$

$\mathrm{C} 20 \mathrm{~B}-\mathrm{C} 20 \mathrm{C}-\mathrm{C} 206$

$\mathrm{C} 20 \mathrm{~B}-\mathrm{C} 20 \mathrm{C}-\mathrm{H} 20$

$\mathrm{C} 20 \mathrm{~B}-\mathrm{C} 20 \mathrm{C}-\mathrm{HH}$

$\mathrm{H} 20-\mathrm{C} 20 \mathrm{C}-\mathrm{HH}$

$\mathrm{C} 216-\mathrm{C} 21 \mathrm{E}-\mathrm{H} 21 \mathrm{Z}$

C216-C21E-HI

$\mathrm{C} 216-\mathrm{C} 21 \mathrm{E}-\mathrm{C} 21 \mathrm{~F}$

$\mathrm{H} 21 \mathrm{Z}-\mathrm{C} 21 \mathrm{E}-\mathrm{HI}$

$\mathrm{C} 21 \mathrm{~F}-\mathrm{C} 21 \mathrm{E}-\mathrm{H} 21 \mathrm{Z}$

$\mathrm{C} 21 \mathrm{~F}-\mathrm{C} 21 \mathrm{E}-\mathrm{HI}$

$\mathrm{C} 21 \mathrm{E}-\mathrm{C} 21 \mathrm{~F}-\mathrm{H} 21$

$\mathrm{C} 21 \mathrm{E}-\mathrm{C} 21 \mathrm{~F}-\mathrm{HJ}$

$\mathrm{H} 21-\mathrm{C} 21 \mathrm{~F}-\mathrm{HJ}$

$\mathrm{C} 21 \mathrm{G}-\mathrm{C} 21 \mathrm{~F}-\mathrm{C} 21 \mathrm{E}$

$\mathrm{C} 21 \mathrm{G}-\mathrm{C} 21 \mathrm{~F}-\mathrm{H} 21$

$\mathrm{C} 21 \mathrm{G}-\mathrm{C} 21 \mathrm{~F}-\mathrm{HJ}$

$\mathrm{C} 21 \mathrm{~F}-\mathrm{C} 21 \mathrm{G}-\mathrm{H} 1$

$\mathrm{C} 21 \mathrm{~F}-\mathrm{C} 21 \mathrm{G}-\mathrm{HK}$

$\mathrm{C} 21 \mathrm{~F}-\mathrm{C} 21 \mathrm{G}-\mathrm{C} 21 \mathrm{H}$

$\mathrm{H} 1-\mathrm{C} 21 \mathrm{G}-\mathrm{HK}$

$\mathrm{C} 21 \mathrm{H}-\mathrm{C} 21 \mathrm{G}-\mathrm{H} 1$

$\mathrm{C} 21 \mathrm{H}-\mathrm{C} 21 \mathrm{G}-\mathrm{HK}$

$\mathrm{C} 21 \mathrm{G}-\mathrm{C} 21 \mathrm{H}-\mathrm{H} 2$

$\mathrm{C} 21 \mathrm{G}-\mathrm{C} 21 \mathrm{H}-\mathrm{HL}$

$\mathrm{C} 21 \mathrm{G}-\mathrm{C} 21 \mathrm{H}-\mathrm{HM}$

$\mathrm{H} 2-\mathrm{C} 21 \mathrm{H}-\mathrm{HL}$

$\mathrm{H} 2-\mathrm{C} 21 \mathrm{H}-\mathrm{HM}$

$\mathrm{HL}-\mathrm{C} 21 \mathrm{H}-\mathrm{HM}$

$\mathrm{C} 79^{\prime}-\mathrm{C} 80 \mathrm{~B}-\mathrm{C} 81^{\prime}$

$\mathrm{C} 79^{\prime}-\mathrm{C} 80 \mathrm{~B}-\mathrm{H} 80 \mathrm{C}$

C79'-C80B-H80D

$\mathrm{C} 81^{\prime}-\mathrm{C} 80 \mathrm{~B}-\mathrm{H} 80 \mathrm{C}$

$\mathrm{C} 81^{\prime}-\mathrm{C} 80 \mathrm{~B}-\mathrm{H} 80 \mathrm{D}$

$\mathrm{H} 80 \mathrm{C}-\mathrm{C} 80 \mathrm{~B}-\mathrm{H} 80 \mathrm{D}$

$\mathrm{C} 211-\mathrm{C} 212-\mathrm{H} 3$
109.5

109.5

109.5

109.5

109.5

109.8

109.8

109 (2)

108.2

109.8

109.8

111.0

111.0

104.0 (19)

111.0

111.0

109.0

105.4

105.4

127 (3)

106.0

105.4

105.4

95.0

95.0

103.2

164 (3)

95.0

95.0

106.0

106.0

125 (3)

106.3

106.0

106.0

109.5

109.5

109.5

109.5

109.5

109.5

103 (2)

111.1

111.1

111.1

111.1

109.1

109.3 


\begin{tabular}{|c|c|}
\hline $\mathrm{N} 2^{\prime}-\mathrm{Ir} 3-\mathrm{C} 19^{\prime}$ & $81.3(5)$ \\
\hline $\mathrm{C}^{\prime}-\mathrm{Ir} 3-\mathrm{N} 01^{\prime}$ & $97.1(4)$ \\
\hline $\mathrm{C} 1^{\prime}-\mathrm{Ir} 3-\mathrm{N} 1^{\prime}$ & $81.9(5)$ \\
\hline $\mathrm{C}^{\prime}-\mathrm{Ir} 3-\mathrm{N} 02^{\prime}$ & $171.5(5)$ \\
\hline $\mathrm{C} 19^{\prime}-\mathrm{Ir} 3-\mathrm{N} 01^{\prime}$ & $166.9(4)$ \\
\hline $\mathrm{C} 19^{\prime}-\mathrm{Ir} 3-\mathrm{N} 1^{\prime}$ & $96.2(5)$ \\
\hline $\mathrm{C} 19^{\prime}-\mathrm{Ir} 3-\mathrm{N} 02^{\prime}$ & $91.9(4)$ \\
\hline $\mathrm{C} 19^{\prime}-\mathrm{Ir} 3-\mathrm{C} 1^{\prime}$ & $92.3(5)$ \\
\hline $\mathrm{N} 02^{\prime}-\mathrm{Ir} 4-\mathrm{N} 01^{\prime}$ & $80.4(3)$ \\
\hline $\mathrm{N} 3^{\prime}-\mathrm{Ir} 4-\mathrm{N} 01^{\prime}$ & $92.6(4)$ \\
\hline $\mathrm{N} 3^{\prime}-\mathrm{Ir} 4-\mathrm{N} 02^{\prime}$ & $95.9(4)$ \\
\hline $\mathrm{N} 4^{\prime}-\mathrm{Ir} 4-\mathrm{N} 01^{\prime}$ & $90.7(4)$ \\
\hline $\mathrm{N}^{\prime}-\mathrm{Ir} 4-\mathrm{N} 02^{\prime}$ & $91.2(4)$ \\
\hline $\mathrm{N} 4{ }^{\prime}-\mathrm{Ir} 4-\mathrm{N} 3^{\prime}$ & $172.6(4)$ \\
\hline $\mathrm{N} 4^{\prime}-\mathrm{Ir} 4-\mathrm{C} 37^{\prime}$ & $96.6(5)$ \\
\hline $\mathrm{N} 4^{\prime}-\mathrm{Ir} 4-\mathrm{C} 55^{\prime}$ & $80.5(5)$ \\
\hline $\mathrm{C} 37^{\prime}-\mathrm{Ir} 4-\mathrm{N} 01^{\prime}$ & $171.0(5)$ \\
\hline $\mathrm{Ir} 1-\mathrm{N} 1-\mathrm{C} 7-\mathrm{C} 6$ & $-3.4(11)$ \\
\hline $\mathrm{Ir} 1-\mathrm{N} 1-\mathrm{C} 7-\mathrm{C} 8$ & $175.1(8)$ \\
\hline $\mathrm{Ir} 1-\mathrm{N} 1-\mathrm{C} 11-\mathrm{C} 10$ & $-174.3(8)$ \\
\hline $\mathrm{Ir} 1-\mathrm{N} 2-\mathrm{C} 25-\mathrm{C} 24$ & $-0.8(12)$ \\
\hline $\mathrm{Ir} 1-\mathrm{N} 2-\mathrm{C} 25-\mathrm{C} 26$ & $176.7(8)$ \\
\hline $\mathrm{Ir} 1-\mathrm{N} 2-\mathrm{C} 29-\mathrm{C} 28$ & $-172.8(8)$ \\
\hline $\mathrm{Ir} 1-\mathrm{C} 1-\mathrm{C} 2-\mathrm{C} 3$ & $-173.0(7)$ \\
\hline $\mathrm{Ir} 1-\mathrm{C} 1-\mathrm{C} 6-\mathrm{C} 5$ & $174.3(8)$ \\
\hline $\mathrm{Ir} 1-\mathrm{C} 1-\mathrm{C} 6-\mathrm{C} 7$ & $-8.2(11)$ \\
\hline $\mathrm{Ir} 1-\mathrm{C} 19-\mathrm{C} 20-\mathrm{C} 21$ & $-171.0(8)$ \\
\hline $\mathrm{Ir} 1-\mathrm{C} 19-\mathrm{C} 24-\mathrm{C} 23$ & $174.8(8)$ \\
\hline $\mathrm{Ir} 1-\mathrm{C} 19-\mathrm{C} 24-\mathrm{C} 25$ & $-10.5(12)$ \\
\hline Ir2-N3-C43-C42 & $2.5(11)$ \\
\hline Ir2-N3-C43-C44 & $-177.9(7)$ \\
\hline Ir2-N3-C47-C46 & $178.3(7)$ \\
\hline Ir2-N4-C61-C60 & $0.3(12)$ \\
\hline Ir2-N4-C61-C62 & $177.7(8)$ \\
\hline Ir2-N4-C65-C64 & $-177.5(8)$ \\
\hline $\mathrm{Ir} 2-\mathrm{C} 37-\mathrm{C} 38-\mathrm{C} 39$ & $-170.6(8)$ \\
\hline $\mathrm{Ir} 2-\mathrm{C} 37-\mathrm{C} 42-\mathrm{C} 41$ & $174.5(8)$ \\
\hline Ir $2-\mathrm{C} 37-\mathrm{C} 42-\mathrm{C} 43$ & $-4.2(11)$ \\
\hline Ir $2-\mathrm{C} 55-\mathrm{C} 56-\mathrm{C} 57$ & $-168.3(8)$ \\
\hline Ir $2-\mathrm{C} 55-\mathrm{C} 60-\mathrm{C} 59$ & $171.1(9)$ \\
\hline $\mathrm{Ir} 2-\mathrm{C} 55-\mathrm{C} 60-\mathrm{C} 61$ & $-9.2(12)$ \\
\hline $\mathrm{O} 3-\mathrm{C} 10-\mathrm{C} 11-\mathrm{N} 1$ & $-179.9(9)$ \\
\hline $\mathrm{O} 4-\mathrm{C} 28-\mathrm{C} 29-\mathrm{N} 2$ & $177.8(10)$ \\
\hline $\mathrm{O} 5-\mathrm{C} 46-\mathrm{C} 47-\mathrm{N} 3$ & $-177.5(9)$ \\
\hline $\mathrm{O} 6-\mathrm{C} 64-\mathrm{C} 65-\mathrm{N} 4$ & $-179.6(10)$ \\
\hline $\mathrm{N} 1-\mathrm{C} 7-\mathrm{C} 8-\mathrm{C} 9$ & $0.0(16)$ \\
\hline $\mathrm{N} 2-\mathrm{C} 25-\mathrm{C} 26-\mathrm{C} 27$ & $-3.0(18)$ \\
\hline
\end{tabular}

$\begin{array}{ll}\mathrm{C} 211-\mathrm{C} 212-\mathrm{HN} & 109.3 \\ \mathrm{H} 3-\mathrm{C} 212-\mathrm{HN} & 107.9 \\ \mathrm{C} 213-\mathrm{C} 212-\mathrm{C} 211 & 112(2) \\ \mathrm{C} 213-\mathrm{C} 212-\mathrm{H} 3 & 109.3 \\ \mathrm{C} 213-\mathrm{C} 212-\mathrm{HN} & 109.3 \\ \mathrm{C} 212-\mathrm{C} 213-\mathrm{H} 4 & 109.2 \\ \mathrm{C} 212-\mathrm{C} 213-\mathrm{HO} & 109.2 \\ \mathrm{C} 212-\mathrm{C} 213-\mathrm{C} 214 & 112(3) \\ \mathrm{H} 4-\mathrm{C} 213-\mathrm{HO} & 107.9 \\ \mathrm{C} 214-\mathrm{C} 213-\mathrm{H} 4 & 109.2 \\ \mathrm{C} 214-\mathrm{C} 213-\mathrm{HO} & 109.2 \\ \mathrm{C} 213-\mathrm{C} 214-\mathrm{H} 5 & 109.5 \\ \mathrm{C} 213-\mathrm{C} 214-\mathrm{HP} & 109.5 \\ \mathrm{C} 213-\mathrm{C} 214-\mathrm{HQ} & 109.5 \\ \mathrm{H} 5-\mathrm{C} 214-\mathrm{HP} & 109.5 \\ \mathrm{H} 5-\mathrm{C} 214-\mathrm{HQ} & 109.5 \\ \mathrm{HP}-\mathrm{C} 214-\mathrm{HQ} & 109.5\end{array}$

$\mathrm{Ir} 3-\mathrm{C} 19^{\prime}-\mathrm{C} 24^{\prime}-\mathrm{C} 25^{\prime} \quad 3.8(14)$

$\mathrm{Ir} 4-\mathrm{N} 3^{\prime}-\mathrm{C} 43^{\prime}-\mathrm{C} 42^{\prime} \quad 5.3(15)$

$\mathrm{Ir} 4-\mathrm{N} 33^{\prime}-\mathrm{C} 43^{\prime}-\mathrm{C} 44^{\prime} \quad-171.0(10)$

$\mathrm{Ir} 4-\mathrm{N} 3^{\prime}-\mathrm{C}^{\prime} 7^{\prime}-\mathrm{C} 46^{\prime} \quad 169.3$ (11)

$\mathrm{Ir} 4-\mathrm{N} 4^{\prime}-\mathrm{C} 61^{\prime}-\mathrm{C} 60^{\prime} \quad-8.3(12)$

Ir4-N4'-C61'-C62' 170.2 (9)

$\mathrm{Ir} 4-\mathrm{N} 4^{\prime}-\mathrm{C} 65^{\prime}-\mathrm{C} 64^{\prime} \quad-174.3$ (9)

$\mathrm{Ir} 4-\mathrm{C} 37^{\prime}-\mathrm{C} 38^{\prime}-\mathrm{C} 39^{\prime} \quad 172.3(9)$

$\mathrm{Ir} 4-\mathrm{C} 37^{\prime}-\mathrm{C} 42^{\prime}-\mathrm{C} 41^{\prime} \quad-172.2(10)$

$\mathrm{Ir} 4-\mathrm{C} 37^{\prime}-\mathrm{C} 42^{\prime}-\mathrm{C} 43^{\prime} \quad 7.2(15)$

$\mathrm{Ir} 4-\mathrm{C} 55^{\prime}-\mathrm{C} 56^{\prime}-\mathrm{C} 57^{\prime} \quad 173.1(8)$

$\mathrm{Ir} 4-\mathrm{C} 55^{\prime}-\mathrm{C} 60^{\prime}-\mathrm{C} 59^{\prime} \quad-173.6(8)$

$\mathrm{Ir} 4-\mathrm{C} 55^{\prime}-\mathrm{C} 60^{\prime}-\mathrm{C} 61^{\prime} \quad 9.4(13)$

$\mathrm{O}^{\prime}-\mathrm{C} 10^{\prime}-\mathrm{C} 11^{\prime}-\mathrm{N} 1^{\prime} \quad 178.2(12)$

$\mathrm{O}^{\prime}{ }^{\prime}-\mathrm{C} 28^{\prime}-\mathrm{C} 29^{\prime}-\mathrm{N} 2^{\prime} \quad-179.0(11)$

$\mathrm{O} 5^{\prime}-\mathrm{C} 46^{\prime}-\mathrm{C} 47^{\prime}-\mathrm{N} 3^{\prime} \quad 179.6(13)$

$\mathrm{O}^{\prime}-\mathrm{C} 64^{\prime}-\mathrm{C} 65^{\prime}-\mathrm{N} 4^{\prime} \quad 177.8(10)$

N01'-Ir4-N4'-C61' $\quad-80.1(8)$

$\mathrm{N} 01^{\prime}-\mathrm{Ir} 4-\mathrm{N} 4^{\prime}-\mathrm{C} 65^{\prime}$

$\mathrm{N} 1^{\prime}-\mathrm{C} 7^{\prime}-\mathrm{C} 8^{\prime}-\mathrm{C} 9^{\prime} \quad-2(2)$

$\mathrm{N} 2^{\prime}-\mathrm{C} 25^{\prime}-\mathrm{C} 26^{\prime}-\mathrm{C} 27^{\prime} \quad 3(2)$

$\mathrm{N} 02^{\prime}-\mathrm{Ir} 4-\mathrm{N} 4^{\prime}-\mathrm{C}^{\prime} 1^{\prime} \quad-160.6(8)$

$\mathrm{N} 02^{\prime}-\mathrm{Ir} 4-\mathrm{N} 4^{\prime}-\mathrm{C} 65^{\prime} \quad 14.7$ (9)

$\mathrm{N} 3{ }^{\prime}-\mathrm{C} 43^{\prime}-\mathrm{C} 44^{\prime}-\mathrm{C} 45^{\prime} \quad 0$ (2)

$\mathrm{N} 4^{\prime}-\mathrm{C} 61^{\prime}-\mathrm{C} 62^{\prime}-\mathrm{C} 63^{\prime} \quad 5.6(18)$

$\mathrm{C} 1^{\prime}-\mathrm{C}^{\prime}-\mathrm{C}^{\prime}-\mathrm{C} 4^{\prime} \quad-4(2)$

$\mathrm{C} 1^{\prime}-\mathrm{C} 2^{\prime}-\mathrm{C} 3^{\prime}-\mathrm{C} 12^{\prime} \quad-180.0$ (12)

$\mathrm{C}^{\prime}-\mathrm{C} 6^{\prime}-\mathrm{C}^{\prime}-\mathrm{N} 1^{\prime} \quad-2.7$ (17)

$\mathrm{C} 1^{\prime}-\mathrm{C}^{\prime}-\mathrm{C}^{\prime}-\mathrm{C}^{\prime} \quad 174.2(13)$

$\mathrm{C} 2^{\prime}-\mathrm{C}^{\prime}-\mathrm{C}^{\prime}-\mathrm{C} 5^{\prime} \quad-4.6$ (19) 


\begin{tabular}{|c|c|}
\hline $\mathrm{N} 3-\mathrm{C} 43-\mathrm{C} 44-\mathrm{C} 45$ & $-0.3(16)$ \\
\hline N4-C61-C62-C63 & $-1.7(17)$ \\
\hline $\mathrm{C} 1-\mathrm{C} 2-\mathrm{C} 3-\mathrm{C} 4$ & $-2.6(16)$ \\
\hline $\mathrm{C} 1-\mathrm{C} 2-\mathrm{C} 3-\mathrm{C} 12$ & $179.2(10)$ \\
\hline $\mathrm{C} 1-\mathrm{C} 6-\mathrm{C} 7-\mathrm{N} 1$ & $7.6(13)$ \\
\hline $\mathrm{C} 1-\mathrm{C} 6-\mathrm{C} 7-\mathrm{C} 8$ & $-170.9(10)$ \\
\hline $\mathrm{C} 2-\mathrm{C} 1-\mathrm{C} 6-\mathrm{C} 5$ & $-1.8(15)$ \\
\hline $\mathrm{C} 2-\mathrm{C} 1-\mathrm{C} 6-\mathrm{C} 7$ & $175.7(9)$ \\
\hline $\mathrm{C} 2-\mathrm{C} 3-\mathrm{C} 4-\mathrm{C} 5$ & $1.7(16)$ \\
\hline $\mathrm{C} 2-\mathrm{C} 3-\mathrm{C} 4-\mathrm{C} 18$ & $-179.3(9)$ \\
\hline $\mathrm{C} 2-\mathrm{C} 3-\mathrm{C} 12-\mathrm{C} 13$ & $-1(2)$ \\
\hline $\mathrm{C} 2-\mathrm{C} 3-\mathrm{C} 12-\mathrm{C} 17$ & $178.9(10)$ \\
\hline $\mathrm{C} 3-\mathrm{C} 4-\mathrm{C} 5-\mathrm{C} 6$ & $-0.9(16)$ \\
\hline $\mathrm{C} 3-\mathrm{C} 4-\mathrm{C} 18-\mathrm{C} 17$ & $0.6(11)$ \\
\hline $\mathrm{C} 3-\mathrm{C} 4-\mathrm{C} 18-\mathrm{C} 73$ & $-117.1(11)$ \\
\hline $\mathrm{C} 3-\mathrm{C} 4-\mathrm{C} 18-\mathrm{C} 79$ & $119.5(11)$ \\
\hline $\mathrm{C} 3-\mathrm{C} 12-\mathrm{C} 13-\mathrm{C} 14$ & $178.8(11)$ \\
\hline $\mathrm{C} 3-\mathrm{C} 12-\mathrm{C} 17-\mathrm{C} 16$ & $-178.6(9)$ \\
\hline $\mathrm{C} 3-\mathrm{C} 12-\mathrm{C} 17-\mathrm{C} 18$ & $-0.1(12)$ \\
\hline $\mathrm{C} 4-\mathrm{C} 3-\mathrm{C} 12-\mathrm{C} 13$ & $-179.6(12)$ \\
\hline $\mathrm{C} 4-\mathrm{C} 3-\mathrm{C} 12-\mathrm{C} 17$ & $0.5(12)$ \\
\hline $\mathrm{C} 4-\mathrm{C} 5-\mathrm{C} 6-\mathrm{C} 1$ & $1.1(16)$ \\
\hline $\mathrm{C} 4-\mathrm{C} 5-\mathrm{C} 6-\mathrm{C} 7$ & $-176.3(10)$ \\
\hline $\mathrm{C} 4-\mathrm{C} 18-\mathrm{C} 73-\mathrm{C} 74$ & 49.7 (14) \\
\hline $\mathrm{C} 4-\mathrm{C} 18-\mathrm{C} 79-\mathrm{C} 80$ & $-61.0(14)$ \\
\hline $\mathrm{C} 5-\mathrm{C} 4-\mathrm{C} 18-\mathrm{C} 17$ & $179.5(11)$ \\
\hline $\mathrm{C} 5-\mathrm{C} 4-\mathrm{C} 18-\mathrm{C} 73$ & $61.8(15)$ \\
\hline $\mathrm{C} 5-\mathrm{C} 4-\mathrm{C} 18-\mathrm{C} 79$ & $-61.6(15)$ \\
\hline $\mathrm{C} 5-\mathrm{C} 6-\mathrm{C} 7-\mathrm{N} 1$ & $-174.9(9)$ \\
\hline $\mathrm{C} 5-\mathrm{C} 6-\mathrm{C} 7-\mathrm{C} 8$ & $6.6(17)$ \\
\hline $\mathrm{C} 6-\mathrm{C} 1-\mathrm{C} 2-\mathrm{C} 3$ & $2.5(14)$ \\
\hline $\mathrm{C} 6-\mathrm{C} 7-\mathrm{C} 8-\mathrm{C} 9$ & $178.3(11)$ \\
\hline $\mathrm{C} 7-\mathrm{N} 1-\mathrm{C} 11-\mathrm{C} 10$ & $-0.4(15)$ \\
\hline $\mathrm{C} 7-\mathrm{C} 8-\mathrm{C} 9-\mathrm{C} 10$ & $-0.8(17)$ \\
\hline $\mathrm{C} 8-\mathrm{C} 9-\mathrm{C} 10-\mathrm{O} 3$ & $-179.5(10)$ \\
\hline $\mathrm{C} 8-\mathrm{C} 9-\mathrm{C} 10-\mathrm{C} 11$ & $1.0(16)$ \\
\hline $\mathrm{C} 9-\mathrm{C} 10-\mathrm{C} 11-\mathrm{N} 1$ & $-0.4(16)$ \\
\hline $\mathrm{C} 11-\mathrm{N} 1-\mathrm{C} 7-\mathrm{C} 6$ & $-178.0(9)$ \\
\hline $\mathrm{C} 11-\mathrm{N} 1-\mathrm{C} 7-\mathrm{C} 8$ & $0.6(14)$ \\
\hline $\mathrm{C} 12-\mathrm{C} 3-\mathrm{C} 4-\mathrm{C} 5$ & $-179.7(10)$ \\
\hline $\mathrm{C} 12-\mathrm{C} 3-\mathrm{C} 4-\mathrm{C} 18$ & $-0.7(12)$ \\
\hline $\mathrm{C} 12-\mathrm{C} 13-\mathrm{C} 14-\mathrm{C} 15$ & $-0.9(17)$ \\
\hline $\mathrm{C} 12-\mathrm{C} 17-\mathrm{C} 18-\mathrm{C} 4$ & $-0.3(11)$ \\
\hline $\mathrm{C} 12-\mathrm{C} 17-\mathrm{C} 18-\mathrm{C} 73$ & $118.8(10)$ \\
\hline $\mathrm{C} 12-\mathrm{C} 17-\mathrm{C} 18-\mathrm{C} 79$ & $-118.9(11)$ \\
\hline $\mathrm{C} 13-\mathrm{C} 12-\mathrm{C} 17-\mathrm{C} 16$ & $1.5(17)$ \\
\hline $\mathrm{C} 13-\mathrm{C} 12-\mathrm{C} 17-\mathrm{C} 18$ & $-180.0(10)$ \\
\hline $\mathrm{C} 13-\mathrm{C} 14-\mathrm{C} 15-\mathrm{C} 16$ & $3.1(18)$ \\
\hline
\end{tabular}

\begin{tabular}{|c|c|}
\hline $\mathrm{C} 2^{\prime}-\mathrm{C} 1^{\prime}-\mathrm{C} 6^{\prime}-\mathrm{C} 7^{\prime}$ & $-178.3(11)$ \\
\hline $\mathrm{C} 2^{\prime}-\mathrm{C} 3^{\prime}-\mathrm{C} 4^{\prime}-\mathrm{C} 5^{\prime}$ & $4(2)$ \\
\hline $\mathrm{C} 2^{\prime}-\mathrm{C} 3^{\prime}-\mathrm{C} 4^{\prime}-\mathrm{C} 18^{\prime}$ & $179.9(12)$ \\
\hline $\mathrm{C} 2^{\prime}-\mathrm{C} 3^{\prime}-\mathrm{C} 12^{\prime}-\mathrm{C} 13^{\prime}$ & $-6(2)$ \\
\hline $\mathrm{C} 2^{\prime}-\mathrm{C} 3^{\prime}-\mathrm{C} 12^{\prime}-\mathrm{C} 17^{\prime}$ & $178.1(13)$ \\
\hline $\mathrm{C} 3^{\prime}-\mathrm{C} 4^{\prime}-\mathrm{C} 5^{\prime}-\mathrm{C} 6^{\prime}$ & $-4(2)$ \\
\hline $\mathrm{C} 3^{\prime}-\mathrm{C} 4^{\prime}-\mathrm{C} 18^{\prime}-\mathrm{C} 17^{\prime}$ & $3.5(16)$ \\
\hline $\mathrm{C} 3^{\prime}-\mathrm{C} 4^{\prime}-\mathrm{C} 18^{\prime}-\mathrm{C} 73^{\prime}$ & $-115.1(13)$ \\
\hline $\mathrm{C} 3^{\prime}-\mathrm{C} 4^{\prime}-\mathrm{C} 18^{\prime}-\mathrm{C} 79^{\prime}$ & $123.0(14)$ \\
\hline $\mathrm{C} 3^{\prime}-\mathrm{C} 12^{\prime}-\mathrm{C} 13^{\prime}-\mathrm{C} 14^{\prime}$ & $-173.5(13)$ \\
\hline $\mathrm{C} 3^{\prime}-\mathrm{C} 12^{\prime}-\mathrm{C} 17^{\prime}-\mathrm{C} 16^{\prime}$ & $174.3(12)$ \\
\hline $\mathrm{C} 3^{\prime}-\mathrm{C} 12^{\prime}-\mathrm{C} 17^{\prime}-\mathrm{C} 18^{\prime}$ & $0.2(16)$ \\
\hline $\mathrm{C} 4^{\prime}-\mathrm{C} 3^{\prime}-\mathrm{C} 12^{\prime}-\mathrm{C} 13^{\prime}$ & $178.4(14)$ \\
\hline $\mathrm{C} 4^{\prime}-\mathrm{C} 3^{\prime}-\mathrm{C} 12^{\prime}-\mathrm{C} 17^{\prime}$ & $2.1(16)$ \\
\hline $\mathrm{C} 4^{\prime}-\mathrm{C} 5^{\prime}-\mathrm{C} 6^{\prime}-\mathrm{C} 1^{\prime}$ & $5(2)$ \\
\hline $\mathrm{C} 4^{\prime}-\mathrm{C} 5^{\prime}-\mathrm{C} 6^{\prime}-\mathrm{C} 7^{\prime}$ & $177.9(13)$ \\
\hline $\mathrm{C} 4^{\prime}-\mathrm{C} 18^{\prime}-\mathrm{C} 73^{\prime}-\mathrm{C} 74^{\prime}$ & $63.8(16)$ \\
\hline $\mathrm{C} 4^{\prime}-\mathrm{C} 18^{\prime}-\mathrm{C} 79^{\prime}-\mathrm{C} 80^{\prime}$ & $-68(2)$ \\
\hline $\mathrm{C} 4^{\prime}-\mathrm{C} 18^{\prime}-\mathrm{C} 79^{\prime}-\mathrm{C} 80 \mathrm{~B}$ & $-35(3)$ \\
\hline $\mathrm{C} 5^{\prime}-\mathrm{C} 4^{\prime}-\mathrm{C} 18^{\prime}-\mathrm{C} 17^{\prime}$ & $178.7(15)$ \\
\hline $\mathrm{C} 5^{\prime}-\mathrm{C} 4^{\prime}-\mathrm{C} 18^{\prime}-\mathrm{C} 73^{\prime}$ & $60(2)$ \\
\hline $\mathrm{C} 5^{\prime}-\mathrm{C} 4^{\prime}-\mathrm{C} 18^{\prime}-\mathrm{C} 79^{\prime}$ & $-62(2)$ \\
\hline $\mathrm{C} 5^{\prime}-\mathrm{C} 6^{\prime}-\mathrm{C} 7^{\prime}-\mathrm{N} 1^{\prime}$ & $-176.2(12)$ \\
\hline $\mathrm{C} 5^{\prime}-\mathrm{C} 6^{\prime}-\mathrm{C} 7^{\prime}-\mathrm{C} 8^{\prime}$ & $1(2)$ \\
\hline $\mathrm{C} 6^{\prime}-\mathrm{C} 1^{\prime}-\mathrm{C} 2^{\prime}-\mathrm{C} 3^{\prime}$ & $4.4(19)$ \\
\hline $\mathrm{C} 6^{\prime}-\mathrm{C} 7^{\prime}-\mathrm{C} 8^{\prime}-\mathrm{C} 9^{\prime}$ & $-179.0(13)$ \\
\hline $\mathrm{C} 7^{\prime}-\mathrm{N} 11^{\prime}-\mathrm{C} 11^{\prime}-\mathrm{C} 10^{\prime}$ & $-1.5(19)$ \\
\hline $\mathrm{C} 7^{\prime}-\mathrm{C} 8^{\prime}-\mathrm{C} 9^{\prime}-\mathrm{C} 10^{\prime}$ & $3(2)$ \\
\hline $\mathrm{C} 8^{\prime}-\mathrm{C} 9^{\prime}-\mathrm{C} 10^{\prime}-\mathrm{O}^{\prime}$ & $-179.1(12)$ \\
\hline $\mathrm{C} 8^{\prime}-\mathrm{C} 9^{\prime}-\mathrm{C} 10^{\prime}-\mathrm{C} 11^{\prime}$ & $-2(2)$ \\
\hline $\mathrm{C} 9^{\prime}-\mathrm{C} 10^{\prime}-\mathrm{C} 11^{\prime}-\mathrm{N} 1^{\prime}$ & $2(2)$ \\
\hline $\mathrm{C} 11^{\prime}-\mathrm{N} 1^{\prime}-\mathrm{C} 7^{\prime}-\mathrm{C} 6^{\prime}$ & $178.8(11)$ \\
\hline $\mathrm{C} 11^{\prime}-\mathrm{N} 1^{\prime}-\mathrm{C} 7^{\prime}-\mathrm{C} 8^{\prime}$ & $1.6(18)$ \\
\hline $\mathrm{C} 12^{\prime}-\mathrm{C} 3^{\prime}-\mathrm{C} 4^{\prime}-\mathrm{C} 5^{\prime}$ & $-179.2(13)$ \\
\hline $\mathrm{C} 12^{\prime}-\mathrm{C} 3^{\prime}-\mathrm{C} 4^{\prime}-\mathrm{C} 18^{\prime}$ & $-3.6(16)$ \\
\hline $\mathrm{C} 12^{\prime}-\mathrm{C} 13^{\prime}-\mathrm{C} 14^{\prime}-\mathrm{C} 15^{\prime}$ & $-6(2)$ \\
\hline $\mathrm{C} 12^{\prime}-\mathrm{C} 17^{\prime}-\mathrm{C} 18^{\prime}-\mathrm{C} 4^{\prime}$ & $-2.1(16)$ \\
\hline $\mathrm{C} 12^{\prime}-\mathrm{C} 17^{\prime}-\mathrm{C} 18^{\prime}-\mathrm{C} 73^{\prime}$ & $116.3(13)$ \\
\hline $\mathrm{C} 12^{\prime}-\mathrm{C} 17^{\prime}-\mathrm{C} 18^{\prime}-\mathrm{C} 79^{\prime}$ & $-122.3(13)$ \\
\hline $\mathrm{C} 13^{\prime}-\mathrm{C} 12^{\prime}-\mathrm{C} 17^{\prime}-\mathrm{C} 16^{\prime}$ & $-2(2)$ \\
\hline $\mathrm{C} 13^{\prime}-\mathrm{C} 12^{\prime}-\mathrm{C} 17^{\prime}-\mathrm{C} 18^{\prime}$ & $-176.6(12)$ \\
\hline $\mathrm{C} 13^{\prime}-\mathrm{C} 14^{\prime}-\mathrm{C} 15^{\prime}-\mathrm{C} 16^{\prime}$ & $9(2)$ \\
\hline $\mathrm{C} 14^{\prime}-\mathrm{C} 15^{\prime}-\mathrm{C} 16^{\prime}-\mathrm{C} 17^{\prime}$ & $-9(2)$ \\
\hline $\mathrm{C} 15^{\prime}-\mathrm{C} 16^{\prime}-\mathrm{C} 17^{\prime}-\mathrm{C} 12^{\prime}$ & $6(2)$ \\
\hline $\mathrm{C} 15^{\prime}-\mathrm{C} 16^{\prime}-\mathrm{C} 17^{\prime}-\mathrm{C} 18^{\prime}$ & $178.4(15)$ \\
\hline $\mathrm{C} 16^{\prime}-\mathrm{C} 17^{\prime}-\mathrm{C} 18^{\prime}-\mathrm{C} 4^{\prime}$ & $-175.4(15)$ \\
\hline $\mathrm{C} 16^{\prime}-\mathrm{C} 17^{\prime}-\mathrm{C} 18^{\prime}-\mathrm{C} 73^{\prime}$ & $-57(2)$ \\
\hline $\mathrm{C} 16^{\prime}-\mathrm{C} 17^{\prime}-\mathrm{C} 18^{\prime}-\mathrm{C} 79^{\prime}$ & $64(2)$ \\
\hline
\end{tabular}




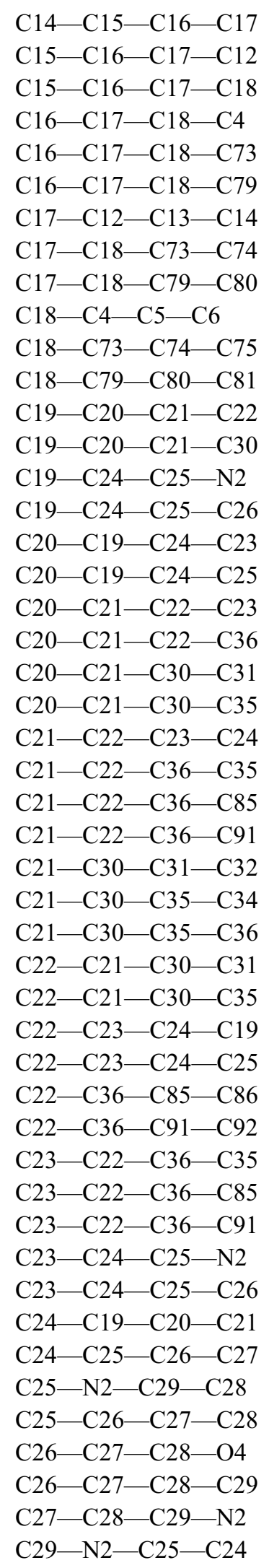

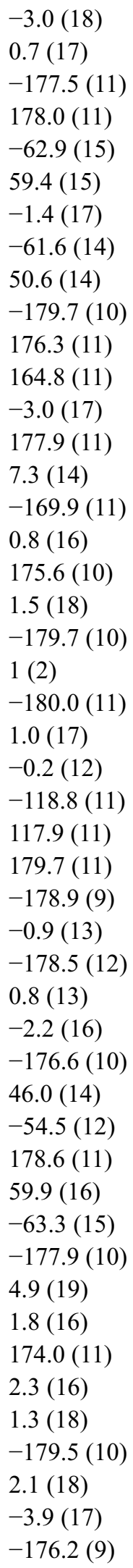

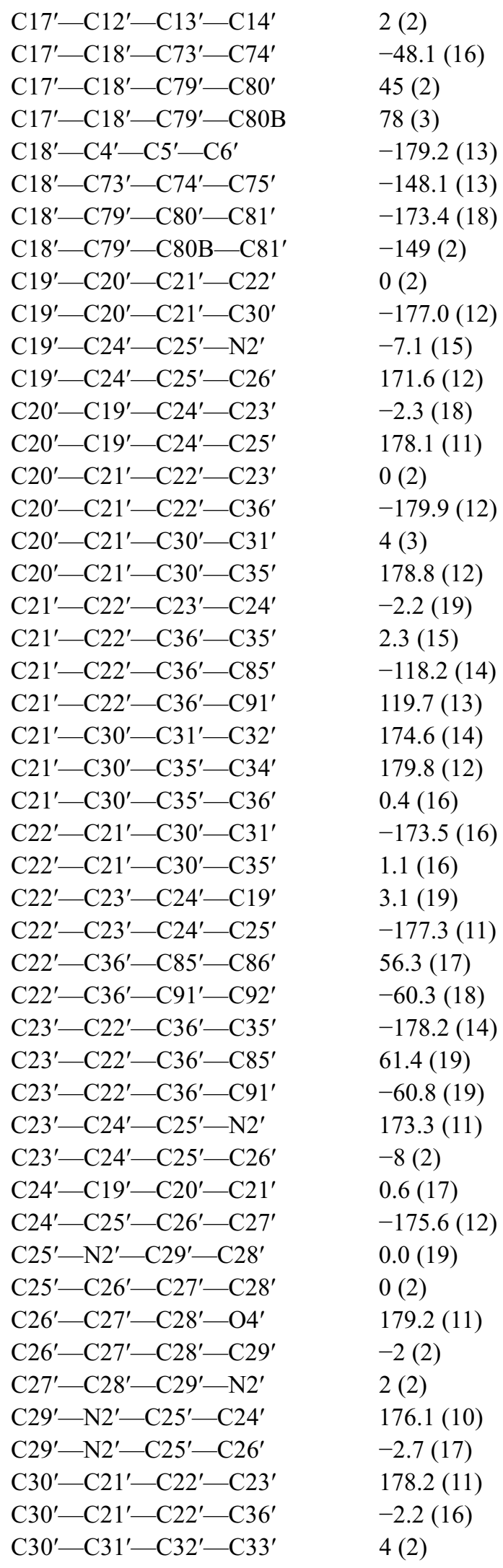

2 (2)

$-48.1(16)$

45 (2)

78 (3)

$-179.2(13)$

$-148.1(13)$

$-173.4(18)$

-149 (2)

0 (2)

$-177.0(12)$

$-7.1(15)$

$171.6(12)$

$-2.3(18)$

$178.1(11)$

0 (2)

$-179.9(12)$

$4(3)$

178.8 (12)

$-2.2(19)$

$2.3(15)$

$-118.2(14)$

119.7 (13)

174.6 (14)

$179.8(12)$

$0.4(16)$

$-173.5(16)$

$1.1(16)$

3.1 (19)

-177.3 (11)

56.3 (17)

$-60.3(18)$

-178.2 (14)

61.4 (19)

$-60.8(19)$

173.3 (11)

$-8(2)$

0.6 (17)

$-175.6(12)$

0.0 (19)

0 (2)

179.2 (11)

-2 (2)

2 (2)

176.1 (10)

-2.7 (17)

178.2 (11)

-2.2 (16)

4 (2) 


\begin{tabular}{|c|c|}
\hline $\mathrm{C} 29-\mathrm{N} 2-\mathrm{C} 25-\mathrm{C} 26$ & $1.2(16)$ \\
\hline $\mathrm{C} 30-\mathrm{C} 21-\mathrm{C} 22-\mathrm{C} 23$ & $-179.2(10)$ \\
\hline $\mathrm{C} 30-\mathrm{C} 21-\mathrm{C} 22-\mathrm{C} 36$ & $-0.4(13)$ \\
\hline $\mathrm{C} 30-\mathrm{C} 31-\mathrm{C} 32-\mathrm{C} 33$ & $-1.0(18)$ \\
\hline $\mathrm{C} 30-\mathrm{C} 35-\mathrm{C} 36-\mathrm{C} 22$ & $0.7(12)$ \\
\hline $\mathrm{C} 30-\mathrm{C} 35-\mathrm{C} 36-\mathrm{C} 85$ & $119.0(10)$ \\
\hline $\mathrm{C} 30-\mathrm{C} 35-\mathrm{C} 36-\mathrm{C} 91$ & $-116.1(11)$ \\
\hline $\mathrm{C} 31-\mathrm{C} 30-\mathrm{C} 35-\mathrm{C} 34$ & $0.5(17)$ \\
\hline $\mathrm{C} 31-\mathrm{C} 30-\mathrm{C} 35-\mathrm{C} 36$ & $178.4(10)$ \\
\hline $\mathrm{C} 31-\mathrm{C} 32-\mathrm{C} 33-\mathrm{C} 34$ & $0.5(19)$ \\
\hline $\mathrm{C} 32-\mathrm{C} 33-\mathrm{C} 34-\mathrm{C} 35$ & $0.6(18)$ \\
\hline $\mathrm{C} 33-\mathrm{C} 34-\mathrm{C} 35-\mathrm{C} 30$ & $-1.1(16)$ \\
\hline $\mathrm{C} 33-\mathrm{C} 34-\mathrm{C} 35-\mathrm{C} 36$ & $-178.7(11)$ \\
\hline $\mathrm{C} 34-\mathrm{C} 35-\mathrm{C} 36-\mathrm{C} 22$ & $178.4(11)$ \\
\hline $\mathrm{C} 34-\mathrm{C} 35-\mathrm{C} 36-\mathrm{C} 85$ & $-63.4(14)$ \\
\hline $\mathrm{C} 34-\mathrm{C} 35-\mathrm{C} 36-\mathrm{C} 91$ & $61.6(15)$ \\
\hline $\mathrm{C} 35-\mathrm{C} 30-\mathrm{C} 31-\mathrm{C} 32$ & $0.6(18)$ \\
\hline $\mathrm{C} 35-\mathrm{C} 36-\mathrm{C} 85-\mathrm{C} 86$ & $-65.5(12)$ \\
\hline $\mathrm{C} 35-\mathrm{C} 36-\mathrm{C} 91-\mathrm{C} 92$ & $56.3(13)$ \\
\hline $\mathrm{C} 36-\mathrm{C} 22-\mathrm{C} 23-\mathrm{C} 24$ & $-177.6(10)$ \\
\hline $\mathrm{C} 36-\mathrm{C} 85-\mathrm{C} 86-\mathrm{C} 87$ & $179.0(10)$ \\
\hline $\mathrm{C} 36-\mathrm{C} 91-\mathrm{C} 92-\mathrm{C} 93$ & $157.6(9)$ \\
\hline $\mathrm{C} 37-\mathrm{C} 38-\mathrm{C} 39-\mathrm{C} 40$ & $-5.2(16)$ \\
\hline $\mathrm{C} 37-\mathrm{C} 38-\mathrm{C} 39-\mathrm{C} 48$ & $174.9(11)$ \\
\hline $\mathrm{C} 37-\mathrm{C} 42-\mathrm{C} 43-\mathrm{N} 3$ & $1.0(13)$ \\
\hline $\mathrm{C} 37-\mathrm{C} 42-\mathrm{C} 43-\mathrm{C} 44$ & $-178.5(10)$ \\
\hline $\mathrm{C} 38-\mathrm{C} 37-\mathrm{C} 42-\mathrm{C} 41$ & $0.4(15)$ \\
\hline $\mathrm{C} 38-\mathrm{C} 37-\mathrm{C} 42-\mathrm{C} 43$ & $-178.3(9)$ \\
\hline $\mathrm{C} 38-\mathrm{C} 39-\mathrm{C} 40-\mathrm{C} 41$ & $5.0(17)$ \\
\hline $\mathrm{C} 38-\mathrm{C} 39-\mathrm{C} 40-\mathrm{C} 54$ & $-176.6(9)$ \\
\hline $\mathrm{C} 38-\mathrm{C} 39-\mathrm{C} 48-\mathrm{C} 49$ & $-3(2)$ \\
\hline $\mathrm{C} 38-\mathrm{C} 39-\mathrm{C} 48-\mathrm{C} 53$ & $179.6(11)$ \\
\hline $\mathrm{C} 39-\mathrm{C} 40-\mathrm{C} 41-\mathrm{C} 42$ & $-2.2(16)$ \\
\hline $\mathrm{C} 39-\mathrm{C} 40-\mathrm{C} 54-\mathrm{C} 53$ & $-4.7(12)$ \\
\hline $\mathrm{C} 39-\mathrm{C} 40-\mathrm{C} 54-\mathrm{C} 97$ & $-123.8(11)$ \\
\hline $\mathrm{C} 39-\mathrm{C} 40-\mathrm{C} 54-\mathrm{C} 103$ & $115.6(11)$ \\
\hline $\mathrm{C} 39-\mathrm{C} 48-\mathrm{C} 49-\mathrm{C} 50$ & $-176.8(12)$ \\
\hline $\mathrm{C} 39-\mathrm{C} 48-\mathrm{C} 53-\mathrm{C} 52$ & $177.9(12)$ \\
\hline $\mathrm{C} 39-\mathrm{C} 48-\mathrm{C} 53-\mathrm{C} 54$ & $-2.9(13)$ \\
\hline $\mathrm{C} 40-\mathrm{C} 39-\mathrm{C} 48-\mathrm{C} 49$ & $177.3(12)$ \\
\hline $\mathrm{C} 40-\mathrm{C} 39-\mathrm{C} 48-\mathrm{C} 53$ & $-0.3(13)$ \\
\hline $\mathrm{C} 40-\mathrm{C} 41-\mathrm{C} 42-\mathrm{C} 37$ & $-0.5(15)$ \\
\hline $\mathrm{C} 40-\mathrm{C} 41-\mathrm{C} 42-\mathrm{C} 43$ & $178.1(10)$ \\
\hline $\mathrm{C} 40-\mathrm{C} 54-\mathrm{C} 97-\mathrm{C} 98$ & $55.5(15)$ \\
\hline $\mathrm{C} 40-\mathrm{C} 54-\mathrm{C} 103-\mathrm{C} 104$ & $-61.1(15)$ \\
\hline $\mathrm{C} 41-\mathrm{C} 40-\mathrm{C} 54-\mathrm{C} 53$ & $173.6(11)$ \\
\hline $\mathrm{C} 41-\mathrm{C} 40-\mathrm{C} 54-\mathrm{C} 97$ & $54.5(16)$ \\
\hline $\mathrm{C} 41-\mathrm{C} 40-\mathrm{C} 54-\mathrm{C} 103$ & $-66.1(15)$ \\
\hline
\end{tabular}

\begin{tabular}{|c|c|}
\hline $\mathrm{C} 30^{\prime}-\mathrm{C} 35^{\prime}-\mathrm{C} 36^{\prime}-\mathrm{C} 22^{\prime}$ & $-1.5(15)$ \\
\hline $\mathrm{C} 30^{\prime}-\mathrm{C} 35^{\prime}-\mathrm{C} 36^{\prime}-\mathrm{C} 85^{\prime}$ & $117.7(13)$ \\
\hline $\mathrm{C} 30^{\prime}-\mathrm{C} 35^{\prime}-\mathrm{C} 36^{\prime}-\mathrm{C} 91^{\prime}$ & $-119.6(13)$ \\
\hline $\mathrm{C} 31^{\prime}-\mathrm{C} 30^{\prime}-\mathrm{C} 35^{\prime}-\mathrm{C} 34^{\prime}$ & $-5(2)$ \\
\hline $\mathrm{C} 31^{\prime}-\mathrm{C} 30^{\prime}-\mathrm{C} 35^{\prime}-\mathrm{C} 36^{\prime}$ & $176.1(12)$ \\
\hline $\mathrm{C} 31^{\prime}-\mathrm{C} 32^{\prime}-\mathrm{C} 33^{\prime}-\mathrm{C} 34^{\prime}$ & $-4(2)$ \\
\hline $\mathrm{C} 32^{\prime}-\mathrm{C} 33^{\prime}-\mathrm{C} 34^{\prime}-\mathrm{C} 35^{\prime}$ & $0(2)$ \\
\hline $\mathrm{C} 33^{\prime}-\mathrm{C} 34^{\prime}-\mathrm{C} 35^{\prime}-\mathrm{C} 30^{\prime}$ & $4(2)$ \\
\hline $\mathrm{C} 33^{\prime}-\mathrm{C} 34^{\prime}-\mathrm{C} 35^{\prime}-\mathrm{C} 36^{\prime}$ & $-176.3(15)$ \\
\hline $\mathrm{C} 34^{\prime}-\mathrm{C} 35^{\prime}-\mathrm{C} 36^{\prime}-\mathrm{C} 22^{\prime}$ & $179.1(15)$ \\
\hline $\mathrm{C} 34^{\prime}-\mathrm{C} 35^{\prime}-\mathrm{C} 36^{\prime}-\mathrm{C} 85^{\prime}$ & $-62(2)$ \\
\hline $\mathrm{C} 34^{\prime}-\mathrm{C} 35^{\prime}-\mathrm{C} 36^{\prime}-\mathrm{C} 91^{\prime}$ & $61(2)$ \\
\hline $\mathrm{C} 35^{\prime}-\mathrm{C} 30^{\prime}-\mathrm{C} 31^{\prime}-\mathrm{C} 32^{\prime}$ & $0(2)$ \\
\hline $\mathrm{C} 35^{\prime}-\mathrm{C} 36^{\prime}-\mathrm{C} 85^{\prime}-\mathrm{C} 86^{\prime}$ & $-57.8(17)$ \\
\hline $\mathrm{C} 35^{\prime}-\mathrm{C} 36^{\prime}-\mathrm{C} 91^{\prime}-\mathrm{C} 92^{\prime}$ & $52.2(19)$ \\
\hline $\mathrm{C} 36^{\prime}-\mathrm{C} 22^{\prime}-\mathrm{C} 23^{\prime}-\mathrm{C} 24^{\prime}$ & $178.3(12)$ \\
\hline $\mathrm{C} 36^{\prime}-\mathrm{C} 85^{\prime}-\mathrm{C} 86^{\prime}-\mathrm{C} 87^{\prime}$ & $-177.3(12)$ \\
\hline $\mathrm{C} 36^{\prime}-\mathrm{C} 91^{\prime}-\mathrm{C} 92^{\prime}-\mathrm{C} 93^{\prime}$ & $-176.2(19)$ \\
\hline $\mathrm{C} 37^{\prime}-\mathrm{Ir} 4-\mathrm{N} 4^{\prime}-\mathrm{C} 61^{\prime}$ & $105.2(9)$ \\
\hline $\mathrm{C} 37^{\prime}-\mathrm{Ir} 4-\mathrm{N} 4^{\prime}-\mathrm{C} 65^{\prime}$ & $-79.6(10)$ \\
\hline $\mathrm{C} 37^{\prime}-\mathrm{C} 38^{\prime}-\mathrm{C} 39^{\prime}-\mathrm{C} 40^{\prime}$ & $0(2)$ \\
\hline $\mathrm{C} 37^{\prime}-\mathrm{C} 38^{\prime}-\mathrm{C} 39^{\prime}-\mathrm{C} 48^{\prime}$ & $-178.0(12)$ \\
\hline $\mathrm{C} 37^{\prime}-\mathrm{C} 42^{\prime}-\mathrm{C} 43^{\prime}-\mathrm{N} 3^{\prime}$ & $-8.3(18)$ \\
\hline $\mathrm{C} 37^{\prime}-\mathrm{C} 42^{\prime}-\mathrm{C} 43^{\prime}-\mathrm{C} 44^{\prime}$ & $167.8(13)$ \\
\hline $\mathrm{C} 38^{\prime}-\mathrm{C} 37^{\prime}-\mathrm{C} 42^{\prime}-\mathrm{C} 41^{\prime}$ & $4.2(19)$ \\
\hline $\mathrm{C} 38^{\prime}-\mathrm{C} 37^{\prime}-\mathrm{C} 42^{\prime}-\mathrm{C} 43^{\prime}$ & $-176.5(12)$ \\
\hline $\mathrm{C} 38^{\prime}-\mathrm{C} 39^{\prime}-\mathrm{C} 40^{\prime}-\mathrm{C} 41^{\prime}$ & $2(2)$ \\
\hline $\mathrm{C} 38^{\prime}-\mathrm{C} 39^{\prime}-\mathrm{C} 40^{\prime}-\mathrm{C} 54^{\prime}$ & $-174.6(12)$ \\
\hline $\mathrm{C} 38^{\prime}-\mathrm{C} 39^{\prime}-\mathrm{C} 48^{\prime}-\mathrm{C} 49^{\prime}$ & $-9(2)$ \\
\hline $\mathrm{C} 38^{\prime}-\mathrm{C} 39^{\prime}-\mathrm{C} 48^{\prime}-\mathrm{C} 53^{\prime}$ & $175.7(13)$ \\
\hline $\mathrm{C} 39^{\prime}-\mathrm{C} 40^{\prime}-\mathrm{C} 41^{\prime}-\mathrm{C} 42^{\prime}$ & $-2(2)$ \\
\hline $\mathrm{C} 39^{\prime}-\mathrm{C} 40^{\prime}-\mathrm{C} 54^{\prime}-\mathrm{C} 53^{\prime}$ & $-3.6(14)$ \\
\hline $\mathrm{C} 39^{\prime}-\mathrm{C} 40^{\prime}-\mathrm{C} 54^{\prime}-\mathrm{C} 97^{\prime}$ & $-119.5(13)$ \\
\hline $\mathrm{C} 39^{\prime}-\mathrm{C} 40^{\prime}-\mathrm{C} 54^{\prime}-\mathrm{C} 203$ & $114.9(13)$ \\
\hline $\mathrm{C} 39^{\prime}-\mathrm{C} 48^{\prime}-\mathrm{C} 49^{\prime}-\mathrm{C} 50^{\prime}$ & $-174.1(13)$ \\
\hline $\mathrm{C} 39^{\prime}-\mathrm{C} 48^{\prime}-\mathrm{C} 53^{\prime}-\mathrm{C} 52^{\prime}$ & $177.2(12)$ \\
\hline $\mathrm{C} 39^{\prime}-\mathrm{C} 48^{\prime}-\mathrm{C} 53^{\prime}-\mathrm{C} 54^{\prime}$ & $0.4(16)$ \\
\hline $\mathrm{C} 40^{\prime}-\mathrm{C} 39^{\prime}-\mathrm{C} 48^{\prime}-\mathrm{C} 49^{\prime}$ & $172.9(13)$ \\
\hline $\mathrm{C} 40^{\prime}-\mathrm{C} 39^{\prime}-\mathrm{C} 48^{\prime}-\mathrm{C} 53^{\prime}$ & $-2.8(15)$ \\
\hline $\mathrm{C} 40^{\prime}-\mathrm{C} 41^{\prime}-\mathrm{C} 42^{\prime}-\mathrm{C} 37^{\prime}$ & $-2(2)$ \\
\hline $\mathrm{C} 40^{\prime}-\mathrm{C} 41^{\prime}-\mathrm{C} 42^{\prime}-\mathrm{C} 43^{\prime}$ & $179.0(12)$ \\
\hline $\mathrm{C} 40^{\prime}-\mathrm{C} 54^{\prime}-\mathrm{C} 97^{\prime}-\mathrm{C} 98^{\prime}$ & $61.1(16)$ \\
\hline $\mathrm{C} 40^{\prime}-\mathrm{C} 54^{\prime}-\mathrm{C} 203-\mathrm{C} 204$ & $-44(2)$ \\
\hline $\mathrm{C} 40^{\prime}-\mathrm{C} 54^{\prime}-\mathrm{C} 203-\mathrm{C} 20 \mathrm{~B}$ & $-64(3)$ \\
\hline $\mathrm{C} 41^{\prime}-\mathrm{C} 40^{\prime}-\mathrm{C} 54^{\prime}-\mathrm{C} 53^{\prime}$ & $179.8(13)$ \\
\hline $\mathrm{C} 41^{\prime}-\mathrm{C} 40^{\prime}-\mathrm{C} 54^{\prime}-\mathrm{C} 97^{\prime}$ & $63.9(18)$ \\
\hline $\mathrm{C} 41^{\prime}-\mathrm{C} 40^{\prime}-\mathrm{C} 54^{\prime}-\mathrm{C} 203$ & $-61.7(19)$ \\
\hline $\mathrm{C} 41^{\prime}-\mathrm{C} 42^{\prime}-\mathrm{C} 43^{\prime}-\mathrm{N} 3^{\prime}$ & $171.0(12)$ \\
\hline
\end{tabular}




\begin{tabular}{|c|c|}
\hline $\mathrm{C} 41-\mathrm{C} 42-\mathrm{C} 43-\mathrm{N} 3$ & $-177.7(9)$ \\
\hline $\mathrm{C} 41-\mathrm{C} 42-\mathrm{C} 43-\mathrm{C} 44$ & $2.8(16)$ \\
\hline $\mathrm{C} 42-\mathrm{C} 37-\mathrm{C} 38-\mathrm{C} 39$ & $2.5(15)$ \\
\hline $\mathrm{C} 42-\mathrm{C} 43-\mathrm{C} 44-\mathrm{C} 45$ & $179.2(10)$ \\
\hline $\mathrm{C} 43-\mathrm{N} 3-\mathrm{C} 47-\mathrm{C} 46$ & $-1.2(15)$ \\
\hline $\mathrm{C} 43-\mathrm{C} 44-\mathrm{C} 45-\mathrm{C} 46$ & $-1.2(16)$ \\
\hline $\mathrm{C} 44-\mathrm{C} 45-\mathrm{C} 46-\mathrm{O} 5$ & $178.8(9)$ \\
\hline $\mathrm{C} 44-\mathrm{C} 45-\mathrm{C} 46-\mathrm{C} 47$ & $1.5(17)$ \\
\hline $\mathrm{C} 45-\mathrm{C} 46-\mathrm{C} 47-\mathrm{N} 3$ & $-0.4(16)$ \\
\hline $\mathrm{C} 47-\mathrm{N} 3-\mathrm{C} 43-\mathrm{C} 42$ & $-178.1(9)$ \\
\hline $\mathrm{C} 47-\mathrm{N} 3-\mathrm{C} 43-\mathrm{C} 44$ & $1.5(15)$ \\
\hline $\mathrm{C} 48-\mathrm{C} 39-\mathrm{C} 40-\mathrm{C} 41$ & $-175.1(10)$ \\
\hline $\mathrm{C} 48-\mathrm{C} 39-\mathrm{C} 40-\mathrm{C} 54$ & $3.4(13)$ \\
\hline $\mathrm{C} 48-\mathrm{C} 49-\mathrm{C} 50-\mathrm{C} 51$ & $0(2)$ \\
\hline $\mathrm{C} 48-\mathrm{C} 53-\mathrm{C} 54-\mathrm{C} 40$ & $4.6(12)$ \\
\hline $\mathrm{C} 48-\mathrm{C} 53-\mathrm{C} 54-\mathrm{C} 97$ & $122.6(11)$ \\
\hline $\mathrm{C} 48-\mathrm{C} 53-\mathrm{C} 54-\mathrm{C} 103$ & $-114.6(11)$ \\
\hline $\mathrm{C} 49-\mathrm{C} 48-\mathrm{C} 53-\mathrm{C} 52$ & $0.0(19)$ \\
\hline $\mathrm{C} 49-\mathrm{C} 48-\mathrm{C} 53-\mathrm{C} 54$ & $179.2(10)$ \\
\hline $\mathrm{C} 49-\mathrm{C} 50-\mathrm{C} 51-\mathrm{C} 52$ & $0(2)$ \\
\hline $\mathrm{C} 50-\mathrm{C} 51-\mathrm{C} 52-\mathrm{C} 53$ & $1(2)$ \\
\hline $\mathrm{C} 51-\mathrm{C} 52-\mathrm{C} 53-\mathrm{C} 48$ & $-1(2)$ \\
\hline $\mathrm{C} 51-\mathrm{C} 52-\mathrm{C} 53-\mathrm{C} 54$ & $-179.8(13)$ \\
\hline $\mathrm{C} 52-\mathrm{C} 53-\mathrm{C} 54-\mathrm{C} 40$ & $-176.3(13)$ \\
\hline $\mathrm{C} 52-\mathrm{C} 53-\mathrm{C} 54-\mathrm{C} 97$ & $-58.3(17)$ \\
\hline $\mathrm{C} 52-\mathrm{C} 53-\mathrm{C} 54-\mathrm{C} 103$ & $64.5(17)$ \\
\hline $\mathrm{C} 53-\mathrm{C} 48-\mathrm{C} 49-\mathrm{C} 50$ & $0.6(18)$ \\
\hline $\mathrm{C} 53-\mathrm{C} 54-\mathrm{C} 97-\mathrm{C} 98$ & $-56.6(15)$ \\
\hline $\mathrm{C} 53-\mathrm{C} 54-\mathrm{C} 103-\mathrm{C} 104$ & $51.8(15)$ \\
\hline $\mathrm{C} 54-\mathrm{C} 40-\mathrm{C} 41-\mathrm{C} 42$ & $179.7(10)$ \\
\hline $\mathrm{C} 54-\mathrm{C} 97-\mathrm{C} 98-\mathrm{C} 99$ & $170.5(12)$ \\
\hline $\mathrm{C} 54-\mathrm{C} 103-\mathrm{C} 104-\mathrm{C} 105$ & $165.6(14)$ \\
\hline $\mathrm{C} 54-\mathrm{C} 103-\mathrm{C} 104-\mathrm{C} 10 \mathrm{~A}$ & $-178(2)$ \\
\hline $\mathrm{C} 55-\mathrm{C} 56-\mathrm{C} 57-\mathrm{C} 58$ & $-2.4(17)$ \\
\hline $\mathrm{C} 55-\mathrm{C} 56-\mathrm{C} 57-\mathrm{C} 66$ & $177.5(10)$ \\
\hline $\mathrm{C} 55-\mathrm{C} 60-\mathrm{C} 61-\mathrm{N} 4$ & $5.7(14)$ \\
\hline $\mathrm{C} 55-\mathrm{C} 60-\mathrm{C} 61-\mathrm{C} 62$ & $-171.5(10)$ \\
\hline $\mathrm{C} 56-\mathrm{C} 55-\mathrm{C} 60-\mathrm{C} 59$ & $-2.3(16)$ \\
\hline $\mathrm{C} 56-\mathrm{C} 55-\mathrm{C} 60-\mathrm{C} 61$ & $177.3(9)$ \\
\hline $\mathrm{C} 56-\mathrm{C} 57-\mathrm{C} 58-\mathrm{C} 59$ & $-1.9(18)$ \\
\hline $\mathrm{C} 56-\mathrm{C} 57-\mathrm{C} 58-\mathrm{C} 72$ & $177.5(10)$ \\
\hline $\mathrm{C} 56-\mathrm{C} 57-\mathrm{C} 66-\mathrm{C} 67$ & $3(2)$ \\
\hline $\mathrm{C} 56-\mathrm{C} 57-\mathrm{C} 66-\mathrm{C} 71$ & $-177.6(11)$ \\
\hline $\mathrm{C} 57-\mathrm{C} 58-\mathrm{C} 59-\mathrm{C} 60$ & $3.9(17)$ \\
\hline $\mathrm{C} 57-\mathrm{C} 58-\mathrm{C} 72-\mathrm{C} 71$ & $1.5(12)$ \\
\hline $\mathrm{C} 57-\mathrm{C} 58-\mathrm{C} 72-\mathrm{C} 109$ & $-117.2(11)$ \\
\hline $\mathrm{C} 57-\mathrm{C} 58-\mathrm{C} 72-\mathrm{C} 115$ & $122.4(11)$ \\
\hline $\mathrm{C} 57-\mathrm{C} 66-\mathrm{C} 67-\mathrm{C} 68$ & $179.3(12)$ \\
\hline
\end{tabular}

\begin{tabular}{|c|c|}
\hline $\mathrm{C} 41^{\prime}-\mathrm{C} 42^{\prime}-\mathrm{C} 43^{\prime}-\mathrm{C} 44^{\prime}$ & $-13(2)$ \\
\hline $\mathrm{C} 42^{\prime}-\mathrm{C} 37^{\prime}-\mathrm{C} 38^{\prime}-\mathrm{C} 39^{\prime}$ & $-3.4(19)$ \\
\hline $\mathrm{C} 42^{\prime}-\mathrm{C} 43^{\prime}-\mathrm{C} 44^{\prime}-\mathrm{C} 45^{\prime}$ & $-176.3(14)$ \\
\hline $\mathrm{C} 43^{\prime}-\mathrm{N} 3^{\prime}-\mathrm{C} 47^{\prime}-\mathrm{C} 46^{\prime}$ & $-1(2)$ \\
\hline $\mathrm{C} 43^{\prime}-\mathrm{C} 44^{\prime}-\mathrm{C} 45^{\prime}-\mathrm{C} 46^{\prime}$ & $1(2)$ \\
\hline $\mathrm{C} 44^{\prime}-\mathrm{C} 45^{\prime}-\mathrm{C} 46^{\prime}-\mathrm{O} 5^{\prime}$ & $-180.0(13)$ \\
\hline $\mathrm{C} 44^{\prime}-\mathrm{C} 45^{\prime}-\mathrm{C} 46^{\prime}-\mathrm{C} 47^{\prime}$ & $-2(3)$ \\
\hline $\mathrm{C} 45^{\prime}-\mathrm{C} 46^{\prime}-\mathrm{C} 47^{\prime}-\mathrm{N} 3^{\prime}$ & $2(2)$ \\
\hline $\mathrm{C} 47^{\prime}-\mathrm{N} 3^{\prime}-\mathrm{C} 43^{\prime}-\mathrm{C} 42^{\prime}$ & $176.6(11)$ \\
\hline $\mathrm{C} 47^{\prime}-\mathrm{N} 3^{\prime}-\mathrm{C} 43^{\prime}-\mathrm{C} 44^{\prime}$ & $0(2)$ \\
\hline $\mathrm{C} 48^{\prime}-\mathrm{C} 39^{\prime}-\mathrm{C} 40^{\prime}-\mathrm{C} 41^{\prime}$ & $-179.1(12)$ \\
\hline $\mathrm{C} 48^{\prime}-\mathrm{C} 39^{\prime}-\mathrm{C} 40^{\prime}-\mathrm{C} 54^{\prime}$ & $4.0(15)$ \\
\hline $\mathrm{C} 48^{\prime}-\mathrm{C} 49^{\prime}-\mathrm{C} 50^{\prime}-\mathrm{C} 51^{\prime}$ & $-2.4(19)$ \\
\hline $\mathrm{C} 48^{\prime}-\mathrm{C} 53^{\prime}-\mathrm{C} 54^{\prime}-\mathrm{C} 40^{\prime}$ & $1.8(15)$ \\
\hline $\mathrm{C} 48^{\prime}-\mathrm{C} 53^{\prime}-\mathrm{C} 54^{\prime}-\mathrm{C} 97^{\prime}$ & $118.9(13)$ \\
\hline $\mathrm{C} 48^{\prime}-\mathrm{C} 53^{\prime}-\mathrm{C} 54^{\prime}-\mathrm{C} 203$ & $-116.6(13)$ \\
\hline $\mathrm{C} 49^{\prime}-\mathrm{C} 48^{\prime}-\mathrm{C} 53^{\prime}-\mathrm{C} 52^{\prime}$ & $1(2)$ \\
\hline $\mathrm{C} 49^{\prime}-\mathrm{C} 48^{\prime}-\mathrm{C} 53^{\prime}-\mathrm{C} 54^{\prime}$ & $-175.7(12)$ \\
\hline $\mathrm{C} 49^{\prime}-\mathrm{C} 50^{\prime}-\mathrm{C} 51^{\prime}-\mathrm{C} 52^{\prime}$ & $2(2)$ \\
\hline $\mathrm{C} 50^{\prime}-\mathrm{C} 51^{\prime}-\mathrm{C} 52^{\prime}-\mathrm{C} 53^{\prime}$ & $1(2)$ \\
\hline $\mathrm{C} 51^{\prime}-\mathrm{C} 52^{\prime}-\mathrm{C} 53^{\prime}-\mathrm{C} 48^{\prime}$ & $-2(2)$ \\
\hline $\mathrm{C} 51^{\prime}-\mathrm{C} 52^{\prime}-\mathrm{C} 53^{\prime}-\mathrm{C} 54^{\prime}$ & $174.2(13)$ \\
\hline $\mathrm{C} 52^{\prime}-\mathrm{C} 53^{\prime}-\mathrm{C} 54^{\prime}-\mathrm{C} 40^{\prime}$ & $-174.7(14)$ \\
\hline $\mathrm{C} 52^{\prime}-\mathrm{C} 53^{\prime}-\mathrm{C} 54^{\prime}-\mathrm{C} 97^{\prime}$ & $-57.6(18)$ \\
\hline $\mathrm{C} 52^{\prime}-\mathrm{C} 53^{\prime}-\mathrm{C} 54^{\prime}-\mathrm{C} 203$ & $66.9(18)$ \\
\hline $\mathrm{C} 53^{\prime}-\mathrm{C} 48^{\prime}-\mathrm{C} 49^{\prime}-\mathrm{C} 50^{\prime}$ & $1.1(19)$ \\
\hline $\mathrm{C} 53^{\prime}-\mathrm{C} 54^{\prime}-\mathrm{C} 97^{\prime}-\mathrm{C} 98^{\prime}$ & $-49.1(15)$ \\
\hline $\mathrm{C} 53^{\prime}-\mathrm{C} 54^{\prime}-\mathrm{C} 203-\mathrm{C} 204$ & $67.8(19)$ \\
\hline $\mathrm{C} 53^{\prime}-\mathrm{C} 54^{\prime}-\mathrm{C} 203-\mathrm{C} 20 \mathrm{~B}$ & $48(3)$ \\
\hline $\mathrm{C} 54^{\prime}-\mathrm{C} 40^{\prime}-\mathrm{C} 41^{\prime}-\mathrm{C} 42^{\prime}$ & $174.8(12)$ \\
\hline $\mathrm{C} 54^{\prime}-\mathrm{C} 97^{\prime}-\mathrm{C} 98^{\prime}-\mathrm{C} 99^{\prime}$ & $-174.4(12)$ \\
\hline $\mathrm{C} 54^{\prime}-\mathrm{C} 203-\mathrm{C} 204-\mathrm{C} 205$ & $175(2)$ \\
\hline $\mathrm{C} 54^{\prime}-\mathrm{C} 203-\mathrm{C} 20 \mathrm{~B}-\mathrm{C} 20 \mathrm{C}$ & $103(4)$ \\
\hline $\mathrm{C} 55^{\prime}-\mathrm{Ir} 4-\mathrm{N} 4^{\prime}-\mathrm{C} 61^{\prime}$ & $11.0(8)$ \\
\hline $\mathrm{C} 55^{\prime}-\mathrm{Ir} 4-\mathrm{N} 4^{\prime}-\mathrm{C} 65^{\prime}$ & $-173.8(10)$ \\
\hline $\mathrm{C} 55^{\prime}-\mathrm{C} 56^{\prime}-\mathrm{C} 57^{\prime}-\mathrm{C} 58^{\prime}$ & $0.5(17)$ \\
\hline $\mathrm{C} 55^{\prime}-\mathrm{C} 56^{\prime}-\mathrm{C} 57^{\prime}-\mathrm{C} 66^{\prime}$ & $-179.1(11)$ \\
\hline $\mathrm{C} 55^{\prime}-\mathrm{C} 60^{\prime}-\mathrm{C} 61^{\prime}-\mathrm{N} 4^{\prime}$ & $-1.4(14)$ \\
\hline $\mathrm{C} 55^{\prime}-\mathrm{C} 60^{\prime}-\mathrm{C} 61^{\prime}-\mathrm{C} 62^{\prime}$ & $-179.8(11)$ \\
\hline $\mathrm{C} 56^{\prime}-\mathrm{C} 55^{\prime}-\mathrm{C} 60^{\prime}-\mathrm{C} 59^{\prime}$ & $3.3(17)$ \\
\hline $\mathrm{C} 56^{\prime}-\mathrm{C} 55^{\prime}-\mathrm{C} 60^{\prime}-\mathrm{C} 61^{\prime}$ & $-173.7(10)$ \\
\hline $\mathrm{C} 56^{\prime}-\mathrm{C} 57^{\prime}-\mathrm{C} 58^{\prime}-\mathrm{C} 59^{\prime}$ & $2.3(17)$ \\
\hline $\mathrm{C} 56^{\prime}-\mathrm{C} 57^{\prime}-\mathrm{C} 58^{\prime}-\mathrm{C} 72^{\prime}$ & $-175.7(10)$ \\
\hline $\mathrm{C} 56^{\prime}-\mathrm{C} 57^{\prime}-\mathrm{C} 66^{\prime}-\mathrm{C} 67^{\prime}$ & $-1(2)$ \\
\hline $\mathrm{C} 56^{\prime}-\mathrm{C} 57^{\prime}-\mathrm{C} 66^{\prime}-\mathrm{C} 71^{\prime}$ & $176.6(11)$ \\
\hline $\mathrm{C} 57^{\prime}-\mathrm{C} 58^{\prime}-\mathrm{C} 59^{\prime}-\mathrm{C} 60^{\prime}$ & $-2.2(16)$ \\
\hline $\mathrm{C} 57^{\prime}-\mathrm{C} 58^{\prime}-\mathrm{C} 72^{\prime}-\mathrm{C} 71^{\prime}$ & $-3.3(13)$ \\
\hline $\mathrm{C} 57^{\prime}-\mathrm{C} 58^{\prime}-\mathrm{C} 72^{\prime}-\mathrm{C} 209$ & $-121.1(12)$ \\
\hline
\end{tabular}




\begin{tabular}{|c|c|}
\hline $\mathrm{C} 57-\mathrm{C} 66-\mathrm{C} 71-\mathrm{C} 70$ & $180.0(10)$ \\
\hline $\mathrm{C} 57-\mathrm{C} 66-\mathrm{C} 71-\mathrm{C} 72$ & $-1.4(13)$ \\
\hline $\mathrm{C} 58-\mathrm{C} 57-\mathrm{C} 66-\mathrm{C} 67$ & $-176.8(12)$ \\
\hline $\mathrm{C} 58-\mathrm{C} 57-\mathrm{C} 66-\mathrm{C} 71$ & $2.3(13)$ \\
\hline $\mathrm{C} 58-\mathrm{C} 59-\mathrm{C} 60-\mathrm{C} 55$ & $-1.8(17)$ \\
\hline $\mathrm{C} 58-\mathrm{C} 59-\mathrm{C} 60-\mathrm{C} 61$ & $178.6(10)$ \\
\hline $\mathrm{C} 58-\mathrm{C} 72-\mathrm{C} 109-\mathrm{C} 110$ & $57.9(14)$ \\
\hline $\mathrm{C} 58-\mathrm{C} 72-\mathrm{C} 115-\mathrm{C} 116$ & $-59.2(14)$ \\
\hline $\mathrm{C} 59-\mathrm{C} 58-\mathrm{C} 72-\mathrm{C} 71$ & $-179.2(11)$ \\
\hline $\mathrm{C} 59-\mathrm{C} 58-\mathrm{C} 72-\mathrm{C} 109$ & $62.1(15)$ \\
\hline $\mathrm{C} 59-\mathrm{C} 58-\mathrm{C} 72-\mathrm{C} 115$ & $-58.3(16)$ \\
\hline $\mathrm{C} 59-\mathrm{C} 60-\mathrm{C} 61-\mathrm{N} 4$ & $-174.6(10)$ \\
\hline $\mathrm{C} 59-\mathrm{C} 60-\mathrm{C} 61-\mathrm{C} 62$ & $8.1(18)$ \\
\hline $\mathrm{C} 60-\mathrm{C} 55-\mathrm{C} 56-\mathrm{C} 57$ & $4.4(15)$ \\
\hline $\mathrm{C} 60-\mathrm{C} 61-\mathrm{C} 62-\mathrm{C} 63$ & $175.3(11)$ \\
\hline $\mathrm{C} 61-\mathrm{N} 4-\mathrm{C} 65-\mathrm{C} 64$ & $-2.4(17)$ \\
\hline C61-C62-C63-C64 & $1.5(18)$ \\
\hline $\mathrm{C} 62-\mathrm{C} 63-\mathrm{C} 64-\mathrm{O} 6$ & $-179.9(10)$ \\
\hline $\mathrm{C} 62-\mathrm{C} 63-\mathrm{C} 64-\mathrm{C} 65$ & $-1.7(19)$ \\
\hline $\mathrm{C} 63-\mathrm{C} 64-\mathrm{C} 65-\mathrm{N} 4$ & $2.3(18)$ \\
\hline $\mathrm{C} 65-\mathrm{N} 4-\mathrm{C} 61-\mathrm{C} 60$ & $-175.3(9)$ \\
\hline $\mathrm{C} 65-\mathrm{N} 4-\mathrm{C} 61-\mathrm{C} 62$ & $2.1(16)$ \\
\hline $\mathrm{C} 66-\mathrm{C} 57-\mathrm{C} 58-\mathrm{C} 59$ & $178.2(10)$ \\
\hline $\mathrm{C} 66-\mathrm{C} 57-\mathrm{C} 58-\mathrm{C} 72$ & $-2.4(13)$ \\
\hline $\mathrm{C} 66-\mathrm{C} 67-\mathrm{C} 68-\mathrm{C} 69$ & $1.4(19)$ \\
\hline $\mathrm{C} 66-\mathrm{C} 71-\mathrm{C} 72-\mathrm{C} 58$ & $0.0(12)$ \\
\hline $\mathrm{C} 66-\mathrm{C} 71-\mathrm{C} 72-\mathrm{C} 109$ & $116.9(11)$ \\
\hline $\mathrm{C} 66-\mathrm{C} 71-\mathrm{C} 72-\mathrm{C} 115$ & $-121.1(11)$ \\
\hline $\mathrm{C} 67-\mathrm{C} 66-\mathrm{C} 71-\mathrm{C} 70$ & $-0.8(18)$ \\
\hline $\mathrm{C} 67-\mathrm{C} 66-\mathrm{C} 71-\mathrm{C} 72$ & $177.8(10)$ \\
\hline $\mathrm{C} 67-\mathrm{C} 68-\mathrm{C} 69-\mathrm{C} 70$ & $-3(2)$ \\
\hline $\mathrm{C} 68-\mathrm{C} 69-\mathrm{C} 70-\mathrm{C} 71$ & $2(2)$ \\
\hline $\mathrm{C} 69-\mathrm{C} 70-\mathrm{C} 71-\mathrm{C} 66$ & $-0.5(19)$ \\
\hline $\mathrm{C} 69-\mathrm{C} 70-\mathrm{C} 71-\mathrm{C} 72$ & $-178.8(12)$ \\
\hline $\mathrm{C} 70-\mathrm{C} 71-\mathrm{C} 72-\mathrm{C} 58$ & $178.4(12)$ \\
\hline $\mathrm{C} 70-\mathrm{C} 71-\mathrm{C} 72-\mathrm{C} 109$ & $-64.7(16)$ \\
\hline $\mathrm{C} 70-\mathrm{C} 71-\mathrm{C} 72-\mathrm{C} 115$ & $57.3(17)$ \\
\hline $\mathrm{C} 71-\mathrm{C} 66-\mathrm{C} 67-\mathrm{C} 68$ & $0.3(18)$ \\
\hline $\mathrm{C} 71-\mathrm{C} 72-\mathrm{C} 109-\mathrm{C} 110$ & $-53.1(14)$ \\
\hline $\mathrm{C} 71-\mathrm{C} 72-\mathrm{C} 115-\mathrm{C} 116$ & $54.2(14)$ \\
\hline $\mathrm{C} 72-\mathrm{C} 58-\mathrm{C} 59-\mathrm{C} 60$ & $-175.3(10)$ \\
\hline $\mathrm{C} 72-\mathrm{C} 109-\mathrm{C} 110-\mathrm{C} 111$ & $-176.1(13)$ \\
\hline $\mathrm{C} 72-\mathrm{C} 115-\mathrm{C} 116-\mathrm{C} 117$ & $179.7(11)$ \\
\hline $\mathrm{C} 73-\mathrm{C} 18-\mathrm{C} 79-\mathrm{C} 80$ & $173.8(10)$ \\
\hline $\mathrm{C} 73-\mathrm{C} 74-\mathrm{C} 75-\mathrm{C} 76$ & $70(2)$ \\
\hline $\mathrm{C} 74-\mathrm{C} 75-\mathrm{C} 76-\mathrm{C} 77$ & $-167.4(15)$ \\
\hline $\mathrm{C} 75-\mathrm{C} 76-\mathrm{C} 77-\mathrm{C} 78$ & $-62(3)$ \\
\hline $\mathrm{C} 79-\mathrm{C} 18-\mathrm{C} 73-\mathrm{C} 74$ & $174.4(10)$ \\
\hline
\end{tabular}

\begin{tabular}{|c|c|}
\hline $\mathrm{C} 57^{\prime}-\mathrm{C} 58^{\prime}-\mathrm{C} 72^{\prime}-\mathrm{C} 215$ & $116.5(12)$ \\
\hline $\mathrm{C} 57^{\prime}-\mathrm{C} 66^{\prime}-\mathrm{C} 67^{\prime}-\mathrm{C} 68^{\prime}$ & $179.9(11)$ \\
\hline $\mathrm{C} 57^{\prime}-\mathrm{C} 66^{\prime}-\mathrm{C} 71^{\prime}-\mathrm{C} 70^{\prime}$ & $-179.0(11)$ \\
\hline $\mathrm{C} 57^{\prime}-\mathrm{C} 66^{\prime}-\mathrm{C} 71^{\prime}-\mathrm{C} 72^{\prime}$ & $1.0(14)$ \\
\hline $\mathrm{C} 58^{\prime}-\mathrm{C} 57^{\prime}-\mathrm{C} 66^{\prime}-\mathrm{C} 67^{\prime}$ & $178.9(12)$ \\
\hline $\mathrm{C} 58^{\prime}-\mathrm{C} 57^{\prime}-\mathrm{C} 66^{\prime}-\mathrm{C} 71^{\prime}$ & $-3.1(13)$ \\
\hline $\mathrm{C} 58^{\prime}-\mathrm{C} 59^{\prime}-\mathrm{C} 60^{\prime}-\mathrm{C} 55^{\prime}$ & $-0.6(17)$ \\
\hline $\mathrm{C} 58^{\prime}-\mathrm{C} 59^{\prime}-\mathrm{C} 60^{\prime}-\mathrm{C} 61^{\prime}$ & $176.2(10)$ \\
\hline $\mathrm{C} 58^{\prime}-\mathrm{C} 72^{\prime}-\mathrm{C} 209-\mathrm{C} 210$ & $53.8(16)$ \\
\hline $\mathrm{C} 58^{\prime}-\mathrm{C} 72^{\prime}-\mathrm{C} 215-\mathrm{C} 216$ & $-53.0(18)$ \\
\hline $\mathrm{C} 59^{\prime}-\mathrm{C} 58^{\prime}-\mathrm{C} 72^{\prime}-\mathrm{C} 71^{\prime}$ & $179.0(11)$ \\
\hline $\mathrm{C} 59^{\prime}-\mathrm{C} 58^{\prime}-\mathrm{C} 72^{\prime}-\mathrm{C} 209$ & $61.2(16)$ \\
\hline $\mathrm{C} 59^{\prime}-\mathrm{C} 58^{\prime}-\mathrm{C} 72^{\prime}-\mathrm{C} 215$ & $-61.2(17)$ \\
\hline $\mathrm{C} 59^{\prime}-\mathrm{C} 60^{\prime}-\mathrm{C} 61^{\prime}-\mathrm{N} 4^{\prime}$ & $-178.4(10)$ \\
\hline $\mathrm{C} 59^{\prime}-\mathrm{C} 60^{\prime}-\mathrm{C} 61^{\prime}-\mathrm{C} 62^{\prime}$ & $3.2(19)$ \\
\hline $\mathrm{C} 60^{\prime}-\mathrm{C} 55^{\prime}-\mathrm{C} 56^{\prime}-\mathrm{C} 57^{\prime}$ & $-3.2(16)$ \\
\hline $\mathrm{C} 60^{\prime}-\mathrm{C} 61^{\prime}-\mathrm{C} 62^{\prime}-\mathrm{C} 63^{\prime}$ & $-176.2(11)$ \\
\hline $\mathrm{C} 61^{\prime}-\mathrm{N} 4^{\prime}-\mathrm{C} 65^{\prime}-\mathrm{C} 64^{\prime}$ & $1.4(16)$ \\
\hline $\mathrm{C} 61^{\prime}-\mathrm{C} 62^{\prime}-\mathrm{C} 63^{\prime}-\mathrm{C} 64^{\prime}$ & $-0.2(18)$ \\
\hline $\mathrm{C} 62^{\prime}-\mathrm{C} 63^{\prime}-\mathrm{C} 64^{\prime}-\mathrm{O} 6^{\prime}$ & $-178.4(10)$ \\
\hline $\mathrm{C} 62^{\prime}-\mathrm{C} 63^{\prime}-\mathrm{C} 64^{\prime}-\mathrm{C} 65^{\prime}$ & $-3.9(19)$ \\
\hline $\mathrm{C} 63^{\prime}-\mathrm{C} 64^{\prime}-\mathrm{C} 65^{\prime}-\mathrm{N} 4^{\prime}$ & $3(2)$ \\
\hline $\mathrm{C} 65^{\prime}-\mathrm{N} 4^{\prime}-\mathrm{C} 61^{\prime}-\mathrm{C} 60^{\prime}$ & $175.8(9)$ \\
\hline $\mathrm{C} 65^{\prime}-\mathrm{N} 4^{\prime}-\mathrm{C} 61^{\prime}-\mathrm{C} 62^{\prime}$ & $-5.8(15)$ \\
\hline $\mathrm{C} 66^{\prime}-\mathrm{C} 57^{\prime}-\mathrm{C} 58^{\prime}-\mathrm{C} 59^{\prime}$ & $-178.0(10)$ \\
\hline $\mathrm{C} 66^{\prime}-\mathrm{C} 57^{\prime}-\mathrm{C} 58^{\prime}-\mathrm{C} 72^{\prime}$ & $4.0(13)$ \\
\hline $\mathrm{C} 66^{\prime}-\mathrm{C} 67^{\prime}-\mathrm{C} 68^{\prime}-\mathrm{C} 69^{\prime}$ & $-3.0(17)$ \\
\hline $\mathrm{C} 66^{\prime}-\mathrm{C} 71^{\prime}-\mathrm{C} 72^{\prime}-\mathrm{C} 58^{\prime}$ & $1.3(13)$ \\
\hline $\mathrm{C} 66^{\prime}-\mathrm{C} 71^{\prime}-\mathrm{C} 72^{\prime}-\mathrm{C} 209$ & $119.3(12)$ \\
\hline $\mathrm{C} 66^{\prime}-\mathrm{C} 71^{\prime}-\mathrm{C} 72^{\prime}-\mathrm{C} 215$ & $-118.0(12)$ \\
\hline $\mathrm{C} 67^{\prime}-\mathrm{C} 66^{\prime}-\mathrm{C} 71^{\prime}-\mathrm{C} 70^{\prime}$ & $-0.7(18)$ \\
\hline $\mathrm{C} 67^{\prime}-\mathrm{C} 66^{\prime}-\mathrm{C} 71^{\prime}-\mathrm{C} 72^{\prime}$ & $179.2(11)$ \\
\hline $\mathrm{C} 67^{\prime}-\mathrm{C} 68^{\prime}-\mathrm{C} 69^{\prime}-\mathrm{C} 70^{\prime}$ & $2.7(19)$ \\
\hline $\mathrm{C} 68^{\prime}-\mathrm{C} 69^{\prime}-\mathrm{C} 70^{\prime}-\mathrm{C} 71^{\prime}$ & $-1.4(19)$ \\
\hline $\mathrm{C} 69^{\prime}-\mathrm{C} 70^{\prime}-\mathrm{C} 71^{\prime}-\mathrm{C} 66^{\prime}$ & $0.4(19)$ \\
\hline $\mathrm{C} 69^{\prime}-\mathrm{C} 70^{\prime}-\mathrm{C} 71^{\prime}-\mathrm{C} 72^{\prime}$ & $-179.6(13)$ \\
\hline $\mathrm{C} 70^{\prime}-\mathrm{C} 71^{\prime}-\mathrm{C} 72^{\prime}-\mathrm{C} 58^{\prime}$ & $-178.7(12)$ \\
\hline $\mathrm{C} 70^{\prime}-\mathrm{C} 71^{\prime}-\mathrm{C} 72^{\prime}-\mathrm{C} 209$ & $-60.8(18)$ \\
\hline $\mathrm{C} 70^{\prime}-\mathrm{C} 71^{\prime}-\mathrm{C} 72^{\prime}-\mathrm{C} 215$ & $62.0(18)$ \\
\hline $\mathrm{C} 71^{\prime}-\mathrm{C} 66^{\prime}-\mathrm{C} 67^{\prime}-\mathrm{C} 68^{\prime}$ & $2.0(17)$ \\
\hline $\mathrm{C} 71^{\prime}-\mathrm{C} 72^{\prime}-\mathrm{C} 209-\mathrm{C} 210$ & $-58.5(16)$ \\
\hline $\mathrm{C} 71^{\prime}-\mathrm{C} 72^{\prime}-\mathrm{C} 215-\mathrm{C} 216$ & $60.5(18)$ \\
\hline $\mathrm{C} 72^{\prime}-\mathrm{C} 58^{\prime}-\mathrm{C} 59^{\prime}-\mathrm{C} 60^{\prime}$ & $175.4(11)$ \\
\hline $\mathrm{C} 72^{\prime}-\mathrm{C} 209-\mathrm{C} 210-\mathrm{C} 211$ & $-179.0(12)$ \\
\hline $\mathrm{C} 72^{\prime}-\mathrm{C} 215-\mathrm{C} 216-\mathrm{C} 217$ & $180(2)$ \\
\hline $\mathrm{C} 72^{\prime}-\mathrm{C} 215-\mathrm{C} 216-\mathrm{C} 21 \mathrm{E}$ & $145(4)$ \\
\hline $\mathrm{C} 73^{\prime}-\mathrm{C} 18^{\prime}-\mathrm{C} 79^{\prime}-\mathrm{C} 80^{\prime}$ & $168.3(15)$ \\
\hline $\mathrm{C} 73^{\prime}-\mathrm{C} 18^{\prime}-\mathrm{C} 79^{\prime}-\mathrm{C} 80 \mathrm{~B}$ & $-159(3)$ \\
\hline
\end{tabular}




\begin{tabular}{|c|c|}
\hline $\mathrm{C} 79-\mathrm{C} 80-\mathrm{C} 81-\mathrm{C} 82$ & $-179.8(11)$ \\
\hline $\mathrm{C} 80-\mathrm{C} 81-\mathrm{C} 82-\mathrm{C} 83$ & $170.5(13)$ \\
\hline $\mathrm{C} 81-\mathrm{C} 82-\mathrm{C} 83-\mathrm{C} 84$ & $-178.9(16)$ \\
\hline $\mathrm{C} 85-\mathrm{C} 36-\mathrm{C} 91-\mathrm{C} 92$ & $-178.0(9)$ \\
\hline $\mathrm{C} 85-\mathrm{C} 86-\mathrm{C} 87-\mathrm{C} 88$ & $172.2(10)$ \\
\hline $\mathrm{C} 86-\mathrm{C} 87-\mathrm{C} 88-\mathrm{C} 89$ & $-61.6(15)$ \\
\hline $\mathrm{C} 87-\mathrm{C} 88-\mathrm{C} 89-\mathrm{C} 90$ & $-174.0(12)$ \\
\hline $\mathrm{C} 91-\mathrm{C} 36-\mathrm{C} 85-\mathrm{C} 86$ & $168.9(10)$ \\
\hline C91-C92-C93-C94 & $179.0(9)$ \\
\hline $\mathrm{C} 92-\mathrm{C} 93-\mathrm{C} 94-\mathrm{C} 95$ & $174.0(10)$ \\
\hline $\mathrm{C} 93-\mathrm{C} 94-\mathrm{C} 95-\mathrm{C} 96$ & $-175.3(12)$ \\
\hline C97-C54-C103-C104 & $176.8(11)$ \\
\hline $\mathrm{C} 97-\mathrm{C} 98-\mathrm{C} 99-\mathrm{C} 100$ & $172.8(13)$ \\
\hline $\mathrm{C} 98-\mathrm{C} 99-\mathrm{C} 100-\mathrm{C} 101$ & $169(2)$ \\
\hline $\mathrm{C} 98-\mathrm{C} 99-\mathrm{C} 100-\mathrm{C} 126$ & $-179(3)$ \\
\hline $\mathrm{C} 99-\mathrm{C} 100-\mathrm{C} 101-\mathrm{C} 102$ & $172(2)$ \\
\hline $\mathrm{C} 99-\mathrm{C} 100-\mathrm{C} 126-\mathrm{C} 125$ & $-66(8)$ \\
\hline $\mathrm{C} 103-\mathrm{C} 54-\mathrm{C} 97-\mathrm{C} 98$ & $178.0(11)$ \\
\hline $\mathrm{C} 103-\mathrm{C} 104-\mathrm{C} 105-\mathrm{C} 106$ & $-177.3(17)$ \\
\hline $\mathrm{C} 103-\mathrm{C} 104-\mathrm{C} 10 \mathrm{~A}-\mathrm{C} 10 \mathrm{~B}$ & $179(3)$ \\
\hline $\mathrm{C} 104-\mathrm{C} 105-\mathrm{C} 106-\mathrm{C} 107$ & $175(2)$ \\
\hline $\mathrm{C} 104-\mathrm{C} 10 \mathrm{~A}-\mathrm{C} 10 \mathrm{~B}-\mathrm{C} 10 \mathrm{C}$ & $54(6)$ \\
\hline $\mathrm{C} 109-\mathrm{C} 72-\mathrm{C} 115-\mathrm{C} 116$ & $179.0(10)$ \\
\hline $\mathrm{C} 109-\mathrm{C} 110-\mathrm{C} 111-\mathrm{C} 112$ & $-167.1(17)$ \\
\hline $\mathrm{C} 110-\mathrm{C} 111-\mathrm{C} 112-\mathrm{C} 113$ & $-67(3)$ \\
\hline $\mathrm{C} 111-\mathrm{C} 112-\mathrm{C} 113-\mathrm{C} 114$ & $-64(3)$ \\
\hline $\mathrm{C} 115-\mathrm{C} 72-\mathrm{C} 109-\mathrm{C} 110$ & $-178.3(11)$ \\
\hline $\mathrm{C} 115-\mathrm{C} 116-\mathrm{C} 117-\mathrm{C} 118$ & $-173.6(10)$ \\
\hline $\mathrm{C} 116-\mathrm{C} 117-\mathrm{C} 118-\mathrm{C} 119$ & $177.9(11)$ \\
\hline $\mathrm{C} 117-\mathrm{C} 118-\mathrm{C} 119-\mathrm{C} 120$ & $-174.3(11)$ \\
\hline $\mathrm{C} 121-\mathrm{O} 3-\mathrm{C} 10-\mathrm{C} 9$ & $174.1(10)$ \\
\hline $\mathrm{C} 121-\mathrm{O} 3-\mathrm{C} 10-\mathrm{C} 11$ & $-6.4(15)$ \\
\hline $\mathrm{C} 122-\mathrm{O} 4-\mathrm{C} 28-\mathrm{C} 27$ & $170.0(11)$ \\
\hline $\mathrm{C} 122-\mathrm{O} 4-\mathrm{C} 28-\mathrm{C} 29$ & $-11.8(17)$ \\
\hline $\mathrm{C} 123-\mathrm{O} 5-\mathrm{C} 46-\mathrm{C} 45$ & $176.6(10)$ \\
\hline $\mathrm{C} 123-\mathrm{O} 5-\mathrm{C} 46-\mathrm{C} 47$ & $-6.2(15)$ \\
\hline $\mathrm{C} 124-\mathrm{O} 6-\mathrm{C} 64-\mathrm{C} 63$ & $171.6(11)$ \\
\hline $\mathrm{C} 124-\mathrm{O} 6-\mathrm{C} 64-\mathrm{C} 65$ & $-6.6(17)$ \\
\hline $\mathrm{C} 105-\mathrm{C} 106-\mathrm{C} 107-\mathrm{C} 108$ & $-176(2)$ \\
\hline $\mathrm{C} 10 \mathrm{~A}-\mathrm{C} 10 \mathrm{~B}-\mathrm{C} 10 \mathrm{C}-\mathrm{C} 10 \mathrm{D}$ & $-176(5)$ \\
\hline $\mathrm{Ir} 3-\mathrm{N} 1^{\prime}-\mathrm{C} 7^{\prime}-\mathrm{C} 6^{\prime}$ & $-3.8(14)$ \\
\hline $\mathrm{Ir} 3-\mathrm{N} 1^{\prime}-\mathrm{C} 7^{\prime}-\mathrm{C} 8^{\prime}$ & $179.1(10)$ \\
\hline $\mathrm{Ir} 3-\mathrm{N} 1^{\prime}-\mathrm{C} 11^{\prime}-\mathrm{C} 10^{\prime}$ & $-178.6(10)$ \\
\hline $\mathrm{Ir} 3-\mathrm{N} 22^{\prime}-\mathrm{C} 25^{\prime}-\mathrm{C} 24^{\prime}$ & $7.5(13)$ \\
\hline $\mathrm{Ir} 3-\mathrm{N} 2^{\prime}-\mathrm{C} 25^{\prime}-\mathrm{C} 26^{\prime}$ & $-171.3(10)$ \\
\hline $\mathrm{Ir} 3-\mathrm{N} 2^{\prime}-\mathrm{C} 29^{\prime}-\mathrm{C} 28^{\prime}$ & $167.3(11)$ \\
\hline $\mathrm{Ir} 3-\mathrm{C} 1^{\prime}-\mathrm{C} 2^{\prime}-\mathrm{C} 3^{\prime}$ & $177.1(9)$ \\
\hline $\mathrm{Ir} 3-\mathrm{C} 1^{\prime}-\mathrm{C} 6^{\prime}-\mathrm{C} 5^{\prime}$ & $-178.5(10)$ \\
\hline
\end{tabular}

\begin{tabular}{|c|c|}
\hline $\mathrm{C} 73^{\prime}-\mathrm{C} 74^{\prime}-\mathrm{C} 75^{\prime}-\mathrm{C} 76^{\prime}$ & $69(2)$ \\
\hline $\mathrm{C} 74^{\prime}-\mathrm{C} 75^{\prime}-\mathrm{C} 76^{\prime}-\mathrm{C} 77^{\prime}$ & $83(2)$ \\
\hline $\mathrm{C} 75^{\prime}-\mathrm{C} 76^{\prime}-\mathrm{C} 77^{\prime}-\mathrm{C} 78^{\prime}$ & $-174.6(14)$ \\
\hline $\mathrm{C} 79^{\prime}-\mathrm{C} 18^{\prime}-\mathrm{C} 73^{\prime}-\mathrm{C} 74^{\prime}$ & $-171.6(13)$ \\
\hline $\mathrm{C} 81^{\prime}-\mathrm{C} 82^{\prime}-\mathrm{C} 83^{\prime}-\mathrm{C} 84^{\prime}$ & $172(3)$ \\
\hline $\mathrm{C} 82^{\prime}-\mathrm{C} 81^{\prime}-\mathrm{C} 80^{\prime}-\mathrm{C} 79^{\prime}$ & $82(3)$ \\
\hline $\mathrm{C} 82^{\prime}-\mathrm{C} 81^{\prime}-\mathrm{C} 80 \mathrm{~B}-\mathrm{C} 79^{\prime}$ & $26(8)$ \\
\hline $\mathrm{C} 85^{\prime}-\mathrm{C} 36^{\prime}-\mathrm{C} 91^{\prime}-\mathrm{C} 92^{\prime}$ & $176.7(14)$ \\
\hline $\mathrm{C} 85^{\prime}-\mathrm{C} 86^{\prime}-\mathrm{C} 87^{\prime}-\mathrm{C} 88^{\prime}$ & $176.7(12)$ \\
\hline $\mathrm{C} 86^{\prime}-\mathrm{C} 87^{\prime}-\mathrm{C} 88^{\prime}-\mathrm{C} 89^{\prime}$ & $-170.1(13)$ \\
\hline $\mathrm{C} 87^{\prime}-\mathrm{C} 88^{\prime}-\mathrm{C} 89^{\prime}-\mathrm{C} 90^{\prime}$ & $173.9(14)$ \\
\hline $\mathrm{C} 91^{\prime}-\mathrm{C} 36^{\prime}-\mathrm{C} 85^{\prime}-\mathrm{C} 86^{\prime}$ & $179.3(12)$ \\
\hline C91'-C92'-C93'-C94' & $174(2)$ \\
\hline C92'-C93'-C94'-C95' & $-78(3)$ \\
\hline $\mathrm{C} 93^{\prime}-\mathrm{C} 94^{\prime}-\mathrm{C} 95^{\prime}-\mathrm{C} 96^{\prime}$ & $-165(2)$ \\
\hline $\mathrm{C} 97^{\prime}-\mathrm{C} 54^{\prime}-\mathrm{C} 203-\mathrm{C} 204$ & $-169.1(16)$ \\
\hline $\mathrm{C} 97^{\prime}-\mathrm{C} 54^{\prime}-\mathrm{C} 203-\mathrm{C} 20 \mathrm{~B}$ & $171(2)$ \\
\hline $\mathrm{C} 97^{\prime}-\mathrm{C} 98^{\prime}-\mathrm{C} 99^{\prime}-\mathrm{C} 200$ & $-174.6(14)$ \\
\hline $\mathrm{C} 98^{\prime}-\mathrm{C} 99^{\prime}-\mathrm{C} 200-\mathrm{C} 201$ & $79(2)$ \\
\hline $\mathrm{C} 99^{\prime}-\mathrm{C} 200-\mathrm{C} 201-\mathrm{C} 202$ & $64(2)$ \\
\hline $\mathrm{C} 203-\mathrm{C} 54^{\prime}-\mathrm{C} 97^{\prime}-\mathrm{C} 98^{\prime}$ & $-173.4(12)$ \\
\hline $\mathrm{C} 203-\mathrm{C} 204-\mathrm{C} 205-\mathrm{C} 206$ & $-173.3(19)$ \\
\hline $\mathrm{C} 203-\mathrm{C} 20 \mathrm{~B}-\mathrm{C} 20 \mathrm{C}-\mathrm{C} 206$ & $174(3)$ \\
\hline $\mathrm{C} 207-\mathrm{C} 206-\mathrm{C} 205-\mathrm{C} 204$ & $95(3)$ \\
\hline $\mathrm{C} 207-\mathrm{C} 206-\mathrm{C} 20 \mathrm{C}-\mathrm{C} 20 \mathrm{~B}$ & $171(4)$ \\
\hline $\mathrm{C} 209-\mathrm{C} 72^{\prime}-\mathrm{C} 215-\mathrm{C} 216$ & $-176.2(13)$ \\
\hline $\mathrm{C} 209-\mathrm{C} 210-\mathrm{C} 211-\mathrm{C} 21 \mathrm{~B}$ & $-164.6(17)$ \\
\hline $\mathrm{C} 209-\mathrm{C} 210-\mathrm{C} 211-\mathrm{C} 212$ & $164.3(19)$ \\
\hline $\mathrm{C} 210-\mathrm{C} 211-\mathrm{C} 21 \mathrm{~B}-\mathrm{C} 21 \mathrm{C}$ & $-72(4)$ \\
\hline $\mathrm{C} 210-\mathrm{C} 211-\mathrm{C} 212-\mathrm{C} 213$ & $-169(3)$ \\
\hline $\mathrm{C} 211-\mathrm{C} 21 \mathrm{~B}-\mathrm{C} 21 \mathrm{C}-\mathrm{C} 21 \mathrm{D}$ & $175(3)$ \\
\hline $\mathrm{C} 211-\mathrm{C} 212-\mathrm{C} 213-\mathrm{C} 214$ & $-168(3)$ \\
\hline $\mathrm{C} 215-\mathrm{C} 72^{\prime}-\mathrm{C} 209-\mathrm{C} 210$ & $177.4(12)$ \\
\hline $\mathrm{C} 215-\mathrm{C} 216-\mathrm{C} 217-\mathrm{C} 218$ & $-164(3)$ \\
\hline $\mathrm{C} 215-\mathrm{C} 216-\mathrm{C} 21 \mathrm{E}-\mathrm{C} 21 \mathrm{~F}$ & $-175(3)$ \\
\hline $\mathrm{C} 216-\mathrm{C} 217-\mathrm{C} 218-\mathrm{C} 219$ & $85(5)$ \\
\hline $\mathrm{C} 216-\mathrm{C} 21 \mathrm{E}-\mathrm{C} 21 \mathrm{~F}-\mathrm{C} 21 \mathrm{G}$ & $152(12)$ \\
\hline $\mathrm{C} 221-\mathrm{O} 3^{\prime}-\mathrm{C} 10^{\prime}-\mathrm{C} 9^{\prime}$ & $-175.9(13)$ \\
\hline $\mathrm{C} 221-\mathrm{O}^{\prime}-\mathrm{C} 10^{\prime}-\mathrm{C} 11^{\prime}$ & $8(2)$ \\
\hline $\mathrm{C} 222-\mathrm{O} 4^{\prime}-\mathrm{C} 28^{\prime}-\mathrm{C} 27^{\prime}$ & $-167.4(12)$ \\
\hline $\mathrm{C} 222-\mathrm{O} 4^{\prime}-\mathrm{C} 28^{\prime}-\mathrm{C} 29^{\prime}$ & $14(2)$ \\
\hline $\mathrm{C} 223-\mathrm{O} 5^{\prime}-\mathrm{C} 46^{\prime}-\mathrm{C} 45^{\prime}$ & $-178.3(14)$ \\
\hline $\mathrm{C} 223-\mathrm{O} 5^{\prime}-\mathrm{C} 46^{\prime}-\mathrm{C} 47^{\prime}$ & $4(2)$ \\
\hline $\mathrm{C} 224-\mathrm{O}^{\prime}-\mathrm{C} 64^{\prime}-\mathrm{C} 63^{\prime}$ & $-179.6(12)$ \\
\hline $\mathrm{C} 224-\mathrm{O} 6^{\prime}-\mathrm{C} 64^{\prime}-\mathrm{C} 65^{\prime}$ & $6.0(18)$ \\
\hline $\mathrm{C} 80^{\prime}-\mathrm{C} 81^{\prime}-\mathrm{C} 82^{\prime}-\mathrm{C} 83^{\prime}$ & $161(3)$ \\
\hline $\mathrm{C} 205-\mathrm{C} 206-\mathrm{C} 207-\mathrm{C} 208$ & $161(2)$ \\
\hline $\mathrm{C} 217-\mathrm{C} 218-\mathrm{C} 219-\mathrm{C} 220$ & $163(4)$ \\
\hline
\end{tabular}




\begin{tabular}{llll}
$\mathrm{Ir} 3-\mathrm{C} 1^{\prime}-\mathrm{C} 6^{\prime}-\mathrm{C} 7^{\prime}$ & $7.8(15)$ & $\mathrm{C} 20 \mathrm{C}-\mathrm{C} 206-\mathrm{C} 207-\mathrm{C} 208$ & $-170(3)$ \\
$\mathrm{Ir} 3-\mathrm{C} 19^{\prime}-\mathrm{C} 20^{\prime}-\mathrm{C} 21^{\prime}$ & $174.0(9)$ & $\mathrm{C} 21 \mathrm{E}-\mathrm{C} 21 \mathrm{~F}-\mathrm{C} 21 \mathrm{G}-\mathrm{C} 21 \mathrm{H}$ & $-146(12)$ \\
$\mathrm{Ir} 3-\mathrm{C} 19^{\prime}-\mathrm{C} 24^{\prime}-\mathrm{C} 23^{\prime}$ & $-176.7(9)$ & $\mathrm{C} 80 \mathrm{~B}-\mathrm{C} 81^{\prime}-\mathrm{C} 82^{\prime}-\mathrm{C} 83^{\prime}$ & $-176(5)$ \\
\hline
\end{tabular}

$\left\{\mu-N, N^{\prime}\right.$-Bis [3,5-bis(trifluoromethyl) phenyl] oxamidato\}bis(bis $\{2-[4-(2,4,6$-trimethylphenyl)pyridin-2-yl] phenyl$\kappa^{2} C^{1}, N^{\prime}$ \}iridium)-chlorobenzene-pentane (1/2.3/0.4) (IV)

Crystal data

$\left[\mathrm{Ir}_{2}\left(\mathrm{C}_{20} \mathrm{H}_{19} \mathrm{~N}\right)_{4}\left(\mathrm{C}_{18} \mathrm{H}_{6} \mathrm{~F}_{12} \mathrm{~N}_{2} \mathrm{O}_{2}\right)\right] \cdot 2.3 \mathrm{C}_{6} \mathrm{H}_{5} \mathrm{Cl} \cdot 0.4 \mathrm{C}_{5} \mathrm{H}_{12}$ $M_{r}=2271.78$

Triclinic, $P \overline{1}$

$a=11.8734(5) \AA$

$b=14.2267(6) \AA$

$c=16.6076(7) \AA$

$\alpha=110.386(2)^{\circ}$

$\beta=106.524(2)^{\circ}$

$\gamma=96.303(2)^{\circ}$

$V=2452.00(18) \AA^{3}$

\section{Data collection}

Bruker D8 Venture diffractometer

Radiation source: microfocus sealed X-ray tube, Incoatec $\mathrm{I} \mu \mathrm{S}$ microsource

Focusing mirrors monochromator

Detector resolution: 10.4 pixels $\mathrm{mm}^{-1}$ $\omega$-scan

Absorption correction: integration

(SADABS; Krause et al., 2015)

Refinement

Refinement on $F^{2}$

Least-squares matrix: full

$R\left[F^{2}>2 \sigma\left(F^{2}\right)\right]=0.028$

$w R\left(F^{2}\right)=0.073$

$S=1.06$

14405 reflections

702 parameters

593 restraints

Primary atom site location: heavy-atom method
$Z=1$

$F(000)=1136$

$D_{\mathrm{x}}=1.538 \mathrm{Mg} \mathrm{m}^{-3}$

Mo $K \alpha$ radiation, $\lambda=0.71073 \AA$

Cell parameters from 9713 reflections

$\theta=2.4-29.9^{\circ}$

$\mu=2.85 \mathrm{~mm}^{-1}$

$T=120 \mathrm{~K}$

Block, yellow

$0.12 \times 0.08 \times 0.04 \mathrm{~mm}$

$T_{\min }=0.770, T_{\max }=0.913$

54411 measured reflections

14405 independent reflections

12696 reflections with $I>2 \sigma(I)$

$R_{\text {int }}=0.032$

$\theta_{\max }=30.1^{\circ}, \theta_{\min }=2.1^{\circ}$

$h=-16 \rightarrow 16$

$k=-20 \rightarrow 20$

$l=-23 \rightarrow 23$

Secondary atom site location: difference Fourier map

Hydrogen site location: mixed

$\mathrm{H}$ atoms treated by a mixture of independent and constrained refinement

$w=1 /\left[\sigma^{2}\left(F_{0}^{2}\right)+(0.0372 P)^{2}+2.3279 P\right]$ where $P=\left(F_{\mathrm{o}}^{2}+2 F_{\mathrm{c}}^{2}\right) / 3$

$(\Delta / \sigma)_{\max }=0.003$

$\Delta \rho_{\max }=0.96$ e $\AA^{-3}$

$\Delta \rho_{\min }=-1.01$ e $\AA^{-3}$

\section{Special details}

Experimental. Data were collected in shutterless mode. Full sphere of reciprocal space was nominally covered by 4 runs of 340 narrow-frame $\omega$-scans (scan width $0.5^{\circ}, 30 \mathrm{~s}$ exposure), every run at a different $\varphi$ angle. Two runs of $358 \varphi$-scans (scan width $1^{\circ}, 3 \mathrm{~s}$ exposure) were used for scaling overflowing intensities. Crystal to detector distance $3.49 \mathrm{~cm}$.

Geometry. All esds (except the esd in the dihedral angle between two 1.s. planes) are estimated using the full covariance matrix. The cell esds are taken into account individually in the estimation of esds in distances, angles and torsion angles; correlations between esds in cell parameters are only used when they are defined by crystal symmetry. An approximate (isotropic) treatment of cell esds is used for estimating esds involving l.s. planes. 
Refinement. A strong $\left(4.2 \mathrm{e}^{-3}\right)$ peak of electron density near the Ir atom was interpreted as an alternative position of the same atom with the occupancy of 0.0183 (6), corresponding to a rotation of the whole molecule by ca. $12^{\circ}$. The alternative positions of light atoms cannot be resolved. The $\mathrm{C}(30) \mathrm{F}_{3}$ group is disordered by rotation and tilt between orientations $\mathrm{A}$ and $\mathrm{B}$ with occupancies $0.586(15)$ and $0.414(15)$, the $\mathrm{C}(31) \mathrm{F}_{3}$ group is disordered by rotation alone [with the $\mathrm{C}(31)$ ordered] between orientations $\mathrm{A}$ and $\mathrm{B}$ with occupancies 0.776 (5) and 0.224 (5), respectively. The opposite $\mathrm{F}$ atoms were refined with identical $\mathrm{ADP}$. The $\mathrm{PhCl}$ molecule $[\mathrm{C}(50)$ to $\mathrm{C}(55)]$ has the $\mathrm{Cl}$ atom disordered between positions $\mathrm{Cl}(1)$ and $\mathrm{Cl}(2)$ with equal occupancies, the former is sterically incompatible with its inversion equivalent. The void of $204 \AA^{3}$ around the inversion centre $(0,0,0)$ is shared by disordered $\mathrm{PhCl}$ and pentane molecules, with the occupancies tentatively estimated as 0.15 and 0.2 , respectively. Methyl group $\mathrm{C}(48) \mathrm{H}_{3}$ was refined as ideally disordered, other methyl groups as rigid bodies rotating around $\mathrm{C}-\mathrm{C}$ bonds, with a common refined $\mathrm{U}$ for three $\mathrm{H}$ atoms. Other $\mathrm{H}$ atoms: riding model.

Fractional atomic coordinates and isotropic or equivalent isotropic displacement parameters $\left(\AA^{2}\right)$

\begin{tabular}{|c|c|c|c|c|c|}
\hline & $x$ & $y$ & $z$ & $U_{\text {iso }} * / U_{\text {eq }}$ & Occ. $(<1)$ \\
\hline $\mathrm{O} 1$ & $0.44213(16)$ & $0.40519(14)$ & $0.53296(12)$ & $0.0202(4)$ & \\
\hline N1 & $0.37318(18)$ & $0.43068(16)$ & $0.39899(14)$ & $0.0168(4)$ & \\
\hline N2 & $0.5291(2)$ & 0.54309 (18) & $0.74258(15)$ & $0.0199(4)$ & \\
\hline N3 & $0.6642(2)$ & $0.35031(17)$ & $0.56890(15)$ & $0.0200(4)$ & \\
\hline $\mathrm{C} 1$ & $0.4488(2)$ & $0.45259(18)$ & $0.48149(16)$ & $0.0164(4)$ & \\
\hline $\mathrm{C} 2$ & $0.7416(2)$ & $0.5027(2)$ & $0.77180(17)$ & $0.0195(5)$ & \\
\hline $\mathrm{C} 3$ & $0.8524(2)$ & $0.4756(2)$ & $0.7863(2)$ & $0.0256(6)$ & \\
\hline $\mathrm{H} 3$ & 0.865589 & 0.424637 & 0.737133 & $0.031^{*}$ & \\
\hline $\mathrm{C} 4$ & $0.9441(3)$ & $0.5214(3)$ & $0.8713(2)$ & $0.0315(6)$ & \\
\hline $\mathrm{H} 4$ & 1.019180 & 0.501879 & 0.879077 & $0.038^{*}$ & \\
\hline $\mathrm{C} 5$ & $0.9276(3)$ & $0.5951(3)$ & $0.9445(2)$ & $0.0354(7)$ & \\
\hline H5 & 0.991573 & 0.627520 & 1.001858 & $0.042 *$ & \\
\hline C6 & $0.8165(3)$ & $0.6212(2)$ & $0.9335(2)$ & $0.0302(6)$ & \\
\hline H6 & 0.803662 & 0.670946 & 0.983629 & $0.036^{*}$ & \\
\hline $\mathrm{C} 7$ & $0.7238(2)$ & $0.5744(2)$ & $0.84862(18)$ & $0.0219(5)$ & \\
\hline $\mathrm{C} 8$ & $0.6009(2)$ & $0.5903(2)$ & $0.83196(18)$ & $0.0210(5)$ & \\
\hline $\mathrm{C} 9$ & $0.5518(3)$ & $0.6384(2)$ & 0.89820 (19) & $0.0248(5)$ & \\
\hline H9 & 0.602846 & 0.672393 & 0.960529 & $0.030 *$ & \\
\hline $\mathrm{C} 10$ & 0.4295 & $0.6375(2)$ & 0.87446 (19) & $0.0242(5)$ & \\
\hline $\mathrm{C} 11$ & 0.3605 & $0.5938(2)$ & $0.7816(2)$ & $0.0287(6)$ & \\
\hline H11 & 0.277713 & 0.595695 & 0.762022 & $0.034^{*}$ & \\
\hline $\mathrm{C} 12$ & $0.4124(3)$ & $0.5480(2)$ & $0.71836(19)$ & $0.0264(6)$ & \\
\hline H12 & 0.364084 & 0.518702 & 0.655240 & $0.032 *$ & \\
\hline $\mathrm{C} 13$ & $0.5551(2)$ & $0.3276(2)$ & $0.67928(18)$ & $0.0202(5)$ & \\
\hline $\mathrm{C} 14$ & $0.5066(3)$ & $0.3197(2)$ & $0.74506(19)$ & $0.0254(5)$ & \\
\hline H14 & 0.496839 & 0.379959 & 0.788143 & $0.030 *$ & \\
\hline $\mathrm{C} 15$ & $0.4726(3)$ & $0.2245(2)$ & $0.7479(2)$ & $0.0303(6)$ & \\
\hline H15 & 0.439964 & 0.220699 & 0.793019 & $0.036^{*}$ & \\
\hline $\mathrm{C} 16$ & $0.4855(3)$ & $0.1355(2)$ & $0.6862(2)$ & $0.0343(7)$ & \\
\hline H16 & 0.460195 & 0.070799 & 0.688127 & $0.041^{*}$ & \\
\hline $\mathrm{C} 17$ & 0.5353 & $0.1407(2)$ & $0.6217(2)$ & $0.0306(6)$ & \\
\hline H17 & 0.545247 & 0.079808 & 0.579427 & $0.037^{*}$ & \\
\hline C18 & $0.5709(2)$ & $0.2363(2)$ & $0.61902(18)$ & $0.0228(5)$ & \\
\hline C19 & $0.6296(2)$ & $0.2496(2)$ & $0.55617(18)$ & $0.0220(5)$ & \\
\hline
\end{tabular}




\begin{tabular}{|c|c|c|c|c|}
\hline $\mathrm{C} 20$ & $0.6518(3)$ & $0.1718(2)$ & $0.48812(19)$ & $0.0264(6)$ \\
\hline $\mathrm{H} 20$ & 0.627753 & 0.102136 & 0.479835 & $0.032 *$ \\
\hline $\mathrm{C} 21$ & $0.7082(3)$ & $0.1937(2)$ & $0.43204(19)$ & $0.0258(5)$ \\
\hline $\mathrm{C} 22$ & $0.7454(3)$ & $0.2967(2)$ & $0.4480(2)$ & $0.0266(6)$ \\
\hline $\mathrm{H} 22$ & 0.786309 & 0.315052 & 0.412247 & $0.032^{*}$ \\
\hline $\mathrm{C} 23$ & $0.7224(2)$ & $0.3720(2)$ & 0.51617 (19) & $0.0234(5)$ \\
\hline $\mathrm{H} 23$ & 0.748595 & 0.442040 & 0.526522 & $0.028^{*}$ \\
\hline $\mathrm{C} 24$ & $0.2774(2)$ & $0.3402(2)$ & $0.36003(17)$ & $0.0190(5)$ \\
\hline $\mathrm{C} 25$ & $0.2970(3)$ & $0.2468(2)$ & $0.3648(2)$ & $0.0259(6)$ \\
\hline $\mathrm{H} 25$ & 0.376434 & 0.241224 & 0.392612 & $0.031^{*}$ \\
\hline $\mathrm{C} 26$ & $0.2006(3)$ & $0.1623(2)$ & $0.3288(2)$ & $0.0318(6)$ \\
\hline $\mathrm{C} 27$ & $0.0840(3)$ & $0.1684(2)$ & $0.2881(2)$ & $0.0334(7)$ \\
\hline $\mathrm{H} 27$ & 0.018131 & 0.110643 & 0.264945 & $0.040^{*}$ \\
\hline $\mathrm{C} 28$ & $0.0652(3)$ & $0.2602(2)$ & $0.2817(2)$ & $0.0289(6)$ \\
\hline $\mathrm{C} 29$ & $0.1609(2)$ & $0.3458(2)$ & $0.31702(18)$ & $0.0225(5)$ \\
\hline $\mathrm{H} 29$ & 0.146642 & 0.408140 & 0.311688 & $0.027^{*}$ \\
\hline $\mathrm{C} 31$ & $-0.0597(3)$ & $0.2692(3)$ & $0.2380(2)$ & $0.0409(8)$ \\
\hline $\mathrm{C} 32$ & $0.3731(2)$ & $0.6731(2)$ & $0.94620(19)$ & $0.0254(5)$ \\
\hline $\mathrm{C} 33$ & $0.3216(3)$ & $0.6002(2)$ & $0.9721(2)$ & $0.0287(6)$ \\
\hline $\mathrm{C} 34$ & $0.2694(3)$ & $0.6321(3)$ & $1.0394(2)$ & $0.0323(6)$ \\
\hline H34 & 0.235092 & 0.582728 & 1.057505 & $0.039^{*}$ \\
\hline $\mathrm{C} 35$ & $0.2665(3)$ & $0.7346(3)$ & $1.0806(2)$ & $0.0329(7)$ \\
\hline $\mathrm{C} 36$ & $0.3169(3)$ & $0.8052(3)$ & $1.0535(2)$ & $0.0374(7)$ \\
\hline H36 & 0.315310 & 0.875588 & 1.081340 & $0.045^{*}$ \\
\hline $\mathrm{C} 37$ & $0.3703(3)$ & $0.7760(2)$ & $0.9860(2)$ & $0.0330(6)$ \\
\hline $\mathrm{C} 38$ & $0.3230(4)$ & $0.4870(3)$ & $0.9286(3)$ & $0.0453(9)$ \\
\hline H38A & 0.278057 & 0.459579 & 0.862655 & $0.086(10)^{*}$ \\
\hline H38B & 0.285261 & 0.448142 & 0.956388 & $0.086(10)^{*}$ \\
\hline $\mathrm{H} 38 \mathrm{C}$ & 0.406693 & 0.480281 & 0.938809 & $0.086(10)^{*}$ \\
\hline C39 & 0.2095 (3) & $0.7662(3)$ & $1.1531(2)$ & $0.0450(9)$ \\
\hline H39A & 0.270928 & 0.813855 & 1.211392 & $0.097(11)^{*}$ \\
\hline H39B & 0.175236 & 0.704936 & 1.160351 & $0.097(11)^{*}$ \\
\hline $\mathrm{H} 39 \mathrm{C}$ & 0.145249 & 0.800287 & 1.135032 & $0.097(11)^{*}$ \\
\hline $\mathrm{C} 40$ & 0.4177 (4) & $0.8541(3)$ & $0.9548(3)$ & $0.0466(9)$ \\
\hline $\mathrm{H} 40 \mathrm{~A}$ & 0.502842 & 0.855475 & 0.961969 & $0.077(9)^{*}$ \\
\hline $\mathrm{H} 40 \mathrm{~B}$ & 0.410483 & 0.922340 & 0.991740 & $0.077(9)^{*}$ \\
\hline $\mathrm{H} 40 \mathrm{C}$ & 0.370914 & 0.835528 & 0.890202 & $0.077(9)^{*}$ \\
\hline $\mathrm{C} 41$ & $0.7266(3)$ & $0.1100(2)$ & $0.3561(2)$ & $0.0306(6)$ \\
\hline $\mathrm{C} 42$ & 0.6447 (4) & $0.0778(3)$ & $0.2669(2)$ & $0.0397(8)$ \\
\hline $\mathrm{C} 43$ & 0.6617 (4) & $-0.0006(3)$ & 0.1963 (3) & $0.0513(10)$ \\
\hline $\mathrm{H} 43$ & 0.605787 & -0.023665 & 0.135712 & $0.062 *$ \\
\hline $\mathrm{C} 44$ & $0.7583(5)$ & -0.0455 & $0.2126(3)$ & $0.0544(11)$ \\
\hline $\mathrm{C} 45$ & $0.8376(4)$ & $-0.0127(3)$ & $0.3002(3)$ & $0.0504(9)$ \\
\hline $\mathrm{H} 45$ & 0.904509 & -0.043287 & 0.311232 & $0.060^{*}$ \\
\hline $\mathrm{C} 46$ & $0.8233(3)$ & 0.0649 (3) & $0.3743(3)$ & $0.0397(8)$ \\
\hline $\mathrm{C} 47$ & $0.5372(4)$ & 0.1248 (4) & $0.2468(3)$ & $0.0555(10)$ \\
\hline H47A & 0.491260 & 0.120515 & 0.286420 & $0.086(10)^{*}$ \\
\hline H47B & 0.485398 & 0.087211 & 0.182531 & $0.086(10)^{*}$ \\
\hline
\end{tabular}




\begin{tabular}{|c|c|c|c|c|c|}
\hline $\mathrm{H} 47 \mathrm{C}$ & 0.565461 & 0.197287 & 0.258418 & $0.086(10)^{*}$ & \\
\hline $\mathrm{C} 48$ & $0.7757(6)$ & $-0.1311(4)$ & $0.1348(4)$ & 0.0853 (19) & \\
\hline $\mathrm{H} 48 \mathrm{~A}$ & 0.711099 & -0.144750 & 0.077264 & $0.102 *$ & $0.49(6)$ \\
\hline H48B & 0.854058 & -0.110100 & 0.129994 & $0.102 *$ & $0.49(6)$ \\
\hline $\mathrm{H} 48 \mathrm{C}$ & 0.773044 & -0.193831 & 0.146991 & $0.102 *$ & $0.49(6)$ \\
\hline H48D & 0.847702 & -0.154371 & 0.158902 & $0.102 *$ & $0.51(6)$ \\
\hline $\mathrm{H} 48 \mathrm{E}$ & 0.704742 & -0.189020 & 0.106172 & $0.102 *$ & $0.51(6)$ \\
\hline $\mathrm{H} 48 \mathrm{~F}$ & 0.785757 & -0.105290 & 0.089175 & $0.102 *$ & $0.51(6)$ \\
\hline C49 & $0.9111(4)$ & $0.0981(3)$ & $0.4691(3)$ & $0.0586(11)$ & \\
\hline $\mathrm{H} 49 \mathrm{~A}$ & 0.939459 & 0.173347 & 0.496631 & $0.088(10)^{*}$ & \\
\hline H49B & 0.980080 & 0.066492 & 0.466851 & $0.088(10)^{*}$ & \\
\hline $\mathrm{H} 49 \mathrm{C}$ & 0.871471 & 0.076294 & 0.506083 & $0.088(10)^{*}$ & \\
\hline Ir1 & $0.60082(2)$ & $0.45280(2)$ & $0.65562(2)$ & $0.01591(4)$ & $0.9817(6)$ \\
\hline F1A & 0.3215 (4) & 0.0447 (4) & $0.3300(6)$ & $0.110(3)$ & $0.774(5)$ \\
\hline $\mathrm{F} 2 \mathrm{~A}$ & $0.2218(10)$ & $0.0665(4)$ & $0.4187(4)$ & $0.133(3)$ & $0.774(5)$ \\
\hline F3A & 0.1348 & $-0.0165(2)$ & $0.2794(4)$ & $0.0836(15)$ & $0.774(5)$ \\
\hline F4A & $-0.0993(8)$ & $0.3252(12)$ & $0.3001(5)$ & $0.132(6)$ & $0.586(15)$ \\
\hline F5A & $-0.0690(5)$ & $0.3071(8)$ & $0.1789(5)$ & $0.071(3)$ & $0.586(15)$ \\
\hline F6A & $-0.1381(6)$ & $0.1809(6)$ & $0.1988(9)$ & $0.124(5)$ & $0.586(15)$ \\
\hline C30A & $0.2207(5)$ & $0.0654(5)$ & $0.3405(4)$ & $0.0432(10)^{*}$ & $0.774(5)$ \\
\hline F1B & 0.1533 (17) & $0.0148(18)$ & $0.350(3)$ & $0.110(3)$ & $0.226(5)$ \\
\hline F2B & $0.275(3)$ & $0.0131(14)$ & $0.2641(13)$ & $0.133(3)$ & $0.226(5)$ \\
\hline F3B & $0.3179(15)$ & $0.0763(9)$ & $0.4024(15)$ & $0.0836(15)$ & $0.226(5)$ \\
\hline F4B & $-0.1086(10)$ & $0.2027(15)$ & $0.1528(5)$ & $0.141(9)$ & $0.414(15)$ \\
\hline F5B & $-0.1364(7)$ & $0.2479(9)$ & $0.2736(8)$ & $0.065(3)$ & $0.414(15)$ \\
\hline F6B & $-0.0671(7)$ & $0.3626(8)$ & $0.2420(15)$ & $0.106(7)$ & $0.414(15)$ \\
\hline C30B & $0.2306(15)$ & $0.0613(16)$ & $0.3244(12)$ & $0.0432(10)^{*}$ & $0.226(5)$ \\
\hline $\mathrm{C} 50$ & $0.0992(4)$ & $0.3843(4)$ & $0.5805(3)$ & $0.0616(11)$ & \\
\hline H50 & 0.072881 & 0.428370 & 0.550858 & $0.074^{*}$ & \\
\hline C51 & $0.0560(4)$ & $0.3792(4)$ & $0.6477(4)$ & $0.0669(13)$ & \\
\hline H51 & 0.001526 & 0.419267 & 0.665464 & $0.080^{*}$ & \\
\hline C52 & $0.0945(4)$ & $0.3146(5)$ & $0.6878(4)$ & $0.0747(15)$ & \\
\hline H52 & 0.063664 & 0.306635 & 0.732506 & $0.090^{*}$ & \\
\hline C53 & $0.1778(4)$ & $0.2602(4)$ & $0.6645(4)$ & $0.0711(13)$ & \\
\hline H53 & 0.206506 & 0.217124 & 0.694538 & $0.085^{*}$ & \\
\hline C54 & $0.2183(4)$ & $0.2691(4)$ & $0.5981(3)$ & 0.0677 (13) & \\
\hline H54 & 0.276172 & 0.232433 & 0.581874 & $0.081^{*}$ & \\
\hline C55 & $0.1770(4)$ & $0.3294(4)$ & $0.5552(3)$ & $0.0642(12)$ & \\
\hline H55 & 0.202888 & 0.333102 & 0.507062 & $0.077^{*}$ & \\
\hline $\mathrm{Cl} 2$ & 0.0335 & $0.2747(2)$ & $0.7559(2)$ & $0.0821(8)$ & 0.5 \\
\hline $\mathrm{Cl1}$ & 0.0600 & $0.4641(2)$ & $0.5229(2)$ & $0.0797(8)$ & 0.5 \\
\hline $\mathrm{Cl3}$ & $0.2563(8)$ & $0.0961(7)$ & $0.0951(6)$ & $0.066(2)$ & 0.15 \\
\hline C56 & $0.1129(12)$ & $0.0482(11)$ & $0.0450(12)$ & $0.055(4) *$ & 0.3 \\
\hline C57 & $0.0353(15)$ & 0.1095 (13) & $0.0364(13)$ & $0.066(5)^{*}$ & 0.3 \\
\hline H57 & 0.059102 & 0.182588 & 0.060265 & $0.079 *$ & 0.3 \\
\hline C58 & $0.0752(13)$ & $-0.0569(11)$ & $0.0089(10)$ & $0.058(4)^{*}$ & 0.3 \\
\hline H58 & 0.136555 & -0.093519 & 0.018609 & $0.069^{*}$ & 0.3 \\
\hline C59 & $0.2059(15)$ & $0.1337(12)$ & $0.0963(8)$ & $0.074(3)$ & 0.4 \\
\hline
\end{tabular}




\begin{tabular}{llllll} 
C60 & $0.0793(19)$ & $0.1029(15)$ & $0.0474(17)$ & $0.060(6)^{*}$ & 0.2 \\
C61 & 0.000000 & 0.000000 & 0.000000 & $0.074(3)$ & 0.4 \\
C62 & $-0.1331(15)$ & $-0.025(2)$ & $-0.0543(16)$ & $0.051(6)^{*}$ & 0.2 \\
Ir2 & $0.5679(7)$ & $0.4090(8)$ & $0.6317(5)$ & $0.01591(4)$ & $0.0183(6)$ \\
\hline
\end{tabular}

Atomic displacement parameters $\left(\AA^{2}\right)$

\begin{tabular}{|c|c|c|c|c|c|c|}
\hline & $U^{11}$ & $U^{22}$ & $U^{33}$ & $U^{12}$ & $U^{13}$ & $U^{23}$ \\
\hline $\mathrm{O} 1$ & $0.0200(9)$ & $0.0217(9)$ & $0.0191(8)$ & $0.0003(7)$ & $0.0056(7)$ & $0.0107(7)$ \\
\hline N1 & $0.0156(9)$ & $0.0173(10)$ & $0.0161(9)$ & $0.0023(8)$ & $0.0043(8)$ & $0.0063(8)$ \\
\hline N2 & $0.0190(10)$ & $0.0264(11)$ & $0.0179(10)$ & $0.0065(9)$ & $0.0070(8)$ & $0.0120(9)$ \\
\hline N3 & $0.0201(10)$ & $0.0223(11)$ & $0.0198(10)$ & $0.0053(8)$ & $0.0078(8)$ & $0.0101(9)$ \\
\hline $\mathrm{C} 1$ & $0.0164(11)$ & $0.0162(11)$ & $0.0171(11)$ & $0.0048(9)$ & $0.0072(9)$ & $0.0058(9)$ \\
\hline $\mathrm{C} 2$ & $0.0172(11)$ & $0.0235(12)$ & $0.0205(12)$ & $0.0023(9)$ & $0.0061(9)$ & $0.0131(10)$ \\
\hline $\mathrm{C} 3$ & $0.0222(13)$ & $0.0329(15)$ & $0.0276(13)$ & $0.0091(11)$ & $0.0109(11)$ & $0.0162(12)$ \\
\hline $\mathrm{C} 4$ & $0.0186(13)$ & $0.0460(18)$ & $0.0323(15)$ & $0.0092(12)$ & $0.0063(11)$ & $0.0197(14)$ \\
\hline $\mathrm{C} 5$ & $0.0212(13)$ & 0.0478 (19) & $0.0266(14)$ & $0.0013(13)$ & $0.0002(11)$ & 0.0113 (14) \\
\hline C6 & $0.0247(13)$ & $0.0363(16)$ & $0.0233(13)$ & $0.0035(12)$ & $0.0058(11)$ & $0.0078(12)$ \\
\hline $\mathrm{C} 7$ & $0.0194(12)$ & $0.0256(13)$ & $0.0207(12)$ & $0.0033(10)$ & $0.0064(10)$ & $0.0101(10)$ \\
\hline $\mathrm{C} 8$ & $0.0209(12)$ & $0.0232(13)$ & $0.0197(12)$ & $0.0044(10)$ & $0.0064(9)$ & $0.0100(10)$ \\
\hline C9 & $0.0237(13)$ & $0.0272(14)$ & $0.0204(12)$ & $0.0053(11)$ & $0.0068(10)$ & $0.0067(11)$ \\
\hline $\mathrm{C} 10$ & $0.0252(13)$ & $0.0266(14)$ & $0.0243(13)$ & $0.0073(11)$ & $0.0112(10)$ & $0.0116(11)$ \\
\hline C11 & $0.0229(13)$ & 0.0407 (17) & $0.0253(13)$ & $0.0138(12)$ & $0.0088(11)$ & $0.0140(12)$ \\
\hline $\mathrm{C} 12$ & $0.0223(13)$ & $0.0365(16)$ & $0.0207(12)$ & $0.0102(11)$ & $0.0052(10)$ & $0.0124(12)$ \\
\hline $\mathrm{C} 13$ & $0.0193(12)$ & $0.0233(12)$ & $0.0200(12)$ & $0.0028(10)$ & $0.0059(9)$ & $0.0124(10)$ \\
\hline $\mathrm{C} 14$ & $0.0244(13)$ & $0.0321(14)$ & $0.0222(13)$ & 0.0057 (11) & $0.0091(10)$ & $0.0132(11)$ \\
\hline $\mathrm{C} 15$ & $0.0307(15)$ & $0.0392(16)$ & $0.0287(14)$ & $0.0044(13)$ & $0.0144(12)$ & 0.0203 \\
\hline $\mathrm{C} 16$ & $0.0418(18)$ & $0.0297(15)$ & 0.0337 (16) & $-0.0015(13)$ & $0.0138(14)$ & $0.0175(13)$ \\
\hline $\mathrm{C} 17$ & $0.0385(16)$ & $0.0235(14)$ & $0.0290(15)$ & $0.0002(12)$ & $0.0126(13)$ & $0.0108(12)$ \\
\hline C18 & $0.0236(13)$ & $0.0240(13)$ & $0.0193(12)$ & $0.0004(10)$ & $0.0060(10)$ & $0.0097(10)$ \\
\hline C19 & $0.0222(12)$ & $0.0232(12)$ & $0.0211(12)$ & $0.0025(10)$ & $0.0063(10)$ & $0.0110(10)$ \\
\hline $\mathrm{C} 20$ & $0.0333(15)$ & $0.0204(13)$ & $0.0260(13)$ & $0.0022(11)$ & $0.0130(12)$ & 0.0087 (11) \\
\hline $\mathrm{C} 21$ & $0.0289(14)$ & $0.0240(13)$ & $0.0236(13)$ & $0.0029(11)$ & $0.0113(11)$ & $0.0077(11)$ \\
\hline $\mathrm{C} 22$ & $0.0308(14)$ & $0.0265(14)$ & $0.0274(14)$ & $0.0054(11)$ & $0.0167(12)$ & 0.0117 (11) \\
\hline $\mathrm{C} 23$ & $0.0249(13)$ & $0.0230(13)$ & $0.0271(13)$ & $0.0048(10)$ & $0.0141(11)$ & $0.0118(11)$ \\
\hline $\mathrm{C} 24$ & $0.0188(11)$ & $0.0198(12)$ & $0.0177(11)$ & $0.0031(9)$ & $0.0073(9)$ & $0.0063(9)$ \\
\hline $\mathrm{C} 25$ & $0.0247(13)$ & $0.0205(13)$ & $0.0310(14)$ & $0.0056(10)$ & $0.0097(11)$ & $0.0083(11)$ \\
\hline $\mathrm{C} 26$ & $0.0333(15)$ & $0.0209(13)$ & $0.0390(17)$ & $0.0038(12)$ & 0.0135 (13) & $0.0094(12)$ \\
\hline $\mathrm{C} 27$ & $0.0280(14)$ & $0.0290(15)$ & $0.0347(16)$ & $-0.0065(12)$ & $0.0090(12)$ & $0.0084(13)$ \\
\hline $\mathrm{C} 28$ & $0.0196(12)$ & $0.0358(16)$ & $0.0274(14)$ & $-0.0016(11)$ & $0.0047(11)$ & $0.0133(12)$ \\
\hline $\mathrm{C} 29$ & $0.0191(12)$ & $0.0269(13)$ & $0.0225(12)$ & $0.0037(10)$ & $0.0070(10)$ & $0.0113(11)$ \\
\hline $\mathrm{C} 31$ & $0.0213(14)$ & $0.054(2)$ & 0.0461 (19) & $-0.0018(14)$ & $0.0052(13)$ & $0.0271(17)$ \\
\hline $\mathrm{C} 32$ & $0.0229(13)$ & $0.0338(15)$ & $0.0215(12)$ & $0.0110(11)$ & $0.0092(10)$ & $0.0111(11)$ \\
\hline $\mathrm{C} 33$ & $0.0281(14)$ & $0.0329(15)$ & $0.0258(14)$ & $0.0088(12)$ & $0.0113(11)$ & $0.0105(12)$ \\
\hline $\mathrm{C} 34$ & $0.0287(15)$ & $0.0444(18)$ & $0.0258(14)$ & 0.0063 (13) & $0.0117(12)$ & $0.0151(13)$ \\
\hline $\mathrm{C} 35$ & $0.0257(14)$ & 0.0445 (18) & $0.0235(14)$ & $0.0084(13)$ & $0.0085(11)$ & 0.0078 (13) \\
\hline $\mathrm{C} 36$ & $0.0415(18)$ & $0.0336(17)$ & $0.0357(17)$ & 0.0125 (14) & $0.0184(14)$ & 0.0069 (14) \\
\hline $\mathrm{C} 37$ & $0.0368(16)$ & $0.0317(15)$ & $0.0332(16)$ & 0.0104 (13) & $0.0156(13)$ & $0.0126(13)$ \\
\hline
\end{tabular}




\begin{tabular}{|c|c|c|c|c|c|c|}
\hline C38 & $0.059(2)$ & $0.0353(18)$ & $0.053(2)$ & $0.0135(17)$ & 0.0338 (19) & $0.0187(16)$ \\
\hline C39 & $0.043(2)$ & $0.055(2)$ & $0.0333(17)$ & 0.0127 (17) & $0.0191(15)$ & $0.0089(16)$ \\
\hline $\mathrm{C} 40$ & $0.060(2)$ & $0.0376(19)$ & $0.057(2)$ & $0.0166(17)$ & $0.034(2)$ & $0.0235(17)$ \\
\hline $\mathrm{C} 41$ & $0.0418(17)$ & 0.0193 & $0.0308(14)$ & $-0.0011(12)$ & $0.0214(13)$ & $0.0054(11)$ \\
\hline $\mathrm{C} 42$ & $0.058(2)$ & $0.0305(16)$ & $0.0300(15)$ & $-0.0022(15)$ & $0.0212(15)$ & $0.0097(13)$ \\
\hline C43 & $0.084(3)$ & 0.0358 (19) & $0.0299(17)$ & -0.0007 (19) & 0.0270 (19) & $0.0067(14)$ \\
\hline C44 & $0.091(3)$ & $0.0269(17)$ & $0.049(2)$ & $0.0002(18)$ & $0.050(2)$ & $0.0044(15)$ \\
\hline $\mathrm{C} 45$ & $0.060(2)$ & $0.0287(17)$ & $0.068(2)$ & $0.0074(16)$ & $0.043(2)$ & 0.0098 (17) \\
\hline $\mathrm{C} 46$ & $0.0448(19)$ & $0.0250(15)$ & $0.0474(19)$ & $0.0051(14)$ & $0.0252(16)$ & $0.0058(14)$ \\
\hline $\mathrm{C} 47$ & $0.064(3)$ & $0.062(3)$ & $0.0316(18)$ & $0.008(2)$ & 0.0095 (18) & $0.0164(18)$ \\
\hline $\mathrm{C} 48$ & $0.147(6)$ & $0.047(3)$ & $0.066(3)$ & $0.018(3)$ & $0.070(4)$ & $0.002(2)$ \\
\hline C49 & $0.048(2)$ & $0.050(2)$ & $0.063(3)$ & 0.0204 (19) & 0.0114 (19) & $0.009(2)$ \\
\hline $\operatorname{Ir} 1$ & $0.01556(6)$ & $0.01830(9)$ & $0.01506(6)$ & $0.00312(6)$ & 0.00533 & $0.00823(6)$ \\
\hline F1A & $0.062(2)$ & $0.066(3)$ & $0.266(8)$ & $0.045(2)$ & $0.090(4)$ & $0.104(4)$ \\
\hline $\mathrm{F} 2 \mathrm{~A}$ & $0.284(10)$ & $0.056(3)$ & $0.090(3)$ & $0.062(4)$ & $0.078(4)$ & $0.049(3)$ \\
\hline F3A & $0.063(2)$ & $0.0151(13)$ & $0.137(4)$ & $-0.0029(13)$ & $0.015(2)$ & $0.0082(18)$ \\
\hline F4A & $0.060(5)$ & $0.264(16)$ & $0.072(4)$ & $0.096(8)$ & $0.028(4)$ & $0.043(6)$ \\
\hline F5A & $0.032(2)$ & $0.120(7)$ & $0.089(5)$ & $0.015(4)$ & $0.008(3)$ & $0.085(5)$ \\
\hline F6A & $0.036(3)$ & $0.084(5)$ & $0.213(12)$ & -0.031 & $-0.036(6)$ & $0.091(7)$ \\
\hline F1B & $0.062(2)$ & $0.066(3)$ & $0.266(8)$ & $0.045(2)$ & $0.090(4)$ & $0.104(4)$ \\
\hline F2B & $0.284(10)$ & $0.056(3)$ & $0.090(3)$ & $0.062(4)$ & $0.078(4)$ & $0.049(3)$ \\
\hline F3B & $0.063(2)$ & $0.0151(13)$ & $0.137(4)$ & $-0.0029(13)$ & $0.015(2)$ & $0.0082(18)$ \\
\hline F4B & $0.057(7)$ & $0.237(18)$ & $0.038(4)$ & $0.070(9)$ & $-0.022(3)$ & $-0.030(6)$ \\
\hline F5B & $0.033(4)$ & $0.100(8)$ & $0.087(7)$ & $0.013(5)$ & $0.034(5)$ & $0.058(6)$ \\
\hline F6B & $0.021(3)$ & $0.089(6)$ & 0.229 (19) & $0.009(4)$ & $0.016(7)$ & $0.111(9)$ \\
\hline $\mathrm{C} 50$ & $0.061(3)$ & $0.066(3)$ & $0.072(3)$ & $0.014(2)$ & $0.033(2)$ & $0.036(2)$ \\
\hline C51 & $0.058(3)$ & $0.087(4)$ & $0.077(3)$ & $0.027(3)$ & $0.044(3)$ & $0.038(3)$ \\
\hline C52 & $0.054(3)$ & $0.116(5)$ & $0.073(3)$ & $0.012(3)$ & $0.025(2)$ & $0.059(3)$ \\
\hline C53 & $0.047(2)$ & $0.075(3)$ & $0.082(3)$ & $0.006(2)$ & $0.001(2)$ & $0.040(3)$ \\
\hline C54 & $0.041(2)$ & $0.076(3)$ & $0.060(3)$ & $0.011(2)$ & $0.007(2)$ & $0.006(2)$ \\
\hline C55 & $0.045(2)$ & $0.079(3)$ & $0.056(3)$ & $0.002(2)$ & $0.027(2)$ & $0.009(2)$ \\
\hline $\mathrm{Cl} 2$ & $0.097(2)$ & $0.105(2)$ & $0.105(2)$ & $0.0543(17)$ & 0.0757 (18) & $0.0701(18)$ \\
\hline C11 & 0.0867 (18) & $0.0660(15)$ & $0.106(2)$ & $0.0037(13)$ & $0.0439(16)$ & $0.0529(15)$ \\
\hline $\mathrm{Cl} 3$ & $0.068(5)$ & $0.070(5)$ & $0.051(4)$ & $0.019(4)$ & $0.025(4)$ & 0.008 (4) \\
\hline C59 & $0.120(10)$ & $0.084(8)$ & $0.046(5)$ & $0.034(7)$ & $0.056(6)$ & $0.031(5)$ \\
\hline C61 & $0.120(10)$ & $0.084(8)$ & $0.046(5)$ & $0.034(7)$ & $0.056(6)$ & $0.031(5)$ \\
\hline $\operatorname{Ir} 2$ & $0.01556(6)$ & $0.01830(9)$ & $0.01506(6)$ & $0.00312(6)$ & $0.00533(4)$ & $0.00823(6)$ \\
\hline
\end{tabular}

Geometric parameters $\left(A,{ }^{o}\right)$

\begin{tabular}{llll}
\hline $\mathrm{O} 1-\mathrm{C} 1$ & $1.273(3)$ & $\mathrm{C} 33-\mathrm{C} 38$ & $1.523(5)$ \\
$\mathrm{O} 1-\mathrm{Ir} 1$ & $2.1757(18)$ & $\mathrm{C} 34-\mathrm{H} 34$ & 0.9500 \\
$\mathrm{~N} 1-\mathrm{C} 1$ & $1.312(3)$ & $\mathrm{C} 34-\mathrm{C} 35$ & $1.386(5)$ \\
$\mathrm{N} 1-\mathrm{C} 24$ & $1.431(3)$ & $\mathrm{C} 35-\mathrm{C} 36$ & $1.380(5)$ \\
$\mathrm{N} 1-\mathrm{Ir} 1{ }^{\mathrm{i}}$ & $2.182(2)$ & $\mathrm{C} 35-\mathrm{C} 39$ & $1.505(4)$ \\
$\mathrm{N} 2-\mathrm{C} 8$ & $1.357(3)$ & $\mathrm{C} 36-\mathrm{H} 36$ & 0.9500 \\
$\mathrm{~N} 2-\mathrm{C} 12$ & $1.346(3)$ & $\mathrm{C} 36-\mathrm{C} 37$ & $1.404(4)$ \\
$\mathrm{N} 2-\mathrm{Ir} 1$ & $2.027(2)$ & $\mathrm{C} 37-\mathrm{C} 40$ & $1.500(5)$
\end{tabular}




\begin{tabular}{|c|c|c|c|}
\hline $\mathrm{N} 3-\mathrm{C} 19$ & $1.371(3)$ & $\mathrm{C} 38-\mathrm{H} 38 \mathrm{~A}$ & 0.9799 \\
\hline $\mathrm{N} 3-\mathrm{C} 23$ & $1.351(3)$ & $\mathrm{C} 38-\mathrm{H} 38 \mathrm{~B}$ & 0.9801 \\
\hline N3-Ir1 & $2.039(2)$ & $\mathrm{C} 38-\mathrm{H} 38 \mathrm{C}$ & 0.9802 \\
\hline $\mathrm{C} 1-\mathrm{C} 1^{\mathrm{i}}$ & $1.521(5)$ & $\mathrm{C} 39-\mathrm{H} 39 \mathrm{~A}$ & 0.9803 \\
\hline $\mathrm{C} 2-\mathrm{C} 3$ & $1.390(4)$ & C39-H39B & 0.9811 \\
\hline $\mathrm{C} 2-\mathrm{C} 7$ & $1.417(4)$ & $\mathrm{C} 39-\mathrm{H} 39 \mathrm{C}$ & 0.9798 \\
\hline $\mathrm{C} 2-\mathrm{Ir} 1$ & $1.993(3)$ & $\mathrm{C} 40-\mathrm{H} 40 \mathrm{~A}$ & 0.9807 \\
\hline $\mathrm{C} 3-\mathrm{H} 3$ & 0.9500 & $\mathrm{C} 40-\mathrm{H} 40 \mathrm{~B}$ & 0.9797 \\
\hline $\mathrm{C} 3-\mathrm{C} 4$ & $1.390(4)$ & $\mathrm{C} 40-\mathrm{H} 40 \mathrm{C}$ & 0.9795 \\
\hline $\mathrm{C} 4-\mathrm{H} 4$ & 0.9500 & $\mathrm{C} 41-\mathrm{C} 42$ & $1.401(5)$ \\
\hline $\mathrm{C} 4-\mathrm{C} 5$ & $1.382(5)$ & $\mathrm{C} 41-\mathrm{C} 46$ & $1.386(5)$ \\
\hline $\mathrm{C} 5-\mathrm{H} 5$ & 0.9500 & $\mathrm{C} 42-\mathrm{C} 43$ & $1.390(5)$ \\
\hline $\mathrm{C} 5-\mathrm{C} 6$ & $1.390(4)$ & $\mathrm{C} 42-\mathrm{C} 47$ & $1.513(6)$ \\
\hline $\mathrm{C} 6-\mathrm{H} 6$ & 0.9500 & $\mathrm{C} 43-\mathrm{H} 43$ & 0.9500 \\
\hline $\mathrm{C} 6-\mathrm{C} 7$ & $1.394(4)$ & $\mathrm{C} 43-\mathrm{C} 44$ & $1.379(7)$ \\
\hline $\mathrm{C} 7-\mathrm{C} 8$ & $1.463(4)$ & $\mathrm{C} 44-\mathrm{C} 45$ & $1.368(6)$ \\
\hline $\mathrm{C} 8-\mathrm{C} 9$ & $1.395(4)$ & $\mathrm{C} 44-\mathrm{C} 48$ & $1.519(5)$ \\
\hline C9-H9 & 0.9500 & $\mathrm{C} 45-\mathrm{H} 45$ & 0.9500 \\
\hline $\mathrm{C} 9-\mathrm{C} 10$ & $1.390(4)$ & $\mathrm{C} 45-\mathrm{C} 46$ & $1.405(5)$ \\
\hline $\mathrm{C} 10-\mathrm{C} 11$ & $1.392(4)$ & $\mathrm{C} 46-\mathrm{C} 49$ & $1.493(6)$ \\
\hline $\mathrm{C} 10-\mathrm{C} 32$ & $1.493(4)$ & $\mathrm{C} 47-\mathrm{H} 47 \mathrm{~A}$ & 0.9800 \\
\hline C11-H11 & 0.9500 & $\mathrm{C} 47-\mathrm{H} 47 \mathrm{~B}$ & 0.9800 \\
\hline $\mathrm{C} 11-\mathrm{C} 12$ & $1.374(4)$ & $\mathrm{C} 47-\mathrm{H} 47 \mathrm{C}$ & 0.9800 \\
\hline $\mathrm{C} 12-\mathrm{H} 12$ & 0.9500 & $\mathrm{C} 48-\mathrm{H} 48 \mathrm{~A}$ & 0.9800 \\
\hline $\mathrm{C} 13-\mathrm{C} 14$ & $1.403(4)$ & C48-H48B & 0.9800 \\
\hline $\mathrm{C} 13-\mathrm{C} 18$ & $1.406(4)$ & $\mathrm{C} 48-\mathrm{H} 48 \mathrm{C}$ & 0.9800 \\
\hline $\mathrm{C} 13-\operatorname{Ir} 1$ & $2.004(3)$ & $\mathrm{C} 48-\mathrm{H} 48 \mathrm{D}$ & 0.9800 \\
\hline $\mathrm{C} 14-\mathrm{H} 14$ & 0.9500 & $\mathrm{C} 48-\mathrm{H} 48 \mathrm{E}$ & 0.9800 \\
\hline $\mathrm{C} 14-\mathrm{C} 15$ & $1.391(4)$ & $\mathrm{C} 48-\mathrm{H} 48 \mathrm{~F}$ & 0.9800 \\
\hline C15-H15 & 0.9500 & $\mathrm{C} 49-\mathrm{H} 49 \mathrm{~A}$ & 0.9800 \\
\hline $\mathrm{C} 15-\mathrm{C} 16$ & $1.380(5)$ & $\mathrm{C} 49-\mathrm{H} 49 \mathrm{~B}$ & 0.9800 \\
\hline $\mathrm{C} 16-\mathrm{H} 16$ & 0.9500 & $\mathrm{C} 49-\mathrm{H} 49 \mathrm{C}$ & 0.9800 \\
\hline $\mathrm{C} 16-\mathrm{C} 17$ & $1.383(4)$ & $\mathrm{F} 1 \mathrm{~A}-\mathrm{C} 30 \mathrm{~A}$ & 1.307 (6) \\
\hline C17- $\mathrm{H} 17$ & 0.9500 & $\mathrm{~F} 2 \mathrm{~A}-\mathrm{C} 30 \mathrm{~A}$ & $1.290(6)$ \\
\hline $\mathrm{C} 17-\mathrm{C} 18$ & $1.400(4)$ & $\mathrm{F} 3 \mathrm{~A}-\mathrm{C} 30 \mathrm{~A}$ & $1.325(6)$ \\
\hline $\mathrm{C} 18-\mathrm{C} 19$ & $1.460(4)$ & $\mathrm{F} 1 \mathrm{~B}-\mathrm{C} 30 \mathrm{~B}$ & $1.326(14)$ \\
\hline $\mathrm{C} 19-\mathrm{C} 20$ & $1.390(4)$ & $\mathrm{F} 2 \mathrm{~B}-\mathrm{C} 30 \mathrm{~B}$ & $1.283(14)$ \\
\hline $\mathrm{C} 20-\mathrm{H} 20$ & 0.9500 & $\mathrm{~F} 3 \mathrm{~B}-\mathrm{C} 30 \mathrm{~B}$ & $1.338(14)$ \\
\hline $\mathrm{C} 20-\mathrm{C} 21$ & $1.388(4)$ & $\mathrm{C} 50-\mathrm{H} 50$ & 0.9500 \\
\hline $\mathrm{C} 21-\mathrm{C} 22$ & $1.390(4)$ & $\mathrm{C} 50-\mathrm{C} 51$ & $1.375(6)$ \\
\hline $\mathrm{C} 21-\mathrm{C} 41$ & $1.491(4)$ & $\mathrm{C} 50-\mathrm{C} 55$ & $1.337(6)$ \\
\hline $\mathrm{C} 22-\mathrm{H} 22$ & 0.9500 & $\mathrm{C} 50-\mathrm{Cl1}$ & $1.743(5)$ \\
\hline $\mathrm{C} 22-\mathrm{C} 23$ & $1.377(4)$ & C51-H51 & 0.9500 \\
\hline $\mathrm{C} 23-\mathrm{H} 23$ & 0.9500 & $\mathrm{C} 51-\mathrm{C} 52$ & $1.358(7)$ \\
\hline $\mathrm{C} 24-\mathrm{C} 25$ & $1.399(4)$ & C52-H52 & 0.9500 \\
\hline $\mathrm{C} 24-\mathrm{C} 29$ & $1.390(4)$ & $\mathrm{C} 52-\mathrm{C} 53$ & $1.379(8)$ \\
\hline $\mathrm{C} 25-\mathrm{H} 25$ & 0.9500 & $\mathrm{C} 52-\mathrm{Cl} 2$ & $1.717(5)$ \\
\hline $\mathrm{C} 25-\mathrm{C} 26$ & $1.386(4)$ & C53-H53 & 0.9500 \\
\hline
\end{tabular}


$\mathrm{C} 26-\mathrm{C} 27$
$\mathrm{C} 26-\mathrm{C} 30 A$
$\mathrm{C} 26-\mathrm{C} 30$
$\mathrm{C} 27-\mathrm{H} 27$
$\mathrm{C} 27-\mathrm{C} 28$
$\mathrm{C} 28-\mathrm{C} 29$
$\mathrm{C} 28-\mathrm{C} 31$
$\mathrm{C} 29-\mathrm{H} 29$
$\mathrm{C} 31-\mathrm{F} 4 \mathrm{~A}$
$\mathrm{C} 31-\mathrm{F} 5 \mathrm{~A}$
$\mathrm{C} 31-\mathrm{F} 6 \mathrm{~A}$
$\mathrm{C} 31-\mathrm{F} 4 \mathrm{~B}$
$\mathrm{C} 31-\mathrm{F} 5 \mathrm{~B}$
$\mathrm{C} 31-\mathrm{F} 6 \mathrm{~B}$
$\mathrm{C} 32-\mathrm{C} 33$
$\mathrm{C} 32-\mathrm{C} 37$
$\mathrm{C} 33-\mathrm{C} 34$

C1-O1-Ir1

$\mathrm{C} 1-\mathrm{N} 1-\mathrm{C} 24$

$\mathrm{C} 1-\mathrm{N} 1-\mathrm{Ir} 1^{\mathrm{i}}$

$\mathrm{C} 24-\mathrm{N} 1-\mathrm{Ir} 1^{\mathrm{i}}$

C8-N2-Ir1

$\mathrm{C} 12-\mathrm{N} 2-\mathrm{C} 8$

C12-N2-Ir1

C19-N3-Ir1

$\mathrm{C} 23-\mathrm{N} 3-\mathrm{C} 19$

C23-N3- Ir 1

$\mathrm{O} 1-\mathrm{C} 1-\mathrm{N} 1$

$\mathrm{O} 1-\mathrm{C} 1-\mathrm{C}^{\mathrm{i}}$

$\mathrm{N} 1-\mathrm{C} 1-\mathrm{C} 1^{\mathrm{i}}$

$\mathrm{C} 3-\mathrm{C} 2-\mathrm{C} 7$

$\mathrm{C} 3-\mathrm{C} 2-\mathrm{Ir} 1$

$\mathrm{C} 7-\mathrm{C} 2-\mathrm{Ir} 1$

$\mathrm{C} 2-\mathrm{C} 3-\mathrm{H} 3$

$\mathrm{C} 4-\mathrm{C} 3-\mathrm{C} 2$

$\mathrm{C} 4-\mathrm{C} 3-\mathrm{H} 3$

$\mathrm{C} 3-\mathrm{C} 4-\mathrm{H} 4$

$\mathrm{C} 5-\mathrm{C} 4-\mathrm{C} 3$

$\mathrm{C} 5-\mathrm{C} 4-\mathrm{H} 4$

$\mathrm{C} 4-\mathrm{C} 5-\mathrm{H} 5$

$\mathrm{C} 4-\mathrm{C} 5-\mathrm{C} 6$

$\mathrm{C} 6-\mathrm{C} 5-\mathrm{H} 5$

$\mathrm{C} 5-\mathrm{C} 6-\mathrm{H} 6$

$\mathrm{C} 5-\mathrm{C} 6-\mathrm{C} 7$

$\mathrm{C} 7-\mathrm{C} 6-\mathrm{H} 6$

$\mathrm{C} 2-\mathrm{C} 7-\mathrm{C} 8$

$\mathrm{C} 6-\mathrm{C} 7-\mathrm{C} 2$
$1.384(4)$

$1.493(7)$

$1.50(2)$

0.9500

$1.383(5)$

$1.393(4)$

1.495 (4)

0.9500

$1.305(7)$

$1.261(6)$

$1.303(6)$

$1.310(8)$

$1.280(7)$

$1.321(8)$

$1.398(4)$

1.389 (4)

1.395 (4)

$114.42(15)$

116.9 (2)

$114.86(16)$

$127.88(16)$

116.55 (17)

119.4 (2)

$123.55(19)$

115.17 (17)

118.8 (2)

125.44 (18)

126.8 (2)

118.3 (3)

114.9 (3)

117.1 (2)

128.4 (2)

114.49 (18)

119.3

121.4 (3)

119.3

119.6

120.9 (3)

119.6

120.3

119.3 (3)

120.3

120.1

119.9 (3)

120.1

114.7 (2)

121.3 (2)
$\mathrm{C} 53-\mathrm{C} 54$

C54-H54

C54-C55

C55-H55

$\mathrm{C} 13-\mathrm{C} 56$

C56-C57

C56-C58

C57-H57

C57-C $58^{\mathrm{ii}}$

C58-H58

C59-C60

$\mathrm{C} 59-\mathrm{C} 62^{\mathrm{ii}}$

C60-C61

$\mathrm{C} 60-\mathrm{C} 62^{\mathrm{ii}}$

$\mathrm{C} 61-\mathrm{C} 62^{\mathrm{ii}}$

C61-C62

$\mathrm{C} 36-\mathrm{C} 37-\mathrm{C} 40$

C33-C $38-\mathrm{H} 38 \mathrm{~A}$

C33-C $38-\mathrm{H} 38 \mathrm{~B}$

C33-C $38-\mathrm{H} 38 \mathrm{C}$

$\mathrm{H} 38 \mathrm{~A}-\mathrm{C} 38-\mathrm{H} 38 \mathrm{~B}$

$\mathrm{H} 38 \mathrm{~A}-\mathrm{C} 38-\mathrm{H} 38 \mathrm{C}$

$\mathrm{H} 38 \mathrm{~B}-\mathrm{C} 38-\mathrm{H} 38 \mathrm{C}$

C35-C39-H39A

C $35-\mathrm{C} 39-\mathrm{H} 39 \mathrm{~B}$

C35-C $39-\mathrm{H} 39 \mathrm{C}$

H39A-C39-H39B

$\mathrm{H} 39 \mathrm{~A}-\mathrm{C} 39-\mathrm{H} 39 \mathrm{C}$

$\mathrm{H} 39 \mathrm{~B}-\mathrm{C} 39-\mathrm{H} 39 \mathrm{C}$

C37- $\mathrm{C} 40-\mathrm{H} 40 \mathrm{~A}$

C37- $\mathrm{C} 40-\mathrm{H} 40 \mathrm{~B}$

C37- $\mathrm{C} 40-\mathrm{H} 40 \mathrm{C}$

$\mathrm{H} 40 \mathrm{~A}-\mathrm{C} 40-\mathrm{H} 40 \mathrm{~B}$

$\mathrm{H} 40 \mathrm{~A}-\mathrm{C} 40-\mathrm{H} 40 \mathrm{C}$

$\mathrm{H} 40 \mathrm{~B}-\mathrm{C} 40-\mathrm{H} 40 \mathrm{C}$

$\mathrm{C} 42-\mathrm{C} 41-\mathrm{C} 21$

$\mathrm{C} 46-\mathrm{C} 41-\mathrm{C} 21$

$\mathrm{C} 46-\mathrm{C} 41-\mathrm{C} 42$

$\mathrm{C} 41-\mathrm{C} 42-\mathrm{C} 47$

$\mathrm{C} 43-\mathrm{C} 42-\mathrm{C} 41$

$\mathrm{C} 43-\mathrm{C} 42-\mathrm{C} 47$

$\mathrm{C} 42-\mathrm{C} 43-\mathrm{H} 43$

$\mathrm{C} 44-\mathrm{C} 43-\mathrm{C} 42$

$\mathrm{C} 44-\mathrm{C} 43-\mathrm{H} 43$

$\mathrm{C} 43-\mathrm{C} 44-\mathrm{C} 48$

$\mathrm{C} 45-\mathrm{C} 44-\mathrm{C} 43$
$1.361(7)$

0.9500

$1.343(7)$

0.9500

1.613 (14)

$1.349(16)$

1.368 (15)

0.9500

1.295 (15)

0.9503

1.423 (17)

1.49 (3)

$1.456(16)$

$1.36(3)$

1.509 (16)

1.509 (16)

120.1 (3)

109.5

109.4

109.5

109.5

109.5

109.4

109.5

109.5

109.6

109.4

109.5

109.4

109.5

109.4

109.5

109.5

109.4

109.5

119.0 (3)

120.1 (3)

120.8 (3)

121.2 (3)

118.9 (4)

119.8 (4)

119.4

121.1 (4)

119.4

$120.6(5)$

$119.1(3)$ 


\begin{tabular}{|c|c|}
\hline $\mathrm{C} 6-\mathrm{C} 7-\mathrm{C} 8$ & $124.0(2)$ \\
\hline $\mathrm{N} 2-\mathrm{C} 8-\mathrm{C} 7$ & $113.3(2)$ \\
\hline $\mathrm{N} 2-\mathrm{C} 8-\mathrm{C} 9$ & $119.8(2)$ \\
\hline $\mathrm{C} 9-\mathrm{C} 8-\mathrm{C} 7$ & $126.5(2)$ \\
\hline $\mathrm{C} 8-\mathrm{C} 9-\mathrm{H} 9$ & 119.5 \\
\hline $\mathrm{C} 10-\mathrm{C} 9-\mathrm{C} 8$ & $121.1(3)$ \\
\hline $\mathrm{C} 10-\mathrm{C} 9-\mathrm{H} 9$ & 119.5 \\
\hline $\mathrm{C} 9-\mathrm{C} 10-\mathrm{C} 11$ & $117.2(2)$ \\
\hline $\mathrm{C} 9-\mathrm{C} 10-\mathrm{C} 32$ & $120.9(2)$ \\
\hline $\mathrm{C} 11-\mathrm{C} 10-\mathrm{C} 32$ & $121.6(3)$ \\
\hline $\mathrm{C} 10-\mathrm{C} 11-\mathrm{H} 11$ & 120.1 \\
\hline $\mathrm{C} 12-\mathrm{C} 11-\mathrm{C} 10$ & $119.9(3)$ \\
\hline $\mathrm{C} 12-\mathrm{C} 11-\mathrm{H} 11$ & 120.1 \\
\hline $\mathrm{N} 2-\mathrm{C} 12-\mathrm{C} 11$ & $122.4(3)$ \\
\hline $\mathrm{N} 2-\mathrm{C} 12-\mathrm{H} 12$ & 118.8 \\
\hline $\mathrm{C} 11-\mathrm{C} 12-\mathrm{H} 12$ & 118.8 \\
\hline $\mathrm{C} 14-\mathrm{C} 13-\mathrm{C} 18$ & $117.2(2)$ \\
\hline $\mathrm{C} 14-\mathrm{C} 13-\mathrm{Ir} 1$ & $128.5(2)$ \\
\hline $\mathrm{C} 18-\mathrm{C} 13-\mathrm{Ir} 1$ & $114.28(18$ \\
\hline $\mathrm{C} 13-\mathrm{C} 14-\mathrm{H} 14$ & 119.6 \\
\hline $\mathrm{C} 15-\mathrm{C} 14-\mathrm{C} 13$ & $120.7(3)$ \\
\hline C15-C14-H14 & 119.6 \\
\hline $\mathrm{C} 14-\mathrm{C} 15-\mathrm{H} 15$ & 119.5 \\
\hline $\mathrm{C} 16-\mathrm{C} 15-\mathrm{C} 14$ & $121.0(3)$ \\
\hline $\mathrm{C} 16-\mathrm{C} 15-\mathrm{H} 15$ & 119.5 \\
\hline $\mathrm{C} 15-\mathrm{C} 16-\mathrm{H} 16$ & 120.1 \\
\hline $\mathrm{C} 15-\mathrm{C} 16-\mathrm{C} 17$ & $119.8(3)$ \\
\hline $\mathrm{C} 17-\mathrm{C} 16-\mathrm{H} 16$ & 120.1 \\
\hline $\mathrm{C} 16-\mathrm{C} 17-\mathrm{H} 17$ & 120.3 \\
\hline $\mathrm{C} 16-\mathrm{C} 17-\mathrm{C} 18$ & $119.5(3)$ \\
\hline $\mathrm{C} 18-\mathrm{C} 17-\mathrm{H} 17$ & 120.3 \\
\hline $\mathrm{C} 13-\mathrm{C} 18-\mathrm{C} 19$ & $115.2(2)$ \\
\hline $\mathrm{C} 17-\mathrm{C} 18-\mathrm{C} 13$ & $121.7(3)$ \\
\hline $\mathrm{C} 17-\mathrm{C} 18-\mathrm{C} 19$ & $123.1(3)$ \\
\hline $\mathrm{N} 3-\mathrm{C} 19-\mathrm{C} 18$ & $113.7(2)$ \\
\hline $\mathrm{N} 3-\mathrm{C} 19-\mathrm{C} 20$ & $119.8(2)$ \\
\hline $\mathrm{C} 20-\mathrm{C} 19-\mathrm{C} 18$ & $126.5(3)$ \\
\hline $\mathrm{C} 19-\mathrm{C} 20-\mathrm{H} 20$ & 119.3 \\
\hline $\mathrm{C} 21-\mathrm{C} 20-\mathrm{C} 19$ & $121.4(3)$ \\
\hline $\mathrm{C} 21-\mathrm{C} 20-\mathrm{H} 20$ & 119.3 \\
\hline $\mathrm{C} 20-\mathrm{C} 21-\mathrm{C} 22$ & $117.6(3)$ \\
\hline $\mathrm{C} 20-\mathrm{C} 21-\mathrm{C} 41$ & $121.4(3)$ \\
\hline $\mathrm{C} 22-\mathrm{C} 21-\mathrm{C} 41$ & $121.0(2)$ \\
\hline $\mathrm{C} 21-\mathrm{C} 22-\mathrm{H} 22$ & 120.2 \\
\hline $\mathrm{C} 23-\mathrm{C} 22-\mathrm{C} 21$ & $119.5(3)$ \\
\hline $\mathrm{C} 23-\mathrm{C} 22-\mathrm{H} 22$ & 120.2 \\
\hline $\mathrm{N} 3-\mathrm{C} 23-\mathrm{C} 22$ & 122.8 \\
\hline & \\
\hline
\end{tabular}

$\begin{array}{ll}\text { C45-C44-C48 } & 120.3(5) \\ \text { C44-C45-H45 } & 119.0 \\ \text { C44-C45-C46 } & 122.0(4) \\ \text { C46-C45-H45 } & 119.0 \\ \text { C41-C46-C45 } & 117.9(4) \\ \text { C41-C46-C49 } & 121.5(3) \\ \text { C45-C46-C49 } & 120.6(4) \\ \text { C42-C47-H47A } & 109.5 \\ \text { C42-C47-H47B } & 109.5 \\ \text { C42-C47-H47C } & 109.5 \\ \text { H47A-C47-H47B } & 109.5 \\ \text { H47A-C47-H47C } & 109.5 \\ \text { H47B-C47-H47C } & 109.5 \\ \text { C44-C48-H48A } & 109.5 \\ \text { C44-C48-H48B } & 109.5 \\ \text { C44-C48-H48C } & 109.5 \\ \text { H48A-C48-H48B } & 109.5 \\ \text { H48A-C48-H48C } & 109.5 \\ \text { H48B-C48-H48C } & 109.5 \\ \text { H48D-C48-H48E } & 109.5 \\ \text { H48D-C48-H48F } & 109.5 \\ \text { H48E-C48-H48F } & 109.5 \\ \text { C46-C49-H49A } & 109.5 \\ \text { C46-C49-H49B } & 109.5 \\ \text { C46-C49-H49C } & 109.5 \\ \text { H49A-C49-H49B } & 109.5 \\ \text { H49A-C49-H49C } & 109.5 \\ \text { H49B-C49-H49C } & 109.5 \\ \text { O1-Ir1-N1 } & 75.81(7) \\ \text { N2-Ir1-O1 } & 96.15(8) \\ \text { N2-Ir1-N1 } & 91.38(8) \\ \text { N2-Ir1-N3 } & 174.60(9) \\ \text { N3-Ir1-O1 } & 82.90(8) \\ \text { N3-Ir1-N1 } & 93.54(8) \\ \text { C2-Ir1-O1 } & 176.58(9) \\ \text { C2-Ir1-N1 } & 103.47(9) \\ \text { C2-Ir1-N2 } & 80.51(10) \\ \text { C2-Ir1-N3 } & 100.49(10) \\ \text { C2-Ir1-C13 } & 87.62(10) \\ \text { C13-Ir1-O1 } & 93.36(9) \\ \text { C13-Ir1-N1 } & 168.24(9) \\ \text { C13-Ir1-N2 } & 94.38(10) \\ \text { C13-Ir1-N3 } & 80.38(10) \\ \text { F1A-C30A-C26 } & 111.9(4) \\ \text { F1A-C30A-F3A } & 105.4(5) \\ \text { F2A-C30A-C26 } & 113.6(5) \\ \text { F2A-C30A-F1A } & \\ & \end{array}$




\begin{tabular}{|c|c|}
\hline $\mathrm{C} 22-\mathrm{C} 23-\mathrm{H} 23$ & 118.6 \\
\hline $\mathrm{C} 25-\mathrm{C} 24-\mathrm{N} 1$ & $122.2(2)$ \\
\hline $\mathrm{C} 29-\mathrm{C} 24-\mathrm{N} 1$ & $118.8(2)$ \\
\hline $\mathrm{C} 29-\mathrm{C} 24-\mathrm{C} 25$ & $119.0(2)$ \\
\hline $\mathrm{C} 24-\mathrm{C} 25-\mathrm{H} 25$ & 120.0 \\
\hline $\mathrm{C} 26-\mathrm{C} 25-\mathrm{C} 24$ & $120.0(3)$ \\
\hline $\mathrm{C} 26-\mathrm{C} 25-\mathrm{H} 25$ & 120.0 \\
\hline $\mathrm{C} 25-\mathrm{C} 26-\mathrm{C} 30 \mathrm{~A}$ & $119.6(3)$ \\
\hline $\mathrm{C} 25-\mathrm{C} 26-\mathrm{C} 30 \mathrm{~B}$ & $116.7(7)$ \\
\hline $\mathrm{C} 27-\mathrm{C} 26-\mathrm{C} 25$ & $121.2(3)$ \\
\hline $\mathrm{C} 27-\mathrm{C} 26-\mathrm{C} 30 \mathrm{~A}$ & $119.0(3)$ \\
\hline $\mathrm{C} 27-\mathrm{C} 26-\mathrm{C} 30 \mathrm{~B}$ & $121.6(7)$ \\
\hline $\mathrm{C} 26-\mathrm{C} 27-\mathrm{H} 27$ & 120.6 \\
\hline $\mathrm{C} 28-\mathrm{C} 27-\mathrm{C} 26$ & $118.8(3)$ \\
\hline $\mathrm{C} 28-\mathrm{C} 27-\mathrm{H} 27$ & 120.6 \\
\hline $\mathrm{C} 27-\mathrm{C} 28-\mathrm{C} 29$ & $120.9(3)$ \\
\hline $\mathrm{C} 27-\mathrm{C} 28-\mathrm{C} 31$ & $120.0(3)$ \\
\hline $\mathrm{C} 29-\mathrm{C} 28-\mathrm{C} 31$ & $119.0(3)$ \\
\hline $\mathrm{C} 24-\mathrm{C} 29-\mathrm{C} 28$ & $120.1(3)$ \\
\hline $\mathrm{C} 24-\mathrm{C} 29-\mathrm{H} 29$ & 119.9 \\
\hline $\mathrm{C} 28-\mathrm{C} 29-\mathrm{H} 29$ & 119.9 \\
\hline $\mathrm{F} 4 \mathrm{~A}-\mathrm{C} 31-\mathrm{C} 28$ & $110.4(4)$ \\
\hline $\mathrm{F} 5 \mathrm{~A}-\mathrm{C} 31-\mathrm{C} 28$ & $114.4(4)$ \\
\hline $\mathrm{F} 5 \mathrm{~A}-\mathrm{C} 31-\mathrm{F} 4 \mathrm{~A}$ & $108.2(6)$ \\
\hline $\mathrm{F} 5 \mathrm{~A}-\mathrm{C} 31-\mathrm{F} 6 \mathrm{~A}$ & $107.1(6)$ \\
\hline $\mathrm{F} 6 \mathrm{~A}-\mathrm{C} 31-\mathrm{C} 28$ & $112.5(4)$ \\
\hline $\mathrm{F} 6 \mathrm{~A}-\mathrm{C} 31-\mathrm{F} 4 \mathrm{~A}$ & $103.6(7)$ \\
\hline $\mathrm{F} 4 \mathrm{~B}-\mathrm{C} 31-\mathrm{C} 28$ & $111.8(5)$ \\
\hline $\mathrm{F} 4 \mathrm{~B}-\mathrm{C} 31-\mathrm{F} 6 \mathrm{~B}$ & $109.3(10)$ \\
\hline $\mathrm{F} 5 \mathrm{~B}-\mathrm{C} 31-\mathrm{C} 28$ & $114.8(5)$ \\
\hline $\mathrm{F} 5 \mathrm{~B}-\mathrm{C} 31-\mathrm{F} 4 \mathrm{~B}$ & $102.3(8)$ \\
\hline $\mathrm{F} 5 \mathrm{~B}-\mathrm{C} 31-\mathrm{F} 6 \mathrm{~B}$ & $103.3(8)$ \\
\hline $\mathrm{F} 6 \mathrm{~B}-\mathrm{C} 31-\mathrm{C} 28$ & $114.3(4)$ \\
\hline $\mathrm{C} 33-\mathrm{C} 32-\mathrm{C} 10$ & $118.5(3)$ \\
\hline $\mathrm{C} 37-\mathrm{C} 32-\mathrm{C} 10$ & $121.2(3)$ \\
\hline $\mathrm{C} 37-\mathrm{C} 32-\mathrm{C} 33$ & 120.3 \\
\hline $\mathrm{C} 32-\mathrm{C} 33-\mathrm{C} 38$ & $121.0(3)$ \\
\hline $\mathrm{C} 34-\mathrm{C} 33-\mathrm{C} 32$ & $119.4(3)$ \\
\hline $\mathrm{C} 34-\mathrm{C} 33-\mathrm{C} 38$ & $119.6(3)$ \\
\hline $\mathrm{C} 33-\mathrm{C} 34-\mathrm{H} 34$ & 119.4 \\
\hline $\mathrm{C} 35-\mathrm{C} 34-\mathrm{C} 33$ & $121.2(3)$ \\
\hline $\mathrm{C} 35-\mathrm{C} 34-\mathrm{H} 34$ & 119.4 \\
\hline $\mathrm{C} 34-\mathrm{C} 35-\mathrm{C} 39$ & $119.8(3)$ \\
\hline $\mathrm{C} 36-\mathrm{C} 35-\mathrm{C} 34$ & $118.6(3)$ \\
\hline $\mathrm{C} 36-\mathrm{C} 35-\mathrm{C} 39$ & $121.7(3)$ \\
\hline $\mathrm{C} 35-\mathrm{C} 36-\mathrm{H} 36$ & 119.1 \\
\hline $\mathrm{C} 35-\mathrm{C} 36-\mathrm{C} 37$ & $121.8(3)$ \\
\hline & \\
\hline
\end{tabular}

$\mathrm{F} 3 \mathrm{~A}-\mathrm{C} 30 \mathrm{~A}-\mathrm{C} 26$

$\mathrm{F} 1 \mathrm{~B}-\mathrm{C} 30 \mathrm{~B}-\mathrm{C} 26$

$\mathrm{F} 1 \mathrm{~B}-\mathrm{C} 30 \mathrm{~B}-\mathrm{F} 3 \mathrm{~B}$

$\mathrm{F} 2 \mathrm{~B}-\mathrm{C} 30 \mathrm{~B}-\mathrm{C} 26$

$\mathrm{F} 2 \mathrm{~B}-\mathrm{C} 30 \mathrm{~B}-\mathrm{F} 1 \mathrm{~B}$

$\mathrm{F} 2 \mathrm{~B}-\mathrm{C} 30 \mathrm{~B}-\mathrm{F} 3 \mathrm{~B}$

$\mathrm{F} 3 \mathrm{~B}-\mathrm{C} 30 \mathrm{~B}-\mathrm{C} 26$

$\mathrm{C} 51-\mathrm{C} 50-\mathrm{H} 50$

$\mathrm{C} 51-\mathrm{C} 50-\mathrm{Cl} 1$

$\mathrm{C} 55-\mathrm{C} 50-\mathrm{H} 50$

$\mathrm{C} 55-\mathrm{C} 50-\mathrm{C} 51$

$\mathrm{C} 55-\mathrm{C} 50-\mathrm{Cl} 1$

$\mathrm{C} 50-\mathrm{C} 51-\mathrm{H} 51$

$\mathrm{C} 52-\mathrm{C} 51-\mathrm{C} 50$

$\mathrm{C} 52-\mathrm{C} 51-\mathrm{H} 51$

$\mathrm{C} 51-\mathrm{C} 52-\mathrm{H} 52$

$\mathrm{C} 51-\mathrm{C} 52-\mathrm{C} 53$

$\mathrm{C} 51-\mathrm{C} 52-\mathrm{Cl} 2$

C53-C52-H52

$\mathrm{C} 53-\mathrm{C} 52-\mathrm{Cl} 2$

$\mathrm{C} 52-\mathrm{C} 53-\mathrm{H} 53$

C $54-\mathrm{C} 53-\mathrm{C} 52$

$\mathrm{C} 54-\mathrm{C} 53-\mathrm{H} 53$

C53-C54-H54

C55-C54-C53

$\mathrm{C} 55-\mathrm{C} 54-\mathrm{H} 54$

$\mathrm{C} 50-\mathrm{C} 55-\mathrm{C} 54$

$\mathrm{C} 50-\mathrm{C} 55-\mathrm{H} 55$

$\mathrm{C} 54-\mathrm{C} 55-\mathrm{H} 55$

$\mathrm{C} 57-\mathrm{C} 56-\mathrm{Cl} 3$

C57-C56-C58

$\mathrm{C} 58-\mathrm{C} 56-\mathrm{Cl} 3$

C56-C57-H57

C58 ii-C57-C56

$\mathrm{C} 58^{\mathrm{ii}}-\mathrm{C} 57-\mathrm{H} 57$

C56-C58-H58

$\mathrm{C} 57^{\mathrm{ii}}-\mathrm{C} 58-\mathrm{C} 56$

$\mathrm{C} 57^{\mathrm{ii}}-\mathrm{C} 58-\mathrm{H} 58$

$\mathrm{C} 60-\mathrm{C} 59-\mathrm{C} 62^{\mathrm{ii}}$

C59-C60-C61

$\mathrm{C} 62^{\mathrm{ii}}-\mathrm{C} 60-\mathrm{C} 59$

C62 $2^{\mathrm{ii}}-\mathrm{C} 60-\mathrm{C} 61$

$\mathrm{C} 60^{\mathrm{ii}}-\mathrm{C} 61-\mathrm{C} 60$

C60 1 - C61-C62

$\mathrm{C} 60^{\mathrm{ii}}-\mathrm{C} 61-\mathrm{C} 62$

$\mathrm{C} 60-\mathrm{C} 61-\mathrm{C} 62^{\mathrm{ii}}$

C60- $661-\mathrm{C} 62$

C62 $-\mathrm{ii} 61-\mathrm{C} 62$
$112.6(4)$

107.8 (13)

88.7 (15)

119.4 (14)

124 (2)

101.8 (18)

109.4 (14)

118.8

$123.1(4)$

118.8

$122.3(5)$

$114.6(4)$

121.4

$117.2(5)$

121.4

119.5

$121.0(5)$

126.3 (4)

119.5

111.9 (4)

120.4

$119.2(5)$

120.4

119.8

120.4 (5)

119.8

119.9 (5)

120.1

120.1

120.9 (13)

122.1 (13)

116.9 (13)

123.9

112.0 (15)

124.1

115.9

125.9 (15)

118.2

55.4 (14)

$129.6(19)$

64.9 (15)

64.8 (12)

180.0

125.5 (14)

54.5 (14)

54.5 (14)

125.5 (14)

180.0 


\begin{tabular}{|c|c|}
\hline $\mathrm{C} 32-\mathrm{C} 37-\mathrm{C} 36$ & $118.7(3)$ \\
\hline $\mathrm{C} 32-\mathrm{C} 37-\mathrm{C} 40$ & $121.1(3)$ \\
\hline $\mathrm{N} 1-\mathrm{C} 24-\mathrm{C} 25-\mathrm{C} 26$ & $177.7(3)$ \\
\hline $\mathrm{N} 1-\mathrm{C} 24-\mathrm{C} 29-\mathrm{C} 28$ & $-177.4(2)$ \\
\hline $\mathrm{N} 2-\mathrm{C} 8-\mathrm{C} 9-\mathrm{C} 10$ & $1.1(4)$ \\
\hline $\mathrm{N} 3-\mathrm{C} 19-\mathrm{C} 20-\mathrm{C} 21$ & $-0.2(4)$ \\
\hline $\mathrm{C} 1-\mathrm{N} 1-\mathrm{C} 24-\mathrm{C} 25$ & $-44.8(3)$ \\
\hline $\mathrm{C} 1-\mathrm{N} 1-\mathrm{C} 24-\mathrm{C} 29$ & $134.4(2)$ \\
\hline $\mathrm{C} 2-\mathrm{C} 3-\mathrm{C} 4-\mathrm{C} 5$ & $0.6(5)$ \\
\hline $\mathrm{C} 2-\mathrm{C} 7-\mathrm{C} 8-\mathrm{N} 2$ & $-7.7(3)$ \\
\hline $\mathrm{C} 2-\mathrm{C} 7-\mathrm{C} 8-\mathrm{C} 9$ & $165.6(3)$ \\
\hline $\mathrm{C} 3-\mathrm{C} 2-\mathrm{C} 7-\mathrm{C} 6$ & $4.6(4)$ \\
\hline $\mathrm{C} 3-\mathrm{C} 2-\mathrm{C} 7-\mathrm{C} 8$ & $-172.7(2)$ \\
\hline $\mathrm{C} 3-\mathrm{C} 4-\mathrm{C} 5-\mathrm{C} 6$ & $1.9(5)$ \\
\hline $\mathrm{C} 4-\mathrm{C} 5-\mathrm{C} 6-\mathrm{C} 7$ & $-1.0(5)$ \\
\hline $\mathrm{C} 5-\mathrm{C} 6-\mathrm{C} 7-\mathrm{C} 2$ & $-2.2(5)$ \\
\hline $\mathrm{C} 5-\mathrm{C} 6-\mathrm{C} 7-\mathrm{C} 8$ & $174.8(3)$ \\
\hline $\mathrm{C} 6-\mathrm{C} 7-\mathrm{C} 8-\mathrm{N} 2$ & $175.1(3)$ \\
\hline $\mathrm{C} 6-\mathrm{C} 7-\mathrm{C} 8-\mathrm{C} 9$ & $-11.6(5)$ \\
\hline $\mathrm{C} 7-\mathrm{C} 2-\mathrm{C} 3-\mathrm{C} 4$ & $-3.7(4)$ \\
\hline $\mathrm{C} 7-\mathrm{C} 8-\mathrm{C} 9-\mathrm{C} 10$ & $-171.8(3)$ \\
\hline $\mathrm{C} 8-\mathrm{N} 2-\mathrm{C} 12-\mathrm{C} 11$ & $-4.1(4)$ \\
\hline $\mathrm{C} 8-\mathrm{C} 9-\mathrm{C} 10-\mathrm{C} 11$ & $-4.9(4)$ \\
\hline $\mathrm{C} 8-\mathrm{C} 9-\mathrm{C} 10-\mathrm{C} 32$ & $169.6(3)$ \\
\hline $\mathrm{C} 9-\mathrm{C} 10-\mathrm{C} 11-\mathrm{C} 12$ & $4.3(4)$ \\
\hline $\mathrm{C} 9-\mathrm{C} 10-\mathrm{C} 32-\mathrm{C} 33$ & $-92.3(4)$ \\
\hline $\mathrm{C} 9-\mathrm{C} 10-\mathrm{C} 32-\mathrm{C} 37$ & $88.4(4)$ \\
\hline $\mathrm{C} 10-\mathrm{C} 11-\mathrm{C} 12-\mathrm{N} 2$ & $0.1(5)$ \\
\hline $\mathrm{C} 10-\mathrm{C} 32-\mathrm{C} 33-\mathrm{C} 34$ & $179.5(3)$ \\
\hline $\mathrm{C} 10-\mathrm{C} 32-\mathrm{C} 33-\mathrm{C} 38$ & $0.2(4)$ \\
\hline $\mathrm{C} 10-\mathrm{C} 32-\mathrm{C} 37-\mathrm{C} 36$ & $-179.6(3)$ \\
\hline $\mathrm{C} 10-\mathrm{C} 32-\mathrm{C} 37-\mathrm{C} 40$ & $3.3(5)$ \\
\hline $\mathrm{C} 11-\mathrm{C} 10-\mathrm{C} 32-\mathrm{C} 33$ & $82.0(4)$ \\
\hline $\mathrm{C} 11-\mathrm{C} 10-\mathrm{C} 32-\mathrm{C} 37$ & $-97.2(4)$ \\
\hline $\mathrm{C} 12-\mathrm{N} 2-\mathrm{C} 8-\mathrm{C} 7$ & $177.3(2)$ \\
\hline $\mathrm{C} 12-\mathrm{N} 2-\mathrm{C} 8-\mathrm{C} 9$ & $3.5(4)$ \\
\hline $\mathrm{C} 13-\mathrm{C} 14-\mathrm{C} 15-\mathrm{C} 16$ & $-0.1(5)$ \\
\hline $\mathrm{C} 13-\mathrm{C} 18-\mathrm{C} 19-\mathrm{N} 3$ & $2.4(3)$ \\
\hline $\mathrm{C} 13-\mathrm{C} 18-\mathrm{C} 19-\mathrm{C} 20$ & $-177.6(3)$ \\
\hline $\mathrm{C} 14-\mathrm{C} 13-\mathrm{C} 18-\mathrm{C} 17$ & $2.6(4)$ \\
\hline $\mathrm{C} 14-\mathrm{C} 13-\mathrm{C} 18-\mathrm{C} 19$ & $-175.8(2)$ \\
\hline $\mathrm{C} 14-\mathrm{C} 15-\mathrm{C} 16-\mathrm{C} 17$ & $1.4(5)$ \\
\hline $\mathrm{C} 15-\mathrm{C} 16-\mathrm{C} 17-\mathrm{C} 18$ & $-0.7(5)$ \\
\hline $\mathrm{C} 16-\mathrm{C} 17-\mathrm{C} 18-\mathrm{C} 13$ & $-1.3(5)$ \\
\hline $\mathrm{C} 16-\mathrm{C} 17-\mathrm{C} 18-\mathrm{C} 19$ & $176.9(3)$ \\
\hline $\mathrm{C} 17-\mathrm{C} 18-\mathrm{C} 19-\mathrm{N} 3$ & $-175.9(3)$ \\
\hline $\mathrm{C} 17-\mathrm{C} 18-\mathrm{C} 19-\mathrm{C} 20$ & $4.1(5)$ \\
\hline
\end{tabular}

$$
\mathrm{C} 59^{\mathrm{ii}}-\mathrm{C} 62-\mathrm{C} 61
$$$$
\text { C60 } 1 \text { ii } 62-\mathrm{C} 61
$$$$
\mathrm{C} 27-\mathrm{C} 28-\mathrm{C} 29-\mathrm{C} 24
$$$$
\mathrm{C} 27-\mathrm{C} 28-\mathrm{C} 31-\mathrm{F} 4 \mathrm{~A}
$$$$
\mathrm{C} 27-\mathrm{C} 28-\mathrm{C} 31-\mathrm{F} 5 \mathrm{~A}
$$$$
\mathrm{C} 27-\mathrm{C} 28-\mathrm{C} 31-\mathrm{F} 6 \mathrm{~A}
$$$$
\mathrm{C} 27-\mathrm{C} 28-\mathrm{C} 31-\mathrm{F} 4 \mathrm{~B}
$$$$
\mathrm{C} 27-\mathrm{C} 28-\mathrm{C} 31-\mathrm{F} 5 \mathrm{~B}
$$$$
\text { C27-C28-C } 31-\mathrm{F} 6 \mathrm{~B}
$$$$
\mathrm{C} 29-\mathrm{C} 24-\mathrm{C} 25-\mathrm{C} 26
$$$$
\mathrm{C} 29-\mathrm{C} 28-\mathrm{C} 31-\mathrm{F} 4 \mathrm{~A}
$$$$
\mathrm{C} 29-\mathrm{C} 28-\mathrm{C} 31-\mathrm{F} 5 \mathrm{~A}
$$$$
\text { C29-C28-C31-F6A }
$$$$
\text { C29-C28-C31-F4B }
$$$$
\mathrm{C} 29-\mathrm{C} 28-\mathrm{C} 31-\mathrm{F} 5 \mathrm{~B}
$$$$
\mathrm{C} 29-\mathrm{C} 28-\mathrm{C} 31-\mathrm{F} 6 \mathrm{~B}
$$$$
\mathrm{C} 31-\mathrm{C} 28-\mathrm{C} 29-\mathrm{C} 24
$$$$
\mathrm{C} 32-\mathrm{C} 10-\mathrm{C} 11-\mathrm{C} 12
$$$$
\mathrm{C} 32-\mathrm{C} 33-\mathrm{C} 34-\mathrm{C} 35
$$$$
\mathrm{C} 33-\mathrm{C} 32-\mathrm{C} 37-\mathrm{C} 36
$$$$
\mathrm{C} 33-\mathrm{C} 32-\mathrm{C} 37-\mathrm{C} 40
$$$$
\mathrm{C} 33-\mathrm{C} 34-\mathrm{C} 35-\mathrm{C} 36
$$$$
\mathrm{C} 33-\mathrm{C} 34-\mathrm{C} 35-\mathrm{C} 39
$$$$
\mathrm{C} 34-\mathrm{C} 35-\mathrm{C} 36-\mathrm{C} 37
$$$$
\mathrm{C} 35-\mathrm{C} 36-\mathrm{C} 37-\mathrm{C} 32
$$$$
\mathrm{C} 35-\mathrm{C} 36-\mathrm{C} 37-\mathrm{C} 40
$$$$
\mathrm{C} 37-\mathrm{C} 32-\mathrm{C} 33-\mathrm{C} 34
$$$$
\mathrm{C} 37-\mathrm{C} 32-\mathrm{C} 33-\mathrm{C} 38
$$$$
\text { C38-C33-C34-C35 }
$$$$
\mathrm{C} 39-\mathrm{C} 35-\mathrm{C} 36-\mathrm{C} 37
$$$$
\mathrm{C} 41-\mathrm{C} 21-\mathrm{C} 22-\mathrm{C} 23
$$$$
\mathrm{C} 41-\mathrm{C} 42-\mathrm{C} 43-\mathrm{C} 44
$$$$
\mathrm{C} 42-\mathrm{C} 41-\mathrm{C} 46-\mathrm{C} 45
$$$$
\mathrm{C} 42-\mathrm{C} 41-\mathrm{C} 46-\mathrm{C} 49
$$$$
\mathrm{C} 42-\mathrm{C} 43-\mathrm{C} 44-\mathrm{C} 45
$$$$
\mathrm{C} 42-\mathrm{C} 43-\mathrm{C} 44-\mathrm{C} 48
$$$$
\mathrm{C} 43-\mathrm{C} 44-\mathrm{C} 45-\mathrm{C} 46
$$$$
\text { C44-C45-C46-C41 }
$$$$
\mathrm{C} 44-\mathrm{C} 45-\mathrm{C} 46-\mathrm{C} 49
$$$$
\mathrm{C} 46-\mathrm{C} 41-\mathrm{C} 42-\mathrm{C} 43
$$$$
\mathrm{C} 46-\mathrm{C} 41-\mathrm{C} 42-\mathrm{C} 47
$$$$
\mathrm{C} 47-\mathrm{C} 42-\mathrm{C} 43-\mathrm{C} 44
$$$$
\mathrm{C} 48-\mathrm{C} 44-\mathrm{C} 45-\mathrm{C} 46
$$$$
\mathrm{Ir} 1-\mathrm{O} 1-\mathrm{C} 1-\mathrm{N} 1
$$$$
\mathrm{Ir} 1-\mathrm{O} 1-\mathrm{C} 1-\mathrm{Cl}^{\mathrm{i}}
$$$$
\mathrm{Ir} 1-\mathrm{N} 1-\mathrm{C} 1-\mathrm{O} 1
$$$$
\mathrm{Ir} 1^{\mathrm{i}}-\mathrm{N} 1-\mathrm{C} 1-\mathrm{C}^{\mathrm{i}}
$$

$120.4(18)$

$60.8(10)$

$-0.5(4)$

$106.7(9)$

$-131.0(6)$

$-8.5(8)$

$-59.8(12)$

$56.2(8)$

$175.3(11)$

$-1.5(4)$

$-72.2(9)$

$50.1(7)$

$172.6(8)$

$121.3(12)$

$-122.7(7)$

$-3.6(11)$

$178.4(3)$

$-170.2(3)$

$0.7(5)$

$1.2(5)$

$-175.9(3)$

$-0.1(5)$

$-179.9(3)$

$0.0(5)$

$-0.6(5)$

$176.5(3)$

-1.2 (4)

179.5 (3)

179.9 (3)

179.9 (3)

$177.2(3)$

-1.1 (5)

1.0 (5)

$-179.8(3)$

0.7 (6)

180.0 (4)

$0.5(6)$

$-1.4(5)$

179.4 (4)

$0.2(5)$

178.6 (3)

-179.5 (4)

-178.7 (4)

$171.2(2)$

-11.4 (3)

$169.4(2)$

-8.1 (3) 


$\begin{array}{ll}\mathrm{C} 18-\mathrm{C} 13-\mathrm{C} 14-\mathrm{C} 15 & -1.9(4) \\ \mathrm{C} 18-\mathrm{C} 19-\mathrm{C} 20-\mathrm{C} 21 & 179.8(3) \\ \mathrm{C} 19-\mathrm{N} 3-\mathrm{C} 23-\mathrm{C} 22 & 2.0(4) \\ \mathrm{C} 19-\mathrm{C} 20-\mathrm{C} 21-\mathrm{C} 22 & 1.9(4) \\ \mathrm{C} 19-\mathrm{C} 20-\mathrm{C} 21-\mathrm{C} 41 & -177.0(3) \\ \mathrm{C} 20-\mathrm{C} 21-\mathrm{C} 22-\mathrm{C} 23 & -1.7(4) \\ \mathrm{C} 20-\mathrm{C} 21-\mathrm{C} 41-\mathrm{C} 42 & 97.9(4) \\ \mathrm{C} 20-\mathrm{C} 21-\mathrm{C} 41-\mathrm{C} 46 & -81.9(4) \\ \mathrm{C} 21-\mathrm{C} 22-\mathrm{C} 23-\mathrm{N} 3 & -0.3(4) \\ \mathrm{C} 21-\mathrm{C} 41-\mathrm{C} 42-\mathrm{C} 43 & -179.7(3) \\ \mathrm{C} 21-\mathrm{C} 41-\mathrm{C} 42-\mathrm{C} 47 & -1.3(5) \\ \mathrm{C} 21-\mathrm{C} 41-\mathrm{C} 46-\mathrm{C} 45 & -179.1(3) \\ \mathrm{C} 21-\mathrm{C} 41-\mathrm{C} 46-\mathrm{C} 49 & 0.1(5) \\ \mathrm{C} 22-\mathrm{C} 21-\mathrm{C} 41-\mathrm{C} 42 & -81.0(4) \\ \mathrm{C} 22-\mathrm{C} 21-\mathrm{C} 41-\mathrm{C} 46 & 99.2(4) \\ \mathrm{C} 23-\mathrm{N} 3-\mathrm{C} 19-\mathrm{C} 18 & 178.2(2) \\ \mathrm{C} 23-\mathrm{N} 3-\mathrm{C} 19-\mathrm{C} 20 & -1.8(4) \\ \mathrm{C} 24-\mathrm{N} 1-\mathrm{C} 1-\mathrm{O} 1 & -4.4(4) \\ \mathrm{C} 24-\mathrm{N} 1-\mathrm{C} 1-\mathrm{C} 1 \mathrm{i} & 178.1(2) \\ \mathrm{C} 24-\mathrm{C} 25-\mathrm{C} 26-\mathrm{C} 27 & -0.2(5) \\ \mathrm{C} 24-\mathrm{C} 25-\mathrm{C} 26-\mathrm{C} 30 \mathrm{~A} & -175.2(3) \\ \mathrm{C} 24-\mathrm{C} 25-\mathrm{C} 26-\mathrm{C} 30 \mathrm{~B} & 171.6(7) \\ \mathrm{C} 25-\mathrm{C} 24-\mathrm{C} 29-\mathrm{C} 28 & 1.9(4) \\ \mathrm{C} 25-\mathrm{C} 26-\mathrm{C} 27-\mathrm{C} 28 & 1.6(5) \\ \mathrm{C} 25-\mathrm{C} 26-\mathrm{C} 30 \mathrm{~A}-\mathrm{F} 1 \mathrm{~A} & -40.4(7) \\ \mathrm{C} 25-\mathrm{C} 26-\mathrm{C} 30 \mathrm{~A}-\mathrm{F} 2 \mathrm{~A} & 82.5(7) \\ \mathrm{C} 25-\mathrm{C} 26-\mathrm{C} 30 \mathrm{~A}-\mathrm{F} 3 \mathrm{~A} & -158.8(5) \\ \mathrm{C} 25-\mathrm{C} 26-\mathrm{C} 30 \mathrm{~B}-\mathrm{F} 1 \mathrm{~B} & 138(2) \\ \mathrm{C} 25-\mathrm{C} 26-\mathrm{C} 30 \mathrm{~B}-\mathrm{F} 2 \mathrm{~B} & -74(2) \\ \mathrm{C} 25-\mathrm{C} 26-\mathrm{C} 30 \mathrm{~B}-\mathrm{F} 3 \mathrm{~B} & 42.8(17) \\ \mathrm{C} 26-\mathrm{C} 27-\mathrm{C} 28-\mathrm{C} 29 & -1.2(5) \\ \mathrm{C} 26-\mathrm{C} 27-\mathrm{C} 28-\mathrm{C} 31 & 179.9(3) \\ \mathrm{C} 27-\mathrm{C} 26-\mathrm{C} 30 \mathrm{~A}-\mathrm{F} 1 \mathrm{~A} & 144.5(6) \\ \mathrm{C} 27-\mathrm{C} 26-\mathrm{C} 30 \mathrm{~A}-\mathrm{F} 2 \mathrm{~A} & -92.7(7) \\ \mathrm{C} 27-\mathrm{C} 26-\mathrm{C} 30 \mathrm{~A}-\mathrm{F} 3 \mathrm{~A} & 26.0(6) \\ \mathrm{C} 27-\mathrm{C} 26-\mathrm{C} 30 \mathrm{~B}-\mathrm{F} 1 \mathrm{~B} & -50(2) \\ \mathrm{C} 27-\mathrm{C} 26-\mathrm{C} 30 \mathrm{~B}-\mathrm{F} 2 \mathrm{~B} & 98.0(19) \\ \mathrm{C} 27-\mathrm{C} 26-\mathrm{C} 30 \mathrm{~B}-\mathrm{F} 3 \mathrm{~B} & -145.4(15) \\ & \end{array}$

\begin{tabular}{|c|c|}
\hline $\mathrm{Ir} 1-\mathrm{N} 1-\mathrm{C} 24-\mathrm{C} 25$ & $142.3(2)$ \\
\hline $\mathrm{Ir} 1-\mathrm{N} 1-\mathrm{C} 24-\mathrm{C} 29$ & $-38.4(3)$ \\
\hline $\mathrm{Ir} 1-\mathrm{N} 2-\mathrm{C} 8-\mathrm{C} 7$ & $5.2(3)$ \\
\hline $\mathrm{Ir} 1-\mathrm{N} 2-\mathrm{C} 8-\mathrm{C} 9$ & $-168.6(2)$ \\
\hline $\mathrm{Ir} 1-\mathrm{N} 2-\mathrm{C} 12-\mathrm{C} 11$ & $167.4(2)$ \\
\hline $\mathrm{Ir} 1-\mathrm{N} 3-\mathrm{C} 19-\mathrm{C} 18$ & $-9.9(3)$ \\
\hline $\mathrm{Ir} 1-\mathrm{N} 3-\mathrm{C} 19-\mathrm{C} 20$ & $170.1(2)$ \\
\hline $\mathrm{Ir} 1-\mathrm{N} 3-\mathrm{C} 23-\mathrm{C} 22$ & $-168.9(2)$ \\
\hline $\mathrm{Ir} 1-\mathrm{C} 2-\mathrm{C} 3-\mathrm{C} 4$ & $176.9(2)$ \\
\hline $\mathrm{Ir} 1-\mathrm{C} 2-\mathrm{C} 7-\mathrm{C} 6$ & $-175.9(2)$ \\
\hline $\mathrm{Ir} 1-\mathrm{C} 2-\mathrm{C} 7-\mathrm{C} 8$ & $6.8(3)$ \\
\hline $\mathrm{Ir} 1-\mathrm{C} 13-\mathrm{C} 14-\mathrm{C} 15$ & $175.7(2)$ \\
\hline $\mathrm{Ir} 1-\mathrm{C} 13-\mathrm{C} 18-\mathrm{C} 17$ & $-175.3(2)$ \\
\hline $\mathrm{Ir} 1-\mathrm{C} 13-\mathrm{C} 18-\mathrm{C} 19$ & $6.3(3)$ \\
\hline $\mathrm{C} 30 \mathrm{~A}-\mathrm{C} 26-\mathrm{C} 27-\mathrm{C} 28$ & $176.6(3)$ \\
\hline $\mathrm{C} 30 \mathrm{~B}-\mathrm{C} 26-\mathrm{C} 27-\mathrm{C} 28$ & $-169.8(8)$ \\
\hline $\mathrm{C} 50-\mathrm{C} 51-\mathrm{C} 52-\mathrm{C} 53$ & $2.9(8)$ \\
\hline $\mathrm{C} 50-\mathrm{C} 51-\mathrm{C} 52-\mathrm{Cl} 2$ & $-166.3(5)$ \\
\hline $\mathrm{C} 51-\mathrm{C} 50-\mathrm{C} 55-\mathrm{C} 54$ & $-2.1(8)$ \\
\hline $\mathrm{C} 51-\mathrm{C} 52-\mathrm{C} 53-\mathrm{C} 54$ & $-2.4(8)$ \\
\hline $\mathrm{C} 52-\mathrm{C} 53-\mathrm{C} 54-\mathrm{C} 55$ & $-0.5(8)$ \\
\hline $\mathrm{C} 53-\mathrm{C} 54-\mathrm{C} 55-\mathrm{C} 50$ & $2.7(8)$ \\
\hline $\mathrm{C} 55-\mathrm{C} 50-\mathrm{C} 51-\mathrm{C} 52$ & $-0.7(8)$ \\
\hline $\mathrm{Cl} 2-\mathrm{C} 52-\mathrm{C} 53-\mathrm{C} 54$ & $168.3(4)$ \\
\hline $\mathrm{C} 11-\mathrm{C} 50-\mathrm{C} 51-\mathrm{C} 52$ & $-179.4(4)$ \\
\hline $\mathrm{C} 11-\mathrm{C} 50-\mathrm{C} 55-\mathrm{C} 54$ & $176.7(4)$ \\
\hline $\mathrm{Cl} 3-\mathrm{C} 56-\mathrm{C} 57-\mathrm{C} 58^{\mathrm{ii}}$ & $177.4(15)$ \\
\hline $\mathrm{Cl} 3-\mathrm{C} 56-\mathrm{C} 58-\mathrm{C} 57^{\mathrm{ii}}$ & $-177.5(16)$ \\
\hline $\mathrm{C} 57-\mathrm{C} 56-\mathrm{C} 58-\mathrm{C} 57^{\mathrm{ii}}$ & $0(3)$ \\
\hline $\mathrm{C} 58-\mathrm{C} 56-\mathrm{C} 57-\mathrm{C} 58^{\mathrm{ii}}$ & $0(3)$ \\
\hline $\mathrm{C} 59-\mathrm{C} 60-\mathrm{C} 61-\mathrm{C} 62$ & $-177(2)$ \\
\hline $\mathrm{C} 59-\mathrm{C} 60-\mathrm{C} 61-\mathrm{C} 62^{\mathrm{ii}}$ & $3(2)$ \\
\hline $\mathrm{C} 60-\mathrm{C} 61-\mathrm{C} 62-\mathrm{C} 59^{\mathrm{ii}}$ & $-177.3(18)$ \\
\hline $\mathrm{C} 60^{\mathrm{ii}}-\mathrm{C} 61-\mathrm{C} 62-\mathrm{C} 59^{\mathrm{ii}}$ & $2.7(18)$ \\
\hline $\mathrm{C} 60-\mathrm{C} 61-\mathrm{C} 62-\mathrm{C} 60^{\mathrm{ii}}$ & 180.0 \\
\hline $\mathrm{C} 62^{\mathrm{ii}}-\mathrm{C} 59-\mathrm{C} 60-\mathrm{C} 61$ & $-3(2)$ \\
\hline $\mathrm{C} 62^{\mathrm{ii}}-\mathrm{C} 60-\mathrm{C} 61-\mathrm{C} 62$ & 180.0 \\
\hline
\end{tabular}

Symmetry codes: (i) $-x+1,-y+1,-z+1$; (ii) $-x,-y,-z$. 